Mesures des excitations par diffusion inélastique de neutrons 
Collection SFN 10 (2010) 159-336

(C) Owned by the authors, published by EDP Sciences, 2010

DOI: $10.1051 / \mathrm{sfn} / 2010003$

\title{
Diffusion des neutrons par la matière cristalline ou amorphe non-magnétique
}

\author{
H. Schober
}

Institut Laue Langevin, 6 rue Jules Horowitz, 38042 Grenoble Cedex 9, France et Université

Joseph Fourier, UFR de Physique, 38041 Grenoble Cedex 9, France

\begin{abstract}
Résumé. Nous présentons une introduction de la diffusion des neutrons par la matière cristalline ou amorphe non-magnétique, en mettant l'accent sur la diffusion inélastique. Le point de départ de notre discussion sera la diffusion d'une particule non-relativiste par un potentiel. Ce cas simple nous permettra d'introduire les notions d'amplitude et de longueur de diffusion, les ondes partielles ainsi que la série de Born. Nous passerons ensuite aux systèmes composés comportant des degrés de liberté internes. L'équation maîtresse établira le lien entre la section efficace de diffusion et les transitions entre les niveaux d'excitation, dans le cadre de l'approximation de Born. Cette équation peut être formulée en terme de fonctions de diffusion cohérentes et incohérentes. Pour illustrer les effets d'interférences, qui constituent la base de la diffusion des neutrons, nous effectuerons un petit détour par la diffraction optique. C'est le potentiel de Fermi qui décrit l'interaction des neutrons thermiques et froids avec les noyaux des atomes. Ce potentiel étant basé sur l'opérateur de densité du système il est possible d'exprimer les résultats de la mesure à l'aide des fonctions de corrélation de la densité. Le formalisme théorique sera ensuite confronté à l'expérience. Nous finirons ce chapitre en montrant comment les fonctions de corrélation de la densité peuvent être calculées pour des systèmes harmoniques en s'appuyant sur le concept des phonons. Nous présenterons les expressions qui relient les mesures de diffusion inélastique des neutrons à la densité d'états de phonons et aux courbes de dispersion. Nous discuterons les effets des multiphonons sur les spectres et nous concluerons par quelques remarques concernant la caractérisation d'effets anharmoniques.
\end{abstract}

1 Le principe de la diffusion

161

2 La section efficace $\quad 164$

3 Le neutron $r$

4 Diffusion par un potentiel statique $\quad 168$

4.1 Fonction d'onde en régime asymptotique . . . . . . . . . . . . . . . . . . . . . . . . . . . . . . . 169

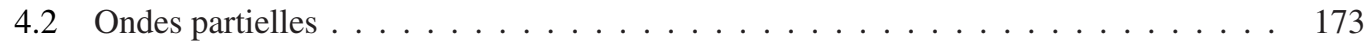

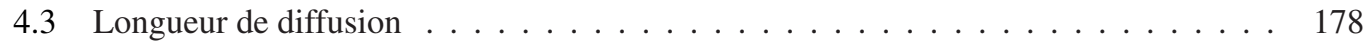

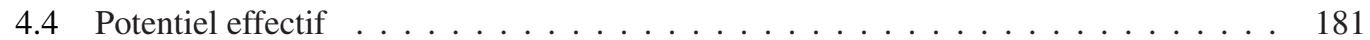

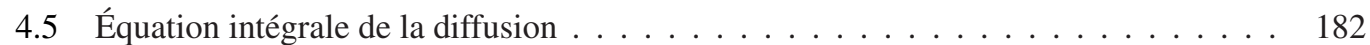

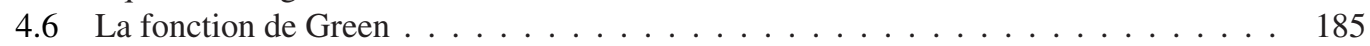

4.7 La série et l'approximation de Born . . . . . . . . . . . . . . . 185

5 Cible composée $\quad 189$

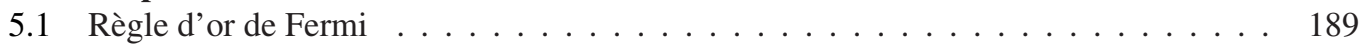

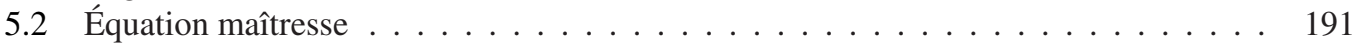

5.3 Facteur de forme du potentiel d'interaction . . . . . . . . . . . . . . . . 192

5.4 Pseudo-potentiel de Fermi . . . . . . . . . . . . . . . . . . . . . . . . . . . . . . 194

This is an Open Access article distributed under the terms of the Creative Commons Attribution-Noncommercial License 3.0, which permits unrestricted use, distribution, and reproduction in any noncommercial medium, provided the original work is properly cited. 
$5.5 \quad$ La fonction de diffusion $S(\vec{Q}, \omega) \ldots \ldots \ldots \ldots \ldots$

5.6 Diffusion cohérente et diffusion incohérente . . . . . . . . . . . . . . . . . . . 197

5.7 La dépendance de la longueur de diffusion du spin nucléaire . . . . . . . . . . 200

5.8 Diffusion d'un neutron par un proton ou un deutérium . . . . . . . . . . . . . . 201

5.9 Section efficace pour des particules libres . . . . . . . . . . . . . . . . 203

5.10 Triangle de diffusion et gamme dynamique . . . . . . . . . . . . . . . . . 204

5.11 Exemple : taux de production pour des neutrons ultra-froids . . . . . . . . . . 206

6 Résumé partiel $\quad 208$

7 Parallèles avec l'optique $\quad 211$

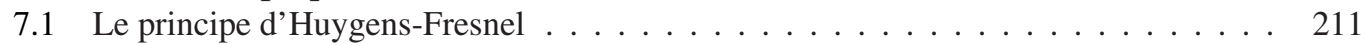

7.2 Diffraction par une fente . . . . . . . . . . . . . . . . . . . . 212

7.3 Diffraction par un ensemble de fentes avec translation aléatoire . . . . . . . . . . 213

7.4 Diffraction par un réseau . . . . . . . . . . . . . . . . . . . . . . . . 214

7.5 Diffraction par un réseau à trois dimensions . . . . . . . . . . . . . . . . . . . 219

7.6 Longueur de cohérence longitudinale . . . . . . . . . . . . . . . . . . 220

7.7 Longueur de cohérence transversale . . . . . . . . . . . . . . . . . . . 221

8 Fonctions de corrélation $\quad \mathbf{2 2 3}$

8.1 La fonction d'auto-corrélation de la densité atomique $G(\vec{r}, t) \ldots \ldots \ldots \ldots 224$

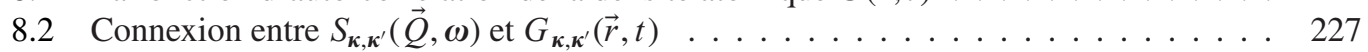

8.3 Relation entre les fonctions de corrélation et la section efficace différentielle partielle 228

8.4 Variation de contraste pour obtenir les fonctions de corrélation partielles . . . . . . 229

8.5 Fonctions de corrélation d'ordre supérieur . . . . . . . . . . . . . . . . . . . 229

8.6 Les fonctions de diffusion intermédiaires $I_{\boldsymbol{\kappa}, \boldsymbol{\kappa}^{\prime}}(\vec{Q}, t)$ et $I_{\boldsymbol{\kappa}}^{s}(\vec{Q}, t) \ldots \ldots \ldots 230$

8.7 Balance détaillée . . . . . . . . . . . . . . . . . . . . . 233

8.8 Fonctions de corrélation symétrisées . . . . . . . . . . . . . . . . . 235

8.9 Comportement asymptotique . . . . . . . . . . . . . . . . . . . 236

8.10 Exemple : ensemble de particules de Boltzmann . . . . . . . . . . . . . . 237

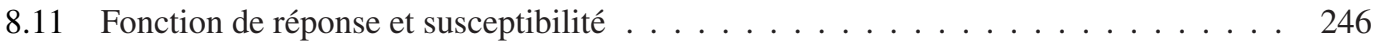

8.12 Moments et règles de somme pour la fonction de diffusion . . . . . . . . . . . . . 249

8.13 Résumé partiel . . . . . . . . . . . . . . . . . . . . . . . . . . . 254

9 Le faisceau de particules 256

9.1 Description statistique du faisceau $\ldots \ldots \ldots \ldots$. . . . . . . . . . . . . . 256

9.2 Éléments optiques et fonction de transfert . . . . . . . . . . . . . . . . . 259

9.3 Échantillons de petite taille . . . . . . . . . . . . . . . . . . 260

9.4 Échantillon de taille finie et diffusion multiple . . . . . . . . . . . . . . 261

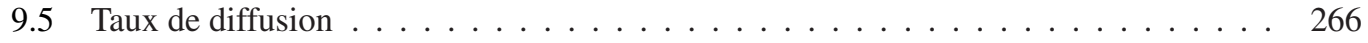

9.6 Résolution . . . . . . . . . . . . . . . . . . . . . . . . . 270

10 Diffusion des neutrons par un système harmonique 272

10.1 Facteur de Debye-Waller . . . . . . . . . . . . . . . . . . . . . . . 274

10.2 Diffusion cohérente élastique . . . . . . . . . . . . . . . . . 276

10.3 Diffusion cohérente à une excitation vibrationnelle . . . . . . . . . . . . . . . . 278

10.4 Règle de somme $f$ pour les vibrations . . . . . . . . . . . . . . . . . . . . . . 282

10.5 Diffusion cohérente à un phonon dans un cristal . . . . . . . . . . . . . . . . . 284

10.6 Diffusion incohérente élastique . . . . . . . . . . . . . . . . . . . . . . 290

10.7 La diffusion diffuse . . . . . . . . . . . . . . . . . . . . . . . . 292 
10.8 Diffusion incohérente à un phonon . . . . . . . . . . . . . . . . . . . . . . . . . . . . . . . . 396

10.9 Approximation incohérente . . . . . . . . . . . . . . . 303

11 La diffusion inélastique au-delà des expressions à un phonon 310

11.1 Expression intégrale pour la fonction de diffusion . . . . . . . . . . . . . . . 311

11.2 L'oscillateur harmonique isotrope . . . . . . . . . . . . . . . . . 311

11.3 Approximation à temps courts ou approximation d'impulsion . . . . . . . . . . . . . . . 314

11.4 Multiphonons dans un monocristal . . . . . . . . . . . . . . . . . . . . . 317

11.5 Calcul itératif pour des systèmes monoatomiques incohérents . . . . . . . . . . 321

11.6 Susceptibilité et anharmonicité . . . . . . . . . . . . . . . . . 328

11.7 Résumé partiel . . . . . . . . . . . . . . . . . . . . 331

Références

\section{LE PRINCIPE DE LA DIFFUSION}

Afin de placer la diffusion des neutrons dans un contexte plus large, et en particulier pour confronter cette technique à celles de la diffusion des électrons, des rayons-X et des atomes d'hélium, nous allons commencer par quelques remarques assez générales sur la diffusion.

Notre conception du monde microscopique est en grande partie issue des expériences de diffusion. C'est particulièrement vrai pour les particules élémentaires : si nous sommes aujourd'hui convaincus que le proton et le neutron possèdent une structure interne, c'est grâce aux expériences de diffusion menées à très haute énergie, comme c'est le cas dans les grands collisionneurs de particules comme le $\mathrm{LHC}_{\mathrm{au}} \mathrm{CERN}^{1}$. Il en est de même à l'échelle atomique, celle de la physique de la matière condensée. En effet, notre compréhension de l'organisation de la matière à cette échelle, ainsi que des mouvements des atomes la constituant, est souvent le fruit d'expériences de diffusion d'ondes électromagnétiques (lumière, rayons-X, rayons- $\gamma$ ) ou de particules massives (électrons, positrons, neutrons, atomes d'hélium).

Evidemment, la manière la plus intuitive de comprendre une structure est en premier lieu de la voir, ce qui est le domaine de la microscopie. Parce qu'elle permet d'aller en deçà de la limite imposée par la diffraction des ondes optiques, la microscopie électronique en transmission était pendant longtemps la technique de prédilection permettant d'étudier directement les structures à l'échelle atomique. Ce domaine s'est maintenant ouvert aux microscopes rayons-X et aux microscopies à champs proches. Ces microscopes font partie d'une classe d'appareils qui travaillent en balayant l'objet d'investigation.

L'invention des microscopes de balayage à effet tunnel ou à force atomique ont radicalement amélioré nos capacités d'analyse mais aussi de manipulation des matériaux à l'échelle atomique. Comparée à ces sondes expérimentales, la diffusion est une technique moins directe parce qu'elle convertit les informations contenues dans l'espace en informations angulaires. Dans les expériences les plus simples, comme celles mettant en jeu des interférences entre deux ondes sphériques, l'information sur la distance entre les sources se trouve "encodée" dans le profil de diffusion. Il faut considérer les positions successives des maxima pour remonter à cette information. En dépit de son caractère indirect la diffusion reste une technique extrêmement utilisée pour les études structurales, en particulier quand il s'agit de sonder le matériau dans son volume et ne pas seulement en surface.

\footnotetext{
${ }^{1}$ http://public.web.cern.ch/Public/fr/LHC/LHC-fr.html
} 
Le principe d'une expérience de diffusion est simple (voir figure 1). Une particule sonde, dont on prépare l'état initial, est envoyée sur un échantillon cible ${ }^{2}$. Dans le cas de la diffusion des neutrons lents, la cible peut changer d'état interne mais reste intacte. En particulier, aucune particule secondaire libre n'est émise ${ }^{3}$. Nous pouvons donc nous limiter à l'étude des particules sondes diffusées. Toute l'information concernant la cible est contenue dans les probabilités de transition entre l'état initial et les états finaux de celle-ci. Quand nous parlerons de diffusion dans ce chapitre, nous nous limiterons toujours à ce cas de figure particulier qui reste bien adapté à l'étude de la matière condensée.

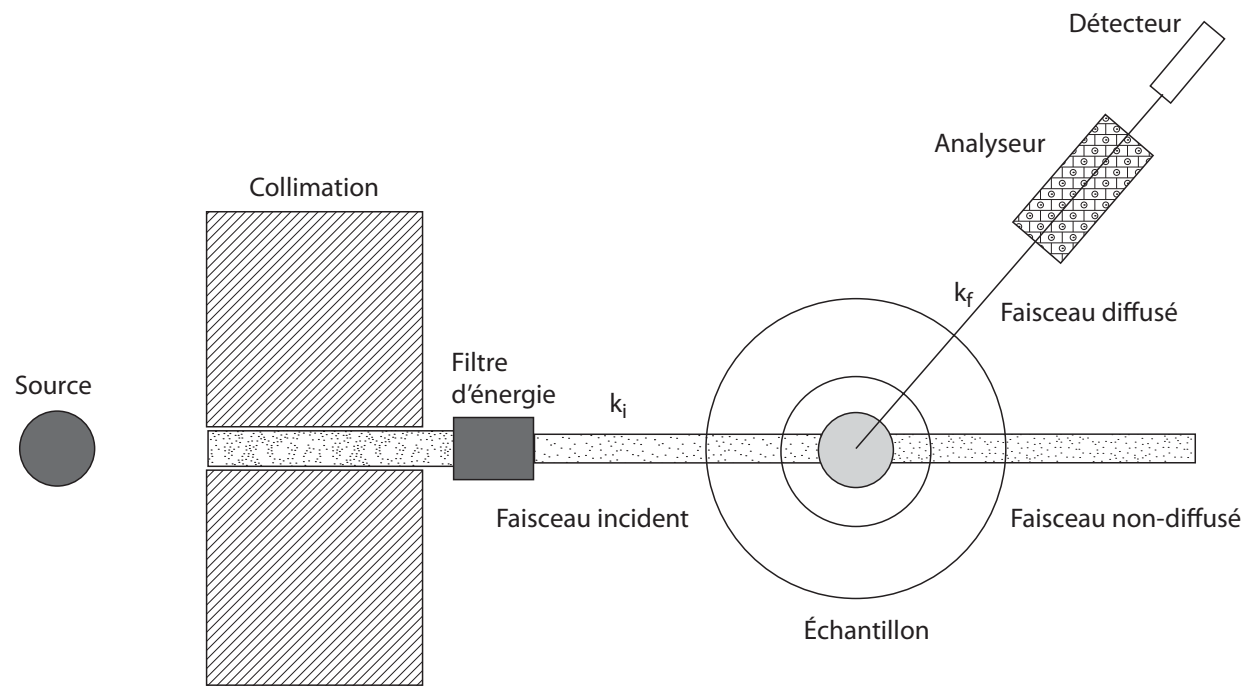

Figure 1. Illustration schématique d'une expérience de diffusion. Une source émet des particules qui passent par un collimateur leur définissant une direction. Un filtre sélectionne une bande d'énergie. Le faisceau monochromatique et collimaté heurte la cible. Celle-ci diffuse les particules dans l'espace; elles sont ensuite analysées (par exemple en fonction de leur énergie et/ou de leur état de spin) et détectées.

La diffusion étant un phénomène d'interférence, elle moyenne sur la partie de la cible illuminée par le faisceau. Ce volume de jauge est grand à l'échelle de la longueur d'onde, contrairement aux informations structurales recherchées qui sont de l'ordre de la longueur d'onde voire de dimension inférieure. C'est la situation inverse de la microscopie, qui elle fonctionne bien en dessus de la limite de diffraction imposée par la longueur d'onde. La diffusion peut donc être associée à la microscopie à balayage. Elle permet de créer du contraste dans des échantillons qui sont homogènes à l'échelle du volume de jauge. C'est donc une microscopie à trois dimensions avec une résolution qui est fixée par le volume illuminé.

\footnotetext{
2 Dans le cas des expériences à très haute énergie il est aussi coutume de faire interagir deux faisceau de particules. Ceci ne change rien au principe.

${ }^{3} \mathrm{La}$ collision de deux particules élémentaires peut aboutir à la création d'une gerbe de particules diffusées à haute énergie. De la même manière la diffusion d'une particule peut être accompagnée par l'émission de particules secondaires. Dans le cas de la diffusion des neutrons thermiques, les énergies mises en jeu sont telles que ce phénomène est pratiquement inexistant. Il ne se produit que dans le cas de l'absorption, qui peut être considerée comme un cas extrême d'une expérience de diffusion. Dans le cas de la diffusion des rayons-X, la sonde peut induire l'émission d'électrons et de photons, à cause de son énergie élevée. Ceci permet une panoplie de méthodes de spectroscopie de rayons-X.
} 
La particule sonde et la nature de son état initial sont choisies soigneusement selon les buts de l'expérience. Ce choix doit satisfaire aux critères non-exhaustifs suivants :

1. La sonde doit interagir de manière suffisamment forte avec la cible pour que les changements d'états soient rendus observables. Les neutrinos, qui traversent sans gêne des kilomètres de matière condensée sont le contre-exemple par excellence d'une particule sonde. En ce qui concerne la matière condensée l'interaction doit se faire avec la partie de la cible qu'on désire étudier: les neutrons interagissent avec les noyaux des atomes ou avec les champs magnétiques. Par conséquent, tout phénomène lié au cortège électronique ne peut être sondé qu'indirectement.

2. L'interaction ne doit pas non plus être trop forte, pour deux raisons. Premièrement l'intensité de l'interaction définit le volume sondé. La sonde doit donc être adaptée au but scientifique : des atomes d'hélium seront diffusés par la surface du matériau, des électrons auront une pénétration limitée, qui dépendra fortement de leur énergie. Les neutrons thermiques constituent des sondes de volume, sauf dans le cas spécifique de la réflectométrie. Deuxièmement une interaction trop forte rend l'interprétation de la diffusion plus complexe. En effet, dans ce cas la diffusion multiple brouille la relation entre les propriétés de l'échantillon et l'état final de la sonde. Une grande partie de ce chapitre sera dédiée à développer un formalisme d'interprétation basé sur la présomption que l'interaction est faible. L'interaction pourra alors être traitée en perturbation.

3. Les particules sondes doivent être diffusées dans leur grande majorité et non pas absorbées. Non seulement l'absorption diminuera le nombre d'évènements de diffusion et affectera ainsi la statistique de la mesure, mais elle pourra aussi -en y "déposant" son énergie cinétique- gravement altérer l'échantillon, voir même aller jusqu'à le détruire. C'est un problème important de la diffusion des rayons-X par la matière molle. Il ne se posera pas pour les neutrons.

4. La longueur d'onde de la particule diffusée définit l'échelle spatiale sur laquelle on pourra sonder la matière. Comme déjà mentionné, la diffusion convertit la mesure d'une distance en mesure d'angle. La longueur d'onde est le paramètre qui régit cette conversion. Dans le cas de la diffusion par les fentes de Young, qui constitue le dispositif interférentiel le plus simple, deux faisceaux de lumière issus d'une même source interfèrent en passant par deux petites fentes percées dans un plan opaque et séparées par une distance $d$. Vu sous l'angle d'une étude structurale, le but est alors de déterminer cette séparation. Le motif d'interférence, qu'on pourra enregistrer sur un écran à une distance $D$ -grande par rapport à $d$ - est donnée par l'expression

$$
I(y) \propto \cos ^{2}\left(\pi \frac{d}{D} \frac{y}{\lambda}\right) .
$$

où $y$ est la distance du point d'observation au centre de l'écran. La distance séparant deux maxima successifs est de

$$
\Delta y=D \frac{\lambda}{d}
$$

correspondant à des angles

$$
\Delta \phi=\frac{\Delta y}{D}=\frac{\lambda}{d}
$$

La distance entre la cible et l'écran sera toujours macroscopique et sera choisie de manière à permettre la mesure de l'angle $\Delta \phi$ avec la précision voulue. Si la longueur d'onde $\lambda$ est beaucoup plus petite que la distance $d$, la déviation angulaire sera minime et risquera d'être indiscernable du faisceau direct (voir la figure 2). Si $\lambda$ augmente, les maxima se déplacent vers des angles plus grands et deviennent observables pour finalement disparaître lorsque $\lambda$ prend des valeurs très supérieures à $d$. La mesure sera donc plus aisée si $\lambda$ est de l'ordre de $d$. Les rayons-X et les neutrons thermiques, avec des longueurs d'onde de quelques $\AA$, sont donc bien adaptés à l'étude de la matière condensée à 
l'échelle atomique. Pour l'étude des particules élémentaires il faudra des longueurs d'onde beaucoup plus courtes et donc des énergies beaucoup plus élevées.

5. L'énergie totale du système composé de la cible et de la sonde est conservée. Le changement de l'énergie de la sonde reflète donc directement le changement d'énergie de la cible. La mesure du changement d'une valeur physique est facile si celui-ci est relativement important, c'est-à-dire s'il est du même ordre de grandeur que la valeur elle-même. Il est ainsi préférable de travailler avec des particules sondes qui ont une énergie comparable à celles des excitations de la matière que l'on veut étudier ${ }^{4}$. Pour la matière condensée nous avons vu dans le chapitre I, que l'échelle d'énergie des excitations du réseau est donnée par la température. Nous avions également constaté que les neutrons modérés peuvent être assimilés à un gaz tenu à la température du modérateur. Comme règle de base nous pouvons retenir que les excitations dans un matériau à température $T$ sont à étudier de préférence avec des neutrons modérés à la même température.

6. Il est en général avantageux de pouvoir faire varier l'intensité de l'interaction entre la sonde et certains atomes de la cible. Ceci permet de marquer le signal provenant de certains constituants de la cible : c'est ce que l'on appelle "la variation de contraste". Dans le cas des neutrons, cette variation s'effectue par substitution isotopique. Dans le cas des rayons-X il est possible de renforcer le signal d'une espèce atomique en choisissant une longueur d'onde proche de son seuil d'absorption.

\section{LA SECTION EFFICACE}

Dans notre définition, la seule observable d'une expérience de diffusion est l'intensité des particules sondes enregistrée par les détecteurs (voir figure 1).

Chaque détecteur est orienté de manière que son aire de détection $\Delta A$ soit placée perpendiculairement au vecteur $\vec{r}=(r, \theta, \phi)$, qui le relie à la cible. Idéalement cette surface est une partie d'une sphère de rayon $r$. Le nombre de particules que le détecteur intercepte ${ }^{5}$ par seconde est donnée par l'expression

$$
I(t)=\Delta A \cdot J_{\mathrm{diff}}(r, \theta, \phi, t)
$$

où $J_{\text {diff }}(r, \theta, \phi, t)$ est le flux (ou courant) de particules diffusées. A grande distance, ce flux est nécessairement radial. Il indique le nombre de particules qui traversent une surface unitaire par unité de temps. Les coordonnées adaptées sont donc les coordonnées sphériques $r, \theta$ et $\phi$.

Loin de la cible, nous pouvons considérer les particules comme libres. Par conséquent, elles sont caractérisées par leur impulsion $\hbar \vec{k}$. Pour un élément de détection donné le vecteur d'onde $\vec{k}_{f}$ des neutrons enregistrés (on les appellera neutrons diffusés) est dirigé parallèlement à $\vec{r}$ (voir figure 3 ). De la même manière le faisceau incident est décrit par un vecteur d'onde $\vec{k}_{i}$. Dans la réalité les faisceaux ne sont jamais idéaux : ils comportent une distribution de vecteur d'onde $p\left(\vec{k}_{i}\right)$ et $p\left(\vec{k}_{f}\right)$ centrés autour des valeurs nominales. Toute l'intelligence d'un instrument bien construit réside dans l'adaptation de ces distributions aux besoin de l'expérience. Si elles sont trop larges elles vont occulter le signal recherché, dans le cas contraire on gaspillera du flux.

\footnotetext{
${ }^{4}$ Il peut exister de bonnes raisons pour ne pas suivre cette règle simple. Ces dernières années la diffusion inélastique des rayons$\mathrm{X}$ a apporté beaucoup de connaissances nouvelles sur les excitations de la matière en dépit du fait que l'énergie des photons utilisés est de quelques dizaines de keV, et donc 6 à 7 ordres de grandeur supérieurs à l'énergie des excitations étudiées. Une des raisons de ce succès tient au fait que les transferts en impulsion, accessibles aux rayons-X, sont plus petits que ceux accessibles aux neutrons. Le domaine dynamique exploré couvre une zone qui n'était pas accessible à l'expérience avant. Ces expériences requièrent néanmoins une préparation extrême du faisceau incident et diffusé en terme de monochromaticité induisant une perte considérable d'intensité. Ce type d'expérience n'est donc faisable qu'auprès des sources de lumière de troisième génération comme l'ESRF.

5 Nous présumons ici que le détecteur est idéal, c'est-à-dire que son efficacité de détection est de 100\%. Dans la réalité les effets dûs à l'efficacité du détecteur doivent être pris en compte.
} 

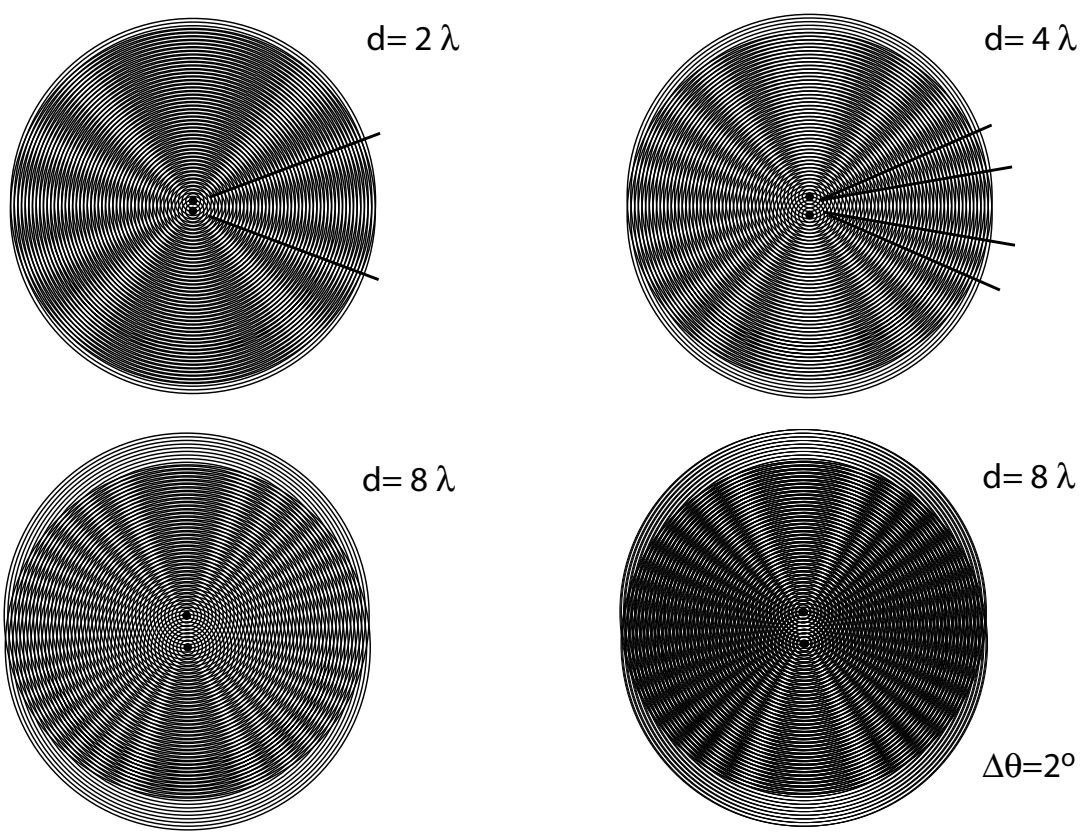

Figure 2. Illustration des motifs d'interférences créés par deux particules (ou fentes) séparées d'une distance $d$ et illuminées par un faisceau perpendiculaire à l'axe joignant ces deux particules. Les deux particules émettent des ondes sphériques en phase. Les maxima correspondent aux cercles tracés autour de ces deux sources de rayonnement. La longueur d'onde $\lambda$ correspond donc à la distance entre les cercles concentriques. Au croisement des cercles, l'interférence sera maximale. On voit que l'angle des premiers maxima augmente avec la longueur d'onde. La détection de ces maxima est aisée quand $\lambda \approx d$. Si $\lambda$ diminue fortement par rapport à $d$ on pourra -en principe- observer un grand nombre de maxima, ce qui rendrait la mesure très précise. Néanmoins le faisceau devra être particulièrement bien préparé dans ce cas. En bas à droite sont représentés les motifs d'interférence superposés pour trois faisceau incidents à 0,2 et -2 degrés. La détection des maxima est pratiquement impossible pour $d=8 \lambda$.

La diffusion correspond donc au transfert d'une particule initialement (c'est-à-dire très en amont de l'impact) dans l'état libre $\vec{k}_{i}$ vers un état final (c'est-à-dire très en aval de l'impact) $\vec{k}_{f}$. La probabilité de cette transition est étroitement liée aux éléments de matrice

$$
S_{\vec{k}_{f}, \vec{k}_{i}}=\left\langle\vec{k}_{f}\left|\mathbf{U}_{I}(+\infty,-\infty)\right| \vec{k}_{i}\right\rangle
$$

de l'opérateur d'évolution $\mathbf{U}_{I}\left(t^{\prime}, t\right)$. Cet opérateur décrit la façon dont un état quantique évolue dans le temps entre $t$ et $t^{\prime}$ et dépend du potentiel d'interaction entre la sonde et la cible. Les limites $t \rightarrow+\infty$ et $t \rightarrow-\infty$ traduisent les notions "initiales" et "finales" dans un scénario idéal. Pour le moment, la relation (2.2) ne fait que transcrire la situation expérimentale en langage mathématique.

Le flux diminuant au fur et à mesure qu'on s'éloigne de la cible, l'intensité enregistrée dans un détecteur de surface donnée diminuera en $1 / r^{2}$. Seule la dépendance du flux diffusé en fonction de la direction, c'est-à-dire au flux détectable dans en élément angulaire $d \Omega=\sin \theta d \theta d \phi$ comporte une information utile. La relation entre l'aire $A(\theta, \phi)$ du détecteur et l'angle solide $\Omega$ formé par le détecteur vu depuis le centre diffuseur est donnée par la relation

$$
\Delta A=r^{2} \Delta \Omega
$$

Concernant la distance $r$ nous supposons que les détecteurs peuvent être placés suffisamment loin de la cible pour être capables de résoudre la direction de diffusion avec la précision désirée. La quantité 


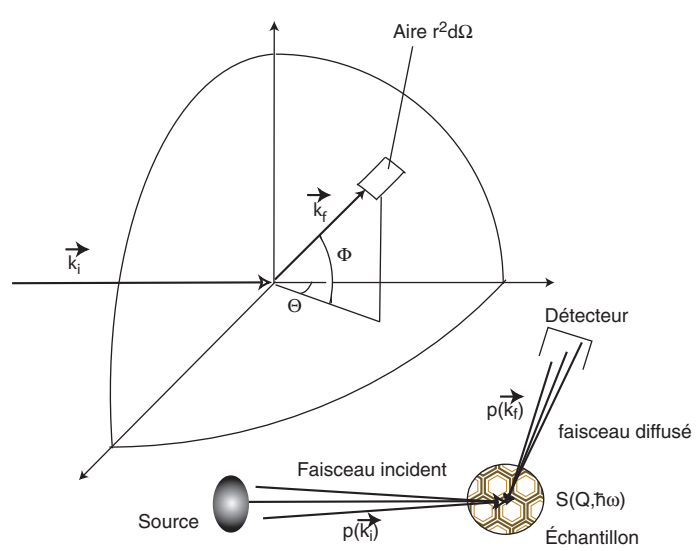

Figure 3. Dans l'approche traditionnelle, la diffusion de particules est décrite par la section efficace différentielle partielle $d^{2} \sigma / d \Omega d E_{f}$. Elle donne la probabilité avec laquelle un flux normalisé de particules, caractérisé par un vecteur d'onde $\vec{k}_{i}$, est diffusé dans un élément angulaire $d \Omega$ perpendiculaire à la direction $\hat{k}_{f}$. L'énergie finale des particules diffusées doit être comprise entre $E_{f}=\hbar^{2} k_{f}^{2} / 2 m$ et $E_{f}+d E_{f}$. Si nous choisissons le système de coordonnées tel que $\hat{k}_{f}=\hat{k}_{z}^{f}$ alors $d \Omega=d k_{x}^{f} d k_{y}^{f} / k_{f}^{2}$. Dans la pratique les particules incidentes ont une distribution $p\left(\vec{k}_{i} ; t\right)$ en énergie et en direction. Cette distribution dépend de la préparation de l'état initial des particules. De la même façon, les particules détectées possédent une distribution $p\left(\vec{k}_{f} ; t\right)$ qui est déterminée par le processus d'analyse des neutrons diffusés, éventuellement positionné sur leur chemin entre la cible et le détecteur. Les caractéristiques des faisceaux décrits par $p\left(\vec{k}_{i} ; t\right)$ et $p\left(\vec{k}_{f} ; t\right)$ sont déterminantes pour la statistique et la résolution qu'on pourra atteindre.

à déterminer est finalement le rapport du flux diffusé dans un élément $d \Omega$ par rapport au flux initial rentrant en collision avec la cible.

$$
d \sigma=\frac{\text { flux diffusé dans un élément } d \Omega}{\text { flux initial sur cible }}=r^{2} d \Omega \frac{J_{\text {diff }}(r, \theta, \phi, t)}{J_{\text {ini }}(t)}
$$

La quantité $\frac{d \sigma}{d \Omega}$ est apellée section efficace de diffusion différentielle. Ce nom est justifié par le fait que $\frac{d \sigma}{d \Omega}$ possède la dimension d'une surface. L'intégrale sur tous les angles donne la totalité du flux diffusé par rapport au flux initial. Cette grandeur physique est appelée section efficace totale de diffusion ou simplement section efficace.

$$
\sigma_{t}=\int \frac{d \sigma}{d \Omega} d \Omega=\int d \sigma .
$$

La section efficace représente la surface que le diffuseur oppose au rayonnement. Pour une sphère classique de rayon $r$, complètement opaque, dans un flux homogène de particules, elle vaut $\pi r^{2}$. Nous allons voir plus loin (section 4.2) que la mécanique quantique modifie ce résultat. Les sections efficaces sont généralement exprimées en barn $=10^{-24} \mathrm{~cm}^{2}$.

Nous avons evoqué dans l'introduction, la possibilité de "filtrer" les particules diffusées préalablement à leur détection. Le filtrage le plus répandu dans le cas de la diffusion des neutrons est un filtrage en énergie. Le filtre permet d'identifier les neutrons qui possèdent une énergie située entre $E_{f}$ et $E_{f}+d E_{f}$. Ceci nous amène à la double différentielle de la section efficace ou section efficace différentielle partielle.

$$
d^{2} \sigma=\frac{\text { flux diffusé dans un élément } d \Omega \text { avec une énergie comprise entre } E_{f} \text { et } E_{f}+d E_{f}}{\text { flux initial sur cible avec une énergie } E_{i}} d \Omega d E_{f}
$$


La quantité

$$
\frac{d^{2} \sigma}{d \Omega d E_{f}}
$$

possède l'unité d'une surface sur une énergie. La section efficace totale s'obtient par une double intégration

$$
\sigma_{t}=\iint \frac{d^{2} \sigma}{d \Omega d E_{f}} d \Omega d E_{f} .
$$

\section{LE NEUTRON}

Avant d'avancer plus le formalisme de la diffusion, il est utile de revenir brièvement sur le neutron. Le neutron est une particule élémentaire découverte par James Chadwick [1,2] en 1932. Son état quantique est déterminé par son impulsion $|\vec{P}\rangle(\vec{P}=\hbar \vec{k}, k=h / \lambda)$ ainsi que par son état de spin $|s\rangle$. Les neutrons qui sont utilisés comme sonde de la matière condensée ont des énergies faibles et peuvent ainsi être traités de manière non-relativiste. Leur énergie en fonction du vecteur d'onde est donc donnée par

$$
E=\frac{\hbar^{2} k^{2}}{2 m} .
$$

La masse du neutron ${ }^{6}$ est de 1.008 unités de masse atomique. Ceci donne la relation entre l'énergie et la longueur d'onde du neutron :

$$
E[\mathrm{meV}] \equiv 2.0725 k^{2}\left[\AA^{-1}\right] \equiv 81.8204 \lambda^{-2}[\AA] \equiv 5.227 v^{2}[\mathrm{~km} / \mathrm{s}],
$$

ou inversement

$$
\lambda[\AA] \equiv \frac{2 \pi}{k}\left[\AA^{-1}\right] \equiv 9.045 \frac{1}{\sqrt{E}}[\mathrm{meV}] \equiv 3.956 \frac{1}{v}[\mathrm{~km} / \mathrm{s}],
$$

On indique entre parenthèses les unités à prendre pour $E, \lambda, k$ et $v$.

Le spin du neutron est de $1 / 2$ avec un moment magnétique associé de -1.9132 magnétons nucléaires.

Les neutrons dans l'univers se trouvent soit confinés dans des noyaux, soit dans les étoiles à neutrons. Pour obtenir des neutrons libres, il faut employer des processus technologiques comme la fission dans un réacteur ou la spallation dans une source pulsée. Une description de ces méthodes peut être trouvée dans [4]. Une fois produits, les neutrons libres ont une durée de vie d' environ 886 secondes.

La production et la modération des neutrons se font par l'intermédiaire de processus aléatoires (voir la figure 4). Comme nous avons vu dans le chapitre I, les neutrons modérés peuvent être assimilés à un gaz. Le rayonnement neutronique est donc de nature complètement incohérent. Les sources de neutrons sont donc comparables à des ampoules incandescentes et non pas à des lasers. Le manque de cohérence implique qu'il n'y a pas d'interférence entre les ondes émanant des diverses régions de la source. La nature incohérente de la source permet de décrire les faiseaux en terme de distribution $p(\vec{k})$ du vecteur d'onde (voir section 9.1).

Nous avons déjà vu dans le chapitre I que la température du modérateur définit la forme du spectre. La distribution se déplace vers des énergies de plus en plus basses et devient de plus en plus étroite au fur et à mesure que nous baissons la température de modération. Un spectre de neutrons plus froids possède de ce fait un flux plus important pour un intervalle restreint $\Delta E$ autour du maximum.

Comme des neutrons moderés sont en équilibre avec un bain thermique de température $T$ leur énergie moyenne sera la même que l'énergie moyenne cinétique de n'importe quel matériau à cette

\footnotetext{
6 Nous utilisons comme source le National Institute of Standards and Technology (http://physics.nist.gov/cuu/Constants/). $h=6.62606896 \times 10^{-34} \mathrm{Js}$ et $m=1.674927211 \times 10^{-27} \mathrm{~kg}$. Comme $e=1.602176487 \times 10^{-19} \mathrm{C}$, un Joule est équivalent à $6.24150965 \times 10^{18} \mathrm{eV}$. De nombreuses informations utiles peuvent être trouvées dans le Neutron Data Booklet [3].
} 


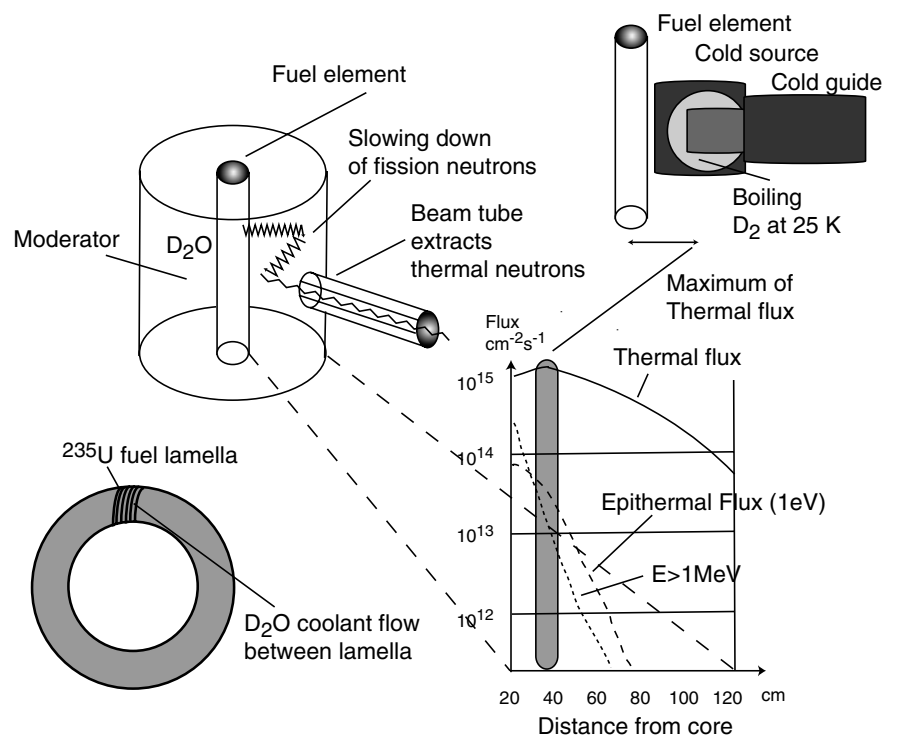

Figure 4. Vue schématique de la source à neutrons de l'Institut Laue-Langevin. Un élément combustible compact est entouré par un modérateur d'eau lourde $\mathrm{D}_{2} \mathrm{O}$. A la suite de collisions inélastiques avec les molécules d'eau, les neutrons très énergétiques $(E \approx 1 \mathrm{MeV})$ provenant de la fission de l'uranium ${ }^{235} \mathrm{U}$ sont rapidement ralentis. Une fois en équilibre thermodynamique avec l'eau lourde, un spectre de neutrons thermiques de flux intégré maximal de $1.5 \times 10^{15} \mathrm{n}$ par $\mathrm{cm}^{2}$ et par seconde est obtenu à $40 \mathrm{~cm}$ du centre du cœur. Des tubes à neutrons dont l' extrémité est proche du maximum du flux permettent leur extraction et leur acheminement vers les instruments. Un tube permet d' alimenter un éventail de guides à neutrons. Pour déplacer le spectre des neutrons vers des énergies encore plus basses, deux cavités remplies de $D_{2}$ en ébullition sont introduites dans la région où se trouve le maximum du flux thermique [5]. Elle sont appelées sources froides. Des guides à neutrons froids extraient les neutrons de ces sources froides. Un bloc de graphite, chauffé par le rayonnement $\gamma$ à $2400 \mathrm{~K}$, déplace le spectre des neutrons produits vers des énergies plus élevées. On parle alors de neutrons chauds.

température. Dans le langage de la mécanique quantique l'énergie des neutrons est comparable aux énergies des excitations peuplées à cette température. L'argument vaut néanmoins pour n'importe quelle particule moderée. La particularité du neutron réside dans sa masse qui, selon (3.3), impose des longueurs d'ondes de l'ordre des distances inter-atomiques pour ces énergies. Ils constituent donc des sondes idéales à l'échelle de l'̊̊.

Dans la table 1 nous donnons la classification généralement utilisée pour les neutrons moderés. Les faisceaux contiennent, en plus des neutrons modérés, des neutrons rapides et épi-thermiques. Des mesures adéquates sont à prendre pour éviter que ces neutrons deviennent une source importante de bruit de fond.

Tableau 1. Classification standard des neutrons selon leur énergie. Les valeurs indiquées correspondent aux spectres froid, thermique et chaud de l'ILL.

\begin{tabular}{|l|r|r|r|}
\hline dénomination & énergie $[\mathrm{meV}]$ & longueur d'onde $[\AA]$ & température $[\mathrm{K}]$ \\
\hline & & & \\
froid & $0.1-10$ & $30-3$ & $1-120$ \\
thermique & $10-100$ & $3-1$ & $120-1200$ \\
chaud & $100-500$ & $1-0.4$ & $1200-6000$ \\
\hline
\end{tabular}

\section{DIFFUSION PAR UN POTENTIEL STATIQUE}

Pour exploiter l'expérience nous avons besoin d'un formalisme qui relie les intensités mesurées aux propriétés des échantillons, de la manière la plus directe possible. Nous commençons notre discussion 
par la diffusion d'une particule ${ }^{7}$ de masse $m$ par un potentiel statique $V(\vec{r})$. La diffusion sera donc nécessairement élastique.

Du point de vue de la mécanique quantique le problème est décrit par l'équation de Schrödinger ${ }^{8}$

$$
i \hbar \frac{\partial}{\partial t} \psi(\vec{r}, t)=\mathbf{H} \psi(\vec{r}, t)=\left[-\frac{\hbar^{2}}{2 m} \Delta+V(\vec{r})\right] \psi(\vec{r}, t) .
$$

Etant donné que le potentiel ne dépend pas explicitement du temps, l'énergie est conservée et les solutions sont stationnaires (voir chapitre I). Elles ont la forme

$$
\psi(\vec{r}, t)=u(\vec{r}) e^{-i(E / \hbar) t}
$$

avec $u(\vec{r})$ une fonction propre du Hamiltonien

$$
\left[-\frac{\hbar^{2}}{2 m} \Delta+V(\vec{r})\right] u(\vec{r})=E u(\vec{r}) .
$$

Les fonctions propres $u(\vec{r})$, à déterminer, ne sont pas des états liés mais des états de diffusion par le potentiel $V(\vec{r})$. La diffusion permet donc aussi la spectroscopie des états non-liés. Elle est particulièrement riche en information quand il n'y a pas d'états liés, ce qui est le cas pour des potentiels répulsifs.

\subsection{Fonction d'onde en régime asymptotique}

Sans connaissance du potentiel, il est impossible de déterminer la fonction d'onde de la particule diffusée dans la région de $V(\vec{r})$. Par conséquent nous allons nous intéresser aux solutions asymptotiques de l'équation 4.3, c'est-à-dire loin du point d'impact. Une expérience de diffusion impose que le potentiel $V(\vec{r})$ décroisse rapidement avec la distance $\vec{r} .^{9}$ Les solutions propres les plus générales sont de la forme

$$
u_{\vec{k}}(\vec{r}) \longrightarrow \frac{1}{\sqrt{V}}\left(e^{i \vec{k} \cdot \vec{r}}+f_{\vec{k}}(\theta, \phi) \frac{e^{i k r}}{r}\right), \quad r \rightarrow \infty,
$$

avec

$$
k=\frac{1}{\hbar} \sqrt{2 m E} .
$$

Pour le démontrer nous remplaçons l'expression (4.4) dans l'equation de Schrödinger. En se souvenant que l'opérateur de Laplace, ici écrit en fonction des coordonnées polaires

$$
\Delta=\frac{\partial^{2}}{\partial r^{2}}+\frac{2}{r} \cdot \frac{\partial}{\partial r}+\frac{1}{r^{2}}\left(\frac{1}{\sin \theta} \cdot \frac{\partial}{\partial \theta} \sin \theta \frac{\partial}{\partial \theta}+\frac{1}{\sin ^{2} \theta} \frac{\partial^{2}}{\partial \phi^{2}}\right),
$$

se réduit à

$$
\frac{\partial^{2}}{\partial r^{2}} \text { pour } \quad r \rightarrow \infty
$$

\footnotetext{
7 Nous pouvons ainsi décrire la diffusion d'une particule sonde par une particule cible fixe. La diffusion par une particule libre doit prendre en compte la possibilité que celle-ci recule. Mathématiquement cette situation requiert de travailler dans le référentiel du centre de masse. Nous ne jugeons pas nécessaire de rajouter cette complication à notre discussion, parce que pour la plupart des problèmes traités en spectroscopie des neutrons, l'effet de recul n'est pas important. Nous donnerons néanmoins un exemple concret pour illustrer la différence entre la diffusion d'un neutron par une particule libre et par une particule liée, quand nous parlerons de la diffusion d'un neutron par un proton dans la section 5.7.

${ }^{8}$ L'equation de Schrödinger est une équation non-relativiste. Il est donc évident que tous les résultats qu'on en déduira ne seront valables que pour des particules de faible vitesse par rapport à la vitesse de la lumière. En dépit des similitudes énoncées dans l'introduction ils ne seront en particulier pas utilisables pour des expériences de collision à très haute énergie.

${ }^{9}$ Nous excluons de ce fait le potentiel de Coulomb. Dans la pratique le potentiel de Coulomb est toujours écranté dans la matière solide.
} 
on obtient

$$
\frac{\partial^{2}}{\partial r^{2}}\left(\frac{e^{i k r}}{r}\right)+\frac{2 m E}{\hbar^{2}}\left(\frac{e^{i k r}}{r}\right)=0+\mathrm{O}\left(\frac{1}{r^{2}}\right) .
$$

Les fonctions (4.4), dont l'existence reste en principe à prouver, sont donc bien des solutions asymptotiques et stationnaires de l'équation de Schrödinger. Le rôle du volume de normalisation $V$ a été discuté dans le chapitre I.

Le processus de diffusion dépend du temps. Une particule incidente créée vers $t \rightarrow-\infty$, se propage, rencontre le potentiel vers $t \approx 0$ et est diffusée avec une certaine probabilité. Elle se propage à nouveau, et est finalement enregistrée avec une certaine probabilité dans un détecteur vers $t \rightarrow+\infty$. Un formalisme capable de rendre compte de cette dépendance s'appuiera nécessairement sur des paquets d'ondes. Ces paquets d'ondes pourront être construits à partir des solutions stationnaires

$$
\psi(\vec{r}, t)=\frac{1}{\sqrt{V}} \int d^{3} k w(\vec{k}) u_{\vec{k}}(\vec{r}) \exp \left(-i \omega_{k} t\right) .
$$

Travailler avec des paquets d'ondes offre l'avantage que le temps apparaît explicitement. Les paquets, comme les particules, voyagent dans le temps. On paye cette facilité d'interprétation avec un formalisme plus lourd. L'information principale concernant les paquets d'ondes est contenue dans la fonction $w(\vec{k})$ qui décrit (voir chapitre I) la distribution en fonctions d'ondes stationnaires. Cette distribution est ellemême indépendante du temps. En particulier, elle n'est pas modifiée par le processus de diffusion. En d'autres termes, chaque fonction $u_{\vec{k}}(\vec{r})$ constitue une voie de diffusion et les différentes voies se superposent. ${ }^{10} \mathrm{C}$ 'est la raison pour laquelle nous pouvons contourner le problème des paquets d'ondes et obtenir une bonne description de la diffusion en nous limitant à des processus stationnaires.

Les fonctions d'ondes stationnaires sont interprétées en terme de courant de probabilité. Ces courants décrivent le flux des particules. Ils nous permettent ainsi de faire le lien avec les sections efficaces définies précédemment. La densité de probabilité de présence d'une particule est, comme on l'a vu dans le chapitre I, donnée par la norme de la fonction d'onde

$$
\rho(\vec{r}, t)=|\psi(\vec{r}, t)| .
$$

La probabilité de trouver la particule à l'instant $t$ dans le volume $d^{3} r$ en $\vec{r}$ est donc $\rho(\vec{r}, t) d^{3} r$. L'intégrale de $\rho(\vec{r}, t)$ sur toute l'espace est constante, mais sa valeur locale peut varier. Ces variations donnent lieu à un courant de probabilité $\vec{J}(\vec{r}, t)$. Il se calcule comme

$$
\vec{J}(\vec{r}, t)=\frac{\hbar}{2 m i}\left(\psi^{*} \vec{\nabla} \psi-\psi \vec{\nabla} \psi^{*}\right)
$$

et obéit à l'équation de continuité

$$
\frac{\partial}{\partial t} \rho(\vec{r}, t)+\vec{\nabla} \cdot \vec{J}(\vec{r}, t)=0 .
$$

Des relations de ce type régissent le flux de toute quantité conservée. Elles stipulent tout simplement que la probabilité de trouver une particule dans une région donnée ne peut évoluer que si cette évolution est accompagnée d'un courant de probabilité, qui traverse la surface enrobant la région.

Nous pouvons écrire toute fonction d'onde sous la forme

$$
\psi(\vec{r}, t)=A(\vec{r}, t) \exp (i \Phi(\vec{r}, t)) .
$$

Dans cette notation, la densité de probabilité est reliée au carré de l'amplitude

$$
\rho(\vec{r}, t)=A^{2}(\vec{r}, t)
$$

\footnotetext{
10 Attention, ce n'est pas le cas si nous composons les paquets d'ondes à partir d'ondes planes $\psi(\vec{r}, t) \propto \exp (i(\vec{k} \cdot \vec{r}-\omega t))$. Dans ce cas, la forme du paquet d'ondes n'est conservée que si l'amplitude de diffusion $f_{\vec{k}}(\theta, \phi)$ varie suffisamment lentement avec $\vec{k}$. Ce n'est pas toujours le cas. Un exemple typique est la diffusion de Bragg par un cristal parfait (voir section 7.5 et 9.4), qui peut rendre le paquet diffusé beaucoup mieux défini que le paquet initial.
} 


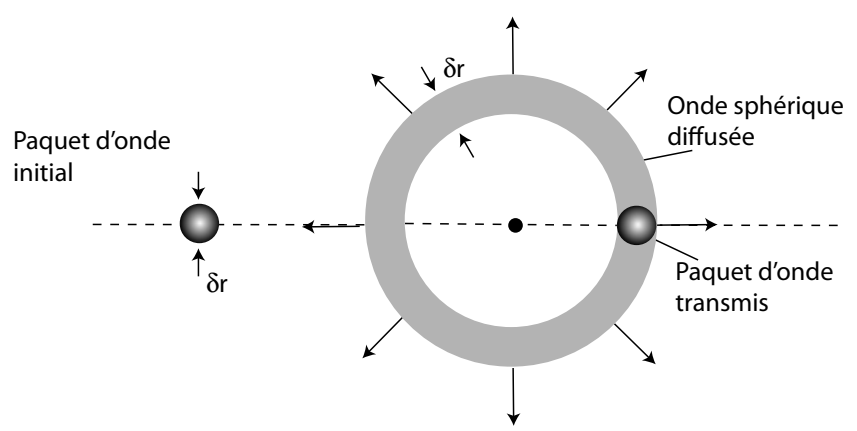

Figure 5. Illustration schématique de la fonction d'onde correspondant à une expérience de diffusion. Une source émet des particules, qui passent ensuite par un collimateur. Celui-ci en définit la direction et l'extension latérale du paquet d'onde. Un filtre approprié sélectionne une bande d'énergie qui, entre autres, influe sur l'extension temporelle du paquet d'onde. Le faisceau ainsi monochromaté et collimaté heurte la cible. Celui-ci diffuse les particules dans l'espace. Les particules diffusées se propagent et sont ensuite analysées (filtrées) en fonction de leur état final (par exemple leur énergie) avant d'être détectées. Tout ce processus est décrit par une onde sphérique.

et le courant de probabilité, à la densité multipliée par le gradient de la phase

$$
\vec{J}(\vec{r}, t)=\rho \vec{\nabla}\left(\frac{\hbar \Phi}{m}\right)
$$

de la fonction d'onde. L'expression $\vec{\nabla}(\hbar \Phi / m)$ est donc à interpréter comme la vitesse d'écoulement du fluide de probabilité. Pour une onde plane

$$
\psi(\vec{r}, t)=A \exp (i(\vec{k} \cdot \vec{r}-\omega t))
$$

nous obtenons

$$
\rho(\vec{r}, t)=|A|^{2}
$$

et

$$
\vec{J}(\vec{r}, t)=|A|^{2} \vec{\nabla}(\hbar \Phi / m)=\rho(\vec{r}, t) \frac{\vec{P}}{m}=\rho(\vec{r}, t) \vec{v}_{g} .
$$

$v_{g}$ est la vitesse de groupe associée à l'impulsion $\hbar \vec{k}$. Le régime d'écoulement du fluide de probabilité, dans le cas d'une onde plane, est un régime permanent. Dans le cas d'un faisceau permanent de particules identiques, qui n'interagissent pas, la probabilité de présence d'une particule est identique à la densité, c'est à dire à la probabilité de trouver n'importe quelle particule dans le volume $d^{3} r$ en $\vec{r}$, divisée par le nombre total de particules.

Les solutions (4.4) de l'équation de Schrödinger s'analysent donc de la manière suivante (voir aussi la figure 5).

1. La fonction

$$
\frac{1}{\sqrt{V}} e^{i \vec{k} \cdot \vec{r}}
$$

décrit un flux de particules libres d'impulsion $\vec{P}=\hbar \vec{k}$. Si nous choisissons de normaliser les fonctions d'onde pour la particule libre dans une boite de dimensions $L_{x}=L_{y}=L_{z}=1$, le volume de normalisation (voir chapitre I) devient

$$
V=(2 \pi)^{3}
$$

et les vecteurs d'onde ont la forme

$$
\vec{k}=\left(k_{x}, k_{y}, k_{z}\right)=\left(n_{x}, n_{y}, n_{z}\right), \quad n_{x}, n_{y}, n_{z}=\ldots-2,-1,0,1,2, \ldots
$$


En utilisant cette normalisation, nous obtenons le flux associé

$$
\vec{J}(\vec{r}, t)=\frac{1}{(2 \pi)^{3}} \frac{\hbar}{m} \vec{k}
$$

Dans le formalisme des paquets d'onde c'est cette partie qui survit à des temps négatifs, c'est-à-dire longtemps avant l'impact.

Formellement, la dimension latérale -c'est-à-dire perpendiculaire à la propagation- est infinie pour une onde plane. Il est évident que ce n'est pas le cas dans la pratique : le faisceau incident doit être suffisamment large pour éclairer complètement la cible. En même temps le faisceau doit être suffisamment étroit pour ne pas interférer avec le faisceau diffusé à l'endroit de la détection.

2. La fonction d'onde

$$
\frac{1}{\sqrt{V}} f_{\vec{k}}(\theta, \phi) \frac{e^{i k r}}{r}
$$

est l'expression mathématique d'une onde sphérique propagative de vecteur d'onde $k=2 \pi / \lambda$. Il est essentiel de remarquer que ce sont les valeurs absolues $r$ et $k$ qui figurent dans cette expression. La direction de propagation est définie de la cible $(r=0)$ vers le détecteur $(r \rightarrow \infty)$. Elle décrit la diffusion de la particule. Etant donné que le potentiel ne dépend pas du temps, la diffusion est élastique, c'est-à-dire que les énergies -et donc les normes des vecteurs d'onde- après et avant la diffusion sont identiques. L'amplitude de cette onde décroît avec $r^{-1}$ de sorte que la norme

$$
\int \rho(\vec{r}, t) r^{2} d r
$$

de la densité

$$
\rho(\vec{r})=\frac{1}{V} \frac{1}{r^{2}}\left|f_{\vec{k}}(\theta, \phi)\right|^{2}
$$

est préservée en fonction de $r$. La probabilité de trouver la particule diffusée ne doit pas dépendre de la distance entre détecteur et cible. Le paquet d'onde associé à l'onde sphérique possède la même vitesse de groupe que la particule initiale. Sa largeur évolue de la même manière que celle du paquet d'onde transmis (voir figure 5). Le flux associé à l'onde diffusée est donc donné par

$$
J_{\text {diff }}=J_{\text {ini }} \frac{1}{r^{2}}|f(\theta, \phi)|^{2}, \quad r \rightarrow \infty .
$$

3. Toute l'information concernant l'interaction de la particule avec le potentiel, qui persiste dans le régime asymptotique est contenue dans la fonction

$$
f_{\vec{k}}(\theta, \phi) .
$$

Elle a la dimension d'une longueur. Nous l'appelons amplitude de diffusion. Elle module l'onde sphérique en fonction des angles $\theta$ et $\phi$. Elle exprime de manière mathématique le fait que la diffusion encode le potentiel d'interaction dans la dépendance angulaire du faisceau diffusé. Il est évident que l'amplitude de diffusion $f_{\vec{k}}(\theta, \phi)$ ne peut pas prendre n'importe quelle forme. Elle doit par exemple assurer que la somme des probabilités de diffusion et de transmission soit égale à l'unité.

En utilisant les expressions 4.25 et 2.4 nous obtenons la relation entre la section efficace et l'amplitude de diffusion

$$
\frac{d \sigma}{d \Omega}=r^{2} \frac{J_{\mathrm{diff}}(r, \theta, \phi, t)}{J_{\mathrm{ini}}(t)}=|f(\theta, \phi)|^{2} .
$$

Nous constatons que le volume de normalisation $V$ ne figure plus dans cette expression. 


\subsection{Ondes partielles}

Pour nous familiariser avec les concepts développés plus haut et en particulier avec l'amplitude de diffusion, il est utile d'illustrer ce formalisme en prenant des potentiels isotropes, c'est-à-dire des potentiels à symétrie sphérique pour lesquels $V(\vec{r})=V(r)$. C'est par exemple la situation que nous rencontrons dans le cas de la diffusion d'une particule sonde par une particule cible isolée dans le référentiel du centre de masse. ${ }^{11}$

Nous allons procéder en nous référant au traitement de l'atome d'hydrogène en mécanique quantique. Il est bien connu que les fonctions d'ondes de l'électron dans le potentiel de Coulomb du proton peuvent être labellées par rapport aux opérateurs de moment cinétique $\vec{L}$. Ceci reflète le fait que, pour un potentiel isotrope, les fonctions propres $u(\vec{r})$ de l'Hamiltonien peuvent toujours s'écrire comme

$$
u(\vec{r})=u_{l m}(r, \theta, \phi)=R_{l}(r) Y_{l m}(\theta, \phi) .
$$

Les fonctions harmoniques sphériques $Y_{l m}(\theta, \phi)$ sont les fonctions propres des opérateurs $\mathbf{L}^{2}$ et $\mathbf{L}_{\mathbf{z}}$.

$$
\mathbf{L}^{2} Y_{l m}(\theta, \phi)=l(l+1) \hbar^{2} Y_{l m}(\theta, \phi)
$$

avec

$$
\mathbf{L}^{2}=\hbar^{2}\left(\frac{1}{\sin \theta} \cdot \frac{\partial}{\partial \theta} \sin \theta \frac{\partial}{\partial \theta}+\frac{1}{\sin ^{2} \theta} \cdot \frac{\partial^{2}}{\partial \phi^{2}}\right)
$$

et

$$
\mathbf{L}_{z} Y_{l m}(\theta, \phi)=m \hbar Y_{l m}(\theta, \phi)
$$

avec

$$
\mathbf{L}_{z}=-i \hbar \frac{\partial}{\partial \phi}
$$

La fonction radiale $R_{l}(r)$ doit satisfaire à l'équation

$$
\left(-\frac{\hbar^{2}}{2 m}\left[\frac{d^{2}}{d r^{2}}+\frac{2}{r} \frac{d}{d r}-\frac{l(l+1)}{r^{2}}\right]+V(r)\right) R_{l}(r)=E R_{l}(r)
$$

avec comme condition aux limites

$$
R_{l}(r) \propto r^{l+1}, \text { pour } \quad r \rightarrow 0 .
$$

Nous pouvons considérablement simplifier cette équation différentielle en factorisant $R_{l}(r)$ en $w_{l}(r) / r$

$$
\left(-\frac{\hbar^{2}}{2 m}\left[\frac{d^{2}}{d r^{2}}-\frac{l(l+1)}{r^{2}}\right]+V(r)\right) w_{l}(r)=E w_{l}(r)
$$

Le terme

$$
\frac{\hbar^{2}}{2 m} \frac{l(l+1)}{r^{2}}
$$

est appelé "barrière centrifuge" en analogie avec la mécanique classique.

Nous pouvons donc séparer la dépendance radiale de la fonction d'onde, de sa dépendance angulaire, ce qui constitue un énorme avantage. En l'absence de potentiel, l'équation radiale (4.33) définit les

\footnotetext{
11 Le formalisme qu'on présentera pourra sembler lourd par endroits. Il est néanmoins inévitable si nous tenons à bien fonder les concepts tel que la longueur de diffusion. Nous conseillons au lecteur principalement interessé par l'utilisation du formalisme, de sauter cette partie et d'aller directement à la section (4.5).
} 
fonctions de Bessel sphériques ${ }^{12} j_{l}(\rho)$ et $n_{l}(\rho)$, pourvu que nous exprimions la distance $r$ en unités de $\lambda / 2 \pi$, c'est-à-dire que nous remplacions $\rho=r \cdot k$.

$$
\left[\frac{d^{2}}{d \rho^{2}}+\frac{2}{\rho} \frac{d}{d \rho}+1-\frac{l(l+1)}{\rho^{2}}\right] R_{l}(\rho)=0
$$

Pour les valeurs de $l$ les plus basses, ces fonctions s'écrivent comme :

$$
\begin{aligned}
& j_{0}(\rho)=\frac{\sin \rho}{\rho} \\
& n_{0}(\rho)=\frac{\cos \rho}{\rho} \\
& j_{1}(\rho)=\frac{\sin \rho}{\rho^{2}}-\frac{\cos \rho}{\rho} \\
& n_{1}(\rho)=\frac{\cos \rho}{\rho^{2}}+\frac{\sin \rho}{\rho} .
\end{aligned}
$$

Les fonctions, pour des valeurs de $l$ plus élevées, peuvent s'obtenir par différenciation.

$$
\begin{aligned}
& j_{l}(\rho)=(-\rho)^{l}\left(\frac{1}{\rho} \cdot \frac{d}{d \rho}\right)^{l} \frac{\sin \rho}{\rho} \\
& n_{l}(\rho)=(-\rho)^{l}\left(\frac{1}{\rho} \cdot \frac{d}{d \rho}\right)^{l} \frac{\cos \rho}{\rho} .
\end{aligned}
$$

La solution la plus générale pour la partie radiale de l'équation de Schrödinger (4.35) est donnée par une superposition de ces fonctions

$$
R_{l}(\rho)=\sum_{l}\left[a_{l} j_{l}(\rho)+b_{l} n_{l}(\rho)\right]
$$

ou alternativement en posant $a_{l}=B_{l} \cos \delta_{l}$ et $b_{l}=B_{l} \sin \delta_{l}$

$$
R_{l}(\rho)=\sum_{l}\left[B_{l}\left(\cos \delta_{l} j_{l}(\rho)+\sin \delta_{l} n_{l}(\rho)\right]\right.
$$

L'utilité de représenter les coefficients du développement en terme d'une amplitude et d'une phase n'est pas évidente à ce stade de la discussion mais se justifiera plus tard.

Les fonctions $j_{l}(\rho)$ sont présentées pour $l \leq 5$ dans la figure 6 . On voit clairement que pour des distances $r \ll \lambda$ la fonction avec $l=0$ (dite de type s) domine toutes les autres. Mathématiquement on obtient pour $\rho \rightarrow 0$

$$
\begin{aligned}
j_{l}(\rho) & =\frac{1}{1 \cdot 2 \cdot 3 \cdot \ldots \cdot(2 l+1)} \rho^{l} \\
n_{l}(\rho) & =\frac{1 \cdot 2 \cdot 3 \cdot \ldots \cdot(2 l+1)}{(2 l+1)} \frac{1}{\rho^{l+1}} .
\end{aligned}
$$

\footnotetext{
12 La base complète des solutions de cette équation comprend à la fois les fonctions de Bessel sphériques dans le sens propre et les fonctions de von Neumann sphériques. Ces fonctions sont connectées aux fonctions de Bessel ordinaires $J(\rho)$ comme suit :

$$
\begin{aligned}
j_{l}(\rho) & =\sqrt{\frac{\pi}{2 \rho}} J_{l+1 / 2}(\rho), \\
n_{l}(\rho) & =(-1)^{l} \sqrt{\frac{\pi}{2 \rho}} J_{-(l+1 / 2)}(\rho) .
\end{aligned}
$$




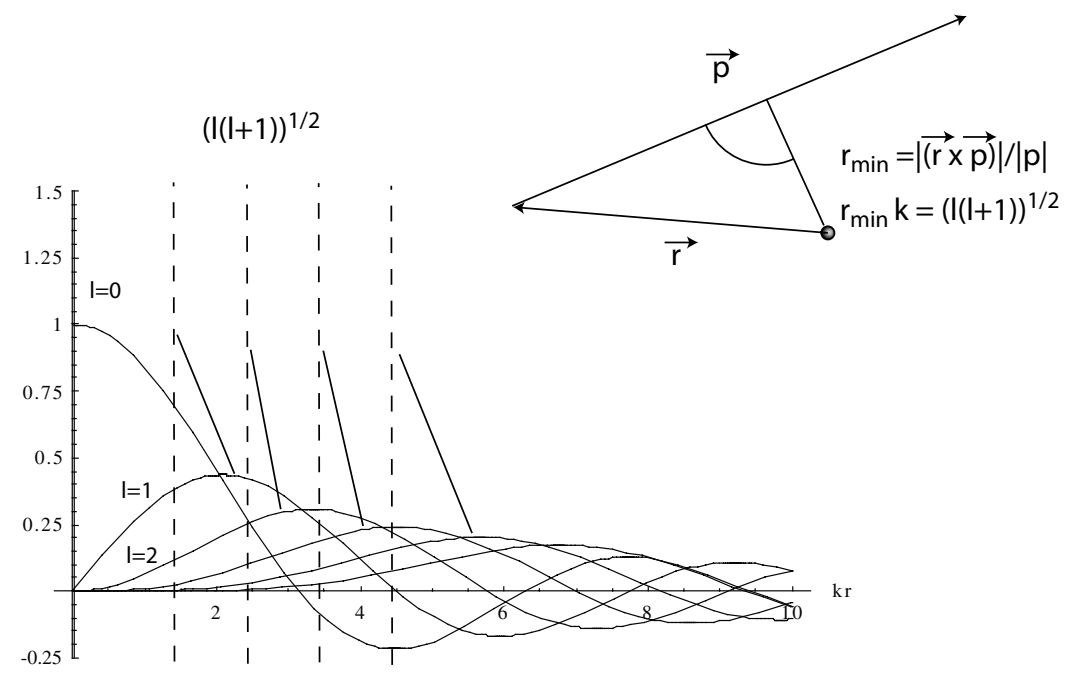

Figure 6. Fonctions de Bessel sphériques. Les maxima pour $l>0$ sont proches de la valeur $r k \approx \sqrt{l(l+1)}$ qui correspond à la distance minimum entre la trajectoire de la particule d'impulsion $\vec{p}$ et l'origine. Le cas $l=0$ doit être assimilé à l'impact centré sur la cible. Pour des cibles très petites par rapport à la longueur d'onde $\lambda$ de la sonde, seule cette fonction -dite de type s- contribue dans la région d'interaction.

Nous voudrions maintenant déterminer les solutions de l'équation de Schrödinger sans potentiel, c'està-dire dans l'espace libre, qui correspondent à un moment angulaire bien défini par rapport à une origine donnée. Cet exercice nous permettra de relier la fonction de la particule incidente à celle de la particule diffusée dans la même base. Les fonctions $n_{l}(\rho)$ divergent à l'origine. Elles ne peuvent donc pas contribuer au développement de $R_{i}(\rho)$ autour de l'origine, et en particulier pas au développement de la fonction d'onde d'une particule libre dans cette base. La base pour tout état d'une particule libre est donc donnée par les fonctions

$$
u_{l m}(r, \theta, \phi)=k \sqrt{\frac{2}{\pi}} j_{l}(k r) Y_{l m}(\theta, \phi)
$$

En particulier la fonction d'onde d'une particule qui possède une impulsion parallèle à la direction $\hat{z}$ s'exprime comme

$$
\frac{1}{\sqrt{V}} e^{i k z}=\frac{1}{\sqrt{V}} e^{i k r \cos \theta}=\sqrt{\frac{4 \pi}{V}} \sum_{l=0}^{\infty} \sqrt{2 l+1} i^{l} j_{l}(k r) Y_{l 0}(\theta) .
$$

En utilisant la connexion

$$
Y_{l 0}(\theta)=\sqrt{\frac{2 l+1}{4 \pi}} P_{l}(\cos \theta)
$$

entre harmoniques sphériques et polynômes de Legendre on peut écrire cette expression sous la forme équivalente

$$
\frac{1}{\sqrt{V}} e^{i k z}=\frac{1}{\sqrt{V}} \sum_{l=0}^{\infty}(2 l+1) i^{l} j_{l}(k r) P_{l}(\cos \theta) .
$$

Dans le cas asymptotique, $\rho \gg l(l+1)$ les fonctions de Bessel se simplifient pour donner :

$$
j_{l}(\rho)=\frac{\sin \left(\rho-l \frac{\pi}{2}\right)}{\rho}
$$




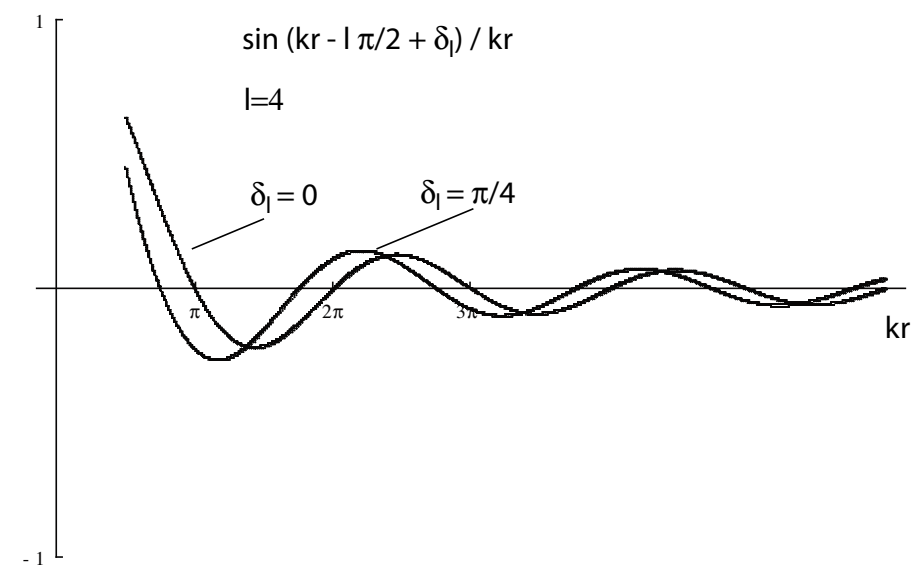

Figure 7. Ondes sphériques stationnaires pour $l=4$ avec et sans déphasage.

$$
n_{l}(\rho)=\frac{\cos \left(\rho-l \frac{\pi}{2}\right)}{\rho} .
$$

Il s'agit de simples ondes sphériques stationnaires ${ }^{13}$ (voir figure 7). Le caractère stationnaire de ces ondes reflète le fait qu'un état correspondant à un moment angulaire précis, ne peut pas se propager dans l'espace.

Nous pouvons utiliser les approximations (4.54) et (4.55) pour reformuler le développement (4.47) de la fonction radiale dans le cas des grandes distances comme

$$
R_{l}(\rho) \approx \sum_{l} B_{l} \frac{\sin \left(\rho-l \frac{\pi}{2}+\delta_{l}\right)}{\rho} .
$$

Si nous nous contentons de traiter la diffusion de particules non polarisées, il est évident que le résultat de l'expérience ne pourra pas dépendre de l'angle $\phi$. En d'autres mots, la symétrie intrinsèque du problème impose que la probabilité de diffusion possède une symétrie cylindrique autour de la direction du faisceau incident. Dans ce cas il suffit de retenir les fonctions $u_{l 0}$, dans le développement (4.28), qui sont les seules fonctions indépendantes de l'angle $\phi$. Loin de la cible nous pouvons donc -en utilisant (4.56) et (4.28)- exprimer la fonction d'onde correspondant au problème de la diffusion comme

$$
u(\vec{r})=\sum_{l=0}^{\infty} B_{l} \frac{\sin \left(\rho-l \frac{\pi}{2}+\delta_{l}\right)}{\rho} Y_{l 0}(\theta) .
$$

La forme de $u(\vec{r})$, obtenue ici pour un potentiel isotrope, en terme de fonctions sphériques doit être conforme à celle qu'on avait déduite pour le cas général en terme de l'amplitude de diffusion. En comparant les deux expressions (4.57) et (4.4) nous obtenons avec l'identité (4.51)

$$
B_{l}=e^{i l \frac{\pi}{2}+i \delta_{l}} \sqrt{4 \pi(2 l+1)}=i^{l} e^{i \delta_{l}} \sqrt{4 \pi(2 l+1)}
$$

et donc pour l'amplitude de diffusion

$$
f(\theta)=\frac{1}{2 i k} \sum_{l=0}^{\infty} \sqrt{4 \pi(2 l+1)}\left(e^{2 i \delta_{l}}-1\right) Y_{l 0}(\theta)
$$

\footnotetext{
${ }^{13}$ L'adjectif stationnaire est ici employé dans le sens des ondes stationnaires (voir chapitre I) et non pas dans le sens de la mécanique quantique.
} 


$$
\begin{aligned}
& =\frac{1}{2 i k} \sum_{l=0}^{\infty}(2 l+1)\left(e^{2 i \delta_{l}}-1\right) P_{l}(\cos \theta) \\
& =\frac{1}{k} \sum_{l=0}^{\infty}(2 l+1)\left(e^{i \delta_{l}} \sin \delta_{l}\right) P_{l}(\cos \theta)
\end{aligned}
$$

Nous avons réussi à développer l'amplitude de diffusion dans la base des fonctions $Y_{l 0}(\theta)$ qui sont aussi appelées ondes partielles. Les coefficients du développement s'expriment en fonction des phases $\Delta_{l}$. C'est un résultat important, qui indique que le processus de diffusion peut être décrit par un jeu de paramètres que nous pouvons déterminer expérimentalement sans connaissance précise du potentiel d'interaction. Les déphasages dépendent explicitement du vecteur d'onde $k$. Dans ce contexte on utilise souvent l'abréviation

$$
S_{l}(k)=e^{2 i \delta_{l}(k)}
$$

$S_{l}(k)$ est appelé élément de matrice $S$ de l'onde partielle $l$. On constate, sans pour autant en donner la preuve, qu'il s'agit des éléments de matrices déjà rencontrés dans la section 2 (voir (2.2)).

Pour élucider le rôle que joue le déphasage dans un processs de diffusion il est utile de comparer (dans la base des ondes partielles) la fonction d'onde $u(\vec{r})(4.57)$ avec celle d'une onde plane. En utilisant l'expression (4.58) pour $B_{l}$, la fonction de diffusion en régime asymptotique s'obtient sous la forme alternative

$$
u(\vec{r})=\frac{i}{2 k} \sum_{l=0}^{\infty}(2 l+1) P_{l}(\cos \theta)\left[(-1)^{l} \frac{e^{-i k r}}{r}-S_{l} \frac{e^{i k r}}{r}\right]
$$

La partie radiale des termes de cette somme est la superposition d'une onde sphérique sortante

$$
u^{\text {out }}(r) \propto \frac{e^{i k r}}{r}
$$

avec une onde sphérique entrante

$$
u^{\text {in }}(r) \propto \frac{e^{-i k r}}{r} .
$$

Cette superposition est pilotée par le facteur $S_{l}$. Pour $V(r)=0$ le processus de diffusion s'éteint. La fonction d'onde doit donc correspondre à une onde plane. Une onde plane peut -en toute analogie avec (4.63)- être écrite dans la base des fonctions sphériques comme

$$
u(\vec{r})=\frac{i}{2 k} \sum_{l=0}^{\infty}(2 l+1) P_{l}(\cos \theta)\left[(-1)^{l} \frac{e^{-i k r}}{r}-\frac{e^{i k r}}{r}\right] .
$$

Il s'agit à nouveau d'une superposition d'une onde entrante avec une onde sortante. En comparant (4.63) avec (4.66) nous constatons que la différence qu'introduit le potentiel est la multiplication de l'onde sortante par le facteur complexe unitaire $S_{l}(k)$. En conséquence, le potentiel déphase l'onde sortante par rapport à l'onde entrante. L'interférence entre les ondes déphasées crée une onde stationnaire sphérique de la même manière que deux phonons peuvent produire une onde stationnaire plane (voir chapitre I). C'est cette onde stationnaire qui décrit la diffusion. Sans potentiel, et donc sans déphasage, la superposition des ondes partielles est telle que l'onde sphérique sortante s'éteint.

En intégrant la section efficace différentielle (4.27) sur l'angle solide nous pouvons calculer la section efficace totale.

$$
\sigma_{t}=2 \pi \int_{0}^{\pi}|f(\theta)|^{2} \sin \theta d \theta
$$


Les polynômes de Legendre sont orthogonaux. Dans le carré de (4.61) il ne reste donc que les termes diagonaux en $l$. Ceux-ci s'intègrent facilement. Avec l'aide de la relation

$$
\int_{0}^{\pi} P_{l}^{2}(\cos \theta) \sin \theta d \theta=\frac{2}{2 l+1}
$$

nous obtenons

$$
\sigma_{t}=\frac{4 \pi}{k^{2}} \sum_{l=0}^{\infty}(2 l+1) \sin ^{2} \delta_{l}=\sum_{l=0}^{\infty} \sigma_{t}(l) .
$$

La section efficace totale est donc la somme des sections efficaces partielles $\sigma_{t}(l)$. En autres termes, chaque valeur $l$ constitue une "voie de diffusion" bien distincte.

Les phases $\delta_{l}$ sont réelles. Cela implique que $\left|S_{l}\right|=1$. Dans l'expression (4.63), l'amplitude associée à l'onde entrante est donc identique à l'amplitude correspondant à l'onde sortante. C'est la condition même pour obtenir une onde stationnaire. Le module unitaire de $S_{l}(k)$ reflète la situation physique que nous décrivons : le nombre de particules sondes est identique avant et après la diffusion. Si nous avions affaire à une cible capable d'absorber la particule sonde, nous aurions $\left|S_{l}\right|<1$ au moins pour quelques $l$. Cela impliquerait que $\delta_{l}$ soit un nombre complexe.

\subsection{Longueur de diffusion}

Les ondes partielles contribuant au développement de $f(\theta)$ sont d'autant plus nombreuses que le potentiel d'interaction est à longue portée par rapport à la longueur d'onde de la sonde. En effet, les fonctions $j_{l}(r k)$ ne contribuent dans la région du potentiel $\left(r<r_{0}\right)$ que si $\sqrt{l(l+1)}<k r$. Les ondes partielles qui ne sont pas présentes dans la région du potentiel ne peuvent évidemment pas être modifiées par le processus de diffusion, et par conséquent ne peuvent pas figurer dans le développement de $f(\theta)$. Dans le cas extrême où la longueur d'onde excède considérablement la portée du potentiel, alors seul l'onde partielle de type $s$ restera dans le développement de l'amplitude de diffusion. Avec

$$
Y_{00}=\frac{1}{\sqrt{4 \pi}}
$$

nous obtenons de (4.59)

$$
f(\theta)=\frac{e^{2 i \delta_{0}}-1}{2 i k}=\frac{1}{k} e^{i \delta_{0}} \sin \delta_{0} .
$$

$f(\theta)$ étant indépendant de l'angle, la diffusion est donc isotrope pour $k \ll r$. Ce résultat confirme que la structure d'une cible ne peut être étudiée qu'à l'échelle de la longueur d'onde de la sonde.

Comme exemple de calcul de déphasage nous allons maintenant étudier la diffusion par des potentiels isotropes concrets. Le cas extrême, le plus simple à traiter, est celui de la "sphère dure".

$$
V(r)=\left\{\begin{array}{cc}
\infty & r \leq r_{0} \\
0 & r>r_{0}
\end{array}\right.
$$

La fonction d'onde ne pénètre pas dans l'intérieur de la sphère. Sous la condition $\lambda \gg r_{0}$ nous ne retenons que l'onde de type $s$. La solution radiale de l'équation de Schrödinger est donnée par

$$
R(r)=w_{0}(r) / r .
$$

En remplaçant cette expression dans (4.35) il vient

$$
\begin{array}{ll}
r>r_{0}: & \left(\frac{d^{2}}{d r^{2}}+k^{2}\right) w_{0}(r)=0 \\
r \leq r_{0} \quad: \quad & w_{0}(r)=0
\end{array}
$$




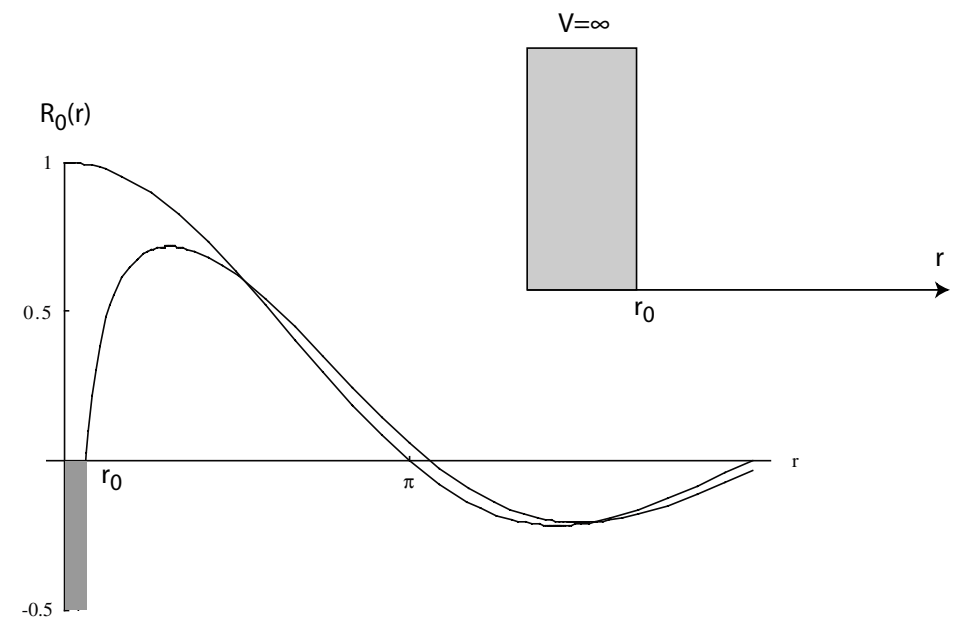

Figure 8. Nous montrons ici la partie radiale $R(r)=\sin (k r+\delta) /(k r)$ pour un déphasage fini et un déphasage nul.

Les solutions de cette équation sont évidemment de la forme (voir figure 8)

$$
\begin{array}{ll}
r>r_{0} \quad: & w_{0}(r)=C \sin \left(k r+\delta_{0}\right) \\
r<r_{0} & : \quad w_{0}(r)=0
\end{array}
$$

Nous déduisons de la condition de continuité de la fonction d'onde, que

$$
k r_{0}+\delta_{0}=n \pi
$$

Pour des $k$ suffisamment petits, cela implique un déphasage linéaire en $k$.

$$
\delta_{0}=-k r_{0} .
$$

On peut montrer en toute rigueur [6], que plus généralement

$$
\delta_{l}(k) \propto k^{(2 l+1)}, \quad \text { pour } \quad k \rightarrow 0,
$$

et cela indépendamment de la forme du potentiel. Par conséquent, seul le déphasage $\delta_{0}$ pour $l=0$ subsistera dans l'amplitude de diffusion dans la limite $k \rightarrow 0$, selon (4.59). Nous pouvons donc définir sans ambiguité

$$
b=-\lim _{k \rightarrow 0} f(\theta)=-\lim _{k \rightarrow 0} \frac{\delta_{0}(k)}{k} .
$$

La grandeur $b$ est appelée longueur de diffusion. Elle est réelle quand la diffusion ne comporte pas d'absorption. Dans le cas contraire, les longueurs de diffusion deviennent complexes et varient en fonction de $k$. Elles doivent donc être spécificiées pour un $k$ donné. Jusqu'à maintenant, nous n'avons pas considéré les effets de recul au niveau de la cible. Les longueurs de diffusion diffèrent entre une cible fixe et une cible libre.

Dans le cas de la sphère dure nous obtenons

$$
b=r_{0} .
$$

La longueur de diffusion a donc été définie de manière à ce qu'elle soit positive dans le cas d'une sphère dure. Pour la section efficace totale nous obtenons avec 4.27

$$
\sigma_{t}=4 \pi b^{2}=4 \pi r_{0}^{2}
$$



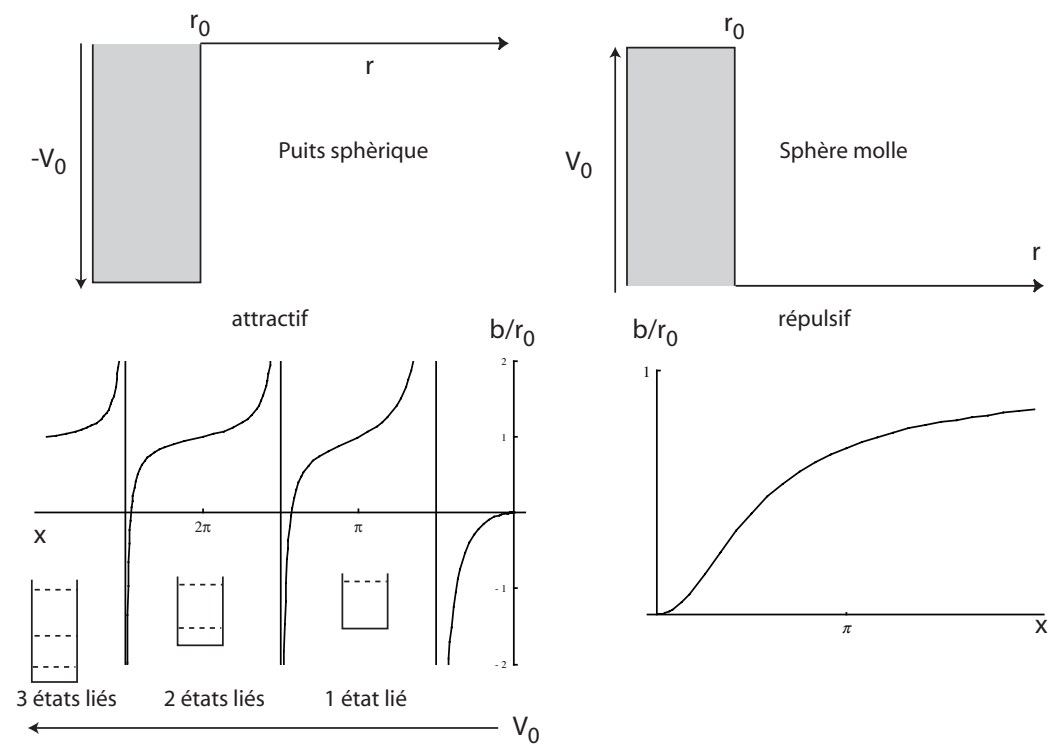

Figure 9. Longueur de diffusion $b$ dans le cas d'un puits de potentiel de profondeur $V_{0}$ et de rayon $r_{0} . b / r_{0}$ est tracé en fonction de $x=k_{0} r_{0}=\sqrt{2 m V_{0} / \hbar^{2}} r_{0}$. On voit que, contrairement au cas d'un potentiel répulsif, le potentiel attractif est conciliable avec toutes les longueurs de diffusion. Le signe de $b$ dépendra de manière cruciale des paramètres $V_{0}$ et $r_{0}$. Des valeurs de $b$ à la fois négatives et très grandes indiquent l'apparition d'un état lié.

Cette section efficace est quatre fois plus grande que la section géométrique de la sphère classique, que nous avions évoquée pour donner une illustration classique de la diffusion dans la section 2 . Un traitement quantique s'impose donc même dans ce cas très simple.

Dans le cas de la sphère dure, la longueur de diffusion est entièrement définie par la portée $r_{0}$ du potentiel. Ce n'est plus le cas quand nous rajoutons un peu plus de complexité en passant à la sphère molle.

$$
V(r)=\left\{\begin{array}{cc}
V_{0} & r \leq r_{0} \\
0 & r>r_{0}
\end{array}\right.
$$

Nous pouvons facilement déterminer les fonctions radiales qui satisfont à l'équation de Schrödinger, en nous limitant soit à l'intérieur soit à l'extérieur de la sphère. En imposant à la fonction totale d'être continue et différenciable au bord de la sphère, nous fixons la phase $\delta_{0}$ pour l'onde diffusée de type $s$. A partir de la phase nous obtenons la longueur de diffusion dans la limite de $k \rightarrow 0$. Le résultat de cet exercice s'exprime comme

$$
b=r_{0}\left(1+\frac{\tanh \left(k_{0} r_{0}\right)}{k_{0} r_{0}}\right)
$$

avec

$$
k_{0}=\sqrt{\frac{2 m V_{0}}{\hbar^{2}}} .
$$

La tangente hyperbolique

$$
\tanh x=\frac{e^{x}+e^{-x}}{e^{x}-e^{-x}}
$$

est toujours plus petite que 1. La longueur de diffusion reste donc positive et toujours plus petite que la portée du potentiel comme on peut voir sur la figure 9 . 
La situation change pour le puits de potentiel sphérique.

$$
V(r)=\left\{\begin{array}{cc}
-V_{0} & r \leq r_{0} \\
0 & r>r_{0}
\end{array}\right.
$$

La longueur de diffusion peut de nouveau être calculée sans grande difficulté à partir des conditions de continuité.

$$
b=r_{0}\left(1+\frac{\tan k_{0} r_{0}}{k_{0} r_{0}}\right)
$$

avec

$$
k_{0}=\sqrt{\frac{2 m V_{0}}{\hbar^{2}}} .
$$

Nous remarquons -sans le prouver- que la longueur de diffusion est toujours positive dans le cas d'un potentiel répulsif. Dans le cas d'un potentiel attractif, ce qui est normalement le cas pour la diffusion des neutrons par des noyaux, le signe de la longueur de diffusion dépendra des détails (portée, profondeur) du potentiel. Cette variabilité de la longueur de diffusion joue un rôle très important pour la diffusion des neutrons. Nous remarquons également que les longueurs de diffusion divergent pour

$$
\sqrt{2 m V_{0} / \hbar^{2}} r_{0} \approx \pi / 2+n \pi
$$

C'est un effet qu' on appelle “diffusion de résonance". Il apparait à chaque fois que le système admet un nouvel état lié, c'est à dire lorsque soit $V_{0}$ soit $r_{0}$ est augmenté.

\subsection{Potentiel effectif}

Dans le cas où l'énergie de la sonde est tellement basse que la diffusion est purement de type $s$, elle est entièrement décrite par la longueur de diffusion $b$. Pour beaucoup de problèmes pratiques, la forme exacte du potentiel est dans cette limite sans importance. Il est donc utile de construire un potentiel qui produise le bon déphasage pour l'onde $s$ et qui soit le plus simple possible. En d'autres termes, nous cherchons un potentiel sans structure interne. Le choix évident est un point. Un potentiel effectif qui correspond à ces critères est le suivant :

$$
V_{\mathrm{eff}}(r) \psi(r)=\left.\frac{2 \pi \hbar^{2}}{m} b \delta(\vec{r}) \frac{d}{d r}\right|_{r=0}(r \psi(r))
$$

En effet, la fonction de l'onde $s$

$$
\psi(r)=\frac{\sin \left(k r+\delta_{0}\right)}{k r}
$$


est une fonction propre ${ }^{14}$ de l'équation de Schrödinger

pourvu que

$$
\frac{\hbar^{2}}{2 m}\left[\Delta+V_{\text {eff }}(r)\right] \psi(r)=\frac{\hbar^{2} k^{2}}{2 m} \psi(r),
$$

$$
k \tan ^{-1} \delta_{0}(k)=-\frac{1}{b}
$$

Dans la limite $k \rightarrow 0$ cette condition devient

$$
\lim _{k \rightarrow 0} \frac{\delta_{0}}{k}=-b,
$$

ce qui correspond à la définition correcte de la longueur de diffusion $b$.

Le potentiel effectif ne dépend que d'un seul paramètre

$$
g=\frac{2 \pi \hbar^{2}}{m} b
$$

qui détermine la force de diffusion sans qu'on ait besoin de connaître la portée ou la profondeur du potentiel. Elle est appelée constante de couplage entre la sonde et la cible.

\section{5 Équation intégrale de la diffusion}

Toute l'information concernant la cible est contenue dans l'amplitude de diffusion $f_{\vec{k}}(\theta, \phi)$. Pour exploiter les résultats expérimentaux nous devons trouver un mode de calcul qui nous permette de relier l'amplitude de diffusion mesurée, aux propriétés microscopiques des échantillons. Des solutions analytiques n'existent que pour des potentiels simples comme ceux que nous avons rencontrés dans la section précédente. Pour tous les autres potentiels nous devons recourir à des calculs numériques ou de perturbation. Dans les deux cas il est souhaitable d'établir un lien direct entre l'amplitude de diffusion et le potentiel d'interaction de la forme

$$
f_{\vec{k}}(\theta, \phi)=\mathbf{L}_{\vec{k}} V .
$$

avec un opérateur $\mathbf{L}$ qui ne contient pas d'opérateur différentiel. On retrouve des relations de ce genre un peu partout en physique. Un exemple bien connu est le calcul du champ électrostatique à partir d'une distribution de charges.

Nous nous proposons maintenant d'établir une telle relation pour la diffusion des neutrons. Pour ne pas alourdir le formalisme inutilement nous préservons, au moins pour le moment, le caractère statique du potentiel, c'est-à-dire que nous cherchons les solutions stationnaires de l'équation de Schrödinger

\footnotetext{
${ }^{14}$ La preuve n'est pas triviale à cause de la présence de la fonction $\Delta$. On peut éliminer le problème en utilisant le fait que
}

$$
\Delta f(r)=\frac{1}{r} \frac{d^{2}}{d r^{2}}(r f(r))
$$

pourvu que $f(r)$ soit régulière en $r=0$. Nous pouvons construire une telle fonction régulière en posant

$$
f(r)=\psi(r)-\psi(0)=\frac{\sin \left(k r-\delta_{0}\right)}{k r}+\frac{\sin \delta_{0}}{k r} .
$$

Pour calculer le terme

$$
\frac{\sin \delta_{0}}{k} \Delta \frac{1}{r}
$$

nous utilisons l'équation bien connue en électrostatique

$$
\Delta \frac{1}{r}=-4 \pi \delta(\vec{r})
$$


(équation 4.3)

$$
\left[-\frac{\hbar^{2}}{2 m} \Delta+V(\vec{r})\right] u(\vec{r})=E u(\vec{r}) .
$$

Pour trouver des solutions approximatives, nous avons recours au calcul de perturbation. Ce calcul requiert la connaissance exacte du système non-perturbé. Dans le cas de la diffusion, ce système correspond aux particules libres. Autrement dit, tout potentiel constitue une perturbation aux yeux de la diffusion. La diffusion est, de ce point de vue, un cas extrême du calcul de perturbation. Comme premier pas vers une solution approximative, nous isolons la partie apparentée au potentiel dans l'équation de Schrödinger :

$$
-\frac{\hbar^{2}}{2 m}\left(\Delta+k^{2}\right) u_{\vec{k}}(\vec{r})=-V(\vec{r}) u_{\vec{k}}(\vec{r}) .
$$

Guidé par le formalisme de l'électrostatique, et conforme au langage mathématique des équations différentielles nous introduisons le concept d'une source $^{15}$

$$
j_{\vec{k}}(\vec{r})=V(\vec{r}) u_{\vec{k}}(\vec{r})
$$

L'équation de Schrödinger devient ainsi

$$
\mathbf{D} u_{\vec{k}}(\vec{r})=j_{\vec{k}}(\vec{r})
$$

avec l'opérateur

$$
\mathbf{D}=-\left(\mathbf{H}_{0}-E\right) \text {. }
$$

La source $j_{\vec{k}}(\vec{r})$ peut être considérée à l'origine du champ de diffusion, qu'il nous reste à déterminer. ${ }^{16}$

L'équation de Schrödinger étant linéaire, les solutions s'additionnent ce qui permet d' intégrer formellement (4.102). Toujours guidé par l'analogie avec l'électrostatique nous partons du principe que tout potentiel lisse $V(\vec{r})$, et donc la source $j_{\vec{k}}(\vec{r})$, peut s'exprimer sous la forme d'une superposition de sources ponctuelles ${ }^{17}$ (fonctions- $\Delta$ ou distributions de Dirac)

$$
j_{\vec{k}}(\vec{r})=\int d^{3} r j_{\vec{k}}\left(\vec{r}^{\prime}\right) \delta\left(\vec{r}-\vec{r}^{\prime}\right) .
$$

Nous introduisons la fonction de Green $G_{0}\left(\vec{r}, \vec{r}^{\prime}\right)$ comme solution de l'équation

$$
\mathbf{D} G_{0}\left(\vec{r}, \vec{r}^{\prime}\right)=\frac{\hbar^{2}}{2 m}\left(\Delta+k^{2}\right) G_{0}\left(\vec{r}, \vec{r}^{\prime}\right)=\delta\left(\vec{r}-\vec{r}^{\prime}\right) .
$$

15 Un exemple typique pour une équation différentielle contenant une source est la première équation de Maxwell

$$
\vec{\nabla} \cdot \vec{E}(\vec{r})=\frac{\rho(\vec{r})}{\epsilon_{0}} .
$$

La densité de charge électrique $\rho(\vec{r})$ y figure comme source du champ électrique $\vec{E}(\vec{r})$.

16 Ce champ de diffusion $u_{\vec{k}}(\vec{r})$ est non-trivial, même en absence de source. Pour $V(\vec{r})=0$ la source du champ de diffusion disparaît mais l'équation de Schrödinger possède toujours des solutions non-triviales

$$
\mathbf{H}_{0} u_{\vec{k}}^{0}(\vec{r})=-\frac{\hbar^{2}}{2 m} \Delta u_{\vec{k}}(\vec{r})=E u_{\vec{k}}^{0}(\vec{r})
$$

${ }^{17}$ Dans le cas de l'équation de Maxwell citée plus haut, ce sont des charges ponctuelles $q \cdot \rho(\vec{r})=q \delta(\vec{r})$ pour une charge qui se trouve à l'origine. 
La fonction de Green est donc la solution de l'équation de Schrödinger pour une source ponctuelle. Si cette fonction est connue, la solution $u_{\vec{k}}(\vec{r})$ pour une source quelconque peut s'obtenir par l'intermédiaire d'une superposition

$$
u_{\vec{k}}(\vec{r})=C(\vec{r})+\int d^{3} r^{\prime} G_{0}\left(\vec{r}, \vec{r}^{\prime}\right) j_{\vec{k}}\left(\vec{r}^{\prime}\right)
$$

En effet, l'opérateur $\mathbf{D}$ agissant sur la variable $\vec{r}$, on obtient en permutant dérivation et intégration, et en utilisant la définition de $G_{0}\left(\vec{r}, \vec{r}^{\prime}\right)$ :

$$
\begin{aligned}
\mathbf{D}_{\vec{r}} u_{\vec{k}}(\vec{r}) & =\mathbf{D}_{\vec{r}} \int d^{3} r^{\prime} G_{0}\left(\vec{r}, \vec{r}^{\prime}\right) j_{\vec{k}}\left(\vec{r}^{\prime}\right) \\
& =\int d^{3} r^{\prime} \mathbf{D}_{\vec{r}} G_{0}\left(\vec{r}, \vec{r}^{\prime}\right) j_{\vec{k}}\left(\vec{r}^{\prime}\right) \\
& =\int d^{3} r^{\prime} \delta\left(\vec{r}-\vec{r}^{\prime}\right) j_{\vec{k}}\left(\vec{r}^{\prime}\right) \\
& =j_{\vec{k}}(\vec{r})
\end{aligned}
$$

sous la condition que

$$
\mathbf{D}_{\vec{r}} C(\vec{r})=\frac{\hbar^{2}}{2 m}\left(\Delta+k^{2}\right) C(\vec{r})=0 .
$$

$C(\vec{r})$ doit donc correspondre à la fonction d'onde de la particule sonde libre

$$
C(\vec{r})=u_{\vec{k}}^{0}(\vec{r})
$$

En utilisant la définition (4.101) pour la source $j_{\vec{k}}(\vec{r})$ le résultat final s'écrit comme

$$
u_{\vec{k}}(\vec{r})=u_{\vec{k}}^{0}(\vec{r})+\int d^{3} r^{\prime} G_{0}\left(\vec{r}, \vec{r}^{\prime}\right) V\left(\vec{r}^{\prime}\right) u_{\vec{k}}\left(\vec{r}^{\prime}\right)
$$

La fonction d'onde (le champ) recherchée est définie par cette équation intégrale. Elle est donc complètement équivalente à l'équation de Schrödinger originale. Le problème n'est pas forcément plus facile étant donné que la fonction d'onde apparaît de manière explicite sous l'intégrale. Le résultat peut sembler à ce stade décevant. 


\subsection{La fonction de Green}

Avant de démontrer que ce pessimisme est tout-à-fait infondé, nous allons donner la forme explicite de la fonction de Green. Elle s'obtient comme ${ }^{18}$

$$
G_{0}\left(\vec{r}, \vec{r}^{\prime}\right)=-\frac{1}{4 \pi} \cdot \frac{2 m}{\hbar^{2}} \cdot \frac{e^{\left(i k \cdot\left|\vec{r}-\vec{r}^{\prime}\right|\right)}}{\left|\vec{r}-\vec{r}^{\prime}\right|} .
$$

Il s'agit donc d'une onde sphérique avec comme origine la source ponctuelle. En remplaçant (4.121) dans l'expression (4.111) nous dégageons le résultat suivant

$$
\begin{aligned}
u_{\vec{k}}(\vec{r}) & =u_{\vec{k}}^{0}(\vec{r})+\int d^{3} r^{\prime} G_{0}\left(\vec{r}, \vec{r}^{\prime}\right) V\left(\vec{r}^{\prime}\right) u_{\vec{k}}\left(\vec{r}^{\prime}\right) \\
& =u_{\vec{k}}^{0}(\vec{r})-\frac{1}{4 \pi} \frac{2 m}{\hbar^{2}} \int d^{3} r^{\prime} \frac{\exp \left(i k \cdot\left|\vec{r}-\vec{r}^{\prime}\right|\right)}{\left|\vec{r}-\vec{r}^{\prime}\right|} V\left(\vec{r}^{\prime}\right) u_{\vec{k}}\left(\vec{r}^{\prime}\right) .
\end{aligned}
$$

\subsection{La série et l'approximation de Born}

L'expression (4.122) se prête à une interprétation physique précise. En absence de potentiel, la sonde se comporte comme une particule libre décrite par la fonction d'onde $u_{\vec{k}}^{0}(\vec{r})$. Le potentiel est alors une source de perturbation. Chaque point $\vec{r}$ de l'espace où le potentiel $V(\vec{r})$ n'est pas nul, est une

18 Pour déduire la fonction de Green nous reformulons l'équation 4.106 comme

$$
\left(E-\mathbf{H}_{0}\right) G_{0}\left(\vec{r}, \vec{r}^{\prime}\right)=\delta\left(\vec{r}-\vec{r}^{\prime}\right) .
$$

Dans la suite il s'avèrera utile d'être très précis dans la notation et d'indiquer clairement la base de l'espace réel dans laquelle nous travaillons. Donc

$$
\left\langle\vec{r}\left|\left(E-\mathbf{H}_{0}\right) G_{0}\left(\vec{r}, \vec{r}^{\prime}\right)\right| \vec{r}^{\prime}\right\rangle=\left\langle\vec{r} \mid \vec{r}^{\prime}\right\rangle .
$$

Ce résultat nous apprend que formellement

$$
\mathbf{G}_{0}=\frac{1}{\left(E-\mathbf{H}_{0}\right)}
$$

c'est-à-dire que l'opérateur de Green est l'inverse de l'opérateur $\left(E-\mathbf{H}_{0}\right)$. Pour la fonction de Green formulée dans l'espace reél cela implique

$$
G_{0}\left(\vec{r}, \vec{r}^{\prime}\right)=\left\langle\vec{r}\left|\frac{1}{\left(E-\mathbf{H}_{0}\right)}\right| \vec{r}^{\prime}\right\rangle .
$$

Les solutions de l'équation de Schrödinger en absence de potentiel sont des ondes planes

$$
\langle\vec{r} \mid \vec{k}\rangle=u_{\vec{k}}^{0}(\vec{r})=\frac{1}{\sqrt{V}} e^{i \vec{k} \cdot \vec{r}}
$$

avec

$$
\mathbf{H}_{\mathbf{0}}|\vec{k}\rangle=\frac{\hbar^{2} k^{2}}{2 m}|\vec{k}\rangle
$$

Utilisant $E=\hbar^{2} k^{2} / 2 m$ le fait que

$$
\left(E-\mathbf{H}_{0}\right) \frac{2 m}{\hbar^{2}\left(k^{2}-k^{\prime 2}\right)}\left|\vec{k}^{\prime}\right\rangle=\left|\vec{k}^{\prime}\right\rangle
$$

identifie $2 m / \hbar^{2}\left(k^{2}-k^{\prime 2}\right)$ avec l'inverse de $\left(E-\mathbf{H}_{0}\right)$ pour les états $\left|\vec{k}^{\prime}\right\rangle$. Nous obtenons la relation

$$
\frac{1}{\left(E-\mathbf{H}_{0}\right)} \int d^{3} k^{\prime} \frac{1}{\left(E-\mathbf{H}_{0}\right)}\left|\vec{k}^{\prime}\right\rangle\left\langle\vec{k}^{\prime}\left|\frac{2 m}{\hbar^{2}} \int d^{3} k^{\prime} \frac{1}{\left(k^{2}-k^{\prime 2}\right)}\right| \vec{k}^{\prime}\right\rangle\left\langle\vec{k}^{\prime}\right|
$$

ce que nous donne dans l'espace reél

$$
\left\langle\vec{r}\left|\frac{1}{\left(E-\mathbf{H}_{0}\right)}\right| \vec{r}^{\prime}\right\rangle \frac{1}{V} \frac{2 m}{\hbar^{2}} \int d^{3} k^{\prime} \frac{e^{i \vec{k}^{\prime} \cdot\left(\vec{r}-\vec{r}^{\prime}\right)}}{\left(k^{2}-k^{\prime 2}\right)} .
$$

La difficulté mathématique réside dans l'évaluation de cette intégrale en présence des pôles aux endroits $k^{2}=k^{\prime 2}$. Ceci est du ressort du calcul des résidus. Le lecteur s'intéressant à ce sujet est prié de consulter les livres specialisés. La solution applicable au problème de la diffusion des particules est alors donnée par l'expression (4.121) avec un volume de normalisation choisi comme $V=(2 \pi)^{3}$ 


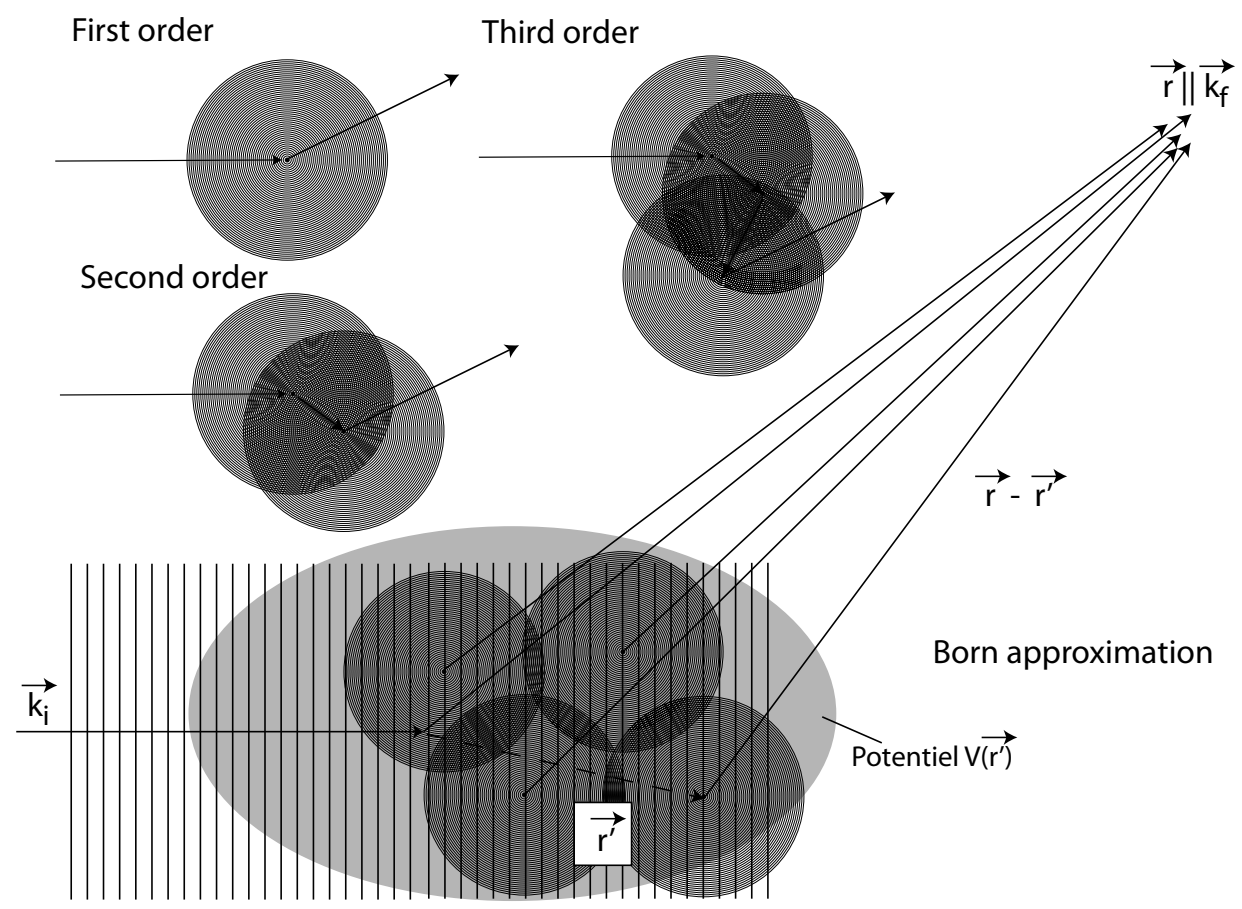

Figure 10. Représentation schématique de la série de Born. Sans potentiel $V\left(\vec{r}^{\prime}\right)$, la sonde est décrite par une onde plane de vecteur d'onde $\vec{k}_{i}$. Chaque point dans la région d'interaction est une source potentielle de diffusion et peut donc engendrer des ondes shériques. La probabilité d'un tel évènement dépend à la fois du potentiel et de la fonction d'onde elle-même. En d'autres termes, la fonction d'onde est couplée à elle-même par l'intermédiaire du potentiel. Les ondes sphériques créées deviennent elles-même des sources de diffusion. Le processus complet peut se résumer à une série infinie de processus locaux de diffusion (voir l'expression 4.126). Cette série peut être tronquée si l'interaction est suffisamment faible. Quand on se contente du premier ordre, on parle de l'approximation de Born (voir l'expression (4.128)). Dans ce cas l'onde diffusée est la somme de toutes les ondes sphériques créées par l'onde entrante non-perturbée. Elle est observée dans une direction $\vec{r}$ qui définit le vecteur d'onde final $\vec{k}_{f}$. Dans cette approximation la sommation des ondes sphériques correspond à la transformée de Fourier du potentiel (voir l'expression (4.141)).

source potentielle d'onde sphérique. L'amplitude de cette onde est proportionnelle, à la fois à la force d'interaction (le potentiel), et à la fonction d'onde en cet endroit. Ceci se comprend si l'on considère que la fonction d'onde donne la probabilité de présence de la particule sonde. Si cette dernière est élevée, la probabilité d'engendrer une perturbation est également élevée. Si, par contre, la probabilité de présence est nulle à un endroit donné, il n'y aura pas de création d'onde même si le potentiel est non-nul. Nous comprenons maintenant pourquoi la fonction d'onde apparaît dans l'expression 4.101 pour la source. Tout le problème mathématique réside dans cette rétroaction: la fonction d'onde est à la fois la source et le résultat de la perturbation.

L'expression intégrale offre, par rapport à l'expression différentielle, l'avantage d'être accessible à l'itération. Nous obtenons ainsi par simple substitution

$$
\begin{aligned}
u_{\vec{k}}(\vec{r})= & u_{\vec{k}}^{0}(\vec{r})+\int d^{3} r^{\prime} G_{0}\left(\vec{r}, \vec{r}^{\prime}\right) V\left(\vec{r}^{\prime}\right) u_{\vec{k}}\left(\vec{r}^{\prime}\right) \\
= & u_{\vec{k}}^{0}(\vec{r})+\int d^{3} r^{\prime} G_{0}\left(\vec{r}, \vec{r}^{\prime}\right) V\left(\vec{r}^{\prime}\right) u_{\vec{k}}^{0}\left(\vec{r}^{\prime}\right) \\
& +\int d^{3} r^{\prime \prime} \int d^{3} r^{\prime} G_{0}\left(\vec{r}, \vec{r}^{\prime}\right) V\left(\vec{r}^{\prime}\right) G_{0}\left(\vec{r}^{\prime}, \vec{r}^{\prime \prime}\right) V\left(\vec{r}^{\prime \prime}\right) u_{\vec{k}}\left(\vec{r}^{\prime \prime}\right) .
\end{aligned}
$$


Cette manière explicite de décrire la fonction d'onde devient très vite laborieuse. En introduisant l'opérateur de Green

$$
\mathbf{G}_{0}\left|u_{\vec{k}}\right\rangle=\int d^{3} r^{\prime} G_{0}\left(\vec{r}, \vec{r}^{\prime}\right) u_{\vec{k}}\left(\vec{r}^{\prime}\right)
$$

nous pouvons reformuler l'expression 4.123 de manière plus compacte

$$
\begin{aligned}
\left|u_{\vec{k}}\right\rangle & =\left|u_{\vec{k}}^{0}\right\rangle+\mathbf{G}_{0} V\left|u_{\vec{k}}\right\rangle \\
& =\left|u_{\vec{k}}^{0}\right\rangle+\mathbf{G}_{0} V\left|u_{\vec{k}}^{0}\right\rangle+\mathbf{G}_{0} V \mathbf{G}_{0} V\left|u_{\vec{k}}\right\rangle .
\end{aligned}
$$

Cette expression est connue sous le nom d' équation de Lippmann-Schwinger. Elle est d'une importance fondamentale pour tout calcul de perturbation.

En poussant l'itération à l'extrême nous aboutissons à la série de Born

$$
\left|u_{\vec{k}}\right\rangle=\sum_{n=0}^{\infty}\left(\mathbf{G}_{0} V\right)^{n}\left|u_{\vec{k}}^{0}\right\rangle
$$

L’opérateur

$$
\Omega=\sum_{n=0}^{\infty}\left(\mathbf{G}_{0} V\right)^{n}
$$

qui produit la fonction de diffusion $\left|u_{\vec{k}}\right\rangle$ à partir de la fonction d'onde non-perturbée $\left|u_{\vec{k}}^{0}\right\rangle$ est appelé opérateur d'onde.

En fonction de l'interaction entre la sonde et la cible il nous sera possible -ou non- de tronquer cette série à un certain ordre. Pour les neutrons lents, l'interaction est plutôt faible. Nous pouvons donc présumer que la perturbation de la fonction d'onde initiale est suffisamment faible pour que l'on puisse utiliser dans l'expression la fonction non-perturbée pour la source. En clair nous présumons que tous les atomes de la cible voient les neutrons arriver comme si les autres atomes étaient absents. En particulier le nombre de neutrons diffusés est tellement faible qu'on n'aura pratiquement aucun effet d'atténuation du flux incident par le volume irradié. ${ }^{19}$ Dans ce cas nous pouvons nous limiter au plus bas ordre de perturbation.

$$
\left|u_{\vec{k}}\right\rangle=\left|u_{\vec{k}}^{0}\right\rangle+\mathbf{G}_{0} V\left|u_{\vec{k}}^{0}\right\rangle
$$

Nous dirons que nous travaillons dans l'approximation de Born. Si l'approximation de Born est applicable, nous sommes alors en mesure de calculer la fonction d'onde des particules diffusées à partir du potentiel d'interaction.

Pour faire le lien avec la section précédente il nous reste à determiner la forme asymptotique de la fonction d'onde donnée par l'expression( 4.122) que nous répétons ici pour convenance :

$$
u_{\vec{k}}(\vec{r})=u_{\vec{k}}^{0}(\vec{r})-\frac{1}{4 \pi} \frac{2 m}{\hbar^{2}} \int d^{3} r^{\prime} \frac{\exp \left(i k \cdot\left|\vec{r}-\vec{r}^{\prime}\right|\right)}{\left|\vec{r}-\vec{r}^{\prime}\right|} V\left(\vec{r}^{\prime}\right) u_{\vec{k}}\left(\vec{r}^{\prime}\right)
$$

Pour des positions $\vec{r}$ très éloignées de la cible, c'est-à-dire pour des valeurs $|\vec{r}|$ grandes par rapport à la portée $\left|\vec{r}^{\prime}\right|$ du potentiel, ${ }^{20}$ nous pouvons développer la distance $\left|\vec{r}-\vec{r}^{\prime}\right|$ :

$$
\left|\vec{r}-\vec{r}^{\prime}\right|=\sqrt{r^{2}+r^{\prime 2}-2 \vec{r} \cdot \vec{r}^{\prime}} \approx r\left(1-\frac{\vec{r} \cdot \vec{r}^{\prime}}{r^{2}}\right) .
$$

\footnotetext{
19 Il y a des situations expérimentales où ce n'est pas le cas et cela en dépit d'une longueur de diffusion faible. Un exemple typique est la diffusion de Bragg par un cristal de grande qualité. La diffusion est tellement forte pour des valeurs de $\vec{Q}$ satisfaisant l'équation de Bragg, que le faisceau incident est fortement attenué après seulement quelques milliers de couches d'atomes. On parle d'effet d'extinction. Dans ce cas on devra utiliser une théorie plus complexe qui est la théorie dynamique de la diffusion (Se référer à la section 9.4).

${ }^{20}$ En optique cette condition correspond à la diffraction de Fraunhofer (voir la section 7.1).
} 
Le dénominateur $\left|\vec{r}-\vec{r}^{\prime}\right|$ de la fonction

$$
\frac{\exp \left(i k \cdot\left|\vec{r}-\vec{r}^{\prime}\right|\right)}{\left|\vec{r}-\vec{r}^{\prime}\right|}
$$

varie très lentement avec $r^{\prime}$. Il peut donc être remplacé par $r$. Ceci n'est pas vrai pour l'exponentielle $\exp \left(i k \cdot\left|\vec{r}-\vec{r}^{\prime}\right|\right)$. Elle varie fortement sur des distances $r^{\prime}$ comparables à la longueur d'onde $\lambda=2 \pi / k$. C'est ici que le choix de la longueur d'onde devient important. Par conséquent nous devons retenir le terme linéaire en $r^{\prime}$ même pour des distances $r$ très grandes. La fonction d'onde devient

$$
r \rightarrow \infty \quad: \quad u_{\vec{k}}(\vec{r})=\frac{1}{(2 \pi)^{3 / 2}} e^{i \vec{k} \cdot \vec{r}}-\frac{1}{4 \pi} \frac{2 m}{\hbar^{2}} \frac{e^{i k \cdot r}}{r} \int d^{3} r^{\prime} \exp \left(i k \cdot\left|\hat{r} \cdot \vec{r}^{\prime}\right|\right) V\left(\vec{r}^{\prime}\right) u_{\vec{k}}\left(\vec{r}^{\prime}\right) .
$$

C'est la forme asymptotique à laquelle nous nous sommes attendus. La comparaison avec l'expression 4.4 nous permet d'identifier l'amplitude de diffusion comme

$$
f_{\vec{k}}(\Omega)=-\frac{\sqrt{2 \pi} m}{\hbar^{2}} \int d^{3} r^{\prime} \exp \left(-i k \hat{r} \cdot \vec{r}^{\prime}\right) V\left(\vec{r}^{\prime}\right) u_{\vec{k}}\left(\vec{r}^{\prime}\right) .
$$

Elle ne dépend que de la direction $\hat{r}$ et du vecteur d'onde $\vec{k}$ de la cible. Dans l'approximation de Born elle se simplifie pour prendre la forme

$$
f_{\vec{k}}(\Omega)=-\frac{1}{2 \pi} \frac{m}{\hbar^{2}} \int d^{3} r^{\prime} e^{-i k \hat{r} \cdot \vec{r}^{\prime}} e^{i \vec{k} \cdot \vec{r}^{\prime}} V\left(\vec{r}^{\prime}\right)
$$

A ce point il est avantageux d'introduire les vecteurs

$$
\begin{aligned}
\vec{k}_{i} & :=\vec{k} \\
\vec{k}_{f} & :=k \hat{r} \\
\vec{Q} & :=\vec{k}_{i}-\vec{k}_{f} .
\end{aligned}
$$

Avec cette notation et

$$
\langle r \mid \vec{k}\rangle=\frac{1}{(2 \pi)^{3 / 2}} e^{i \vec{k} \cdot \vec{r}}
$$

nous obtenons

$$
f\left(\vec{k}_{f}, \vec{k}_{i}\right)=-\frac{4 \pi^{2} m}{\hbar^{2}}\left\langle\vec{k}_{f}|V| u_{\vec{k}_{i}}\right\rangle=-\frac{4 \pi^{2} m}{\hbar^{2}}\left\langle\vec{k}_{f}|V \Omega| \vec{k}_{i}\right\rangle
$$

Cette expression nous permet de définir l'opérateur de transition $\mathbf{T}$.

$$
f\left(\vec{k}_{f}, \vec{k}_{i}\right)=-\frac{4 \pi^{2} m}{\hbar^{2}}\left\langle\vec{k}_{f}|\mathbf{T}| \vec{k}_{i}\right\rangle .
$$

Dans l'approximation de Born $(\Omega \rightarrow 1, \mathbf{T} \rightarrow V)$ et donc

$$
f\left(\vec{k}_{f}, \vec{k}_{i}\right)=-\frac{4 \pi^{2} m}{\hbar^{2}}\left\langle\vec{k}_{f}|V| \vec{k}_{i}\right\rangle
$$

ce que nous pouvons écrire comme

$$
f_{\vec{Q}}(\Omega)=-\frac{1}{2 \pi} \frac{m}{\hbar^{2}} \int d^{3} r^{\prime} e^{i \vec{Q} \cdot \vec{r}^{\prime}} V\left(\vec{r}^{\prime}\right)
$$

C'est un résultat remarquable. Il stipule que l'amplitude de diffusion est simplement proportionnelle à la transformée de Fourier du potentiel d'interaction. L'approximation de Born permet ainsi de relier l'amplitude de diffusion à une propriété intrinsèque de l'échantillon. 


\section{CIBLE COMPOSÉE}

Un échantillon de matière condensée est composé d'un grand nombre de diffuseurs (noyaux des atomes et moments magnétiques pour les neutrons, électrons pour les rayons-X, etc). Pour calculer comment une telle cible diffuse une sonde, il faut en principe résoudre l'équation de Schrödinger pour le système combiné incluant la cible et la sonde comme entités dynamiques. Le problème ressemble étroitement à celui des électrons couplés aux noyaux traité dans le chapitre I. Pour traiter ce couplage, nous avions utilisé l'approximation adiabatique qui nous a permis de prendre en compte l'effet des électrons par un potentiel. Dans le cas de la diffusion nous allons suivre une démarche identique : nous présumons que l'interaction sera tellement faible, que la présence de la sonde n'influera pas sur les états $|\lambda\rangle$ de la cible. Elle ne pourra -au plus- qu'induire le passage d'un état vers un autre. Ceci est certainement vrai pour les neutrons lents hors résonance. Dans ce cas, il est évident que l'interaction pourra se décrire sous la forme d'un potentiel

$$
V\left(\vec{r} ; \vec{R}_{1}, \ldots, \vec{R}_{N}\right)
$$

dans lequel les coordonnées des diffuseurs $\vec{R}_{i}(i=1, \ldots N)$ figurent comme paramètres et non pas comme variables dynamiques et $\vec{r}$ décrit les coordonnées de la sonde. Ceci nous permet d'utiliser le formalisme développé pour le cas d'un potentiel statique comme point de départ du calcul de la section efficace d'une cible composée.

\subsection{Règle d'or de Fermi}

Pour traiter le cas d'un échantillon constitué de plusieurs diffuseurs nous reprenons l'expression 4.139

$$
f_{\vec{Q}}(\Omega)=-\frac{4 \pi^{2} m}{\hbar^{2}}\left\langle\vec{k}_{f}|\mathbf{T}| \vec{k}_{i}\right\rangle .
$$

Dans cette écriture formelle la section efficace différentielle devient

$$
\frac{d \sigma}{d \Omega}=\frac{(2 \pi)^{4} m^{2}}{\hbar^{4}}\left|\left\langle\vec{k}_{f}|\mathbf{T}| \vec{k}_{i}\right\rangle\right|^{2} .
$$

La section efficace différentielle peut néanmoins également s'exprimer comme

$$
d \sigma_{\vec{k}_{i} \rightarrow \vec{k}_{f}}=\frac{w_{\vec{k}_{i} \rightarrow \vec{k}_{f}}}{J_{\vec{k}_{i}}} d \Omega .
$$

où $w_{\vec{k}_{i} \rightarrow \vec{k}_{f}}$ désigne la densité de probabilité de transition d'un état $\vec{k}_{i}$ vers un état $\vec{k}_{f}$. En comparant les expressions 5.3 et 5.4 et en nous rappelant la forme (4.17) pour le flux $J_{\vec{k}_{i}}$ nous identifions

$$
w_{\vec{k}_{i} \rightarrow \vec{k}_{f}}=\frac{2 \pi}{\hbar}\left|\left\langle\vec{k}_{f}|\mathbf{T}| \vec{k}_{i}\right\rangle\right|^{2} \rho\left(E_{f}\right)
$$

où nous avons introduit la densité d'états

$$
\rho_{\vec{k}}\left(E_{f}\right)=\frac{m k}{\hbar^{2}} .
$$

L'expression 5.5 est connue sous le nom de règle d'or de Fermi.

A part le facteur $2 \pi / \hbar$, les termes composant la règle d'or de Fermi se prêtent à une interprétation intuitive. L'élément de matrice $\left\langle\vec{k}_{f}|\mathbf{T}| \vec{k}_{i}\right\rangle$ au carré donne la probabilité de la transition de l'état $\left|\vec{k}_{i}\right\rangle$ vers l'état $\left|\vec{k}_{f}\right\rangle$ dans le sens propre du terme. Cette transition est induite par le potentiel d'interaction $V$. Si les états $|\vec{k}\rangle$ étaient discrets nous pourrions nous arrêter là. Les états $|\vec{k}\rangle$ formant un continuum nous devons nous assurer que toute expression les contenant soit proprement normalisée. La continuité des états $|\vec{k}\rangle$ implique, pour l'expérimentateur, l'impossibilité de les déterminer individuellement. Toute mesure 


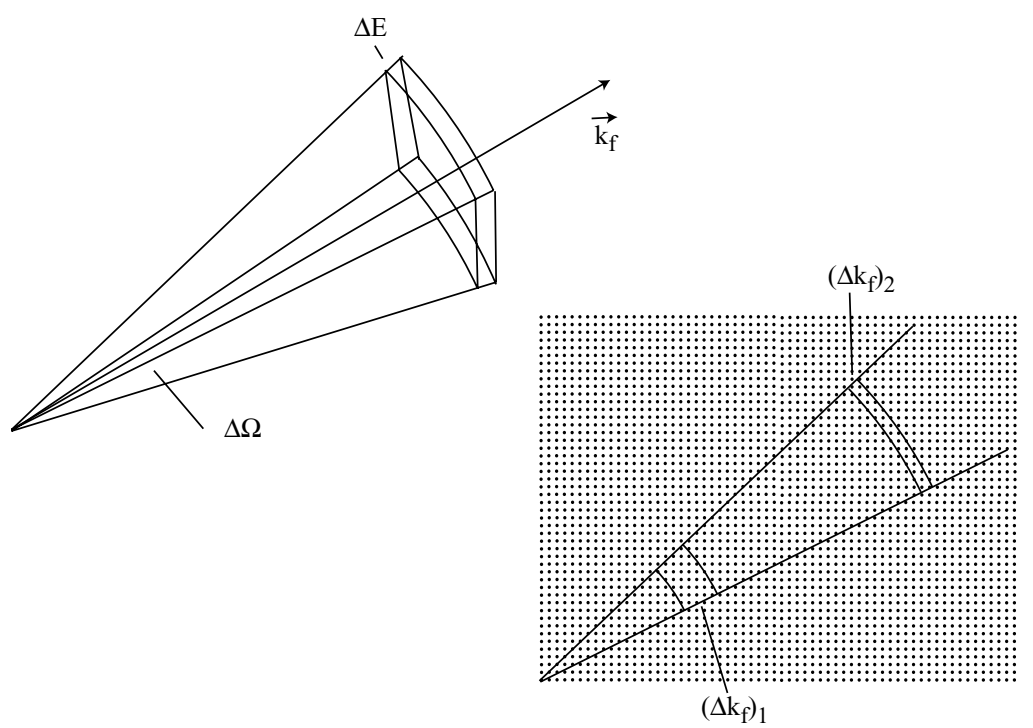

Figure 11. Illustration de la fonction $\rho_{\vec{k}}\left(E_{f}\right)$. Cette fonction est la densité d'états $\left|\vec{k}_{f}\right\rangle$ dans l'espace $\left(\Omega, E_{f}\right)$. Pour la déterminer nous devons compter le nombre d'états $\left|\vec{k}_{f}\right\rangle$ dans un élément $d \Omega d E_{f}$. Cet élément est montré dans la figure à gauche dans l'espace des $\left|\vec{k}_{f}\right\rangle$. La figure à droite correspond à une coupe bi-dimensionnelle de cet élément tri-dimensionnel. Les états $\left|\vec{k}_{f}\right\rangle$ sont schématisés par des points uniformément répartis dans l'espace. A une épaisseur radiale $d k_{f}$ constante, le volume de $d \Omega d k_{f}$ augmentera comme la surface d'une sphère, c'est-àdire comme $k_{f}^{2}$. Etant donné que l'épaisseur devra correspondre à un $d E_{f}$ fixe, $d k_{f}$ diminuera avec $k_{f}$. Avec $E_{f}=\hbar^{2} k_{f}^{2} /(2 m)$ on calcule $d k_{f}\left(E_{f}\right)=\hbar^{2} /\left(m k_{f}\right)$, c'est-à-dire $d k_{f}\left(E_{f}\right)$ est proportionnel à $k_{f}^{-1}$. La densité d'états (nombre de points dans $d \Omega d E_{f}$ ) n'est donc que linéaire en $k_{f}$. C'est le résultat de l'expression 5.6. La présence de $\rho_{\vec{k}}\left(E_{f}\right)$ dans l'expression pour la section efficace, a des conséquences pratiques importantes. Si $k_{f}$ devient très petit, comme c'est le cas pour les neutrons qui sont presque arrêtés par le processus de diffusion, il y aura très peu d'états finaux. En conséquence, le taux de diffusion pour une bande d'énergie finale donnée sera très faible. A l'inverse, la section efficace augmentera si $k_{i}$ diminue. Le flux étant proportionnel à la densité multipliée par la vitesse (4.15), la densité augmentera à flux constant avec l'inverse de la vitesse. Il y aura de plus en plus de neutrons simultanément présents dans la cible au fur et à mesure que la vitesse baisse.

correspondra impérativement à une intégration sur un groupe d'états finaux défini par les conditions expérimentales. C'est aussi la raison pour laquelle nous avons choisi d'appeler $w_{\vec{k}_{i} \rightarrow \vec{k}_{f}}$ une densité de probabilité de transition et non pas une simple probabilité de transition. Dans le cas de la diffusion, l'espace d'intégration est donné par l'énergie et l'angle solide. Formellement, nous imposons que la section efficace double différentielle, une fois intégrée sur les états finaux, donne la section efficace totale. Toute fonction, y compris la section efficace double différentielle, doit donc être définie par élément de stéradian $d \Omega$ et d'énergie finale $d E_{f}$. Pour passer de $d^{3} k$ à $d \Omega d E_{f}$ il faut la densité d'états $|\vec{k}\rangle$ c'est à dire la fonction qui nous donne le nombre d'états $|\vec{k}\rangle$ se trouvant dans un élément $d \Omega d E_{f}$. Cette fonction, notée $\rho_{\vec{k}}\left(E_{f}\right)$ est illustrée dans la figure 11 .

Penchons nous un peu plus en détail sur la notion de transition que nous venons d'introduire. Dans les sections précédentes nous avions toujours considéré les solutions du problème de diffusion comme stationnaires, le temps n'apparaissant pas explicitement. En particulier l'expression (4.139) sur laquelle nous basons notre discussion est le fruit d'un traitement stationnaire. L'état $\left|\vec{k}_{i}\right\rangle$ qui y figure, correspond à un flux continu de particules d' impulsion $\vec{p}=\hbar \vec{k}_{i}$. C'est le flux qui subsiste en l'absence de perturbation. Il est donc naturel de le considérer comme point de départ de la diffusion. C'est à partir de ce flux initial que nous créons l'onde de diffusion $\left|u_{\vec{k}_{i}}\right\rangle=\Omega\left|\vec{k}_{i}\right\rangle$ en introduisant la cible. Ceci est analogue à placer un bâton dans un ruisseau. Loin de la cible, l'onde de diffusion ne peut plus être 
distinguée d'une onde plane $\left|\vec{k}_{f}\right\rangle$. C'est l'état final de la diffusion. Pour que le courant de probabilité atteigne un régime stationnaire, il faudra "laisser du temps". La transition dont nous parlons correspond donc formellement à l' évolution du système perturbé entre $t \rightarrow-\infty$ vers $t \rightarrow+\infty$. C'est ce que nous avons déjà indiqué avec l'expression (2.2). Cette évolution assurera que la diffusion reste purement élastique.

\section{2 Équation maîtresse}

Passons maintenant à un système possédant des degrés de liberté internes. Dans ce but nous reformulons la section efficace double différentielle comme

$$
\frac{d^{2} \sigma_{\vec{k}_{i} \rightarrow \vec{k}_{f}}}{d \Omega d E_{f}}=\frac{2 \pi}{\hbar} \frac{\rho\left(E_{f}\right)}{J_{\vec{k}_{i}}}\left|\left\langle\vec{k}_{f}|\mathbf{T}| \vec{k}_{i}\right\rangle\right|^{2} \delta\left(E_{f}-E_{i}\right) .
$$

Cette formulation est justifiée par le caractère purement élastique de la diffusion en régime stationnaire.

Une cible avec des degrés de liberté internes peut subir des changements d'état pendant la collision. Les états du système (cible plus sonde) avant et après la collision sont des produits directs des états non-perturbés de la cible $|\vec{k}\rangle$ avec les états de la sonde $|\lambda\rangle$

$$
\begin{aligned}
& |i\rangle=\left|\vec{k}_{i}\right\rangle \otimes\left|\lambda_{i}\right\rangle=\left|\vec{k}_{i} \lambda_{i}\right\rangle, \\
& |f\rangle=\left|\vec{k}_{f}\right\rangle \otimes\left|\lambda_{f}\right\rangle=\left|\vec{k}_{f} \lambda_{f}\right\rangle .
\end{aligned}
$$

La diffusion pourra ainsi s' accompagner du passage de la cible de l'état $\left|\lambda_{i}\right\rangle$ vers l'état $\left|\lambda_{f}\right\rangle$, mais elle n'entraînera aucune autre modification. Si nous supposons de plus que les états de la cible sont liés, c'est-à-dire discrets, la probabilité de transition devient

$$
w_{\vec{k}_{i} \rightarrow \vec{k}_{f}, \lambda_{i} \rightarrow \lambda_{f}}=\frac{2 \pi}{\hbar}\left|\left\langle\lambda_{f}, \vec{k}_{f}|\mathbf{T}| \lambda_{i}, \vec{k}_{i}\right\rangle\right|^{2} \rho\left(E_{f}\right) .
$$

La transition interne pouvant impliquer un changement d'énergie, la conservation de l'énergie s'exprime par

$$
\delta\left(\left(E_{f}-E_{i}\right)-\left(E_{\lambda_{i}}-E_{\lambda_{f}}\right)\right) .
$$

La densité des états finaux est à prendre pour $k_{f}$ (qui n'est plus nécessairement identique à $k_{i}$, qui détermine selon (4.21) le flux incident). En recombinant ces résultats nous obtenons

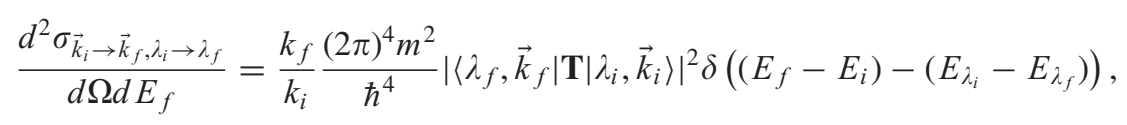

ou dans l'approximation de Born

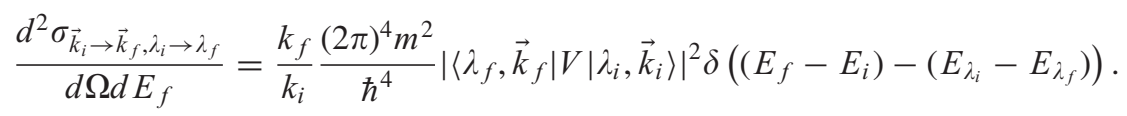

Dans l'expérience de diffusion nous n'observons pas les états finaux de la cible. Les sections efficaces conditionnelles — la diffusion ne se fera qu'à condition que la cible passe de $\left|\lambda_{i}\right\rangle$ à $\left|\lambda_{f}\right\rangle$ — sont par conséquent inaccessibles dans une expérience de diffusion simple. ${ }^{21} \mathrm{Ne}$ connaissant pas les états finaux, nous devons donc sommer ${ }^{22}$ les sections efficaces leur correspondant.

Un argument similaire vaut pour les états initiaux. Nous n'en connaissons que leur occupation statistique $p\left(\lambda_{i}\right)$ (voir chapitre I). Un exemple concret est l'occupation des phonons en fonction de la

\footnotetext{
21 On pourrait imaginer une expérience de diffusion complexe où l'état final de la cible serait identifié par une mesure supplémentaire, comme l'émission d'une autre particule (par exemple d'un photon ou d'un électron).

22 Nous pouvons toujours présumer qu'il s'agit d'états liés et donc discrets. Là où ce n'est pas le cas, la sommation est à remplacer par une intégration.
} 
température. Pour obtenir la section efficace observable nous devons par conséquent effectuer également une somme sur les états initiaux mais en prenant en compte leur poids statistique $p\left(\lambda_{i}\right)$. Etant donné que le système doit obligatoirement se trouver dans un de ces états, la somme des poids doit être égale à un.

Avec ces considérations, nous aboutissons à l'expression

$$
\frac{d^{2} \sigma_{\vec{k}_{i} \rightarrow \vec{k}_{f}}}{d \Omega d E_{f}}=\frac{k_{f}}{k_{i}} \frac{(2 \pi)^{4} m^{2}}{\hbar^{4}} \sum_{\lambda_{i}, \lambda_{f}} p\left(\lambda_{i}\right) \mid\left\langle\lambda_{f}, \vec{k}_{f}|V| \lambda_{i}, \vec{k}_{i}>\left.\right|^{2} \delta\left(\left(E_{f}-E_{i}\right)-\left(E_{\lambda_{i}}-E_{\lambda_{f}}\right)\right) .\right.
$$

Cette expression est appelée équation maîtresse la diffusion. Nous rapellons qu'elle est basée sur l'approximation de Born et que les fonctions $\langle\vec{r} \mid \vec{k}\rangle$ sont normalisées selon 4.19. Nous allons voir que pratiquement toutes les expériences de diffusion de neutrons de basse énergie peuvent être interprétées dans le cadre de cette équation.

\subsection{Facteur de forme du potentiel d'interaction}

Le premier pas dans l'évaluation de l'élément de matrice qui figure dans l'équation maîtresse, est son intégration par rapport à la variable $\vec{r}$ du neutron. En général, la diffusion de la particule sonde par un diffuseur individuel ne sera pas influencée par la présence d'autres diffuseurs. Nous pouvons donc décomposer le potentiel d'interaction en contributions individuelles

$$
V(\vec{r})=\sum_{j=1}^{N} V_{j}\left(\vec{r}-\vec{R}_{j}\right)=\int_{V} d r^{\prime 3} \sum_{j=1}^{N} V_{j}\left(\vec{r}^{\prime}\right) \delta\left(\vec{r}-\vec{R}_{j}\right)
$$

avec $V_{j}\left(\vec{r}-\vec{R}_{j}\right)$ le potentiel d'interaction d'un diffuseur caractérisé par son opérateur de position $\vec{R}_{j}$. La deuxième égalité souligne le fait qu'il s'agit d'une convolution des positions avec les potentiels d'interaction.

Nous devons intégrer l'élément de matrice $\left\langle\lambda_{f}, \vec{k}_{f}|V| \lambda_{i}, \vec{k}_{i}\right\rangle$, qui constitue le cœur de l'équation maîtresse.

$$
\begin{aligned}
& \left\langle\lambda_{f}, \vec{k}_{f}|V| \lambda_{i}, \vec{k}_{i}\right\rangle \\
& =\sum_{j=1}^{N} \frac{1}{(2 \pi)^{3}} \int d^{3} R_{1} \ldots \int d^{3} R_{N} \int d^{3} r \psi_{\lambda_{f}}^{*}\left(e^{-i \vec{k}_{f} \cdot \vec{r}} V_{j}\left(\vec{r}-\vec{R}_{j}\right) e^{i \vec{k}_{i} \cdot \vec{r}}\right) \psi_{\lambda_{i}} .
\end{aligned}
$$

Si nous présumons que le neutron est une perturbation faible, alors les fonctions propres de la cible

$$
\psi_{\lambda}=\langle r \mid \lambda\rangle=\psi_{\lambda}\left(\left\{\vec{R}_{j}\right\}\right)
$$

ne dépendent que des coordonnées $\vec{R}_{j}$.

Etant donné que l'intégrale s'étend sur tout l'espace, nous pouvons choisir librement l'origine de la variable d'intégration correspondant à la sonde pour chaque terme $j$ de la somme de l'expression 5.16. En particulier, en définissant (voir figure 12)

$$
\vec{r}_{j}=\vec{r}-\vec{R}_{j}
$$

nous obtenons

$$
\begin{aligned}
& \int d^{3} R_{1} \ldots \int d^{3} R_{N} \int d^{3} r \psi_{\lambda_{f}}^{*}\left(e^{-i \vec{k}_{f} \cdot \vec{r}} V_{j}\left(\vec{r}-\vec{R}_{j}\right) e^{i \vec{k}_{i} \cdot \vec{r}}\right) \psi_{\lambda_{i}} \\
& =\int d^{3} R_{1} \ldots \int d^{3} R_{N} \int d^{3} r_{j} \psi_{\lambda_{f}}^{*}\left(e^{-i \vec{k}_{f} \cdot\left(\vec{r}_{j}+\vec{R}_{j}\right)} V_{j}\left(\vec{r}_{j}\right) e^{i \vec{k}_{i} \cdot\left(\vec{r}_{j}+\vec{R}_{j}\right)}\right) \psi_{\lambda_{i}} \\
& =\int d^{3} R_{1} \ldots \int d^{3} R_{N} \psi_{\lambda_{f}}^{*} e^{i \vec{Q} \cdot \vec{R}_{j}} \psi_{\lambda_{i}} \int d^{3} r_{j} e^{-i \vec{Q} \cdot \vec{r}_{j}} V_{j}\left(\vec{r}_{j}\right) .
\end{aligned}
$$




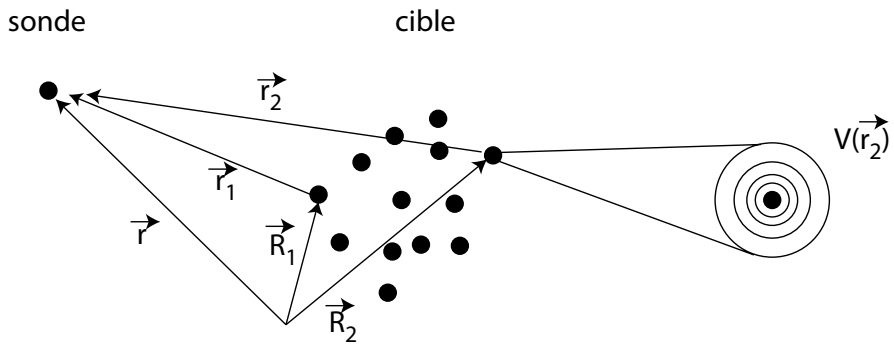

Figure 12. Coordonnées utilisées pour le calcul de $V_{j}(\vec{Q})(5.21)$ à partir du potentiel d'interaction $V_{j}\left(\vec{R}_{j}\right)$.

Grâce à (5.19), l'élément de matrice apparaissant dans l'équation maîtresse peut s'écrire de manière plus compacte

$$
\left\langle\lambda_{f}, \vec{k}_{f}|V| \lambda_{i}, \vec{k}_{i}\right\rangle=\frac{1}{(2 \pi)^{3}} \sum_{j=1}^{N} V_{j}(\vec{Q})\left\langle\lambda_{f}\left|e^{i \vec{Q} \cdot \vec{R}_{j}}\right| \lambda_{i}\right\rangle
$$

La fonction

$$
V_{j}(\vec{Q})=\int d^{3} r_{j} e^{-i \vec{Q} \cdot \vec{r}_{j}} V_{j}\left(\vec{r}_{j}\right)
$$

est la transformée de Fourier du potentiel d'interaction du diffuseur $j$. Elle rappelle l'expression analogue (4.141) que nous avions obtenue pour la diffusion par un potentiel statique. La fonction $V_{j}(\vec{Q})$ peut êre assimilée à un facteur de forme du potentiel $V_{j}\left(\vec{r}_{j}\right)$ du diffuseur $j$.

C'est de nouveau un résultat important. ${ }^{23}$ L'élément de matrice est factorisé en deux termes. Le premier, le facteur de forme du potentiel

$$
V_{j}(\vec{Q})
$$

est une fonction qui caractérise les diffuseurs individuellement. ${ }^{24}$ L'interaction d'un diffuseur avec la sonde est entièrement contenue dans son facteur de forme. Ainsi, le potentiel d'interaction ne figure plus dans le deuxième facteur

$$
\left\langle\lambda_{f}\left|e^{i \vec{Q} \cdot \vec{R}_{j}}\right| \lambda_{i}\right\rangle
$$

qui est une fonction qui dépend uniquement de la cible.

En utilisant les facteurs de forme $V_{j}(\vec{Q})$ du potentiel d'interaction, l'équation maîtresse (5.14) devient

$$
\frac{d \sigma_{\vec{k}_{i} \rightarrow \vec{k}_{f}}}{d \Omega d E_{f}}=\frac{k_{f}}{k_{i}} \frac{m^{2}}{(2 \pi)^{2} \hbar^{4}} \sum_{\lambda_{i}, \lambda_{f}} p\left(\lambda_{i}\right)\left|\sum_{j=1}^{N} V_{j}(\vec{Q})\left\langle\lambda_{f}\left|e^{i \vec{Q} \cdot \vec{R}_{j}}\right| \lambda_{i}\right\rangle\right|^{2} \delta\left(\left(E_{f}-E_{i}\right)-\left(E_{\lambda_{i}}-E_{\lambda_{f}}\right)\right) .
$$

\footnotetext{
${ }^{23}$ Nous aurions pu anticiper ce résultat en se rappelant que dans l'approximation de Born, l'amplitude de diffusion est la transformée de Fourier du potentiel (voir (4.141)). Pour un potentiel qui est, comme le montre (5.15), la convolution de deux fonctions, la transformée de Fourier est le produit des transformées de Fourier individuelles. Nous reviendrons sur ce point à l'occasion de la discussion des analogies avec la diffraction optique (section 7).

${ }^{24}$ Il n'y a aucune ambiguité dans la définition du potentiel si le diffuseur est une particule élémentaire sans dégré de liberté interne (nous rappelons que nous négligeons le spin dans cette discussion). En général, ce que nous appelons un diffuseur est néanmoins un objet composé. C'est à la fois vrai pour les noyaux de la diffusion nucléaire et pour les nuages électroniques des ions magnétiques. Nous ne pouvons négliger ces structures internes que sous la condition qu'elles soient stables en regard du processus de diffusion. En d'autres termes, l'interaction avec l'objet composé peut être décrite par un potentiel effectif pourvu que le neutron ne déclenche pas de transitions entre les niveaux d'excitations. Pour les noyaux c'est le cas hors résonance. Pour les niveaux électroniques, c'est en général le cas pour les énergies typiques d'une expérience de diffusion, inférieures à $100 \mathrm{meV}$. Au dessus de $100 \mathrm{meV}$, les transitions entre multiplets deviennent possibles et doivent être prises en compte dans le traitement formel de la diffusion (voir [7]).
} 


\subsection{Pseudo-potentiel de Fermi}

Nous voudrions maintenant évaluer le facteur de forme pour des potentiels de très courte portée. C'est notamment le cas pour la diffusion des neutrons par les noyaux, qui se joue sur l'échelle nucléaire du femtomètre. Si nous utilisons le potentiel effectif développé dans la section 4.4 nous obtenons ${ }^{25}$

$$
V_{j}(\vec{Q}) \int d^{3} r V(\vec{r}) e^{-i \vec{Q} \cdot \vec{r}}=\left.\frac{2 \pi \hbar^{2}}{m} b_{j} \int d^{3} r \delta(\vec{r}) \frac{d}{d r}\right|_{r=0} r e^{-i \vec{Q} \cdot \vec{r}} .
$$

Nous pouvons évaluer cette expression en développant la fonction $e^{-i \vec{Q} \cdot \vec{r}}$ en ondes partielles (voir 4.51) et en nous souvenant du comportement de la partie radiale de ces ondes pour $\rho \rightarrow 0$ (voir 4.48).

$$
V_{j}(\vec{Q})=\frac{2 \pi \hbar^{2}}{m} b_{j}
$$

La transformée de Fourier d'un potentiel ponctuel devant donner une constante, ce résultat n'est donc pas étonnant. Nous aurions obtenu le même résultat avec le potentiel plus simple

$$
V_{\mathrm{eff}}(r) \psi(r)=\frac{2 \pi \hbar^{2}}{m} b_{j} \delta(\vec{r}),
$$

qui est connu sous le nom de pseudo-potentiel de Fermi. La section efficace conditionnelle (5.22) prend donc la forme

$$
\left.\frac{d^{2} \sigma_{\vec{k}_{i} \rightarrow \vec{k}_{f}, \lambda_{i} \rightarrow \lambda_{f}}}{d \Omega d E_{f}}=\frac{k_{f}}{k_{i}}\left|\sum_{j=1}^{N} b_{j}\left\langle\lambda_{f}\left|e^{i \vec{Q}^{\vec{R}_{j}}}\right| \lambda_{i}\right\rangle\right|^{2} \delta\left(\left(E_{\lambda_{i}}-E_{\lambda_{f}}\right)+\hbar \omega\right)\right),
$$

où nous avons introduit

$$
\hbar \omega=E_{i}-E_{f}
$$

pour définir l'énergie cédée par le neutron à la cible.

\subsection{La fonction de diffusion $S(\vec{Q}, \omega)$}

L'équation maîtresse (5.22), que nous avons obtenue pour la section efficace différentielle partielle, contient une somme qui porte sur les états excités de la cible. Une solution qui nous aurait permis de calculer la section efficace à partir de l'état d'équilibre de la cible aurait été préférable. On pourrait être tenté d'invoquer la relation de fermeture

$$
\sum_{\lambda_{f}}\left|\lambda_{f}\right\rangle\left\langle\lambda_{f}\right|=\mathbf{I}
$$

avec I l'opérateur d'identité, pour remédier à cet inconvénient. La présence de la fonction $\left.\Delta\left(\left(E_{\lambda_{i}}-E_{\lambda_{f}}\right)+\hbar \omega\right)\right)$ dans l'expression (5.14) hélas nous en empêche. Pour contourner cet obstacle nous devons utiliser l'astuce qui consiste à exprimer une fonction de Dirac en énergie comme la transformée de Fourier d'une constante dans le temps.

$$
\begin{aligned}
\delta\left(E_{\lambda_{i}}-E_{\lambda_{f}}+\hbar \omega\right) & =\frac{1}{2 \pi \hbar} \int_{-\infty}^{\infty} d t \exp \left(-\frac{i}{\hbar}\left(E_{\lambda_{i}}-E_{\lambda_{f}}+\hbar \omega\right) t\right) \\
& =\frac{1}{2 \pi \hbar} \int_{-\infty}^{\infty} d t \exp \left(-\frac{i}{\hbar}\left(E_{\lambda_{i}}-E_{\lambda_{f}}\right) t\right) \cdot \exp (-i \omega t) .
\end{aligned}
$$

\footnotetext{
25 Nous plaçons le diffuseur à l'origine par commodité.
} 
Les énergies $E_{\lambda}$, qui figurent dans cette expression, sont les valeurs propres de l'Hamiltonien de la cible

$$
\mathbf{H}|\lambda\rangle=E_{\lambda}|\lambda\rangle .
$$

Pour une fonction $f(\mathbf{H})$ quelconque de cet Hamiltonien, agissant sur une de ses fonctions propres, nous obtenons

$$
f(\mathbf{H})|\lambda\rangle=f\left(E_{\lambda}\right)|\lambda\rangle .
$$

En particulier,

$$
\exp \left(\frac{i}{\hbar} \mathbf{H} t\right)|\lambda\rangle=\exp \left(\frac{i}{\hbar} E_{\lambda} t\right)|\lambda\rangle
$$

Retournons maintenant à l'équation maîtresse (5.26). La partie centrale de cette équation est le carré de la somme des éléments de matrice. Nous exprimons ce carré en somme double

$$
\left|\sum_{j=1}^{N} b_{j}\left\langle\lambda_{f}\left|e^{i \vec{Q} \cdot \vec{R}_{j}}\right| \lambda_{i}\right\rangle\right|^{2}=\sum_{j, j^{\prime}=1}^{N} b_{j} b_{j^{\prime}}^{*}\left\langle\lambda_{f}\left|e^{i \vec{Q} \cdot \vec{R}_{j}}\right| \lambda_{i}\right\rangle\left\langle\lambda_{i}\left|e^{-i \vec{Q} \cdot \vec{R}_{j^{\prime}}}\right| \lambda_{f}\right\rangle .
$$

A chaque terme, assorti de sa fonction $\delta(E)$, nous appliquons l'expression (5.29) de la manière suivante :

$$
\begin{aligned}
& \left\langle\lambda_{f}\left|e^{i \vec{Q} \cdot \vec{R}_{j}}\right| \lambda_{i}\right\rangle\left\langle\lambda_{i}\left|e^{-i \vec{Q} \cdot \vec{R}_{j^{\prime}}}\right| \lambda_{f}\right\rangle \delta\left(E_{\lambda_{i}}-E_{\lambda_{f}}-\hbar \omega\right) \\
& =\frac{1}{2 \pi \hbar} \int_{-\infty}^{\infty} d t\left\langle\lambda_{f}\left|e^{i \frac{E_{\lambda_{f}}}{\hbar} t} e^{i \vec{Q} \cdot \vec{R}_{j}} e^{-i \frac{E_{\lambda_{i}}}{\hbar} t}\right| \lambda_{i}\right\rangle\left\langle\lambda_{i}\left|e^{-i \vec{Q} \cdot \vec{R}_{j^{\prime}}}\right| \lambda_{f}\right\rangle e^{-i \omega t} .
\end{aligned}
$$

Les énergies figurant dans les exponentielles peuvent selon (5.32) être remplacées par l'opérateur de Hamilton. Nous obtenons

$$
\begin{aligned}
& \left\langle\lambda_{f}\left|e^{i \vec{Q} \cdot \vec{R}_{j}}\right| \lambda_{i}\right\rangle\left\langle\lambda_{i}\left|e^{-i \vec{Q} \cdot \vec{R}_{j^{\prime}}}\right| \lambda_{f}\right\rangle \delta\left(E_{\lambda_{i}}-E_{\lambda_{f}}-\hbar \omega\right) \\
& =\frac{1}{2 \pi \hbar} \int_{-\infty}^{\infty} d t\left\langle\lambda_{f}\left|e^{i \frac{\mathrm{H}}{\hbar} t} e^{i \vec{Q} \cdot \vec{R}_{j}} e^{-i \frac{\mathrm{H}}{\hbar} t}\right| \lambda_{i}\right\rangle\left\langle\lambda_{i}\left|e^{-i \vec{Q} \cdot \vec{R}_{j^{\prime}}}\right| \lambda_{f}\right\rangle e^{-i \omega t} \\
& =\frac{1}{2 \pi \hbar} \int_{-\infty}^{\infty} d t\left\langle\lambda_{i}\left|e^{-i \vec{Q} \cdot \vec{R}_{j^{\prime}}}\right| \lambda_{f}\right\rangle\left\langle\lambda_{f}\left|e^{i \frac{\mathrm{H}}{\hbar} t} e^{i \vec{Q} \cdot \vec{R}_{j}} e^{-i \frac{\mathrm{H}}{\hbar} t}\right| \lambda_{i}\right\rangle e^{-i \omega t}
\end{aligned}
$$

où nous avons interverti l'ordre des éléments de matrice dans la dernière ligne. Nous sommes ainsi parvenus au stade où nous pouvons appliquer la relation de fermeture (5.28)

$$
\begin{aligned}
& \sum_{\lambda_{f}}\left\langle\lambda_{f}\left|e^{i \vec{Q} \cdot \vec{R}_{j}}\right| \lambda_{i}\right\rangle\left\langle\lambda_{i}\left|e^{-i \vec{Q} \cdot \vec{R}_{j^{\prime}}}\right| \lambda_{f}\right\rangle \delta\left(E_{\lambda_{i}}-E_{\lambda_{f}}-\hbar \omega\right) \\
& =\frac{1}{2 \pi \hbar} \int_{-\infty}^{\infty} d t\left\langle\lambda_{f}\left|e^{-i \vec{Q} \cdot \vec{R}_{j^{\prime}}} e^{i \frac{\mathrm{H}}{\hbar} t} e^{i \vec{Q} \cdot \vec{R}_{j}} e^{-i \frac{\mathrm{H}}{\hbar} t}\right| \lambda_{f}\right\rangle e^{-i \omega t} .
\end{aligned}
$$

A ce point il est important de mentionner que l'ordre dans lequel apparaissent les $\vec{R}_{j}$ est figé parce que les opérateurs $\vec{R}_{j}$ ne commutent pas avec l' Hamiltonien $\mathbf{H}^{26}$ Les opérateurs $\exp (-(i / \hbar) \mathbf{H} t) \operatorname{sont}$ bien connus en mécanique quantique. Il s'agit des opérateurs d'évolution dont nous avions parlé dans la section 2

$$
\mathbf{U}\left(t, t_{0}\right)=e^{-\frac{i}{\hbar} \mathbf{H}\left(t-t_{0}\right)} .
$$

Appliqué à un opérateur $\mathbf{A}$ de la façon suivante

$$
\mathbf{A}(t)=\mathbf{U}^{+}\left(t, t_{0}\right) \mathbf{A}\left(t_{0}\right) \mathbf{U}\left(t, t_{0}\right)=e^{\frac{i}{\hbar} \mathbf{H}\left(t-t_{0}\right)} \mathbf{A}\left(t_{0}\right) e^{-\frac{i}{\hbar} \mathbf{H}\left(t-t_{0}\right)}
$$

\footnotetext{
${ }^{26} \mathbf{H}$ contient les opérateurs d'impulsion $\vec{P}_{j}$, qui ne commutent pas avec $\vec{R}_{j}$.
} 
l'opérateur $\mathbf{U}$ en décrit l'évolution temporaire. ${ }^{27}$ En identifiant $\mathbf{A}$ avec $\overrightarrow{\mathbf{R}}_{j}^{0}=\overrightarrow{\mathbf{R}}_{j}(t=0)$ nous en déduisons ${ }^{28}$

$$
e^{i \frac{\mathrm{H}}{\hbar} t} e^{i \vec{Q} \cdot \vec{R}_{j}} e^{-i \frac{\mathrm{H}}{\hbar} t}=e^{i \vec{Q} \cdot \vec{R}_{j}(t)} .
$$

Les termes de l'équation maîtresse prennent la forme

$$
\begin{gathered}
\sum_{\lambda_{f}}\left\langle\lambda_{f}\left|e^{i \vec{Q} \cdot \vec{R}_{j}}\right| \lambda_{i}\right\rangle\left\langle\lambda_{i}\left|e^{-i \vec{Q} \cdot \vec{R}_{j^{\prime}}}\right| \lambda_{f}\right\rangle \delta\left(E_{\lambda_{i}}-E_{\lambda_{f}}-\hbar \omega\right) \\
=\frac{1}{2 \pi \hbar} \int_{-\infty}^{\infty} d t\left\langle\lambda_{i}\left|e^{-i \vec{Q} \cdot \vec{R}_{j^{\prime}}^{0}} e^{i \vec{Q}^{\prime} \cdot \vec{R}_{j}(t)}\right| \lambda_{i}\right\rangle e^{-i \omega t} .
\end{gathered}
$$

La section efficace différentielle partielle devient

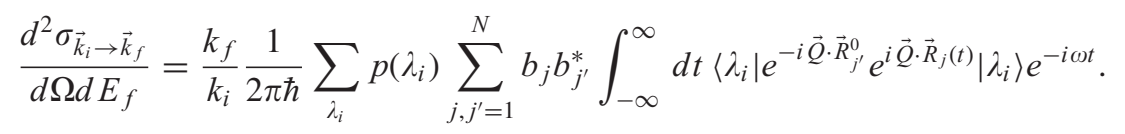

Nous écrivons ce résultat dans la forme plus compacte

avec

$$
\frac{d^{2} \sigma_{\vec{k}_{i} \rightarrow \vec{k}_{f}}}{d \Omega d E_{f}}=\frac{k_{f}}{k_{i}} S(\vec{Q}, \omega)
$$

$$
S(\vec{Q}, \omega)=\frac{1}{2 \pi \hbar} \sum_{\lambda_{i}} p\left(\lambda_{i}\right) \sum_{j, j^{\prime}=1}^{N} b_{j} b_{j^{\prime}}^{*} \int_{-\infty}^{\infty} d t\left\langle\lambda_{i}\left|e^{-i \vec{Q} \cdot \vec{R}_{j^{\prime}}^{0}} e^{i \vec{Q} \cdot \vec{R}_{j}(t)}\right| \lambda_{i}\right\rangle e^{-i \omega t}
$$

$S(\vec{Q}, \omega)$ est appelée la fonction de diffusion.

Nous allons brièvement commenter ce résultat.

- Nous rappelons que l'expression (5.43) décrit la diffusion dans l'approximation de Born.

- L'expression (5.42) est valable pour des potentiels d'interaction de très courte portée qui sont décrits par des longueurs de diffusion $b_{j}$. Le passage à un potentiel d'interaction plus général se fait à partir de (5.22) en substituant les longueurs de diffusions $b_{j}$ par les facteurs de forme du potentiel d'interaction individuel (5.21) selon

$$
b_{j} \rightarrow \frac{m}{2 \pi \hbar^{2}} V_{j}(\vec{Q})
$$

- La fonction de diffusion est une grandeur physique extensive de la cible. En d'autres termes, elle est proportionnelle au nombre de diffuseurs. Elle est souvent rapportée normalisée, soit au nombre d'atomes présents dans l'unité chimique, soit au nombre d'atomes dans la maille primitive (voir chapitre I).

- La proportionnalité de la fonction de diffusion au nombre de diffuseurs montre clairement les limites de l'approximation de Born. Il n'est pas possible que le nombre de particules diffusées croisse sans limite pour un flux incident donné. A partir du moment où la grande majorité des particules est diffusée il ne servira plus à rien d' augmenter le volume de l'échantillon. On parle d'effets d'extinction. Dans la pratique l'extinction est observée lors de la diffusion de Bragg. Quand un cristal est de bonne qualité, le réservoir de neutrons obéissant à la condition de

\footnotetext{
27 Le lecteur soucieux de connaître la justification formelle de cette relation en trouvera l'explication dans tous les livres de mécanique quantique.

28 Pour ne pas alourdir trop le formalisme nous nous abstenons, dans la plupart des formules, à explicitement indiquer le caractère d'opérateur pour les $\vec{R}_{j}$.
} 
diffusion élastique dans une direction de Bragg donnée est épuisé par quelques milliers de plans atomiques. Il est évident qu'au-delà, l'onde incidente ne pourra plus être considérée comme invariante sur le volume de l'échantillon. Mais c'est exactement ce que nous présumons en utilisant l'approximation de Born. On devra alors se tourner vers la théorie dynamique de la diffusion $[9,10]$. Nous allons revenir sur ce problème dans la section 9.4 . Un autre cas où l'approximation de Born n'est pas, par définition, adaptée est celui de la diffusion totale ou rasante. Dans ce cas, la probabilité de diffusion est de $100 \%$ et on ne pourra plus considérer la diffusion comme une simple perturbation de l'onde incidente. Ce cas est traîté avec la Distorted Born Approximation [11,12].

- La fonction de diffusion ne dépend plus explicitement des états finaux. En équilibre thermodynamique ces états sont toujours présents dans les facteurs $p_{\lambda_{i}}$. Comme nous avons vu dans le chapitre I ces probabilités s'expriment comme

$$
p_{n}=\frac{1}{Z} \exp \left(-\frac{E_{n}}{k_{\mathrm{b}} T}\right)
$$

avec la fonction de partition canonique $Z$ définie par

$$
Z=\sum_{n} \exp \left(-\frac{E_{n}}{k_{\mathrm{b}} T}\right)
$$

Pour calculer $Z$ on a besoin de connaître le spectre complet des excitations.

- La fonction de diffusion est une grandeur de l'état d'équilibre de la cible. Nous allons revenir sur ce point dans le contexte de la susceptibilité.

- Si la sonde possède des degrés de liberté internes, ceci rajoute des processus supplémentaires de diffusion. Pour les neutrons, ces canaux sont liés aux deux états de spin $|\sigma\rangle=| \pm\rangle$. Soit $p\left(\sigma_{i}\right)$, la probabilité de trouver un neutron dans l'état de spin $\left|\sigma_{i}\right\rangle$ avant la collision. Supposons que l'état final du spin $\left|\sigma_{f}\right\rangle$ ne soit pas analysé. La fonction de diffusion devient alors

$$
S(\vec{Q}, \omega)=\frac{1}{2 \pi \hbar} \sum_{\lambda_{i}, \sigma_{i}, \sigma_{f}} p\left(\lambda_{i}\right) p\left(\sigma_{i}\right) \sum_{j, j^{\prime}=1}^{N} b_{j} b_{j^{\prime}}^{*} \int_{-\infty}^{\infty} d t\left\langle\sigma_{f} ; \lambda_{i}\left|e^{-i \vec{Q} \cdot \vec{R}_{j^{\prime}}^{0}} e^{i \vec{Q} \cdot \vec{R}_{j}(t)}\right| \lambda_{i} ; \sigma_{i}\right\rangle e^{-i \omega t}
$$

\subsection{Diffusion cohérente et diffusion incohérente}

Dans l'expression (5.43), que nous avons obtenue pour la fonction de diffusion, nous rencontrons une double somme sur les diffuseurs que nous pouvons formellement exprimer comme

$$
S(\vec{Q}, \omega)=\sum_{j, j^{\prime}=1}^{N}\left(b_{j} b_{j^{\prime}}^{*}\right) S_{j, j^{\prime}}(\vec{Q}, \omega)
$$

avec

$$
S_{j, j^{\prime}}(\vec{Q}, \omega) \frac{1}{2 \pi \hbar} \sum_{\lambda_{i}} p\left(\lambda_{i}\right) \int_{-\infty}^{\infty} d t\left\langle\lambda_{i}\left|e^{-i \vec{Q} \cdot \vec{R}_{j^{\prime}}^{0}} e^{i \vec{Q} \cdot \vec{R}_{j}(t)}\right| \lambda_{i}\right\rangle e^{-i \omega t}
$$

Tandis que le terme $b_{j} b_{j^{\prime}}^{*}$ dépend des noyaux, la fonction $S_{j, j^{\prime}}(\vec{Q}, \omega)$ reflète l'état chimique de la cible. La diffusion des neutrons a ceci de particulier que le potentiel de diffusion nucléaire peut varier d'un atome à l'autre pour le même élément chimique. Ces variations sont dûes au fait que les neutrons sont sensibles aux isotopes et à l'état de spin du noyau. Les isotopes, ainsi que les états de spin, sont en général distribués de manière aléatoire. Les interactions entre noyaux, qui pourraient aligner les spins, sont très faibles à cause de leur nature indirecte. Dans la matière condensée, ces interactions 


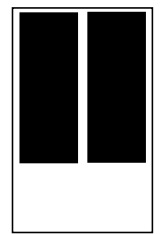

(1) 2

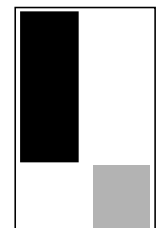

12

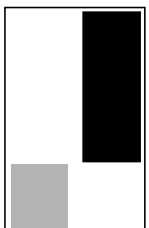

12

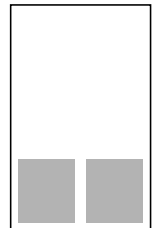

12

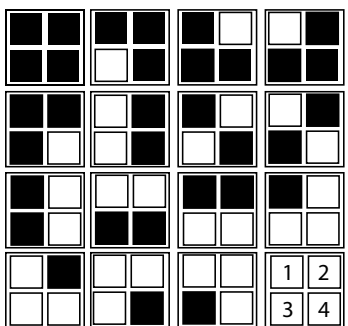

Figure 13. Illustration de la distribution aléatoire des longueurs de diffusions. Dans l'exemple en haut nous nous imaginons un système composé de deux atomes identiques (par exemple une molécule diatomique). Nous présumons que le noyau de l'atome possède deux isotopes également probables. Les deux isotopes possèdent des longueurs de diffusion différentes. L'une est présumée positive et l'autre négative. Il n'y a aucune corrélation entre la position de l'atome et l'isotope. Quand nous effectuons le calcul de la section efficace, nous devons moyenner sur les 4 configurations possibles. Pour reprendre l'image de la molécule, nous additionnons la section efficace sur un ensemble de molécules, représentatif pour de la distribution isotopique. Dans la figure en bas nous traitons le cas de 4 atomes avec deux longueurs de diffusion équi-réparties. Les seize configurations données sont représentatives de cette distribution. Nous pouvons nous imaginer que les 4 atomes correspondent au volume de corrélation de la cible. En faisant balayer ce volume sur la cible nous moyennons parfaitement la distribution d'isotopes.

sont relayées par les électrons (interaction hyperfine). ${ }^{29}$ On voit que l'argument ne s'applique pas à la diffusion des rayons- $\mathrm{X}$ : la diffusion des neutrons se fait alors par le cortège électronique ce qui la rend ainsi sensible qu'à la seule nature chimique du diffuseur.

Une répartition aléatoire des longueurs de diffusion sur les sites $j$ des atomes implique qu'on ne pourra pas déduire d'information sur la longueur de diffusion à partir de la position $\vec{R}_{j}$. Mathématiquement cet enoncé se traduit par l'absence de corrélation entre $b_{j}$ et $\vec{R}_{j}$. Le désordre présent dans le système des noyaux concerne donc exclusivement les termes $\left(b_{j} b_{j^{\prime}}^{*}\right)$ et non pas $S_{j, j^{\prime}}(\vec{Q}, \omega)$.

Nous voudrions illustrer cette situation par un exemple simple. Nous considérons une cible composée de deux atomes du même type chimique. Les noyaux de ces deux atomes sont présents sous deux formes isotopiques, dont nous étiquetons les longueurs de diffusion associées avec $c_{1}$ et $c_{2}$, respectivement. Ces longueurs de diffusion sont présumées réelles. Nous recopions le système dans ses quatre combinaisons isotopiques possibles. Chaque copie correspondra à une cible pour laquelle nous calculons la fonction de diffusion. A partir de ces fonctions de diffusion particulières nous déterminons la fonction de diffusion moyenne. Nous regardons d'abord les membres distincts de la somme (5.48). Pour une paire $j, j^{\prime}$ nous avons à traiter quatre cas de figure(voir figure 13)

$$
j \neq j^{\prime} \quad: \quad b_{j} b_{j^{\prime}}=c_{1} \cdot c_{1}, \quad b_{j} b_{j^{\prime}}=c_{1} \cdot c_{2}, \quad b_{j} b_{j^{\prime}}=c_{2} \cdot c_{1}, \quad b_{j} b_{j^{\prime}}=c_{2} \cdot c_{2} .
$$

Nous pouvons donc écrire

$$
j \neq j^{\prime} \quad: \quad\left(b_{j} b_{j^{\prime}}\right) S_{j, j^{\prime}}(\vec{Q}, \omega)=\frac{1}{4}\left(c_{1} \cdot c_{1}+2 c_{1} \cdot c_{2}+c_{2} \cdot c_{2}\right) S_{j, j^{\prime}}(\vec{Q}, \omega)(\bar{c})^{2} S_{j, j^{\prime}}(\vec{Q}, \omega)
$$

\footnotetext{
29 Nous voudrions néanmoins souligner qu'il existe des systèmes où les spins des noyaux sont corrélés à très basse température.
} 
Les termes distincts de la fonction de diffusion sont donc proportionnels à la longueur de diffusion moyenne $\bar{c}=(1 / 2)\left(c_{1}+c_{2}\right)$ au carré. Pour les termes propres $j=j^{\prime}$ nous obtenons par analogie

$$
j=j^{\prime} \quad: \quad b_{j} b_{j}=c_{1} \cdot c_{1}(2 \text { fois }), \quad b_{j} b_{j}=c_{2} \cdot c_{2}(2 \text { fois }),
$$

ce qui nous amène à

$$
j=j^{\prime} \quad: \quad\left(b_{j} b_{j}\right) S_{j, j}(\vec{Q}, \omega)=\frac{1}{2}\left(c_{1} \cdot c_{1}+c_{2} \cdot c_{2}\right) S_{j, j}(\vec{Q}, \omega) \overline{c^{2}} S_{j, j}(\vec{Q}, \omega) .
$$

Les termes propres de la fonction de diffusion sont donc proportionnels à la moyenne des carrés des longueurs de diffusion $\overline{c^{2}}=(1 / 2)\left(c_{1}^{2}+c_{2}^{2}\right)$.

Nous allons maintenant généraliser ce résultat. Nous partons d'un système macroscopique avec un grand nombre de diffuseurs. A priori ce système correspond à une fonction de diffusion précise, qu'il nous faut calculer. ${ }^{30}$ Comme nous allons voir plus loin (voir section 9.4) la partie donnant lieu à des interférences, le volume de corrélation, est néanmoins de dimension microscopique, en particulier pour des faisceaux de faible cohérence. Nous pouvons donc considérer que le système macroscopique est un ensemble de répliques du volume de corrélation. Nous pouvons imaginer que parmi ces répliques, nous trouvons toutes les distributions possibles d'isotopes ou de spins nucléaires avec la même fréquence et qu'il n'y a aucune corrélation spatiale et temporelle des occupations isotopiques et de spin nucléaire. Comme dans notre exemple, nous obtenons la fonction de diffusion en moyennant sur les répliques

$$
S(\vec{Q}, \omega)=\sum_{j, j^{\prime}=1}^{N} \overline{\left(b_{j} b_{j^{\prime}}^{*}\right)} S_{j, j^{\prime}}(\vec{Q}, \omega) .
$$

En l'absence de corrélation entre la longueur de diffusion et la position des noyaux

$$
\begin{aligned}
& j \neq j^{\prime} \quad: \quad \overline{\left(b_{j} b_{j^{\prime}}\right)}=\overline{b_{j}} \overline{b_{j^{\prime}}^{*}}, \\
& j=j^{\prime} \quad: \quad \overline{\left(b_{j} b_{j}\right)}=\overline{b_{j}^{2}},
\end{aligned}
$$

avec

$$
\begin{aligned}
& \overline{b_{j}}=\sum_{i} p_{j}^{i} \cdot b_{j}^{i}, \\
& \overline{b_{j}^{2}}=\sum_{i} p_{j}^{i} \cdot\left(b_{j}^{i}\right)^{2} .
\end{aligned}
$$

Les coefficients $p_{j}^{i}$ donnent la probabilité que la longueur de diffusion de l'élément occupant la position $\vec{R}_{j}$ possède la valeur $b_{j}^{i}$. Naturellement

$$
\sum_{i} p_{j}^{i}=1
$$

Avec ce résultat nous pouvons calculer la fonction de diffusion à partir des distributions $p\left(b_{j}\right)$ des longueurs de diffusion, qui sont en général bien connues.

$$
S(\vec{Q}, \omega)=\sum_{j \neq j^{\prime}}^{N} \overline{b_{j}} \overline{b_{j^{\prime}}^{*}} S_{j, j^{\prime}}(\vec{Q}, \omega)+\sum_{j=1}^{N} \overline{b_{j}^{2}} S_{j, j}(\vec{Q}, \omega)
$$

\footnotetext{
30 L'interférence de toutes les ondelettes produites dans le volume d'échantillon illuminé est exploitée dans le cas de la spectroscopie par corrélation de photons (PCS). Elle nécessite de la lumière, qui est cohérente sur ce volume (se référer à la section 7.6).
} 


$$
=\sum_{j, j^{\prime}=1}^{N} \overline{b_{j}} \overline{b_{j^{\prime}}^{*}} S_{j, j^{\prime}}(\vec{Q}, \omega)+\sum_{j=1}^{N}\left(\overline{b_{j}^{2}}-\left(\bar{b}_{j}\right)^{2}\right) S_{j, j}(\vec{Q}, \omega)
$$

Pour obtenir la dernière ligne nous avons fait passer le terme $\sum_{j=1}^{N}\left(\bar{b}_{j}\right)^{2} S_{j, j}(\vec{Q}, \omega)$ de la seconde à la première somme.

Nous définissons

$$
\frac{d^{2} \sigma}{d \Omega d E_{f}}=\left(\frac{d^{2} \sigma}{d \Omega d E_{f}}\right)_{\mathrm{coh}}+\left(\frac{d^{2} \sigma}{d \Omega d E_{f}}\right)_{\mathrm{inc}}
$$

avec la contribution cohérente à la section efficace donnée par

$$
\left(\frac{d^{2} \sigma}{d \Omega d E_{f}}\right)_{\mathrm{coh}}=\frac{k_{f}}{k_{i}} \frac{1}{2 \pi \hbar} \sum_{\lambda_{i}} p\left(\lambda_{i}\right) \sum_{j, j^{\prime}=1}^{N} \overline{b_{j}} \overline{b_{j^{\prime}}^{*}} \int_{-\infty}^{\infty} d t\left\langle\lambda_{i}\left|e^{-i \vec{Q} \cdot \vec{R}_{j^{\prime}}^{0}} e^{i \vec{Q} \cdot \vec{R}_{j}(t)}\right| \lambda_{i}\right\rangle e^{-i \omega t},
$$

ainsi que la contribution incohérente

$$
\left(\frac{d^{2} \sigma}{d \Omega d E_{f}}\right)_{\mathrm{inc}}=\frac{k_{f}}{k_{i}} \frac{1}{2 \pi \hbar} \sum_{\lambda_{i}} p\left(\lambda_{i}\right) \sum_{j}^{N}\left(\overline{b_{j}^{2}}-\left(\bar{b}_{j}\right)^{2}\right) \int_{-\infty}^{\infty} d t\left\langle\lambda_{i}\left|e^{-i \vec{Q} \cdot \vec{R}_{j}^{0}} e^{i \vec{Q} \cdot \vec{R}_{j}(t)}\right| \lambda_{i}\right\rangle e^{-i \omega t} .
$$

Si le système est monoatomique, nous pouvons simplifier ces expressions en introduisant les sections efficaces totales cohérentes et incohérentes

$$
\begin{aligned}
\sigma_{\mathrm{coh}} & =4 \pi(\bar{b})^{2}, \\
\sigma_{\mathrm{inc}} & =4 \pi\left[\overline{b^{2}}-(\bar{b})^{2}\right] .
\end{aligned}
$$

Les expressions (5.63) et (5.64) deviennent

$$
\left(\frac{d^{2} \sigma}{d \Omega d E_{f}}\right)_{\mathrm{coh}}=\frac{\sigma_{\mathrm{coh}}}{4 \pi} \frac{k_{f}}{k_{i}} \frac{1}{2 \pi \hbar} \sum_{\lambda_{i}} p\left(\lambda_{i}\right) \sum_{j, j^{\prime}=1}^{N} \int_{-\infty}^{\infty} d t\left\langle\lambda_{i}\left|e^{-i \vec{Q} \cdot \vec{R}_{j^{\prime}}^{0}} e^{i \vec{Q} \cdot \vec{R}_{j}(t)}\right| \lambda_{i}\right\rangle e^{-i \omega t}
$$

et

$$
\left(\frac{d^{2} \sigma}{d \Omega d E_{f}}\right)_{\mathrm{inc}}=\frac{\sigma_{\mathrm{inc}}}{4 \pi} \frac{k_{f}}{k_{i}} \frac{1}{2 \pi \hbar} \sum_{\lambda_{i}} p\left(\lambda_{i}\right) \sum_{j}^{N} \int_{-\infty}^{\infty} d t\left\langle\lambda_{i}\left|e^{-i \vec{Q} \cdot \vec{R}_{j}^{0}} e^{i \vec{Q} \cdot \vec{R}_{j}(t)}\right| \lambda_{i}\right\rangle e^{-i \omega t}
$$

La diffusion cohérente est qualitativement très différente de la diffusion incohérente. Nous allons nous en rendre compte lors de la discussion des fonctions de corrélation.

Il est important de distinguer la diffusion incohérente de la diffusion diffuse que nous allons discuter un peu plus en détail dans la section 10.7.

\subsection{La dépendance de la longueur de diffusion du spin nucléaire}

Comme evoqué, la distribution statistique des longueurs de diffusion nucléaires peut avoir son origine dans les isotopes mais aussi dans le spin nucléaire. La distribution isotopique est un sujet compliqué de la physique nucléaire dans lequel nous ne voulons pas nous aventurer. La distribution des voies de diffusion dûe au spin nucléaire est au contraire une conséquence de la conservation du moment angulaire, qui elle relève de la mécanique quantique de base $[13,14]$. Nous allons déduire des expressions explicites pour $\bar{b}$ et $\overline{b^{2}}$ pour un isotope donné de spin nucléaire $I$.

Comme nous l'avons déjà évoqué lors de la discussion des ondes partielles, le moment cinétique est conservé lors de la diffusion par un potentiel isotrope. Quand à la fois la sonde et la cible possèdent des spins, cet argument s'applique au moment angulaire total $\vec{J}$. Pour une diffusion de type $s$ il n'y a pas de 
contribution orbitale de la sonde. L'opérateur de moment angulaire total est donc la somme du spin $\overrightarrow{\mathbf{S}}$ du neutron et du spin nucléaire du noyau $\overrightarrow{\mathbf{I}}$

$$
\overrightarrow{\mathbf{J}}=\overrightarrow{\mathbf{I}}+\overrightarrow{\mathbf{S}} .
$$

Le neutron possédant un spin $S=1 / 2$ le système combiné prendra les valeurs

$$
\begin{aligned}
& J^{+}=I+1 / 2 \text { et } \\
& J^{-}=I-1 / 2 .
\end{aligned}
$$

Pour chaque valeur de $J$ nous nous attendons en principe à une longueur de diffusion différente. La dégénérescence des niveaux de $\overrightarrow{\mathbf{J}}$ est donnée par

$$
n_{J}=2 J+1 .
$$

Nous obtenons

$$
\begin{aligned}
& n^{+}=2(I+1 / 2)+1=2 I+2, \\
& n^{-}=2(I-1 / 2)+1=2 I .
\end{aligned}
$$

Si le faisceau incident n'est pas polarisé, et si les états de spin de la cible sont distribués de manière aléatoire, chacun des états de $\overrightarrow{\mathbf{J}}$ est également probable. La distribution de probabilité conditionnelle qui indique avec quelle probabilité la diffusion passe par une voie qui correspond soit à l'une (+) soit à l'autre (-) des deux longueurs de diffusion est donc

$$
\begin{aligned}
& p^{+}=\frac{2 I+2}{4 I+2}=\frac{I+1}{2 I+1}, \\
& p^{-}=\frac{2 I}{4 I+2}=\frac{I}{2 I+1} .
\end{aligned}
$$

Nous sommes maintenant en mesure de calculer les moyennes

$$
\begin{aligned}
\bar{b} & =\frac{1}{2 I+1}\left((I+1) b^{+}+I b^{-}\right), \\
\overline{b^{2}} & =\frac{1}{2 I+1}\left((I+1)\left(b^{+}\right)^{2}+I\left(b^{-}\right)^{2}\right) .
\end{aligned}
$$

\subsection{Diffusion d'un neutron par un proton ou un deutérium}

Comme exemple concret de ce formalisme nous regardons la diffusion d'un neutron par un proton. Tous les deux possèdent un spin $S=1 / 2$. L'espace de Hilbert du système commun est le produit tensoriel des espaces à deux dimensions des deux spins. Il possède donc quatre dimensions et une base possible est donnée par

$$
\begin{aligned}
& |\uparrow \uparrow\rangle=|\uparrow\rangle_{n} \otimes|\uparrow\rangle_{p}, \\
& |\downarrow \uparrow\rangle=|\downarrow\rangle_{n} \otimes|\uparrow\rangle_{p}, \\
& |\uparrow \downarrow\rangle=|\uparrow\rangle_{n} \otimes|\downarrow\rangle_{p}, \\
& |\downarrow \downarrow\rangle=|\downarrow\rangle_{n} \otimes|\downarrow\rangle_{p} .
\end{aligned}
$$

Les indices $n$ et $p$ se réfèrent au neutron et au proton, respectivement. L'opérateur $\overrightarrow{\mathbf{S}}^{2}$ avec $\overrightarrow{\mathbf{S}}=\overrightarrow{\mathbf{S}}_{n}+\overrightarrow{\mathbf{S}}_{p}$ n'est pas diagonal dans cette base. Elle nous n'est donc pas très utile parce que c'est le spin total qui est la grandeur conservée et non pas les spins isolés du neutron et du proton. Appliquant les règles de 
composition de spin, nous obtenons deux valeurs propres pour $\overrightarrow{\mathbf{S}}^{2}$

$$
S=S_{n}+S_{p}=1 \quad \text { et } \quad S=\left|S_{n}-S_{p}\right|=0 .
$$

A chaque valeur de $s$ correspond un sous-espace de vecteurs propres de dimension $2 S+1$. Dans le cas de deux spin $1 / 2$ ces vecteurs propres sont donnés par

$$
\begin{array}{ll}
S=1 \quad: \quad|1,1\rangle=|\uparrow \uparrow\rangle \\
\quad: \quad|1,0\rangle=\frac{1}{\sqrt{2}}(|\uparrow \downarrow\rangle+|\downarrow \uparrow\rangle) \\
: \quad|1,-1\rangle=|\downarrow \downarrow\rangle \\
S=0 \quad: \quad|0,0\rangle=\frac{1}{\sqrt{2}}(|\uparrow \downarrow\rangle-|\downarrow \uparrow\rangle)
\end{array}
$$

Les fonctions à l'intérieur d'un multiplet se distinguent par leur projection sur $S_{z}$.

$$
\mathbf{S}_{z}\left|S, M_{s}\right\rangle=M_{s}\left|S, M_{s}\right\rangle .
$$

Pour le système neutron-proton nous obtenons un triplet et un singulet.

Dans un système sans préférence pour une direction donnée ${ }^{31}$ il est évident que la valeur de $M_{s}$ ne pourra pas influencer la diffusion. La longueur de diffusion sera donc uniquement une fonction de $S$ seul. Dans le cas présent elle pourra prendre deux valeurs

$$
b^{+}=b(S=1)
$$

et

$$
b^{-}=b(S=0) .
$$

Ces quantités se déterminent expérimentalement, et on trouve que pour des protons liés dans la matière condensée elles valent

$$
\begin{aligned}
& b^{+}\left({ }^{1} H\right)=1.082 \times 10^{-12} \mathrm{~cm}=10.82 \mathrm{fm}, \quad p^{+}=\frac{3}{4}, \\
& b^{-}\left({ }^{1} H\right)=-4.74 \times 10^{-12} \mathrm{~cm}=-47.4 \mathrm{fm}, \quad p^{-}=\frac{1}{4} .
\end{aligned}
$$

La longueur de diffusion du singulet est grande et négative. Nous pouvons, selon la section 4.2 , en déduire que le système est proche de la formation d'un état lié. Dans l'état triplet il existe un vrai état lié (n-p) le deutéron, ce qui explique que la longueur de diffusion associée au triplet est appréciable et positive. A partir des longueurs de diffusion nous obtenons

$$
\begin{aligned}
\bar{b} & =\frac{3}{4} b^{+}+\frac{1}{4} b^{-}=-0.38 \times 10^{-12} \mathrm{~cm}, \\
\overline{|b|^{2}} & =\frac{3}{4}\left|b^{+}\right|^{2}+\frac{1}{4}\left|b^{-}\right|^{2}=6.49 \text { barn. }
\end{aligned}
$$

Le fait que

$$
\frac{b^{+}}{b^{-}} \approx-\frac{p^{+}}{p^{-}}=-\frac{1}{3}
$$

a comme conséquence que la longueur de diffusion moyenne est proche de zéro. Nous nous attendons donc à une diffusion cohérente assez faible. La diffusion incohérente s'avére par contre importante à

\footnotetext{
${ }^{31}$ La seule direction qui brise la symétrie est la direction du faisceau incident, qui n'a néanmoins pas d'influence sur le système des spins. La situation ne changerait qu'en présence d'un champ magnétique externe.
} 
cause de la grande section efficace de l'état singulet. Avec (5.77)(5.78) nous calculons numériquement les sections efficaces totales cohérente et incohérente

$$
\begin{aligned}
\sigma_{\text {coh }}\left({ }^{1} \mathrm{H}\right) & =1.8 \text { barn, } \\
\sigma_{\text {inc }}\left({ }^{1} \mathrm{H}\right) & =79.8 \text { barn, } \\
\sigma_{\text {tot }}\left({ }^{1} \mathrm{H}\right) & =81.7 \text { barn. }
\end{aligned}
$$

Si nous passons au deutérium, la situation change radicalement. Le spin du deutérium est $I=1$. Le neutron se combine avec le deutérium dans les états $S=3 / 2$ et $S=1 / 2$. Nous avons donc affaire à un quadruplet et un doublet. Les longueurs de diffusion expérimentales sont

$$
\begin{aligned}
& b^{+}\left({ }^{2} H\right)=0.95 \times 10^{-12} \mathrm{~cm} \quad p^{+}=\frac{4}{6} \\
& b^{-}\left({ }^{2} H\right)=0.10 \times 10^{-12} \mathrm{~cm} \quad p^{-}=\frac{2}{6} .
\end{aligned}
$$

Nous calculons

$$
\begin{aligned}
\bar{b} & =\frac{2}{3} b^{+}+\frac{1}{3} b^{-}=0.67 \times 10^{-12} \mathrm{~cm}, \\
\overline{|b|^{2}} & =\frac{2}{3}\left|b^{+}\right|^{2}+\frac{1}{3}\left|b^{-}\right|^{2}=0.61 \text { barn. }
\end{aligned}
$$

et pour les sections efficaces

$$
\begin{aligned}
\sigma_{\text {coh }}\left({ }^{2} \mathrm{H}\right) & =5.6 \text { barn, } \\
\sigma_{\text {inc }}\left({ }^{2} \mathrm{H}\right) & =2.0 \text { barn, } \\
\sigma_{\text {tot }}\left({ }^{2} \mathrm{H}\right) & =7.6 \text { barn. }
\end{aligned}
$$

En substituant le deutérium pour le proton nous pouvons considérablement changer les caractéristiques de diffusion d'un échantillon sans pour autant influer beaucoup sur ses propriétés physiques et chimiques. Cette possibilité est souvent mise en application dans la pratique, par exemple pour changer le contraste entre diverses parties d'un échantillon ou pour simplement jouer sur le rapport entre diffusion cohérente et incohérente.

Les isotopes de l'hydrogène diffèrent aussi pour leur section d' absorption. L'absorption est très faible pour le deutérium mais n'est plus négligeable pour le proton $\left(\sigma_{\mathrm{abs}} \approx 0.33\right.$ barn pour des neutrons de $2200 \mathrm{~m} / \mathrm{s}$ ). C'est une des raisons pour laquelle on préfère utiliser le deutérium pour la modération des neutrons dans les réacteurs de recherche.

Le vanadium est aussi un élément chimique très intéressant en diffusion de neutrons. Sa section efficace de $\sigma_{\text {tot }}=4.95$ barn est pratiquement purement incohérente. La diffusion à basse température d'un échantillon de vanadium est donc isotrope et sert à étalonner le spectromètre.

\subsection{Section efficace pour des particules libres}

Si le proton (ou n'importe quel autre diffuseur) était libre, il nous faudrait travailler dans le référentiel du centre de masse pour garantir une description simple du processus de diffusion. Ainsi le caractère isotrope de la diffusion serait conservé pour une diffusion de type $s$. La constante de couplage $g$ figurant dans le potentiel effectif serait par contre à calculer avec la masse réduite

$$
\mu=\left(\frac{1}{m_{p}}+\frac{1}{m_{n}}\right)^{-1} \approx \frac{1}{2} m,
$$


ce qui implique que la longueur de diffusion à utiliser pour le proton dans le référentiel du centre de masse ne fait que la moitié de la longueur de diffusion du proton lié. La diffusion étant isotrope dans le référentiel du centre de masse elle ne l'est plus dans le référentiel du laboratoire.

Nous allons regarder un peu plus dans le détail la relation entre les deux descriptions. Soit

$$
\gamma=\frac{m_{n}}{m_{s}}
$$

le rapport entre la masse du neutron et la masse de la particule cible. L'origine du référentiel du laboratoire coïncide avec la coordonnée de la cible, c'est-à-dire que la cible est sensée être au repos dans ce référentiel. Dans ce cas la section efficace pour la collision élastique dans le référentiel du laboratoire est reliée à la section efficace dans le référentiel du centre de masse par [6,8]

$$
\left(\frac{d \sigma}{d \Omega}\right)_{\mathrm{lab}}=\frac{\left(1+\gamma^{2}+2 \gamma \cos \theta\right)^{\frac{3}{2}}}{|1+\gamma \cos \theta|}\left(\frac{d \sigma}{d \Omega}\right)_{\mathrm{c} . \mathrm{m} .},
$$

où l'angle de diffusion $\theta$ est à prendre dans le référentiel du centre de masse. Cet angle est relié à l'angle de diffusion dans le référentiel du laboratoire par

$$
\cos \theta_{\mathrm{lab}}=\frac{\gamma+\cos \theta}{\sqrt{1+2 \gamma \cos \theta+\gamma^{2}}} .
$$

Dans le cas du proton $\gamma \approx 1$ et nous déduisons que la diffusion dans le référentiel du laboratoire prend la forme

$$
\begin{aligned}
\left(\frac{d \sigma}{d \Omega}\right)_{\mathrm{lab}} & =\overline{|b|^{2}} \cos \theta_{\mathrm{lab}} ; & \theta_{\mathrm{lab}} & \leq \frac{\pi}{2}, \\
& =0 ; & \theta_{\mathrm{lab}} & >\frac{\pi}{2} .
\end{aligned}
$$

$\theta_{\text {lab }}$ est l'angle de diffusion entre $\vec{k}_{i}$ et $\vec{k}_{f} . b$ est la longueur de diffusion du proton lié. Comme pour la collision de deux particules classiques la diffusion se limite dans le référentiel du laboratoire à l'hémisphère en aval. Pour $\theta_{\mathrm{lab}}=\pi / 2$ le neutron transmet toute son énergie au proton et s'arrête.

En intégrant l'équation 5.107 sur $d \Omega$ nous obtenons pour la section efficace totale

$$
\sigma_{\text {free }}=\pi \overline{|b|^{2}}=\frac{1}{4} \sigma_{\text {bound }} .
$$

Elle est donc égal au quart de la section efficace totale du proton lié. Nous obtenons le même résultat qu'en appliquant les expressions (5.65) et (5.66) dans le référentiel du centre de masse, c'est-à-dire en employant la longueur de diffusion obtenue avec la masse réduite. Ceci est normal car la section efficace totale doit être indépendante du référentiel utilisé.

Dans tout le reste de ce chapitre nous travaillerons avec les longueurs de diffusion pour des particules liées. L' effet de recul sera automatiquement pris en compte dans le traitement des effets inélastiques (voir section 8.10).

\subsection{Triangle de diffusion et gamme dynamique}

La fonction de diffusion (5.43) ne dépend plus des propriétés intrinsèques du neutron (ou de la sonde en général). En oubliant le spin, les propriétés du neutron se résument au vecteur d'onde $\vec{k}$. La fonction de diffusion contient néanmoins toujours le potentiel d'interaction du diffuseur avec la sonde, en l'occurrence les longueurs de diffusion nucléaires $b_{j}$. Cette dépendance ne peut être levée que dans le cas où l'interaction est identique pour tous les diffuseurs (voir les expressions 5.67 et 5.68 pour le cas d'une cible monoatomique). La fonction de diffusion est une fonction de la cible et ne dépend que des positions et des mouvements des diffuseurs. 

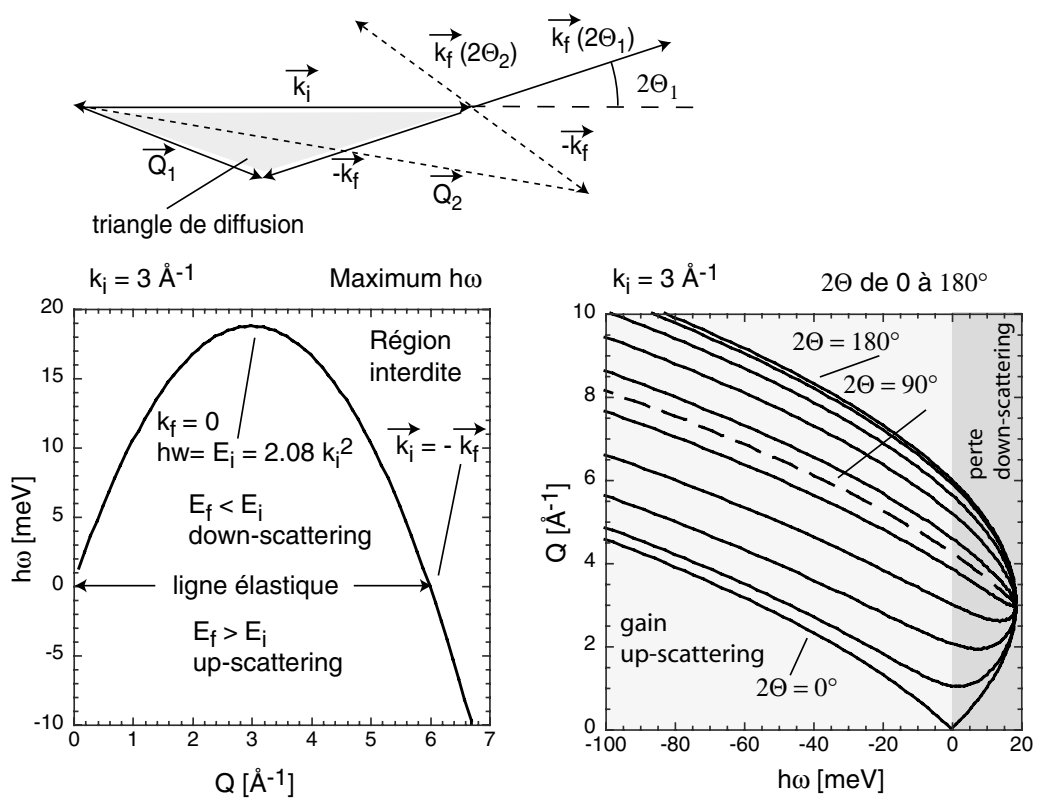

Figure 14. Gamme dynamique d'une mesure de diffusion avec des neutrons. Pour une valeur de $k_{i}$ fixée, il existe toujours des points $(\vec{Q}, \hbar \omega)$ pour lesquels le triangle de diffusion (montré dans la figure en haut) ne se ferme pas, c'est-à-dire pour lesquels les conditions cinématiques ne peuvent pas être satisfaites. Figure de gauche: Transfert d'énergie maximal en fonction de $Q=|\vec{Q}|$ pour un $k_{i}$ de $3 \AA^{-1}$. Figure de droite: transfert d'énergie associé à une valeur de $Q$ pour un angle de diffusion $2 \Theta$ (l'angle augmente de 0 à $180^{\circ}$ en pas de $20^{\circ}$ ). Les lignes correspondent aux trajectoires $(Q, \hbar \omega)$ pour un $k_{i}$ et un angle $\Theta$ fixes. Toute la région se trouvant à l'extérieur des lignes pour $\Theta=0$ et $\theta=180^{\circ}$ n'est pas accessible. Ces lignes définissent ainsi le domaine dynamique accessible à la mesure.

La discussion ayant été assez abstraite jusqu'ici, il est utile d'aborder quelques aspects pratiques. Nous commençons avec quelques définitions.

Nous avions déjà introduit le vecteur de diffusion

$$
\begin{gathered}
\vec{Q}=\vec{k}_{i}-\vec{k}_{f}, \\
Q^{2}=k_{i}^{2}+k_{f}^{2}-2 k_{i} k_{f} \cos 2 \Theta,
\end{gathered}
$$

ainsi que le transfert d'énergie

$$
\hbar \omega=E_{i}-E_{f}=\frac{\hbar^{2}}{2 m}\left(k_{i}^{2}-k_{f}^{2}\right) .
$$

La fonction de diffusion est définie dans l'espace des $(\vec{Q}, \hbar \omega)$. Cet espace à 4 dimensions est aussi appelé espace réciproque (voir chapitre I). On utilise souvent le terme d'espace $(\vec{Q}, \omega)$. L'espace $(\vec{Q}, \hbar \omega)$ est infini. D'un point de vue purement mathématique la fonction $S(\vec{Q}, \hbar \omega)$ est définie partout. Néanmoins nous devons respecter les conditions de validité (approximation de Born) qui nous ont amenés à la fonction de diffusion. Ces conditions peuvent se traduire en terme de "région de validité" dans l'espace $(\vec{Q}, \hbar \omega)$.

Le triplet de vecteurs $\left(\vec{Q}, \vec{k}_{i}, \vec{k}_{f}\right)$ forme un triangle dans ce qu'on appelle la plaine de diffusion (voir figure 14). Dans une expérience, les vraies variables sont les vecteurs d'onde $\vec{k}_{i}$ initiaux et $\vec{k}_{f}$ finaux. En général, il existe plusieurs combinaisons de $\left(\vec{k}_{i}, \vec{k}_{f}\right)$ qui donnent accès au même point $(\hbar \omega, \vec{Q})$ de l'espace réciproque. On peut, par exemple, fixer la direction et l'énergie du faisceau incident et atteindre 
un point dans le plan $(\vec{Q}, \hbar \omega)$ en détectant l'énergie finale

$$
E_{f}=\frac{\hbar^{2}}{2 m} k_{f}^{2}=E_{i}-\hbar \omega
$$

sous l'angle de diffusion

$$
\cos 2 \theta=\frac{k_{i}^{2}+k_{f}^{2}-Q^{2}}{2 k_{i} k_{f}} .
$$

On parlera d'une configuration de mesure à $\vec{k}_{i}$ constant. En toute analogie on pourra aussi travailler à $\vec{k}_{f}$ constant (voir le cours de B. Hennion consacré aux spectromètres 3 axes, dans le même ouvrage).

Pour un $k_{i}$ ou un $k_{f}$ donné, seul un domaine restreint de l'espace réciproque sera accessible expérimentalement. Cette restriction est dûe au fait que les conditions cinématiques (5.112) et (5.113) ne peuvent pas toujours être satisfaites. Un exemple simple est la diffusion élastique. Pour $\hbar \omega=0$ nous obtenons de (5.112) que $k_{i}=k_{f}$. Le $Q=|\vec{Q}|$ le plus grand qu'on pourra atteindre est égal à $2 k_{i}$ en rétrodiffusion. La raison physique est simple. Le neutron ne pourra transférer que deux fois son impulsion initiale. De la même manière il ne pourra perdre -au maximum- que son énergie initiale dans une expérience inélastique.

\subsection{Exemple : taux de production pour des neutrons ultra-froids}

Dans cette partie, nous allons donner un exemple concret pour illustrer l'intérêt d'exprimer la section efficace à l'aide de la fonction de diffusion $S(\vec{Q}, \omega)$. Nous souhaitons en particulier souligner la différence fondamentale, qui existe entre ces deux grandeurs bien qu'elles soient liées par une relation très simple. La section efficace donne le taux de diffusion en barn pour une expérience concrète. Ce taux peut être calculé avec l'aide de la fonction de diffusion. La fonction de diffusion elle-même peut être déterminée pour des conditions complètement différentes de celles de l'experience envisagée. La seule condition préalable à la transférabilité de la fonction de diffusion est la validité de l'approximation de Born.

L'exemple choisi traite de la production de neutrons ultra-froids à partir d'un faisceau de neutrons froids ou thermiques. Les neutrons ultra-froids étant de grande importance pour des études de physique fondamentale, l'amélioration de leur taux de production est un problème de grande actualité [16]. La quantité qui intéresse le scientifique est le nombre total de neutrons ultra-froids (c'est-à-dire d' énergie inférieure à $E_{\mathrm{u}}^{\max } \approx 300 \mathrm{neV}$ ), obtenus à partir d'un faisceau de neutrons froids ou thermiques. Ce seuil est choisi parce que les neutrons en dessous de quelques $300 \mathrm{neV}$ ne pénètrent plus dans la plupart des matériaux et peuvent donc être piégés facilement, par exemple pour mesurer leur durée de vie.

Soit $E_{i}$ l'énergie incidente des neutrons. La section efficace de production de neutrons ultra-froids — c'est-à-dire le nombre de neutrons ultra-froids produits par seconde et par unité de flux incident est donnée par

$$
\begin{aligned}
\sigma_{\text {prod }}\left(E_{i}\right) & =\int_{0}^{E_{\mathrm{u}}^{\max }} \frac{k_{f}}{k_{i}} S(Q, \omega) d E_{f} d \Omega \\
& =4 \pi \int_{0}^{E_{\mathrm{u}}^{\max }} \frac{k_{f}}{k_{i}} S(Q, \omega) d E_{f},
\end{aligned}
$$

où nous avons pris en compte le fait que la production se fasse sur un angle de $4 \pi$ et que la diffusion soit isotrope dans un liquide ou dans une poudre.

Le rapport de l'énergie $E_{i}$ des neutrons froids incidents (quelques meV) sur l'énergie $E_{\mathrm{u}}$ des neutrons ultra-froids (quelques neV) est très faible. La fonction de diffusion - convoluée avec la résolution (voir section 9.6) — ne varie que très peu sur cette échelle d'énergie. Nous pouvons donc 
supposer que

$$
\begin{gathered}
Q \approx k_{i} \\
\hbar \omega \approx \frac{\hbar^{2} k_{i}^{2}}{2 m} .
\end{gathered}
$$

Les valeurs de $Q$ et de $\omega$ qui nous intéressent, correspondent à la parabole $\hbar \omega=\hbar^{2} Q^{2} / 2 m$ des neutrons libres (voir chapitre I et figure 15). Ainsi

$$
\begin{aligned}
\sigma_{\text {prod }}\left(E_{0}\right) & =4 \pi \int_{0}^{E_{\mathrm{u}}^{\max }} \frac{k_{f}}{k_{i}} S\left(k_{i}, \frac{\hbar^{2} k_{i}^{2}}{2 m}\right) d E \\
& =4 \pi \frac{1}{k_{i}} S\left(k_{i}, \frac{\hbar^{2} k_{i}^{2}}{2 m}\right) \int_{0}^{E_{\mathrm{u}}^{\max }} \frac{k_{f}^{2} \hbar^{2}}{m} d k_{f} \\
& =4 \pi \frac{1}{k_{i}} S\left(k_{i}, \frac{\hbar^{2} k_{i}^{2}}{2 m}\right) \frac{2}{3} k_{\mathrm{u}}^{\max } E_{\mathrm{u}}^{\max } .
\end{aligned}
$$

Cette expression est le produit de trois facteurs. Le premier facteur

$$
\frac{8 \pi}{3} k_{\mathrm{u}}^{\max } E_{\mathrm{u}}^{\max }
$$

mesure le volume de l'espace des phases des états finaux. Ce volume dépend fortement du vecteur d'onde des neutrons convertis. Comme l'énergie finale neutrons ultra-froids est petite, ce volume est également faible. Le deuxième facteur

$$
\frac{1}{k_{i}}
$$

mesure le flux incident. Lors du calcul du taux de production absolu, on multipliera la section efficace avec le spectre du flux incident. Ce facteur va donc disparaître. Le dernier terme

$$
S\left(k_{i}, \frac{\hbar^{2} k_{i}^{2}}{2 m}\right)
$$

est la fonction de diffusion évaluée le long de la parabole du neutron libre. Il donne la probabilité de produire un neutron ultra-froid à partir d'un neutron froid d'énergie $E_{i}$. La fonction de diffusion peut être extraite de n'importe quelle expérience de diffusion pourvu que l'énergie utilisée dépasse $E_{i}$ et sous la condition que l'expérience satisfasse aux critères de l'approximation de Born.

Un convertisseur très prometteur est le deutérium solide [16]. Dans la figure 15 nous donnons la fonction $S(Q, \omega)$ déterminée à partir de la section efficace différentielle partielle. L'échantillon est une poudre de $\mathrm{D}_{2}$ solide contenant $33 \%$ de para-deutérium. L'énergie incidente s'élève à $17.4 \mathrm{meV}$. La section efficace totale vaut $\sigma_{\text {tot }}\left(E_{i}=17.4 \mathrm{meV}\right) \approx 23$ barn. Elle dépend naturellement de l'énergie incidente. La fonction de diffusion nous permet de calculer les sections efficaces différentielles partielles pour n'importe quelle énergie incidente, y compris pour celles nécessaires à la production des neutrons ultra-froids. Par exemple, pour calculer le nombre de neutrons ultra-froids produits à partir de neutrons froids d'une énergie de $5 \mathrm{meV}$, il faut connaître $S\left(Q=1.55 \AA^{-1}, \hbar \omega=5 \mathrm{meV}\right)$. Cette valeur s'extrait de la section efficace différentielle partielle mesurée en considérant la transition des neutrons froids de 17.4 à $12.4 \mathrm{meV}$ et de $\left|\vec{k}_{i}-\vec{k}_{f}\right|=1.55 \AA^{-1}$.

La situation change beaucoup quand on regarde le processus inverse, c'est-à-dire la perte d'énergie d'un neutron ultra-froid par un processus inélastique. Dans ce cas l'espace des phases des états finaux possibles est très large. Pour que la perte puisse être limitée, la fonction de diffusion doit tendre vers 

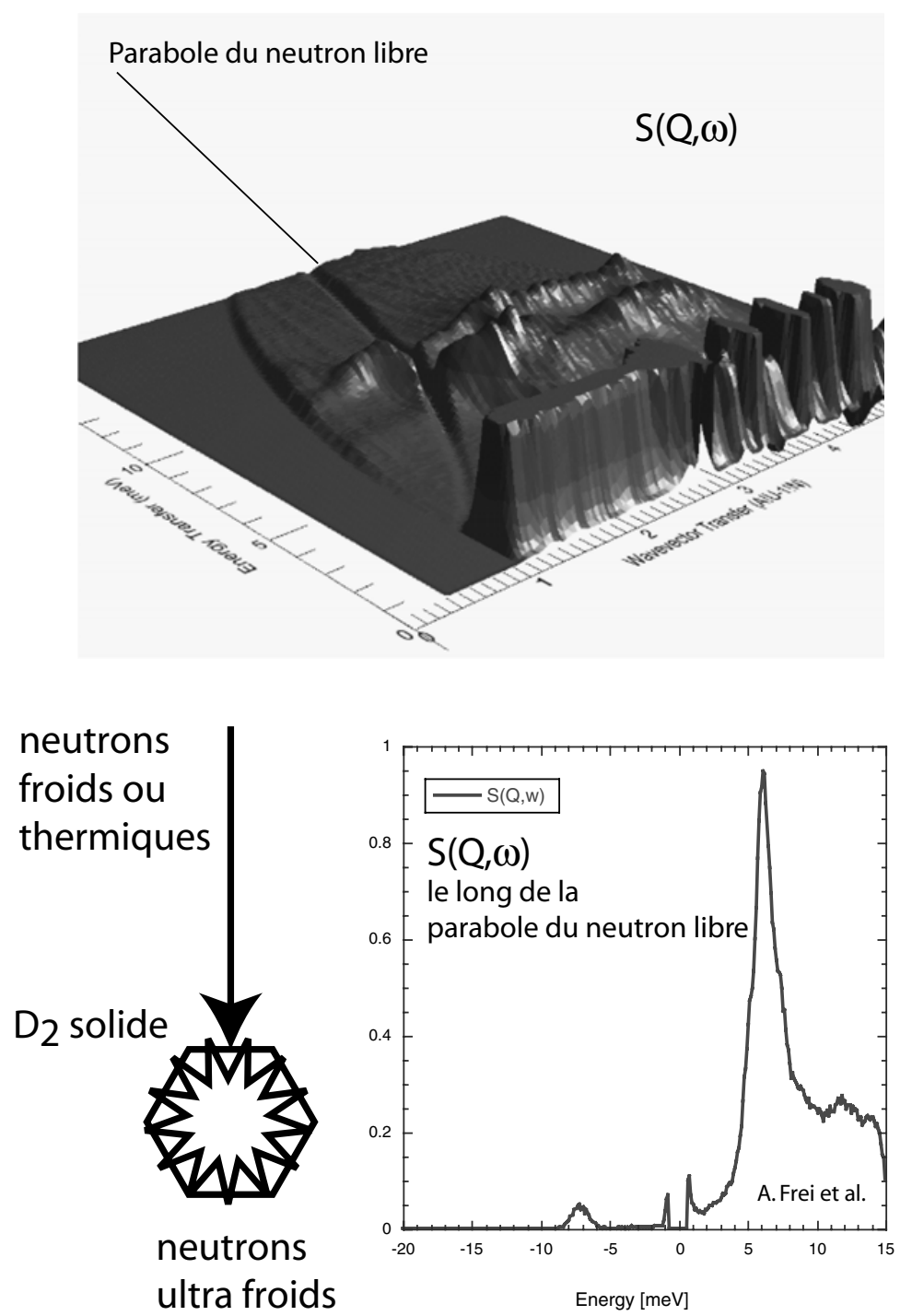

Figure 15. $S(Q, \omega)$ pour un échantillon de poudre de $\mathrm{D}_{2}$ solide mesuré avec une énergie incidente de $E_{i}=$ $17.288 \mathrm{meV} \equiv k_{i}=2.888 \AA^{-1}$ sur l'instrument IN4C à l'ILL [16]. Le contenu en para-deuterium est de $33 \%$ et on ne montre que le coté du spectre qui correspond à une perte d'énergie du neutron diffusé. La probabilité qu'un neutron perde toute son énergie $\hbar \omega$ est proportionnelle à la fonction de diffusion $S(Q, \omega)$ le long de la parabole du neutron libre. A partir de cette mesure nous pouvons estimer le taux de production pour toute énergie inférieure à $\approx 15 \mathrm{meV}$.

zéro du côté gain d'énergie. C'est le cas pour les basses températures à cause du facteur de balance detaillée (voir section 8.7).

\section{RÉSUMÉ PARTIEL}

Nous allons succinctement résumer le formalisme développé jusqu'ici.

La grandeur caractérisant un processus de diffusion est la section efficace différentielle. Elle est définie 
$\operatorname{par}(2.4)$

$$
d \sigma=\frac{\text { flux diffusé dans un élément } d \Omega}{\text { flux initial sur la cible }}=r^{2} d \Omega \frac{J_{\text {diff }}(r, \theta, \phi, t)}{J_{\text {ini }}(t)} .
$$

Pour un potentiel statique la diffusion est élastique. La fonction d'onde qui décrit la diffusion des neutrons doit satisfaire à l'équation de Schrödinger stationnaire (4.3)

$$
\left[-\frac{\hbar^{2}}{2 m} \Delta+V(\vec{r})\right] u_{\vec{k}}(\vec{r})=E(k) u_{\vec{k}}(\vec{r}) .
$$

Dans le régime asymptotique elle possède la forme (4.4)

$$
u_{\vec{k}}(\vec{r}) \longrightarrow \frac{1}{\sqrt{V}}\left(e^{i \vec{k} \cdot \vec{r}}+f_{\vec{k}}(\theta, \phi) \frac{e^{i k r}}{r}\right), \quad r \rightarrow \infty,
$$

avec $f_{\vec{k}}(\theta, \phi)$ l'amplitude de diffusion. Cette amplitude est directement reliée à la section efficace comme (4.27)

$$
\frac{d \sigma}{d \Omega}=r^{2} \frac{J_{\mathrm{diff}}(r, \theta, \phi, t)}{J_{\mathrm{ini}}(t)}=|f(\theta, \phi)|^{2}
$$

Pour des potentiels statiques qui sont de plus isotropes, l'amplitude de diffusion s'exprime en termes de fonctions sphériques (ou ondes partielles) comme (4.59)

$$
f(\theta)=\frac{1}{2 i k} \sum_{l=0}^{\infty} \sqrt{4 \pi(2 l+1)}\left(e^{2 i \delta_{l}}-1\right) Y_{l 0}(\theta) .
$$

Pour des longueurs d'onde très petites par rapport à l'extension spatiale du potentiel, il suffit de retenir l'onde partielle de type s. L'amplitude de diffusion s'exprime en fonction du déphasage de cette onde comme (4.71)

$$
f(\theta)=\frac{e^{2 i \delta_{0}}-1}{2 i k}=\frac{1}{k} e^{i \delta_{0}} \sin \delta_{0} .
$$

La longueur de diffusion est définie par la valeur limite du rapport entre le déphasage et le vecteur d'onde (4.78)

$$
b=-\lim _{k \rightarrow 0} f(\theta)=-\lim _{k \rightarrow 0} \frac{\delta_{0}(k)}{k} .
$$

Pour une sphère dure elle est identique au rayon (4.79)

$$
b=r_{0} .
$$

En général la longueur de diffusion peut prendre des valeurs positives ainsi que des valeurs négatives. L'absorption hors résonance est prise en compte par une longueur de diffusion complexe.

Pour permettre un calcul en perturbation, l'équation de Schrödinger, qui définit la fonction d'onde du processus de diffusion, est transcrite en forme intégrale (4.111)

$$
u_{\vec{k}}(\vec{r})=u_{\vec{k}}^{0}(\vec{r})+\int d^{3} r^{\prime} G_{0}\left(\vec{r}, \vec{r}^{\prime}\right) V\left(\vec{r}^{\prime}\right) u_{\vec{k}}\left(\vec{r}^{\prime}\right),
$$

avec la fonction de Green (4.121)

$$
G_{0}\left(\vec{r}, \vec{r}^{\prime}\right)=-\frac{1}{4 \pi} \cdot \frac{2 m}{\hbar^{2}} \cdot \frac{e^{\left(i k \cdot \vec{r}-\vec{r}^{\prime} \mid\right)}}{\left|\vec{r}-\vec{r}^{\prime}\right|} .
$$


En remplaçant la fonction $u_{\vec{k}}(\vec{r})$ par l'onde non-perturbée $u_{\vec{k}}^{0}(\vec{r})$ dans l'intégrale

$$
u_{\vec{k}}(\vec{r})=u_{\vec{k}}^{0}(\vec{r})-\frac{1}{4 \pi} \frac{2 m}{\hbar^{2}} \int d^{3} r^{\prime} \frac{\exp \left(i k \cdot\left|\vec{r}-\vec{r}^{\prime}\right|\right)}{\left|\vec{r}-\vec{r}^{\prime}\right|} V\left(\vec{r}^{\prime}\right) u_{\vec{k}}^{0}\left(\vec{r}^{\prime}\right)
$$

équivaut à travailler dans l'approximation de Born. Dans cette approximation, l'amplitude de diffusion se calcule comme la transformée de Fourier du potentiel (4.141)

$$
f_{\vec{Q}}(\Omega)=-\frac{1}{2 \pi} \frac{m}{\hbar^{2}} \int d^{3} r^{\prime} e^{i \vec{Q} \cdot \vec{r}^{\prime}} V\left(\vec{r}^{\prime}\right) .
$$

Des cibles composées possèdant une dynamique interne permettent la diffusion inélastique des neutrons. Elle est accompagnée de transitions quantiques au sein de la cible. La règle d'or de Fermi permet d'établir le lien entre les transitions et la section efficace différentielle partielle. Dans l'approximation de Born ce lien est donné par (5.14)

$$
\frac{d^{2} \sigma_{\vec{k}_{i} \rightarrow \vec{k}_{f}}}{d \Omega d E_{f}}=\frac{k_{f}}{k_{i}} \frac{(2 \pi)^{4} m^{2}}{\hbar^{4}} \sum_{\lambda_{i}, \lambda_{f}} p\left(\lambda_{i}\right)\left|\left\langle\lambda_{f}, \vec{k}_{f}|V| \lambda_{i}, \vec{k}_{i}\right\rangle\right|^{2} \delta\left(\left(E_{f}-E_{i}\right)-\left(E_{\lambda_{i}}-E_{\lambda_{f}}\right)\right) .
$$

Cette expression est appelée équation maîtresse de la diffusion. Le potentiel d'interaction entre neutron et cible étant en général une somme de contributions individuelles indépendantes, l'équation maîtresse devient (5.22)

$$
\frac{d \sigma_{\vec{k}_{i} \rightarrow \vec{k}_{f}}}{d \Omega d E_{f}}=\frac{k_{f}}{k_{i}} \frac{m^{2}}{(2 \pi)^{2} \hbar^{4}} \sum_{\lambda_{i}, \lambda_{f}} p\left(\lambda_{i}\right)\left|\sum_{j=1}^{N} V_{j}(\vec{Q})\left\langle\lambda_{f}\left|e^{i \vec{Q} \cdot \vec{R}_{j}}\right| \lambda_{i}\right\rangle\right|^{2} \delta\left(\left(E_{f}-E_{i}\right)-\left(E_{\lambda_{i}}-E_{\lambda_{f}}\right)\right),
$$

avec les facteurs de forme des diffuseurs définis en terme de leurs transformées de Fourier (5.21)

$$
V_{j}(\vec{Q})=\int d^{3} r_{j} e^{-i \vec{Q} \cdot \vec{r}_{j}} V_{j}\left(\vec{r}_{j}\right)
$$

Pour la diffusion nucléaire, le potentiel adapté est le pseudo-potentiel de Fermi (5.25)

$$
V_{\mathrm{eff}}(r) \psi(r)=\frac{2 \pi \hbar^{2}}{m} b_{j} \delta(\vec{r})
$$

Le facteur de forme devient une simple constante

$$
V_{j}(\vec{Q})=\frac{2 \pi \hbar^{2}}{m} b_{j}
$$

La somme sur les états finaux dans l'équation maîtresse peut être éliminée au profit d'une transformation de Fourier dans le temps. Nous obtenons pour la section efficace différentielle partielle (5.42)

$$
\frac{d^{2} \sigma_{\vec{k}_{i} \rightarrow \vec{k}_{f}}}{d \Omega d E_{f}}=\frac{k_{f}}{k_{i}} S(\vec{Q}, \omega)
$$

$\operatorname{avec}(5.43)$

$$
S(\vec{Q}, \omega) \frac{1}{2 \pi \hbar} \sum_{\lambda_{i}} p\left(\lambda_{i}\right) \sum_{j, j^{\prime}=1}^{N} b_{j} b_{j^{\prime}}^{*} \int_{-\infty}^{\infty} d t\left\langle\lambda_{i}\left|e^{-i \vec{Q} \cdot \vec{R}_{j^{\prime}}^{0}} e^{i \vec{Q} \cdot \vec{R}_{j}(t)}\right| \lambda_{i}\right\rangle e^{-i \omega t}
$$

$S(\vec{Q}, \omega)$ est appelée la fonction de diffusion.

La diffusion nucléaire des neutrons dépend de la composition isotopique et des états de spin des noyaux. Il faut alors distinguer la diffusion cohérente de la diffusion incohérente. La diffusion cohérente 
dépend de la longueur de diffusion moyenne d'un diffuseur (5.63)

$$
\left(\frac{d^{2} \sigma}{d \Omega d E_{f}}\right)_{\mathrm{coh}}=\frac{k_{f}}{k_{i}} \frac{1}{2 \pi \hbar} \sum_{\lambda_{i}} p\left(\lambda_{i}\right) \sum_{j, j^{\prime}=1}^{N} \overline{b_{j}} \overline{b_{j^{\prime}}^{*}} \int_{-\infty}^{\infty} d t\left\langle\lambda_{i}\left|e^{-i \vec{Q} \cdot \vec{R}_{j^{\prime}}^{0}} e^{i \vec{Q} \cdot \vec{R}_{j}(t)}\right| \lambda_{i}\right\rangle e^{-i \omega t} .
$$

La diffusion incohérente est determinée par la dispersion des longueurs de diffusion par rapport aux valeurs moyennes (dispersion caractérisée par son écart type) (5.64)

$$
\left(\frac{d^{2} \sigma}{d \Omega d E_{f}}\right)_{\text {inc }}=\frac{k_{f}}{k_{i}} \frac{1}{2 \pi \hbar} \sum_{\lambda_{i}} p\left(\lambda_{i}\right) \sum_{j}^{N}\left(\overline{b_{j}^{2}}-\left(\bar{b}_{j}\right)^{2}\right) \int_{-\infty}^{\infty} d t\left\langle\lambda_{i}\left|e^{-i \vec{Q} \cdot \vec{R}_{j}^{0}} e^{i \vec{Q} \cdot \vec{R}_{j}(t)}\right| \lambda_{i}\right\rangle e^{-i \omega t} .
$$

\section{PARALLÈLES AVEC L'OPTIQUE}

Dans cette partie, nous allons effectuer une petite excursion dans le monde de l'optique. Nous nous intéressons en particulier à la diffraction de la lumière.

\subsection{Le principe d'Huygens-Fresnel}

L'interprétation de la diffraction s'appuie sur le principe d'Huygens-Fresnel [17]. Selon ce principe chaque élément de surface atteint par le front d'une onde lumineuse se comporte comme une source secondaire qui émet des ondelettes sphériques dont l'amplitude est proportionnelle à l'aire de cet élément. L'amplitude complexe produite en un point par toutes ces sources secondaires, est la somme des amplitudes complexes des ondelettes. Sans perturbation, les interférences produites correspondent à la propagation rectiligne de la lumière, définissant le régime de l'optique géométrique. Dès que le front lumineux rencontre un obstacle il est perturbé. L'absence d'ondelettes aux endroits de la perturbation produit des effets de diffraction. Selon ce principe, la fonction d'onde ${ }^{32}$ au point $\vec{r}$ engendré par une onde monochromatique $(k=2 \pi / \lambda)$, s'obtient à partir de la fonction d'onde sur une surface $S$ selon [17]

$$
\psi(\vec{r})=\int_{S} \psi_{0}(\vec{r}) \Gamma \frac{\exp \left(i k \cdot\left|\vec{r}-\vec{r}^{\prime}\right|\right)}{\left|\vec{r}-\vec{r}^{\prime}\right|} d S
$$

Les vecteurs $\vec{r}$ et $\vec{r}^{\prime}$ sont définis comme dans la figure 10 avec la différence qu'ils ne parcourent pas un volume de diffusion $V$ mais une surface de diffraction $S$, qui enrobe le front lumineux. Le facteur $\Gamma$ prend en compte l'inclinaison de la surface à l'endroit $\vec{r}^{\prime}$. Nous constatons que le principe de HuygensFresnel ressemble beaucoup à l'expression intégrale de l'équation de Schrödinger (voir 4.122). La différence principale réside dans le fait que les interférences, dans le cas de la diffusion, se font par création d'ondelettes et non par omission comme c'est le cas pour la diffraction. Une conséquence majeure de cette différence porte sur l'absence d'effet d'ordre supérieur dans le formalisme de la diffraction. La diffraction n'étant pas le résultat d'une interaction de l'onde lumineuse avec la matière, il n'existe pas d'interactions multiples. Nous sommes donc d'office dans le régime de l'approximation de Born.

Dans l'approximation de Fraunhofer, on place le plan d'observation à l'infini. En d'autres termes, la distance entre l'objet diffractant et l'observateur est très grande devant toutes les autres dimensions du problème. Les rayons à considérer se rencontrent à l'infini et doivent donc être parallèles en sortant du plan diffractant. Ils sont donc pleinement caractérisés par le vecteur $\vec{k}_{f} \| \hat{r}$. Ceci correspond exactement aux conditions de la diffusion. Dans la pratique, ils peuvent être ramenés dans un plan d'observation par une lentille. Dans l'approximation de Fraunhofer, la fonction d'onde diffractée à un point $\vec{r}$ du plan

\footnotetext{
32 Comme dans le cas des neutrons, nous ignorons ici les problèmes liés à la polarisation. Ceci nous permet de décrire la lumière à l'aide d'une amplitude scalaire, complexe, qui correspond à l'une quelconque de ses composantes.
} 
d'observation est donnée pour $\vec{k}_{i} \| \hat{z}$ par

$$
\psi(\vec{r}) \propto \Gamma \frac{\exp \left(i \vec{k}_{f} \cdot \vec{r}\right)}{r} \int_{S} \psi_{0}(\vec{r}) \exp \left(i \vec{k}_{f} \cdot \vec{r}^{\prime}\right) d S .
$$

C'est l'équivalent de l'expression (4.141) pour la diffusion. L'image de la lumière diffractée donne, dans l'approximation de Fraunhofer, le carré du module de la transformée de Fourier du motif diffractant.

\subsection{Diffraction par une fente}

Nous allons illustrer le formalisme par deux exemples pédagogiques : la fente simple et le réseau de fentes. Considérons une fente $F=2 a \times 2 b$ illuminée par une onde de lumière monochromatique plane d'incidence normale $\left(\vec{k}_{i}=(2 \pi / \lambda) \hat{z}\right.$; voir figure 16$)$. La fonction d'onde $\psi_{0}$, qui est la source des ondelettes diffractées, s'identifie à la transmittance de la fente dans le plan diffractant. Elle est donnée par le rapport des amplitudes complexes juste avant et juste après le diaphragme.

$$
t(x, y)=\frac{\psi_{0}^{\text {avant }}(x, y)}{\psi_{0}^{\text {après }}(x, y)} .
$$

Dans le cas d'une fente $F$ simple, c'est-à-dire caractérisée par une parfaite transparence à l'intérieur de l'ouverture, la transmittance est binaire. Elle vaut un pour tout point à l'intérieur de $F$ et zéro autrement.

$$
t(x, y)=\operatorname{rect}\left(\frac{x}{2 a}\right) \operatorname{rect}\left(\frac{y}{2 b}\right)=\left\{\begin{array}{ll}
1, & -a<x<a \\
0 & \text { autrement }
\end{array} \text { et }-b<y<b\right.
$$

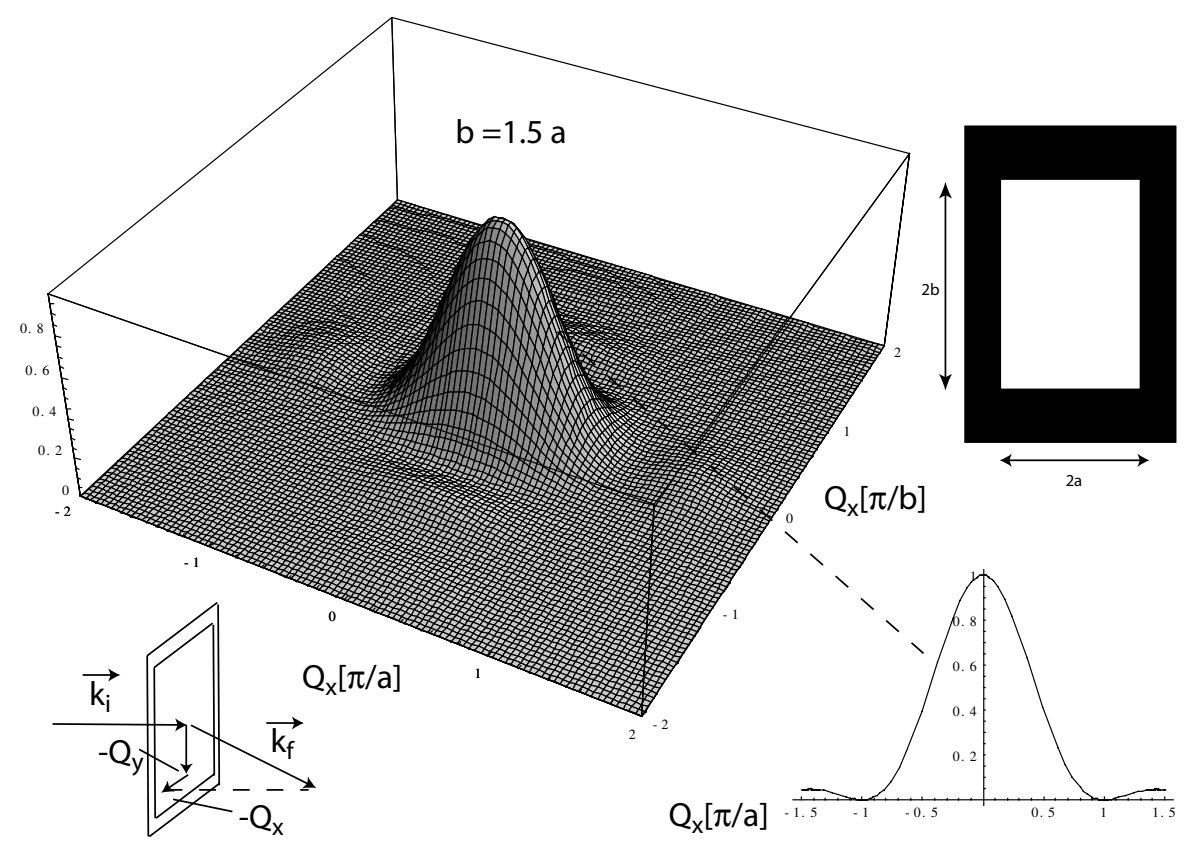

Figure 16. Figure de diffraction d'une fente simple. La largeur des pics est inversement proportionnelle à la largeur de la fente. Dans le cas présenté $b=1.5 a$. Le pic central est donc plus fin le long de $Q_{y}$ que le long de $Q_{x}$. 
En définissant $\vec{Q}=\vec{k}_{i}-\vec{k}_{f}$ nous obtenons, en incidence normale pour les projections de $\vec{k}_{f}$ sur le plan diffractant

$$
k_{x}^{f}=-Q_{x}=\frac{2 \pi}{\lambda} \sin \theta_{x} \quad \text { et } \quad k_{y}^{f}=-Q_{y}=\frac{2 \pi}{\lambda} \sin \theta_{y} .
$$

Cette notation est très proche de la notation utilisée pour la diffusion. En plus, formulée en terme de $\vec{Q}$, les expressions seront aussi valables pour une incidence oblique.

La transformée de Fourier de la transmittance de la fente est donnée par

$$
\begin{aligned}
\tilde{t}\left(Q_{x}, Q_{y}\right)=F(t(x, y)) & =\int_{-a}^{a} \int_{-b}^{b} \exp \left(i\left(Q_{x} x+Q_{y} y\right)\right) d x d y \\
& =\int_{-a}^{a} \exp \left(i Q_{x} x\right) d x \int_{-b}^{b} \exp \left(i Q_{y} y\right) d y \\
& =4 a b\left[\frac{\sin \left(Q_{x} a\right)}{Q_{x} a}\right]\left[\frac{\sin \left(Q_{y} b\right)}{Q_{y} b}\right] .
\end{aligned}
$$

La figure de diffraction, c'est-à-dire l'intensité sur l'écran d'observation, s'obtient comme le carré de l'amplitude

$$
I\left(Q_{x}, Q_{y}\right)=16 a^{2} b^{2}\left[\frac{\sin \left(Q_{x} a\right)}{Q_{x} a}\right]^{2}\left[\frac{\sin \left(Q_{y} b\right)}{Q_{y} b}\right]^{2} .
$$

Elle est représentée sur la figure 16. La largeur des pics de diffraction est inversement proportionnelle à la largeur de la fente. La quasi-totalité de l'intensité est concentrée dans le pic central qui possède une largeur à mi-hauteur de $0.89 \pi \cdot a^{-1}$ et $0.89 \pi \cdot b^{-1}$, respectivement. La déviation angulaire correspondant à ces largeurs se calcule comme

$$
\theta_{x}=\arcsin \left(\frac{0.89 \cdot \lambda}{2 a}\right) \quad \text { et } \quad \theta_{y}=\arcsin \left(\frac{0.89 \cdot \lambda}{2 b}\right)
$$

La diffraction est donc importante quand les dimensions de la fente sont proches de la longueur d'onde de la lumière.

Pour la suite, nous présumons qu'à la fois $b \gg a$ et $b \gg \lambda$. La diffraction aura donc exclusivement lieu selon la direction $Q_{x}$. Si nous déplaçons la fente d'une distance $\Delta x$ selon la direction $\hat{x}$ nous introduisons un facteur de phase dans l'amplitude de diffraction

$$
\tilde{t}\left(Q_{x}\right)=2 a \exp \left(i Q_{x} \Delta x\right)\left[\frac{\sin \left(Q_{x} a\right)}{Q_{x} a}\right] .
$$

\subsection{Diffraction par un ensemble de fentes avec translation aléatoire}

La translation laisse donc la figure de diffraction inchangée. Comme nous alllons le montrer maintenant, cela vaut aussi pour un grand nombre $N$ de fentes avec translation aléatoire selon $\hat{x}$.

L'amplitude de l'onde diffractée est la somme des amplitudes propagées par chaque ouverture. Soient $x_{m}$ l'abscisse du centre de l'ouverture $m$. La transformée de Fourier de la transmittance de l'ensemble s'obtient comme

$$
\tilde{t}\left(Q_{x}\right)=2 a\left[\frac{\sin \left(Q_{x} a\right)}{Q_{x} a}\right] \sum_{m=1}^{N} \exp \left(i Q_{x} x_{m}\right)
$$


En prenant le carré de cette expression pour aboutir à la figure de diffraction $I$, nous obtenons la double somme

$$
I \propto \sum_{m=1}^{N} \exp \left(i Q_{x} x_{m}\right) \sum_{n=1}^{N} \exp \left(-i Q_{x} x_{n}\right) .
$$

Pour $m=n$ nous avons affaire à $N$ termes, chacun contribuant la valeur 1 à la somme. Dans la terminologie que nous avions introduite pour décrire la diffusion, ce sont les contributions propres ou self. Il reste la partie distincte

$$
I \propto 2 \operatorname{Re}\left[\sum_{m \neq n} \exp \left(i Q_{x}\left(x_{m}-x_{n}\right)\right)\right] .
$$

Pour un grand nombre de fentes avec positions aléatoires ce terme s'annulera. La multiplication des fentes augmentera donc l'intensité proportionnellement au nombre de fentes. La figure de diffraction restera par contre inchangée, parce que la superposition aléatoire des phases ne produira pas d'interférences constructives entre les amplitudes provenant de fentes distinctes. C'est l'analogue de la diffusion incohérente.

\subsection{Diffraction par un réseau}

La situation change complètement quand nous passons aux réseaux ordonnés. Considérons un arrangement régulier de $\mathrm{N}$ fentes de largeur $2 a$ et espacées d'une distance $d$ (voir figure 17). La

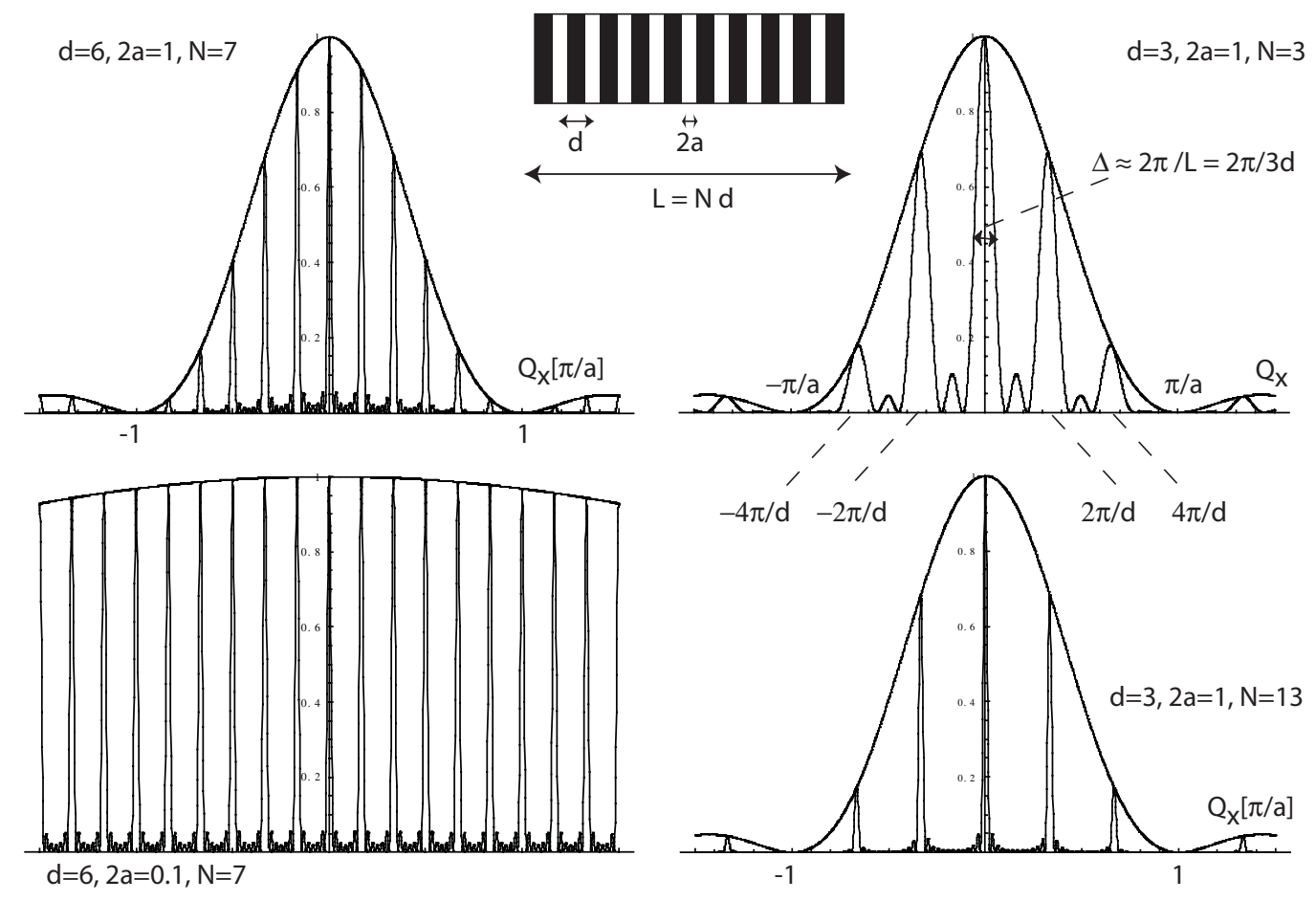

Figure 17. Figures de diffraction en fonction de $Q_{x}$ pour divers réseaux. 
transmittance de l'ensemble est la somme de la transmittance $t_{a}$ des fentes individuelles

$$
t(x)=\sum_{m=-n}^{m=n} t_{a}\left(x-x_{m}\right),
$$

avec $N=2 n+1 . x_{m}$ désigne la coordonnée du centre de la fente de rang $m$. La transformée de Fourier s'écrit comme

$$
\begin{aligned}
\tilde{t}\left(Q_{x}\right) & =\int t(x) \exp \left(i Q_{x} x\right) d x \\
& =\sum_{m=-n}^{m=n} \int t_{a}\left(x-x_{m}\right) \exp \left(i Q_{x} x\right) d x \\
& =\sum_{m=-n}^{m=n} \exp \left(i Q_{x} x_{m}\right) \int t_{a}\left(x-x_{m}\right) \exp \left(i Q_{x}\left(x-x_{m}\right)\right) d\left(x-x_{m}\right) \\
& =\tilde{t}_{a}\left(Q_{x}\right) \sum_{m=-n}^{m=n} \exp \left(i Q_{x} x_{m}\right) \\
& =\tilde{t}_{a}\left(Q_{x}\right) \sum_{m=-n}^{m=n}\left(\exp \left(i Q_{x} d\right)\right)^{m} \\
& =2 a\left[\frac{\sin \left(Q_{x} a\right)}{Q_{x} a}\right]_{m=-n}^{m=n}\left(\exp \left(i Q_{x} d\right)\right)^{m} .
\end{aligned}
$$

L'expression

$$
\sum_{m=-n}^{m=n}\left(\exp \left(i Q_{x} d\right)\right)^{m}
$$

est une série géométrique

$$
\sum_{k=-n}^{n} r^{k}=\frac{r^{n+1}-r^{-n}}{r-1}=r^{-n} \frac{1-r^{N}}{1-r}
$$

Elle a donc comme solution

$$
\sum_{m=-n}^{m=n}\left(\exp \left(i Q_{x} d\right)\right)^{m}=\exp \left(i n Q_{x} d\right) \frac{1-\exp \left(i N Q_{x} d\right)}{1-\exp \left(i Q_{x} d\right)}=\frac{\sin \left(N Q_{x} d / 2\right)}{\sin \left(Q_{x} d / 2\right)}
$$

Selon (7.14) le résultat final pour l'amplitude de diffraction du réseau s'obtient comme

$$
\tilde{t}\left(Q_{x}\right)=N 2 a\left[\frac{\sin \left(Q_{x} a\right)}{Q_{x} a}\right]\left[\frac{\sin \left(N Q_{x} d / 2\right)}{N \sin \left(Q_{x} d / 2\right)}\right] .
$$

L'intensité $I\left(Q_{x}\right)$ est le carré de l'amplitude

$$
I\left(Q_{x}\right)=4 N^{2} a^{2}\left[\frac{\sin \left(Q_{x} a\right)}{Q_{x} a}\right]^{2}\left[\frac{\sin \left(N Q_{x} d / 2\right)}{N \sin \left(Q_{x} d / 2\right)}\right]^{2} .
$$

La figure 17 donne différentes images de diffraction en fonction de $Q_{x}$ et la figure 18 les présente en fonction de $\theta_{x}$. 

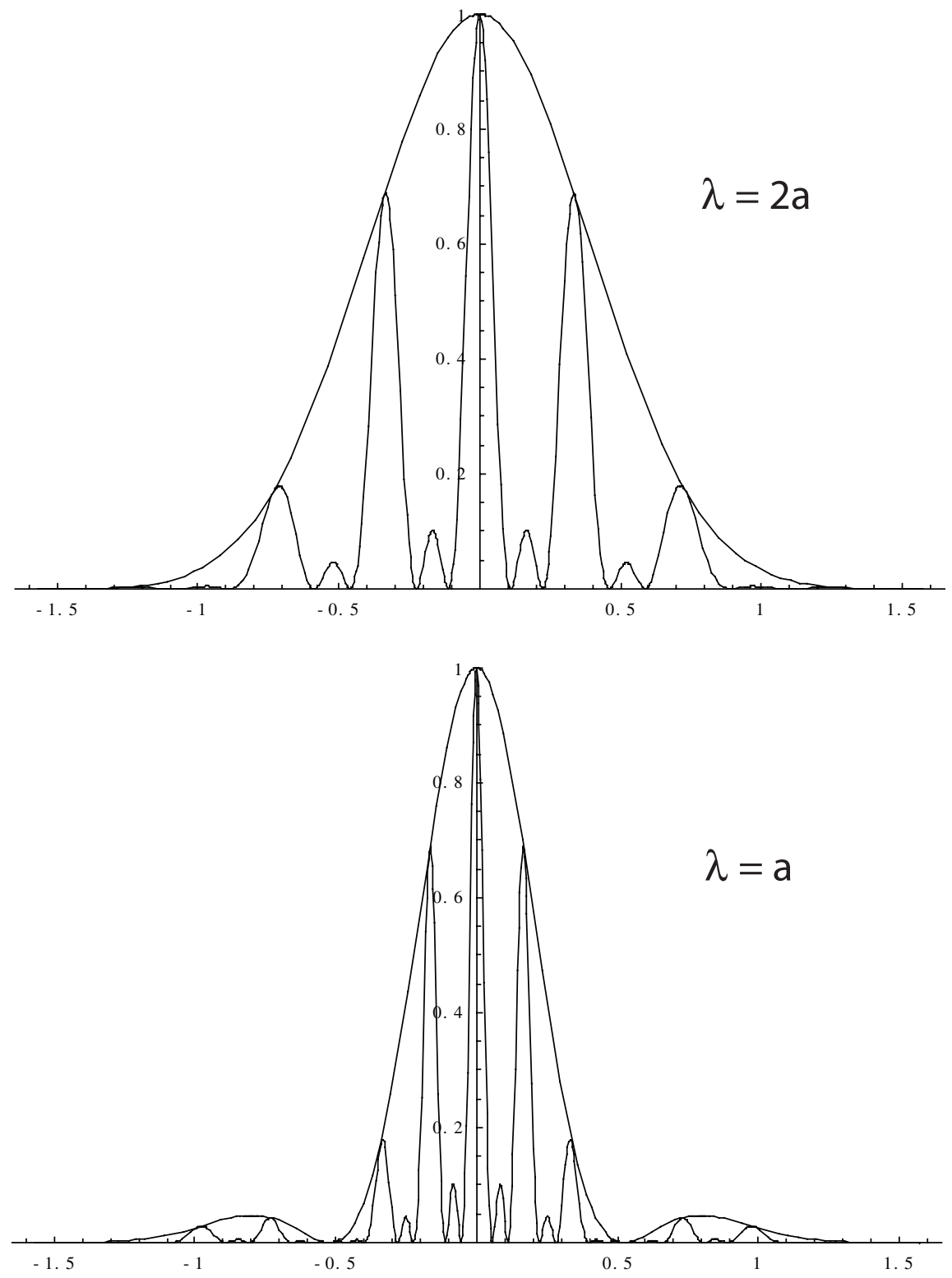

Figure 18. Figures de diffraction en fonction de $\theta_{x}$ pour deux longueurs d'onde. Il s'agit d'un réseau composé de trois fentes de largeur $2 a$ espacées de $d=6 a$. Pour $\lambda=2 a$ seul le pic central de l'enveloppe est observable. La gamme dynamique de l'expérience de diffraction est très restreinte. Quand la longueur d'onde diminue la gamme dynamique augmente.

Avant d'interpréter ce résultat nous voudrions l'établir d'une manière alternative. Nous introduisons le peigne de Dirac comme la suite périodique de fonctions de Dirac

$$
\sum_{m=-\infty}^{\infty} \delta(x-m \cdot d)
$$


Ensuite nous rappelons que la convolution de deux fonctions $g(x)$ et $h(x)$ est définie comme

$$
g(x) \star h(x)=\int g\left(x^{\prime}\right) h\left(x-x^{\prime}\right) d x^{\prime},
$$

et que la transformée de Fourier de deux fonctions convoluées est le produit des transformées de Fourier de chacune d'elles

$$
\mathrm{TF}[(g(x) \star h(x)]=\mathrm{TF}[(g(x)] \cdot \operatorname{TF}[(h(x)] .
$$

La transmittance du réseau peut s'écrire sous la forme

$$
\begin{aligned}
t(x) & =\operatorname{rect}\left(\frac{x}{L}\right) \cdot\left[\int_{-\infty}^{\infty} d x^{\prime} \operatorname{rect}\left(\frac{x^{\prime}}{2 a}\right) \sum_{m=-\infty}^{\infty} \delta\left(x^{\prime}-(x-m \cdot d)\right)\right] \\
& =\operatorname{rect}\left(\frac{x}{L}\right) \cdot\left[\operatorname{rect}\left(\frac{x}{2 a}\right) \star \sum_{m=-\infty}^{\infty} \delta(x-m \cdot d)\right] .
\end{aligned}
$$

Le peigne de Dirac correspondant aux centres des fentes est convolué avec la transmittance d'une fente individuelle et ensuite multiplié avec la transmittance d'une fonction rectangle, qui introduit la taille finie du réseau. C'est un peu comme si on posait du carrelage. Les carreaux correspondent aux fentes, le peigne de Dirac est la grille qui indique où il faut les placer, et l'ensemble est délimité par les murs de la pièce. La transformée de Fourier de la fonction (7.23) est la convolution de la fonction de diffraction de l'enveloppe (la pièce)

$$
\mathrm{FT}\left[\operatorname{rect}\left(\frac{x}{L}\right)\right]=L\left[\frac{\sin \left(Q_{x} L / 2\right)}{\left(Q_{x} L / 2\right)}\right]
$$

avec le produit des fonctions de diffraction de la fente (le carreau)

$$
\mathrm{FT}\left[\operatorname{rect}\left(\frac{x}{2 a}\right)\right]=2 a\left[\frac{\sin \left(Q_{x} a\right)}{Q_{x} a}\right]
$$

et du peigne de Dirac (la grille)

$$
\mathrm{FT}[t]=\mathrm{FT}\left[\operatorname{rect}\left(\frac{x}{L}\right)\right] \star\left\{\mathrm{FT}\left[\operatorname{rect}\left(\frac{x}{2 a}\right)\right] \cdot \mathrm{FT}\left[\sum_{m=-\infty}^{\infty} \delta(x-m \cdot d)\right]\right\} .
$$

La transformée de Fourier d'un peigne de Dirac est un peigne de Dirac dans l'espace réciproque avec une période de $2 \pi / d$. Formellement

$$
\mathrm{FT}\left[\sum_{m=-\infty}^{\infty} \delta(x-m \cdot d)\right]=\frac{1}{d} \sum_{m=-\infty}^{\infty} \delta\left(\frac{Q_{x}}{2 \pi}-\frac{m}{d}\right) .
$$

Les images de diffraction s'obtiennent à partir du carré de l'expression (7.26), qui n'est rien d'autre que l'expression (7.19). Nous obtenons une figure de diffraction riche en effets d'interférences. Dans la terminologie de la diffusion, nous sommes en régime purement cohérent. Les pics se trouvent à une distance de $2 \pi / d$. Leurs positions nous renseignent en conséquence sur la périodicité du réseau. La largeur d'un pic est donnée par la largeur $2 \pi / L$ de la figure de diffraction de l'enveloppe du réseau. Ainsi les pics s'affinent soit avec le nombre de fentes, soit avec la distance entre les fentes. Mais c'est en fin de compte l'extension du réseau, et non pas le nombre de fentes, qui définit la largeur des pics. Dans la limite d'un réseau qui est infiniment large sur l'échelle de la longueur d'onde de la lumière nous obtenons des pics $\delta$ (parce que dans ce cas la transformée de Fourier de l'enveloppe devient une fonction $\delta$ ). Par conséquent, la largeur des pics contient des informations sur la taille des régions diffractantes. L'intensité des pics est modulée par la fonction de diffraction ou le facteur de forme de la fente. Si la fente est très fine, la periodicité $\pi / a$ de cette modulation est très grande. Pour des fentes 


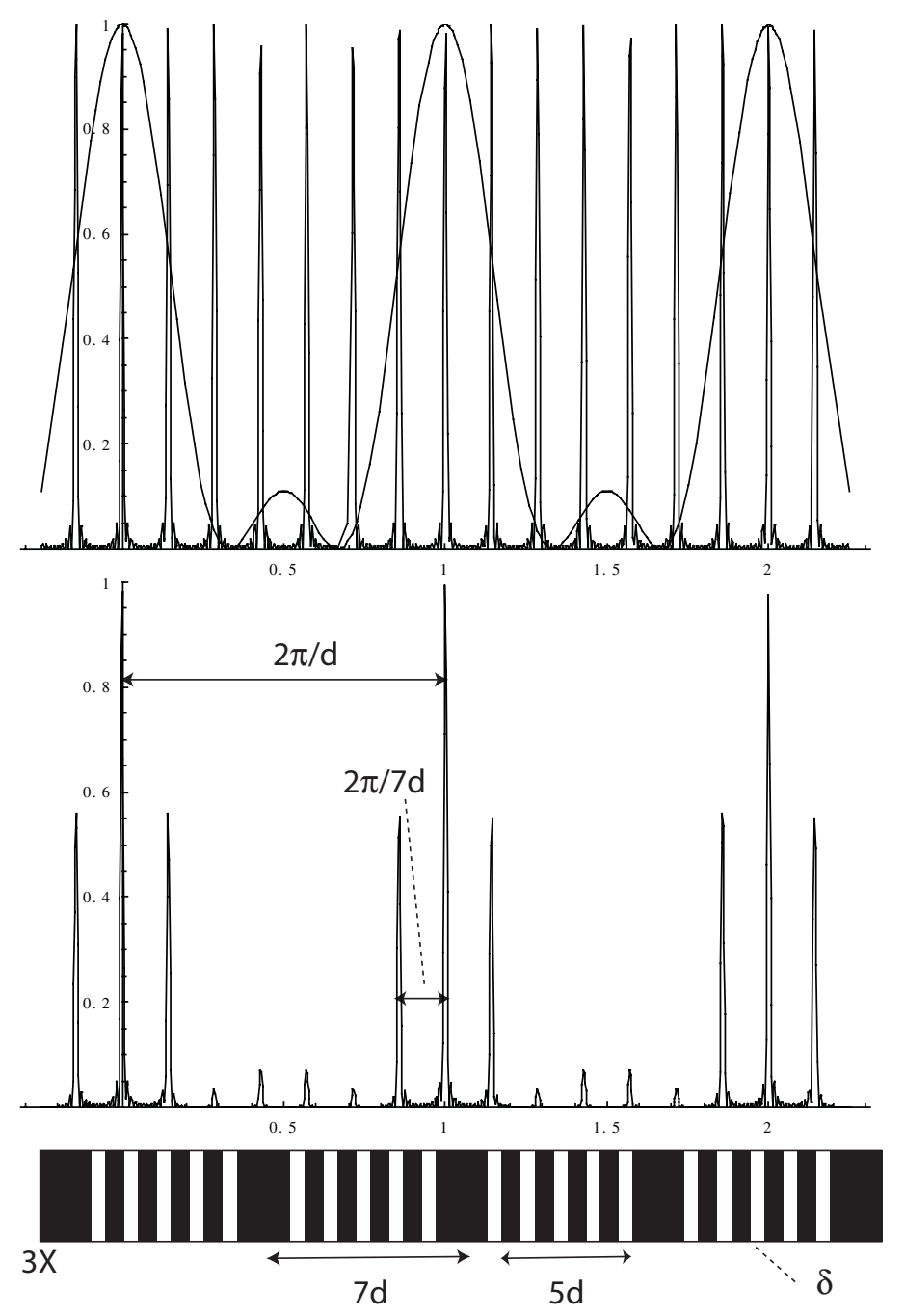

Figure 19. Figure de diffraction d'un réseau avec motif. Si nous négligeons l'extension finie du réseau, la figure de diffraction s'obtient en multipliant la fonction de diffraction du motif (ici un système de 5 fentes de Dirac de distance $d$ ), avec la figure de diffraction du réseau périodique (ici un peigne de Dirac de distance $7 d$ ). La figure de diffraction du réseau périodique infini est un peigne de Dirac de distance $2 \pi / 7 d$. La fonction de diffraction du motif est une fonction périodique qui modifie la hauteur de ces pics. Pour des fentes d'extension finie, cette fonction est à multiplier par la fonction de diffraction de la fente. C'est l'équivalent du facteur de forme d'un atome en diffusion des rayons X. Elle entraînera la perte d'intensité vers les grands vecteurs $Q$. L'information sur la périodicité du réseau (la maille) est donc contenue dans la position des pics. L'information sur la forme du motif, ou dans le cas d'un cristal sur la position des atomes dans la maille, s'obtient à partir de l'intensité des pics.

décrites par une fonction de Dirac le facteur de forme de la fente est une simple constante. C'est le cas pour le pseudo-potentiel de Fermi en diffusion. Dans le cas des rayons-X, la diffusion est faite par le nuage électronique. Ceci introduit un facteur de forme qui fait décroître l'intensité pour les grands vecteurs d'onde $\vec{Q}$, comme c'est le cas pour les fentes optiques. Pour pousser plus loin l'analogie avec la diffusion, regardons ce qui se passe si nous remplaçons la simple fente par un motif. Une manière très simple et instructive de former un tel motif consiste à ôter périodiquement des fentes pour obtenir un réseau de plus grande périodicité (voir figures 19 et 20). Les pics principaux apparaissent à des 

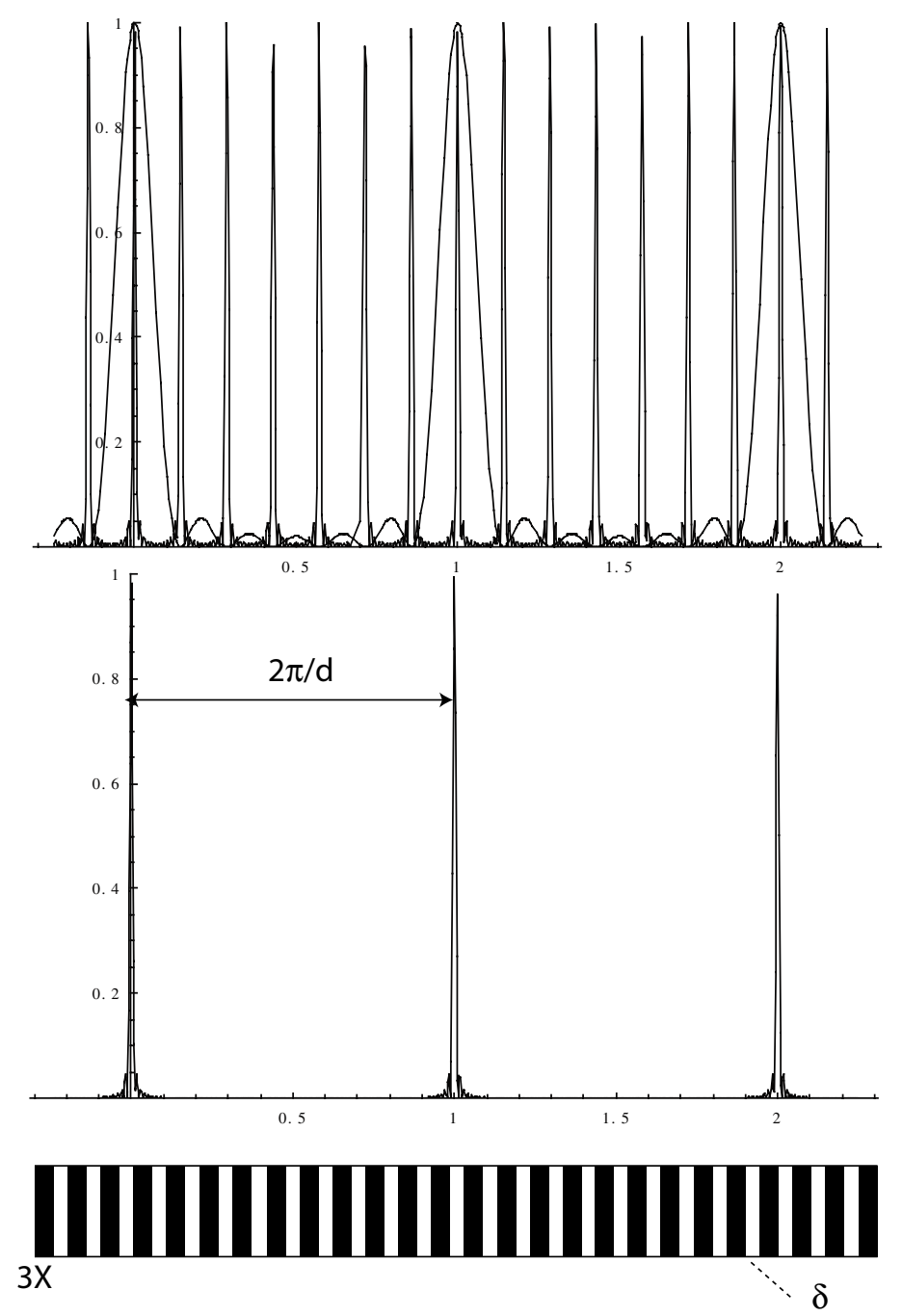

Figure 20. Figure de diffraction d'un réseau avec un motif qui restaure la periodicité à distance $d$. Tous les pics intermédiaires sont éteints par l'action du facteur de forme du motif.

intervalles plus petits. Comme le réseau peut de nouveau être consideré comme la convolution d'un peigne de Dirac avec la transmittance du motif, l'image de diffraction s'obtient par le produit de la fonction de diffraction du motif et de la fonction de diffraction du réseau. L'intensité des pics est donc modulée selon la fonction de diffraction du motif et il est possible de déterminer la forme du motif en étudiant ces intensités.

\subsection{Diffraction par un réseau à trois dimensions}

Si nous passons des réseaux plans aux réseaux tridimensionnels, nous obtenons la diffraction de Bragg. La relation fondamentale entre l'angle de diffusion $2 \Theta$ (voir figure 21) et la longueur d'onde de la lumière diffractée est donnée par l'équation de Bragg

$$
n \lambda=2 \cdot d \cdot \sin \theta,
$$




\section{$\mathrm{Na}_{2} \mathrm{Ca}_{3} \mathrm{Al}_{2} \mathrm{~F}_{14}$ mesuré sur super-D2B}
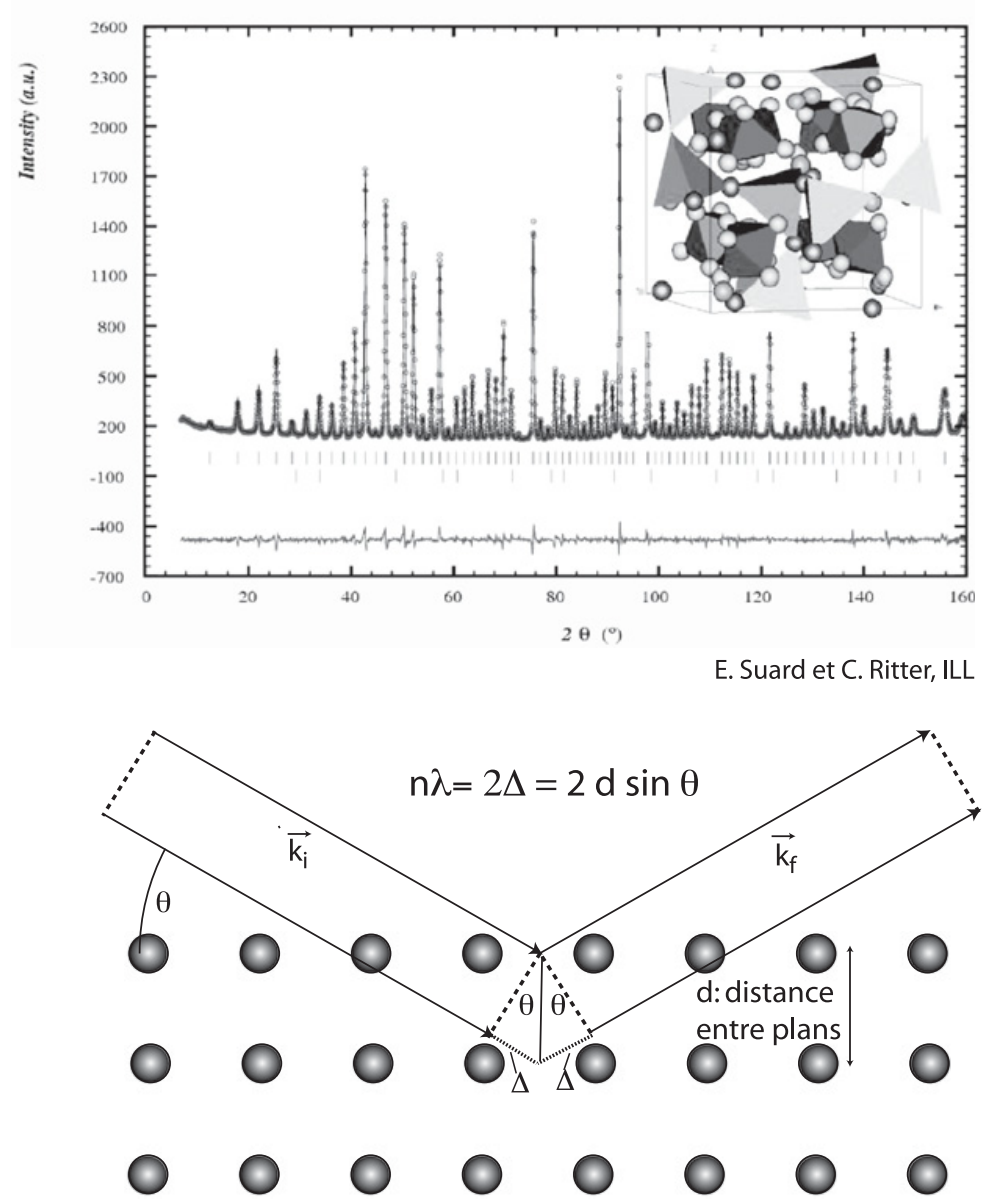

Figure 21. Diffraction de Bragg. La figure du haut donne un exemple typique d'une figure de diffraction d'une poudre cristalline mesurée sur un diffractomètre à neutrons de haute résolution. La figure du bas justifie l'équation de Bragg à partir des différences de chemin optique entre deux plans cristallins.

où $d$ est la distance entre les plans diffractants, et $n$ dénote l'ordre des réflexions de Bragg. L'équation de Bragg exprime le fait que, pour aboutir à une interférence positive, les chemins optiques impliquant les divers plans ne doivent différer que d'un multiple $n$ de la longueur d'onde (voir figure 21). De tels réseaux tridimensionnels sont, par exemple, fournis par des monocristaux. La diffusion des rayons-X et/ou aux neutrons thermiques est bien adaptée à l'étude de ces réseaux du fait de la distance entre atomes qui est de l'ordre du nanomètre.

\subsection{Longueur de cohérence longitudinale}

Jusqu'ici, nous avons considéré que la lumière incidente était décrite par des ondes monochromatiques planes parfaites. Dans la pratique ce n'est jamais le cas. Les imperfections sont dûes, soit à l'étendue de la source lumineuse primaire, soit à la distribution spectrale de celle-ci. Les interférences ont comme origine, la différence de phase entre les ondelettes provenant des divers endroits de l'objet diffractant. Cette différence de phase est engendrée par la variation du chemin optique entre l'origine de l'ondelette 
et son point d'observation. Les images d'interférence seront nettes pourvu que l'excitation des ondelettes aux divers endroits est synchronisée, c'est-à-dire effectuée avec des relations de phase bien determinées. C'est le cas pour un front d'onde unique, par exemple produit par une source ponctuelle. Toute variation aléatoire dans le front d'onde aura comme conséquence l'affaiblissement des effets d'interférence. De telles fluctuations sont inévitables pour une source étendue dont l'émission de la lumière n'est pas cohérente, c'est-à-dire non-synchronisée. C'est le cas des ampoules incandescentes ainsi que des étoiles. Les lasers par contre sont des sources cohérentes. De plus, les images de diffraction dépendent de la longueur d'onde utilisée. Si la source de lumière primaire contient un spectre de fréquences, la superposition des images pour les différentes longueurs d'onde du faisceau aura également une tendance à "brouiller" les interférences.

Discutons, dans un premier temps, de la polychromacité de la source primaire. Pour une fréquence $\nu$ donnée, la phase de l'amplitude complexe de la lumière change d'un facteur $2 \pi$ sur une distance de $L=\lambda=c / v$, avec $c$ la vitesse de la lumière. Deux ondes engendrées simultanément au même endroit, dont les fréquences diffèrent de $\Delta v$, se trouveront donc complètement dephasées quand elles auront parcouru un chemin optique d'une longueur

$$
L_{c}=\frac{c}{\Delta v}=\frac{\lambda^{2}}{\Delta \lambda}
$$

$L_{c}$ est appelé longueur de cohérence temporelle ou longueur de cohérence longitudinale (voir figure 22). Si les différences dans le chemin optique parcouru par les ondelettes à un point $P$ du plan d'observation dépassent la longueur de cohérence de la source primaire, alors les interférences deviennent inobservables. Pour illustrer ce phénomène, prenons comme exemple le cas du réseau de fentes. Les maxima de diffraction se trouvent à

$$
Q_{m}=m \frac{2 \pi \sin \theta}{\lambda}=m \frac{2 \pi}{d} .
$$

Si nous choisissons l'incertitude en longueur d'onde égale à $\Delta \lambda=\frac{\lambda^{2}}{d}$, nous obtenons avec (7.29) une longueur de corrélation longitudinale qui est égale à la distance $d$ entre les fentes. Pour un ordre $m$ de diffraction donné, les maxima de l'image de diffraction balayent toutes les valeurs entre $Q(\lambda)$ et $Q\left(\lambda^{\prime}\right)$ avec

$$
\lambda^{\prime}=\lambda \pm \frac{\Delta \lambda}{2}=\lambda \pm \frac{\lambda^{2}}{2 d}
$$

Pour $\Delta \lambda \ll \lambda$ on obtient

$$
Q_{m=1}\left(\lambda^{\prime}\right)=\frac{2 \pi \sin \theta}{\lambda \pm \frac{\lambda^{2}}{2 d}} \approx Q \pm \frac{1}{2} \frac{2 \pi \sin \theta}{d} .
$$

Pour $\theta=\pi / 4$ nous obtenons $\sin \theta=1 / \sqrt{2}$ et donc $Q_{m=1}\left(\lambda^{\prime}\right) \approx Q \pm \pi / d$. Le balayage comprend dans ce cas toute la région entre les pics, et les interférences devraient être à peine observables (voir figure 22). Les maxima correspondant à une fréquence se superposent aux minima de l'autre en effaçant ainsi les effets d'interférences. La longueur de cohérence définit donc la résolution du dispositif de diffraction dans l'espace réel.

\subsection{Longueur de cohérence transversale}

Même si la source est monochromatique, la résolution du dispositif optique sera limitée du fait de l'extension de la source. Pour illustrer ce phénomène nous allons étudier comment le déplacement d'une source ponctuelle se répercute sur les interférences dans le plan d'observation (voir figure 23). On prend des déplacements grands à l'échelle de la longueur d'onde, ce qui nous permettra de proposer une argumentation basée sur des fronts d'onde. Au premier ordre, seul des déplacements latéraux vont 


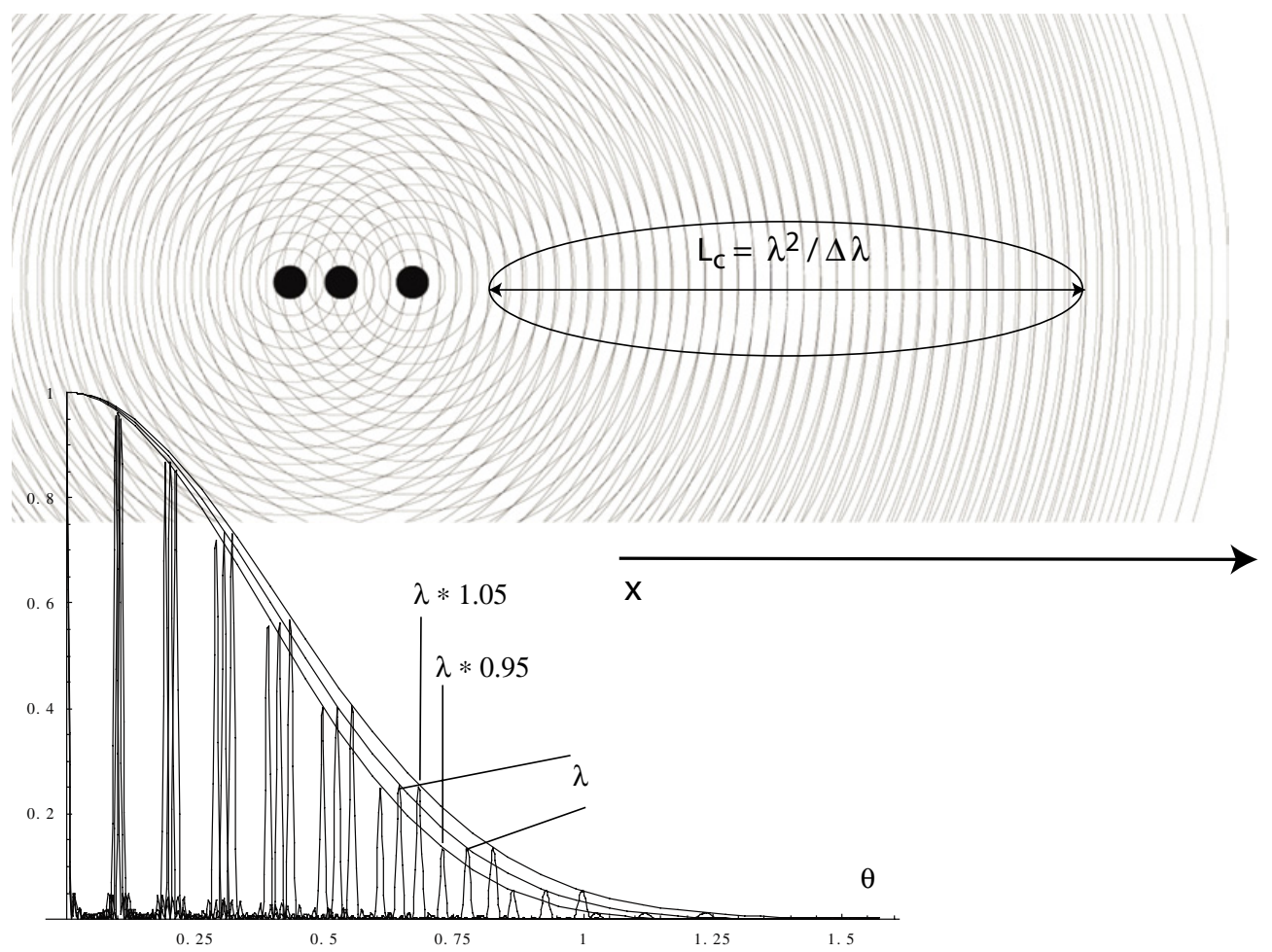

Figure 22. Longueur de cohérence longitudinale. L'objet à illuminer se trouve à une certaine distance le long de la direction $x$. Les ondes polychromatiques se déphasent les unes par rapport aux autres sur une longueur $L_{c}$. Dans ce contexte la relation de phase absolue entre les ondelettes ne joue aucune importance parce que chacune produit sa propre image de diffraction (Il n'y a pas d'interférence entre des ondelettes dont la longueur d'onde diffère parce que de telles interférences s'annulent en intégrant sur le temps). Les sources des ondes primaires ont ici été déplacées horizontalement de manière à illustrer le déphasage à une certaine distance de l'origine. Le choix de cette distance est arbitraire. C'est la superposition des images de diffraction qui brouille les interférences. Nous montrons en bas trois courbes de diffraction (en fonction de l'angle de diffraction $\theta$ ) calculées pour un système de 11 fentes de largeurs $2 a=\lambda$ et espacées de $d=10 \cdot \lambda$. Un $\Delta \lambda$ de $10 \%$ donne une longueur de cohérence longitudinale $L_{\mathrm{c}}=\lambda$. Pour les grands angles de diffraction, on observe de l'intensité partout. Les interférences sont brouillées. Pour des petits angles de diffraction les différences entre les chemins optiques des ondelettes donnant lieu à interférence sont plus petites et la longueur de cohérence sera suffisante pour séparer les franges.

contribuer à la modification des chemins optiques. Des déplacements purement longitudinaux, c'est-àdire le long de l'axe qui lie la source à l'objet diffractant, ne modifient pas les différences de phases, comme nous l'avons déjà explicité plus haut. Nous prenons comme système diffractant, les fentes de Young, que nous avons déjà abordées dans l'introduction. Considérons aussi une distance $L$ entre la source primaire et les fentes de Young grande à la fois par rapport à l'extension $L_{S}$ de la source et par rapport à la distance $L_{F}$ entre les fentes. La partie secondaire du dispositif restant inchangée, toute modification des chemins optiques doit se faire dans la partie primaire, c'est-à-dire entre la source primaire et les fentes. Les fronts d'ondes correspondant aux divers points de la source heurteront les deux fentes avec un décalage spatial $\Delta$ de

$$
\Delta \approx \frac{L_{F}}{L} L_{S} .
$$


Dès que cette différence de chemin optique équivaut à la longueur d'onde, les effets d'interférence vont disparaître. Définissons la longueur de cohérence latérale comme

$$
L_{c}=\lambda \frac{L}{L_{s}}=\frac{\lambda}{\theta}
$$

avec $\Theta$ l'angle interceptant la source, vue à la position des fentes. La longueur de cohérence latérale augmente avec la distance entre la source primaire et l'objet diffractant. On dit que la distance crée la cohérence. On obtient par exemple une longueur de cohérence latérale de $60 \mu \mathrm{m}$ pour le soleil. Si nous utilisons la diffraction comme méthode analytique il nous sera impossible de déterminer la distance entre les fentes dès que celles-ci sont espacées de plus de $L_{c}$. Comme dans le cas de la longueur de cohérence longitudinale, la longueur de cohérence latérale définit la résolution dans l'espace réel. Nous allons revenir sur ce point lors de la discussion de la résolution dans le cas de la diffusion des neutrons (voir section 9.6).

\section{FONCTIONS DE CORRÉLATION}

Lors de la discussion de la diffusion cohérente et incohérente nous avons fait allusion à la connexion entre la fonction de diffusion et les fonctions de corrélation. Il reste à établir le cadre mathématique précis de cette relation. Pour cela, nous suivrons de près la voie proposée par Van Hove [18].

On rappelle l'expression générale (5.43) pour la fonction de diffusion $S(\vec{Q}, \omega)$

$$
S(\vec{Q}, \omega)=\frac{1}{2 \pi \hbar} \sum_{\lambda_{i}} p\left(\lambda_{i}\right) \sum_{j, j^{\prime}=1}^{N} V_{j}(\vec{Q}) V_{j^{\prime}}^{*}(\vec{Q}) \int_{-\infty}^{\infty} d t\left\langle\lambda_{i}\left|e^{-i \vec{Q} \cdot \vec{R}_{j^{\prime}}^{0}} e^{i \vec{Q} \cdot \vec{R}_{j}(t)}\right| \lambda_{i}\right\rangle e^{-i \omega t} .
$$

ainsi (voir chapitre I) que l'expression de la moyenne d'une grandeur physique A définie par

$$
\langle A\rangle=\sum p_{n}\left\langle\psi_{n}|\mathbf{A}| \psi_{n}\right\rangle .
$$

Les états $\left|\lambda_{i}\right\rangle$ doivent constituer une base complète du système étudié. Ceci nous permet d'écrire

$$
\sum_{\lambda_{i}} p\left(\lambda_{i}\right)\left\langle\lambda_{i}\left|e^{-i \vec{Q} \cdot \vec{R}_{j^{\prime}}^{0}} e^{i \vec{Q} \cdot \vec{R}_{j}(t)}\right| \lambda_{i}\right\rangle=\left\langle e^{-i \vec{Q} \cdot \vec{R}_{j^{\prime}}^{0}} e^{i \vec{Q} \cdot \vec{R}_{j}(t)}\right\rangle
$$

La fonction de diffusion devient

$$
S(\vec{Q}, \omega)=\sum_{j, j^{\prime}}\left(V_{j}(\vec{Q}) V_{j^{\prime}}^{*}(\vec{Q})\right) S_{j, j^{\prime}}(\vec{Q}, \omega)
$$

avec

$$
S_{j, j^{\prime}}(\vec{Q}, \omega)=\frac{1}{2 \pi \hbar} \sum_{j, j^{\prime}=1}^{N} \int_{-\infty}^{\infty} d t\left\langle e^{-i \vec{Q} \cdot \vec{R}_{j^{\prime}}^{0}} e^{i \vec{Q} \cdot \vec{R}_{j}(t)}\right\rangle e^{-i \omega t} .
$$

La fonction de diffusion totale est donc une superposition de fonctions qui impliquent des paires de diffuseurs $\left(j, j^{\prime}\right)$. En général, le nombre de potentiels d'interaction $V(\vec{Q})$ distincts reste relativement limité. Dans le cas d'un système monoatomique il n'existe même qu'un seul potentiel. Nous allons distinguer les differents types de diffuseur par l'indice $\kappa$. Chacun des $N_{\kappa}$ diffuseurs appartenant à un type $\kappa$ seront étiquetés par $j_{\kappa}$. La somme sur les diffuseurs devient donc

$$
\sum_{j}=\sum_{\kappa} \sum_{j_{\kappa}}=\sum_{\kappa} \sum_{j \in\left\{j_{\kappa}\right\}}
$$

Nous pouvons reformuler la fonction de diffusion (8.4) comme

$$
S(\vec{Q}, \omega)=\sum_{\kappa, \kappa^{\prime}}\left(V_{\kappa}(\vec{Q}) V_{\kappa^{\prime}}^{*}(\vec{Q})\right) S_{\kappa, \kappa^{\prime}}(\vec{Q}, \omega)
$$



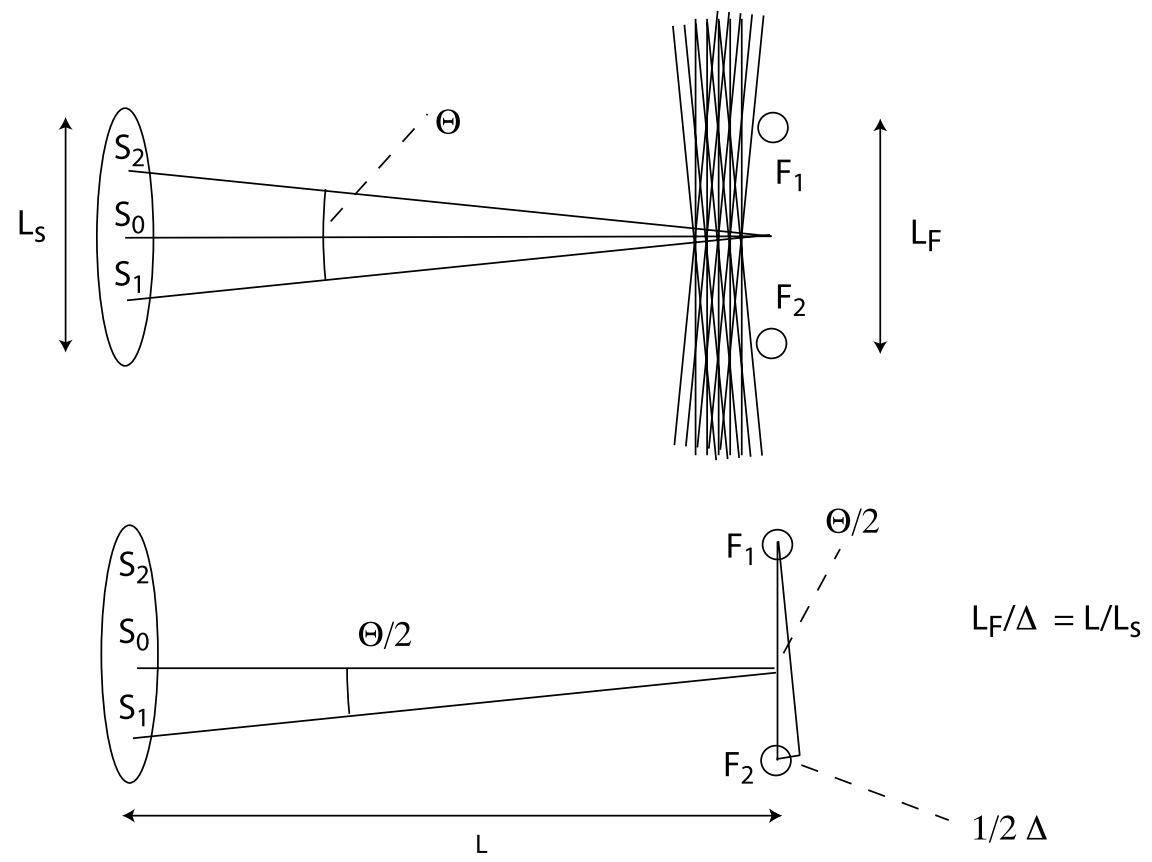

Figure 23. Longueur de cohérence latérale dûe à l'étendue de la source primaire. $L_{s}$ et $L$ sont grandes à l'échelle de $L_{F}$ et $L \gg L_{S}$. Les fronts d'onde correspondant aux divers points d'émission de la source primaire sont inclinés les uns par rapport aux autres au moment où ils heurtent les deux fentes. Ceci se traduit par un décalage spatial (ou mieux une différence de phase) lors de l'émission des ondelettes qui doivent interférer dans le plan d'observation. Si cette différence $\Delta$ devient de l'ordre de la longueur d'onde $\lambda$ les franges d'interférence seront brouillées. Ceci définit la longueur de cohérence latérale.

avec les fonctions de diffusion partielles définies comme

$$
S_{\kappa, \kappa^{\prime}}(\vec{Q}, \omega)=\frac{1}{2 \pi \hbar} \sum_{j \in\left\{j_{k}\right\}, j^{\prime} \in\left\{j_{\kappa^{\prime}}\right\}} \int_{-\infty}^{\infty} d t\left\langle e^{-i \vec{Q} \cdot \vec{R}_{j^{\prime}}^{0}} e^{i \vec{Q} \cdot \vec{R}_{j}(t)}\right\rangle e^{-i \omega t}
$$

De la même manière nous pouvons définir des fonctions de diffusion partielles propres

$$
S_{\kappa}^{S}(\vec{Q}, \omega)=\frac{1}{2 \pi \hbar} \sum_{j \in\left\{j_{\kappa}\right\}} \int_{-\infty}^{\infty} d t\left\langle e^{-i \vec{Q} \cdot \vec{R}_{j}^{0}} e^{i \vec{Q} \cdot \vec{R}_{j}(t)}\right\rangle e^{-i \omega t}
$$

Ces fonctions nous seront utiles dans le contexte de la diffusion incohérente.

Nous pourrions considérablement alléger le formalisme en nous concentrant sur des systèmes monoatomiques. Dans la pratique, les systèmes étudiés ne le sont que très rarement. C'est la raison pour laquelle nous avons fait le choix de faire apparaître la diversité des diffuseurs dans les formules, au détriment de l'aspect pédagogique du cas monoatomique.

\subsection{La fonction d'auto-corrélation de la densité atomique $G(\vec{r}, t)$}

Nous allons montrer que les fonctions de diffusion partielles $S_{\kappa, \kappa^{\prime}}(\vec{Q}, \omega)$ peuvent être exprimées en termes de fonctions de corrélation à deux points. 
En ignorant son spin, un diffuseur est caractérisé par sa position dans l'espace $\vec{R}_{j}$. La fonction d'autocorrélation ${ }^{33}$ d'une grandeur physique $A$ est définie comme la corrélation croisée continue de $A$ avec elle-même. Formellement ceci équivaut à une convolution dans le temps et dans l'espace de $A$ avec elle-même (voir chapitre I).

$$
\Gamma_{A, A}(\vec{r}, t)=\lim _{T \rightarrow \infty} \frac{1}{T} \frac{1}{V} \int_{0}^{T} d t^{\prime} \int d^{3} r^{\prime}\left\langle A\left(\vec{r}^{\prime}, t^{\prime}\right) A^{*}\left(\vec{r}^{\prime}-\vec{r}, t^{\prime}-t\right)\right\rangle .
$$

en supposant que le système est ergodique pour que la limite existe. L'autocorrélation est un outil puissant pour détecter des régularités -comme des oscillations fondamentales- dans un signal.

Pour un système stationnaire l'origine du temps peut être choisie arbitrairement. L'expression $\left\langle A\left(\vec{r}^{\prime}, t^{\prime}\right) A^{*}\left(\vec{r}^{\prime}-\vec{r}, t^{\prime}+t\right)\right\rangle$ ne dépendra donc pas de $t^{\prime}$. La moyenne sur $t^{\prime}$ est par conséquent triviale et la fonction d'auto-corrélation se simplifie.

$$
\Gamma_{A, A}(\vec{r}, t)=\frac{1}{V} \int d^{3} r^{\prime}\left\langle A\left(\vec{r}^{\prime}, t=0\right) A^{*}\left(\vec{r}^{\prime}+\vec{r}, t\right)\right\rangle .
$$

Dans le cas d'une seule particule, les postulats de la mécanique quantique donnent la probabilité de rencontrer la particule à l'endroit $\vec{R}$ au moment $t$ quand le système se trouve dans l'état $|\lambda\rangle$. Celle-ci s'exprime comme

$$
\begin{aligned}
\psi_{\lambda}^{*}(\vec{R}(t)) \psi_{\lambda}(\vec{R}(t)) & =\int d^{3} r \int d^{3} r^{\prime} \delta(\vec{r}-\vec{R}(t)) \delta\left(\vec{r}^{\prime}-\vec{R}(t)\right) \psi_{\lambda}^{*}(\vec{r}) \psi_{\lambda}\left(\vec{r}^{\prime}\right) \\
& =\int d^{3} r \delta(\vec{r}-\vec{R}(t)) \psi_{\lambda}^{*}(\vec{r}) \psi_{\lambda}(\vec{r}) \\
& =\langle\lambda|\hat{\rho}(\vec{r}, t)| \lambda\rangle
\end{aligned}
$$

avec

$$
\hat{\rho}(\vec{r}, t)=\delta(\vec{r}-\vec{R}(t)) .
$$

33 En statistique, l'autocorrélation d'un processus $X_{t}$, par exemple discret dans le temps, est définie comme la corrélation
normalisée de l'écart $X_{t}-\langle X\rangle$ par rapport à une version décalée dans le temps de lui-même. Formellement
\[ R(t)=\frac{\left\langle\left(X_{t^{\prime}}-\langle X\rangle\right)\left(X_{t^{\prime}+t}-\langle X\rangle\right)\right\rangle}{\left\langle\left(X_{t^{\prime}}-\langle X\rangle\right)^{2}\right\rangle} \]
Sans normalisation, on parle plutôt d'autocovariance. Deux variables peuvent être considerées décorrélées si
\[ \langle X \cdot Y\rangle=\langle X\rangle\langle Y\rangle \text {. } \]
Ceci nous amène à des fonctions de corrélation strictement nulles, si et seulement si elles sont formulées en terme d'écart par
rapport à la moyenne, comme c'est le cas dans l'expression (8.10). Les fonctions de corrélation définies dans la théorie de la
diffusion ne satisfont en général pas à ce critère.
La notion d'indépendance statistique qui est directement liée à la notion de corrélation de deux variables aléatoires n'est pas
toujours intuitive. Sur ce sujet, le lecteur est invité à consulter le livre de Konstantin Protassov [29] et en particulier le passage
concernant l'exemple du "roi de cœur" qui y figure. Dans un jeu de 52 cartes l'évènement "roi" et l'évènement "cœur" sont
statistiquement indépendants. Formellement cette indépendance s'exprime par le fait que la probabilité de tirer un "roi de cœur"
est le produit de la probabilité de tirer un "roi" multipliée par la probabilité de tirer la "couleur cœur".

$$
P\left(\text { (roi de cœur") }=P(\text { (roi") }) \cdot P(\text { “cœur" })=\frac{4}{52} \frac{13}{52}=\frac{1}{52} .\right.
$$

Cette relation n'est plus vraie si nous rajoutons un joker au jeu

$$
P\left(\text { (roi de cœur") }=\frac{1}{53}>P(\text { (roi”) }) \cdot P(\text { (cœur" })=\frac{4}{53} \frac{13}{53}=\frac{52}{53} \frac{1}{53} .\right.
$$

Le joker modifie ainsi l'indépendance statistique de deux évènements dont il ne fait partie ni de l'un ni de l'autre. Cette corrélation s'explique par le fait qu'à partir du moment où nous savons que la carte possède une couleur, nous éliminons immédiatement la possibilité qu'il s'agisse d'un joker, et ainsi nous augmentons la possibilité de trouver un roi. 
$\hat{\rho}(\vec{r}, t)$ peut donc être consideré comme un opérateur, dont les éléments de matrice donnent la probabilité que la position de la particule soit comprise dans l'élément $d^{3} r$ autour de $\vec{r}$.

Nous pouvons généraliser ce concept à un système à plusieurs particules en introduisant l'opérateur

$$
\hat{\rho}_{\kappa}(\vec{r}, t)=\sum_{j \in\left\{j_{k}\right\}} \delta\left(\vec{r}-\vec{R}_{j}(t)\right) .
$$

$\left\{j_{\kappa}\right\}$ est l'ensemble des indices des diffuseurs de type $\kappa$. L'opérateur $\hat{\rho}_{\kappa}(\vec{r}, t)$ "projette" la probabilité de trouver un diffuseur de type $\kappa$ dans l'élément $d^{3} r$ autour de $\vec{r} . \hat{\rho}_{\kappa}(\vec{r}, t)$ est appelé opérateur du nombre densité. Il ne doit pas être confondu avec l'opérateur de densité statistique (voir chapitre I).

La transformée de Fourier spatiale de $\hat{\rho}_{\kappa}(\vec{r}, t)$ s'obtient comme

$$
\hat{\rho}_{\kappa}(\vec{Q}, t)=\int_{V} d^{3} r \exp (-i \vec{Q} \cdot \vec{r}) \hat{\rho}_{\kappa}(\vec{r}, t)=\sum_{j \in\left\{j_{k}\right\}} \exp \left(-i \vec{Q} \cdot \vec{R}_{j}(t)\right) .
$$

C'est le même type d'expression que nous trouvons dans les moyennes qui composent la fonction de diffusion (voir (8.8)).

Nous pouvons maintenant définir la fonction d'autocorrélation de cet opérateur

$$
\begin{aligned}
G_{\kappa, \kappa^{\prime}}(\vec{r}, t) & =\int d^{3} \vec{r}^{\prime}\left\langle\hat{\rho}_{\kappa}\left(\vec{r}^{\prime}-\vec{r}\right) \hat{\rho}_{\kappa^{\prime}}\left(\vec{r}^{\prime}, t\right)\right\rangle \\
& =\sum_{j \in\left\{j_{\kappa}\right\}, j^{\prime} \in\left\{j_{\kappa^{\prime}}\right\}} \int d^{3} \vec{r}^{\prime}\left\langle\delta\left(\vec{r}^{\prime}-\vec{r}-\vec{R}_{j}(t=0)\right) \delta\left(\vec{r}^{\prime}-\vec{R}_{j^{\prime}}(t)\right)\right\rangle
\end{aligned}
$$

$G_{\kappa, \kappa^{\prime}}(\vec{r}, t)$ est une fonction, qui relie la probabilité de trouver un diffuseur de type $\kappa$ au moment $t$ autour de $\vec{r}^{\prime}-\vec{r}$ à la présence d'un diffuseur de type $\kappa^{\prime}$ à $t=0$ autour de $\vec{r}^{\prime}$.

Avant de relier les fonctions de corrélation aux fonctions de diffusion il faut introduire des notions supplémentaires.

- Il s'avère utile de définir une fonction qui ne prend en compte que les corrélations d'un diffuseur avec lui-même.

$$
G_{\kappa}^{s}(\vec{r}, t):=\sum_{j \in\left\{j_{\kappa}\right\}} \int d^{3} \vec{r}^{\prime}\left\langle\delta\left(\vec{r}^{\prime}-\vec{r}-\vec{R}_{j}(t=0)\right) \delta\left(\vec{r}^{\prime}-\vec{R}_{j}(t)\right)\right\rangle
$$

En anglais $G_{\kappa}^{s}(\vec{r}, t)$ est appelé self pair correlation function ce que nous pouvons traduire comme fonction de d'autocorrélation. Il s'agit d'une fonction, qui relie la probabilité de trouver un diffuseur de type $\kappa$ au moment $t$ autour de $\vec{r}^{\prime}-\vec{r}$ alors que ce même diffuseur se trouvait à $t=0$ autour de $\vec{r}^{\prime}$.

- En introduisant l'opérateur de densité $\hat{\rho}$ nous avons utilisé la représentation de Heisenberg pour les opérateurs de position $[13,14]$. Les opérateurs de Heisenberg $\overrightarrow{\mathbf{R}}_{j}(t)$ sont particulièrement adaptés à la formulation des fonctions de corrélation dans l'espace et dans le temps. Dans la représentation de Heisenberg, le système est décrit par une fonction d'onde stationnaire et c'est l'opérateur qui suit (à la manière de la trajectoire classique) l'évolution des particules dans l'espace.

- Rien ne nous oblige à placer l'opérateur $\overrightarrow{\mathbf{R}}_{j}(t=0)$ à gauche de $\overrightarrow{\mathbf{R}}_{j^{\prime}}(t)$ en définissant $G(\vec{r}, t)$. Notre choix doit être considéré arbitraire. Les deux options sont reliées l'une à l'autre par la relation

$$
G(\vec{r}, t)=G(-\vec{r},-t)
$$

ce qu'on peut démontrer avec les règles de calcul de base de la mécanique quantique. 
- On pourrait s'étonner de la forme compliquée que prennent les fonctions de corrélation (8.18) et (8.19) quand on sait que

$$
\int d^{3} \vec{r}^{\prime} \delta\left(\vec{r}^{\prime}-\vec{r}\right) \delta\left(\vec{r}^{\prime}\right)=\delta(\vec{r})
$$

C'est le caractère d'opérateur de $\mathbf{R}_{j}(t)$ qui nous empêche d'utiliser cette relation pour réaliser l'intégration apparaîssant dans les expressions (8.18) et (8.19). Les deux fonctions $\Delta$ ne commutent en général pas l'une avec l'autre pour des temps différents. Cela se voit facilement à partir de leur transformées de Fourier (8.17). Si nous nous libérons de cette contrainte imposée par la mécanique quantique nous obtenons les fonctions de corrélation dites classiques

$$
\begin{aligned}
{ }^{\mathrm{cl}} G_{\kappa, \kappa^{\prime}}(\vec{r}, t) & =\sum_{j \in\left\{j_{\kappa}\right\}, j^{\prime} \in\left\{j_{\kappa^{\prime}}\right\}}\left\langle\delta\left(\vec{r}+\vec{R}_{j}(t=0)-\vec{R}_{j^{\prime}}(t)\right)\right\rangle, \\
{ }^{\mathrm{cl}} G_{\kappa}^{s}(\vec{r}, t) & =\sum_{j \in\left\{j_{\kappa}\right\}}\left\langle\delta\left(\vec{r}+\vec{R}_{j}(t=0)-\vec{R}_{j}(t)\right)\right\rangle .
\end{aligned}
$$

L'interprétation des fonctions de corrélation classiques est considérablement simplifiée. A part un facteur qui prend en compte le nombre total de diffuseurs de type $\kappa$, cette fonction donne tout simplement la probabilité de trouver un diffuseur de type $\kappa$ au moment $t$ autour de $\vec{r}^{\prime}-\vec{r}$ sous la condition qu'un diffuseur de type $\kappa^{\prime}$ se trouvait à $t=0$ dans les environs de $\vec{r}^{\prime}$.

\subsection{Connexion entre $S_{\kappa, \kappa^{\prime}}(\vec{Q}, \omega)$ et $G_{\kappa, \kappa^{\prime}}(\vec{r}, t)$}

Tout comme nous avons converti les fonctions $\delta\left(E_{i}-E_{f}-\hbar \omega\right)$ en intégrale sur le temps, nous pouvons exprimer l'opérateur de densité $\hat{\rho}(\vec{r}, t)$ comme une intégrale sur $\vec{Q}$

$$
\delta\left(\vec{r}^{\prime}-\vec{r}-\vec{R}_{j}(t)\right)=\frac{1}{(2 \pi)^{3}} \int d^{3} Q \exp \left(i \vec{Q} \cdot\left(\vec{r}^{\prime}-\vec{r}-\vec{R}_{j}(t)\right)\right) .
$$

Ceci nous permet de reformuler la fonction de corrélation $G(\vec{r}, t)$ de la manière suivante

$$
\begin{aligned}
G_{\kappa, \kappa^{\prime}}(\vec{r}, t)= & \int d^{3} r^{\prime}\left\langle\hat{\rho}_{\kappa}\left(\vec{r}^{\prime}-\vec{r}\right) \hat{\rho}_{\kappa^{\prime}}\left(\vec{r}^{\prime}, t\right)\right\rangle \\
= & \sum_{j \in\left\{j_{k}\right\}, j^{\prime} \in\left\{j_{\kappa^{\prime}}\right\}} \int d^{3} r^{\prime}\left\langle\delta\left(\vec{r}^{\prime}-\vec{r}-\vec{R}_{j}\right) \delta\left(\vec{r}^{\prime}-\vec{R}_{j^{\prime}}(t)\right)\right\rangle \\
= & \sum_{j \in\left\{j_{k}\right\}, j^{\prime} \in\left\{j_{K^{\prime}}\right\}} \int d^{3} r^{\prime}\left\langle\frac{1}{(2 \pi)^{3}} \int d^{3} Q \exp \left(i \vec{Q} \cdot\left(\vec{r}^{\prime}-\vec{r}-\vec{R}_{j}\right)\right) \delta\left(\vec{r}^{\prime}-\vec{R}_{j^{\prime}}(t)\right)\right\rangle \\
= & \frac{1}{(2 \pi)^{3}} \int d^{3} Q \exp (-i \vec{Q} \cdot \vec{r}) \\
& \times \sum_{j \in\left\{j_{k}\right\}, j^{\prime} \in\left\{j_{\kappa^{\prime}}\right\}} \int d^{3} r^{\prime}\left\langle\exp \left(i \vec{Q} \cdot \vec{r}^{\prime}-i \vec{Q} \cdot \vec{R}_{j}\right) \delta\left(\vec{r}^{\prime}-\vec{R}_{j^{\prime}}(t)\right)\right\rangle \\
= & \frac{1}{(2 \pi)^{3}} \int d^{3} Q \exp (-i \vec{Q} \cdot \vec{r})
\end{aligned}
$$




$$
\begin{aligned}
& \times \sum_{j \in\left\{j_{k}\right\}, j^{\prime} \in\left\{j_{k^{\prime}}\right\}}\left\langle\exp \left(-i \vec{Q} \cdot \vec{R}_{j}\right) \int d^{3} r^{\prime} \exp \left(i \vec{Q} \cdot \vec{r}^{\prime}\right) \delta\left(\vec{r}^{\prime}-\vec{R}_{j^{\prime}}(t)\right)\right\rangle \\
= & \frac{1}{(2 \pi)^{3}} \int d^{3} Q \exp (-i \vec{Q} \cdot \vec{r}) \sum_{j \in\left\{j_{k}\right\}, j^{\prime} \in\left\{j_{k^{\prime}}\right\}}\left\langle\exp \left(-i \vec{Q} \cdot \vec{R}_{j}\right) \exp \left(i \vec{Q} \cdot \vec{R}_{j^{\prime}}(t)\right)\right\rangle .
\end{aligned}
$$

Nous avons de nouveau pris soin de ne pas commuter des expressions contenant $\overrightarrow{\mathbf{R}}_{j}(t)$ à des temps différents.

En comparant l'expression obtenue pour $G(\vec{r}, t)$ avec les expressions (8.8) pour $S_{\kappa, \kappa^{\prime}}(\vec{Q}, \omega)$ nous arrivons (en utilisant les règles de calcul pour les transformées de Fourier) à

$$
S_{\kappa, \kappa^{\prime}}(\vec{Q}, \omega)=\frac{1}{2 \pi \hbar} \int_{V} d^{3} r \int_{-\infty}^{\infty} d t e^{i(\vec{Q} \cdot \vec{r}-\omega t)} G_{\kappa, \kappa^{\prime}}(\vec{r}, t) .
$$

La fonction de diffusion est identifiée comme la double transformée de Fourier dans l'espace et dans le temps de la fonction d'autocorrélation de la densité. L'expérience de diffusion nous livre donc une analyse de Fourier des corrélations temporelles et spatiales. C'est un résultat extrêmement important pour l'analyse des résultats.

Grâce à la transformation de Fourier inverse nous pouvons intervertir les rôles de la fonction de diffusion $S_{\kappa, \kappa^{\prime}}(\vec{Q}, \omega)$ et de la fonction d'auto-correlation $G_{\kappa, \kappa^{\prime}}(\vec{r}, t)$

$$
G_{\kappa, \kappa^{\prime}}(\vec{r}, t)=\frac{\hbar}{(2 \pi)^{3}} \int_{V_{\underline{Q}}} d^{3} Q \int_{-\infty}^{\infty} d t e^{-i(\vec{Q} \cdot \vec{r}-\omega t)} S_{\kappa, \kappa^{\prime}}(\vec{Q}, \omega) .
$$

Ainsi $G_{\kappa, \kappa^{\prime}}(\vec{r}, t)$ se révèle être la double transformée de Fourier de $S_{\kappa, \kappa^{\prime}}(\vec{Q}, \omega)$.

\subsection{Relation entre les fonctions de corrélation et la section efficace différentielle partielle}

La section efficace différentielle partielle peut s' exprimer å l'aide de la fonction de diffusion $S(\vec{Q}, \omega)$ comme

$$
\frac{d^{2} \sigma_{\vec{k}_{i} \rightarrow \vec{k}_{f}}}{d \Omega d E_{f}}=\frac{k_{f}}{k_{i}} S(\vec{Q}, \omega)
$$

avec

$$
S(\vec{Q}, \omega)=\sum_{\kappa, \kappa^{\prime}}\left(V_{\kappa}(\vec{Q}) V_{\kappa^{\prime}}^{*}(\vec{Q})\right) S_{\kappa, \kappa^{\prime}}(\vec{Q}, \omega)
$$

et

$$
S_{\kappa, \kappa^{\prime}}(\vec{Q}, \omega)=\frac{1}{2 \pi \hbar} \sum_{j \in\left\{j_{k}\right\}, j^{\prime} \in\left\{j_{k^{\prime}}\right\}} \int_{-\infty}^{\infty} d t\left\langle\lambda_{i}\left|e^{-i \vec{Q} \cdot \vec{R}_{j^{\prime}}^{0}} e^{i \vec{Q} \cdot \vec{R}_{j}(t)}\right| \lambda_{i}\right\rangle e^{-i \omega t} .
$$

Avec (8.26) nous pouvons donc établir un lien entre les sections efficaces et les fonctions de corrélation

$$
\frac{d^{2} \sigma_{\vec{k}_{i} \rightarrow \vec{k}_{f}}}{d \Omega d E_{f}}=\frac{k_{f}}{k_{i}} \frac{1}{2 \pi \hbar} \sum_{\kappa, \kappa^{\prime}}\left[\left(V_{\kappa}(\vec{Q}) V_{\kappa^{\prime}}^{*}(\vec{Q})\right) \int_{V} d^{3} r \int_{-\infty}^{\infty} d t e^{i(\vec{Q} \cdot \vec{r}-\omega t)} G_{\kappa, \kappa^{\prime}}(\vec{r}, t)\right] .
$$

Si nous avons connaissance des fonctions de corrélation, par exemple à partir d'un calcul ou d'un modèle théorique, nous sommes en mesure de déterminer la section efficace, et ainsi d'anticiper les résultats des expériences de diffusion. L'expression (8.31) joue par conséquent un rôle crucial dans l'interprétation des données.

Malheureusement l'expression (8.31) ne peut pas être inversée, c'est-à-dire que la mesure des sections efficaces ne permet pas de déterminer les fonctions de corrélation $G_{\kappa, \kappa^{\prime}}(\vec{r}, t)$ de manière 
univoque. L'information nécessaire à cette inversion est perdue dans la superposition des contributions pour les différents types de diffuseurs (éléments chimiques) dans (8.31). C'est un problème dont l'importance varie beaucoup selon la question scientifique. Si l'information contenue dans le signal cohérent est en général suffisante pour déterminer avec précision les positions des atomes dans la maille d'un cristal, ce n'est plus le cas pour les amorphes et pour les liquides. La perte de la symétrie de translation a pour conséquence d'étaler le signal mesuré dans l'espace réciproque. Extraire de ce signal les fonctions de corrélation nécessaires à la description de la structure des système désordonnés est une tâche d'une grande complexité, qui ne possède pas de solution univoque.

La situation change évidemment quand nous avons affaire à un système monoatomique. Dans ce cas c'est la fonction de diffusion elle-même qui est reliée à la fonction de corrélation par une transformation de Fourier. Les fonctions de corrélation peuvent donc être determinées de manière directe à partir des mesures. Pour la diffusion totale nous obtenons

$$
\frac{d^{2} \sigma_{\vec{k}_{i} \rightarrow \vec{k}_{f}}}{d \Omega d E_{f}}=\frac{\sigma}{4 \pi} \frac{k_{f}}{k_{i}} \frac{1}{2 \pi \hbar} \int_{V} d^{3} r \int_{-\infty}^{\infty} d t e^{i(\vec{Q} \cdot \vec{r}-\omega t)} G(\vec{r}, t) .
$$

Les contributions cohérentes (5.67) et incohérentes (5.68) sont données par

$$
\left(\frac{d^{2} \sigma_{\vec{k}_{i} \rightarrow \vec{k}_{f}}}{d \Omega d E_{f}}\right)_{\mathrm{coh}}=\frac{\sigma_{\mathrm{coh}}}{4 \pi} \frac{k_{f}}{k_{i}} \frac{1}{2 \pi \hbar} \int_{V} d^{3} r \int_{-\infty}^{\infty} d t e^{i(\vec{Q} \cdot \vec{r}-\omega t)} G(\vec{r}, t)
$$

et

$$
\left(\frac{d^{2} \sigma_{\vec{k}_{i} \rightarrow \vec{k}_{f}}}{d \Omega d E_{f}}\right)_{\mathrm{inc}}=\frac{\sigma_{\mathrm{inc}}}{4 \pi} \frac{k_{f}}{k_{i}} \frac{1}{2 \pi \hbar} \int_{V} d^{3} r \int_{-\infty}^{\infty} d t e^{i(\vec{Q} \cdot \vec{r}-\omega t)} G^{s}(\vec{r}, t) .
$$

La diffusion cohérente nous renseigne sur les corrélations qui existent entre tous les diffuseurs. La diffusion incohérente est, par contre, beaucoup plus restrictive. Elle est reliée à la fonction d'autocorrélation et de ce fait ne contient des informations que sur la corrélation d'une particule avec elle-même. Pour une particule immobile, cette information se résume à une preuve d'existence et est dénuée d'intérêt. Par contre, dès que la position des particules évolue dans le temps, la diffusion incohérente est alors une sonde précise de leur dynamique.

\subsection{Variation de contraste pour obtenir les fonctions de corrélation partielles}

La seule voie pour contourner les difficultés associées à la superposition des fonctions partielles est la variation du contraste, c'est-à-dire le changement de la visibilité des différents types de diffuseur. Dans un scénario idéal, il est possible d'éteindre tous les éléments chimiques et de les rallumer un par un. Ce processus permettrait d'isoler les différentes contributions à (8.31). Dans le cas des neutrons il existe deux possibilités pour y arriver : (i) jouer avec la composition isotopique ou (ii) utiliser la dépendance de la longueur de diffusion avec le spin nucléaire. Il ne faut surtout pas intervenir dans le système chimique de l'échantillon car ceci ne changerait pas seulement le contraste mais aussi les grandeurs physiques que l'expérience est sensée révéler. La variation de contraste peut s'avérer indispensable dans tous les systèmes qui comportent de l'hydrogène (matière molle, chimie organique, biologie et biomatériaux). La variation de contraste est un sujet qui peut être très complexe dans le détail. Nous n'allons pas en poursuivre la discussion dans cette introduction.

\subsection{Fonctions de corrélation d'ordre supérieur}

Nous voudrions conclure avec une remarque concernant les fonctions de corrélation d'ordre supérieur. Jusqu'ici nous n'avons évoqué que des fonctions à deux points, c'est-à-dire des fonctions qui ne dépendent que de la distance $\vec{r}$. Rien n'empêche de définir des fonctions de corrélation plus complexes, 
par exemple du type $G\left(\vec{r}, \vec{r}^{\prime}, t\right)$. Ces fonctions prennent en compte l'influence de l'entourage sur la corrélation de paires. Les mesurer serait d'un grand intérêt pour comprendre la structure des liquides moléculaires. Elles pourraient, par exemple, nous renseigner sur l'orientation d'une molécule d'eau par rapport à ses voisines. Dans l'approximation de Born, les fonctions de corrélation d'ordre supérieur ne sont pas accessibles. On peut démontrer qu'elles entrent en jeu quand on rajoute des termes d'ordre supérieur à la série de Born (4.126) [19]. Il sera donc nécessaire de travailler avec des volumes de cohérence très grands (voir section 9.6) ce qui pose des problèmes expérimentaux considérables. Même si on arrivait à réunir les conditions nécessaires pour observer les effets des termes d'ordre deux de la série de Born, l'exploit serait dans la pratique d'un impact limité. La contribution d'ordre deux serait superposée à la contribution d'ordre un. On se heurterait alors à une difficulté similaire à l'extraction des fonctions de corrélation à deux points pour un système composé de plusieurs types d'atomes. Et même si on arrivait à surmonter cet obstacle, les effets d'ordre supérieur ne seraient pas reliées de manière simple aux fonctions de corrélation multi-points recherchées. Il semble donc que cette information restera encore longtemps inaccessible aux expériences classiques de diffusion de neutrons. ${ }^{34}$

\subsection{Les fonctions de diffusion intermédiaires $I_{\kappa, \kappa^{\prime}}(\vec{Q}, t)$ et $I_{\kappa}^{s}(\vec{Q}, t)$}

Si nous n'effectuons que la transformation de Fourier de $G(\vec{r}, t)$ dans l'espace nous obtenons la fonction intermédiaire de diffusion $I_{\kappa, \kappa^{\prime}}(\vec{Q}, t)[20] .{ }^{35}$ Chacune des expressions suivantes pourra servir de définition.

$$
\begin{aligned}
I_{\kappa, \kappa^{\prime}}(\vec{Q}, t) & =\sum_{j \in\left\{j_{\kappa}\right\}, j^{\prime} \in\left\{j_{\kappa^{\prime}}\right\}}\left\langle\exp \left(-i \vec{Q} \cdot \vec{R}_{j}\right) \exp \left(i \vec{Q} \cdot \vec{R}_{j^{\prime}}(t)\right)\right\rangle . \\
& =\left\langle\hat{\rho}_{\kappa}(\vec{Q}, t=0) \hat{\rho}_{\kappa^{\prime}}(-\vec{Q}, t)\right\rangle \\
I_{\kappa, \kappa^{\prime}}(\vec{Q}, t) & =\int_{V} d^{3} r e^{-i \vec{Q} \cdot \vec{r}} G_{\kappa, \kappa^{\prime}}(\vec{r}, t) . \\
I_{\kappa, \kappa^{\prime}}(\vec{Q}, t) & =\hbar \int_{-\infty}^{\infty} d \omega e^{i \omega t} S_{\kappa, \kappa^{\prime}}(\vec{Q}, \omega) .
\end{aligned}
$$

La première définit la fonction de corrélation directement en termes de transformée de Fourier de l'opérateur de densité. Nous nous rappelons que la fonction de corrélation est une convolution (voir (8.18)) de $\rho_{\kappa}(\vec{r}, t=0)$ avec $\rho_{\kappa^{\prime}}(-\vec{r}, t)$ dans l'espace. Sa transformée de Fourier est donc le produit des transformées de Fourier individuelles (se référer à (7.21) et (7.22)).

En inversant la dernière ligne nous obtenons l'expression de la fonction de diffusion en fonction de la fonction intermédiaire.

$$
\begin{aligned}
S_{\kappa, \kappa^{\prime}}(\vec{Q}, \omega) & =\frac{1}{2 \pi \hbar} \int_{-\infty}^{\infty} d t I_{\kappa, \kappa^{\prime}}(\vec{Q}, t) e^{-i \omega t} \\
& =\frac{1}{2 \pi \hbar} \int_{-\infty}^{\infty} d t\left\langle\hat{\rho}_{\kappa}(\vec{Q}, t=0) \hat{\rho}_{\kappa^{\prime}}(-\vec{Q}, t)\right\rangle e^{-i \omega t}
\end{aligned}
$$

\footnotetext{
34 Comme proposé par H. Rauch, une possibilité serait donnée par la manipulation directe de paquets d'ondes. Ceci permettrait de mesurer des fonctions de corrélation d'ordre supérieur en analogie avec le spin-echo qui permet la mesure de la fonction de corrélation à deux points.

35 Comme dans le cas des fonctions $G(\vec{r}, t)$ nous tentons de rester le plus général possible et ainsi nous définissons des fonctions intermédiaires de diffusion partielles. Ces fonctions ne sont logiquement pas normalisées aux nombres de diffuseurs. Cette caractéristique, qui est commune à $S_{\kappa, \kappa^{\prime}}(\vec{r}, t), I_{\kappa, \kappa^{\prime}}(\vec{r}, t)$ et $G_{\kappa, \kappa^{\prime}}(\vec{r}, t)$, est à prendre en compte si on compare les expressions impliquant les fonctions partielles avec celles que l'on trouve dans la littérature pour les fonctions totales $(S(\vec{r}, t), I(\vec{r}, t)$ et $G(\vec{r}, t))$, qui elles sont en général normalisées à $N$.
} 
La fonction de diffusion totale s'obtient comme

$$
\begin{aligned}
S(\vec{Q}, \omega) & =\sum_{\kappa, \kappa^{\prime}}\left(V_{\kappa}(\vec{Q}) V_{\kappa^{\prime}}^{*}(\vec{Q})\right) S_{\kappa, \kappa^{\prime}}(\vec{Q}, \omega) \\
& =\frac{1}{2 \pi \hbar} \sum_{\kappa, \kappa^{\prime}}\left(V_{\kappa}(\vec{Q}) V_{\kappa^{\prime}}^{*}(\vec{Q})\right) \int_{-\infty}^{\infty} d t I_{\kappa, \kappa^{\prime}}(\vec{Q}, t) e^{-i \omega t} \\
& =\frac{1}{2 \pi \hbar} \int_{-\infty}^{\infty} d t I(\vec{Q}, t) e^{-i \omega t}
\end{aligned}
$$

avec la fonction de diffusion intermédiaire totale définie comme

$$
I(\vec{Q}, t)=\sum_{\kappa, \kappa^{\prime}}\left(V_{\kappa}(\vec{Q}) V_{\kappa^{\prime}}^{*}(\vec{Q})\right) I_{\kappa, \kappa^{\prime}}(\vec{Q}, t) .
$$

De la même manière, nous pouvons introduire la fonction de diffusion intermédiaire propre.

$$
\begin{aligned}
& I_{\kappa}^{S}(\vec{Q}, t)=\sum_{j \in\left\{j_{\kappa}\right\}}\left\langle\exp \left(-i \vec{Q} \cdot \vec{R}_{j}\right) \exp \left(i \vec{Q} \cdot \vec{R}_{j}(t)\right)\right\rangle . \\
& I_{\kappa}^{S}(\vec{Q}, t)=\int_{V} d^{3} r e^{-i \vec{Q} \cdot \vec{r}} G_{\kappa}^{S}(\vec{r}, t) .
\end{aligned}
$$

En partant de sa définition en terme de fonctions de corrélation, nous pouvons déduire quelques relations pertinentes pour la fonction de diffusion intermédiaire.

- Nous commençons par

$$
I_{\kappa, \kappa^{\prime}}(\vec{Q}, t)=I_{\kappa^{\prime}, \kappa}^{*}(\vec{Q},-t) .
$$

Cette relation vient du fait que pour n'importe quel couple d'opérateurs

$$
\begin{aligned}
\langle\mathbf{A B}\rangle & =\sum_{\lambda} p_{\lambda}\langle\lambda|\mathbf{A} \mathbf{B}| \lambda\rangle \\
& =\sum_{\lambda} p_{\lambda}\left\langle\lambda\left|\mathbf{B}^{+} \mathbf{A}^{+}\right| \lambda\right\rangle^{*} \\
& =\left\langle\mathbf{B}^{+} \mathbf{A}^{+}\right\rangle^{*}
\end{aligned}
$$

Avec

$$
\hat{\rho}_{\kappa}^{+}(\vec{Q}, t)=\frac{1}{(2 \pi)^{3}} \sum_{j_{\kappa}} \exp \left(i \vec{Q} \cdot \vec{R}_{j}(t)\right)=\hat{\rho}_{\kappa}(-\vec{Q}, t)
$$

nous obtenons

$$
\begin{aligned}
I_{\kappa, \kappa^{\prime}}(\vec{Q}, t) & =\left\langle\hat{\rho}_{\kappa}(\vec{Q}, t=0) \hat{\rho}_{\kappa^{\prime}}(-\vec{Q}, t)\right\rangle \\
& =\left\langle\hat{\rho}_{\kappa^{\prime}}^{+}(-\vec{Q}, t) \hat{\rho}_{\kappa}^{+}(\vec{Q}, t=0)\right\rangle^{*} \\
& =\left\langle\hat{\rho}_{\kappa^{\prime}}(\vec{Q}, t) \hat{\rho}_{\kappa}(-\vec{Q}, t=0)\right\rangle^{*} \\
& =\left\langle\hat{\rho}_{\kappa^{\prime}}(\vec{Q}, t=0) \hat{\rho}_{\kappa}(-\vec{Q},-t)\right\rangle^{*} \\
& =I_{\kappa^{\prime}, \kappa}^{*}(\vec{Q},-t)
\end{aligned}
$$

où le passage de la troisième vers la quatrième ligne est lié à l'homogénéité du système dans le temps. En d'autres termes, l'origine du temps est sans importance. Seul compte le délai entre les 
évènements pour le calcul des éléments de matrice :

$$
\left\langle\lambda\left|\mathbf{A}(t) \mathbf{B}\left(t^{\prime}\right)\right| \lambda\right\rangle=\left\langle\lambda\left|\mathbf{A}(t=0) \mathbf{B}\left(t^{\prime}-t\right)\right| \lambda\right\rangle .
$$

La relation (8.43) indique qu'il suffit de connaître les fonctions intermédiaires de diffusion sur l'axe positif du temps.

- Jusqu'ici, la fonction intermédiaire de diffusion était définie sur l'espace réel des temps. Nous pouvons la reformuler avec l'aide de l'opérateur d'évolution

$$
\mathbf{U}(t)=\exp (-i \mathbf{H} t / \hbar)
$$

Nous arrivons à

$$
\begin{aligned}
I_{\kappa, \kappa^{\prime}}(\vec{Q}, t) & =\left\langle\hat{\rho}_{\kappa}(\vec{Q}, t=0) \hat{\rho}_{\kappa^{\prime}}(-\vec{Q}, t)\right\rangle \\
& =\sum_{\lambda} p_{\lambda}\left\langle\lambda\left|\hat{\rho}_{\kappa}(\vec{Q}, 0) \hat{\rho}_{\kappa^{\prime}}(-\vec{Q}, t)\right| \lambda\right\rangle \\
& \left.=\sum_{\lambda} p_{\lambda}\langle\lambda| \hat{\rho}_{\kappa}(\vec{Q}) \mathbf{U}^{+}(t) \hat{\rho}_{\kappa^{\prime}}(-\vec{Q}) \mathbf{U}(t)\right)|\lambda\rangle
\end{aligned}
$$

Cette définition permet de porter la fonction d'une manière naturelle à l'espace complexe. Nous posons

$$
\begin{aligned}
I_{\kappa, \kappa^{\prime}}(\vec{Q}, t+i \tau) & \left.=\sum_{\lambda} p_{\lambda}\langle\lambda| \hat{\rho}_{\kappa}(\vec{Q}) \mathbf{U}^{+}(t+i \tau) \hat{\rho}_{\kappa^{\prime}}(-\vec{Q}) \mathbf{U}(t+i \tau)\right)|\lambda\rangle \\
& =\sum_{\lambda} p_{\lambda}\left\langle\lambda\left|\hat{\rho}_{\kappa}(\vec{Q}) \exp (i \mathbf{H}(t+i \tau) / \hbar) \hat{\rho}_{\kappa^{\prime}}(-\vec{Q}) \exp (-i \mathbf{H}(t+i \tau))\right| \lambda\right\rangle
\end{aligned}
$$

Le concept devient très puissant en identifiant le temps complexe avec l'inverse de la température comme

$$
\tau=\hbar \beta=\frac{\hbar}{k_{B} T} .
$$

Pour toute moyenne en équilibre thermodynamique nous dérivons la relation

$$
\begin{aligned}
\langle\mathbf{A}(t=0) \mathbf{B}(t)\rangle & =\sum_{\lambda} p_{\lambda}\langle\lambda|\mathbf{A}(0) \mathbf{B}(t)| \lambda\rangle \\
& \left.=\frac{1}{Z} \sum_{\lambda} \exp \left(-\beta E_{\lambda}\right)\langle\lambda| \mathbf{A} \mathbf{U}^{+}(t) \mathbf{B} \mathbf{U}(t)\right)|\lambda\rangle \\
& \left.=\frac{1}{Z} \sum_{\lambda}\langle\lambda| \mathbf{A} \mathbf{U}^{+}(t) \mathbf{B} \mathbf{U}(t)\right) \exp (-\beta \mathbf{H})|\lambda\rangle \\
& =\frac{1}{Z} \sum_{\lambda, \lambda^{\prime}}\left\langle\lambda|\mathbf{A}| \lambda^{\prime}\right\rangle\left\langle\lambda^{\prime}\left|\left(\mathbf{U}^{+}(t) \mathbf{B} \mathbf{U}(t)\right) \exp (-\beta \mathbf{H})\right| \lambda\right\rangle \\
& =\frac{1}{Z} \sum_{\lambda, \lambda^{\prime}}\left\langle\lambda^{\prime}\left|\left(\mathbf{U}^{+}(t) \mathbf{B} \mathbf{U}(t)\right) \exp (-\beta \mathbf{H})\right| \lambda\right\rangle\left\langle\lambda|\mathbf{A}| \lambda^{\prime}\right\rangle \\
& =\frac{1}{Z} \sum_{\lambda}\left\langle\lambda\left|\left(\mathbf{U}^{+}(t) \mathbf{B} \mathbf{U}(t)\right) \exp (-\beta \mathbf{H}) \mathbf{A}\right| \lambda\right\rangle \\
& =\frac{1}{Z} \sum_{\lambda} \exp \left(-\beta E_{\lambda}\right)\left\langle\lambda\left|\exp (\beta \mathbf{H})\left(\mathbf{U}^{+}(t) \mathbf{B} \mathbf{U}(t)\right) \exp (-\beta \mathbf{H}) \mathbf{A}\right| \lambda\right\rangle
\end{aligned}
$$




$$
\begin{aligned}
& =\sum_{\lambda} p_{\lambda}\left\langle\lambda\left|\left(\mathbf{U}^{+}(t-i \hbar \beta) \mathbf{B} \mathbf{U}(t-i \hbar \beta)\right) \mathbf{A}(0)\right| \lambda\right\rangle \\
& =\sum_{\lambda} p_{\lambda}\langle\lambda|\mathbf{B}(t-i \hbar \beta) \mathbf{A}(0)| \lambda\rangle \\
& =\langle\mathbf{B}(t-i \hbar \beta) \mathbf{A}(0)\rangle \\
& =\langle\mathbf{B}(t) \mathbf{A}(i \hbar \beta)\rangle
\end{aligned}
$$

Si le système n'est pas en équilibre, les probabilités $p_{\lambda}$ ne sont pas exprimées par les facteurs de Boltzmann et donc le raisonnement qui nous avons employé pour déduire la relation (8.54) n'est pas applicable.

Appliqué à la fonction de diffusion intermédiaire, l'expression (8.54 ) se traduit en

$$
I_{\kappa, \kappa^{\prime}}(\vec{Q}, t)=I_{\kappa^{\prime}, \kappa}(-\vec{Q},-t+i \hbar \beta)
$$

et pour la fonction intermédiaire de diffusion totale en

$$
\begin{aligned}
& I(\vec{Q}, t) \\
& \quad=\sum_{\kappa \leq \kappa^{\prime}}\left[\left(V_{\kappa}(\vec{Q}) V_{\kappa^{\prime}}^{*}(\vec{Q})\right) I_{\kappa, \kappa^{\prime}}(\vec{Q}, t)+\left(V_{\kappa^{\prime}}(\vec{Q}) V_{\kappa}^{*}(\vec{Q})\right) I_{\kappa^{\prime}, \kappa}(\vec{Q}, t)\right] \\
& \quad=\sum_{\kappa \leq \kappa^{\prime}}\left[\left(V_{\kappa}(\vec{Q}) V_{\kappa^{\prime}}^{*}(\vec{Q})\right) I_{\kappa^{\prime}, \kappa}(-\vec{Q},-t+i \hbar \beta)+\left(V_{\kappa^{\prime}}(\vec{Q}) V_{\kappa}^{*}(\vec{Q})\right) I_{\kappa, \kappa^{\prime}}(-\vec{Q},-t+i \hbar \beta)\right] \\
& \quad=I(-\vec{Q},-t+i \hbar \beta),
\end{aligned}
$$

pourvu que $V_{\kappa}(\vec{Q})=V_{\kappa}^{*}(\vec{Q}), \forall \kappa$, c'est-à-dire en absence d'absorption.

Il existe donc une relation exacte entre la fonction intermédiaire de diffusion, définie sur l'axe des temps réels, avec celle définie sur l'espace des temps complexes. En connaissant l'une nous connaissons automatiquement l'autre.

Nous allons maintenant utiliser ces relations pour déduire une propriété importante de la fonction de diffusion.

\subsection{Balance détaillée}

En utilisant (8.38) et (8.55), nous obtenons la relation suivante pour la fonction de diffusion

$$
\begin{aligned}
S_{\kappa, \kappa^{\prime}}(\vec{Q}, \omega) & =\frac{1}{2 \pi \hbar} \int_{-\infty}^{\infty} d t I_{\kappa, \kappa^{\prime}}(\vec{Q}, t) e^{-i \omega t} \\
& =\frac{1}{2 \pi \hbar} \int_{-\infty}^{\infty} d t I_{\kappa^{\prime}, \kappa}(-\vec{Q},-t+i \hbar \beta) e^{-i \omega t} \\
& =\frac{1}{2 \pi \hbar} \int_{-\infty}^{\infty} d t^{\prime} I_{\kappa^{\prime}, \kappa}\left(-\vec{Q}, t^{\prime}\right) e^{-i \omega\left(-t^{\prime}+i \hbar \beta\right)} \\
& =e^{\hbar \omega \beta} \frac{1}{2 \pi \hbar} \int_{-\infty}^{\infty} d t I_{\kappa^{\prime}, \kappa}(-\vec{Q}, t) e^{i \omega t} \\
& =e^{\hbar \omega \beta} S_{\kappa^{\prime}, \kappa}(-\vec{Q},-\omega) .
\end{aligned}
$$


Si le système est invariant par inversion des directions $\vec{r}$ et donc $\vec{Q}$, cette relation se simplifie

$$
S_{\kappa, \kappa^{\prime}}(\vec{Q},-\omega)=e^{-\hbar \omega \beta} S_{\kappa^{\prime}, \kappa}(\vec{Q}, \omega) .
$$

C'est notamment le cas pour les systèmes désordonnés comme les liquides et les gaz ainsi que pour les cristaux avec un centre d'inversion.

Appliqué à la fonction totale de diffusion nous obtenons

$$
\begin{aligned}
& S(\vec{Q}, \omega) \\
& \quad=\sum_{\kappa \leq \kappa^{\prime}}\left[\left(V_{\kappa}(\vec{Q}) V_{\kappa^{\prime}}^{*}(\vec{Q})\right) S_{\kappa, \kappa^{\prime}}(\vec{Q}, \omega)+\left(V_{\kappa^{\prime}}(\vec{Q}) V_{\kappa}^{*}(\vec{Q})\right) S_{\kappa^{\prime}, \kappa}(\vec{Q}, \omega)\right] \\
& \quad=e^{\hbar \omega \beta} \sum_{\kappa \leq \kappa^{\prime}}\left[\left(V_{\kappa}(\vec{Q}) V_{\kappa^{\prime}}^{*}(\vec{Q})\right) S_{\kappa^{\prime}, \kappa}(-\vec{Q},-\omega)+\left(V_{\kappa^{\prime}}(\vec{Q}) V_{\kappa}^{*}(\vec{Q})\right) S_{\kappa, \kappa^{\prime}}(-\vec{Q},-\omega)\right] \\
& =e^{\hbar \omega \beta} \sum_{\kappa \leq \kappa^{\prime}}\left[\left(V_{\kappa}(\vec{Q}) V_{\kappa^{\prime}}^{*}(\vec{Q})\right) S_{\kappa^{\prime}, \kappa}^{*}(-\vec{Q},-\omega)+\left(V_{\kappa^{\prime}}(\vec{Q}) V_{\kappa}^{*}(\vec{Q})\right) S_{\kappa, \kappa^{\prime}}^{*}(-\vec{Q},-\omega)\right] \\
& =e^{\hbar \omega \beta} S^{*}(-\vec{Q},-\omega)=e^{\hbar \omega \beta} S(-\vec{Q},-\omega),
\end{aligned}
$$

où nous avons exploité le fait que les fonctions de diffusion sont réelles.

Comme nous pouvons le constater, la fonction de diffusion est asymétrique par rapport à la fréquence $\omega$. Le facteur $e^{-\hbar \omega \beta}$ étant toujours inférieur à un pour $\omega>0$, le côté négatif de $S(\vec{Q}, \omega)$ sera toujours rabaissé par rapport au côté positif.

Pour mieux comprendre la signification de ce résultat, nous retournons aux sections efficaces partielles. En comparant

$$
\frac{d^{2} \sigma_{\vec{k}_{i} \rightarrow \vec{k}_{f}}}{d \Omega d E_{f}}=\frac{k_{f}}{k_{i}} S(\vec{Q}, \omega)
$$

avec

$$
\frac{d^{2} \sigma_{\vec{k}_{i} \rightarrow \vec{k}_{f}}}{d \Omega d E_{f}}=\frac{k_{f}}{k_{i}} \frac{m^{2}}{(2 \pi)^{2} \hbar^{4}} \sum_{\lambda_{i}, \lambda_{f}} p\left(\lambda_{i}\right)\left|\sum_{j=1}^{N} V_{j}(\vec{Q})\left\langle\lambda_{f}\left|e^{i \vec{Q} \cdot \vec{R}_{j}}\right| \lambda_{i}\right\rangle\right|^{2} \delta\left(\hbar \omega+\left(E_{\lambda_{i}}-E_{\lambda_{f}}\right)\right)
$$

nous rappelons que la fonction de diffusion $S(\vec{Q}, \omega)$ donne (à part le facteur $k_{f} / k_{i}$ ) la probabilité que le neutron passe de l'état $\vec{k}_{i}$ à l'état $\vec{k}_{f}$ en échangeant l'énergie $\hbar \omega=E_{f}-E_{i}$ avec la cible. Si $\hbar \omega<0$ alors $E_{f}>E_{i}$ et le neutron a absorbé de l'énergie tandis que la cible en a perdu. Le neutron est excité et la cible désexcitée suite au processus de diffusion. En utilisant le vocabulaire de la spectroscopie optique nous sommes du côté anti-Stokes du spectre. Si au contraire $\hbar \omega>0$ alors $E_{f}<E_{i}$ et le neutron cède de l'énergie à la cible. Nous nous trouvons du côté Stokes du spectre. La probabilité intrinsèque de passer d'un état à l'autre est identique pour l'excitation et pour la désexcitation. La différence réside dans le taux d'occupation des états de départ dans la cible. ${ }^{36}$ Ce taux est exprimé par le facteur $e^{-\hbar \omega \beta}$. A très basse température, la cible se trouve dans son état fondamental. Aucune désexcitation n'est possible et $S(\vec{Q}, \omega)=0$ pour $\omega<0$. A très haute température $(T \gg \hbar \omega)$, le facteur $e^{-\hbar \omega \beta} \approx 1$ et les spectres deviennent de plus en plus symétriques.

\footnotetext{
36 Même pour des flux élevés, le neutron est une perturbation tellement faible qu'il ne changera pas l'équilibre de la cible.
} 


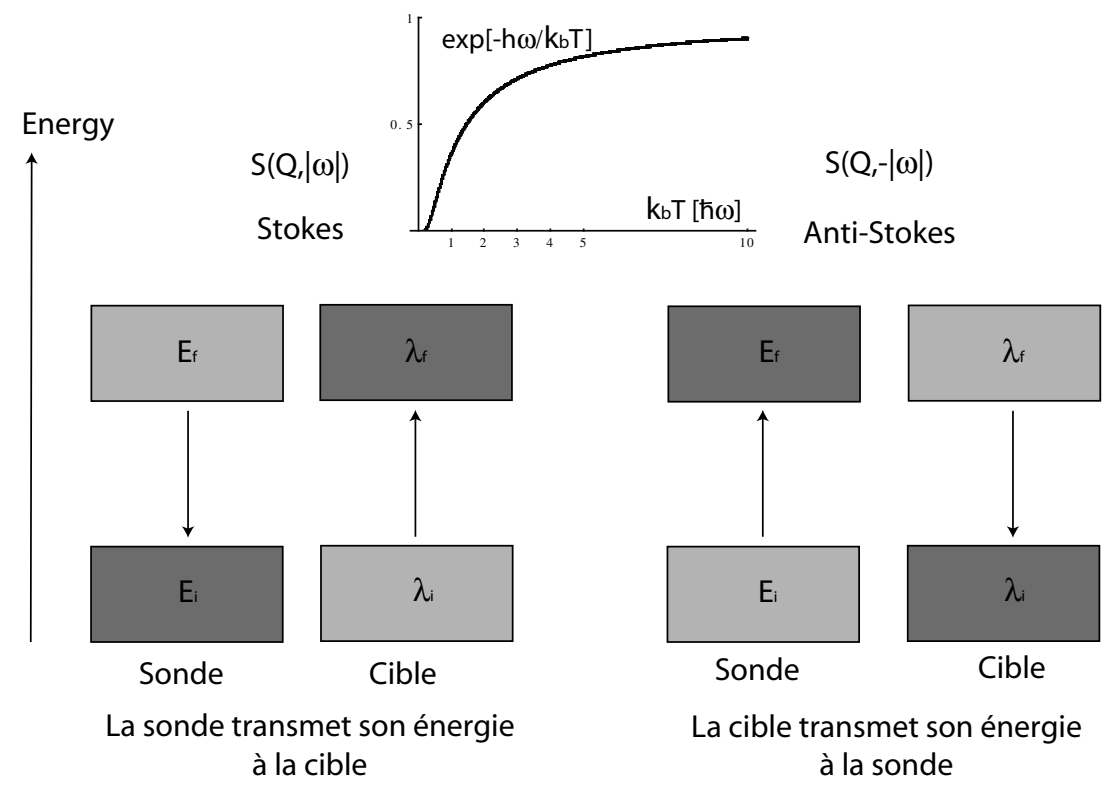

Figure 24. Présentation schématique du principe de balance détaillée. Pour des températures largement en-dessous du transfert d'énergie, le côté anti-Stokes de la diffusion est ramené à zéro. Il n'y a pas d'état occupé aux énergies requises. Pour $k_{\mathrm{B}} T \approx \hbar \omega$ l'intensité du signal mesuré du côté anti-Stokes atteint à peu près $30 \%$ de celui du côté Stokes. A très haute température, le rapport entre les deux côtés est égale à l'unité.

\subsection{Fonctions de corrélation symétrisées}

La fonction de diffusion $S(\vec{Q}, \omega)$ est asymétrique par rapport à la variable $\omega$. Nous pouvons en déduire une version symétrique [20] en posant

En effet

$$
S^{\mathrm{sym}}(\vec{Q}, \omega)=\exp \left(-\frac{1}{2} \hbar \omega \beta\right) S(\vec{Q}, \omega) .
$$

$$
S^{\mathrm{sym}}(\vec{Q}, \omega)=S^{\mathrm{sym}}(-\vec{Q},-\omega) .
$$

De la même manière nous définissons

$$
I_{\kappa, \kappa^{\prime}}^{\mathrm{sym}}(\vec{Q}, t)=I_{\kappa, \kappa^{\prime}}\left(\vec{Q}, t+\frac{1}{2} \hbar \omega \beta\right)
$$

ce qui donne une fonction

$$
I^{\mathrm{sym}}(\vec{Q}, t)=I^{\mathrm{sym}}(-\vec{Q},-t)\left(=I^{\mathrm{sym} *}(\vec{Q},-t)\right),
$$

qui est symétrique en $t$ et réelle pour des systèmes à symétrie d'inversion.

La valeur pratique de ces fonctions se manifeste quand nous devons comparer les mesures de diffusions à des calculs ou à des prédictions théoriques. La connaissance des fonctions d'onde de la cible, nécessaire aux calculs des valeurs moyennes figurant dans les expressions de $S(\vec{Q}, \omega)$, est plutôt l'exception que la règle. Dans un grand nombre de cas, les mesures doivent être comparées à des calculs classiques, par exemple issus de simulations de dynamique moléculaire ou de modèles stochastiques (modèles de diffusion). Ces calculs permettent le calcul des fonctions de corrélation classiques ${ }^{\mathrm{cl}} G(\vec{r}, t)$ (8.22) et (8.23). Souvent ces fonctions sont réelles et paires dans le temps, ce qui implique un $S(\vec{Q}, \omega)$ symétrique dans $\omega$ en contradiction avec la balance détaillée. Une possibilité de redresser la balance est de comparer les prévisions classiques avec les fonctions de diffusion symétrisées. Ce procédé a été proposé pour la première fois par Schofield [21]. 


\subsection{Comportement asymptotique}

Il est souvent très instructif d'étudier le comportement d'une fonction aux limites. Nous pouvons ainsi espérer identifier des relations utiles, qui sont indépendantes de la forme exacte des fonctions.

Considérons la fonction de diffusion intermédiaire $I_{\kappa, k^{\prime}}(\vec{Q}, t)$. Pour des temps très longs, les corrélations deviendront nécessairement indépendantes du temps. Formellement ceci implique que la limite $I_{\kappa, \kappa^{\prime}}(\vec{Q}, t \rightarrow \pm \infty)$ existe. $^{37}$

Posons

$$
I_{\kappa, \kappa^{\prime}}^{\prime}(\vec{Q}, t)=I_{\kappa, \kappa^{\prime}}(\vec{Q}, t)-I_{\kappa, \kappa^{\prime}}(\vec{Q}, \infty) .
$$

La fonction de diffusion s'obtiendra comme la transformée de Fourier de la fonction intermédiaire

$$
\begin{aligned}
S_{\kappa, \kappa^{\prime}}(\vec{Q}, \omega) & =\frac{1}{2 \pi \hbar} \int_{-\infty}^{\infty} d t I_{\kappa, \kappa^{\prime}}(\vec{Q}, t) e^{-i \omega t} \\
& =\frac{1}{2 \pi \hbar} \int_{-\infty}^{\infty} d t\left[I_{\kappa, \kappa^{\prime}}^{\prime}(\vec{Q}, t)+I_{\kappa, \kappa^{\prime}}(\vec{Q}, \infty)\right] e^{-i \omega t} \\
& =I_{\kappa, \kappa^{\prime}}(\vec{Q}, \infty) \delta(\hbar \omega)+\int_{-\infty}^{\infty} d t I_{\kappa, \kappa^{\prime}}^{\prime}(\vec{Q}, t) e^{-i \omega t} .
\end{aligned}
$$

Avec (8.28) nous en déduisons que la section efficace différentielle partielle cohérente contient une composante élastique, qui est donnée par

$$
\left(\frac{d^{2} \sigma}{d \Omega d E_{f}}\right)_{\mathrm{el}}^{\mathrm{coh}}=\sum_{\kappa, \kappa^{\prime}} \bar{b}_{\kappa} \bar{b}_{\kappa^{\prime}} I_{\kappa, \kappa^{\prime}}(\vec{Q}, \infty) \delta(\hbar \omega)
$$

La différence entre la section efficace différentielle partielle et la section efficace élastique correspond à la section efficace inélastique. Attention, la diffusion inélastique n'est pas zéro à $\hbar \omega=0$. On parle d'intensité quasi-élastique. ${ }^{38}$ Elle se distingue clairement de la diffusion élastique du fait que

$$
\int_{-\epsilon}^{\epsilon} d(\hbar \omega)\left(\frac{d^{2} \sigma}{d \Omega d E_{f}}\right)_{\mathrm{el}}=\sum_{\kappa, \kappa^{\prime}} \bar{b}_{\kappa} \bar{b}_{\kappa^{\prime}}^{*} I_{\kappa, \kappa^{\prime}}(\vec{Q}, \infty)
$$

indépendamment du choix de $\epsilon$, pendant que

$$
\int_{-\epsilon}^{\epsilon} d(\hbar \omega)\left(\frac{d^{2} \sigma}{d \Omega d E_{f}}\right)_{\mathrm{el}} \rightarrow 0
$$

pour $\epsilon \rightarrow 0$. Dans la pratique, cette différence se manifeste par l'observation que l'intensité de la diffusion élastique est indépendante de la résolution (se référer à la section 9.6) avec laquelle la mesure est faite.

Regardons un peu plus dans le détail la fonction $I_{\kappa, \kappa^{\prime}}(\vec{Q}, \infty)$. Elle est d'après (8.37) définie comme

$$
\begin{aligned}
I_{\kappa, \kappa^{\prime}}(\vec{Q}, \infty) & =\sum_{j \in\left\{j_{k}\right\}, j^{\prime} \in\left\{j_{\kappa^{\prime}}\right\}}\left\langle\exp \left(-i \vec{Q} \cdot \vec{R}_{j}\right) \exp \left(i \vec{Q} \cdot \vec{R}_{j^{\prime}}(\infty)\right)\right\rangle \\
& =\left\langle\hat{\rho}_{\kappa}(\vec{Q}, t=0) \hat{\rho}_{\kappa^{\prime}}(-\vec{Q}, \infty)\right\rangle \\
& =\int_{V} d^{3} r e^{-i \vec{Q} \cdot \vec{r}} \int d^{3} \vec{r}^{\prime}\left\langle\hat{\rho}_{\kappa}\left(\vec{r}^{\prime}-\vec{r}\right) \hat{\rho}_{\kappa^{\prime}}\left(\vec{r}^{\prime}, \infty\right)\right\rangle .
\end{aligned}
$$

\footnotetext{
${ }^{37}$ En théorie on peut construire des situations où la limite n'existe pas. Par exemple, pour un système décrit par une excitation harmonique, la fonction de diffusion intermédiaire oscillera pour tout $t$ autour de sa valeur moyenne. C'est d'ailleurs la raison pour laquelle la réponse dynamique d'un tel système est donnée par des fonctions de Dirac en énergie (se référer aux sections 10.3 et 10.5). Dans la réalité les systèmes sont immergés dans un bain thermique et les oscillations sont amorties, ce qui garantit l'existence de la valeur limite.

${ }^{38}$ L'expression semble avoir été introduite en [20].
} 


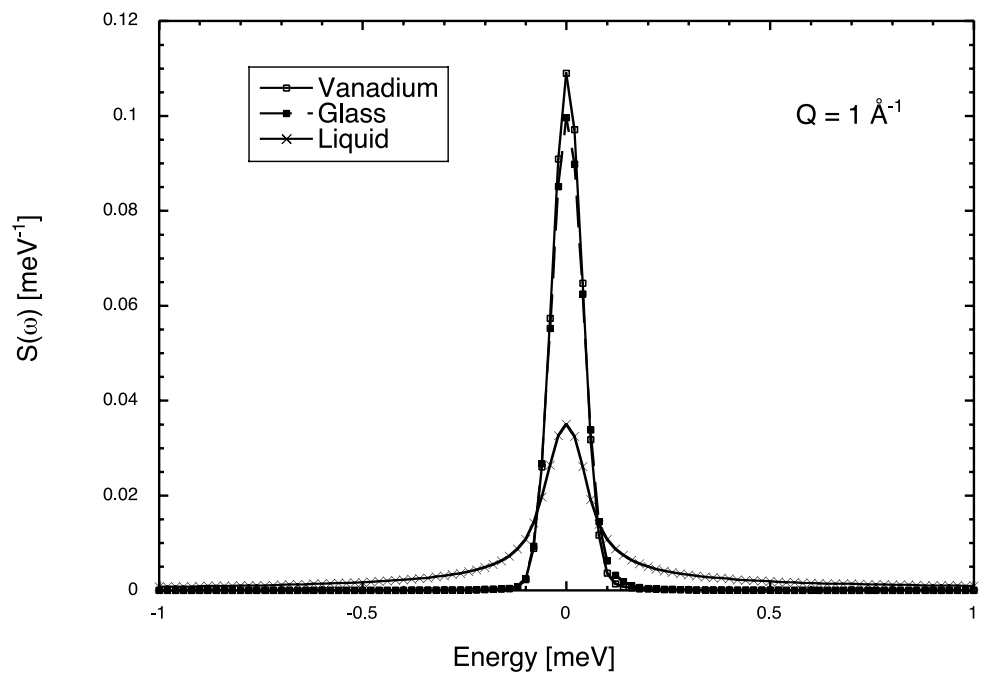

Figure 25. Dans la figure, nous comparons la diffusion du composé trans-Décaline dans l'état liquide et vitreux. Les molécules $\mathrm{C}_{10} \mathrm{H}_{18}$ sont constituées de deux cycles cyclohexaniques accolés. Le signal quasi-élastique caractérisant le liquide disparaît dans le verre au profit d'un pic élastique, dont la largeur est donnée par la résolution de l'instrument, cette dernière étant mesurée avec un vanadium. Contrairement au liquide, les atomes gardent leurs positions d'équilibre dans le verre.

Si la limite $I_{K, \kappa^{\prime}}(\vec{Q}, \infty)$ existe, la corrélation entre $\vec{R}_{j}$ et $\vec{R}_{j^{\prime}}(t)$ doit devenir indépendante de $t$ pour $t \rightarrow \infty$. En d'autres termes $\vec{R}_{j}$ et $\vec{R}_{j^{\prime}}(t)$ doivent se décorréler pour $t \rightarrow \infty$. Formellement

$$
\int d^{3} \vec{r}^{\prime}\left\langle\hat{\rho}_{K}\left(\vec{r}^{\prime}-\vec{r}\right) \hat{\rho}_{\kappa^{\prime}}\left(\vec{r}^{\prime}, \infty\right)\right\rangle=\int d^{3} \vec{r}^{\prime}\left\langle\hat{\rho}_{K}\left(\vec{r}^{\prime}-\vec{r}\right)\right\rangle\left\langle\hat{\rho}_{K^{\prime}}\left(\vec{r}^{\prime}\right)\right\rangle,
$$

où nous avons exploité le fait que $\left\langle\hat{\rho}_{K}\left(\vec{r}^{\prime}-\vec{r}\right)\right\rangle$ est indépendant de $t$.

Dans un liquide, la densité $\rho=N / V$ est homogène, c'est à-dire qu'elle ne dépend pas du lieu. Donc

$$
\int d^{3} \vec{r}^{\prime}\left\langle\hat{\rho}_{\kappa}\left(\vec{r}^{\prime}-\vec{r}\right)\right\rangle\left\langle\hat{\rho}_{\kappa^{\prime}}\left(\vec{r}^{\prime}\right)\right\rangle=\int d^{3} \vec{r}^{\prime} \rho^{2}=V \rho^{2}=N \rho .
$$

La transformée de Fourier de cette expression donne la fonction de diffusion intermédiaire

$$
I_{\kappa, \kappa^{\prime}}(\vec{Q}, \infty) \propto \delta(\vec{Q}) .
$$

Une densité constante dans l'espace ne donne donc pas lieu à de la diffusion. On obtient le même résultat pour la diffusion incohérente. Un liquide ou un gaz ne produiront donc pas de diffusion élastique. Quand le liquide cristallise ou se vitrifie, les atomes deviennent piégés dans des positions d'équilibre qui sont stables à l'échelle de l'expérience. Cette stabilité donne lieu à une valeur finie de $I_{\kappa, \kappa^{\prime}}(\vec{Q}, \infty)$ et donc à une diffusion élastique (voir la figure 25).

\subsection{Exemple : ensemble de particules de Boltzmann}

Nous suivons Squires [36] et Lovesey [15] pour illustrer les fonctions de corrélation que nous venons de définir, avec l'exemple du gaz parfait. Dans un gaz parfait il n'y a pas d'interaction entre les particules. Les fonctions d'onde sont donc celles d'un ensemble de particules libres. Dans la description de Heisenberg

$$
|\lambda\rangle=|\vec{P}(1), \ldots, \vec{P}(N)\rangle=|\vec{P}(1)\rangle \cdot \ldots \cdot|\vec{P}(N)\rangle
$$


$\operatorname{avec}^{39}$

$$
|\vec{P}(j)\rangle=\frac{1}{\sqrt{V}} \exp \left(i \vec{q}(j) \cdot \vec{R}_{j}\right), \quad \vec{q}(j)=\frac{\vec{P}(j)}{\hbar} .
$$

L'ensemble des particules est couplé à un bain thermique. Nous supposons que toutes les particules peuvent être distinguées. Par conséquent la probabilité qu'une particule $j$ se trouve dans l'état $\vec{P}_{j}$ est donnée par la distribution de Boltzmann

$$
f_{\vec{P}} d^{3} p=f\left(p_{x}, p_{y}, p_{z}\right) d p_{x} d p_{y} d p_{z}=\sqrt{\left(\frac{1}{2 \pi m k_{\mathrm{B}} T}\right)^{3}} \exp \left(-\frac{p_{x}^{2}+p_{y}^{2}+p_{z}^{2}}{2 m k_{\mathrm{B}} T}\right) d^{3} p .
$$

Si nous procédons à un calcul direct de la section efficace partielle différentielle nous devons évaluer

$$
\begin{aligned}
\frac{d^{2} \sigma_{\vec{k}_{i} \rightarrow \vec{k}_{f}}}{d \Omega d E_{f}}= & \frac{k_{f}}{k_{i}} \sum_{\lambda_{i}, \lambda_{f}} p\left(\lambda_{i}\right)\left|\sum_{j=1}^{N} b_{j}\left\langle(\vec{P}(1), \ldots, \vec{P}(N))_{f}\left|e^{i \vec{Q} \cdot \vec{R}_{j}}\right|(\vec{P}(1), \ldots, \vec{P}(N))_{i}\right\rangle\right|^{2} \\
& \times \delta\left(\left(E_{\lambda_{i}}-E_{\lambda_{f}}\right)+\hbar(\omega)\right) .
\end{aligned}
$$

Les éléments de matrice figurant dans cette expression se calculent avec (8.76) comme

$$
\begin{aligned}
& \left\langle(\vec{P}(1), \ldots, \vec{P}(N))_{f}\left|e^{i \vec{Q} \cdot \vec{R}_{j}}\right|(\vec{P}(1), \ldots, \vec{P}(N))_{i}\right\rangle \\
& =\left\langle\vec{P}_{f}(1) \mid \vec{P}_{i}(1)\right\rangle \cdot \ldots \cdot\left\langle\vec{P}_{f}(j)\left|e^{i \vec{Q} \cdot \vec{R}_{j}}\right| \vec{P}_{i}(j)\right\rangle \cdot \ldots \cdot\left\langle\vec{P}_{f}(N) \mid \vec{P}_{i}(N)\right\rangle \\
& =\frac{1}{V^{N}} \int_{V} d^{3} R_{1} \exp \left(i\left(\vec{q}_{i}(1)-\vec{q}_{f}(1)\right) \cdot \vec{R}_{1}\right) \ldots \int_{V} d^{3} R_{j} \exp \left(i\left(\vec{Q}+\vec{q}_{i}(j)-\vec{q}_{f}(j)\right) \cdot \vec{R}_{j}\right) \ldots \\
& \quad \times \int_{V} d^{3} R_{N} \exp \left(i\left(\vec{q}_{i}(N)-\vec{q}_{f}(N)\right) \cdot \vec{R}_{N}\right) \\
& =\delta\left(\vec{q}_{i}(1)-\vec{q}_{f}(1)\right) \cdot \ldots \cdot \delta\left(\vec{Q}+\vec{q}_{i}(j)-\vec{q}_{f}(j)\right) \cdot \ldots \cdot \delta\left(\vec{q}_{i}(N)-\vec{q}_{f}(N)\right) .
\end{aligned}
$$

C'est l'expression de la conservation de l'impulsion dans une collision, qui n'implique qu'une particule. Donc, si la fonction d'onde initiale et la fonction d'onde finale diffèrent de plus d'un nombre quantique, la contribution à la section efficace sera zéro. Dans tous les autres cas, l'élément de matrice est égal à l'unité à condition que

$$
\hbar \omega=-\left(E_{\lambda_{i}}-E_{\lambda_{f}}\right)=-\frac{\hbar^{2}}{2 m_{j}}\left(q_{i}^{2}-q_{f}^{2}\right)=\frac{\hbar^{2}}{2 m}\left(Q^{2}+2 \vec{Q} \cdot \vec{q}_{i}(j)\right) .
$$

En remplaçant (8.79) et (8.80) dans (8.78) nous obtenons

$$
\begin{aligned}
\frac{d^{2} \sigma_{\vec{k}_{i} \rightarrow \vec{k}_{f}}}{d \Omega d E_{f}} & =\frac{k_{f}}{k_{i}} \sum_{j=1}^{N} b_{j} b_{j}^{*} \sum_{\vec{q}_{i}(j)} p\left(\vec{q}_{i}(j)\right) \delta\left(\hbar \omega-\frac{\hbar^{2}}{2 m_{j}}\left(Q^{2}+2 \vec{Q} \cdot \vec{q}_{i}(j)\right)\right) \\
& =\frac{k_{f}}{k_{i}} \sum_{j=1}^{N} b_{j} b_{j}^{*} \int d^{3} q_{i}(j) p\left(\vec{q}_{i}(j)\right) \delta\left(\hbar \omega-\frac{\hbar^{2}}{2 m_{j}}\left(Q^{2}+2 \vec{Q} \cdot \vec{q}_{i}(j)\right)\right) .
\end{aligned}
$$

\footnotetext{
${ }^{39}$ Pour éviter la confusion entre les vecteurs d'onde du neutron nous noterons $\vec{q}$ les vecteurs d'onde associés aux particules des diffuseurs.
} 
L'intégrale y figurant s'exprime avec la distribution de probabilité de Boltzmann (8.77) comme

$$
\begin{aligned}
& \int d^{3} q p(\vec{q}) \delta\left(\hbar \omega-\frac{\hbar^{2}}{2 m_{j}}\left(Q^{2}+2 \vec{Q} \cdot \vec{q}\right)\right)= \\
& \hbar^{3} \sqrt{\left(\frac{1}{2 \pi m_{j} k_{\mathrm{B}} T}\right)^{3}} \int d^{3} q \exp \left(-\frac{\hbar^{2} q^{2}}{2 m_{j} k_{\mathrm{B}} T}\right) \delta\left(\hbar \omega-\frac{\hbar^{2}}{2 m_{j}}\left(Q^{2}+2 \vec{Q} \vec{Q} \cdot \vec{q}\right)\right) .
\end{aligned}
$$

Cette intégrale peut être évaluée en utilisant un référentiel cartésien avec un des axes aligné dans la direction de $\vec{Q} \cdot{ }^{40}$ Nous obtenons

$$
\frac{d^{2} \sigma_{\vec{k}_{i} \rightarrow \vec{k}_{f}}}{d \Omega d E_{f}}=\frac{k_{f}}{k_{i}} \sum_{j=1}^{N} b_{j} b_{j}^{*} \frac{1}{\sqrt{2 \pi} \sigma_{j}} \exp \left(-\frac{\left(\hbar \omega-E_{r}(j)\right)^{2}}{2 \sigma_{j}^{2}}\right)
$$

avec l'énergie de recul de la particule $j$

$$
E_{r}(j)=\frac{\hbar^{2} Q^{2}}{2 m_{j}}
$$

et l'écart type

$$
\sigma_{j}=\sqrt{2 K_{\mathrm{B}} T E_{r}(j)} .
$$

L'énergie de recul se calcule avec (3.2) comme

$$
E_{r}(j)[\mathrm{meV}]=\left(\frac{m_{n}}{m_{j}}\right) 2.0725 \cdot Q^{2}\left[\AA^{-1}\right]
$$

${ }^{40}$ Nous choisissons $\hat{z} \| \vec{Q}$. La fonction de Dirac s'écrit comme

$$
\begin{aligned}
\delta\left(\hbar \omega-\frac{\hbar^{2}}{2 m_{j}}\left(Q^{2}+2 \vec{Q} \cdot \vec{q}\right)\right) & =\delta\left(\hbar \omega-\frac{\hbar^{2}}{2 m_{j}}\left(Q^{2}+2 Q \cdot q_{z}\right)\right) \\
& =\delta\left(\hbar \omega-E_{r}(j)-2 \frac{E_{r}(j)}{Q} \cdot q_{z}\right) \\
& =\frac{Q}{2 E_{r}(j)} \delta\left(\left(\frac{\hbar \omega Q}{2 E_{r}(j)}-\frac{Q}{2}\right)-q_{z}\right)
\end{aligned}
$$

L'intégration sur $q_{x}$ et $q_{y}$ fournit deux fois le facteur

$$
\hbar \sqrt{\frac{1}{2 \pi m_{j} k_{\mathrm{B}} T}}
$$

C'est une conséquence directe de la normalisation des probabilités. L'intégration sur $q_{z} \mathrm{~s}$ 'effectue avec la fonction de Dirac. Nous obtenons

$$
\begin{aligned}
& \hbar \sqrt{\frac{1}{2 \pi m_{j} k_{\mathrm{B}} T}} \int d q_{z} \exp \left(-\frac{\hbar^{2} q_{z}^{2}}{2 m_{j} k_{\mathrm{B}} T}\right) \frac{Q}{2 E_{r}(j)} \delta\left(\left(\frac{\hbar \omega Q}{2 E_{r}(j)}-\frac{Q}{2}\right)-q_{z}\right)= \\
& \hbar \sqrt{\frac{1}{2 \pi m_{j} k_{\mathrm{B}} T}} \frac{Q}{2 E_{r}(j)} \exp \left(-\frac{\hbar^{2}}{2 m_{j} k_{\mathrm{B}} T}\left(\frac{\hbar \omega Q}{2 E_{r}(j)}-\frac{Q}{2}\right)^{2}\right)= \\
& \sqrt{\frac{1}{4 \pi E_{r}(j) k_{\mathrm{B}} T}} \exp \left(\frac{\left(\hbar \omega-E_{r}(j)\right)^{2}}{4 E_{r}(j) k_{\mathrm{B}} T}\right) .
\end{aligned}
$$




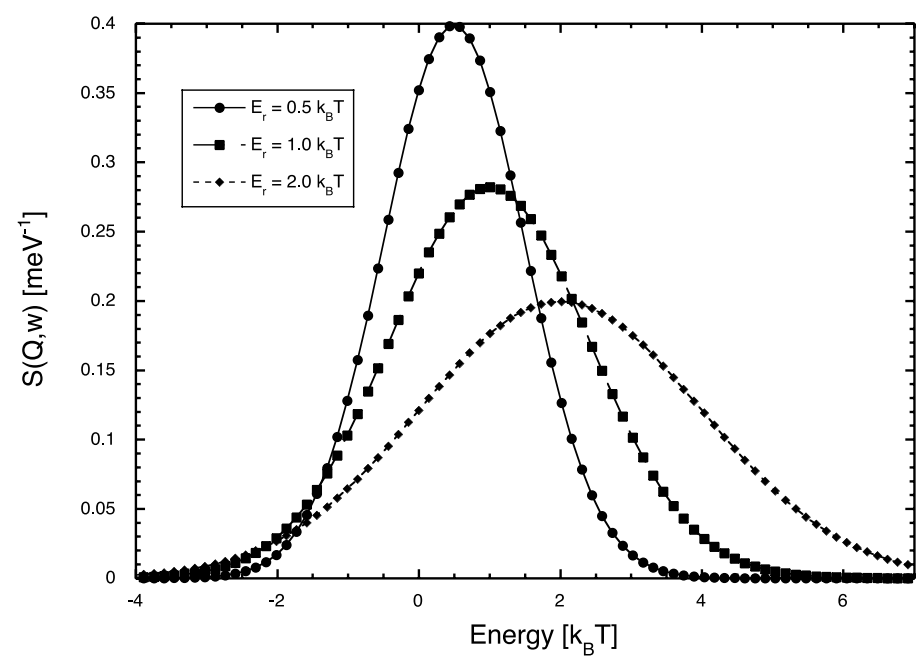

Figure 26. Fonction de diffusion pour une particule de Boltzmann pour différentes valeurs de l'énergie de recul $E_{r}$. L'unité naturelle de l'énergie est $\left(k_{\mathrm{B}} T\right)$.

avec $m_{n}$ la masse du neutron. La section efficace différentielle partielle est donc la somme de $N$ gaussiennes. Chaque gaussienne est centrée sur l'énergie de recul de la particule concernée. La largeur des gaussiennes varie comme la racine carrée de la température et de l'énergie de recul. La réponse dynamique d'une particule lourde sera donc toujours plus centrée que celle d'une particule légère. Avec

$$
\delta(x)=\lim _{\sigma \rightarrow 0} \frac{1}{\sqrt{2 \pi \sigma}} \exp \left(-\frac{x^{2}}{2 \sigma}\right)
$$

nous obtenons dans la limite $T \rightarrow 0$

$$
\frac{d^{2} \sigma_{\vec{k}_{i} \rightarrow \vec{k}_{f}}}{d \Omega d E_{f}}=\frac{k_{f}}{k_{i}} \sum_{j=1}^{N} b_{j} b_{j}^{*} \delta\left(\hbar \omega-E_{r}(j)\right) .
$$

C'est la réponse d'un ensemble de particule de Boltzmann à température nulle.

Nous constatons qu'il n'y a pas de terme d'interférence entre les contributions des particules. Nous avons affaire à des ondes planes et chaque particule est distribuée de manière homogène sur le volume du gaz. Le manque d'interaction d'échange fait qu'une particule donnée n'est pas perturbée par l'existence des autres. Il n'y a par conséquence aucune corrélation entre les particules. De ce fait la diffusion cohérente pour un ensemble de particules de Boltzmann sans interaction ne se distingue pas de sa diffusion incohérente. On dit souvent que la réponse du système est incohérente même si l'adjectif correct serait décorrélée.

Nous devons néanmoins garder à l'esprit le fait qu'à très basse température, l'occupation des niveaux n'est plus petite. La distribution de Boltzmann devient alors une mauvaise approximation (se référer au chapitre I). Nous ne pouvons plus négliger les corrélations introduites par les interactions d'échange entre particules. Ces effets sont particulièrement prononcés pour les fermions. Nous devons alors remplacer le gaz de Boltzmann par un liquide de Fermi. Une particule dans un liquide de Fermi est liée aux autres par le principe d'exclusion de Pauli qui induit des corrélations d'occupation de niveau. 
En dépit de ces limitations, nous restons dans cette section avec des particules de Boltzmann. Dans la limite $m_{j} \rightarrow \infty$ nous pouvons déduire que

$$
\frac{d^{2} \sigma_{\vec{k}_{i} \rightarrow \vec{k}_{f}}}{d \Omega d E_{f}}=\frac{k_{f}}{k_{i}} \sum_{j=1}^{N} b_{j} b_{j}^{*} \delta(\hbar \omega) .
$$

Nous retrouvons le résultat pour des particules fixes.

Il faut maintenant déduire les fonctions de corrélation pour le gaz de Boltzmann. En l'absence d'interaction, nous pouvons nous limiter aux fonctions propres. Au lieu de procéder à un calcul direct de ces fonctions, nous passons par la section efficace (8.86) dans laquelle nous identifions d'abord les fonctions de diffusion partielles propres comme

$$
S_{\kappa}^{S}(\vec{Q}, \omega)=\frac{N_{\kappa}}{\sqrt{2 \pi} \sigma_{\kappa}} \exp \left(-\frac{\left(\hbar \omega-E_{r}(\kappa)\right)^{2}}{2 \sigma_{\kappa}^{2}}\right) .
$$

Elles sont représentées figure 26. Les $S_{\kappa}^{S}(\vec{Q}, \omega)$ satisfont à la condition de balance détaillée. Le fait que nous ayons utilisé pour ce calcul la distribution de Boltzmann, qui n'est valable que pour des particules classiques, ne change rien à ce constat. Ceci se comprend si on considère que nous aurions obtenu les mêmes expressions pour une particule isolée ou pour un ensemble de particules toutes différentes. La fonction symétrisée se calcule comme

$$
\begin{aligned}
S_{\kappa}^{\mathrm{sym}}(\vec{Q}, \omega) & =\exp \left(-\frac{1}{2} \hbar \omega \beta\right) S_{\kappa}^{2}(\vec{Q}, \omega) \\
& =\frac{N_{\kappa}}{\sqrt{2 \pi} \sigma_{\kappa}} \exp \left(-\frac{\hbar \omega}{2 k_{\mathrm{B}} T}\right) \exp \left(-\frac{\left(\hbar \omega-E_{r}(\kappa)\right)^{2}}{2 \sigma_{\kappa}^{2}}\right) \\
& =\frac{N_{\kappa}}{\sqrt{2 \pi} \sigma_{\kappa}} \exp \left(\frac{-2 E_{r}(\kappa) \hbar \omega-\left(\hbar \omega-E_{r}(\kappa)\right)^{2}}{2 \sigma_{\kappa}^{2}}\right) \\
& =\frac{N_{\kappa}}{\sqrt{2 \pi} \sigma_{\kappa}} \exp \left(-\frac{\hbar^{2} \omega^{2}+E_{r}(\kappa)^{2}}{2 \sigma_{\kappa}^{2}}\right) .
\end{aligned}
$$

Attention, ces fonctions ne sont plus normalisées (voir la figure 27). Les fonctions de diffusion intermédiaires s'obtiennent à partir des fonctions de diffusion partielles par transformation de Fourier. Avec

$$
\int_{-\infty}^{\infty} d x \exp \left(-a x^{2}+b x\right)=\sqrt{\frac{\pi}{a}} \exp \left(\frac{b^{2}}{4 a}\right)
$$

il vient

$$
I_{\kappa}^{S}(\vec{Q}, t)=\hbar \int_{-\infty}^{\infty} d \omega \exp (-i \omega t) S_{\kappa}^{S}(\vec{Q}, \omega)=\exp \left(-\frac{1}{2} Q^{2} \sigma_{\kappa}(t)^{2}\right)
$$

avec

$$
\sigma_{\kappa}(t)^{2}=\frac{k_{\mathrm{B}} T}{m_{\kappa}} \cdot t\left(t-i \frac{\hbar}{k_{\mathrm{B}} T}\right) .
$$

Nous aurions obtenu le même résultat en évaluant directement

$$
I_{\kappa}^{s}(\vec{Q}, t)=\sum_{j \in\left\{j_{\kappa}\right\}}\left\langle\exp \left(-i \vec{Q} \cdot \vec{R}_{j}\right) \exp \left(i \vec{Q} \cdot \vec{R}_{j}(t)\right)\right\rangle
$$

avec les méthodes de calcul de la mécanique quantique [15]. La fonction de diffusion intermédiaire pour une particule de Boltzmann est donc une fonction intrinsèquement complexe. La forme symétrisée de 


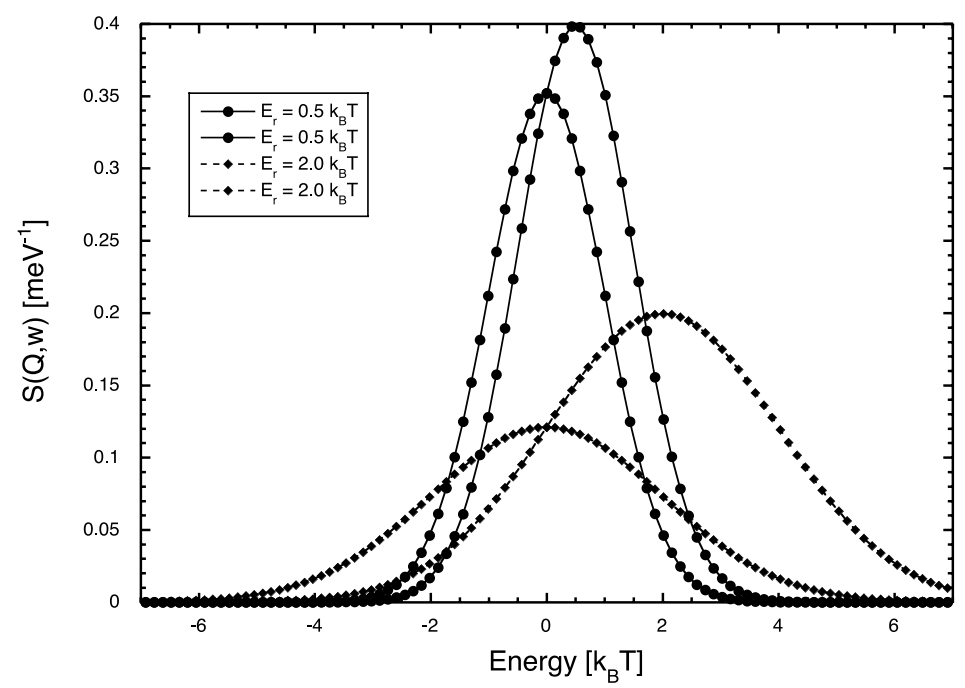

Figure 27. Comparaison entre les fonctions de diffusion et les fonctions de diffusion symétrisées pour deux valeurs de l'énergie de recul $E_{r}$.

cette fonction se calcule comme

$$
I_{\kappa}^{\mathrm{sym}}(\vec{Q}, t)=I_{\kappa}^{s}\left(\vec{Q}, t+\frac{1}{2} \hbar \omega \beta\right)=\exp \left(-\frac{1}{2} Q^{2} \sigma_{\mathrm{sym}}^{2}(\kappa)\right)
$$

avec

$$
\sigma_{\mathrm{sym}}^{2}(\kappa)=\frac{k_{\mathrm{B}} T}{m_{\kappa}}\left(t^{2}+\frac{\hbar^{2}}{4\left(k_{\mathrm{B}} T\right)^{2}}\right) .
$$

Il s'agit d'une fonction réelle et paire. Finalement, la fonction de corrélation de paires $G_{\kappa}^{s}(\vec{r}, t)$ se calcule à partir de la fonction de diffusion intermédiaire en effectuant une transformation de Fourier dans l'espace

$$
\begin{aligned}
G_{\kappa}^{S}(\vec{r}, t) & =\frac{1}{(2 \pi)^{3}} \int d^{3} Q e^{-i \vec{Q} \cdot \vec{r}} I_{\kappa}^{S}(\vec{Q}, t) \\
& =\frac{1}{\sqrt{\left(2 \pi \sigma_{\kappa}(t)^{2}\right)^{3}}} \exp \left(-\frac{r^{2}}{2 \sigma_{\kappa}(t)^{2}}\right) .
\end{aligned}
$$

C'est une gaussienne normalisée dont la largeur est complexe et augmente avec le temps. La version symétrisée de $G_{\kappa}^{s}(\vec{r}, t)$ s'obtient comme

$$
G_{\kappa}^{\text {sym }}(\vec{r}, t)=\frac{1}{\sqrt{\left(2 \pi \sigma_{\kappa}^{\text {sym }}(t)^{2}\right)^{3}}} \exp \left(-\frac{r^{2}}{2 \sigma_{\kappa}^{\text {sym }}(t)^{2}}\right) .
$$

L'interprétation d'une fonction de corrélation complexe n'est pas facile. Revenons donc aux systèmes classiques. Pour un gaz parfait la fonction de corrélation de paires à la même forme que (8.101). Elle se détermine comme

$$
G_{\kappa}^{\mathrm{cl}}(\vec{r}, t)=\frac{1}{\sqrt{\left(2 \pi \sigma_{\kappa}^{\mathrm{cl}}(t)^{2}\right)^{3}}} \exp \left(-\frac{r^{2}}{2 \sigma_{\kappa}^{\mathrm{cl}}(t)^{2}}\right) .
$$




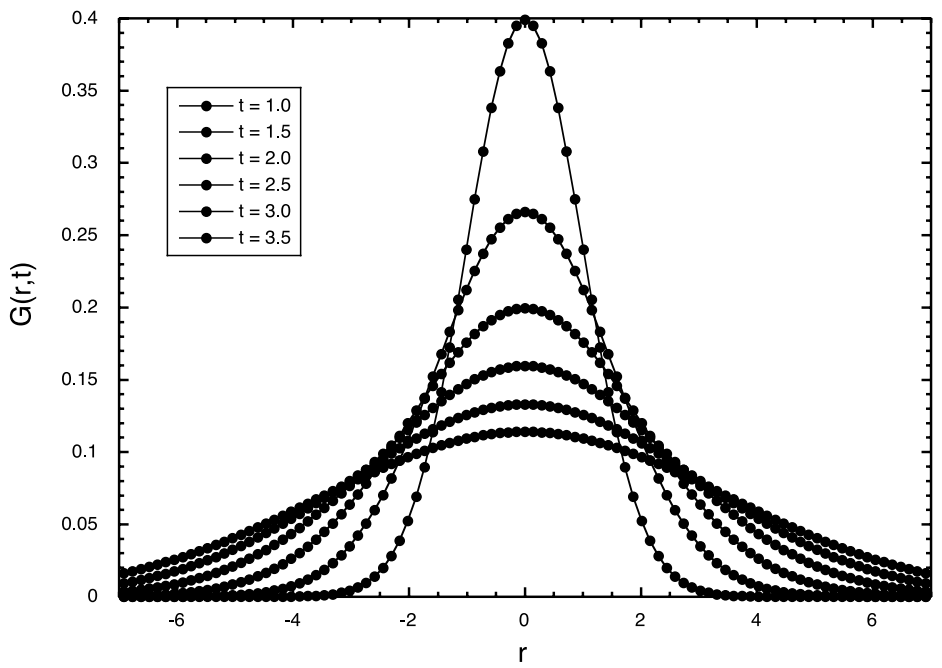

Figure 28. La fonction $G_{\kappa}^{\mathrm{cl}}(\vec{r}, t)$ pour différents temps. Les unités sont choisies e telle manière que la vitesse $\sqrt{k_{\mathrm{B}} T / m_{\kappa}}=1$.

L'écart type se calcule comme

$$
\sigma_{\kappa}^{\mathrm{cl}}(t)=\sqrt{\frac{k_{\mathrm{B}} T}{m_{\kappa}}} t .
$$

Les deux fonctions $G_{\kappa}^{s}(\vec{r}, t)$ et $G_{\kappa}^{\mathrm{cl}}(r, t)$ se correspondent par un changement de variable

$$
G_{\kappa}^{s}(\vec{r}, t)=G_{\kappa}^{\mathrm{cl}}(r, \sqrt{t(t+i \beta \hbar)}) .
$$

Cette substitution constitue une méthode alternative à l'approche de Schofield pour obtenir une fonction de diffusion respectant la balance détaillée à partir d'un calcul classique. Elle à été proposée par Egelstaff [22]. Sa validité est discutée dans [23].

La fonction $G_{\kappa}^{\mathrm{cl}}(\vec{r}, t)$ constitue la limite classique de $G_{\kappa}^{s}(\vec{r}, t)$. Comme on peut facilement le vérifier avec (8.97), elle s'obtient à partir de $G_{\kappa}^{s}(\vec{r}, t)$ soit pour $\hbar \rightarrow 0$ ou pour $\beta \rightarrow 0(T \rightarrow \infty)$. La signification de la fonction $G_{\kappa}^{\mathrm{cl}}(r, t)$ est simple (voir la figure 28 ).

$$
4 \pi r^{2} G_{\kappa}^{\mathrm{cl}}(\vec{r}, t) d r
$$

donne la probabilité de trouver une particule de type $\kappa$ à $t$ à une distance située entre $r$ et $r+d r$, si la même particule était à l'origine à $t=0$. Dans le régime balistique, cette probabilité est directement reliée à la probabilité $p(v) d v$ que la particule possède une vitesse comprise entre $v$ et $v+d v$ avec $v=r / t . p(v)$ est donnée par la distribution de Maxwell (voir chapitre I)

$$
p(v) \propto v^{2} \exp \left(-\frac{m_{\kappa} v^{2}}{2 k_{\mathrm{B}} T}\right) .
$$

La largeur de $G_{\kappa}^{\mathrm{cl}}(\vec{r}, t)$ augmente donc avec $v$, ce qui équivaut pour un $r$ donné à augmenter avec $t$.

L'exemple des particules de Boltzmann est important d'un point de vue pédagogique. Il est par contre d'un intérêt scientifique limité. Si on ne veut pas ou si on ne peut pas traiter les interactions entre particules au niveau atomique, il est possible de prendre en compte leur existence en introduisant les mouvements de diffusion. Nous avions déjà évoqué que la dynamique d'un fluide est gouvernée par une 
équation de continuité (4.12)

$$
\frac{\partial}{\partial t} n(\vec{r}, t)+\vec{\nabla} \cdot \vec{J}(\vec{r}, t)=0,
$$

qui relie le changement de densité atomique $n(r, t)$ à un courant. La première loi de Fick stipule que ce courant est proportionnel au gradient de concentration

$$
\vec{J}(\vec{r}, t)=\vec{\nabla} n(\vec{r}, t) .
$$

Il est évident que cette loi ne peut s'appliquer que pour des temps assez longs. Vers des temps très courts on restera nécessairement dans un régime balistique. Si nous combinons l'équation de continuité avec la première loi de Fick nous obtenons l'équation de la diffusion ou deuxième loi de Fick

$$
\frac{\partial n(\vec{r}, t)}{\partial t}=D \vec{\nabla} \cdot \vec{\nabla} n(\vec{r}, t)=D \Delta n(\vec{r}, t) .
$$

avec $D$ la constante de diffusion. Cette équation ressemble beaucoup -dans sa forme- à l'équation de Schrödinger libre. Les solutions sont néanmoins très différentes.Si nous ne retenons que celles qui sont isotropes $^{41}$ les fonctions de corrélation classiques correspondant à l'équation de diffusion s'obtiennent comme

$$
G_{\kappa}^{\mathrm{cl}}(\vec{r}, t)=\frac{1}{\sqrt{4 \pi D|t|}^{3}} \exp \left(-\frac{r^{2}}{4 D|t|}\right) .
$$

Nous voudrions souligner que $G_{\kappa}^{\mathrm{cl}}(\vec{r}, t)$ décrit la corrélation d'une particule avec elle-même. La fonction de corrélation est toujours une gaussienne comme pour les particules de Boltzmann. Néanmoins nous constatons que la largeur de cette gaussienne n'augmente qu'avec $\sqrt{t}$. Nous pouvons déterminer la

${ }^{41}$ L'opérateur $\nabla^{2}$ est bien connu dansà partir de l'équation de Schrödinger. Les solutions isotropes de la loi de Fick sont de type $s$ (voir section 4.2). Dans ce cas nous pouvons poser (voir (4.33))

$$
\Delta=\left[\frac{\partial^{2}}{\partial r^{2}}+\frac{2}{r} \frac{\partial}{\partial r}\right]
$$

Utilisant l'Ansatz

$$
n(\vec{r}, t)=\frac{1}{\sqrt{(2 \pi)^{3}}} \frac{1}{\sigma(t)^{3}} \exp \left(-\frac{r^{2}}{2 \sigma(t)^{2}}\right)
$$

nous vérifions que

$$
\left[\frac{\partial^{2}}{\partial r^{2}}+\frac{2}{r} \frac{\partial}{\partial r}\right] n(r, t)=\left[-\frac{3}{\sigma(t)^{2}}-\frac{r^{2}}{\sigma(t)^{4}}\right] n(\vec{r}, t)
$$

ainsi que

$$
\frac{\partial}{\partial t} n(r, t)=\left[-\frac{3 \frac{d \sigma(t)}{d t}}{\sigma(t)}-\frac{r^{2} \frac{d \sigma(t)}{d t}}{\sigma(t)^{3}}\right] n(\vec{r}, t) .
$$

Nous obtenons donc une solution de l'équation de diffusion (8.109) pourvu que

$$
\sigma(t) \frac{d \sigma(t)}{d t}=D
$$

C'est le cas si

$$
\frac{d}{d t} \sigma(t)^{2}=2 D
$$

ou

$$
\sigma(t)=2 D|t|+C
$$

Pour des temps longs, qui nous intéressent ici, nous négligeons la constante $C$. La fonction de corrélation de paires s'obtient à partir de $n(\vec{r}, t)$ avec la condition aux limites $n(0, t)=n_{0} \delta(\vec{r})$. 


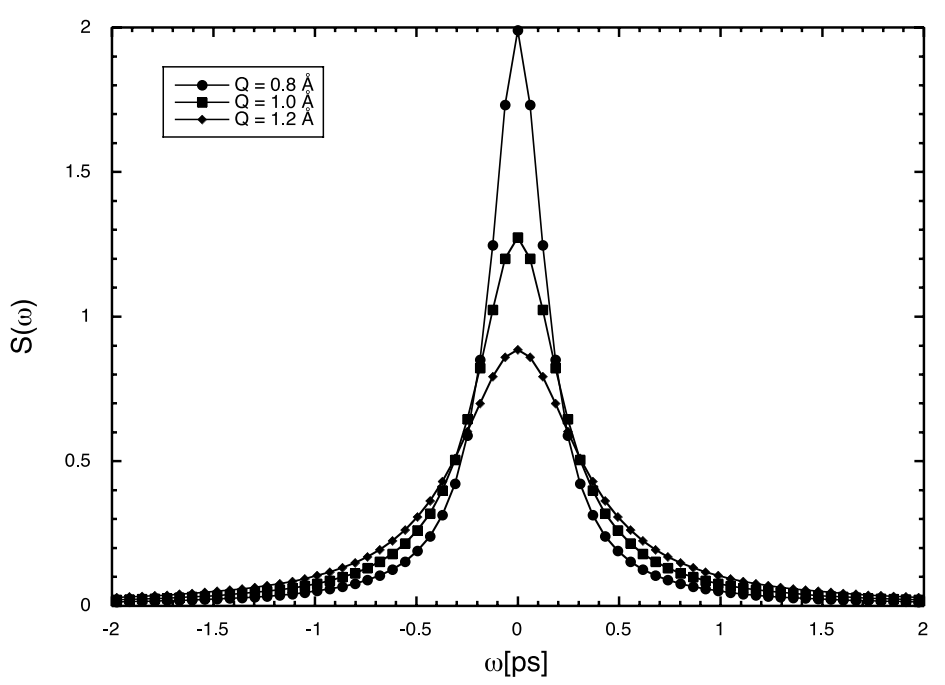

Figure 29. La fonction $S_{\kappa}^{\mathrm{cl}}(Q, \omega)$ définie dans (8.119) pour différents $Q$. La constante de diffusion a été choisie comme $2.5 \cdot 10^{-5} \mathrm{~cm}^{2} / \mathrm{s}$ égal à $0.25 \AA^{2} / \mathrm{ps}$. Elle est proche de celle de l'eau à 25 degrés. On peut comparer la forme de ces courbes avec celle mesurée dans la figure 25 pour la décaline liquide.

fonction de diffusion intermédiaire propre classique par transformation de Fourier

$$
\begin{aligned}
I_{s}^{\mathrm{cl}}(\vec{r}, t) & =\int d^{3} r \exp (i \vec{Q} \cdot \vec{r}) G_{\kappa}^{\mathrm{cl}}(\vec{r}, t) \\
& =\exp \left(-\frac{1}{2} Q^{2} \sigma^{2}(t)\right) \\
& =\exp \left(-Q^{2} D|t|\right) .
\end{aligned}
$$

La transformée de Fourier de cette fonction, et par conséquent la fonction de diffusion, est une lorentzienne

$$
\begin{aligned}
S_{s}^{\mathrm{cl}}(\vec{r}, t) & =\frac{1}{2 \pi \hbar} \int_{-\infty}^{\infty} I_{s}^{\mathrm{cl}}(\vec{r}, t) \exp (-i \omega t) d t \\
& =\frac{1}{2 \pi \hbar}\left\{\int_{0}^{\infty} \exp \left(-\left(Q^{2} D+i \omega\right) t\right) d t+\int_{-\infty}^{0} \exp \left(\left(Q^{2} D-i \omega\right) t\right) d t\right\} \\
& =\frac{1}{\pi \hbar} \frac{D Q^{2}}{\left(D Q^{2}\right)^{2}+\omega^{2}} .
\end{aligned}
$$

La fonction (voir figure 29) est centrée sur $\omega=0$ et possède une largeur (FWHM) de

$$
\hbar \Delta \omega=2 \hbar D Q^{2} .
$$

Nous pouvons donc clairement distinguer un régime balistique d'un régime de diffusion à partir simplement de la forme de la réponse dynamique.

Si nous identifions $S_{S}^{\mathrm{cl}}(\vec{r}, t)$ avec la fonction de diffusion propre $S^{S}(\vec{r}, t)$, cette fonction sera symétrique et par conséquent violera la balance détaillée. Une solution pour échapper au problème serait d'identifier $S_{s}^{\mathrm{cl}}(\vec{r}, t)$ avec la fonction de diffusion propre symétrisée $S_{S}^{\mathrm{sym}}(\vec{r}, t)$ et d'en déduire la fonction de diffusion propre. 
Nous abordons enfin la section efficace totale d'un ensemble de particules de Boltzmann. Il faut calculer

$$
\sigma_{\mathrm{tot}}=4 \pi \frac{k_{f}}{k_{i}} \int_{-\infty}^{\infty} d(\hbar \omega) \sum_{\kappa} \overline{\left|b_{\kappa}\right|^{2}} S_{\kappa}(Q, \omega) .
$$

La présence du facteur $k_{f} / k_{i}$ rend ce calcul assez fastidieux. Le lecteur désireux de le développer est prié de se référer à Lovesey [15]. Nous nous contentons ici d'en donner le résultat dans la limite d'une énergie incidente grande

$$
E_{i} \gg \frac{m_{\kappa}}{m_{n}}\left(k_{\mathrm{B}} T\right)
$$

Dans ce cas nous obtenons

$$
\sigma_{\mathrm{tot}} \approx 4 \pi \sum_{\kappa} N_{\kappa} \overline{\left|b_{\kappa}\right|^{2}}\left(1+\frac{m_{n}}{m_{\kappa}}\right)^{-2} .
$$

Pour des protons, cette section efficace est égale au quart de la section efficace liée. Pour des énergies incidentes très élevées, le mouvement des particules peut être négligé. Nous retrouvons la situation des particules libres de la section 5.9. L'expression (8.122) confirme donc que dans notre formalisme nous devons toujours travailler avec les longueurs de diffusion liées.

\subsection{Fonction de réponse et susceptibilité}

Dans le chapitre I nous avions déjà introduit la théorie de la réponse linéaire. Le neutron étant une perturbation faible, nous allons utiliser cette théorie pour la description de la diffusion. Nous rappelons que la théorie est basée sur l'hypothèse que la variation d'une observable est linéaire avec la perturbation si celle-ci est faible. ${ }^{42}$

Pour $t \rightarrow-\infty$, c'est-à-dire bien avant l'interaction avec le neutron, le système est décrit par l'Hamiltonien non-perturbé $\hat{H}_{0}$. La perturbation est supposée se faire de façon adiabatique, c'est àdire suffisamment lentement pour que le système reste en permanence en état d'équilibre. Le terme de perturbation $\hat{H}_{p}=-\hat{B} F(t)$ se rajoute à l'Hamiltonien non perturbé $H_{0}$ de sorte que l'Hamiltonien du système s'écrive

$$
\hat{H}=\hat{H}_{0}+\hat{B} F(t)
$$

$B$ est un opérateur constant dans le temps. Le changement survenant dans le système à la suite de la perturbation peut être observé au travers d' une variable $\hat{A}$. Nous avons vu dans le chapitre I que la valeur moyenne de $\hat{A}$ se détermine grâce à l'opérateur de densité statistique. Sans perturbation

$$
\begin{aligned}
& \langle\hat{A}\rangle=\operatorname{Tr}\left\{\rho_{0} \hat{A}\right\} \\
& \rho_{0}=\frac{1}{Z} \exp \left(-\frac{\hat{H}_{0}}{k_{\mathrm{B}} T}\right) ; \quad Z=\operatorname{Tr} \exp \left(-\frac{\hat{H}_{0}}{k_{\mathrm{B}} T}\right) ;
\end{aligned}
$$

$\langle\hat{A}\rangle$ est alors indépendante du temps. Avec la perturbation, l'opérateur de densité statistique devient dépendant du temps, et la valeur moyenne de $\hat{A}$ s'écrit

$$
\begin{aligned}
& \langle\hat{A}(t)\rangle=\operatorname{Tr}\{\rho(t) \hat{A}\} \\
& \rho(t)=\frac{1}{Z} \exp \left(-\frac{\hat{H}}{k_{\mathrm{B}} T}\right) ; \quad Z=\operatorname{Tr} \exp \left(-\frac{\hat{H}}{k_{\mathrm{B}} T}\right) .
\end{aligned}
$$

\footnotetext{
42 Ce sujet est très bien exposé dans le livre de Jensen et Mackintosh [24]
} 
Ce changement peut être décrit à l'aide d'une fonction de réponse $\Phi_{A, B}\left(t-t^{\prime}\right)$ de la manière suivante

$$
\langle\hat{A}(t)\rangle-\langle\hat{A}\rangle=\int_{-\infty}^{t} d t^{\prime} \Phi_{A, B}\left(t-t^{\prime}\right) F\left(t^{\prime}\right) .
$$

Dans le souci d'alléger la notation nous n'indiquons pas explicitement la dépendance en fonction de $\vec{r}$.

En utilisant les lois de Kubo (voir chapitre I) la fonction de réponse se calcule comme ${ }^{43}$

$$
\Phi_{A, B}\left(t-t^{\prime}\right)=\frac{i}{\hbar}\left\langle\left[\hat{A}(t), \hat{B}\left(t^{\prime}\right)\right]\right\rangle \Theta\left(t-t^{\prime}\right)
$$

avec le cas particulier

$$
\Phi_{A, B}(t)=\frac{i}{\hbar}\langle[\hat{A}(t), \hat{B}]\rangle \Theta(t) .
$$

La présence de $\Theta(t)$ assure la causalité. Ces fonctions sont donc appelées fonctions de réponse causales. Pour le calcul qui suit il est utile d'introduire les fonctions de réponse généralisées

$$
K_{A, B}(t)=\frac{i}{\hbar}\langle[\hat{A}(t), \hat{B}]\rangle
$$

On vérifie que

$$
K_{A, B}(t)=\left\{\begin{array}{c}
K_{A, B}(t) \text { pour } t>0 \\
-K_{B, A}(-t) \text { pour } t<0 .
\end{array}\right.
$$

Dans le cas de la diffusion non-magnétique des neutrons, la perturbation est créée par le potentiel de Fermi (5.25). Cette interaction implique l'opérateur de densité atomique $\hat{\rho}_{\kappa}(\vec{r}, t)(8.16)$. Afin de travailler dans l'espace réciproque nous identifions

$$
B(t) \equiv \hat{\rho}_{\kappa}(\vec{Q}, t)
$$

Nous choisissons d'observer l'effet de la perturbation également en termes de densité atomique ${ }^{44}$. L'opérateur conjugé de $\hat{\rho}_{\kappa}(\vec{Q}, t)$ est $\hat{\rho}_{\kappa}^{+}(\vec{Q}, t)=\hat{\rho}_{\kappa}(-\vec{Q}, t)$. On a alors

$$
A(t) \equiv \hat{B}^{+}(t)=\hat{\rho}_{\kappa}(-\vec{Q}, t)
$$

où nous permettons implicitement que l'indice $\kappa$ varie entre $A=B^{+}$et $B$, c'est-à-dire que nous autorisons l'observation des effets de la perturbation dûs à l'interaction du neutron avec les atomes du sous-système de type $\kappa$ dans le sous-système de type $\kappa^{\prime}$.

Dans cette notation les fonctions de diffusion partielles (8.38) s'écrivent comme

$$
\begin{aligned}
S_{\kappa, \kappa^{\prime}}(\vec{Q}, \omega) & =\frac{1}{2 \pi \hbar} \int_{-\infty}^{\infty} d t \exp (-i \omega t) I_{\kappa, \kappa^{\prime}}(\vec{Q}, t) \\
& =\frac{1}{2 \pi \hbar} \int_{-\infty}^{\infty} d t \exp (-i \omega t)\left\langle\hat{B}, \hat{B}^{+}(t)\right\rangle .
\end{aligned}
$$

Pour établir une relation entre la fonction de diffusion et la fonction de réponse $\Phi_{B^{+}{ }_{B}}(t)$ nous évaluons la transformée de Fourier de $K_{B^{+} B}(t)$.

$$
\begin{aligned}
\frac{1}{2 \pi} \int_{-\infty}^{\infty} d t \exp (-i \omega t) K_{B^{+} B}(t) & =\frac{i}{2 \pi \hbar} \int_{-\infty}^{\infty} d t \exp (-i \omega t)\left\langle\hat{B}^{+}(t) \hat{B}-\hat{B} \hat{B}^{+}(t)\right\rangle \\
& =-i S_{\kappa, \kappa^{\prime}}(\vec{Q}, \omega)+\frac{i}{2 \pi \hbar} \int_{-\infty}^{\infty} d t \exp (-i \omega t)\left\langle\hat{B}^{+}(t) \hat{B}\right\rangle .
\end{aligned}
$$

\footnotetext{
${ }^{43}$ La fonction de Heaviside est définie par $\Theta(t)=1$ pour $t>0$ et est nulle ailleurs.

44 Par exemple par la création ou l'absorption d'un phonon etc.
} 
Avec (8.54) nous pouvons renverser l'ordre des opérateurs. On obtient

$$
\left\langle\hat{B}^{+}(t) \hat{B}\right\rangle=\left\langle\hat{B} \hat{B}^{+}\left(t+i \hbar \frac{\hbar \omega}{k_{\mathrm{B}} T}\right)\right\rangle
$$

et donc

$$
\begin{aligned}
\frac{1}{2 \pi \hbar} \int_{-\infty}^{\infty} d t \exp (-i \omega t)\left\langle\hat{B}^{+}(t) \hat{B}\right\rangle & =\frac{1}{2 \pi \hbar} \int_{-\infty}^{\infty} d t \exp (-i \omega t)\left\langle\hat{B} \hat{B}^{+}\left(t+i \frac{\hbar}{k_{\mathrm{B}} T}\right)\right\rangle \\
& =\frac{1}{2 \pi \hbar} \exp \left(-\frac{\hbar \omega}{k_{\mathrm{B}} T}\right) \int_{-\infty}^{\infty} d t \exp (-i \omega t)\left\langle\hat{B} \hat{B}^{+}(t)\right\rangle \\
& =\frac{1}{2 \pi \hbar} \exp \left(-\frac{\hbar \omega}{k_{\mathrm{B}} T}\right) S_{\kappa, \kappa^{\prime}}(\vec{Q}, \omega) .
\end{aligned}
$$

En combinant les expressions (8.133) et (8.135) nous dérivons une relation importante

$$
S_{\kappa, \kappa^{\prime}}(\vec{Q}, \omega)=(1+n(\omega)) \frac{i}{2 \pi} \int_{-\infty}^{\infty} d t \exp (-i \omega t) K_{B^{+} B}(t)
$$

Nous avons déduit de manière explicite une forme du théorème de fluctuation-dissipation pour les neutrons. Cette expression a l'avantage d'être formulée en terme d'une transformation de Fourier normale. Pour aboutir à la forme usuelle du théorème de fluctuation-dissipation, nous rappelons que la susceptibilité généralisée est définie à l'aide d'une transformation de Laplace ${ }^{45}$ monolatérale de la fonction de réponse causale (voir chapitre I)

$$
\chi_{B^{+} B}[\omega]=\lim _{\epsilon \rightarrow 0^{+}} \chi_{B^{+} B}[i p=\omega+i \epsilon]=\lim _{\epsilon \rightarrow 0^{+}} \int_{0}^{\infty} d t \Phi_{B^{+} B}(t) \exp (p t) .
$$

La notation $[\omega]$ souligne, comme dans le chapitre I, qu'il s'agit d'une transformation de Laplace monolatérale et non pas d'une transformation de Fourier.

La susceptibilité est composée de 2 termes

$$
\chi_{B^{+} B}[p]=\chi_{B^{+} B}^{\prime}[p]+i \chi_{B^{+} B}^{\prime \prime}[p] .
$$

L'une

$$
\chi_{B^{+}{ }_{B}}^{\prime}[p]=\frac{1}{2}\left(\chi_{B^{+} B}[p]+\chi_{B B^{+}}\left[-p^{*}\right]\right)
$$

est réactive et l'autre

$$
\chi_{B^{+} B}^{\prime \prime}[p]=\frac{1}{2 i}\left(\chi_{B^{+} B}[p]-\chi_{B B^{+}}\left[-p^{*}\right]\right)
$$

est dissipative. Les deux fonctions sont reliées par la relation de Kramers-Kronig (voir chapitre I).

La transformée de Fourier de la fonction $K_{B^{+} B}(t)$ figurant dans l'expression (8.136) est étroitement liée à la susceptibilité. Il vient

$$
\begin{aligned}
& \int_{-\infty}^{\infty} d t \exp (-i \omega t) K_{B^{+} B}(t) \\
& \quad=\lim _{\epsilon \rightarrow 0^{+}} \int_{-\infty}^{\infty} d t \exp (-i \omega t-\epsilon|t|) K_{B^{+} B}(t)
\end{aligned}
$$

45 la transformée de Laplace monolatérale d'une fonction $f(t)$ d'une variable réelle positive $t$, est la fonction $F(p)$ de la variable complexe $p$, définie par

$$
F(p)=\int_{0}^{\infty} d t f(t) \exp (-p t)
$$




$$
\begin{aligned}
& =\lim _{\epsilon \rightarrow 0^{+}}\left\{\int_{0}^{\infty} d t \exp (-i \omega t-\epsilon t) K_{B^{+} B}(t)+\int_{-\infty}^{0} d t \exp (-i \omega t+\epsilon t) K_{B^{+} B}(t)\right\} \\
& =\lim _{\epsilon \rightarrow 0^{+}}\left\{\int_{0}^{\infty} d t \exp (-i \omega t-\epsilon t) K_{B^{+} B}(t)-\int_{0}^{\infty} d t \exp (+i \omega t-\epsilon t) K_{B B^{+}}(t)\right\} \\
& =\chi_{B^{+} B_{B}}[\omega]-\chi_{B B^{+}}[-\omega] \\
& =2 i \chi^{\prime \prime}{ }_{B^{+} B}[\omega],
\end{aligned}
$$

où nous avons exploité l'identité

$$
K_{B^{+} B}(-t)=-K_{B B^{+}}(t),
$$

que l'on peut vérifier facilement en s'appuyant sur (8.131).

En remplaçant (8.142) dans (8.136) nous obtenons

$$
S_{\kappa, \kappa^{\prime}}(\vec{Q}, \omega)=(1+n(\omega)) \frac{1}{\pi} \chi_{\vec{Q}}^{\prime \prime}[\omega]
$$

C'est la forme la plus connue du théorème de fluctuation-dissipation.

\subsection{Moments et règles de somme pour la fonction de diffusion}

Pour une fonction $f(x)$, qui est continue sur un intervalle $I$ (non réduit à un point), nous définissons (à condition qu'il existe) le n-ième moment de $f$ par

$$
m_{n}(f)=\int_{I} x^{n} f(x) d x
$$

avec $n$ un entier naturel. Pour la fonction de diffusion partielle, les moments par rapport à l'énergie s'écrivent comme

$$
S_{\kappa, \kappa^{\prime}}^{n}(\vec{Q}, \omega)=\int_{-\infty}^{\infty} S_{\kappa, \kappa^{\prime}}(\vec{Q}, \omega)(\hbar \omega)^{n} d(\hbar \omega)
$$

D’après (8.37) le moment d'ordre zéro est rélié directement à la fonction de diffusion intermédiaire

$$
\begin{aligned}
S_{\kappa, \kappa^{\prime}}(\vec{Q})=S_{\kappa, \kappa^{\prime}}^{0}(\vec{Q}, \omega) & =\int_{-\infty}^{\infty} S_{\kappa, \kappa^{\prime}}(\vec{Q}, \omega) d(\hbar \omega) \\
& =\int_{-\infty}^{\infty} d t I_{\kappa, \kappa^{\prime}}(\vec{Q}, t) \frac{1}{2 \pi \hbar} \int_{\infty}^{\infty} d(\hbar \omega) e^{-i \omega t} \\
& =\int_{-\infty}^{\infty} d t I_{\kappa, \kappa^{\prime}}(\vec{Q}, t) \delta(t) \\
& =I_{\kappa, \kappa^{\prime}}(\vec{Q}, t=0) .
\end{aligned}
$$

Nous montrons un exemple de fonction intermédiaire $I(Q, t)$ dans la figure 30. Cette fonction est issue d'une simulation de dynamique moléculaire classique. Pour $t=0$ elle donne le facteur de structure $S(Q)$.

$S_{\kappa, \kappa^{\prime}}(\vec{Q})$ est le facteur de structure statique partiel ou dans le cas d'un système monoatomique le facteur de structure (statique) simplement. Il est d'une grande importance pour les études structurales. 


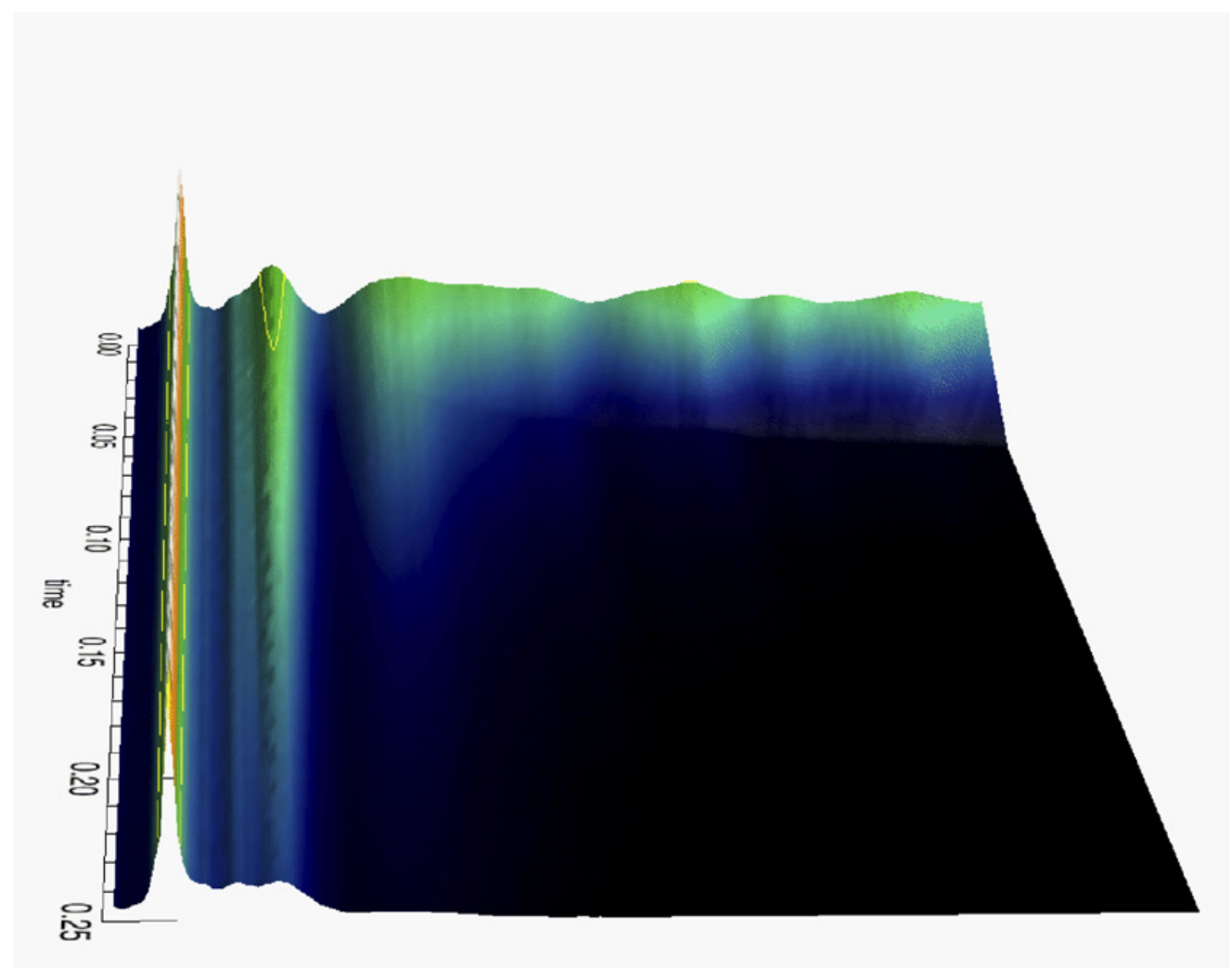

Figure 30. Fonction de diffusion intermédiaire normalisée au nombre de diffuseurs pour le composé cis-Décaline deuteré $\mathrm{C}_{10} \mathrm{D}_{18}$ dans l'état liquide. L'unité de temps est la pico-seconde. $Q$ couvre la gamme de 1 à $25 \AA^{-1}$. Pour $t=0$ cette fonction correspond au facteur de structure $S(Q)$. Avec le temps les corrélations disparaissent. La relaxation est plus lente pour les valeurs de $Q$ qui correspondent aux maxima de $S(Q)$. Pour des $Q$ importants le facteur de structure s'approche de l'unité et décroît très vite avec le temps. La valeur un est aussi la valeur attendue pour la diffusion incohérente (voir (8.151)). On en déduit qu'il n’y a plus de corrélation spatiale à très courtes distances. On ne voit que la densité moyenne (image fournie par S. Eibl).

Lors de la diffraction, l'énergie finale n'est pas analysée et la mesure correspond donc à une intégration du signal sur l'énergie. Mais c'est une intégration à angle de diffusion constant et la section efficace contient le facteur $k_{f} / k_{i}$. Elle ne donnera le facteur de structure que dans le cas ou $k_{f} \approx k_{i}$ sur toute la gamme d'énergie des excitations. ${ }^{46} \mathrm{C}$ 'est certainement le cas pour les rayons-X. On s'en approche dans le cas des neutrons pour des $k_{i}$ suffisamment grands. Mais même dans ce cas, des corrections doivent être mises en place afin de corriger des contributions inélastiques. Ces corrections sont connues sous le nom de corrections de Placzek [25].

Il est utile de souligner que la diffraction, en intégrant sur l'énergie, correspond à la superposition de clichés du système pris avec des expositions courtes. On parle de snapshots. Le temps d'exposition sera défini par l'étendue de l'intégration en énergie. Plus cette étendue sera importante, plus courts seront les temps d'exposition. Se limiter à l'intensité purement élastique correspondra alors à une exposition de durée infinie.

\footnotetext{
46 Souvent ce facteur est implicitement corrigé par l'efficacité des détecteurs, qui diminue plus ou moins en proportion avec la vitesse des neutrons.
} 
Les expressions pour les moments ont avantage d'être des fonctions d'opérateurs à un temps donné. Pour des temps égaux les opérateurs de position commutent. Nous obtenons alors

$$
\begin{aligned}
I_{\kappa, \kappa^{\prime}}(\vec{Q}, t=0) & =\sum_{j \in\left\{j_{\kappa}\right\}, j^{\prime} \in\left\{j_{\kappa^{\prime}}\right\}}\left\langle\exp \left(-i \vec{Q} \cdot \vec{R}_{j}\right) \exp \left(i \vec{Q} \cdot \vec{R}_{j^{\prime}}\right)\right\rangle \\
& =\sum_{j \in\left\{j_{k}\right\}, j^{\prime} \in\left\{j_{k^{\prime}}\right\}}\left\langle\exp \left(-i \vec{Q} \cdot\left(\vec{R}_{j}-\vec{R}_{j^{\prime}}\right)\right)\right\rangle \\
& =\int_{V} d^{3} r \exp (-i \vec{Q} \cdot \vec{r}) G_{\kappa, \kappa^{\prime}}(\vec{r}, t=0) \\
& =\int_{V} d^{3} r \exp (-i \vec{Q} \cdot \vec{r}){ }^{\mathrm{cl}} G_{\kappa, \kappa^{\prime}}(\vec{r}, t=0),
\end{aligned}
$$

avec la version classique de la fonction $G_{\kappa, \kappa^{\prime}}(\vec{r}, t)$ définie en (8.22). Si le système étudié est monoatomique, ce résultat peut être formulé en termes de la distribution de paires statique $g(\vec{r})$

$$
\frac{I(\vec{Q}, t=0)}{N}=1+\int_{V} d^{3} r \exp (-i \vec{Q} \cdot \vec{r}) g(\vec{r})
$$

avec

$$
{ }^{\mathrm{cl}} G(\vec{r}, t=0)=\delta(\vec{r})+g(\vec{r})=\delta(\vec{r})+\sum_{j \neq 0}\left\langle\delta\left(\vec{r}-\left(\vec{R}_{j}-\vec{R}_{j=0}\right)\right)\right\rangle .
$$

$g(\vec{r})$ donne la densité moyenne d'atome en fonction de la distance.

Pour le moment de la fonction de diffusion propre nous obtenons

$$
\begin{aligned}
S_{\kappa}^{s}(\vec{Q}) & :=\int_{\infty}^{\infty} S_{\kappa}^{s}(\vec{Q}, \omega) d(\hbar \omega) \\
& =I_{\kappa, \kappa^{\prime}}^{s}(\vec{Q}, t=0) \\
& \left.=\int_{V} d^{3} r \exp (-i \vec{Q} \cdot \vec{r})\right) G_{\kappa}^{s}(\vec{r}, t=0) \\
& =N_{\kappa} .
\end{aligned}
$$

L'intégrale sur l'énergie de la fonction de diffusion incohérente normalisée au nombre de diffuseurs est donc égale à l'unité. Le signal perdu dans le canal élastique doit impérativement apparaître comme signal inélastique à la même valeur de $\vec{Q}$. Attention, cela ne veut pas dire que le signal intégré pour un angle de diffusion donné soit constant. En plus du fait que l'intégration n'est pas effectuée à $\vec{Q}$ constant, on ne couvrira en général qu'une partie du signal inélastique à cause des contraintes cinématiques.

Avec les règles de calcul pour les transformations de Fourier, nous pouvons immédiatement relier les moments supérieurs de la fonction de diffusion aux dérivées de la fonction intermédiaire à $t=0$ [26]

$$
S_{\kappa, \kappa^{\prime}}^{n}(\vec{Q}, \omega)=\left(\frac{\hbar}{i}\right)^{n}\left[\frac{\partial^{n}}{\partial t^{n}} I_{\kappa, \kappa^{\prime}}(\vec{Q}, t)\right]_{t=0}
$$

Ces moments sont d'une grande utilité pratique. Ils permettent à la fois de tester la qualité des données expérimentales et des modèles utilisés pour les interpréter. 
Parmi les moments d'ordre supérieur, le moment d'ordre un a une importance particulière. Il est relié à la dérivée simple de la fonction intermédiaire de diffusion

$$
\begin{aligned}
\frac{\partial}{\partial t} I_{\kappa, \kappa^{\prime}}(\vec{Q}, t) & =\left\langle\hat{\rho}_{\kappa}(\vec{Q}) \frac{\partial}{\partial t} \hat{\rho}_{\kappa^{\prime}}(-\vec{Q}, t)\right\rangle \\
& =-\frac{i}{\hbar}\left\langle\hat{\rho}_{\kappa}(\vec{Q})\left[\hat{\rho}_{\kappa^{\prime}}(-\vec{Q}), \hat{H}\right]\right\rangle,
\end{aligned}
$$

où, pour arriver à la dernière ligne, nous avons appliqué la formule usuelle qui exprime la dérivée d'un opérateur de Heisenberg par l'intermédiaire de son commutateur avec l'Hamiltonien.

Pour calculer ce moment, il est néanmoins préférable de passer par la fonction de réponse noncausale

$$
K_{\hat{\rho}_{\kappa}(-\vec{Q}) \hat{\rho}_{\kappa^{\prime}}(\vec{Q})}(t)=\frac{i}{\hbar}\left\langle\left[\hat{\rho}_{\kappa}(-\vec{Q}, t), \hat{\rho}_{\kappa^{\prime}}(\vec{Q})\right]\right\rangle
$$

que nous venons d'introduire dans la section 8.11, et non pas par la fonction intermédiaire de diffusion. En inversant la transformation de Fourier, nous obtenons du théorème de fluctuation-dissipation (8.136) une expression pour $K_{\kappa, \kappa^{\prime}}(\vec{Q}, t)$ en terme de la fonction de diffusion

$$
-i \int_{-\infty}^{\infty} d \omega \exp (i \omega t)(1+n(\omega))^{-1} S_{\kappa, \kappa^{\prime}}(\vec{Q}, \omega)=K_{\kappa, \kappa^{\prime}}(\vec{Q}, t) .
$$

Alors

$$
\int_{-\infty}^{\infty} d \omega \omega(1+n(\omega))^{-1} S_{\kappa, \kappa^{\prime}}(\vec{Q}, \omega)=\left.\frac{\partial}{\partial t} K_{\kappa, \kappa^{\prime}}(\vec{Q}, t)\right|_{t=0} .
$$

Avec la balance détaillée (8.58), nous pouvons démontrer que

$$
\int_{-\infty}^{\infty} d \omega \omega(1+n(\omega))^{-1} S_{\kappa, \kappa^{\prime}}(\vec{Q}, \omega)=2 \int_{-\infty}^{\infty} d \omega \omega S_{\kappa, \kappa^{\prime}}(\vec{Q}, \omega)
$$

à condition que $S_{\kappa, \kappa^{\prime}}(\vec{Q}, \omega)=S_{\kappa, \kappa^{\prime}}(-\vec{Q}, \omega)$. Nous obtenons donc une relation directe entre la dérivée de la fonction de réponse et le premier moment de la fonction de diffusion

$$
\int_{-\infty}^{\infty} d \omega \omega S_{\kappa, \kappa^{\prime}}(\vec{Q}, \omega)=\left.\frac{1}{2} \frac{\partial}{\partial t} K_{\kappa, \kappa^{\prime}}(\vec{Q}, t)\right|_{t=0}
$$

Calculons la dérivée par rapport au temps avec l'aide du commutateur avec l'Hamiltonien

$$
\begin{aligned}
\frac{\partial}{\partial t} K_{\kappa, \kappa^{\prime}}(\vec{Q}, t) & =\frac{i}{\hbar}\left\langle\left[\frac{\partial}{\partial t} \hat{\rho}_{\kappa^{\prime}}(-\vec{Q}, t), \hat{\rho}_{\kappa}(\vec{Q})\right]\right\rangle \\
& =\frac{1}{\hbar^{2}}\left\langle\left[\left[\hat{\rho}_{\kappa^{\prime}}(-\vec{Q}), \hat{H}\right], \hat{\rho}_{\kappa}(\vec{Q})\right]\right\rangle .
\end{aligned}
$$

Dans beaucoup de cas nous pouvons supposer que l'interaction entre les atomes est indépendante des moments. Dans ce cas, l'Hamiltonien possède la forme

$$
\hat{H}=\sum_{\kappa} \sum_{j \in\left\{j_{\kappa}\right\}} \frac{\left(\vec{P}_{j}\right)^{2}}{2 m_{\kappa}}+V\left(\left\{\vec{R}_{j}\right\}\right)
$$

Le commutateur de l'operateur de densité avec l'Hamiltonien est défini comme

$$
\left[\hat{\rho}_{\kappa^{\prime}}(-\vec{Q}), \hat{H}\right]=\sum_{\kappa} \sum_{j^{\prime} \in\left\{j_{\kappa}\right\}}\left[\exp \left(i \vec{Q} \cdot \vec{R}_{j^{\prime}}\right), \hat{H}\right] .
$$


Dans cette expression, tous les opérateurs sont des opérateurs de Schrödinger. Par conséquent

$$
\left[\exp \left(i \vec{Q} \cdot \vec{R}_{j^{\prime}}\right), V\left(\left\{\vec{R}_{j}\right\}\right)\right]=0 \quad \forall \vec{R}_{j}
$$

et donc

$$
\begin{aligned}
{\left[\hat{\rho}_{\kappa^{\prime}}(-\vec{Q}), \hat{H}\right] } & =\sum_{\kappa} \sum_{j \in\left\{j_{\kappa}\right\}, j^{\prime} \in\left\{j_{\kappa^{\prime}}\right\}} \frac{1}{2 m_{\kappa}}\left[\exp \left(i \vec{Q} \cdot \vec{R}_{j^{\prime}}\right),\left(\vec{P}_{j}\right)^{2}\right] \\
& =\sum_{j \in\left\{j_{\kappa^{\prime}}\right\}} \frac{1}{2 m_{\kappa^{\prime}}}\left[\exp \left(i \vec{Q} \cdot \vec{R}_{j}\right),\left(\vec{P}_{j}\right)^{2}\right] \\
& =\sum_{j \in\left\{j_{\kappa^{\prime}}\right\}} \frac{1}{2 m_{\kappa^{\prime}}}\left\{\left[\exp \left(i \vec{Q} \cdot \vec{R}_{j}\right), \vec{P}_{j}\right] \vec{P}_{j}+\vec{P}_{j}\left[\exp \left(i \vec{Q} \cdot \vec{R}_{j}\right), \vec{P}_{j}\right]\right\},
\end{aligned}
$$

ou nous avons employé l'identité

$$
\left[A, B^{2}\right]=[A, B] B-B[B, A]=[A, B] B+B[A, B] .
$$

Sachant que le commutateur d'une fonction $F\left(\vec{R}_{j}\right)$ avec $\vec{P}_{j}$ donne le gradient de cette fonction selon

$$
\left[f\left(\vec{R}_{j}\right), \vec{P}_{j}\right]=i \hbar \vec{\nabla}_{\vec{R}_{j}} f\left(\vec{R}_{j}\right)
$$

le commutateur se calcule comme

$$
\begin{aligned}
{\left[\hat{\rho}_{\kappa^{\prime}}(-\vec{Q}), \hat{H}\right] } & =\sum_{j \in\left\{j_{\kappa^{\prime}}\right\}} \frac{1}{2 m_{\kappa^{\prime}}}\left\{-\hbar \vec{Q} \cdot \exp \left(i \vec{Q} \cdot \vec{R}_{j}\right) \vec{P}_{j}-\hbar \vec{Q} \cdot \vec{P}_{j} \exp \left(i \vec{Q} \cdot \vec{R}_{j}\right)\right\} \\
& =-\sum_{j \in\left\{j_{\kappa^{\prime}}\right\}} \frac{1}{2 m_{\kappa^{\prime}}} \exp \left(i \vec{Q} \cdot \vec{R}_{j}\right)\left(2 \hbar \vec{Q} \cdot \vec{P}_{j}+\hbar^{2} Q^{2}\right) .
\end{aligned}
$$

En remplaçant (8.165) dans (8.153) nous obtenons

$$
\begin{aligned}
{\left[\left[\hat{\rho}_{\kappa^{\prime}}(-\vec{Q}), \hat{H}\right], \hat{\rho}_{\kappa}(\vec{Q})\right] } & =-\left[\hat{\rho}_{\kappa}(\vec{Q}),\left[\hat{\rho}_{\kappa^{\prime}}(-\vec{Q}), \hat{H}\right]\right] \\
& =\sum_{j \in\left\{j_{k}\right\}} \sum_{j^{\prime} \in\left\{j_{\kappa^{\prime}}\right\}} \frac{1}{2 m_{\kappa^{\prime}}} \exp \left(i \vec{Q} \cdot \vec{R}_{j^{\prime}}\right)\left(2 \hbar \vec{Q} \cdot\left[\exp \left(-i \vec{Q} \cdot \vec{R}_{j}\right), \vec{P}_{j^{\prime}}\right]\right) \\
& =\sum_{j \in\left\{j_{\kappa}\right\}} \sum_{j^{\prime} \in\left\{j_{\kappa^{\prime}}\right\}} \frac{\hbar^{2} Q^{2}}{m_{\kappa^{\prime}}} \delta_{j, j^{\prime}} \exp \left(i \vec{Q} \cdot \vec{R}_{j^{\prime}}\right) \exp \left(-i \vec{Q} \cdot \vec{R}_{j}\right) \\
& =\sum_{j \in\left\{j_{\kappa}\right\}} \delta_{\kappa, \kappa^{\prime}} \frac{1}{m_{\kappa^{\prime}}} \hbar^{2} Q^{2}=\delta_{\kappa, \kappa^{\prime}} N_{\kappa} \frac{\hbar^{2} Q^{2}}{m_{\kappa}},
\end{aligned}
$$

où nous avons appliqué l'identité (8.164) une deuxième fois. Nous sommes finalement en mesure d'exprimer le premier moment. Selon (8.157)

$$
\int_{-\infty}^{\infty} d \hbar \omega \hbar \omega S_{\kappa, \kappa^{\prime}}(\vec{Q}, \omega)=\left.\frac{\hbar^{2}}{2} \frac{\partial}{\partial t} K_{\kappa, \kappa^{\prime}}(\vec{Q}, t)\right|_{t=0}=\delta_{\kappa, \kappa^{\prime}} N_{\kappa} \frac{\hbar^{2} Q^{2}}{2 m_{\kappa}}=\delta_{\kappa, \kappa^{\prime}} N_{\kappa} E_{\kappa}^{r}(Q)
$$

avec

$$
E_{\kappa}^{r}(Q):=\frac{\hbar^{2} Q^{2}}{2 m_{\kappa}}
$$


l'énergie de recul des atomes de type $\kappa$. C'est l'énergie qui est échangée en moyenne lors d'une collision si cette collision correspond à un changement d'impulsion égal à $\hbar \vec{Q}$. Il faut souligner que ce résultat est indépendant des interactions, à la condition -assez peu restrictive- que celles-ci ne dépendent pas de $\vec{P}_{j}$. On obtiendrait le même résultat pour des particules sans interaction, c'est-à-dire pour le cas d'un gaz parfait. Il n'y a donc pas d'effet de cohérence dans le moment d'ordre un. L'expression (8.167) est connue sous le nom de règle de somme $f$ (f-sum rule). Elle est particulièrement importante pour la physique des liquides. Elle permet par exemple de conclure que la dispersion des excitations dans le ${ }^{4}$ He doit avoir un minimum, le roton, là où le facteur de structure possède un maximum [27].

Le moment d'ordre un est strictement nul pour des fonctions symétriques. Il constitue ainsi une mesure de l'asymétrie de la fonction de diffusion. D'après (8.167) cette asymétrie est indépendante de la température. En particulier, le moment peut toujours être évalué à $T=0$. Dans ce cas $S(\vec{Q}, \omega)=0$ pour $\omega<0 . S(\vec{Q}, \omega)=0$ à $T=0$ constitue le point d'ancrage à partir duquel le signal à température plus élevée se construit de manière symétrique. Avec le théorème de fluctuation-dissipation (8.144) nous obtenons une règle de somme pour la susceptibilité

$$
\int_{0}^{\infty} d \hbar \omega \hbar \omega S_{\kappa, \kappa^{\prime}}(\vec{Q}, \omega ; T=0)=\frac{1}{\pi} \int_{0}^{\infty} d \hbar \omega \hbar \omega \chi_{\vec{Q}}{ }^{\prime \prime}[\omega ; T=0]=\delta_{\kappa, \kappa^{\prime}} N_{\kappa} E_{\kappa}^{r} .
$$

Les expressions pour les moments supérieurs peuvent être trouvées dans [20,26,28]. On doit néanmoins faire très attention à distinguer les moments pour les fonctions de diffusion classiques de ceux que l'on obtient pour les expressions quantiques. Comme nous l'avons déjà mentionné, le $S^{\mathrm{cl}}(\vec{Q}, \omega)$ classique est en général une fonction paire de $\omega$. Par conséquent tous les moments $S_{\mathrm{cl}}^{n}(\vec{Q}, \omega)$ pour $n$ impair sont nécessairement nuls. Le moment classique qui s'approche le plus du "f-sum rule" est le moment d'ordre deux. Dans l'hypothèse où les vitesses des différents atomes ne sont pas corrélées, ce qui est une bonne approximation pour les liquides, il se calcule comme [26]

$$
\int_{-\infty}^{\infty} d \omega \omega^{2} S^{\mathrm{cl}}(\vec{Q}, \omega)=N \frac{Q^{2} k_{\mathrm{B}} T}{m}
$$

Pierre Gilles de Gennes a employé les règles de somme pour déduire le ralentissement de la relaxation structurale dans les liquides pour des valeurs de $Q$ qui correspondent aux maxima du facteur de structure (De Gennes narrowing) [26] (voir figure 30).

\subsection{Résumé partiel}

Nous allons de nouveau succinctement résumer les principales fonctions introduites dans cette section, ainsi que les relations entre elles.

La diffusion nucléaire donne accès à la fonction d'autocorrélation de la densité atomique (8.18)

$$
G_{\kappa, \kappa^{\prime}}(\vec{r}, t)=\int d^{3} \vec{r}^{\prime}\left\langle\hat{\rho}_{\kappa}\left(\vec{r}^{\prime}-\vec{r}\right) \hat{\rho}_{\kappa^{\prime}}\left(\vec{r}^{\prime}, t\right)\right\rangle
$$

Elle est reliée à la fonction de diffusion intermédiaire (8.37)

$$
I_{\kappa, \kappa^{\prime}}(\vec{Q}, t)=\left\langle\hat{\rho}_{\kappa}(\vec{Q}, t=0) \hat{\rho}_{\kappa^{\prime}}(-\vec{Q}, t)\right\rangle
$$

par une transformation de Fourier dans l'espace

$$
I_{\kappa, \kappa^{\prime}}(\vec{Q}, t)=\int_{V} d^{3} r e^{-i \vec{Q} \cdot \vec{r}} G_{\kappa, \kappa^{\prime}}(\vec{r}, t) .
$$


La fonction de diffusion s'obtient à partir de la fonction de diffusion intermédiaire par le biais d'une transformation de Fourier dans le temps (8.38)

$$
S_{\kappa, \kappa^{\prime}}(\vec{Q}, \omega)=\frac{1}{2 \pi \hbar} \int_{-\infty}^{\infty} d t I_{\kappa, \kappa^{\prime}}(\vec{Q}, t) e^{-i \omega t} .
$$

La fonction de diffusion est donc la double transformée de Fourier dans l'espace et dans le temps de la fonction d'autocorrélation de la densité (8.26)

$$
S_{\kappa, \kappa^{\prime}}(\vec{Q}, \omega)=\frac{1}{2 \pi \hbar} \int_{V} d^{3} r \int_{-\infty}^{\infty} d t e^{i(\vec{Q} \cdot \vec{r}-\omega t)} G_{\kappa, \kappa^{\prime}}(\vec{r}, t) .
$$

Cette relation nous permet d'exprimer les sections efficaces différentielles partielles cohérentes en terme de fonctions d'autocorrélation de la densité (8.31)

$$
\frac{d^{2} \sigma}{d \Omega d E_{f}}=\frac{k_{f}}{k_{i}} \frac{1}{2 \pi \hbar} \sum_{\kappa, \kappa^{\prime}}\left[\left(V_{\kappa}(\vec{Q}) V_{\kappa^{\prime}}^{*}(\vec{Q})\right) \int_{V} d^{3} r \int_{-\infty}^{\infty} d t e^{i(\vec{Q} \cdot \vec{r}-\omega t)} G_{\kappa, \kappa^{\prime}}(\vec{r}, t)\right] .
$$

Inversement la fonction d'autocorrélation de la densité est la double transformée de Fourier dans l'espace et dans le temps de la fonction de diffusion (8.27)

$$
G_{\kappa, \kappa^{\prime}}(\vec{r}, t)=\frac{\hbar}{(2 \pi)^{3}} \int_{V_{Q}} d^{3} Q \int_{-\infty}^{\infty} d t e^{-i(\vec{Q} \cdot \vec{r}-\omega t)} S_{\kappa, \kappa^{\prime}}(\vec{Q}, \omega) .
$$

Quand on a affaire à la diffusion incohérente les fonctions de corrélation sont à remplacer par les fonctions de corrélation propres ou self.

Les propriétés analytiques de la fonction de diffusion imposent la balance détaillée (8.57)

$$
S_{\kappa, \kappa^{\prime}}(\vec{Q}, \omega)=e^{\hbar \omega \beta} S_{\kappa^{\prime}, \kappa}(-\vec{Q},-\omega) .
$$

Les fonctions de corrélation sont étroitement liées aux fonctions de réponse. En mécanique statistique les fonctions de réponse décrivent la réaction d'un système à une perturbation dans le régime linéaire. Nous définissons une fonction de réponse généralisée

$$
K_{\hat{\rho}_{\kappa^{\prime}}^{+}, \hat{\rho}_{\kappa}}(t)=\frac{i}{\hbar}\left\langle\left[\hat{\rho}_{\kappa^{\prime}}(-\vec{Q}, t), \hat{\rho}_{\kappa}(\vec{Q}, t=0)\right]\right\rangle .
$$

Cette fonction est liée à la fonction de diffusion par (8.136)

$$
S_{\kappa, \kappa^{\prime}}(\vec{Q}, \omega)=(1+n(\omega)) \frac{i}{2 \pi} \int_{-\infty}^{\infty} d t \exp (-i \omega t) K_{\hat{\rho}_{\kappa^{\prime}}^{+}, \hat{\rho}_{\kappa}}(t)
$$

Nous rendons la fonction de réponse causale en imposant que la réaction se limite à des temps positifs (8.129)

$$
\Phi_{\hat{\rho}_{\kappa^{\prime}}^{+}, \hat{\rho}_{\kappa}}(t)=\frac{i}{\hbar}\left\langle\left[\hat{\rho}_{\kappa^{\prime}}(-\vec{Q}, t), \hat{\rho}_{\kappa}(\vec{Q}, t=0)\right]\right\rangle \Theta(t) .
$$

Le passage au régime des fréquences s'effectue par l'intermédiaire d'une transformation de Laplace (8.138)

$$
\chi_{\hat{\rho}_{\kappa^{\prime}}^{+}, \hat{\rho}_{\kappa}}[\omega]=\lim _{\epsilon \rightarrow 0^{+}} \chi_{\hat{\rho}_{\kappa^{\prime}}^{+}, \hat{\rho}_{\kappa}}[i p=\omega+i \epsilon]=\lim _{\epsilon \rightarrow 0^{+}} \int_{0}^{\infty} d t \Phi_{\hat{\rho}_{k^{\prime}}^{+}, \hat{\rho}_{\kappa}}(t) \exp (p t) .
$$

La susceptibilité généralisée $\chi_{\hat{\rho}_{\kappa^{\prime}}^{+}, \hat{\rho}_{\kappa}}[\omega]$ est connectée à la fonction de diffusion par le théorème de fluctuation-dissipation (8.144)

$$
S_{\kappa, \kappa^{\prime}}(\vec{Q}, \omega)=(1+n(\omega)) \frac{1}{\pi} \chi_{\hat{\rho}_{\kappa^{\prime}}^{+}, \hat{\rho}_{\kappa}}^{\prime \prime}[\omega] .
$$




\section{LE FAISCEAU DE PARTICULES}

Jusqu'ici nous avons traité le faisceau de neutrons comme un flux de probabilité décrit par une onde stationnaire. Ce choix était motivé par la facilité avec laquelle nous pouvons manipuler les ondes stationnaires et aussi par la nécessité de décrire des phénomènes d'interférence, qui sont à la base de la diffusion des particules. Le flux stationnaire est très loin de la réalité expérimentale d'un faisceau de particules de basse intensité. Les neutrons arrivent plutôt individuelement et au "compte-gouttes" sur l'échantillon. Le formalisme de la description ondulatoire deviennent inutilement encombrant pour décrire des situations où les interférences ne jouent aucun rôle. Ce problème est récurrent en l'optique où l'on jongle en permanence entre la description corpusculaire et la description ondulatoire [17]. La théorie des rayons lumineux est une approximation de la théorie ondulatoire de la lumière. Comme en optique, le paramètre pilotant le choix de la théorie est en général le rapport entre la longueur d'onde de la sonde et la distance entre les diffuseurs. Pour la diffusion des neutrons lents par la matière condensée, ce rapport est proche de un, ce qui nécessite de travailler avec des ondes pour décrire la diffusion. Nous allons voir dans la suite que même cet énoncé doit être nuancé pour des échantillons macroscopiques. Par contre, le passage d'un neutron par une fente peut être traité en considérant des simples rayons de neutrons.

Dans cette section nous développerons tout d'abord le formalisme permettant de donner une description du faisceau de particules. Nous continuons à décrire les faisceaux de manière statistique, c'est-à-dire en termes de distributions de probabilité ${ }^{47}$. Cette description est imposée par la mécanique quantique (la détection restera par exemple toujours un évènement quantique) mais aussi par le caractère aléatoire de la production et du transport des neutrons. Les distributions sont aussi la base des logiciels de simulations [30]. Elles nous permettront de donner une signification précise aux concepts de valeurs nominales, monochromaticité et divergence du faisceau.

\subsection{Description statistique du faisceau}

Nous avons vu dans le chapitre I, qu'une particule localisée dans l'espace doit être décrite par un paquet d'onde. Ce paquet peut être construit en superposant des ondes planes ${ }^{48}$,

$$
\psi(x, t)=\frac{1}{\sqrt{2 \pi}} \int w(k) e^{i(k x-\omega(k) t)} d k .
$$

Si nous prenons comme distribution des vecteurs d'onde $w(k)$, une fonction gaussienne de largeur $\Delta k$, alors nous obtenons pour $t=0$ une largeur du paquet d'onde dans l'espace réel qui est inversement proportionnelle à la largeur $\Delta k$

$$
(\Delta x)_{0}=\frac{1}{2} \frac{1}{(\Delta k)_{0}} .
$$

Toute description corpusculaire du faisceau doit rendre compte des incertitudes quantiques dans la position et dans l'impulsion d'une particule. Dans cet esprit $\vec{r}$ et $\vec{P}(=\hbar \vec{k})$ dénoteront dans la suite les valeurs moyennes de la position et de l'impulsion d'un paquet d'onde.

Les paquets d'onde sont accessibles à l'expérience. Les interféromètres à neutrons permettent de superposer la fonction d'onde d'un neutron à elle-même par division d'amplitude [10], de façon similaire à ce que fait un interféromètre de Michelson ou de Mach-Zehnder pour la lumière. Lors de la diffraction de Bragg sur un cristal parfait la fonction d'onde devient la somme de deux parties, l'une réfléchie et l'autre transmise. En les superposant correctement on peut déterminer la forme du paquet d'onde, sorte

47 Pour toute question relative aux notions de la statistique le lecteur est prié de se référer au livre de Konstantin Protassov [29].

48 Dans un souci de simplicité nous nous limitons à une dimension. 

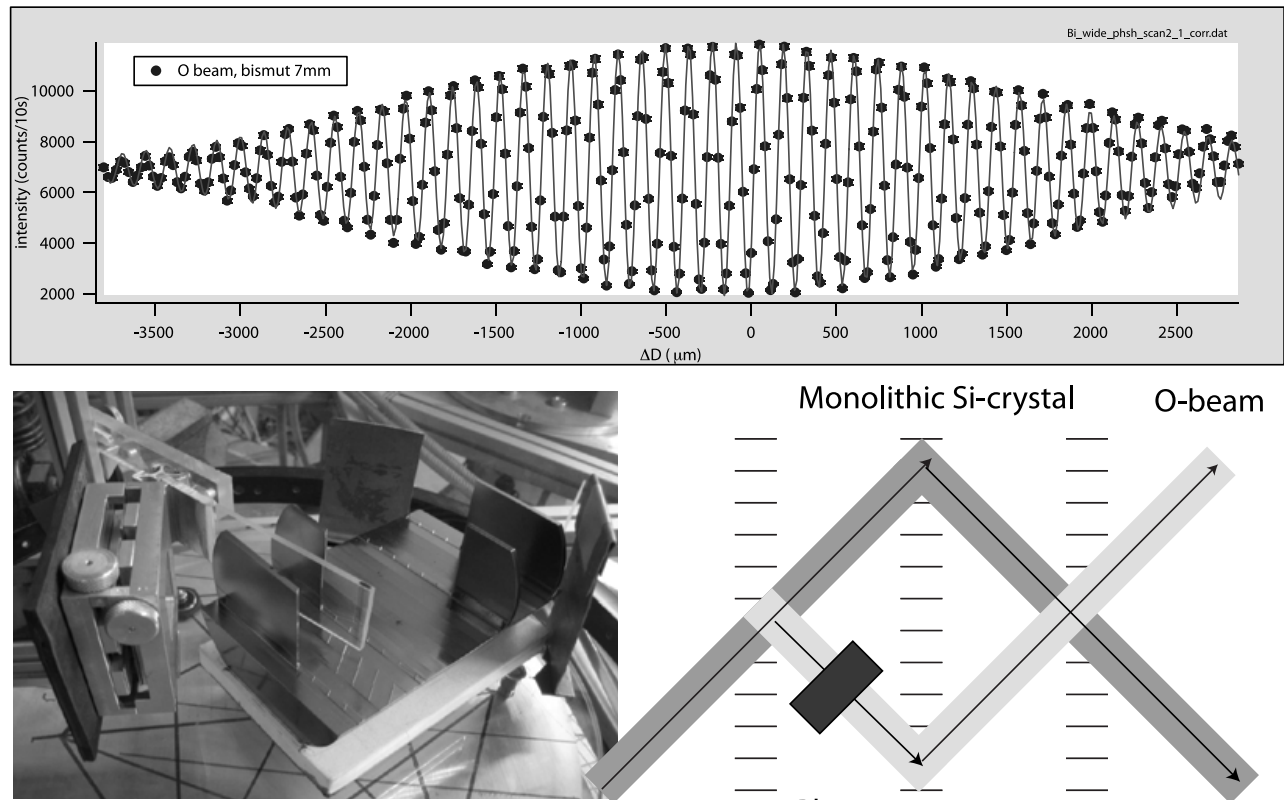

Monolithic Si-crystal

O-beam

\section{Incident}

beam

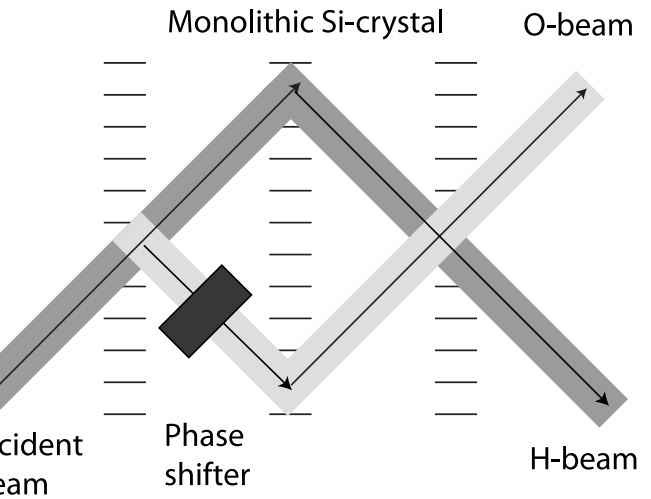

Figure 31. Figure d'interférence d'un faisceau de neutrons diffracté par un cristal de silicium pur. Pour que les plans cristallins soient parfaitement alignés les uns par rapport aux autres, l'interféromètre est coupé dans un seul bloc de silicium parfait. En introduisant de la matière dans un bras de l'interféromètre nous changeons les phases relatives et ainsi la superposition des fonctions d'onde. C'est cette différence de "chemin optique" qui donne lieu aux interférences. Comme en optique (voir section 7), une oscillation équivaut à un changement du chemin optique pour une longueur d'onde, qui est ici proche de $2 \AA$. Dans ce cas précis, on obtient une extension du paquet d'onde de plus ou moins $100 \AA$ A. Il s'agit de la longueur de cohérence latérale du faisceau (figures par H. Rauch).

de "miroir" du faisceau qui permet d'en observer toutes les imperfections. La figure 31 en montre un exemple.

Les neutrons extraits du modérateur peuvent être considerés comme un gaz en expansion ${ }^{49}$. Comme les neutrons n'interagissent ni entre eux, ni avec un bain thermique, ce gaz est hors équilibre. D'un point de vue classique, l'état du gaz est caractérisé par la distribution

$$
p(\vec{k} ;(\vec{r}, t))
$$

qui donne la probabilité de détecter un neutron avec un vecteur de propagation $\hat{k}=\vec{k} /|\vec{k}|$ et avec une énergie $E=\hbar^{2} k^{2} / 2 m$ à l'instant $t$ à l'endroit $\vec{r}$. Cette distribution est aussi appelée densité de l'espace des phases des neutrons. En mode stationnaire le faisceau ne dépend pas explicitement du temps, et nous pouvons ignorer la variable $t$. Ce n'est naturellement plus le cas quand le faisceau est pulsé.

La distribution de probabilité doit être normalisée de telle manière que l'intégrale

$$
N(t)=\int_{K} \int_{R} p(\vec{k} ;(\vec{r}, t)) d^{3} k d^{3} r
$$

\footnotetext{
49 Cette description est basée sur la nature incohérente du faisceau de neutrons (attention, le mot incohérent ici n'a pas la même signification que quand il est utilisé dans le contexte de la diffusion neutronique). Elle exclue la description des phénomènes d'interférence entre les faisceaux de neutrons, qu'on peut observer en séparant un faisceau en parties cohérentes à l'aide de cristaux parfaits [10].
} 
donne le nombre de neutrons que l'on pourra détecter à un moment donné dans l'élément d'espace réel $R=\Delta r_{x} \Delta r_{y} \Delta r_{z}$ autour de $\vec{r}$, et qui possèdent des vecteurs d'onde à l'intérieur de l'élément $K=\Delta k_{x} \Delta k_{y} \Delta k_{z}$ autour de $\vec{k}$. L'espace des vecteurs d'onde est également appelé espace $K$. Le principe d'incertitude de Heisenberg impose que $\Delta r_{\alpha} \cdot \Delta k_{\alpha}>1$ pour $\alpha=x, y, z$. Les volumes $R$ et $K$ ne peuvent donc pas être arbitrairement petits sinon $p(\vec{k} ;(\vec{r}, t))$ ne sera plus définie. Dans la pratique il est rare que le vecteur d'onde d'un neutron soit mieux défini qu'à $10^{-4} \AA$ près ${ }^{50}$. De ce fait nous ne courons aucun risque de définition pourvu que nous nous limitions à regarder le faisceau sur une échelle dépassant le $\mu \mathrm{m}$. En-dessous de cette valeur $p(\vec{k} ;(\vec{r}, t))$ ne varie significativement.

La densité de neutrons s'obtient à partir de la densité de l'espace des phases en intégrant sur le vecteur d'onde

$$
\rho_{n}(\vec{r}, t)=\int d^{3} k p(\vec{k} ;(\vec{r}, t))
$$

Le courant de particules traversant une surface $S$ est déterminé par l'intégrale de surface

$$
I(t)=\int\left[\int_{S} p(\vec{k} ;(\vec{r}, t))\left(\vec{v}_{k} \cdot d \vec{a}\right)\right] d^{3} k=\frac{\hbar}{m} \int\left[\int_{S} p(\vec{k} ;(\vec{r}, t))(\vec{k} \cdot d \vec{a})\right] d^{3} k,
$$

avec $d \vec{a}$ le vecteur de surface infinitésimal perpendiculaire à $S$.

Dans le cas d'une surface rectangulaire $A$ perpendiculaire à $\hat{z}$, l'expression $(9.5)$ se réduit à

$$
I(t)=\frac{\hbar}{m} \int_{A} d x d y \int p(\vec{k} ;(x, y, t)) k_{z} d^{3} k .
$$

Si les vecteurs d'onde, c'est-à-dire les vitesses, sont distribuées de manière homogène sur la section $A$ nous obtenons une simplification supplémentaire :

$$
I(t)=A \frac{\hbar}{m} \int p(\vec{k} ; t) k_{z} d^{3} k=A \cdot \rho_{n} \cdot \bar{v}_{z} .
$$

Dans l'expression finale nous avons introduit la vitesse moyenne du faisceau dans la direction $\hat{z}$. En divisant cette expression par l'aire $A$ de la surface nous obtenons la densité de courant. Dans un système isotrope, comme par exemple à l'interieur du modérateur, la densité de courant est égale à zéro. Il y a autant de particules qui traversent la surface dans un sens que dans l'autre. Dans ce cas il est utile d'introduire la notion de $f l u x \Phi(t)$

$$
\Phi(t)=\frac{\hbar}{m} \int p(\vec{k} ; t)\left|k_{z}\right| d^{3} k=\rho_{n} \cdot \overline{\left|v_{z}\right|},
$$

$\Phi(t)$ est égal au nombre de particules traversant une surface unitaire dans la direction $\hat{z}$ par seconde.

Un faisceau bien défini possède des distributions de probabilité étroites. Un faisceau monochromatique ne contient par exemple que des neutrons d'une seule énergie ou d'une seule longueur d'onde. Un tel faisceau aurait une intensité très faible. Il est donc souhaitable que la monochromaticité ne soit pas parfaite et que les énergies des neutrons soient distribuées autour d'une valeur nominale ${ }^{51}$

$$
E_{0}(t)=\frac{1}{N} \int_{K} \int_{R} p(\vec{k} ;(\vec{r}, t)) \frac{\hbar^{2} k^{2}}{2 m} d^{3} k d^{3} r,
$$

\footnotetext{
${ }^{50}$ C'est la valeur que nous obtenons sur un spectromètre de rétro-diffusion (voir [4]) qui est optimisé pour produire un faisceau hautement monochromatique.

51 Nous allons indiquer les valeurs nominales par l'indice zéro et les variations (largeur totale de la distribution à mi-hauteur FWHM) par le préfixe $\Delta$.
} 
avec la variation

$$
\Delta E(t)=\sqrt{\frac{1}{N} \int_{K} \int_{R} p(\vec{k} ;(\vec{r}, t))\left(\frac{\hbar^{2} k^{2}}{2 m}-E_{0}\right)^{2} d^{3} k d^{3} r}
$$

$\Delta E$ est la largeur absolue en énergie du faisceau. La largeur relative est donnée par

$$
\frac{\Delta E}{E_{0}}=2 \frac{\Delta \lambda}{\lambda_{0}}
$$

Les largeurs évoluent en général en fonction du lieu et du temps.

D'une manière analogue il est possible d'introduire la direction du vecteur d'onde nominal $\hat{k}_{0}(\vec{r}, t)$. Les déviations angulaires par rapport à cette direction $\left(\Delta \hat{k}_{x}(\vec{r}, t), \Delta \hat{k}_{y}(\vec{r}, t), \Delta \hat{k}_{z}(\vec{r}, t)\right)$ sont appelées divergences du faisceau.

Pour déterminer la distribution $p(\vec{k} ;(\vec{r}, t))$ nous devons déterminer le nombre de neutrons dans $R$ autour de $\vec{r}$, qui possèdent des vecteurs d'onde à l'intérieur de $K$ autour de $\vec{k}$. Ce nombre va fluctuer autour de la valeur moyenne que nous voulons déterminer. En répétant les mesures nous allons réduire les incertitudes. La probabilité de la détection par unité de temps étant constante pour des particules sans interaction, la distribution statistique correspondant à la détection est une distribution de Poisson (voir chapitre I et [29]). L'erreur de mesure sera donc donnée par la racine du nombre d'évènements détectés. En doublant le temps de mesure, on diminuera l'erreur d'un facteur $\sqrt{2}$.

\section{2 Éléments optiques et fonction de transfert}

Pour faciliter la description du transport des neutrons, nous décomposons le trajet en éléments optiques. Cette approche est facilitée quand le passage des neutrons d'un élément à un autre est uni-directionel. Un exemple typique est celui d'un guide de neutrons. Les neutrons entrent par la face en amont du guide pour sortir par la face en aval; toute sortie est considérée définitive. L'emploi de ce concept est même indispensable pour décrire le fonctionnement de tout dispositif optique en théorie ondulatoire. C'est par exemple le cas pour un cristal monochromateur.

Tout élément optique, qui se trouve sur le chemin des neutrons, change la distribution $p(\vec{k} ; \vec{r}, t)$. Nous dénommons les interfaces à l'entrée et à la sortie d'un élément optique par $S_{i}$ et $S_{f}$, de surface respectives $A_{i}$ et $A_{f}$. Les distributions correspondantes sont données par

$$
p\left(\vec{k} ; \vec{r}_{i}, t_{i}\right), \quad \vec{r} \in S_{i}
$$

et

$$
p\left(\vec{k} ; \vec{r}_{f}, t_{f}\right), \quad \vec{r}_{f} \in S_{f} .
$$

Un élément optique est pleinement caractérisé si nous connaissons la fonction de transfert $T_{i f}\left(\vec{k}_{f}, \vec{k}_{i} ; \vec{r}_{f}, t_{f}, \vec{r}_{i}, t_{i}\right)$, qui permet de calculer la probabilité avec laquelle un courant incident donné produit un neutron $\vec{k}_{f}$ à la sortie. Mathématiquement la fonction de transfert est définie par

$$
p\left(k_{f} ; \vec{r}_{f}, t_{f}\right) d^{3} k_{f}=\iiint_{S_{i}}\left[\frac{\hbar}{m}\left(\vec{k}_{i} \cdot d \vec{a}\right) p\left(\vec{k}_{i} ; \vec{r}_{i}, t_{i}\right)\right] T_{i f}\left(\vec{k}_{f}, \vec{k}_{i} ; \vec{r}_{f}, t_{f}, \vec{r}_{i}, t_{i}\right) d^{3} k_{i} d t_{i} .
$$

En mode stationnaire nous pouvons nous affranchir de la dépendance en $t$. De plus, pour un faisceau incident homogène, monochromatique et sans divergence, on peut simplifier considérablement l'expression et utiliser l'expression du courant (9.7) donné par

$$
\frac{\hbar}{m} \int_{S_{i}}\left(\vec{k}_{i} \cdot d \vec{a}\right) p\left(\vec{k}_{i} ; \vec{r}_{i}, t_{i}\right)=A \frac{\hbar}{m} k_{z} \rho_{n} \delta\left(\vec{k}_{i}-\vec{k}_{i}^{0}\right) .
$$


pour en déduire que

$$
\begin{aligned}
p\left(k_{f} ; \vec{r}_{f}, t_{f}\right) d^{3} k_{f} & =\rho_{n} A_{i} \frac{\hbar}{m} \int d^{3} k_{i} T_{i f}\left(\vec{k}_{f}, \vec{k}_{i} ; \vec{r}_{f}\right) k_{z} \delta\left(\vec{k}_{i}-\vec{k}_{i}^{0}\right) \\
& =\rho_{n} A_{i} \frac{\hbar}{m} k_{z}^{0} T_{i f}\left(\vec{k}_{f}, \vec{k}_{i}^{0}\right) \\
& =\rho_{n}\left(\vec{k}_{0}\right) A v_{z} T_{i f}\left(\vec{k}_{f}, \vec{k}_{i}^{0}\right) \\
& =I_{0} T\left(\vec{k}_{f}, \vec{k}_{i}^{0}\right) .
\end{aligned}
$$

Ceci confirme que la fonction de transfert $T\left(\vec{k}_{f}, \vec{k}_{i}^{0}\right)$ indique la manière dont l'élement optique redistribue le courant de neutrons. Pour décrire une ligne de neutrons constituée d'une suite d'éléments optiques nous injecterons la distribution obtenue à la sortie de l'élément $n$ comme source dans l'élément $n+1$. Cette méthode est utilisée pour simuler le fonctionnement des spectromètres [30].

\section{3 Échantillons de petite taille}

L'échantillon est à priori un élément optique comme les autres ${ }^{52}$. Sa particularité en termes de transport réside dans le fait que

- l'échantillon est en général suffisamment petit pour que le flux incident puisse être considéré comme homogène sur toute sa surface

- les détecteurs sont suffisamment éloignés de l'échantillon pour que la direction du vecteur d'onde final coïncide avec le vecteur de position du détecteur $\left(\hat{k}_{f}=\hat{r}_{f}\right)$.

Ceci nous permet de nous affranchir des variables $\vec{r}_{i}$ et $\vec{r}_{f}$. L'influence de l'échantillon sur le faisceau peut alors être décrit de manière plus simple par une fonction

$$
f\left(\vec{k}_{f}, \vec{k}_{i}\right) d^{3} k_{f},
$$

qui donne la probabilité avec laquelle un neutron $\vec{k}_{i}$ présent dans l'échantillon est diffusé par unité de temps dans l'élement $d^{3} k_{f}$ de l'espace réciproque $K$.

Dans le régime de l'approximation de Born, le flux de neutrons incidents n'est pas sensiblement attenué par la diffusion. En pratique ceci implique que notre échantillon doive être de petite taille par rapport aux dimensions du spectromètre. La densité de neutrons $\rho_{n}$ sera alors homogène sur tout le volume de l'échantillon. La section efficace est la probabilité par unité de temps et par unité de flux incident de diffuser un neutron $\vec{k}_{i}$ dans $d^{3} k_{f}$. Nous obtenons

$$
\begin{aligned}
d^{2} \sigma & =\frac{\rho_{n} V}{\Phi_{i}} f\left(\vec{k}_{f}, \vec{k}_{i}\right) d^{3} k_{f} \\
& =\frac{\rho_{n} V}{\rho_{n} v_{i}} f\left(\vec{k}_{f}, \vec{k}_{i}\right) \frac{m}{\hbar} k_{f} d \Omega d E_{f} \\
& =V \frac{m^{2}}{\hbar^{2}} \frac{k_{f}}{k_{i}} f\left(\vec{k}_{f}, \vec{k}_{i}\right) d \Omega d E_{f}
\end{aligned}
$$

où nous avons utilisé

$$
d^{3} k_{f}=k_{f}^{2} d k_{f} d \Omega=\frac{m k_{f}}{\hbar} d \Omega d E_{f}
$$

\footnotetext{
52 La diffusion de Bragg est aussi bien utilisée pour monochromatiser les faisceaux que pour étudier la structure des cristaux.
} 
et

$$
\Phi_{i}=\rho_{n} \frac{\hbar}{m}\left|\vec{k}_{i}\right|=\rho_{n} \frac{\hbar}{m} k_{i} .
$$

$\rho_{n}$ est la densité de neutrons instantanée et $V$ le volume de l'échantillon. Avec ${ }^{53}$

$$
\frac{d^{2} \sigma}{d \Omega d E_{f}}=N \frac{\sigma}{4 \pi} \frac{k_{f}}{k_{i}} S(\vec{Q}, \omega)
$$

nous obtenons l'identité

$$
f\left(\vec{k}_{f}, \vec{k}_{i}\right)=\frac{N}{V} \frac{\hbar^{2}}{m^{2}} \frac{\sigma}{4 \pi} S(\vec{Q}, \omega) .
$$

Nous retrouvons donc la fonction de diffusion $S(\vec{Q}, \omega)$. Nous constatons le résultat important que $f\left(\vec{k}_{f}, \vec{k}_{i}\right)$ ne dépend que de $\vec{Q}$ et $\omega$ dans l'approximation de Born.

\section{4 Échantillon de taille finie et diffusion multiple}

Le but d'une expérience de diffusion de neutrons est de déterminer la fonction de diffusion $S(\vec{Q}, \omega)$. C'est elle qui renseigne sur l'existence de corrélations dans l'échantillon (voir la section 8.2). Déterminer $S(\vec{Q}, \omega)$ revient à mesurer la distribution des neutrons $p(\vec{k} ; \vec{r}, t)$ après diffusion. La précision de la mesure dépendra (i) de la préparation du faisceau incident et en particulier de son flux ainsi que (ii) de l'intensité détectée et de la précision de détection. La discussion que nous avons menée jusqu'ici concernant la diffusion était basée sur l'approximation de Born. Elle était assujettie à deux conditions majeures: $(\alpha)$ le faisceau incident reste pratiquement inchangé et $(\beta)$ le faisceau diffusé n'est qu' une perturbation faible du faisceau ne devant pas donner lieu à des processus de diffusion secondaires. Se placer dans ces conditions impose donc d'utiliser des échantillons qui ne diffusent que faiblement. Cette contrainte est un dilemme pour l'expérimentateur. Le flux de neutrons est fixe, c'est-à-dire qu'il est déterminé par la source ainsi que par l'efficacité de l'extraction, du transport et du conditionnement du faisceau. Ce flux est souvent faible, même auprès des sources de neutrons les plus puissantes ${ }^{54}$. De plus, le signal recherché n'est souvent pas celui dont l'intensité domine le spectre expérimental. La seule variable à la disposition du chercheur afin améliorer la statistique des données sans détériorer pour autant la résolution est souvent le volume de l'échantillon. Malheureusement, comme nous allons voir, le fait d'agrandir le volume de l'échantillon et de sortir du cadre de l'approximation de Born produit des effets secondaires indésirables. Ces effets sont à mettre en balance avec le gain espéré en statistique.

Il est essentiel de comprendre ces effets secondaires pour éviter le risque de mal interpréter les données expérimentales. Travailler avec des ordres supérieurs de la série de Born quand on analyse les données est un processus très lourd. Nous allons décrire le processus de diffusion comme le parcours d'une particule supportant des collisions statistiques. Pour cela il faut diviser mentalement l'échantillon. Chaque partie doit être suffisamment petite pour justifier l'approximation de Born. La diffusion produite par élément donné est traitée comme une source supplémentaire de diffusion de tous les autres éléments. Il est évident que la diffusion d'une partie ne peut pas excéder la diffusion de l'ensemble.

Même si cette approche est beaucoup plus simple que de travailler avec une série de Born d'ordre supérieur elle n'est pas sans pièges. A première vue elle peut même sembler paradoxale : Nous avons souligné tout au long de ce chapitre que la diffusion est un processus d'interférence. L'interférence est justement le type de phénomène qui ne pourra pas être décrit par une théorie corpusculaire. Le neutron en tant qu' onde de matière engendre le long de son chemin des ondelettes sphériques qui possèdent entre elles des relations de phase bien déterminées. La superposition de ces ondelettes donne

\footnotetext{
53 Nous nous limitons ici à des échantillons monoatomiques.

54 On dit souvent que la diffusion des neutrons est une méthode expérimentale limitée par le flux. Ceci est de moins en moins vrai pour des spectromètres de dernière génération auprès de sources puissantes comme l'ILL.
} 
lieu à des effets d'interférence. C'est le principe de Huygens-Fresnel appliqué aux ondes de matière. A partir de ces interférences on déduit les corrélations dans l'espace et dans le temps entre diffuseurs. L'exemple canonique est la diffusion de Bragg d'un monocristal, qui nous permet de déterminer sa structure. Les plans d'atomes diffusent les neutrons d'une longueur d'onde donnée dans des directions spécifiques. Si le monocristal est très pur, ces directions sont très bien définies. Nous pouvons observer expérimentalement que la diffusion de Bragg dépend des dimensions macroscopiques d'un cristal pur comme le silicium. Les effets d'interférence s'étendent donc sur des distances de plusieurs millimètres (voir la figure 32). Dans ce cas nous sommes très loin de la validité de l'approximation de Born. Le neutron doit être décrit par la superposition d'ondes incidentes et d'ondes diffusées qui, à cause de la périodicité, prennent la forme d'ondes de Bloch $^{55}$ (voir chapitre I). C'est la base de la théorie de la diffusion dynamique que nous n'allons pas aborder dans ce chapitre. Il faut néanmoins souligner que ces phenomènes d'interférence sur de très grandes distances ont lieu pour des faisceaux dont les paquets d'ondes initiaux ont des extensions spatiales beaucoup plus faibles. Il faudra donc résister à la tentation de considérer cette extension comme un bon paramètre pour délimiter une zone d'interaction. C'est le cristal parfait qui sélectionne une bande étroite de vecteurs d'onde dans le paquet d'onde de la particule sonde permettant ainsi l'observation d'interférences sur des distances macroscopiques.

Nous pouvons résumer ce résultat comme suit: lors de la diffusion, le neutron interagit avec l'échantillon dans sa totalité. En d'autres termes, la diffusion n'est pas un phénomène local. Le problème suivant se pose alors : comment justifier l'approximation de Born, retenue à la base de l'interprétation de la diffusion des neutrons, tout en travaillant avec des échantillons macroscopiques pour des raisons de statistique? Et comment pouvons nous parler de libre parcours et de collision si la diffusion n'est pas un événement local?

La solution vient du fait que la grande majorité des échantillons n'est pas parfaite. Le cas extrême est le gaz parfait dans lequel il n'y a aucune corrélation entre les positions des différents atomes. Comme nous avons vu dans la section 8.10 la diffusion se décrit alors comme la superposition de collisions avec des atomes individuels. Dans les liquides, la portée des corrélations est limitée à quelques couches d'atomes voisins. Les interférences dues à la superposition des ondelettes diffusées disparaissent relativement vite avec la distance entre diffuseurs. Ceci nous permet de parler d'un volume de corrélation (voir figure 33).

Ce n'est pas le cas dans un cristal. Dans l'approximation de Born chaque plan rajouté affine le diagramme de diffusion, rendant les pics de Bragg mieux définis. Nous avons explicitement démontré cet affinement pour les réseaux optiques (se référer à la section 7.4). La saturation a lieu quand toute l'intensité initiale est passée dans l'onde diffusée. Alors, l'onde diffusée prend le relais et joue elle-même le rôle d'une onde incidente. En augmentant encore plus l'épaisseur du cristal le courant de neutrons sera progressivement redirigé dans la direction de l'onde incidente originale. Ce phénomène, qui ne sert ici que d'illustration, est connu comme Pendellösung [10]. Sa manifestation expérimentale est illustrée dans la figure 32. La longueur pour laquelle l'onde incidente disparaît est appelée longueur d'extinction primaire. L'approximation de Born n'est plus valable pour des cristaux dont la taille excède la longueur d'extinction primaire.

La plupart des cristaux qu' on retrouve sur les spectromètres sont loin d'être parfaits. ${ }^{56}$ Ils possèdent en particulier des dislocations (voir figure 33). Les plans d'atomes ne sont parfaitement alignés qu'à l'intérieur des cristallites. On parle alors de cristaux mosaïques. Les effets d'interférence sont par définition très prononcés dans un cristallite. L'assemblage des cristallites n'affine par contre plus les pics de Bragg. Il rajoute des réflexions à des angles de diffusion voisins ou pour des longueurs d'onde légèrement différentes. Nous pouvons donc identifier le volume de corrélation dans un cristal mosaïque

\footnotetext{
55 C'est la fonction d'onde imposée par la symétrie à une particule dans un potentiel périodique.

56 La matière cristalline n'est généralement pas présente à l'état de monocristal. Les monocristaux de silicium produits pour l'industrie électronique sont des exceptions remarquables. Un polycristal est composé de monocristaux, que nous appelons cristallites.
} 

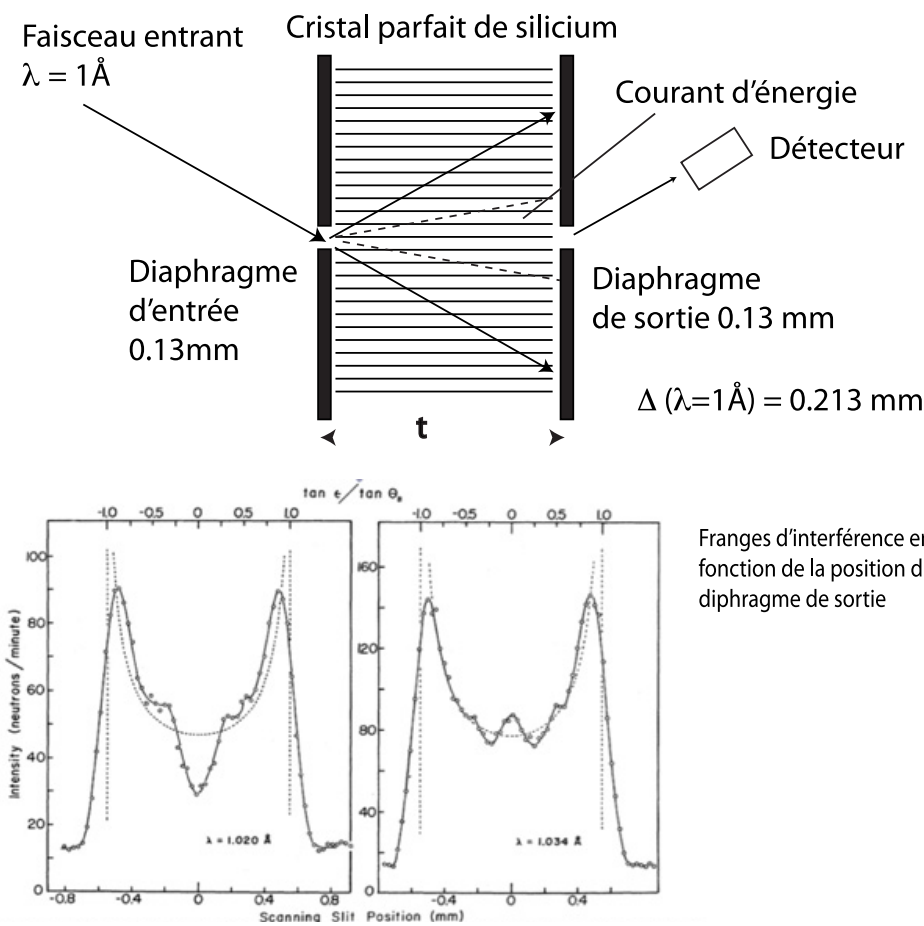

Franges d'interférence en fonction de la position du diphragme de sortie

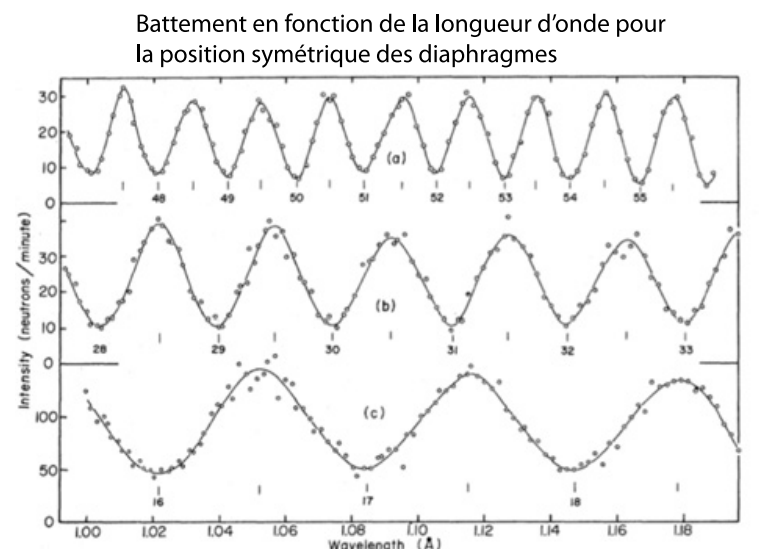

Épaisseur t de

la lame de cristal

$10.0 \mathrm{~mm}$

$5.9 \mathrm{~mm}$

$3.3 \mathrm{~mm}$

Figure 32. Illustraton schématique de l'expérience de Shull, qui a démontré l'existence de battements dans la diffusion de Bragg des neutrons (Pendellösung) [31]. Un faisceau monochromatique de neutrons subit une réflexion de Bragg par un cristal parfait de silicium. Pour une incidence parfaite, le courant d'énergie est dirigé parallèlement aux plans cristallins. Pour un faisceau divergent, le cône de transmission peut s'élargir pour atteindre $\pm \theta_{\text {Bragg. }}$. Pour éviter que cet élargissement n'obscurcisse les interférences, une bonne résolution spatiale est assurée par des diaphragmes. Le flux détecté en réflexion varie pour une longueur d'onde donnée périodiquement avec l'épaisseur du cristal. Dans le silicium, pour la réflexion (111) la période dite de Pendellösung est de $\Delta=0.213 \mathrm{~mm}$. Cette période peut être identifiée avec la longueur d'extinction primaire. Chaque battement correspond à un décalage supplémentaire d'une longueur d'onde entre les paquets d'ondes cohérents se propageant dans le cristal.

comme la taille des cristallites. Si cette taille est plus petite que la longueur d'extinction, l'approximation de Born est applicable à l'intérieur d'un cristallite, ce que facilite considérablement le formalisme. Il est important de souligner que la situation est différente dans un cristal mosaïque et dans un liquide : le 

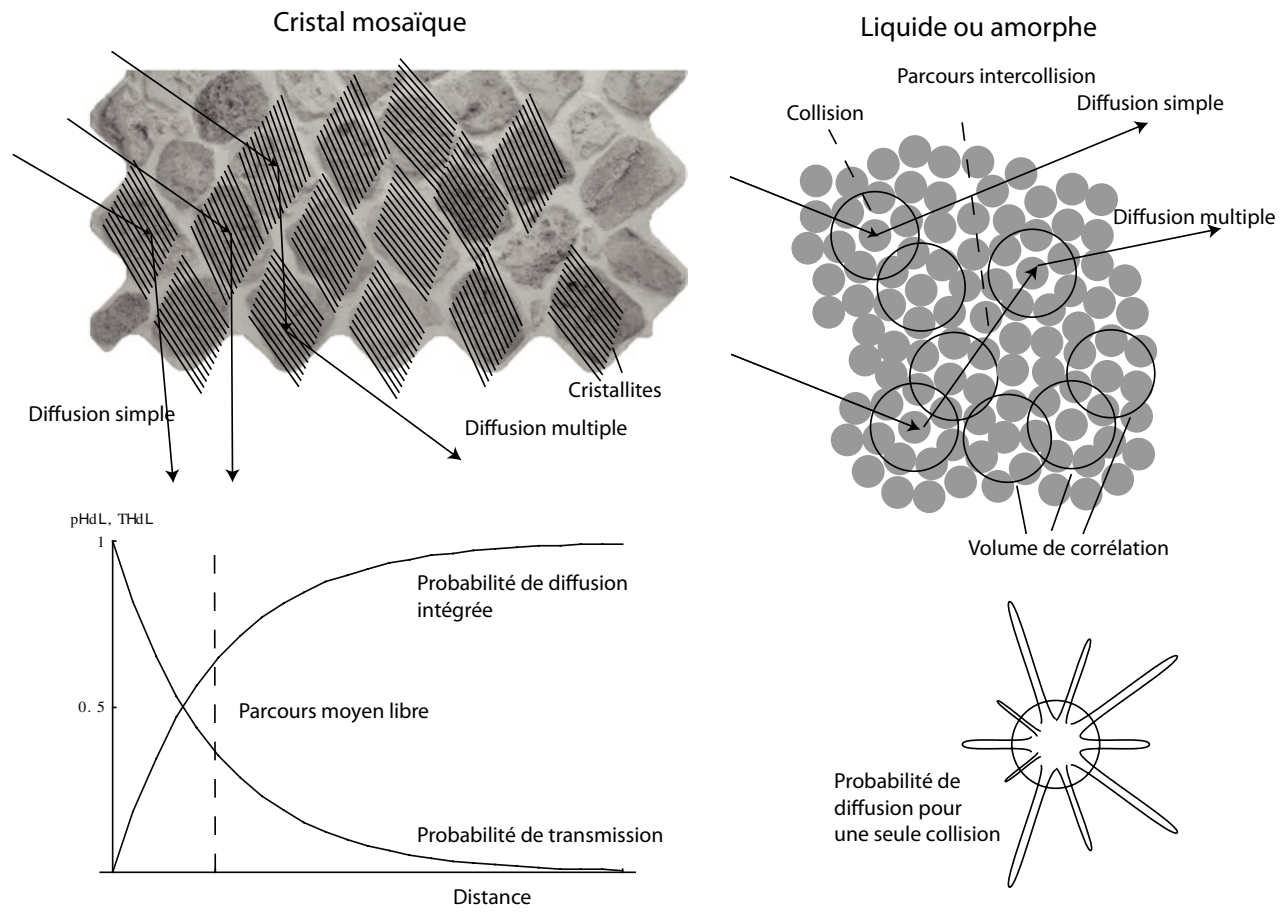

Figure 33. Présentation schématique de la diffusion d'un neutron par un échantillon de taille finie. Lors de son passage, le neutron subit un certain nombre de collisions. Chacune d'elles change le vecteur d'onde du neutron (soit la direction, soit l'énergie, soit les deux à la fois). La probabilité d'un tel changement se calcule dans l'approximation de Born sur le volume de corrélation qui entoure le lieu de la collision. Après une collision unique, le neutron parcours une distance $d$. Celle-ci est reliée à la probabilité de diffusion intégrée. Si la probabilité de diffusion est élevée les collisions seront fréquentes et le libre parcours moyen sera très court. Dans le cas d'un cristal mosaïque, la diffusion est hautement directionnelle. Les raies de Bragg s'obtiennent en traitant les cristallites dans l'approximation de Born, pourvu que la taille linéaire des cristallites soit plus faible que la longueur d'extinction primaire. Dans le cas d'un liquide ou d'un solide amorphe, les volumes de corrélation sont plus petits. Contrairement aux cristaux, tous les points sont équivalents puisque le liquide est isotrope et homogène. Dans les deux cas, le libre parcours moyen doit être beaucoup plus long que chacune des dimensions des cristallites ou des volumes de corrélation pour que le concept d'une diffusion par collision soit applicable.

liquide est homogène. Nous pouvons tracer une sphère contenant le même volume de corrélation autour de n'importe quel point $\vec{r}$. Pour un cristal mosaïque l'entourage est complètement différent selon que l'on se trouve au centre d'un cristallite ou proche d'un joint de grain. Cette inhomogénéité engendre des effets particuliers dans le processus de la diffusion et doit être prise en compte lors de l'interprétation des données.

Ce sont finalement les volumes de corrélation qui nous permettent de décrire la diffusion comme un processus de collisions multiples (voir figure 33). Du point de vue expérimental nous ne pouvons que difficilement distinguer (i) une diffusion localisée dans le volume de corrélation avec propagation de la particule diffusée d'un volume à l'autre et (ii) une diffusion sur tout le volume de l'échantillon traitée en ordre supérieur de la série de Born (noter les similitudes entre les figures 10 et 33). Les taux de diffusion et la propagation sont à calculer dans l'approximation de Born pour les volumes de corrélation (toujours sous la condition que les dimensions linéaires de ces volumes sont inférieures à la longueur d'extinction).

Pour décrire la propagation, nous allons d'abord introduire la notion de libre parcours moyen qui est la distance moyenne que parcourt le neutron entre deux collisions. Selon (9.18), la probabilité par unité 
de temps qu'un neutron $\vec{k}_{i}$ soit diffusé est égale à

$$
f\left(\vec{k}_{i}\right)=\int f\left(\vec{k}_{f}, \vec{k}_{i}\right) d^{3} k_{f}
$$

Ainsi $f\left(\vec{k}_{i}\right)^{-1}$ est le temps moyen de séjour d'un neutron dans l'échantillon sans être diffusé. Pendant ce temps le neutron se déplace de

$$
l\left(\vec{k}_{i}\right)=\frac{v\left(\vec{k}_{i}\right)}{f\left(\vec{k}_{i}\right)} .
$$

$l\left(\vec{k}_{i}\right)$ est le libre parcours moyen recherché. Selon (9.25) et en utilisant (9.22)

$$
\begin{aligned}
l\left(\vec{k}_{i}\right)^{-1} & =\frac{1}{v\left(\vec{k}_{i}\right)} \int f\left(\vec{k}_{f}, \vec{k}_{i}\right) d^{3} k_{f} \\
& =\frac{\hbar}{m k_{i}}\left[\frac{N}{V} \frac{m^{2}}{\hbar^{2}} \frac{\sigma}{4 \pi} \int S(\vec{Q}, \omega) d^{3} k_{f}\right] \\
& =\frac{N}{V} \frac{k_{f}}{k_{i}} \frac{\sigma}{4 \pi} \int S(\vec{Q}, \omega) d \Omega d E_{f} \\
& =\frac{N}{V} \sigma_{\text {tot }}
\end{aligned}
$$

Le libre parcours moyen peut donc être calculé à partir de la section efficace totale. Ce résultat est valable même en dehors de la validité de l'approximation de Born.

Le nombre de neutrons diffusés à un endroit $z$ par élément $d z$ le long de la trajectoire est proportionnel au taux de diffusion (9.28) ainsi qu'au nombre de neutrons $n_{t}(z)$ transmis jusqu'à $z$

$$
d n_{d}(z)=n_{t}(z) \frac{1}{l} d z=n_{t}(z) \frac{N \sigma_{\text {tot }}}{V} d z
$$

avec

$$
d n_{t}(z)=-d n_{d}(z)
$$

Attention de bien faire la distinction entre le nombre de neutrons $n$ et de diffuseurs $N$. L'équation (9.29) possède une solution simple (voir figure 33)

$$
n_{t}(z)=n_{t}(0) \exp \left(-\frac{z}{l}\right)
$$

pour le nombre de neutrons transmis jusqu'à $z$. Ainsi le nombre de neutrons diffusés jusqu'à $z$ est donné par

$$
n_{d}(z)=n_{i}(0)\left(1-\exp \left(-\frac{z}{l}\right)\right)
$$

Dans le cas d'une diffusion très faible

$$
z \ll l=\frac{N V}{\sigma_{\text {tot }}} \quad: \quad \frac{n_{d}(z)}{n_{i}(0)}=\frac{\sigma_{\text {tot }}}{N V} z .
$$

Une fois que le neutron se sera propagé jusqu'à l'endroit $\vec{r}$, il subira avec une certaine probabilité une collision. Lors de cette collision, le neutron changera de direction et d'énergie. La distribution de vecteurs d'onde qui en résulte, se calcule avec $f\left(\vec{k}_{f}, \vec{k}_{i}\right)(9.25)$. Après la collision, le neutron peut soit sortir de l'échantillon, soit être diffusé une deuxième fois, et ainsi de suite. On parlera de diffusion multiple.

En général on ne souhaite pas travailler avec des échantillons qui possèdent une transmission inférieure à $90 \%$. Dans ce cas, un raisonnement simple permet d'estimer que l'intensité de la diffusion 
multiple se situera en dessous de $10 \%$ de l'intensité de la diffusion simple, qui elle-même ne pourra pas dépasser $10 \%$ du flux incident. En autres termes, sur 10 neutrons qui arrivent dans le détecteur, un neutron au maximum aura subi plus d'une collision. La diffusion multiple est toujours une nuisance. Elle brise la relation entre le signal mesuré et les fonctions de corrélation à deux points. Les vecteurs $\vec{k}_{i}-\vec{k}_{f}$ ne peuvent alors plus être associés avec un vecteur $\vec{Q}$ de l'approximation de Born, la sonde ayant subi plusieurs “collisions". Comme nous l'avons discuté dans la section 8.5, la diffusion multiple devrait ouvrir une voie vers les fonctions de corrélation à trois points et plus. Néanmoins ceci n'a pas encore été démontré dans la pratique.

Les volumes de corrélation nous permettent aussi de mieux définir les moyennes qui nous ont permis de séparer la diffusion en partie cohérente et incohérente (section 5.6). L'échantillon macroscopique est un ensemble de répliques du volume de corrélation. Parmi ces répliques, on trouve toutes les distributions possibles d'isotopes ou de spins nucléaires. Lors d'une collision, une de ces répliques est sondée par le neutron. Le signal de diffusion s'obtient en moyennant sur l'ensemble des collisions.

\subsection{Taux de diffusion}

Dans le formalisme que nous avons développé, le taux de diffusion par collision se calcule à partir de la fonction de diffusion (9.25)

$$
f\left(\vec{k}_{i}\right)=\int d^{3} k_{f} f\left(\vec{k}_{f}, \vec{k}_{i}\right)=\frac{N}{V} \frac{m^{2}}{\hbar^{2}} \frac{\sigma}{4 \pi} \int d^{3} k_{f} S(\vec{Q}, \omega) .
$$

L'intégration porte sur tout l'espace des vecteurs $\vec{k}_{f}$. La fonction de diffusion étant le résultat recherché de l'expérience, ce calcul n'est pas réalisable en amont, par exemple pour estimer la taille idéale de l'échantillon. C'est la raison pour laquelle nous allons introduire une méthode approximative simple pour estimer le taux de diffusion.

Nous allons voir plus loin que la diffusion élastique est largement dominante pour la grande majorité des conditions expérimentales. La diffusion élastique est reliée à la partie des corrélations entre diffuseurs qui persiste pour $t \rightarrow \infty$ (se référer à la section 8.9). Une manière approximative pour décrire ces corrélations consiste à supposer que les positions des diffuseurs sont figées dans le temps. Dans ce cas, les expressions pour les sections efficaces se simplifient énormément. Cette simplification vient du fait que toute fonction d'un opérateur de position $\overrightarrow{\mathbf{R}}_{j}$ à $t=0$ ne fait que multiplier les fonctions d'ondes. Ce n'est qu'au moment où l'opérateur de Hamilton entre en jeu via

$$
\overrightarrow{\mathbf{R}}_{j}(t)=\exp (i \mathbf{H} t / \hbar) \overrightarrow{\mathbf{R}}_{j} \exp (-i \mathbf{H} t / \hbar)
$$

que le problème se complique. Nous obtenons en particulier

$$
\left\langle\lambda_{i}\left|e^{i \vec{Q} \cdot\left(\vec{R}_{j}(t=0)-\vec{R}_{j^{\prime}}^{0}\right)}\right| \lambda_{i}\right\rangle \rightarrow e^{i \vec{Q} \cdot\left(\vec{R}_{j}-\vec{R}_{j^{\prime}}\right)}\left\langle\lambda_{i} \mid \lambda_{i}\right\rangle=e^{i \vec{Q} \cdot\left(\vec{R}_{j}-\vec{R}_{j^{\prime}}\right)} .
$$

Nous rappelons que

$$
\sum_{\lambda_{i}} p\left(\lambda_{i}\right)=1
$$

et que

$$
\frac{1}{2 \pi \hbar} \int_{-\infty}^{\infty} d t e^{-i \omega t}=\delta(\hbar \omega)
$$

Pour un système monoatomique, ces trois relations permettent de reformuler les sections efficaces différentielles partielles (5.67) et (5.68). On obtient

$$
\left(\frac{d \sigma}{d \Omega d E_{f}}\right)_{\mathrm{coh}}=\frac{\sigma_{\mathrm{coh}}}{4 \pi} \frac{k_{f}}{k_{i}} \sum_{j, j^{\prime}=1}^{N} e^{i \vec{Q} \cdot\left(\vec{R}_{j}-\vec{R}_{j^{\prime}}\right)} \delta(\hbar \omega)
$$


et

$$
\left(\frac{d \sigma}{d \Omega d E_{f}}\right)_{\mathrm{inc}}=\frac{\sigma_{\mathrm{inc}}}{4 \pi} \frac{k_{f}}{k_{i}} \sum_{j}^{N} e^{i \vec{Q} \cdot\left(\vec{R}_{j}-\vec{R}_{j^{\prime}}\right)} \delta(\hbar \omega)=N \frac{\sigma_{\mathrm{inc}}}{4 \pi} \frac{k_{f}}{k_{i}} \delta(\hbar \omega)
$$

En effectuant l'intégration sur l'énergie nous aboutissons aux sections efficaces différentielles

$$
\begin{aligned}
\left(\frac{d \sigma}{d \Omega}\right)_{\mathrm{coh}} & =\frac{\sigma_{\mathrm{coh}}}{4 \pi} \sum_{j, j^{\prime}=1}^{N} e^{i \vec{Q} \cdot\left(\vec{R}_{j}-\vec{R}_{j^{\prime}}\right)} \\
& =\frac{\sigma_{\mathrm{coh}}}{4 \pi}\left|\sum_{j=1}^{N} e^{i \vec{Q} \cdot \vec{R}_{j}}\right|^{2}
\end{aligned}
$$

et

$$
\left(\frac{d \sigma}{d \Omega}\right)_{\text {inc }} N \frac{\sigma_{\text {inc }}}{4 \pi}
$$

La diffusion cohérente est donc donnée par le facteur de structure

$$
F(\vec{Q})=\left|\sum_{j=1}^{N} e^{i \vec{Q} \cdot \vec{R}_{j}}\right|^{2}
$$

Pour un cristal monoatomique infini, on peut montrer (voir section 10.2) que

$$
F(\vec{Q})=N \frac{(2 \pi)^{3}}{V_{\text {maille }}} \sum_{\vec{G}} \delta(\vec{Q}-\vec{G}) .
$$

$V_{\text {maille }}$ est le volume de la maille primitive et les $\vec{G}$ correspondent aux vecteurs du réseau réciproque (voir chapitre I). La réponse cohérente est donc finement structurée. On obtient des pics aux vecteurs $\vec{Q}$ du réseau réciproque à priori infiniment bien définis (voir figure 21). Nous avons déjà discuté que c'est un artefact de l'approximation de Born et que la largeur des pics est reliée à la longueur d'extinction primaire. La réponse incohérente, à l'inverse de la réponse cohérente, ne montre aucune dépendance en $\vec{Q}$. Sa section efficace est simplement proportionnelle au nombre $N$ de diffuseurs. C'est évident car la diffusion incohérente est le produit des corrélations d'un diffuseur avec lui-même. Pour des atomes figés ces corrélations sont triviales.

Dans le but de rendre les sections efficaces plus intuitives nous proposons d'estimer le taux de diffusion (voir aussi figure 34). En l'absence de connaissance du facteur de structure, il est évident que nous devons nous contenter de diffuseurs incohérents. ${ }^{57}$ Le volume de corrélation se résume dans ce cas à un seul diffuseur et le neutron peut être considéré comme une particule individuelle rentrant en collision avec les atomes.

Notre échantillon est caractérisé par :

1. sa masse molaire $m_{\mathrm{mol}}$,

2. sa densité de masse $\rho_{m}$,

3. sa section efficace incohérente $\sigma_{\text {inc }}$.

\footnotetext{
57 Dans la pratique on peut souvent remplacer dans les expressions que nous allons développer la section efficace totale incohérente par la section efficace totale. Mais attention, ceci implique que les longueurs d'onde soient suffisamment petites. Quand au contraire, les longueurs d'onde utilisées pour l'expérience sont très longues, il se peut qu'aucun pic de Bragg ne soit atteint même en rétrodiffusion. On travaille alors en dessous du seuil de Bragg. Un échantillon purement cohérent devient dans ces conditions quasiment transparent.
} 


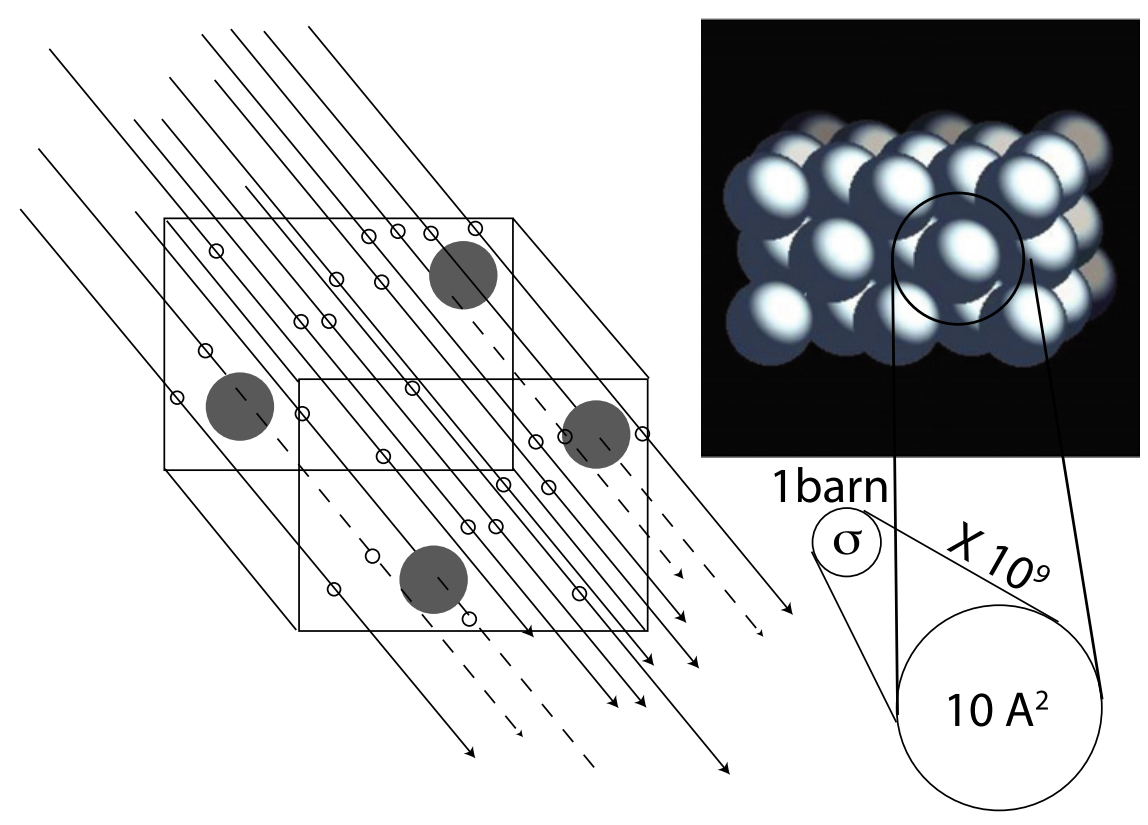

Figure 34. Illustration schématique de la section efficace. Chaque diffuseur, symbolisé par une sphère a une section efficace correspondant à la section qu'il oppose au flux de neutrons. Un neutron qui heurte cette sphère est supposé être diffusé. Les autres passent. Le rapport entre la section efficace et la section d'une sphère qui correspond au volume occupé par l'atome est typiquement de l'ordre de $10^{7}$ à $10^{9}$. Il faudra donc $\sim 1$ milliard de couches d'atomes pour rendre l'échantillon "opaque" aux neutrons. Ceci correspond à une dimension macroscopique entre quelques dixièmes de $\mathrm{mm}$ et quelques $\mathrm{cm}$.

Le volume microscopique correspondant à un diffuseur individuel est donné par

$$
V_{\text {ind }}=\frac{m_{\text {mol }}}{N_{A} \rho_{m}}
$$

avec la constante d'Avogadro $N_{A}=6.022 \cdot 10^{23}$. Nous pouvons supposer que $V_{\text {ind }}$ est de forme cubique. Les aires des surfaces de ce volume ont donc pour valeur

$$
a_{\text {ind }}=V_{\text {ind }}^{\frac{2}{3}} \text {. }
$$

Le taux $\xi_{\text {ind }}$ de diffusion est -pour une seule particule- déterminé par le rapport entre la section efficace et l'aire $a_{\text {ind }}$ qu'il oppose au flux des neutrons

$$
\xi_{\text {ind }}=\frac{\sigma_{\text {inc }}}{a_{\text {ind }}}
$$

Etudions la situation quand un grand nombre d'atomes est aligné dans la direction du faisceau incident que nous choisissons suivant $\hat{z}=\hat{k}_{i}$. Si la diffusion est faible, nous pouvons négliger le fait que le flux incident diminuera avec la profondeur (approximation de Born). Pour atteindre un certain taux de diffusion $\xi$ il nous faudra tapisser la surface $a_{\text {ind }}$ avec

$$
n=\xi \frac{a_{\text {ind }}}{\sigma_{\text {inc }}}
$$


plaquettes, chacune correspondant à la section efficace $\sigma_{\text {inc }}$. Cette condition nous amène à une longueur de

$$
l=n \cdot \sqrt{a_{\text {ind }}}=\xi \frac{\sqrt{a_{\text {ind }}} a_{\text {ind }}}{\sigma_{\text {inc }}}=\frac{V_{\text {ind }}}{\sigma_{\text {inc }}} .
$$

C'est le libre parcours moyen, que nous avions déjà rencontré dans l'expression plus générale (9.28). Dans le contexte de la diffusion multiple, l'expression (9.49) est une formule très simple pour calculer l'épaisseur correcte d'un échantillon.

Traitons deux exemples qui jouent un rôle important pour la diffusion des neutrons: l'eau et le vanadium.

1. L'eau $\left(\mathrm{H}_{2} \mathrm{O}\right)$ est un solvant universel en chimie et un composant indispensable de la matière biologique. Elle est caractérisée par les paramètres :

$1 m_{\mathrm{mol}}=16 \mathrm{~g}$,

$2 \rho_{m}=1 \mathrm{~g} / \mathrm{cm}^{3}$,

$3 \sigma_{\text {inc }} \approx 165$ barn $=165 \times 10^{-10} \mathrm{~nm}^{2}$,

$4 V_{\mathrm{H}_{2} \mathrm{O}}=3 \times 10^{-2} \mathrm{~nm}^{3}$,

533 molécules de $\mathrm{H}_{2} \mathrm{O}$ dans un $\mathrm{nm}^{3}$.

Nous obtenons

$$
\frac{V_{\mathrm{H} 2 \mathrm{O}}}{\sigma_{\text {inc }}}=1.875 \mathrm{~mm}
$$

Pour obtenir $10 \%$ de diffusion et donc $90 \%$ de transmission il ne faut pas que l'épaisseur d'eau traversée par le neutron incident excède environ $0.2 \mathrm{~mm}$. L'eau est parmi les diffuseurs les plus forts. Nous constatons que même dans ce cas extrême, les neutrons possèdent un grand pouvoir de pénétration. Ce pouvoir de pénétration constitue l'un des atouts majeurs de la diffusion des neutrons. Attention, la section efficace totale que nous avons utilisée est celle pour les atomes liés. Si nous travaillons avec des neutrons de très courte longueur d'onde il faudra prendre en compte le fait que la section efficace diminue (voir section 5.9) pour calculer la bonne épaisseur de l'échantillon ${ }^{58}$.

2. Le vanadium est un diffuseur complètement incohérent. Il est beaucoup utilisé pour calibrer les spectromètres.

$1 m_{\mathrm{mol}}=51 \mathrm{~g}$,

$2 \rho_{m}=6.1 \mathrm{~g} / \mathrm{cm}^{3}$,

$3 \sigma_{\text {inc }}=5.08$ barn $=5.08 \times 10^{-10} \mathrm{~nm}^{2}$,

$4 V_{\mathrm{V}}=1.4 \times 10^{-2} \mathrm{~nm}^{3}$,

571 atomes de Vanadium dans un $\mathrm{nm}^{3}$.

Nous obtenons donc

$$
\frac{V_{\mathrm{V}}}{\sigma_{\mathrm{inc}}}=28 \mathrm{~mm} .
$$

Pour atteindre $10 \%$ de diffusion, l'épaisseur d'une lame de vanadium le long de la trajectoire des neutrons incidents devra être d'environ $2.8 \mathrm{~mm}$. De plus, il faut souligner que la forme de l'échantillon est également un paramètre à prendre en compte pour évaluer la diffusion multiple (voir [32]).

\footnotetext{
58 Merci à Bruno Dorner pour avoir attiré mon attention sur ce point.
} 


\subsection{Résolution}

La fonction de diffusion défini la fonction de transfert de l'échantillon selon (9.25). L'intensité enregistrée dans le détecteur par intervalle d'énergie peut donc s'obtenir comme (voir aussi [33])

$$
I\left(E_{f}\right) d E_{f} \propto \iint p\left(\vec{k}_{i}\right) S(\vec{Q}, \hbar \omega) p\left(\vec{k}_{f}\right) d^{3} k_{i} d^{3} k_{f}
$$

La distribution $p\left(\vec{k}_{i}\right)$ a déjà été introduite. Elle donne la probabilité qu'un neutron soit produit à la source et ensuite transmis à l'échantillon en passant par les éléments optiques se trouvant sur son chemin. La distribution $p\left(\vec{k}_{f}\right)$ décrit la probabilité qu'un neutron sortant de l'échantillon trouve le chemin du détecteur. Elle peut s'obtenir en créant une population de neutrons à l'endroit du détecteur qui reflète les caractéristiques de celui-ci. Le détecteur joue alors le rôle d'une source inversée. Ces neutrons sont ensuite propagés vers l'échantillon en retraçant le chemin qu'auraient pris les neutrons diffusés ${ }^{59}$.

Pour faciliter la discussion, les distributions $p\left(\vec{k}_{i}\right)$ et $p\left(\vec{k}_{f}\right)$ sont de nouveau assumées homogènes sur le volume de l'échantillon et indépendantes du temps.

L'expression (9.50) peut être écrite sous la forme d'une convolution [34]

$$
I\left(E_{f}\right) d E_{f} \propto \iint R\left(\vec{Q}-\vec{Q}_{0}, \hbar \omega-\hbar \omega_{0}\right) S(\vec{Q}, \hbar \omega) d^{3} Q d \hbar \omega,
$$

avec la fonction de résolution définie comme

$$
R(\vec{Q}, \hbar \omega)=\iint p\left(\vec{k}_{i}\right) p\left(\vec{k}_{f}\right) \delta\left(\vec{Q}-\left(\vec{k}_{i}-\vec{k}_{f}\right)\right) \delta\left(\hbar \omega-\frac{\hbar^{2}}{2 m}\left(k_{i}^{2}-k_{f}^{2}\right)\right) d^{3} k_{i} d^{3} k_{f} .
$$

$R(\vec{Q}, \hbar \omega)$ mesure le nombre de combinaisons $\vec{k}_{i}$ et $\vec{k}_{f}$ qui sont compatibles avec les conditions $\vec{k}_{i}-\vec{k}_{f}=\vec{Q}$ et $E_{i}-E_{f}=\hbar \omega$. Les variables $\vec{Q}_{0}$ et $\hbar \omega_{0}$ dénotent les valeurs centrales de $\vec{Q}$ et $\hbar \omega$. Elles constituent le centre de gravité de la fonction de résolution autour duquel la mesure est effectuée. Pour extraire la quantité $S\left(\vec{Q}_{0}, \hbar \omega_{0}\right)$ à laquelle nous nous intéressons finalement, la mesure doit être déconvoluée de la fonction de résolution. En particulier la variation du nombre total de neutrons dans le faisceau incident et dans le faisceau transmis au détecteur doit être corrigée. Ces quantités sont formellement décrites par des volumes d'espace des phases

$$
V_{i}=\int p\left(\vec{k}_{i}\right) d^{3} k_{i} \quad \text { et } \quad V_{f}=\int p\left(\vec{k}_{f}\right) d^{3} k_{f}
$$

Nous trouverons des exemples concrets de fonctions de résolution dans les chapitres qui traitent de l'instrumentation. La déconvolution est une opération mathématiquement délicate. Dans le cas où la mesure sert à vérifier un modèle il est préférable de calculer la fonction de diffusion pour la convoluer ensuite avec la fonction de résolution. Dans tous les cas de figure, il est souhaitable de travailler avec des résolutions aussi fines que possible, c'est-à-dire avec des fonctions de résolution qui sont bien centrées autour de $\left(\vec{Q}_{0}, \hbar \omega_{0}\right)$. Ceci implique des distributions fines de $p\left(\vec{k}_{i}\right)$ et de $p\left(\vec{k}_{f}\right)$ avec comme résultat une intensité fortement réduite (voir (9.51)). La résolution se négocie donc aux dépens du flux. Les spectromètres sont construits de telle manière que l'expérimentateur ait la possibilité d'adapter la résolution à ses besoins en sacrifiant un minimum de flux.

Pour donner une idée des volumes d'espace des phases et de la résolution, penchons-nous sur les aspects pratiques de la diffusion de Bragg. La diffraction de Bragg est l'un des moyens les plus répandus pour rendre un faisceau monochromatique. Elle a été introduite dans la section 7 et sera de nouveau discutée dans la section 10.2. Reformulons l'équation de Bragg dans l'espace réciproque

$$
\vec{\tau}=\vec{k}_{f}-\vec{k}_{i}, \quad\left|\vec{k}_{i}\right|=\left|\vec{k}_{f}\right| \text {. }
$$

\footnotetext{
59 C'est possible parce que les équations régissant la propagation des neutrons sont symétriques par inversion du temps en absence de dissipation.
} 

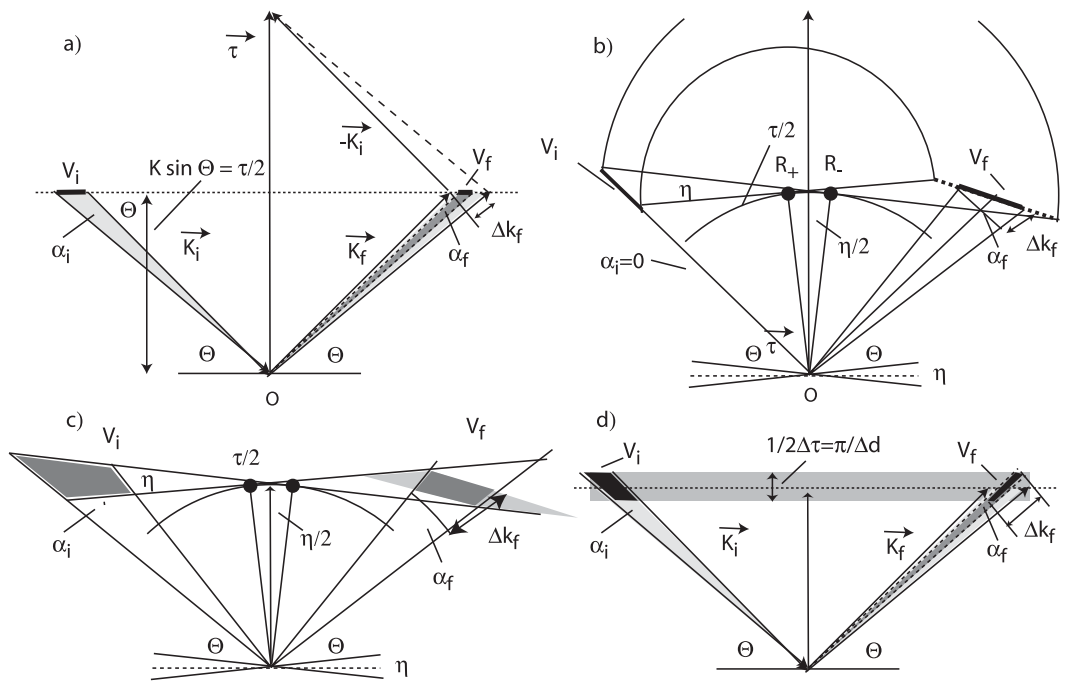

d)

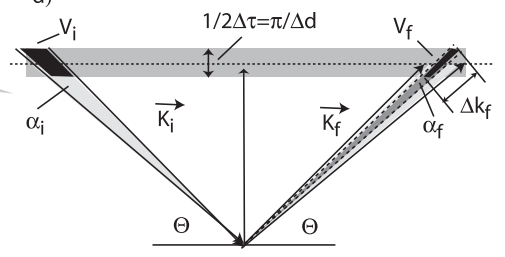

Figure 35. Description de la diffraction de Bragg dans l'espace réciproque. Les conditions pour la diffraction de Bragg sont réunies si la projection du faisceau incident et du faisceau diffracté sur le vecteur du réseau $\vec{\tau}$ est égale à $k \sin \theta=\tau / 2$. Cette contrainte donne une relation exacte entre $\vec{k}_{i}$ et $\vec{k}_{f}$. Le faisceau incident possède nécessairement une divergence finie $\alpha_{i}$. Dans le cas d'un faisceau polychromatique cette divergence entraîne une divergence du faisceau diffusé. Ceci est indiqué par les régions légèrement ombragées de la figure a). La divergence du faisceau incident se traduit par une distribution spectrale $\Delta k_{f}$ du faisceau diffracté. La largeur spectrale peut être contrôlée en réduisant la divergence du faisceau diffracté avec l'aide d'un collimateur (région fortement ombragée de la figure a)). Ainsi le volume de l'espace des phases $V_{i}$ se traduira en volume d'espace des phases $V_{f}$ défini par l'intersection de cette région avec la ligne $\tau / 2$. Il devient immédiatement évident que pour une divergence donnée, la résolution spectrale (monochromaticité) augmentera avec l'angle de Bragg $\Theta$ et dépendra alors fortement de la distance entre plans $d$. Pour un cristal avec mosaïque il y aura une distribution d'orientation des cristallites, que nous pouvons décrire par le paramètre $\eta$. Dans notre schéma, la mosaïque revient à tourner le diagramme de la figure a) de $\pm \eta / 2$ autour de l'origine $O$. Dans la figure b) nous montrons comment l'élément $V_{f}$ peut être obtenu à partir de $V_{i}$ pour un faisceau incident sans divergence. On construit les images du vecteur $\vec{k}_{i}$ le plus court et le plus long par rapport aux lignes $O-R_{-}$et $O-R_{+}$, respectivement. On notera que $V_{f}$ sera incliné par rapport à $\vec{k}_{f}$. Dans la figure c) nous montrons la situation générale d'un faisceau incident avec divergence $\alpha_{i}$ réfléchi par un cristal mosaïque dans un faisceau sortant de divergence $\alpha_{f}$. Le volume $V_{f}$ reflète l'intensité qu'on pourra obtenir pourvu que $V_{i}$ soit complétement rempli. Une configuration expérimentale est optimale si $\mathrm{V}_{f}$ est maximal pour un $p\left(\vec{k}_{f}\right)$ requis, c'est-à-dire pour un $\vec{k}_{f}$ et $\Delta k_{f}$. La figure d) montre la situation d'un cristal avec une distribution en $d$. Une telle variation peut être obtenue par un gradient de composition chimique ou par une distorsion mécanique ou thermique.

$\vec{\tau}_{h k l}=h \vec{a}_{1}^{*}+k \vec{a}_{2}^{*}+l \vec{a}_{3}^{*}$ dénote un vecteur du réseau réciproque perpendiculaire aux plans de norme $\left|\vec{\tau}_{h k l}\right|=2 \pi / d_{h k l}$. La distance entre les plans est $d_{h k l}$. La diffusion de Bragg a lieu quand les vecteurs $\vec{k}_{i}$, $\vec{k}_{f}$ et $\vec{\tau}$ forment un triangle isocèle avec $\tau$ comme base. Comme on peut le voir dans la figure 35 il y a une forte corrélation entre la longueur du vecteur $\vec{k}$ et l'angle qu'il fait avec $\vec{\tau}$. Dans le cas d'un cristal parfait, seuls les vecteurs $\vec{k}_{i}$ et $\vec{k}_{f}$ faisant partie d'une fine lame dans l'espace des phases peuvent participer à la diffraction de Bragg. Ceci équivaut à une intensité diffractée infiniment faible. L'introduction d'une distribution de cristallites (mosaïque) ou de constante de maille (distorsions, défauts etc.) permettra l'obtention de volumes d'espace des phases non-nuls et donc des intensités raisonnables ${ }^{60}$.

Pour mieux comprendre comment une distribution de neutrons influe sur la mesure nous allons regarder la résolution sous un angle légèrement différent. Comme nous avons vu, la diffusion d'un

${ }^{60}$ Dans la réalité la diffraction de Bragg n’est jamais idéale du fait des effets d'extinction dont nous avons déjà parlé. 
neutron peut être décrite dans l'approximation de Born par la superposition d'ondes sphériques.

$$
\begin{aligned}
\psi_{\text {out }}(\vec{r}) & \propto \sum_{l} \frac{b_{l}}{\left|\vec{r}-\vec{r}_{l}\right|} e^{i k\left|\vec{r}-\vec{r}_{l}\right|} \psi_{\text {in }}\left(\vec{r}_{l}\right) \\
& \propto \int p\left(\vec{k}_{i}\right) \sum_{l} \frac{b_{l}}{\left|\vec{r}-\vec{r}_{l}\right|} e^{i k\left|\vec{r}-\vec{r}_{l}\right|} e^{i \vec{k}_{i} \vec{r}_{l}} d^{3} k_{i},
\end{aligned}
$$

avec $\vec{r}_{l}$ la position du diffuseur $l$ et $b_{l}$ sa longueur de diffusion. C'est le principe d'Huygens-Fresnel appliqué à la diffusion des neutrons (voir section 7). La source des ondes sphériques est la fonction d'onde incidente $\psi_{\mathrm{in}}$, qui, dans notre description, s'exprime comme une superposition pondérée d'onde planes. Comme dans le cas de la diffraction optique, les diffuseurs se trouvent sur le front d'onde, c'està-dire possèdent des positions pour lesquelles $\vec{k}_{i} \cdot \vec{r}_{l}$ est constant et diffuseront en phase ${ }^{61}$. Du fait de la distribution en direction et en longueur d'onde de $\vec{k}_{i}$ (et $\vec{k}_{f}$ ), les interférences sont brouillées dès que la distance entre les diffuseurs devient trop importante. La fonction de résolution doit donc être considérée comme l'image des volumes de cohérence (voir section 7) dans l'espace réciproque [35]. Les volumes de cohérence sont, d'une certaine manière, les champs de vision de l'instrument dans l'espace réel.

Nous allons voir dans les divers chapitres de ce livre que la résolution $\Delta Q_{i}, i=x, y, z$ et $\hbar \Delta \omega$, et ainsi les volumes de cohérence, augmentent considérablement avec la longueur d'onde. Ce n'est pas vraiment surprenant du fait que la définition d'un faisceau est normalement faite par rapport aux valeurs nominales. Par conséquent, ce sont les résolutions relatives $\Delta Q / Q$ et $\Delta \omega / \omega$ qui sont semblables sur une large gamme d'instruments. Des longueurs d'onde plus importantes limitent néanmoins les points $(\vec{Q}, \hbar \omega)$ accessibles à la mesure. Par exemple, dans le cas de la diffusion élastique $\left|\vec{k}_{i}\right|=\left|\vec{k}_{f}\right|$, la valeur maximale de $Q$ est de $2 \vec{k}_{i}$ pour $\vec{k}_{f}=-\vec{k}_{i}$. En réduisant $\left|\vec{k}_{i}\right|$, nous limitons la gamme dynamique du dispositif expérimental, c'est-à-dire que nous réduisons le champ de vision dans l'espace réciproque. Des champs de vision larges dans l'espace direct et dans l'espace réciproque sont donc des conditions conflictuelles.

\section{DIFFUSION DES NEUTRONS PAR UN SYSTÈME HARMONIQUE}

Nous allons maintenant calculer le facteur de structure dynamique pour un cristal harmonique. Notre point de départ est la fonction de diffusion (8.7) que nous rappelons ici

$$
S(\vec{Q}, \omega)=\sum_{\kappa, \kappa^{\prime}} b_{\kappa} b_{\kappa^{\prime}}^{*} S_{\kappa, \kappa^{\prime}}(\vec{Q}, \omega)
$$

avec les fonctions de diffusion partielles définies comme

$$
S_{\kappa, \kappa^{\prime}}(\vec{Q}, \omega)=\frac{1}{2 \pi \hbar} \sum_{j \in\left\{j_{\kappa}\right\}, j^{\prime} \in\left\{j_{\kappa^{\prime}}\right\}} \int_{-\infty}^{\infty} d t\left\langle e^{-i \vec{Q} \cdot \vec{R}_{j^{\prime}}(t=0)} e^{i \vec{Q} \cdot \vec{R}_{j}(t)}\right\rangle e^{-i \omega t} .
$$

Le matériau étant harmonique, le mouvement des ions est décrit par des vibrations autour de leurs positions d'équilibre $\vec{R}_{j}^{0}, j=1, \ldots N$. Attention, nous avons introduit ici un changement de notation important pour rester conforme au chapitre I. $\vec{R}_{j}^{0}$ désigne à partir de maintenant la position d'équilibre de l'ion $j$ et ne fait plus figure d'abréviation pour $\vec{R}_{j}(t=0)$. En général, les positions d'équilibre ne coïncideront pas avec les positions des ions à $t=0$, c'est-à-dire $\vec{R}_{j}^{0} \neq \vec{R}_{j}(t=0)$. Les déplacements

61 Comme nous observons la fonction d'onde diffusée $\psi_{\text {out }}$ dans le détecteur, nous devons en principe inclure dans l'expression (9.55) la description du faisceau diffusé en fonction de $\vec{k}_{f}$. Ceci inclut la probabilité avec laquelle un neutron $\vec{k}_{f}$ atteint le détecteur et nous amène vers une expression du type (9.51). Dans le cas de la diffraction optique, on a affaire à un problème similaire. Le faisceau diffracté doit être projeté sur le plan d'observation. Les lentilles utilisées à cet effet introduisent des erreurs d'image. 
ioniques par rapport à ces positions s'expriment comme

$$
\vec{u}_{j}(t)=\vec{R}_{j}(t)-\vec{R}_{j}^{0}, j=1, \ldots N .
$$

Ainsi

$$
\left\langle e^{-i \vec{Q} \cdot \vec{R}_{j^{\prime}}(t=0)} e^{i \vec{Q} \cdot \vec{R}_{j}(t)}\right\rangle=\left\langle e^{-i \vec{Q} \cdot\left(\vec{R}_{j^{\prime}}^{0}+\vec{u}_{j^{\prime}}(t=0)\right)} e^{i \vec{Q} \cdot\left(\vec{R}_{j}^{0}+\vec{u}_{j}(t)\right)}\right\rangle .
$$

Les positions d'équilibre $R_{j}^{0}$ ne sont pas des opérateurs et peuvent donc être traitées comme de simples chiffres. ${ }^{62}$ Il vient

$$
\left\langle e^{-i \vec{Q} \cdot\left(\vec{R}_{j^{\prime}}^{0}+\vec{u}_{j^{\prime}}(t=0)\right)} e^{i \vec{Q} \cdot\left(\vec{R}_{j}^{0}+\vec{u}_{j}(t)\right)}\right\rangle=e^{-i \vec{Q} \cdot\left(\vec{R}_{j^{\prime}}^{0}-\vec{R}_{j}^{0}\right)}\left\langle e^{-i \vec{Q} \cdot \vec{u}_{j^{\prime}}(t=0)} e^{i \vec{Q} \cdot \vec{u}_{j}(t)}\right\rangle .
$$

Pour faciliter la notation, nous noterons $\vec{u}_{j}$ au lieu de $\vec{u}_{j}(t)$ dès que le temps n'est pas mentionné explicitement.

L'approximation harmonique est fondée sur l'hypothèse que les déplacements sont petits à l'échelle interatomique. Il est donc logique de procéder au développement de l'expression (10.5) en terme de déplacements $\vec{u}$. Pour cela, il est souhaitable de la convertir en exponentielle simple. Pour y parvenir, nous utilisons une relation générale de l'algèbre des opérateurs

$$
\exp \mathbf{A} \cdot \exp \mathbf{B}=\exp (\mathbf{A}+\mathbf{B}+\mathbf{C})
$$

avec

$$
\mathbf{C}=\frac{1}{2}[\mathbf{A}, \mathbf{B}]+\frac{1}{12}[[\mathbf{A}, \mathbf{B}], \mathbf{B}]+\frac{1}{12}[[\mathbf{B}, \mathbf{A}], \mathbf{A}]+\ldots
$$

Pour appliquer cette relation à notre cas particulier nous posons

$$
\begin{aligned}
& \mathbf{A}=-i \vec{Q} \cdot \overrightarrow{\mathbf{u}}_{j^{\prime}}, \\
& \mathbf{B}=i \vec{Q} \cdot \overrightarrow{\mathbf{u}}_{j}(t) .
\end{aligned}
$$

Les phonons étant des bosons, les commutateurs des opérateurs $\overrightarrow{\mathbf{u}}$ sont de simples chiffres. La série de commutateurs imbriqués en (10.7) est donc tronquée après le premier terme. On obtient la relation simplifiée

$$
\exp \mathbf{A} \cdot \exp \mathbf{B}=\exp \left(\mathbf{A}+\mathbf{B}+\frac{1}{2}[\mathbf{A}, \mathbf{B}]\right)=\exp \left(\frac{1}{2}[\mathbf{A}, \mathbf{B}]\right) \exp (\mathbf{A}+\mathbf{B})
$$

Nous avons ainsi réussi à reformuler les fonctions de corrélation de l'expression (10.5) sous la forme d'exponentielles. Il va falloir maintenant convertir la valeur moyenne de la fonction exponentielle en fonction exponentielle d'une valeur moyenne. Autrement dit, les \langle\rangle doivent être transférés dans l'exposant. Cette opération est possible en s'appuyant sur l'identité de Bloch.

Soit $\mathbf{L}$ une combinaison linéaire quelconque d'opérateurs d'annihilation $\hat{a}$ et de création $\hat{a}^{+}$de phonons

$$
\mathbf{L}=\sum_{j} a_{j} \hat{a}_{j}+b_{j} \hat{a}_{j}^{+}
$$

L'identité de Bloch stipule que

$$
\langle\exp (\mathbf{L})\rangle=\exp \left(\frac{1}{2}\left\langle\mathbf{L}^{2}\right\rangle\right)
$$

\footnotetext{
${ }^{62}$ Les positions d'équilibre ne sont pas des variables dynamiques. L'Hamiltonien ne contient donc pas de dérivée selon ces positions et les $\vec{R}_{j}^{0}$ commutent donc avec $\mathbf{H}$ et ainsi avec $\vec{R}_{j}(t)$.
} 
Le lecteur soucieux de trouver la preuve de cette identité pourra consulter les livres de Squires [36] ou de Lovesey [15].

Dans le chapitre I nous avons montré que les déplacements ioniques s'exprimaient en fonction des opérateurs de création et d'annihilation selon

$$
\overrightarrow{\mathbf{u}}(\alpha, j)=\sum_{i} \sqrt{\frac{\hbar}{2 m_{j} \omega_{i}}}\left[e_{i}(\alpha, j) \mathbf{a}_{i}+e_{i}^{*}(\alpha, j) \mathbf{a}_{i}^{+}\right]
$$

avec $\omega_{i}$ les fréquences et $e_{i}(\alpha, j)(\alpha=x, y, z, j=1, \ldots, N)$ les vecteurs propres des $3 N$ vibrations. L'identité de Bloch est donc pleinement applicable à l'expression (10.5). Nous obtenons

$$
\begin{aligned}
\langle\exp \mathbf{A} \cdot \exp \mathbf{B}\rangle & =\left\langle\exp \left(\mathbf{A}+\mathbf{B}+\frac{1}{2}[\mathbf{A}, \mathbf{B}]\right)\right\rangle \\
& =\exp \left(\frac{1}{2}[\mathbf{A}, \mathbf{B}]\right)\langle\exp (\mathbf{A}+\mathbf{B})\rangle \\
& =\exp \left(\frac{1}{2}[\mathbf{A}, \mathbf{B}]\right) \exp \left(\frac{1}{2}\left\langle(\mathbf{A}+\mathbf{B})^{2}\right\rangle\right) \\
& =\exp \left(\frac{1}{2}\left\langle(\mathbf{A}+\mathbf{B})^{2}+[\mathbf{A}, \mathbf{B}]\right\rangle\right) \\
& =\exp \left(\frac{1}{2}\left\langle\mathbf{A}^{2}+\mathbf{B}^{2}+\mathbf{A B}+\mathbf{B A}+\mathbf{A B}-\mathbf{B A}\right\rangle\right) \\
& =\exp \left(\frac{1}{2}\left\langle\mathbf{A}^{2}+\mathbf{B}^{2}+2 \mathbf{A B}\right\rangle\right),
\end{aligned}
$$

ou de manière explicite

$$
\begin{aligned}
& \left\langle\exp \left(-i \vec{Q} \cdot \vec{u}_{j^{\prime}}\right) \exp \left(i \vec{Q} \cdot \vec{u}_{j}(t)\right)\right\rangle= \\
& \exp \left(\frac{1}{2}\left\langle-\left(\vec{Q} \cdot \vec{u}_{j^{\prime}}\right)^{2}-\left(\vec{Q} \cdot \vec{u}_{j}(t)\right)^{2}+2\left(\vec{Q} \cdot \vec{u}_{j^{\prime}}\right)\left(\vec{Q} \cdot \vec{u}_{j}(t)\right)\right\rangle\right)= \\
& \exp \left(-\frac{1}{2}\left\langle\left(\vec{Q} \cdot \vec{u}_{j^{\prime}}\right)^{2}\right\rangle\right) \exp \left(-\frac{1}{2}\left\langle\left(\vec{Q} \cdot \vec{u}_{j}(t)\right)^{2}\right\rangle\right) \exp \left(\left\langle\left(\vec{Q} \cdot \vec{u}_{j^{\prime}}\right)\left(\vec{Q} \cdot \vec{u}_{j}(t)\right)\right\rangle\right) .
\end{aligned}
$$

Il faut souligner que ce résultat n'est valable que dans l'approximation harmonique du fait que pour y arriver nous avons invoqué l'identité de Bloch.

\subsection{Facteur de Debye-Waller}

La quantité

$$
W_{j}(\vec{Q})=\frac{1}{2}\left\langle\left(\vec{Q} \cdot \vec{u}_{j}\right)^{2}\right\rangle
$$

est proportionnelle à la valeur moyenne du déplacement de l'ion $j$ projeté sur la direction $\vec{Q}$ au carré. Nous appelons $W(\vec{Q})$ la fonction de Debye-Waller de la particule $j$ et

$$
\exp \left(-2 W_{j}(\vec{Q})\right)=\exp \left(\left\langle\left(\vec{Q} \cdot \vec{u}_{j}\right)^{2}\right\rangle\right)
$$


le facteur de Debye-Waller [37,38]. Nous faisons cette distinction explicite entre fonction et facteur de Debye-Waller pour faciliter la discussion ultérieure.

Avec cette définition les fonctions de diffusions partielles (10.2) deviennent

$$
\begin{aligned}
& S_{K, \kappa^{\prime}}(\vec{Q}, \omega)= \\
& \frac{1}{2 \pi \hbar} \sum_{j \in\left\{j_{k}\right\}, j^{\prime} \in\left\{j_{K^{\prime}}\right\}} \int_{-\infty}^{\infty} d t\left\langle e^{-i \vec{Q} \cdot \vec{R}_{j^{\prime}}(t=0)} e^{i \vec{Q} \cdot \vec{R}_{j}(t)}\right\rangle e^{-i \omega t}= \\
& \frac{1}{2 \pi \hbar} \sum_{j \in\left\{j_{k}\right\}, j^{\prime} \in\left\{j_{K^{\prime}}\right\}} \int_{-\infty}^{\infty} d t e^{-i \vec{Q} \cdot\left(\vec{R}_{j^{\prime}}^{0}-\vec{R}_{j}^{0}\right)}\left\langle e^{-i \vec{Q} \cdot \vec{u}_{j^{\prime}}(t=0)} e^{i \vec{Q}^{-} \vec{u}_{j}(t)}\right\rangle= \\
& \frac{1}{2 \pi \hbar} \sum_{j \in\left\{j_{k}\right\}, j^{\prime} \in\left\{j_{K^{\prime}}\right\}} \int_{-\infty}^{\infty} d t e^{-i \vec{Q} \cdot\left(\vec{R}_{j^{\prime}}^{0}-\vec{R}_{j}^{0}\right)} e^{-W_{j^{\prime}}(\vec{Q})} e^{-W_{j}(\vec{Q})} e^{\left\langle\left(\vec{Q} \cdot \vec{u}_{j^{\prime}}\right)\left(\vec{Q} \cdot \vec{u}_{j}(t)\right)\right\rangle .}
\end{aligned}
$$

Nous avons vu dans le chapitre I que l'amplitude quadratique moyenne de la particule $j$ suivant la direction $\alpha$ se calcule comme

$$
\begin{aligned}
\overline{u(\alpha, j)^{2}} & =\left\langle\mathbf{u}(\alpha, j) \mathbf{u}(\alpha, j)^{+}\right\rangle \\
& =\sum_{i=1}^{3 N} \frac{\left|e_{i}(\alpha, j)\right|^{2} \hbar}{2 m_{j} \omega_{i}}\left\langle\mathbf{a}_{i} \mathbf{a}_{i}^{+}+\mathbf{a}_{i}^{+} \mathbf{a}_{i}\right\rangle \\
& =\sum_{i=1}^{3 N} \frac{\left|e_{i}(\alpha, j)\right|^{2} \hbar}{2 m_{j} \omega_{i}}\left(1+2\left\langle\mathbf{a}_{i}^{+} \mathbf{a}_{i}\right\rangle\right) \\
& =\sum_{i=1}^{3 N} \frac{\left|e_{i}(\alpha, j)\right|^{2} \hbar}{2 m_{j} \omega_{i}}\left(2 n\left(\omega_{i}\right)+1\right) .
\end{aligned}
$$

avec

$$
2 n\left(\omega_{i}\right)+1=2 \frac{1}{\exp \left(\frac{\hbar \omega_{i}}{k_{\mathrm{B}} T}\right)-1}+1=\operatorname{coth}\left(\frac{1}{2} \frac{\hbar \omega_{i}}{k_{\mathrm{B}} T}\right) .
$$

Nous pouvons immédiatement utiliser ce résultat pour déterminer la fonction de Debye-Waller

$$
2 W_{j}(\vec{Q})=\left\langle\left(\vec{Q} \cdot \vec{u}_{j}\right)^{2}\right\rangle=\sum_{\alpha=x, y, z} \sum_{j=1}^{3 N} \frac{\left|Q_{\alpha} \cdot e_{i}(\alpha, j)\right|^{2} \hbar}{2 m_{j} \omega_{i}} \operatorname{coth}\left(\frac{1}{2} \frac{\hbar \omega}{k_{\mathrm{B}} T}\right) .
$$

Il est évident que cette moyenne ne dépend pas du temps. Ainsi l'expression (10.15) se simplifie comme

$$
\left\langle e^{-i \vec{Q} \cdot \vec{u}_{j^{\prime}}} e^{i \vec{Q} \cdot \vec{u}_{j}(t)}\right\rangle=e^{-W_{j^{\prime}}(\vec{Q})} e^{-W_{j}(\vec{Q})} e^{\left\langle\left(\vec{Q} \cdot \vec{u}_{j^{\prime}}\right)\left(\vec{Q} \cdot \vec{u}_{j}(t)\right)\right\rangle} .
$$

Il faut remarquer que la fonction de Debye-Waller est en général spécifique à un site atomique et qu'elle dépend de la direction de $\vec{Q}$. Comme nous avons vu dans le chapitre I la symétrie du système peut restreindre la forme de la fonction de Debye-Waller en la rendant par exemple isotrope. En particulier, pour un système cubique monoatomique, nous pouvons exprimer la fonction de Debye-Waller en fonction de la densité d'états

$$
2 W=\overline{u^{2}}=\frac{\hbar Q^{2}}{2 m} \int_{0}^{\infty} \frac{G(\omega)}{\omega}(2 n(\omega)+1) d \omega .
$$


Pour donner une signification physique à la fonction de Debye-Waller nous nous intéressons à la probabilité $f(\vec{u} \cdot \hat{Q})$ de rencontrer un ion le long de la direction $\hat{Q}$ entre $\vec{u} \hat{Q}$ et $\vec{u} \hat{Q}+d(\vec{u} \cdot \hat{Q})$. Si la dynamique est harmonique, cette probabilité peut être calculée à partir des fonctions d'onde de l'oscillateur harmonique. Bloch [36] a montré que la fonction $f(\vec{u} \cdot \hat{Q})$ est une gaussienne

$$
f(\vec{u} \cdot \hat{Q})=C \exp \left(-\frac{(\vec{u} \cdot \hat{Q})^{2}}{2 \sigma(\hat{Q})^{2}}\right)
$$

avec $\sigma(\hat{Q})^{2}=\overline{(\vec{u} \cdot \hat{Q})^{2}}$ le déplacement carré moyen le long de la direction $\hat{Q}$ et $C$ une constante de normalisation.

La transformée de Fourier de la fonction $f(\vec{u} \cdot \hat{Q})$ s'obtient

$$
\int_{-\infty}^{\infty} \exp \left(-\frac{(\vec{u} \cdot \hat{Q})^{2}}{2 \sigma^{2}}\right) \exp (-i \vec{Q} \cdot \vec{u}) d(\vec{u} \cdot \hat{Q})=\sqrt{2 \pi \sigma^{2}} \exp \left(-\frac{1}{2}(\vec{Q} \cdot \vec{u})^{2}\right) \text {. }
$$

L'expression

$$
\exp \left(-\frac{1}{2}(\vec{Q} \cdot \vec{u})^{2}\right)
$$

n'est donc rien d'autre que le facteur de forme de la probabilité de résidence de l'ion. Si l'ion est très délocalisé, c'est-à-dire si les amplitudes de vibration sont élevées, le facteur de forme va décroître rapidement avec $|\vec{Q}|$. Si par contre l'ion est localisé, alors le facteur de forme variera peu (voir figure 36). Dans l'analogie avec la diffraction optique par un réseau de fentes, le facteur de forme correspond à la transformée de Fourier de la fente individuelle.

\subsection{Diffusion cohérente élastique}

L'exponentielle

$$
\exp \left(\left\langle\left(\vec{Q} \cdot \vec{u}_{j^{\prime}}\right)\left(\vec{Q} \cdot \vec{u}_{j}(t)\right)\right\rangle\right)
$$

peut maintenant être développée en fonction de la fonction de corrélation ${ }^{63}$

$$
\aleph_{j^{\prime}, j}(\vec{Q}, t)=\left\langle\left(\vec{Q} \cdot \vec{u}_{j^{\prime}}\right)\left(\vec{Q} \cdot \vec{u}_{j}(t)\right)\right\rangle .
$$

Le premier terme est indépendant du temps et de ce fait persiste à $t=\infty$. Il donnera (se référer à la section (8.9)) une diffusion élastique. Avec (10.2) et

$$
\int_{-\infty}^{\infty} \exp (i \omega t) d t=2 \pi \hbar \delta(\hbar \omega)
$$

nous obtenons

$$
S_{\kappa, \kappa^{\prime}}^{\mathrm{el}}(\vec{Q}, \omega)=\sum_{j \in\left\{j_{\kappa}\right\}, j^{\prime} \in\left\{j_{\kappa^{\prime}}\right\}} \exp \left(-W_{j^{\prime}}(\vec{Q})-W_{j}(\vec{Q})\right) \exp \left(-i \vec{Q} \cdot\left(\vec{R}_{j^{\prime}}^{0}-\vec{R}_{j}^{0}\right)\right) \delta(\hbar \omega) .
$$

Quand le système diffractant est un cristal, les positions d'équilibre sont définies par rapport à une maille : 64

$$
\vec{R}_{j}^{0}=\vec{R}_{d}^{0}(\vec{l})=\vec{l}+\vec{d}
$$

\footnotetext{
63 Cette fonction prendra une telle importance lors de notre discussion que nous lui accordons un symbole spécifique, le aleph $\aleph$. 64 Par manque de symboles nous nous voyons dans l'obligation de changer légèrement la notation par rapport au chapitre I. Comme nous avons utilisé l'indice $\kappa$ dans ce chapitre pour distinguer les différents types de diffuseurs, nous employons ici l'indice $d$ pour distinguer les atomes dans la maille.
} 


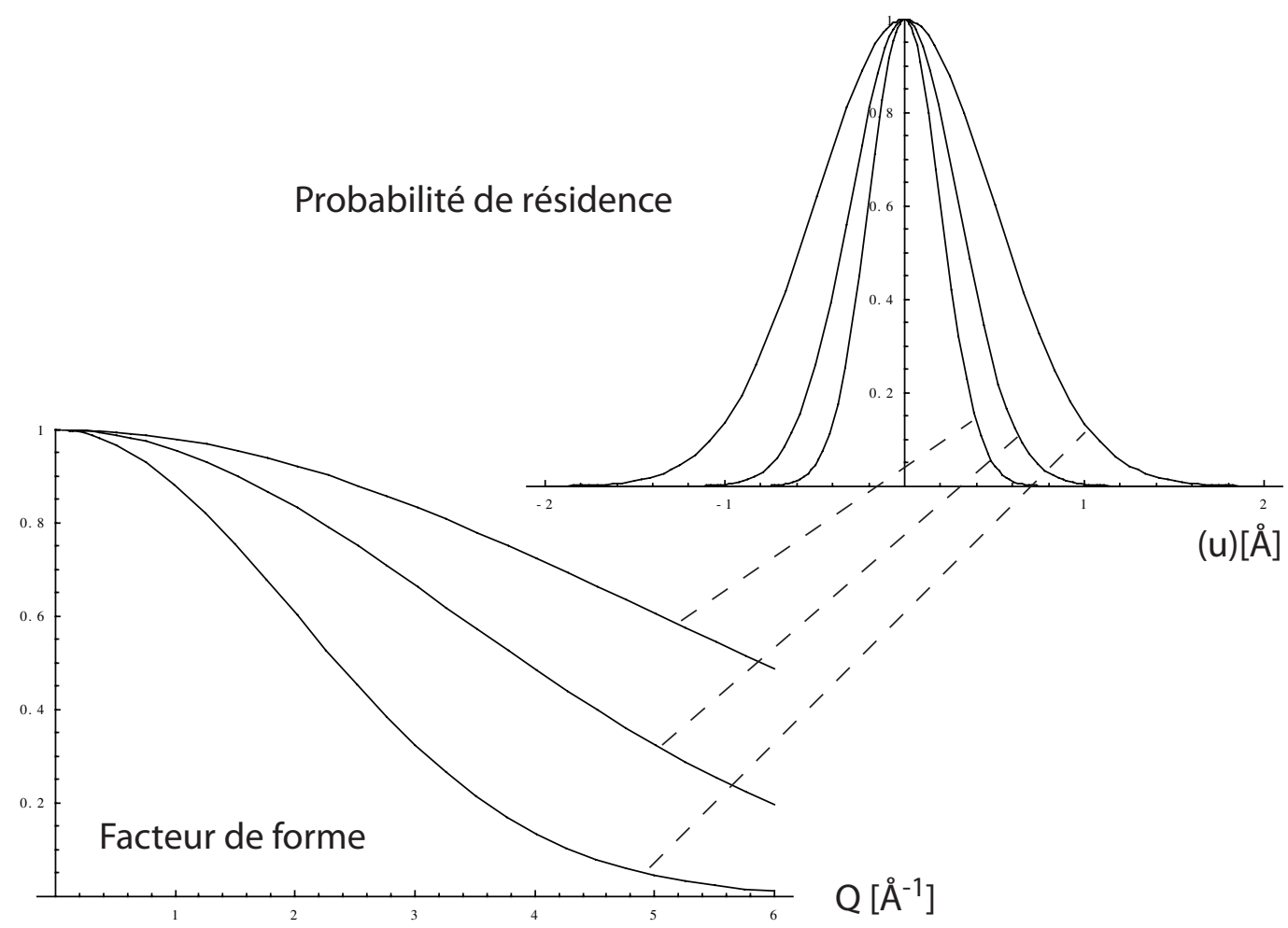

Figure 36. Probabilité de déplacement pour un ion le long d'une direction $\vec{Q}$ pour differentes valeurs de $\sigma^{2}=$ $\overline{(\vec{u} \cdot \hat{Q})^{2}}$. Facteurs de forme correspondants.

avec $\vec{l}$ la position de la maille et $\vec{d}$ la position de l'ion dans la maille. Pour la diffusion élastique $k_{i}=k_{f}$. La section efficace différentielle partielle cohérente devient

$$
\begin{aligned}
\left(\frac{d^{2} \sigma}{d \Omega d E_{f}}\right)_{\mathrm{coh}}^{\mathrm{el}}= & S_{\mathrm{coh}}(\vec{Q}, \hbar \omega=0) \\
= & \sum_{\kappa, \kappa^{\prime}} \bar{b}_{\kappa} \bar{b}_{\kappa^{\prime}}^{*} S_{\kappa, \kappa^{\prime}}(\vec{Q}, \hbar \omega=0) \\
= & \sum_{\vec{l}, \vec{l}^{\prime}} \exp \left(i \vec{Q} \cdot\left(\vec{l}-\vec{l}^{\prime}\right)\right) \\
& \left.\times \sum_{d, d^{\prime}} \bar{b}_{d} \bar{b}_{d^{\prime}}^{*} \exp \left(-W_{d}(\vec{Q})\right) \exp \left(-W_{d^{\prime}}(\vec{Q})\right) \exp (i \vec{Q} \cdot \vec{d})\right) \exp \left(-i \vec{Q} \cdot \vec{d}^{\prime}\right) \delta(\hbar \omega) \\
= & \frac{N}{r} \sum_{\vec{l}} \exp (i \vec{Q} \cdot \vec{l}) \\
& \left.\times \sum_{d, d^{\prime}} \bar{b}_{d} \bar{b}_{d^{\prime}}^{*} \exp \left(-W_{d}(\vec{Q})\right) \exp \left(-W_{d^{\prime}}(\vec{Q})\right) \exp (i \vec{Q} \cdot \vec{d})\right) \exp \left(-i \vec{Q} \cdot \vec{d}^{\prime}\right) \delta(\hbar \omega)
\end{aligned}
$$




$$
\begin{aligned}
= & \frac{N}{r} \sum_{\vec{l}} \exp (i \vec{Q} \cdot \vec{l}) \\
& \left.\times \mid \sum_{d} \bar{b}_{d} \exp \left(-W_{d}(\vec{Q})\right) \exp (i \vec{Q} \cdot \vec{d})\right)\left.\right|^{2} \delta(\hbar \omega),
\end{aligned}
$$

où nous avons utilisé

$$
\left.\sum_{\vec{l}} \exp \left(i \vec{Q} \cdot\left(\vec{l}-\vec{l}^{\prime}\right)\right)=\sum_{\vec{l}} \exp (i \vec{Q} \cdot \vec{l})\right)
$$

en exploitant la symétrie de translation de l'espace. ${ }^{65} r$ désigne comme dans le chapitre I le nombre d'ions dans la maille. L'expression

$$
F(\vec{Q})=\sum_{d} \bar{b}_{d} \exp \left(-W_{d}(\vec{Q})\right) \exp (i \vec{Q} \cdot \vec{d})
$$

est appelée le facteur de forme (nucléaire) de la maille. La grandeur analogue dans la diffraction par un réseau est la transformée de Fourier du motif multipliée par la transformée de Fourier de la fente individuelle.

La somme sur le réseau réciproque peut s'écrire sous la forme

$$
\sum_{\vec{l}} \exp (i \vec{Q} \cdot \vec{l})=\frac{(2 \pi)^{3}}{V_{\text {maille }}} \sum_{\vec{G}} \delta(\vec{Q}-\vec{G}) .
$$

Nous arrivons au résultat final pour la diffusion élastique

$$
\left(\frac{d^{2} \sigma}{d \Omega d E_{f}}\right)_{\text {coh }}^{\text {el }}=N_{\text {maille }} \frac{(2 \pi)^{3}}{V_{\text {maille }}}|F(\vec{Q})|^{2} \sum_{\vec{G}} \delta(\vec{Q}-\vec{G}) \delta(\hbar \omega) .
$$

$N_{\text {maille }}=N / r$ est le nombre de mailles dans le cristal.

Nous obtenons donc des pics de Bragg pour les directions de diffusion $\vec{Q}$ qui correspondent aux vecteurs du réseau réciproque $\vec{G}$. L'intensité de ces pics est modulée par le facteur de forme $F(\vec{Q})$. La situation est identique à celle des réseaux optiques tridimensionels que nous avions discutés dans la section 7. Ce résultat coïncide avec ce que nous avons trouvé pour un cristal composé d'atomes figés (voir (9.44)) à la différence que les facteurs de forme contiennent les facteurs de Debye-Waller. Quand les positions sont figées, les facteurs de forme en tant que transformées de Fourier de fonctions de Dirac deviennent des constantes.

\subsection{Diffusion cohérente à une excitation vibrationnelle}

Le prochain terme dans l'expansion de

$$
\exp \left(\left\langle\left(\vec{Q} \cdot \vec{u}_{j^{\prime}}\right)\left(\vec{Q} \cdot \vec{u}_{j}(t)\right)\right\rangle\right)
$$

est proportionnel à

$$
\aleph_{j^{\prime}, j}(\vec{Q}, t)=\left\langle\left(\vec{Q} \cdot \vec{u}_{j^{\prime}}\right)\left(\vec{Q} \cdot \vec{u}_{j}(t)\right)\right\rangle .
$$

Il implique le mouvement des ions et va de ce fait donner lieu à de la diffusion inélastique. Pour évaluer cette expression dans la base des phonons nous devons exprimer les opérateurs de création

\footnotetext{
65 Dans un cristal toutes les mailles sont équivalentes. Le choix de l'origine ne doit pas influer sur une fonction qui ne dépend que des distances entre les mailles.
} 
et d'annihilation des vibrations en tant qu'opérateurs de Heisenberg. Formellement,

$$
\begin{aligned}
\mathbf{a}_{i}(t) & =\exp \left(i \mathbf{H} \frac{t}{\hbar}\right) \mathbf{a}_{i} \exp \left(-i \mathbf{H} \frac{t}{\hbar}\right), \\
\mathbf{a}_{i}^{+}(t) & =\exp \left(i \mathbf{H} \frac{t}{\hbar}\right) \mathbf{a}_{i}^{+} \exp \left(-i \mathbf{H} \frac{t}{\hbar}\right) .
\end{aligned}
$$

Les opérateurs de Heisenberg satisfont à la relation $[13,14]$

$$
\frac{d}{d t} \mathbf{A}(t)=\frac{i}{\hbar}[\mathbf{H}, \mathbf{A}(t)]
$$

Dans le cas des phonons (voir chapitre I) l'Hamiltonien s'écrit comme

$$
\mathbf{H}=\sum_{i=1}^{3 N} \hbar \omega_{i}\left(\mathbf{a}_{i}^{+} \mathbf{a}_{i}+\frac{1}{2}\right)
$$

En utilisant les relations de commutation

$$
\left[\mathbf{a}_{i}, \mathbf{a}_{i^{\prime}}^{+}\right]=\delta_{i, i^{\prime}}
$$

on obtient

$$
\begin{aligned}
& \frac{d}{d t} \mathbf{a}_{i}(t)=\frac{i}{\hbar}\left[\mathbf{H}, \mathbf{a}_{i}(t)\right]=-i \omega_{i} \mathbf{a}_{i}(t) \\
& \frac{d}{d t} \mathbf{a}_{i}^{+}(t)=\frac{i}{\hbar}\left[\mathbf{H}, \mathbf{a}_{i}^{+}(t)\right]=i \omega_{i} \mathbf{a}_{i}^{+}(t)
\end{aligned}
$$

et donc

$$
\begin{aligned}
& \left.\left.\mathbf{a}_{i}(t)=\mathbf{a}_{i}(t=0) \exp \left(-i \omega_{i} t\right)\right)=\mathbf{a}_{i} \exp \left(-i \omega_{i} t\right)\right) \\
& \left.\left.\mathbf{a}_{i}(t)^{+}=\mathbf{a}_{i}^{+}(t=0) \exp \left(i \omega_{i} t\right)\right)=\mathbf{a}_{i}^{+} \exp \left(i \omega_{i} t\right)\right)
\end{aligned}
$$

Ceci nous amène à

$$
\mathbf{u}_{\alpha}(j ; t)=\sum_{i} \sqrt{\frac{\hbar}{2 m_{j} \omega_{i}}}\left[e_{i}(\alpha, j) \mathbf{a}_{i} \exp \left(-i \omega_{i} t\right)+e_{i}^{*}(\alpha, j) \mathbf{a}_{i}^{+} \exp \left(i \omega_{i} t\right)\right] .
$$

Cette expression nous permet de calculer la fonction de corrélation $\aleph_{j^{\prime}, j}(\vec{Q}, t)$.

$$
\begin{aligned}
\left\langle\left(\vec{Q} \cdot \vec{u}_{j^{\prime}}\right)\left(\vec{Q} \cdot \vec{u}_{j}(t)\right)\right\rangle= & \sum_{\alpha, \beta} Q_{\alpha} Q_{\beta}\left\langle\mathbf{u}_{\alpha}\left(j^{\prime} ; t=0\right) \mathbf{u}_{\beta}(j ; t)\right\rangle \\
= & \frac{\hbar}{2} \sum_{\alpha, \beta} \frac{Q_{\alpha} Q_{\beta}}{\sqrt{m_{j} m_{j^{\prime}}}} \sum_{i, i^{\prime}} \sqrt{\frac{1}{\omega_{i} \omega_{i^{\prime}}}} \\
& \times\left\langle[ e _ { i } ( \alpha , j ^ { \prime } ) \mathbf { a } _ { i } + e _ { i } ^ { * } ( \alpha , j ^ { \prime } ) \mathbf { a } _ { i } ^ { + } ] \left[ e_{i^{\prime}}(\beta, j) \mathbf{a}_{i^{\prime}} \exp \left(-i \omega_{i^{\prime}} t\right)\right.\right. \\
& \left.\left.+e_{i^{\prime}}^{*}(\beta, j) \mathbf{a}_{i^{\prime}}^{+} \exp \left(i \omega_{i^{\prime}} t\right)\right]\right\rangle .
\end{aligned}
$$

Cette expression se simplifie considérablement si nous prenons en compte les règles de calcul pour les valeurs moyennes de produits d'opérateurs de création et d'annihilation (voir chapitre I) :

$$
\begin{aligned}
\left\langle\mathbf{a}_{i} \mathbf{a}_{i^{\prime}}\right\rangle & =0, \\
\left\langle\mathbf{a}_{i}^{+} \mathbf{a}_{i^{\prime}}^{+}\right\rangle & =0, \\
\left\langle\mathbf{a}_{i}^{+} \mathbf{a}_{i^{\prime}}\right\rangle & =n\left(\omega_{i}\right) \delta_{i, i^{\prime}},
\end{aligned}
$$




$$
\left\langle\mathbf{a}_{i} \mathbf{a}_{i^{\prime}}^{+}\right\rangle=\left(1+n\left(\omega_{i}\right)\right) \delta_{i, i^{\prime}}
$$

avec $n\left(\omega_{i}\right)$ le facteur d'occupation de l'oscillateur de fréquence $\omega_{i}$. Ainsi

$$
\begin{aligned}
\langle(\vec{Q} & \left.\left.\cdot \vec{u}_{j^{\prime}}\right)\left(\vec{Q} \cdot \vec{u}_{j}(t)\right)\right\rangle \\
= & \frac{\hbar}{2} \sum_{\alpha, \beta} \frac{Q_{\alpha} Q_{\beta}}{\sqrt{m_{j} m_{j^{\prime}}}} \sum_{i, i^{\prime}} \sqrt{\frac{1}{\omega_{i} \omega_{i^{\prime}}} \times \delta_{i, i^{\prime}}} \\
& \times\left\{\left[e_{i}\left(\alpha, j^{\prime}\right) e_{i^{\prime}}^{*}(\beta, j)\left\langle\mathbf{a}_{i} \mathbf{a}_{i^{\prime}}^{+}\right\rangle \exp \left(i \omega_{i^{\prime}} t\right)\right]+\left[e_{i}^{*}\left(\alpha, j^{\prime}\right) e_{i^{\prime}}(\beta, j)\left\langle\mathbf{a}_{i}^{+} \mathbf{a}_{i^{\prime}}\right) \exp \left(-i \omega_{i^{\prime}} t\right)\right]\right\} \\
= & \frac{\hbar}{2} \sum_{\alpha, \beta} \frac{Q_{\alpha} Q_{\beta}}{\sqrt{m_{j} m_{j^{\prime}}}} \sum_{i} \frac{1}{\omega_{i}} \\
& \times\left\{\left[e_{i}\left(\alpha, j^{\prime}\right) e_{i}^{*}(\beta, j)\left(1+n\left(\omega_{i}\right)\right) \exp \left(i \omega_{i} t\right)\right]+\left[e_{i}^{*}\left(\alpha, j^{\prime}\right) e_{i}(\beta, j) n\left(\omega_{i}\right) \exp \left(-i \omega_{i} t\right)\right]\right\} \\
= & \frac{\hbar}{2} \sum_{i}\left\{\left[\frac{A_{i}\left(\vec{Q} ; j^{\prime}, j\right)}{\omega_{i}}\left(1+n\left(\omega_{i}\right)\right) \exp \left(i \omega_{i} t\right)\right]+\left[\frac{A_{i}\left(\vec{Q} ; j, j^{\prime}\right)}{\omega_{i}} n\left(\omega_{i}\right) \exp \left(-i \omega_{i} t\right)\right]\right\}
\end{aligned}
$$

avec

$$
A_{i}\left(\vec{Q} ; j^{\prime}, j\right)=\frac{\left(\vec{Q} \cdot \vec{e}_{i}\left(j^{\prime}\right)\right) \cdot\left(\vec{Q} \cdot \vec{e}_{i}(j)\right)^{*}}{\sqrt{m_{j^{\prime}} m_{j}}}
$$

En remplaçant cette expression dans (10.18) nous obtenons pour les fonctions de diffusion partielles

$$
\begin{aligned}
S_{\kappa, \kappa^{\prime}}^{1-\mathrm{ph}}(\vec{Q}, \omega) & \frac{1}{2 \pi \hbar} \sum_{j \in\left\{j_{k}\right\}, j^{\prime} \in\left\{j_{\kappa^{\prime}}\right\}} e^{-i \vec{Q} \cdot\left(\vec{R}_{j^{\prime}}^{0}-\vec{R}_{j}^{0}\right)} e^{-W_{j^{\prime}}(\vec{Q})} e^{-W_{j}(\vec{Q})} \int_{-\infty}^{\infty} d t\left\langle\left(\vec{Q} \cdot \vec{u}_{j^{\prime}}\right)\left(\vec{Q} \cdot \vec{u}_{j}(t)\right)\right\rangle \\
= & \frac{1}{4 \pi} \sum_{j \in\left\{j_{k}\right\}, j^{\prime} \in\left\{j_{k^{\prime}}\right\}} e^{-i \vec{Q} \cdot\left(\vec{R}_{j^{\prime}}^{0}-\vec{R}_{j}^{0}\right)} e^{-W_{j^{\prime}}(\vec{Q})} e^{-W_{j}(\vec{Q})} \\
& \times \int_{-\infty}^{\infty} d t \sum_{i}\left\{\left[\frac{A_{i}\left(\vec{Q} ; j^{\prime}, j\right)}{\omega_{i}}\left(1+n\left(\omega_{i}\right)\right) \exp \left(i \omega_{i} t\right)\right]+\left[\frac{A_{i}\left(\vec{Q} ; j, j^{\prime}\right)}{\omega_{i}} n\left(\omega_{i}\right) \exp \left(-i \omega_{i} t\right)\right]\right\} \\
= & \left.\left.\frac{1}{2} \sum_{j \in\left\{j_{k}\right\}, j^{\prime} \in\left\{j_{\kappa^{\prime}}\right\}} e^{-i \vec{Q} \cdot\left(\vec{R}_{j^{\prime}}^{0}-\vec{R}_{j}^{0}\right)} e^{-W_{j^{\prime}}(\vec{Q})} e^{-W_{j}(\vec{Q})}\right]\right\} \\
& \times \sum_{i}\left\{\left[\frac{A_{i}\left(\vec{Q} ; j^{\prime}, j\right)}{\omega_{i}}\left(1+n\left(\omega_{i}\right)\right) \delta\left(\omega-\omega_{i}\right)\right]+\left[\frac{A_{i}\left(\vec{Q} ; j, j^{\prime}\right)}{\omega_{i}} n\left(\omega_{i}\right) \delta\left(\omega+\omega_{i}\right)\right]\right\}
\end{aligned}
$$

où nous avons utilisé

$$
\int_{-\infty}^{\infty} \exp (i \omega t) d t=2 \pi \delta(\omega)
$$


Avec ce résultat nous pouvons calculer la fonction de diffusion cohérente.

$$
\begin{aligned}
& S_{\mathrm{coh}}^{1-\mathrm{ph}}(\vec{Q}, \omega)=\sum_{\kappa, \kappa^{\prime}} \bar{b}_{\kappa} \cdot \bar{b}_{\kappa^{\prime}}^{*} S_{\kappa, \kappa^{\prime}}^{1-\mathrm{ph}}(\vec{Q}, \omega) \\
& =\frac{1}{2} \sum_{\kappa, \kappa^{\prime}} \bar{b}_{\kappa} \cdot \bar{b}_{\kappa^{\prime}}^{*} \sum_{j \in\left\{j_{\kappa}\right\}, j^{\prime} \in\left\{j_{\kappa^{\prime}}\right\}} e^{-i \vec{Q} \cdot\left(\vec{R}_{j^{\prime}}^{0}-\vec{R}_{j}^{0}\right)} e^{-W_{j^{\prime}}(\vec{Q})} e^{-W_{j}(\vec{Q})} \\
& \quad \times \sum_{i}\left\{\left[\frac{A_{i}\left(\vec{Q} ; j^{\prime}, j\right)}{\omega_{i}}\left(1+n\left(\omega_{i}\right)\right) \delta\left(\omega-\omega_{i}\right)\right]+\left[\frac{A_{i}\left(\vec{Q} ; j, j^{\prime}\right)}{\omega_{i}} n\left(\omega_{i}\right) \delta\left(\omega+\omega_{i}\right)\right]\right\} .
\end{aligned}
$$

Une manière alternative d'écrire cette expression est

$$
S_{\mathrm{coh}}^{1-\mathrm{ph}}(\vec{Q}, \omega)=\frac{1}{2} \sum_{i} \frac{\left|F_{i}(\vec{Q})\right|^{2}}{\omega_{i}}\left\{\left[\left(1+n\left(\omega_{i}\right)\right) \delta\left(\omega-\omega_{i}\right)\right]+\left[n\left(\omega_{i}\right) \delta\left(\omega+\omega_{i}\right)\right]\right\}
$$

avec

$$
\begin{aligned}
& \left|F_{i}(\vec{Q})\right|^{2} \\
& \quad=\sum_{\kappa, \kappa^{\prime}} \frac{\bar{b}_{\kappa} \cdot \bar{b}_{\kappa^{\prime}}^{*}}{\sqrt{m_{\kappa^{\prime}} m_{\kappa}}} \sum_{j \in\left\{j_{\kappa}\right\}, j^{\prime} \in\left\{j_{\kappa^{\prime}}\right\}} e^{-i \vec{Q} \cdot \vec{R}_{j^{\prime}}^{0}} e^{i \vec{Q} \cdot \vec{R}_{j}^{0}} e^{-W_{j^{\prime}}(\vec{Q})} e^{-W_{j}(\vec{Q})}\left(\vec{Q} \cdot \vec{e}_{i}\left(j^{\prime}\right)\right) \cdot\left(\vec{Q} \cdot \vec{e}_{i}(j)\right)^{*} \\
& \quad=\left|\sum_{\kappa} \frac{\bar{b}_{\kappa}}{\sqrt{m_{\kappa}}} \sum_{j \in\left\{j_{\kappa}\right\}} e^{-i \vec{Q} \cdot \vec{R}_{j}^{0}} e^{-W_{j}(\vec{Q})}\left(\vec{Q} \cdot \vec{e}_{i}(j)\right)\right|^{2} \cdot
\end{aligned}
$$

La fonction $F_{i}(\vec{Q})$ peut être considerée comme le facteur de forme de la vibration $i$.

Les fonctions de Dirac assurent que la diffusion des neutrons n'a lieu que quand le transfert d'energie $\pm \hbar \omega$ correspond à une fréquence de vibration. Quand $\hbar \omega$ est positif ${ }^{66}$ le neutron va perdre son énergie lors de la diffusion en créant une excitation dans la cible. Le niveau d'occupation d'un état vibrationnel est indiqué par les facteurs $n(\omega)$ (voir figure 37). Du fait que la création d'une excitation est proportionnelle à $n\left(\omega_{i}\right)+1$ ce canal de diffusion est toujours opérationnel ${ }^{67}$ même à très basse température, quand le système se trouve dans son état fondamental et $n\left(\omega_{i}\right)$ tend vers zéro. Pour $\hbar \omega<0$ le neutron gagne de l'énergie lors de la diffusion. Ce processus est soumis à la condition que les oscillateurs soient excités.

L'intensité de la diffusion dépendra entre autres des termes

$$
\frac{\left(\vec{Q} \cdot \vec{e}_{i}\left(j^{\prime}\right)\right) \cdot\left(\vec{Q} \cdot \vec{e}_{i}(j)\right)^{*}}{\sqrt{m_{j^{\prime}} m_{j}}}\left(1+n\left(\omega_{i}\right)\right), \quad \omega>0
$$

et

$$
\frac{\left(\vec{Q} \cdot \vec{e}_{i}(j)\right) \cdot\left(\vec{Q} \cdot \vec{e}_{i}\left(j^{\prime}\right)\right)^{*}}{\sqrt{m_{j^{\prime}} m_{j}}} n\left(\omega_{i}\right), \quad \omega<0
$$

\footnotetext{
66 Attention: cette convention n'est pas toujours respectée dans la littérature. Il est toujours utile d'ajouter les indicateurs "perte" ou "gain d'énergie pour le neutron".

67 Ce n'est évidemment vrai que si les conditions cinématiques (voir section 5.10) sont réunies.
} 


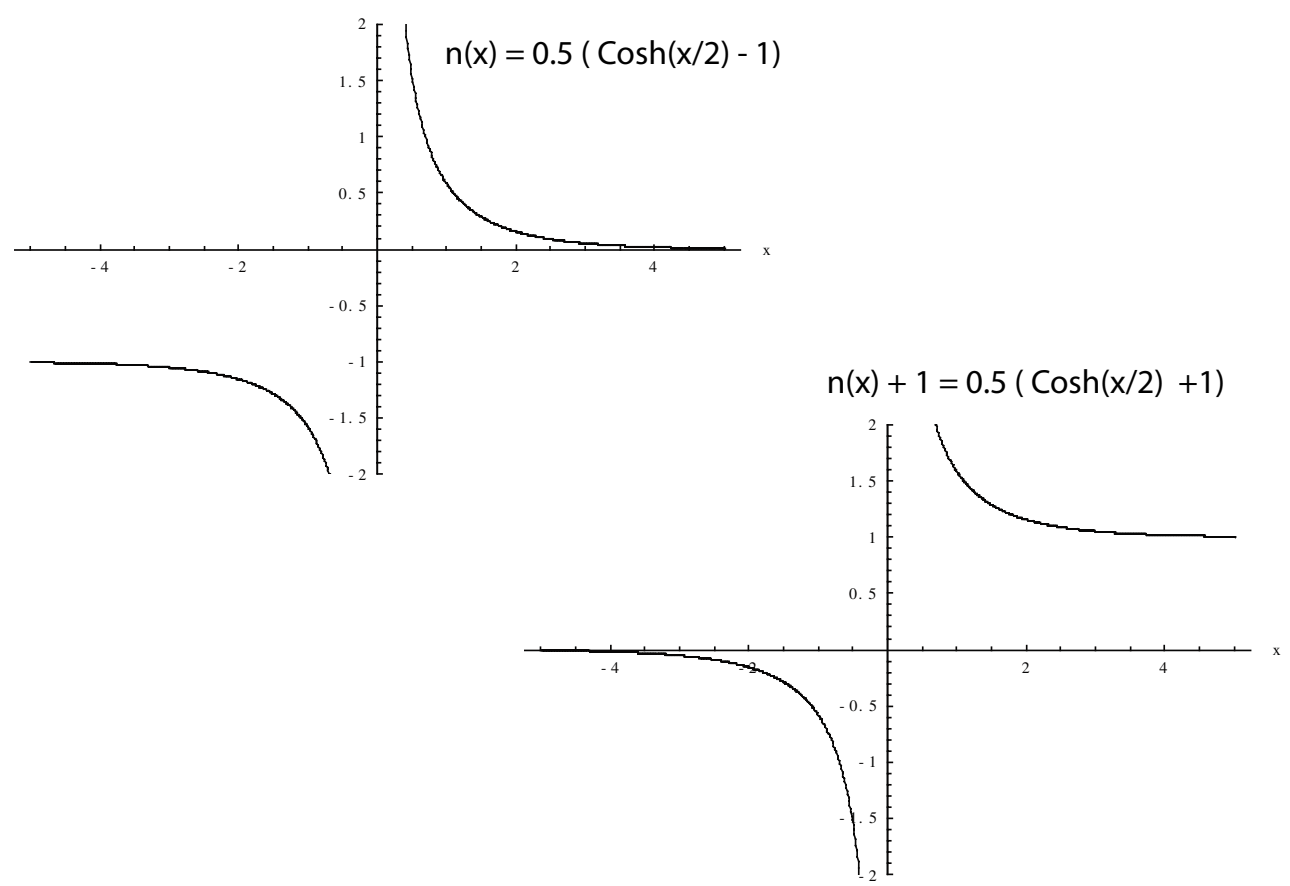

Figure 37. Facteur d'occupation $n(x)=(1 / 2)(\cosh (x / 2)-1)$ et $n(x)+1=(1 / 2)(\cosh (x / 2)+1)$ avec $x=$ $(\hbar \omega) /\left(k_{\mathrm{B}} T\right)$. On notera que $n(x)+1=n(-x)$. Dans notre notation $\hbar \omega>0$ correspond à une perte d'énergie pour le neutron. Le 1 dans $n(x)+1$ est l'expression mathématique du fait que la création d'une excitation est toujours possible, même si $n(x)$ tend vers zéro pour $T \rightarrow 0$. Du côté gain d'énergie, c'est-à-dire pour $\hbar \omega<0$, la diffusion cesse d'être opérationnelle pour $\hbar \omega \gg k_{\mathrm{B}} T$. Mais attention, cette affirmation n'est vraie que si le système se trouve en équilibre. L'hydrogène ainsi que le deutérium sont des exemples bien connus de systèmes qui prennent beaucoup de temps pour s'équilibrer à basse température. Le signal du côté perte peut alors servir comme indicateur de l'état d'équilibre de l'échantillon [16].

En comparant ces expressions avec celles que nous avons obtenues pour les déplacements moyens (10.19)

$$
\left\langle\mathbf{u}(\alpha, j) \mathbf{u}(\alpha, j)^{+}\right\rangle=\sum_{i=1}^{3 N} \frac{\left|e_{i}(\alpha, j)\right|^{2} \hbar}{2 m_{j} \omega_{i}}\left(2 n\left(\omega_{i}\right)+1\right)
$$

nous pouvons déduire que l'intensité de la diffusion à un phonon est étroitement liée aux amplitudes de fluctuations (projetées sur le vecteur $\vec{Q}$ ) associées aux modes de vibration ${ }^{68}$. En d'autres termes, les neutrons sondent les amplitudes des fluctuations. La présence de fluctuations de point zéro assure la diffusion inélastique à très basse température.

\subsection{Règle de somme $f$ pour les vibrations}

Pour donner une application concrète de la règle de somme f nous allons maintenant calculer le premier moment pour la fonction de diffusion à un phonon. Du fait que l'Hamiltonien pour le cristal harmonique

68 Selon l'expression (10.19). Les facteurs de température sont liés aux amplitudes carrées moyennes d'une vibration

$$
\left(n\left(\omega_{i}\right)+1\right)+n\left(\omega_{i}\right) \propto\left\langle u_{i}^{2}\right\rangle .
$$


ne contient que des interactions qui dépendent des coordonnées des ions, la règle de somme $f$ doit être applicable.

Nous obtenons à partir de (10.57)

$$
\begin{aligned}
& \int_{-\infty}^{\infty} d(\hbar \omega)(\hbar \omega) S_{\mathrm{coh}}^{1-\mathrm{ph}}(\vec{Q}, \omega) \\
& =\frac{\hbar^{2}}{2} \sum_{i}\left|F_{i}(\vec{Q})\right|^{2} \int_{-\infty}^{\infty} d \omega \frac{\omega}{\omega_{i}}\left\{\left[\left(1+n\left(\omega_{i}\right)\right) \delta\left(\omega-\omega_{i}\right)\right]+\left[n\left(\omega_{i}\right) \delta\left(\omega+\omega_{i}\right)\right]\right\} \\
& =\frac{\hbar^{2}}{2} \sum_{i}\left|F_{i}(\vec{Q})\right|^{2}\left\{\left(1+n\left(\omega_{i}\right)-n\left(\omega_{i}\right)\right\}\right. \\
& =\frac{\hbar^{2}}{2} \sum_{i}\left|F_{i}(\vec{Q})\right|^{2} .
\end{aligned}
$$

Le moment d'ordre un est donc équivalent à la somme des facteurs de forme pris au carré. Cette somme se calcule selon (10.58) comme

$$
\begin{aligned}
& \sum_{i}\left|F_{i}(\vec{Q})\right|^{2} \\
& \quad=\sum_{\kappa, \kappa^{\prime}} \frac{\bar{b}_{\kappa} \cdot \vec{b}_{\kappa^{\prime}}^{*}}{\sqrt{m_{\kappa^{\prime}} m_{\kappa}}} \sum_{j \in\left\{j_{\kappa}\right\}, j^{\prime} \in\left\{j_{k^{\prime}}\right\}} e^{-i \vec{Q} \cdot \vec{R}_{j^{\prime}}^{0}} e^{i \vec{Q} \cdot \vec{R}_{j}^{0}} e^{-W_{j^{\prime}}(\vec{Q})} e^{-W_{j}(\vec{Q})} \sum_{i}\left(\vec{Q} \cdot \vec{e}_{i}\left(j^{\prime}\right)\right) \cdot\left(\vec{Q} \cdot \vec{e}_{i}(j)\right)^{*} .
\end{aligned}
$$

Les vecteurs propres forment une base complète dans laquelle tout mouvement des ions doit pouvoir s'exprimer. Ils doivent donc formellement satisfaire à la condition de fermeture (voir chapitre I)

$$
\sum_{i} \vec{e}_{i}\left(\alpha, j^{\prime}\right) \vec{e}_{i}(\beta, j)^{*}=\delta_{\alpha, \beta} \delta_{j, j^{\prime}} .
$$

Alors

$$
\begin{aligned}
\sum_{i}\left(\vec{Q} \cdot \vec{e}_{i}\left(j^{\prime}\right)\right) \cdot\left(\vec{Q} \cdot \vec{e}_{i}(j)\right)^{*} & =\sum_{\alpha, \beta} Q_{\alpha} Q_{\beta} \sum_{i} \vec{e}_{i}\left(\alpha, j^{\prime}\right) \vec{e}_{i}(\beta, j)^{*} \\
& =\sum_{\alpha, \beta} Q_{\alpha} Q_{\beta} \delta_{\alpha, \beta} \delta_{j, j^{\prime}} \\
& =Q^{2} \delta_{j, j^{\prime}},
\end{aligned}
$$

et donc

$$
\begin{aligned}
\int_{-\infty}^{\infty} d(\hbar \omega)(\hbar \omega) S_{\mathrm{coh}}^{1-\mathrm{ph}}(\vec{Q}, \omega) & =\frac{\hbar^{2}}{2} \sum_{i}\left|F_{i}(\vec{Q})\right|^{2} \\
& =\frac{\hbar^{2} Q^{2}}{2} \sum_{\kappa} N_{\kappa} \frac{\bar{b}_{\kappa} \cdot \bar{b}_{\kappa}^{*}}{m_{\kappa}} e^{-2 W_{\kappa}(\vec{Q})} \\
& =\sum_{\kappa} N_{\kappa} \bar{b}_{\kappa} \cdot \bar{b}_{\kappa}^{*} e^{-2 W_{\kappa}(\vec{Q})} E_{\kappa}^{r}(Q) .
\end{aligned}
$$

Cette expression pour la partie phonon est à comparer avec celle obtenue pour la fonction de diffusion totale (8.167). Nous remarquons que la seule différence est le facteur de Debye-Waller. Ce facteur introduit une dépendance en température et fait en sorte que le premier moment pour les phonons reste 
systématiquement en dessous du premier moment total. La différence est à attribuer à la contribution des multiphonons, dont nous parlerons dans la section 11 .

La règle de somme nous apprend -sans aucun calcul- que pour n'importe quel vecteur de diffusion $\vec{Q}$, on obtiendra toujours un signal de chaque atome. L'intensité de ce signal dépendra, en dehors des longueurs de diffusion, de l'inverse de la masse et de l'inverse de la fréquence des modes auxquels l'atome participe. Si nous avions voulu, nous aurions pratiquement pu déduire la forme de la fonction de diffusion pour les phonons à partir de la seule règle de somme.

\subsection{Diffusion cohérente à un phonon dans un cristal}

Les expressions obtenues jusqu'ici sont valables pour n'importe quel système harmonique. Elles peuvent sembler complexes mais elles permettent le calcul exact de la diffusion inélastique sur la seule base des fréquences et des vecteurs propres. Naturellement, le calcul de ces fréquences et de ces vecteurs propres constitue une tâche gigantesque pour un amorphe. Extraire des mesures des informations sur le caractère des modes sans passer par un calcul ou même par un modèle n'est pas complètement impossible mais restera toujours un procédé assez arbitraire pour des systèmes amorphes complexes. On pourra, au mieux, espérer identifier grossièrement la nature des mouvements (acoustique, optique, mouvement d'unités rigides etc.) à partir de la dépendance en $Q$ d'une bande de fréquence donnée $[39,40]$.

La situation devient beaucoup plus favorable quand nous travaillons avec des cristaux. Du fait de la symétrie de translation, les opérateurs de déplacement s'écrivent comme (voir chapitre I) ${ }^{69}$

$$
\begin{aligned}
\mathbf{u}_{\alpha}(\vec{l}, d) & =\sqrt{\frac{r}{N}} \sum_{i, \vec{k}} \sqrt{\frac{\hbar}{2 m_{d} \omega_{i}(\vec{k})}}\left[e_{i}(\alpha, d \mid \vec{k}) e^{i \vec{k} \cdot \vec{l}} \mathbf{a}_{i}(\vec{k})+e_{i}^{*}(\alpha, d \mid \vec{k}) e^{-i \vec{k} \cdot \vec{l}} \mathbf{a}_{i}^{+}(\vec{k})\right] \\
& =\sqrt{\frac{r}{N}} \sum_{i, \vec{k}} \sqrt{\frac{\hbar}{2 m_{\kappa} \omega_{i}(\vec{k})}} e_{i}(\alpha, \kappa \mid \vec{k})\left(\mathbf{a}_{i}(\vec{k})+\mathbf{a}_{i}^{+}(-\vec{k})\right) e^{i \vec{k} \cdot \vec{l}},
\end{aligned}
$$

où la deuxième ligne est assujettie à la condition $e_{j}(\vec{k})=e_{j}(-\vec{k})$. Avec cette expression pour l'opérateur de déplacement, nous pouvons calculer $\aleph_{j^{\prime}, j}(\vec{Q}, t)=\aleph_{\vec{l}^{\prime}, l, d^{\prime}, d}(\vec{Q}, t)$.

$$
\begin{aligned}
\langle(\vec{Q} & \left.\left.\cdot \overrightarrow{\mathbf{u}}\left(\vec{l}^{\prime}, d^{\prime}\right)\right)(\vec{Q} \cdot \overrightarrow{\mathbf{u}}(\vec{l}, d ; t))\right\rangle \\
= & \frac{\hbar}{2} \frac{r}{N} \sum_{\alpha, \beta} \frac{Q_{\alpha} Q_{\beta}}{\sqrt{m_{d} m_{d^{\prime}}}} \sum_{i, i^{\prime}} \sum_{\vec{k}_{,} \vec{k}^{\prime}} \sqrt{\frac{1}{\omega_{i}(\vec{k}) \omega_{i^{\prime}}\left(\vec{k}^{\prime}\right)} \times \delta_{i, i^{\prime}} \times \delta_{\vec{k}, \vec{k}^{\prime}}} \\
& \times\left\{\left[e_{i}\left(\alpha, d^{\prime} \mid \vec{k}\right) e_{i^{\prime}}^{*}\left(\beta, d \mid \vec{k}^{\prime}\right) \exp \left(i\left(\vec{k} \cdot \vec{l}^{\prime}-\vec{k}^{\prime} \cdot \vec{l}\right)\right) \exp \left(i \omega_{i^{\prime}} t\right)\right]\left\langle\mathbf{a}_{i}\left(\vec{k}^{\prime}\right) \mathbf{a}_{i^{\prime}}^{+}(\vec{k})\right\rangle\right. \\
& \left.+\left[e_{i}^{*}\left(\alpha, d^{\prime} \mid \vec{k}\right) e_{i^{\prime}}\left(\beta, d \mid \vec{k}^{\prime}\right) \exp \left(i\left(-\vec{k} \cdot \vec{l}^{\prime}+\vec{k}^{\prime} \cdot \vec{l}\right)\right) \exp \left(-i \omega_{i^{\prime}} t\right)\right]\left\langle\mathbf{a}_{i}^{+}(\vec{k}) \mathbf{a}_{i^{\prime}}\left(\vec{k}^{\prime}\right)\right\rangle\right\} \\
= & \frac{\hbar}{2} \frac{r}{N} \sum_{\alpha, \beta} \frac{Q_{\alpha} Q_{\beta}}{\sqrt{m_{d} m_{d^{\prime}}}} \sum_{i, \vec{k}} \frac{1}{\omega_{i}(\vec{k})} \\
& \times\left\{\left[e_{i}\left(\alpha, d^{\prime} \mid \vec{k}\right) e_{i}^{*}(\beta, d \mid \vec{k}) \exp \left(i\left(\vec{k} \cdot\left(\vec{l}^{\prime}-\vec{l}\right)\right)\right)\left(1+n\left(\omega_{i}\right)\right) \exp \left(i \omega_{i} t\right)\right]\right. \\
& \left.+\left[e_{i}^{*}\left(\alpha, d^{\prime} \mid \vec{k}\right) e_{i}(\beta, d \mid \vec{k}) \exp \left(-i\left(\vec{k} \cdot\left(\vec{l}^{\prime}-\vec{l}\right)\right)\right) n\left(\omega_{i}\right) \exp \left(-i \omega_{i} t\right)\right]\right\}
\end{aligned}
$$

${ }^{69}$ Attention, le vecteur $\vec{k}$ est le vecteur d'onde du phonon. Il est défini dans la première zone de Brillouin et n'a aucun lien avec le vecteur d'onde du neutron. 


$$
\begin{aligned}
= & \frac{\hbar}{2} \frac{r}{N} \sum_{i, \vec{k}}\left\{\left[\frac{A_{i, \vec{k}}\left(\vec{Q} ; d^{\prime}, d\right)}{\omega_{i}(\vec{k})}\left(1+n\left(\omega_{i}\right)\right) \exp \left(i\left(\vec{k} \cdot\left(\vec{l}^{\prime}-\vec{l}\right)\right)\right) \exp \left(i \omega_{i} t\right)\right]\right. \\
& \left.+\left[\frac{A_{i, \vec{k}}\left(\vec{Q} ; d, d^{\prime}\right)}{\omega_{i}(\vec{k})} n\left(\omega_{i}\right) \exp \left(-i\left(\vec{k} \cdot\left(\vec{l}^{\prime}-\vec{l}\right)\right)\right) \exp \left(-i \omega_{i} t\right)\right]\right\}
\end{aligned}
$$

avec

$$
A_{i, \vec{k}}\left(\vec{Q} ; d^{\prime}, d\right)=\frac{\left(\vec{Q} \cdot \vec{e}_{i}\left(d^{\prime} \mid \vec{k}\right)\right)\left(\vec{Q} \cdot \vec{e}_{i}(d \mid \vec{k})^{*}\right)}{\sqrt{m_{d} m_{d^{\prime}}}} .
$$

Dans le cas du cristal, nous avons au maximum $r$ types d'atome dans notre système, avec $r$ le nombre d'atomes dans la maille primitive. Sans information supplémentaire concernant d'éventuelles symétries du cristal, nous devons les considérer tous distincts. Nous obtenons alors les fonctions de diffusion partielles suivantes:

$$
\begin{aligned}
S_{d, d^{\prime}}^{1-\mathrm{ph}}(\vec{Q}, \omega) & =\frac{1}{2 \pi \hbar} \sum_{\vec{l}, \vec{l}^{\prime}} e^{\left.-i \vec{Q} \cdot \vec{l}^{\prime}+\vec{d}^{\prime}-\vec{l}-\vec{d}\right)} e^{-W_{d^{\prime}}(\vec{Q})} e^{-W_{d}(\vec{Q})} \int_{-\infty}^{\infty} d t\left\langle\left(\vec{Q} \cdot \overrightarrow{\mathbf{u}}\left(\vec{l}^{\prime}, d^{\prime}\right)\right)(\vec{Q} \cdot \overrightarrow{\mathbf{u}}(\vec{l}, d ; t))\right\rangle \\
= & \frac{r}{4 \pi N} e^{-W_{d^{\prime}}(\vec{Q})} e^{-W_{d}(\vec{Q})} e^{-i \vec{Q} \cdot\left(\overrightarrow{d^{\prime}}-\vec{d}\right)} \sum_{\vec{l}, \vec{l}^{\prime}} e^{-i \vec{Q} \cdot\left(\overrightarrow{l^{\prime}}-\vec{l}\right)} \\
& \times \sum_{i, \vec{k}}\left\{\left[\frac{A_{i, \vec{k}}\left(\vec{Q} ; d^{\prime}, d\right)}{\omega_{i}(\vec{k})}\left(1+n\left(\omega_{i}(\vec{k})\right)\right) \exp \left(i\left(\vec{k} \cdot\left(\vec{l} l^{\prime}-\vec{l}\right)\right)\right) \int_{-\infty}^{\infty} d t \exp \left(i \omega_{i}(\vec{k}) t\right)\right]\right. \\
& \left.+\left[\frac{A_{i, \vec{k}}\left(\vec{Q} ; d, d^{\prime}\right)}{\omega_{i}(\vec{k})} n\left(\omega_{i}(\vec{k})\right) \exp \left(-i\left(\vec{k} \cdot\left(\overrightarrow{l^{\prime}}-\vec{l}\right)\right)\right) \int_{-\infty}^{\infty} d t \exp \left(-i \omega_{i}(\vec{k}) t\right)\right]\right\} \\
= & \frac{(2 \pi)^{3}}{2 V_{\text {maille }}} e^{-W_{d^{\prime}}(\vec{Q})} e^{-W_{d}(\vec{Q})} e^{-i \vec{Q} \cdot\left(\overrightarrow{d^{\prime}}-\vec{d}\right)} \\
& \times \sum_{i, \vec{k}} \sum_{\vec{G}}\left\{\left[\frac{A_{i, \vec{k}}\left(\vec{Q} ; d^{\prime}, d\right)}{\omega_{i}(\vec{k})}\left(1+n\left(\omega_{i}(\vec{k})\right)\right) \delta(\vec{k}-\vec{Q}-\vec{G}) \delta\left(\omega-\omega_{i}(\vec{k})\right)\right]\right. \\
& \left.+\left[\frac{A_{i, \vec{k}}\left(\vec{Q} ; d, d^{\prime}\right)}{\omega_{i}(\vec{k})} n\left(\omega_{i}(\vec{k})\right) \delta(\vec{k}+\vec{Q}-\vec{G}) \delta\left(\omega+\omega_{i}(\vec{k})\right)\right]\right\}
\end{aligned}
$$


où nous avons utilisé les relations (10.32) et (10.34). Avec ce résultat nous pouvons calculer la fonction de diffusion cohérente

$$
\begin{aligned}
S_{\mathrm{coh}}^{1-\mathrm{ph}}(\vec{Q}, \omega)= & \sum_{d, d^{\prime}} \bar{b}_{d} \cdot \bar{b}_{d^{\prime}}^{*} S_{d, d^{\prime}}^{1-\mathrm{ph}}(\vec{Q}, \omega) \\
= & \frac{(2 \pi)^{3}}{V_{\text {maille }}} \sum_{d, d^{\prime}} \overline{b_{d}} \cdot{\overline{b_{d^{\prime}}}}^{*} e^{-W_{d}(\vec{Q})} e^{-W_{d^{\prime}}(\vec{Q})} e^{i \vec{Q} \cdot \vec{d}} e^{-i \vec{Q} \cdot \vec{d}^{\prime}} \\
& \times \sum_{i, \vec{k}} \sum_{\vec{G}}\left\{\left[\frac{A_{i, \vec{k}}\left(\vec{Q} ; d^{\prime}, d\right)}{\omega_{i}(\vec{k})}\left(1+n\left(\omega_{i}(\vec{k})\right)\right) \delta(\vec{k}-\vec{Q}-\vec{G}) \delta\left(\omega-\omega_{i}(\vec{k})\right)\right]\right. \\
& \left.+\left[\frac{A_{i, \vec{k}}\left(\vec{Q} ; d, d^{\prime}\right)}{\omega_{i}(\vec{k})} n\left(\omega_{i}(\vec{k})\right) \delta(\vec{k}+\vec{Q}-\vec{G}) \delta\left(\omega+\omega_{i}(\vec{k})\right)\right]\right\} .
\end{aligned}
$$

La structure intrinsèque de cette expression devient plus claire si nous l'écrivons comme

$$
\begin{aligned}
S_{\text {coh }}^{1-\text { ph }}(\vec{Q}, \omega)= & \frac{(2 \pi)^{3}}{2 V_{\text {maille }}} \sum_{\vec{G}} \sum_{i, \vec{k}} \frac{\left|F_{i}(\vec{Q} \mid \vec{k})\right|^{2}}{\omega_{i}(\vec{k})} \\
& \times\left[\left(1+n\left(\omega_{i}(\vec{k})\right)\right) \delta(\vec{k}-(\vec{Q}+\vec{G})) \delta\left(\omega-\omega_{i}(\vec{k})\right)\right. \\
& \left.+n\left(\omega_{i}(\vec{k})\right) \delta(\vec{k}+(\vec{Q}+\vec{G})) \delta\left(\omega+\omega_{i}(\vec{k})\right)\right]
\end{aligned}
$$

avec

$$
\begin{aligned}
\left|F_{i}(\vec{Q} \mid \vec{k})\right|^{2} & =\sum_{d, d^{\prime}}^{r} \frac{\bar{b}_{d} \cdot \vec{b}_{d^{\prime}}^{*}}{\sqrt{m_{d} m_{d^{\prime}}}} e^{-W_{d}(\vec{Q})} e^{-W_{d^{\prime}}(\vec{Q})}\left(\vec{Q} \cdot \vec{e}_{i}\left(d^{\prime} \mid \vec{k}\right)\right)\left(\vec{Q} \cdot \vec{e}_{i}(d \mid \vec{k})\right)^{*} e^{i \vec{Q} \cdot \vec{d}} e^{-i \vec{Q} \cdot \overrightarrow{d^{\prime}}} \\
& =\left|\sum_{d=1}^{r} \frac{\overline{b_{d}}}{\sqrt{m_{d}}} e^{-W_{d}(\vec{Q})}\left(\vec{Q} \cdot \vec{e}_{i}(d \mid \vec{k})\right) e^{-i \vec{Q} \cdot \vec{d}}\right|
\end{aligned}
$$

La fonction $F_{i}(\vec{Q} \mid \vec{k})$ est appelée facteur de structure du phonon de la branche $i$ et du vecteur d'onde $\vec{k}$. L'expression obtenue pour la diffusion à un phonon ne dépend pas explicitement du nombre $N$ de diffuseurs. Une augmentation du volume de l'échantillon doit, dans l'approximation de Born, entraîner une augmentation proportionnelle de la diffusion. Cette augmentation est néanmoins implicite dans l'expression (10.72) qui est proportionnelle au nombre de phonons. Ce nombre est géré par la densité des vecteurs $\vec{k}$ dans la première zone de Brillouin. A cause des conditions aux limites (voir chapitre I), un cristal plus grand aura des espacements plus petits entre des $\vec{k}$ voisins. Le nombre de modes total correspond à $3 N$ avec $N$ le nombre d'atomes dans le cristal.

Les lois fondamentales de la physique exigent la conservation de l'impulsion. Le changement dans l'impulsion du neutron $\left(\hbar \vec{Q}=\hbar \vec{k}_{i}-\hbar \vec{k}_{f}\right)$ lors de la diffusion doit donc être compensé par un changement opposé de l'impulsion de l'échantillon. Ce constat vaut aussi bien pour le cristal que pour l'amorphe. Les fonctions de Dirac

$$
\begin{aligned}
& \delta(\vec{k}-(\vec{Q}+\vec{G})), \\
& \delta(\vec{k}+(\vec{Q}+\vec{G})),
\end{aligned}
$$


que nous rencontrons dans la fonction de diffusion (10.72) du cristal, impliquent que la diffusion cohérente à un phonon soit aussi assujettie à la conservation du moment cristallin. Pour que la diffusion puisse avoir lieu, il doit exister des vecteurs $\vec{G}$ du réseau réciproque tels que $\mathrm{e}^{70}$

$$
\begin{aligned}
& \vec{Q}=\vec{k}+\vec{G}, \\
& \vec{Q}=-\vec{k}+\vec{G} .
\end{aligned}
$$

En clair, lors du processus de diffusion, le changement d'impulsion du neutron $\left(\hbar \vec{Q}=\hbar \vec{k}_{i}-\hbar \vec{k}_{f}\right)$ doit être absorbé (10.76) ou fourni (10.77) par le phonon $(\hbar \vec{k})$ à une quantité $\hbar \vec{G}$ près (voir figure 38 ). On parlera de création ou d'émission d'un phonon ainsi que d'annihilation ou d'absorption d'un phonon. La diffusion des neutrons est de ce point de vue identique à la diffusion d'un électron de Bloch, que nous avions évoquée dans le chapitre I. La conservation du moment cristallin est une contrainte imposée à n'importe quelle particule exposée à un potentiel périodique du réseau.
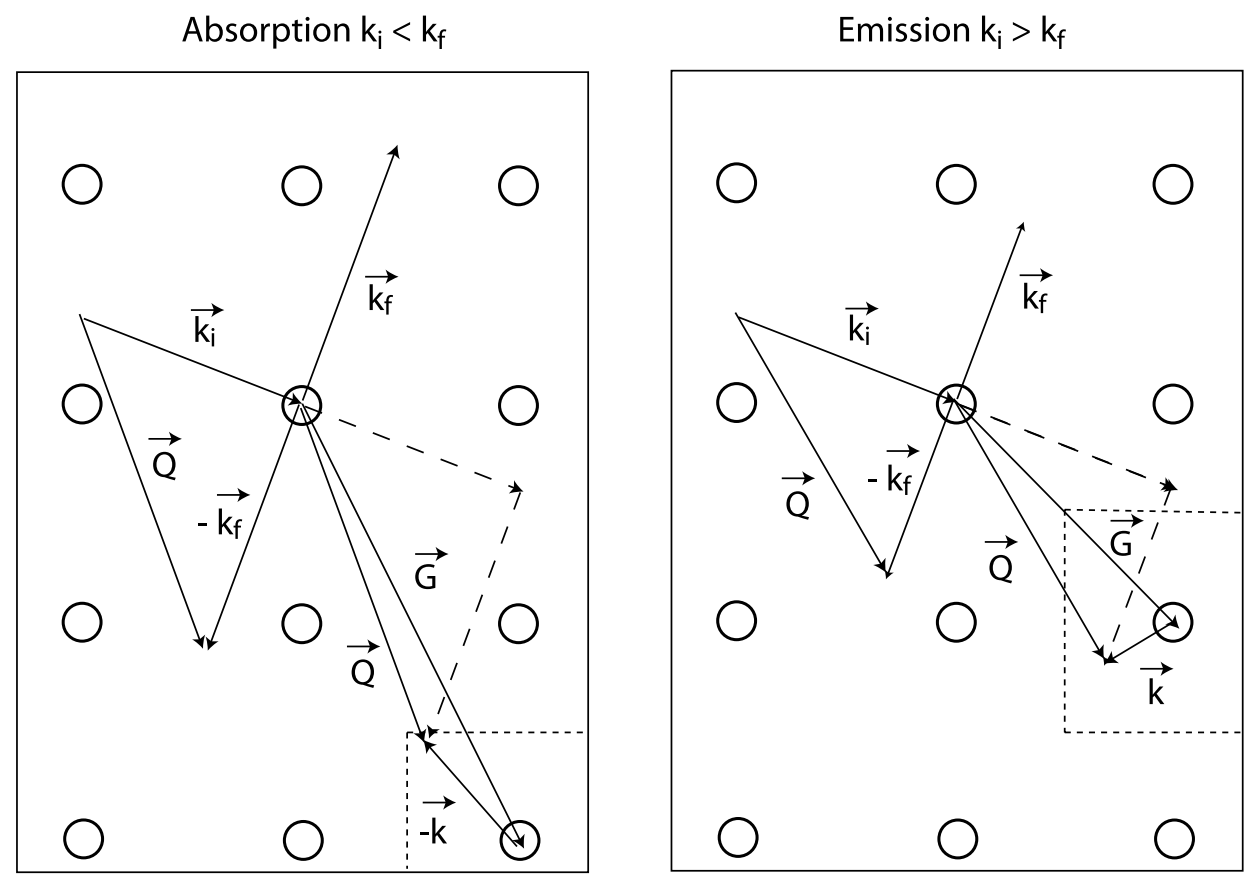

Figure 38. Représentation schématique de la conservation du moment cristallin lors de l'absorption (10.77) et de l'émission (10.76) d'un phonon.

Pour illustrer les expressions obtenues pour la diffusion cohérente à un phonon nous nous inspirons de Squires [36] et rappelons qu'un phonon représente une onde progressive. Dans un référentiel qui se déplace le long de $\vec{k}$ avec la vitesse de phase du phonon, $\left(\omega_{i}(\vec{k}) /|\vec{k}|\right)$ les mouvements des atomes sont figés. La structure du cristal apparaît modulée de manière sinusoïdale. Il est connu (par exemple pour des systèmes incommensurables; voir chapitre de Roland Currat) qu'une telle modulation statique induit des satellites aux positions $\vec{G} \pm \vec{k}$ autour des pics de Bragg. Ceci nous amène aux expressions (10.76) et (10.77). L'intensité de ces satellites est donnée par l'amplitude de la modulation au carré. La transformation du référentiel en mouvement vers le référentiel du laboratoire nous apprend que

\footnotetext{
${ }^{70}$ En passant de $(10.74,10.75)$ à $(10.76,10.77)$ nous utilisons le fait que le réseau réciproque est un réseau de Bravais et qu'il existe pour tout vecteur $\vec{G}$, un $-\vec{G}$.
} 
les satellites correspondent à une diffusion inélastique. Les transferts d'énergies sont donnés par les expressions:

$$
\begin{aligned}
\frac{\hbar}{2 m_{n}}\left(k_{i}^{2}-k_{f}^{2}\right) & =\hbar \omega_{i}(\vec{k}), \\
\frac{\hbar}{2 m_{n}}\left(k_{f}^{2}-k_{i}^{2}\right) & =\hbar \omega_{i}(\vec{k}) .
\end{aligned}
$$

L'intensité de la diffusion sera pilotée par l'amplitude de la modulation.

La conservation du moment cristallin a des conséquences importantes pour la diffusion des neutrons. Considérons un faisceau incident décrit par le vecteur d'onde $\vec{k}_{i}$, et une direction de détection $\hat{k}_{f}$. La diffusion inélastique sera nulle sauf pour les valeurs de $k_{f}$ qui correspondent aux transferts d'énergie définis par les expression (10.78) et (10.79). Etant donné que le spectre des phonons est discret pour un $\vec{k}$ donné la diffusion sera discrète (voir figure 39). Chaque pic inélastique correspondra, soit à l'émission, soit à l'absorption d'un phonon dont la fréquence et le vecteur d'onde sont déterminés par les relations (10.76) à (10.79). La seule inconnue est l'indice de la branche. En effectuant un grand nombre de mesures afin de balayer des régions étendues de l'espace $(\vec{Q}, \omega)$, il est à priori possible de déterminer les relations de dispersions dans des composés complexes. Nous en avons donné plusieurs exemples dans le chapitre I. D'autres exemples suivront dans les chapitres de ce livre.

Point $\Gamma$ à $Q=(6,1,1) * 2 \pi / a$

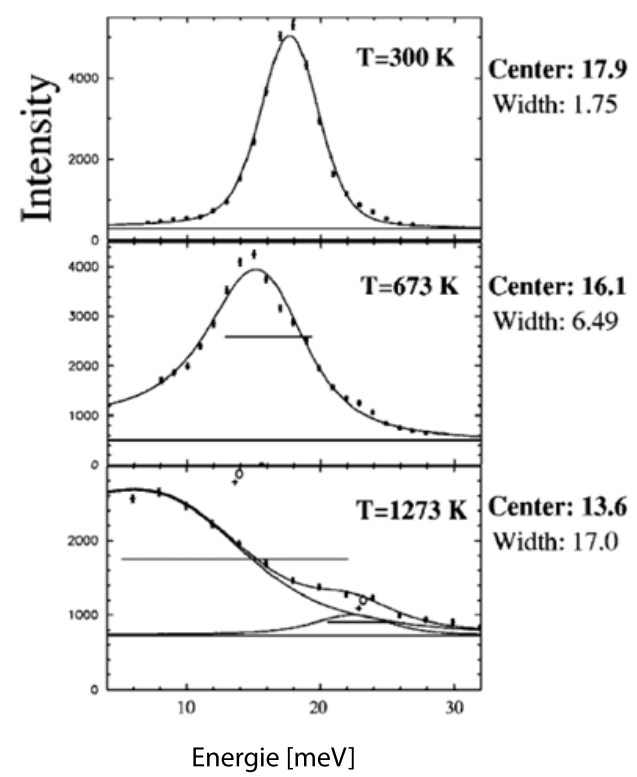

Point $\mathrm{X}$ à $\mathrm{Q}=(7,0,0) * 2 \pi / \mathrm{a}$

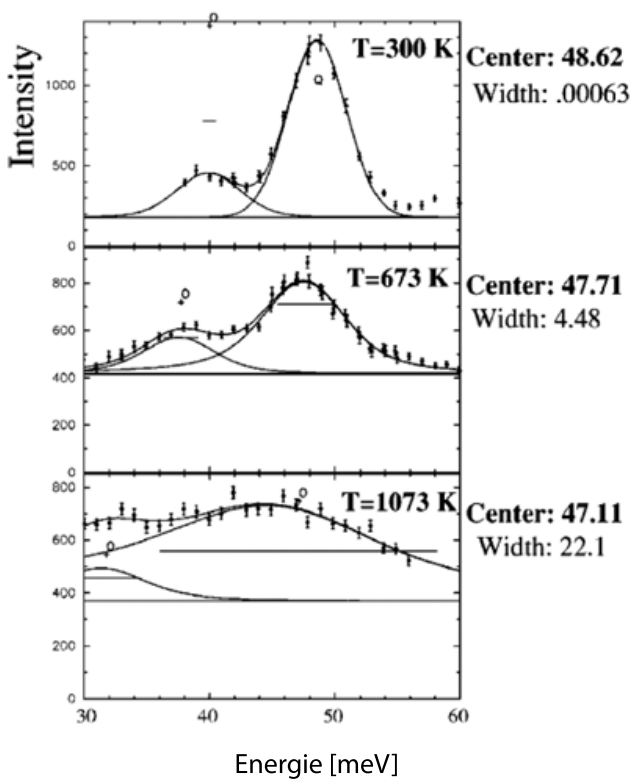

Figure 39. Exemples de pics de phonons obtenus avec un spectromètre à trois axes sur le conducteur super-ionic $\mathrm{CaF}_{2}$ dont nous avions déjà montré les courbes de dispersion ainsi que la figure de droite dans le chapitre I. Les mesures sont effectuées telles que $\vec{Q}$ reste constant. Les valeurs de $\vec{Q}$ sont indiquées au dessus des figures. Les pics sont décrits par des fonctions d'oscillateurs amortis (se référer à la section 11.6). Les centres ainsi que les largeurs obtenues sont indiqués à droite des pics. Ils sont aussi inclus graphiquement dans les figures. A $300 \mathrm{~K}$ les largeurs sont données par la résolution instrumentale. A plus haute température les pics s'amortissent et s'élargissent (voir chapitre I). On voit aussi que le bruit de fond dû à la diffusion multiphonons (voir section 11.4) augmente considérablement avec la température. 
Les relations (10.76) à (10.79) limitent l'observation d'un mode à la région cinématique accessible pour une configuration expérimentale donnée. L'exemple typique est la mesure de la dispersion des modes acoustiques dans la première zone de Brillouin. ${ }^{71}$ La région accessible pour un $k_{i}$ fixe est définie par (voir la section 5.10 et la figure 40)

$$
\hbar \omega(Q) \leq \frac{\hbar}{2 m}\left(k_{i}^{2}-\left(Q-k_{i}\right)^{2}\right) .
$$

La pente de l'enveloppe de ce domaine à $Q=0$ se calcule comme

$$
\left(\frac{d(\hbar \omega(Q))}{d Q}\right)_{Q=0}=\frac{\hbar}{m} k_{i}=v_{n} .
$$

$v_{n}$ est la vitesse du neutron incident. La pente de la courbe de dispersion est donnée par la vitesse du son $c$. Si $c>v_{n}$ les modes ne contribueront pas à la diffusion en absorption, c'est-à-dire pour $\omega>0$. En émission aucune contrainte n'existe à priori. Néanmoins, les angles de diffusion nécessaires à la mesure des phonons acoustiques en émission deviennent de plus en plus petits au fur et à mesure que $c$ augmente. Le signal disparaîtra alors dans le bruit de fond crée par le faisceau direct. Dans un cristal, le problème peut être évité en travaillant dans une autre zone de Brillouin (voir la figure 40). Cette possibilité n'existe pas dans un amorphe. La situation est complètement différente dans le cas des rayons-X du fait que la vitesse du son est toujours plus petite que la vitesse de la lumière.

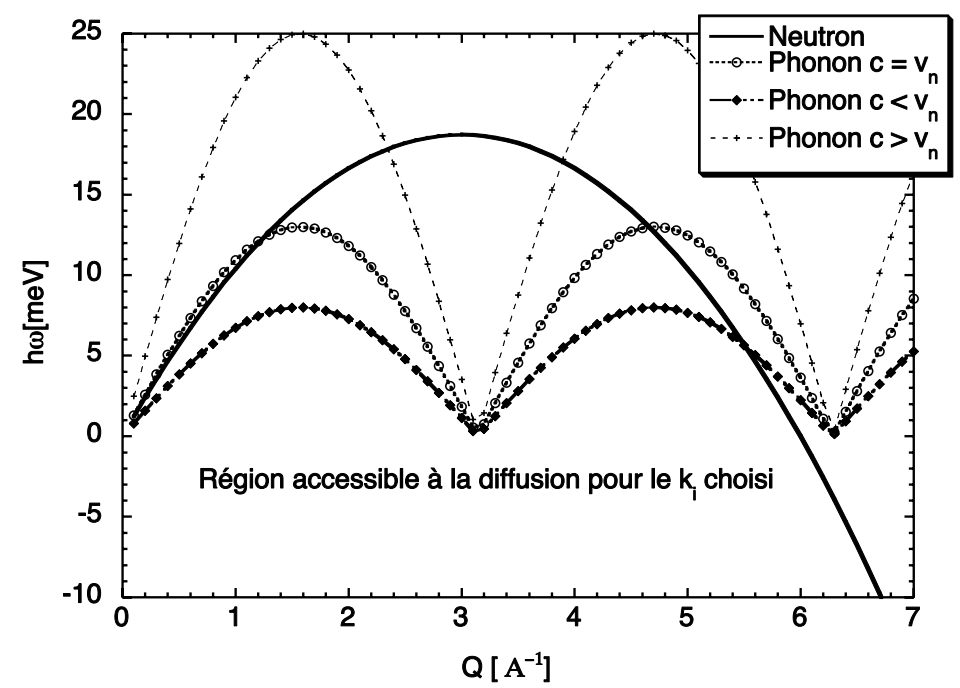

Figure 40. Pour qu'un phonon contribue à la section efficace, la configuration expérimentale doit permettre de satisfaire à la fois la conservation de l'énergie et du moment cristallin. En mode d'absorption ceci nécessite de respecter simultanément (10.78) et (10.76). Dans la première zone de Brillouin ceci est possible si la vitesse du son de la branche concernée est inférieure à la vitesse $v_{n}$ du neutron. La situation tracée correspond (comme dans la figure 5.10) à un $k_{i}$ fixe de $3 \AA^{-1}$.

Les relations (10.76) à (10.79) sont des conditions nécessaires mais pas suffisantes. Pour que la diffusion ait lieu il faut aussi que le facteur de forme $F_{i}(\vec{Q} \mid \vec{k})$ soit suffisamment fort pour que le signal devienne observable. L'exemple le plus simple pour illustrer ceci concerne les phonons purement transverses dans la première zone de Brillouin. Pour ces phonons

$$
\vec{e}_{i}(d \mid \vec{k}) \cdot \vec{k}=0 \quad \forall d .
$$

\footnotetext{
71 On parle alors souvent de diffusion Brillouin.
} 
Dans la première zone de Brillouin $\vec{G}=0$ et donc $\vec{Q}=\vec{k}$. Il vient que

$$
\left|F_{i}(\vec{Q} \mid \vec{k})\right|^{2}=\left|\sum_{d=1}^{r} \frac{\overline{b_{d}}}{\sqrt{m_{d}}} e^{-W_{d}(\vec{Q})}\left(\vec{k} \cdot \vec{e}_{i}(d \mid \vec{k})\right) e^{-i \vec{Q} \cdot \vec{d}}\right|=0 .
$$

Les modes transverses ne donnent donc aucun signal dans la première zone de Brillouin.

Dans la pratique on doit être conscient que le caractère d'un mode est généralement mixte dans un matériau complexe. Néanmoins la symétrie peut imposer des extinctions systématiques pour certaines directions de l'espace réciproque, et cela pas seulement dans la première zone de Brillouin. Le long de ces directions, le facteur de forme est rigoureusement nul pour une partie des phonons. Nous n'allons pas poursuivre la discussion de ces règles de sélection, qui peuvent être déduites avec l'aide de la théorie des groupes. Le lecteur interessé pourra se référer à la littérature [41,42].

Le facteur $\left|F_{i}(\vec{Q} \mid \vec{k})\right|^{2}$ donne l'intensité avec laquelle un phonon peut être observé pour un $\vec{Q}$ donné. Ce facteur est une fonction du vecteur propre $\vec{e}_{i}(d \mid \vec{k})(d=1, \ldots r)$ du phonon. En général il est très difficile de remonter à $\vec{e}_{i}(d \mid \vec{k})(d=1, \ldots r)$ à partir d'une mesure de $\left|F_{i}(\vec{Q} \mid \vec{k})\right|^{2}$. La détermination des $\vec{e}_{i}(d \mid \vec{k})(d=1, \ldots r)$ est donc plutôt l'exception que la règle [43]. Il est néanmoins possible de tirer des renseignements intéressants sur le caractère d'un mode de phonon à partir d'un jeux limité de mesures de $\left|F_{i}(\vec{Q} \mid \vec{k})\right|^{2}$. Un cas particulier concerne les modes à fort caractère polarisé. La diffusion des neutrons n'est sensible qu'à la projection des mouvements sur $\vec{Q}$. Mathématiquement ceci se traduit par la présence des produits scalaires

$$
\left(\vec{Q} \cdot \vec{e}_{i}(d \mid \vec{k})\right)
$$

dans le facteur de forme des phonons. Si nous choisissons des configurations de mesure pour lesquelles $\vec{Q} \| \vec{k}$ ou $\vec{Q} \perp \vec{k}$ alors nous pouvons séparer les modes longitudinaux $e_{i}(d \mid \vec{k}) \| \vec{k}$ des modes transverses $e_{i}(d \mid \vec{k}) \perp \vec{k}$ (voir figure 41).

A cause de la présence du facteur $Q^{2}$ dans $\left|F_{i}(\vec{Q} \mid \vec{k})\right|^{2}$, il est préférable de travailler dans des zones plutôt éloignées de l'espace réciproque. Il faut néanmoins veiller à ce que ce gain en $Q^{2}$ ne soit pas contrebalancé par les facteurs de Debye-Waller

$$
e^{-W_{d}(\vec{Q})}
$$

qui eux dépendent des fonctions de Debye-Waller $W_{d}(\vec{Q})$ et qui donc diminuent avec $Q$. Comme nous verrons dans la section 11, pour des très grands $Q$ l'intensité est transférée vers des processus de diffusion qui impliquent plusieurs phonons.

Même si les spectromètres de temps de vol en sont aussi capables l'instrument de prédilection pour l'étude des phonons dans un cristal reste le spectromètre à trois axes. Il sera presenté ainsi que les divers modes de mesure ( $\vec{Q}$ constant, $E$ constant) dans le chapitre de Bernard Hennion.

\subsection{Diffusion incohérente élastique}

Tournons nous maintenant vers la diffusion incohérente. Notre point de départ est la section efficace différentielle partielle (5.68)

$$
\left(\frac{d \sigma}{d \Omega d E_{f}}\right)_{\mathrm{inc}}=\frac{k_{f}}{k_{i}} \frac{1}{2 \pi \hbar} \sum_{\lambda_{i}} p\left(\lambda_{i}\right) \sum_{j}^{N}\left(\overline{b_{\kappa}^{2}}-\left(\bar{b}_{\kappa}\right)^{2}\right) \int_{-\infty}^{\infty} d t<\lambda_{i}\left|e^{-i \vec{Q} \cdot \vec{R}_{j}^{0}} e^{i \vec{Q} \cdot \vec{R}_{j}(t)}\right| \lambda_{i}>e^{-i \omega t}
$$

que nous pouvons exprimer avec l'aide de la fonction de diffusion incohérente

$$
S_{\mathrm{inc}}(\vec{Q}, \omega)=\sum_{\kappa}\left(\overline{b_{\kappa}^{2}}-\left(\bar{b}_{\kappa}\right)^{2}\right) S_{\kappa}^{S}(\vec{Q}, \omega)
$$




\section{Configuration longitudinale $\overrightarrow{\mathrm{k}}$ est parallèle à $\overrightarrow{\mathrm{Q}}$}

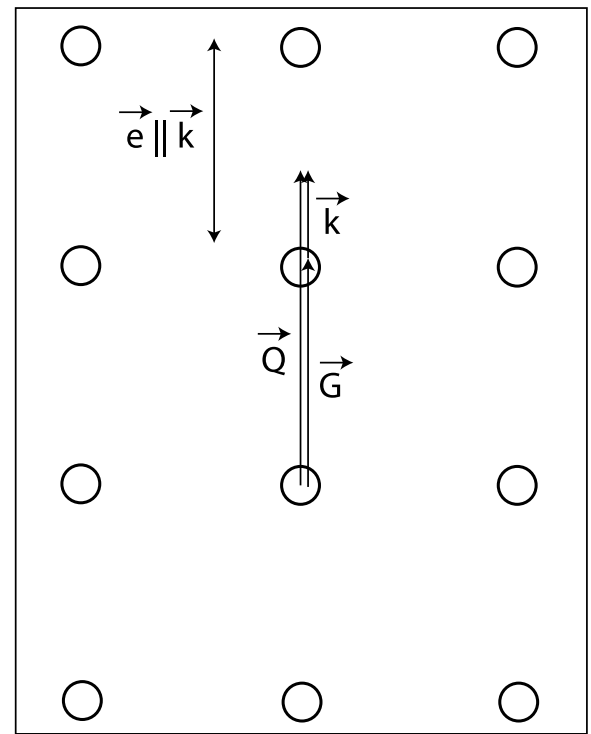

Configuration transversale $\overrightarrow{\mathrm{k}}$ est plus ou moins perpendiculaire à $\vec{Q}$

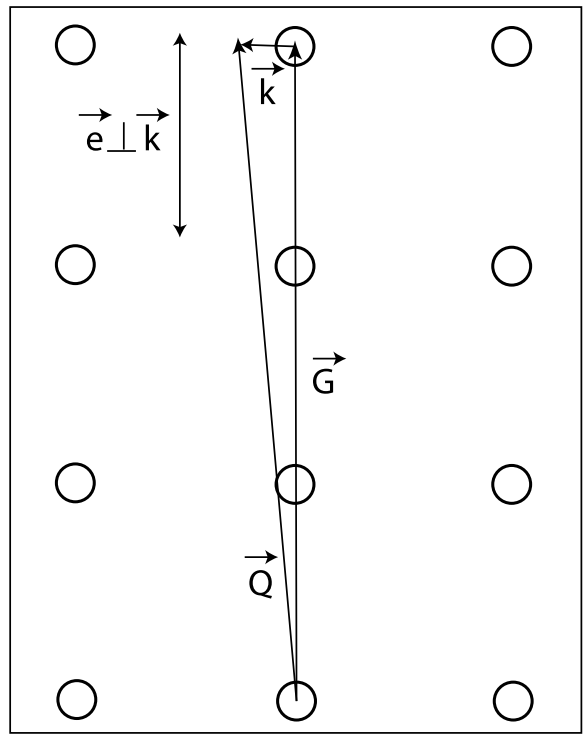

Figure 41. Dans le facteur de forme d'un phonon, les composantes du vecteur propre $\vec{e}_{i}(d \mid \vec{k})(d=1, \ldots r)$ sont projetées sur $\vec{Q}$. Un mode sera d'autant plus visible que cette projection est grande. Cette dépendance permet de sélectionner les modes dans une configuration de mesure donnée. Si $\vec{Q}$ est parallèle à $\vec{k}$, un mode transverse pour lequel les $\vec{e}_{i}(d \mid \vec{k})(d=1, \ldots r)$ sont tous perpendiculaires à $\vec{k}$ (et donc également à $\vec{Q}$ ) sera invisible. Dans une telle configuration, on ne mesurera que des modes qui possèdent des composantes de mouvement longitudinales. Cette configuration longitudinale est présentée sur la figure de gauche. La configuration transverse (figure de droite) gardera en général un caractère mixte pour des directions de haute symétrie, c'est-à-dire qu'on ne pourra pas rendre $\vec{Q}$ et $\vec{k}$ parfaitement perpendiculaires. La composante longitudinale diminuera pour un $\vec{k}$ donné avec $\vec{Q}$. Il sera donc préférable de travailler dans des zones lointaines de l'espace réciproque.

Les fonctions de diffusion partielles propres sont définies comme

$$
S_{\kappa}^{S}(\vec{Q}, \omega)=\frac{1}{2 \pi \hbar} \sum_{j \in\left\{j_{k}\right\}} \int_{-\infty}^{\infty} d t\left\langle e^{-i \vec{Q} \cdot \vec{R}_{j}(t=0)} e^{i \vec{Q} \cdot \vec{R}_{j}(t)}\right\rangle e^{-i \omega t} .
$$

La fonction de corrélation à un atome

$$
\left\langle e^{-i \vec{Q} \cdot \vec{R}_{j}(t=0)} e^{i \vec{Q} \cdot \vec{R}_{j}(t)}\right\rangle
$$

est un cas spécial de la fonction de corrélation à deux atomes

$$
\left\langle e^{-i \vec{Q} \cdot \vec{R}_{j^{\prime}}(t=0)} e^{i \vec{Q} \cdot \vec{R}_{j}(t)}\right\rangle
$$

De ce fait nous pouvons nous appuyer sur le formalisme développé dans le contexte de la réponse cohérente. L'identité de Born nous apprend que

$$
\left\langle e^{-i \vec{Q} \cdot \vec{u}_{j}} e^{i \vec{Q}^{\cdot} \cdot \vec{u}_{j}(t)}\right\rangle=e^{-2 W_{j}(\vec{Q})} e^{\left\langle\left(\vec{Q} \cdot \vec{u}_{j}\right)\left(\vec{Q} \cdot \vec{u}_{j}(t)\right)\right\rangle}
$$


Le premier terme dans le développement de cette expression en fonction de

$$
\aleph_{j, j}(\vec{Q}, t)=\left\langle\left(\vec{Q} \cdot \vec{u}_{j}\right)\left(\vec{Q} \cdot \vec{u}_{j}(t)\right)\right\rangle
$$

donne la diffusion incohérente élastique

$$
\left(\frac{d^{2} \sigma}{d \Omega d E_{f}}\right)_{\mathrm{inc}}^{\mathrm{el}}=\sum_{\kappa}\left(\overline{b_{\kappa}^{2}}-\left(\overline{b_{\kappa}}\right)^{2}\right) S_{\kappa}(\vec{Q}, \hbar \omega=0)=\sum_{\kappa} \sum_{j \in\left\{j_{\kappa}\right\}}\left(\overline{b_{\kappa}^{2}}-\left(\bar{b}_{\kappa}\right)^{2}\right) e^{-2 W_{j}(\vec{Q})} \delta(\hbar \omega) .
$$

Si pour un type d'atome $\kappa$ tous les sites occupés sont équivalents, le facteur de Debye-Waller ne dépend plus que de $\kappa$ et nous obtenons

$$
\left(\frac{d^{2} \sigma}{d \Omega d E_{f}}\right)_{\text {inc }}^{\mathrm{el}}=\sum_{\kappa} \sum_{j \in\left\{j_{\kappa}\right\}}\left(\overline{b_{\kappa}^{2}}-\left(\overline{b_{\kappa}}\right)^{2}\right) e^{-2 W_{\kappa}(\vec{Q})} \delta(\hbar \omega)=\sum_{\kappa} N_{\kappa} \frac{\sigma_{\mathrm{inc}}(\kappa)}{4 \pi} e^{-2 W_{\kappa}(\vec{Q})} \delta(\hbar \omega),
$$

avec $N_{\kappa}$ le nombre d'atomes de type $\kappa$. C'est l'équivalent de l'expression (9.42) que nous avions obtenue pour les atomes figés. La seule différence se trouve dans la présence des facteurs $\exp \left(-2 W_{\kappa}(\vec{Q})\right)$ dans (10.87), qui introduisent une dépendance en $\vec{Q}$. Cette dépendance reflète le fait que les atomes sont distribués autour de leurs sites d'équilibre à cause de l'agitation thermique. La diffusion incohérente cesse ainsi d'être isotrope et cette tendance devient plus prononcée à haute température. Elle est néanmoins également présente à basse température à cause des fluctuations du point zéro. Elle doit être prise en compte si un diffuseur incohérent comme le vanadium est utilisé à des fins d'étalonnage, par exemple pour normaliser l'efficacité de détecteurs situés à des positions angulaires différentes.

\subsection{La diffusion diffuse}

Il est important de distinguer la diffusion incohérente de la diffusion diffuse. On parle de diffusion diffuse dans le cas d'un désordre chimique qui est défini par l'occupation aléatoire d'un site $\vec{R}_{j}$ par un ou plusieurs éléments chimiques. Un exemple de désordre chimique est donné par les solutions solides pour lesquelles les positions $\vec{R}_{j}$ dans la maille d'un cristal sont occupées aléatoirement par plusieurs éléments chimiques. La différence par rapport à la diffusion incohérente réside dans le fait que les grandeurs physiques que nous voulons extraire par la diffusion des neutrons relève d'un désordre au niveau chimique de l'échantillon et non pas d'un désordre au niveau des états de spin nucléaire. La diffusion diffuse nous livre donc des informations précieuses sur la composition chimique (type de désordre, distribution et corrélation des éléments chimiques occupant un site). La diffusion incohérente nous permettrait de faire la même chose concernant la distribution des isotopes. Hélas, la distribution des isotopes ou des spins nucléaires n'est en général pas corrélée avec la distribution chimique. Elle sera donc considerée comme une nuisance. Cependant elle nous permet d'extraire des informations qui ne concernent que l'évolution temporelle d'un seul diffuseur avec beaucoup plus d'aisance que la diffusion cohérente. Nous en verrons la raison dans la section suivante.

Comment pouvons nous incorporer le désordre chimique dans notre formalisme? Suivant le raisonnement employé lors de la discussion de la diffusion incohérente nous devons effectuer des moyennes prenant en compte tous les arrangements possibles des espèces atomiques

$$
S(\vec{Q}, \omega)=\left\{\sum_{j, j^{\prime}=1}^{N}\left(b_{j} b_{j^{\prime}}^{*}\right) S_{j, j^{\prime}}(\vec{Q}, \omega)\right\}_{\mathrm{av}} .
$$


Les sommes sont à effectuer sur les volumes de corrélation comme nous l'avons discuté dans la section (9.4). Contrairement à la diffusion incohérente la moyenne impliquant un désordre chimique ne concerne pas seulement le produit des longueurs de diffusion $\left(b_{j} b_{j^{\prime}}^{*}\right)$ mais également les fonctions de corrélation

$$
S_{j, j^{\prime}}(\vec{Q}, \omega)=\frac{1}{2 \pi \hbar} \sum_{\lambda_{i}} p\left(\lambda_{i}\right) \int_{-\infty}^{\infty} d t\left\langle\lambda_{i}\left|e^{-i \vec{Q} \cdot \vec{R}_{j^{\prime}}^{0}} e^{i \vec{Q} \cdot \vec{R}_{j}(t)}\right| \lambda_{i}\right\rangle e^{-i \omega t}
$$

Il est évident que $S_{j, j^{\prime}}(\vec{Q}, \omega)$ changera selon les éléments chimiques qui occupent les sites $j$ et $j^{\prime}$. En dehors des masses, les interactions qui déterminent les distances entre atomes ainsi que le spectre des excitations dépendront du type d'atome. Pour décrire la diffusion diffuse on devra donc avoir recours à un modèle concret du désordre.

Le modèle le plus simple consiste à présumer que la structure et le désordre sont décorrélés. En d'autres termes, nous considérons que la structure peut être modélisée par un réseau de positions $\vec{R}_{j}$. Le désordre est pris un compte en introduisant des facteurs d'occupation ainsi que des facteurs de Debye-Waller spécifiques sur chaque site. Dans ces conditions, la fonction de diffusion élastique s'écrira comme

$$
S(\vec{Q}, \omega)=\sum_{j, j^{\prime}=1}^{N} \overline{\left(f_{j} f_{j^{\prime}}\right)} S_{j, j^{\prime}}^{\mathrm{el}}(\vec{Q}, \omega)
$$

avec

$$
f_{j}=b_{j} e^{-W_{j}(\vec{Q})}
$$

et selon (10.29)

$$
S_{j, j^{\prime}}^{\mathrm{el}}(\vec{Q}, \omega)=\sum_{j, j^{\prime}} \exp \left(-i \vec{Q} \cdot\left(\vec{R}_{j^{\prime}}^{0}-\vec{R}_{j}^{0}\right)\right) \delta(\hbar \omega) .
$$

Cette expression est formellement identique à (5.48). Nous pouvons donc immédiatement donner le résultat pour les moyennes:

$$
\begin{aligned}
& j \neq j^{\prime}: \overline{\left(f_{j} f_{j^{\prime}}\right)}={\overline{f_{j}}}^{2}, \\
& j=j^{\prime} \quad: \quad \overline{\left(f_{j} f_{j}\right)}=\overline{f_{j}^{2}},
\end{aligned}
$$

avec

$$
\begin{aligned}
& \overline{f_{j}}=\sum_{\kappa} c_{j}^{\kappa} e^{-W_{j}^{\kappa}(\vec{Q})} \sum_{i} p_{j}^{i} \cdot b_{j}^{i}=\sum_{\kappa} c_{j}^{\kappa} \overline{b_{j}} e^{-W_{j}^{\kappa}(\vec{Q})}, \\
& \overline{f_{j}^{2}}=\sum_{\kappa} c_{j}^{\kappa} e^{-2 W_{j}^{\kappa}(\vec{Q})} \sum_{i} p_{j}^{i} \cdot\left(f_{j}^{i}\right)^{2}=\sum_{\kappa} c_{j}^{\kappa} e^{-2 W_{j}^{\kappa}(\vec{Q})} \overline{b_{j}^{2}} .
\end{aligned}
$$

Comme dans la section 5.6 les coefficients $p_{j}^{i}$ donnent la probabilité que la longueur de diffusion de l'élément occupant la position $\vec{R}_{j}$ possède la valeur $b_{j}^{i}$. Les facteurs $c_{j}^{\kappa}$ donnent les occupations fractionnelles (concentrations) des sites $j$ pour des atomes de type $\kappa$. Nous avons alors

$$
\begin{aligned}
& \sum_{i} p_{j}^{i}=1, \\
& \sum_{\kappa} c_{j}^{\kappa}=1 .
\end{aligned}
$$


Nous sommes maintenant en mesure de donner des expressions pour les sections efficaces. Commençons avec la section efficace incohérente. Selon (10.87) elle est donnée par

$$
\begin{aligned}
\left(\frac{d^{2} \sigma}{d \Omega d E_{f}}\right)_{\mathrm{inc}}^{\mathrm{el}} & =\sum_{\kappa} N_{\kappa} \frac{\sigma_{\mathrm{inc}}(\kappa)}{4 \pi} e^{-2 W_{\kappa}(\vec{Q})} \delta(\hbar \omega) \\
& =N \sum_{\kappa} c_{\kappa} \frac{\sigma_{\mathrm{inc}}(\kappa)}{4 \pi} e^{-2 W_{\kappa}(\vec{Q})} \delta(\hbar \omega),
\end{aligned}
$$

où nous avons fait l'hypothèse que les facteurs de Debye-Waller ne dépendent que du type d'atome et non pas du site, ce qui n'est pas toujours le cas. Pour donner plus de transparence à la discussion nous considérons un cas très simple. Deux types d'atomes sont distribués de manière aléatoire sur les positions d'un réseau de Bravais. Il pourrait s'agir par exemple d'un alliage métallique. Dans ce cas

$$
\begin{aligned}
\left(\frac{d^{2} \sigma}{d \Omega d E_{f}}\right)_{\mathrm{inc}}^{\mathrm{el}} & =N\left(c_{1} \sigma_{\mathrm{inc}}(1) e^{-2 W_{1}(\vec{Q})}+c_{2} \sigma_{\mathrm{inc}}(2) e^{-2 W_{2}(\vec{Q})}\right) \frac{\delta(\hbar \omega)}{4 \pi} \\
& =N\left((1-c) \sigma_{\mathrm{inc}}(1) e^{-2 W_{1}(\vec{Q})}+c \sigma_{\mathrm{inc}}(2) e^{-2 W_{2}(\vec{Q})}\right) \frac{\delta(\hbar \omega)}{4 \pi}
\end{aligned}
$$

avec $c_{1}$ et $c=c_{2}$ les concentrations fractionnelles des deux éléments. La présence du désordre sur les sites du réseau ne change donc rien à la réponse incohérente en dehors du fait que les sections efficaces incohérentes $\sigma_{\kappa}$ doivent être pondérées correctement. En effet, nous n'avions même pas besoin du formalisme impliquant les facteurs $f_{j}$ pour obtenir ce résultat.

Nous nous tournons maintenant vers la diffusion élastique cohérente. A partir des expressions (10.90) et (10.29) nous obtenons pour la fonction de diffusion totale

$$
\begin{aligned}
\left(\frac{d^{2} \sigma}{d \Omega d E_{f}}\right)_{\text {total }}^{\mathrm{el}} & =S^{\mathrm{el}}(\vec{Q}, \omega) \\
& =\sum_{j, j^{\prime}=1}^{N} \overline{\left(f_{j} f_{j^{\prime}}\right)} S_{j, j^{\prime}}(\vec{Q}, \hbar \omega=0) \\
& =\sum_{j \neq j^{\prime}} \overline{f_{j}} \overline{f_{j^{\prime}}^{*}} \exp \left(-i \vec{Q} \cdot\left(\vec{R}_{j^{\prime}}^{0}-\vec{R}_{j}^{0}\right)\right) \delta(\hbar \omega)+\sum_{j} \overline{f_{j}^{2}} \delta(\hbar \omega) \\
& =\sum_{j, j^{\prime}} \overline{f_{j}} \overline{f_{j^{\prime}}^{*}} \exp \left(-i \vec{Q} \cdot\left(\vec{R}_{j^{\prime}}^{0}-\vec{R}_{j}^{0}\right)\right) \delta(\hbar \omega)+\sum_{j}\left(\overline{f_{j}^{2}}-\overline{f_{j}} \overline{f_{j}^{*}}\right) \delta(\hbar \omega)
\end{aligned}
$$

Le premier terme de cette expression correspond parfaitement à la fonction de diffusion élastique de la structure non-perturbée si nous remplaçons les longueurs de diffusion cohérentes ainsi que les facteurs de Debye-Waller par leurs valeurs ponderées. Dans le cas d'un cristal, les pics de Bragg ne vont donc pas changer de position. Seules leurs hauteurs vont être affectées par le désordre. Le deuxième terme ressemble étroitement à de la diffusion incohérente. Il ne dépend de $\vec{Q}$ qu'à travers des facteurs de Debye-Waller. Si nous soustrayons de ce terme l'expression (10.99) que nous avions obtenue pour la diffusion incohérente, nous obtenons ce qu'on appelle la diffusion diffuse monotone de type Laue. Du 
point de vue expérimental ${ }^{72}$ la diffusion diffuse de ce type est souvent indiscernable de la diffusion incohérente. $^{73}$

Pour illustrer la discussion générale, nous revenons à notre exemple de deux types d'atomes distribués sur un réseau de Bravais. Les moyennes sur les longueurs de diffusion se calculent comme

$$
\begin{aligned}
\bar{f} & =(1-c) e^{-W_{1}(\vec{Q})} \overline{b_{1}}+c e^{-W_{2}(\vec{Q})} \overline{b_{2}} \\
\overline{f^{2}} & =(1-c) e^{-2 W_{1}(\vec{Q})} \overline{\left(b_{1}\right)^{2}}+c e^{-2 W_{2}(\vec{Q}) \overline{\left(b_{2}\right)^{2}}}
\end{aligned}
$$

Nous obtenons pour la section efficace élastique totale

$$
\left.\left(\frac{d^{2} \sigma}{d \Omega d E_{f}}\right)_{\mathrm{coh}}^{\mathrm{el}}=f^{2} \mid \sum_{l} \exp (i \vec{Q} \cdot \vec{l})\right)\left.\right|^{2} \delta(\hbar \omega)+N\left(\overline{f^{2}}-\bar{f}^{2}\right) \delta(\hbar \omega)
$$

La première partie de cette expression donne lieu aux pics de Bragg. La deuxième mérite d'être regardée de plus près. En utilisant les expressions (10.95) et (10.96) et en négligeant les facteurs de Debye-Waller par souci de clarté, nous calculons

$$
\begin{aligned}
\left(\overline{f^{2}}-\bar{f}^{2}\right)= & (1-c) \overline{b_{1}^{2}}+c \overline{b_{j}^{2}}-\left((1-c) \overline{b_{1}}+c \overline{b_{2}}\right)^{2} \\
= & \left\{(1-c)\left(\overline{b_{1}^{2}}-{\overline{b_{1}}}^{2}\right)+c\left(\overline{b_{2}^{2}}-{\overline{b_{2}}}^{2}\right)\right\} \\
& +\left\{(1-c){\overline{b_{1}}}^{2}+c{\overline{b_{2}}}^{2}-(1-c)^{2}{\overline{b_{1}}}^{2}-c^{2}{\overline{b_{2}}}^{2}+\left(c-c^{2}\right) \overline{b_{1}} \overline{b_{2}}\right\} \\
= & \left\{(1-c)\left({\overline{b_{1}^{2}}}^{2}{\overline{b_{1}}}^{2}\right)+c\left({\overline{b_{2}^{2}}}^{2}{\overline{b_{2}}}^{2}\right)\right\} \\
& +\left\{\left(c-c^{2}\right){\overline{b_{1}}}^{2}+\left(c-c^{2}\right){\overline{b_{2}}}^{2}+\left(c-c^{2}\right) \overline{b_{1}} \overline{b_{2}}\right\} \\
= & \left\{(1-c)\left(\overline{b_{1}^{2}}-{\overline{b_{1}}}^{2}\right)+c\left({\overline{b_{2}^{2}}}^{2}{\overline{b_{2}}}^{2}\right)\right\} \\
& +\left\{c(c-1)\left(\overline{b_{1}}-\overline{b_{2}}\right)^{2}\right\}
\end{aligned}
$$

L'expression

$$
N\left\{(1-c)\left(\overline{b_{1}^{2}}-{\overline{b_{1}}}^{2}\right)+c\left(\overline{b_{2}^{2}}-{\overline{b_{2}}}^{2}\right)\right\}
$$

n'est rien d'autre que la diffusion incohérente ponderée par les concentrations. Mis à part l'absence des facteurs de Debye-Waller, elle est identique à (10.100) La diffusion diffuse est contenue dans le terme

$$
N\left\{c(c-1)\left(\overline{b_{1}}-\overline{b_{2}}\right)^{2}\right\} .
$$

Comme la diffusion incohérente, la diffusion diffuse est indépendante de $\vec{Q}$ pourvu qu'on néglige les facteurs de Debye-Waller. Elle s'observe sous la conditions qu'il existe une variation parmi les longueurs de diffusion des atomes qui se partagent un site du réseau, et cela même si tous les atomes ne diffusent que de manière cohérente.

\footnotetext{
$72 \mathrm{Si}$ la diffusion incohérente est due uniquement aux spins nucléaires, les neutrons polarisés peuvent être employés pour la séparer de la diffusion cohérente ainsi que de la diffusion diffuse.

73 C'est à ce point vrai qu'on confond souvent volontairement la diffusion diffuse et la diffusion incohérente dans des systèmes multi-composant amorphes.[25] La diffusion cohérente est alors définie comme la variance totale de la distribution des longueurs de diffusion $b$, à la fois de spin nucléaire, de composition isotopique et chimique. On parle dans ce contexte de diffusion incohérente même pour les rayons-X, ce qui objectivement n'a pas de sens selon la définition stricte que nous avons donnée pour la diffusion incohérente dans la section 5.6.
} 
En général, le modèle employé ne rend pas bien compte de la réalité. Le désordre chimique, même quand il s'agit d'un désordre très dilué, introduira des distorsions et ainsi influera sur la position des atomes. En ce qui concerne la diffusion de Bragg dans un réseau de Bravais, on écrira plutôt

$$
\left(\frac{d^{2} \sigma}{d \Omega d E_{f}}\right)_{\text {coh }}^{\mathrm{el}}=f^{2}\left|\sum_{l} \exp \left(i \vec{Q} \cdot\left(\vec{l}+\vec{u}_{\text {static }}(\vec{l})\right)\right)\right|^{2} \delta(\hbar \omega)
$$

où nous avons décrit les distortions comme

$$
\vec{l} \rightarrow \vec{l}+\vec{u}_{\text {static }}(\vec{l})
$$

Le choix de la variable $u$ est volontaire pour souligner l'analogie avec les phonons. Nous pouvons déduire de cette analogie que pour des distorsions faibles l'effet du désordre pourra se résumer à un facteur de Debye-Waller. Pour une distribution gaussienne de distorsions ce facteur de Debye-Waller statique aura la forme que nous avions déduite pour les phonons

$$
\exp \left(\frac{1}{2} \overline{\left(\vec{Q} \cdot \vec{u}_{\text {static }}\right)^{2}}\right)=\exp \left(\frac{1}{6} Q^{2} \overline{u_{\text {static }}^{2}}\right) .
$$

Entre les pics de Bragg nous trouverons la diffusion diffuse comme le carré de la transformée de Fourier du champ des distorsions. Ces champs peuvent de nouveau être assimilés à un jeu de phonons gelés et ainsi décrits par des vecteurs propres. C'est un sujet que nous ne voulons pas approfondir ici.

\subsection{Diffusion incohérente à un phonon}

Après ce petit détour par la diffusion diffuse, nous revenons maintenant à la discussion de la diffusion incohérente. Le prochain terme dans le développement de (10.85) contient la fonction de corrélation

$$
\begin{aligned}
\aleph_{j, j}(\vec{Q}, t) & =\left\langle\left(\vec{Q} \cdot \vec{u}_{j}\right)\left(\vec{Q} \cdot \vec{u}_{j}(t)\right)\right\rangle \\
& =\frac{\hbar}{2} \sum_{i}\left\{\left[\frac{A_{i}(\vec{Q} ; j)}{\omega_{i}}\left(1+n\left(\omega_{i}\right)\right) \exp \left(i \omega_{i} t\right)\right]+\left[\frac{A_{i}(\vec{Q} ; j)}{\omega_{i}} n\left(\omega_{i}\right) \exp \left(-i \omega_{i} t\right)\right]\right\}
\end{aligned}
$$

avec

$$
A_{i}(\vec{Q} ; j)=\frac{\left(\vec{Q} \cdot \vec{e}_{i}(j)\right) \cdot\left(\vec{Q} \cdot \vec{e}_{i}(j)\right)^{*}}{m_{j}}=\frac{\left|\vec{Q} \cdot \vec{e}_{i}(j)\right|^{2}}{m_{j}}
$$

Cette expression nous permet de calculer la fonction de diffusion incohérente pour un type d'atome $\kappa$

$$
\begin{aligned}
S_{\kappa}^{1-\mathrm{ph}}(\vec{Q}, \omega) & =\sum_{j \in\left\{j_{\kappa}\right\}} \frac{e^{-2 W_{j}(\vec{Q})}}{2 \pi \hbar} \int_{-\infty}^{\infty} d t\left\langle\left(\vec{Q} \cdot \vec{u}_{j}\right)\left(\vec{Q} \cdot \vec{u}_{j}(t)\right)\right\rangle e^{-i \omega t} \\
& =\sum_{j \in\left\{j_{\kappa}\right\}} \frac{e^{-2 W_{j}(\vec{Q})}}{2} \sum_{i} \frac{\left|\vec{Q} \cdot \vec{e}_{i}(j)\right|^{2}}{m_{\kappa} \omega_{i}}\left\{\left[\left(1+n\left(\omega_{i}\right)\right) \delta\left(\omega-\omega_{i}\right)\right]+\left[n\left(\omega_{i}\right) \delta\left(\omega+\omega_{i}\right)\right]\right\},
\end{aligned}
$$


et à partir de là, la section efficace différentielle partielle

$$
\begin{aligned}
& \left(\frac{d^{2} \sigma}{d \Omega d E_{f}}\right)_{\mathrm{inc}}^{1-\mathrm{ph}}=\frac{k_{f}}{k_{i}} \sum_{\kappa}\left(\overline{b_{\kappa}^{2}}-\left(\bar{b}_{\kappa}\right)^{2}\right) S_{\kappa}(\vec{Q}, \hbar \omega) \\
& =\frac{k_{f}}{k_{i}} \sum_{\kappa} \frac{\sigma_{\mathrm{inc}}}{8 \pi m_{\kappa}} \sum_{j \in\left\{j_{\kappa}\right\}} e^{-2 W_{j}(\vec{Q})} \sum_{i} \frac{\left|\vec{Q} \cdot \vec{e}_{i}(j)\right|^{2}}{\omega_{i}}\left\{\left[\left(1+n\left(\omega_{i}\right)\right) \delta\left(\omega-\omega_{i}\right)\right]+\left[n\left(\omega_{i}\right) \delta\left(\omega+\omega_{i}\right)\right]\right\} .
\end{aligned}
$$

Nous retrouvons la structure de la section efficace cohérente avec deux fonctions de Dirac.

L'une

$$
\begin{aligned}
\left(\frac{d^{2} \sigma}{d \Omega d E_{f}}\right)_{\mathrm{inc}}^{1-\mathrm{ph}(+)} & =\frac{k_{f}}{k_{i}} \sum_{\kappa}\left(\overline{b_{\kappa}^{2}}-\left(\bar{b}_{\kappa}\right)^{2}\right) S_{\kappa}(\vec{Q}, \hbar \omega) \\
& =\frac{k_{f}}{k_{i}} \sum_{\kappa} \frac{\sigma_{\mathrm{inc}}(\kappa)}{8 \pi m_{\kappa}} \sum_{j \in\left\{j_{\kappa}\right\}} e^{-2 W_{j}(\vec{Q})} \sum_{i} \frac{\left|\vec{Q} \cdot \vec{e}_{i}(j)\right|^{2}}{\omega_{i}}\left[\left(1+n\left(\omega_{i}\right)\right) \delta\left(\omega-\omega_{i}\right)\right]
\end{aligned}
$$

correspond à la création d'une excitation vibrationelle et l'autre

$$
\begin{aligned}
\left(\frac{d^{2} \sigma}{d \Omega d E_{f}}\right)_{\mathrm{inc}}^{1-\mathrm{ph}(-)} & =\frac{k_{f}}{k_{i}} \sum_{\kappa}\left(\overline{b_{\kappa}^{2}}-\left(\bar{b}_{\kappa}\right)^{2}\right) S_{\kappa}(\vec{Q}, \hbar \omega) \\
& =\frac{k_{f}}{k_{i}} \sum_{\kappa} \frac{\sigma_{\mathrm{inc}}(\kappa)}{8 \pi m_{\kappa}} \sum_{j \in\left\{j_{\kappa}\right\}} e^{-2 W_{j}(\vec{Q})} \sum_{i} \frac{\left|\vec{Q} \cdot \vec{e}_{i}(j)\right|^{2}}{\omega_{i}}\left[n\left(\omega_{i}\right) \delta\left(\omega+\omega_{i}\right)\right]
\end{aligned}
$$

à son annihilation.

\subsubsection{Relation avec les densités d'états partielles dans des systèmes cubiques ou isomorphes}

Pour un système quelconque, donc à priori anisotrope, les vecteurs propres dépendront explicitement des directions $\vec{Q}$ sur lesquelles on les projette. C'est même évident dans un cristal stratifié comme le graphite ${ }^{74}$. Les modes qui décrivent les vibrations dans les couches n'ont pas d'équivalents perpendiculaires aux couches, du moins pas à la même fréquence. La situation change si le système est cubique et monoatomique. Pour n'importe quel vecteur propre, nous trouverons deux autres modes d'énergies identiques tels que les trois vecteurs $\vec{e}_{i_{m}}(j)(m=1,2,3)$ forment un système orthonormal. La somme des projections de ces trois vecteurs sur $\vec{Q}$ donnera $^{75}$

$$
\sum_{i_{m}}\left|\vec{Q} \cdot \vec{e}_{i_{m}}(j)\right|^{2}=\frac{1}{3} Q^{2}\left|\vec{e}_{i}(j)\right|^{2}=\frac{1}{3} Q^{2} .
$$

On peut être tenté de penser qu'en effectuant la moyenne sur toutes les directions $\vec{Q}$, par exemple en travaillant avec une poudre, on peut se ramener au cas monoatomique isotrope. Ce n'est

\footnotetext{
${ }^{74}$ Le graphite n'est pas un très bon exemple parce qu'il ne diffuse que de manière cohérente.

75 Cette relation devient particulièrement évidente si $\vec{e}_{i_{1}}(j)$ est aligné avec $\vec{Q}$. Dans ce cas spécifique $\left|\vec{Q} \cdot \vec{e}_{i_{1}}(j)\right|^{2}=Q^{2}$ et $\left|\vec{Q} \cdot \vec{e}_{i_{2}}(j)\right|^{2}=\left|\vec{Q} \cdot \vec{e}_{i_{3}}(j)\right|^{2}=0$.
} 
malheureusement pas le cas. Pour une poudre, de même que pour un amorphe, la fonction de diffusion ne pourra en effet pas dépendre de la direction de $\vec{Q}$. Pour une bande de fréquence $[\omega, \omega+\Delta \omega]$ donnée, la moyenne des projections des vecteurs propres sur $\vec{Q}$ a donc forcément la forme

$$
\left\{\sum_{i}\left|\vec{Q} \cdot \vec{e}_{i}(j)\right|^{2}\right\}_{\left.\left.\omega_{i} \in\right] \omega, \omega+\Delta \omega\right]}=f(Q, \omega)\left\{\sum_{i}\left|\vec{e}_{i}(j)\right|^{2}\right\}_{\left.\left.\omega_{i} \in\right] \omega, \omega+\Delta \omega\right]} .
$$

Le problème est alors que la fonction $f(Q, \omega)$ dépendra du système étudié. Dans la pratique, on observe que même des systèmes de basse dimensionnalité comme les nanotubes peuvent être interprétés correctement en supposant que $f(Q, \omega)=f(Q)$, c'est-à-dire que $f(q, \omega)=f(q)$ est indépendante de $\omega$.

Inspiré par les systèmes monoatomiques cubiques, nous allons travailler avec la relation

$$
\left\{\sum_{i}\left|\vec{Q} \cdot \vec{e}_{i}(j)\right|^{2}\right\}_{\left.\left.\omega_{i} \in\right] \omega, \omega+\Delta \omega\right]}=\frac{1}{3} Q^{2}\left\{\sum_{i}\left|\vec{e}_{i}(j)\right|^{2}\right\}_{\left.\left.\omega_{i} \in\right] \omega, \omega+\Delta \omega\right]},
$$

Pour faciliter l'écriture et pour donner une signification plus précise à l'expression (10.116) nous introduisons la densité d'états spécifiques à un atome

$$
F_{j}(\omega)=\sum_{i}\left|\vec{Q} \cdot \vec{e}_{i}(j)\right|^{2} \delta\left(\omega-\omega_{i}\right)
$$

Elle donne une mesure du nombre de modes présents dans une bande d'énergie, qui impliquent la participation de l'atome $j$. Avec ces densités d'états nous pouvons écrire les sections efficaces différentielles comme

$$
\begin{aligned}
\left(\frac{d^{2} \sigma}{d \Omega d E_{f}}\right)_{\mathrm{inc}}^{1-\mathrm{ph}(+)} & =\frac{k_{f}}{k_{i}} \frac{Q^{2}}{3} \sum_{\kappa} \frac{\sigma_{\mathrm{inc}}(\kappa)}{8 \pi m_{\kappa}} \sum_{j \in\left\{j_{\kappa}\right\}} e^{-2 W_{j}(\vec{Q})} \frac{F_{j}(\omega)}{\omega}(1+n(\omega)), \quad \omega>0 \\
\left(\frac{d^{2} \sigma}{d \Omega d E_{f}}\right)_{\mathrm{inc}}^{1-\mathrm{ph}(-)} & =\frac{k_{f}}{k_{i}} \frac{Q^{2}}{3} \sum_{\kappa} \frac{\sigma_{\mathrm{inc}}(\kappa)}{8 \pi m_{\kappa}} \sum_{j \in\left\{j_{\kappa}\right\}} e^{-2 W_{j}(\vec{Q})} \frac{F_{j}(-\omega)}{-\omega} n(-\omega) \\
& =\frac{k_{f}}{k_{i}} \frac{Q^{2}}{3} \sum_{\kappa} \frac{\sigma_{\mathrm{inc}}(\kappa)}{8 \pi m_{\kappa}} \sum_{j \in\left\{j_{\kappa}\right\}} e^{-2 W_{j}(\vec{Q})} \frac{F_{j}(\omega)}{\omega}(n(\omega)+1), \quad \omega<0 .
\end{aligned}
$$

Nous avons exploité le fait que $n(-x)=n(x)+1$ (voir figure 37) et définissons

$$
F_{j}(-\omega)=F_{j}(\omega), \quad \omega \geq 0 .
$$

Dans cette convention nous ne sommes donc plus obligés de faire une distinction entre les expressions pour le gain et pour la perte d'énergie. ${ }^{76}$ Jusqu'ici, l'indice $\kappa$ s'est référé à l'élément chimique. Cette

$$
\begin{aligned}
& 76 \text { Nous voudrions néanmoins souligner qu'une autre convention existe. La fréquence } \omega \text { est toujours présumée positive et la } \\
& \text { diffusion incohérente s'obtient comme } \\
& \qquad\left(\frac{d^{2} \sigma}{d \Omega d E_{f}}\right)_{\text {inc }}^{1-\mathrm{ph}(+)}=\frac{k_{f}}{k_{i}} \frac{Q^{2}}{3} \sum_{\kappa} \frac{\sigma_{\mathrm{inc}}(\kappa)}{8 \pi m_{\kappa}} \sum_{j \in\left\{j_{\kappa}\right\}} e^{-2 W_{j}(\vec{Q})} \frac{F_{j}(\omega)}{\omega}(1+n(\omega)), \quad \omega>0 \\
& \left(\frac{d^{2} \sigma}{d \Omega d E_{f}}\right)_{\mathrm{inc}}^{1-\mathrm{ph}(-)}=\frac{k_{f}}{k_{i}} \frac{Q^{2}}{3} \sum_{\kappa} \frac{\sigma_{\mathrm{inc}}(\kappa)}{8 \pi m_{\kappa}} \sum_{j \in\left\{j_{\kappa}\right\}} e^{-2 W_{j}(\vec{Q})} \frac{F_{j}(\omega)}{\omega} n(\omega), \quad \omega<0 .
\end{aligned}
$$


distinction nous était imposée par le fait que les longueurs de diffusion cohérentes et incohérentes étaient liées au type d'atome. A ce point il s'avère astucieux de distinguer également les atomes de même type mais qui ne sont pas symétriquement équivalents. L'équivalence symétrique est bien définie dans un cristal. Dans un amorphe on pourra souvent considérer comme équivalent tous les atomes du même élément chimique. Les expressions pour la section efficace incohérente se simplifient davantage en introduisant les densités d'états partielles pour les atomes symétriquement équivalents distinguées par l'indice $\kappa$

$$
F_{\kappa}(\omega)=\frac{1}{3 N_{\kappa}} \sum_{j \in\left\{j_{\kappa}\right\}} \sum_{i}\left|\vec{e}_{i}(j)\right|^{2} \delta\left(\omega-\omega_{i}\right)
$$

avec $N_{\kappa}$ le nombre d'atomes de ce type. Les densités d'états partielles peuvent être évaluées à partir de n'importe quel représentant de la classe des atomes équivalents

$$
F_{\kappa}(\omega)=\frac{1}{3} \sum_{i}\left|\vec{e}_{i}\left(j \in\left\{j_{\kappa}\right\}\right)\right|^{2} \delta\left(\omega-\omega_{i}\right)
$$

Nous obtenons pour la section efficace

$$
\left(\frac{d^{2} \sigma}{d \Omega d E_{f}}\right)_{\mathrm{inc}}^{1-\mathrm{ph}}=\frac{k_{f}}{k_{i}} Q^{2} \frac{1}{8 \pi} \sum_{\kappa} N_{\kappa} \frac{\sigma_{\mathrm{inc}}(\kappa)}{m_{\kappa}} e^{-2 W_{\kappa}(\vec{Q})} F_{\kappa}(\omega) \frac{(1+n(\omega))}{\omega} .
$$

Dans un cristal, le nombre d'atomes non-équivalents est au maximum égal au nombre $r$ d'atomes dans la maille. Ainsi le résultat s'écrit comme

$$
\left(\frac{d^{2} \sigma}{d \Omega d E_{f}}\right)_{\mathrm{inc}}^{1-\mathrm{ph}}=\frac{k_{f}}{k_{i}} Q^{2} \frac{N}{8 \pi r} \sum_{d} \frac{\sigma_{\mathrm{inc}}(d)}{m_{d}} e^{-2 W_{k}(\vec{Q})} F_{d}(\omega) \frac{(1+n(\omega))}{\omega} .
$$

Il faut noter que pour aboutir à cette expression, nous n'avons à aucun moment utilisé le fait que dans un cristal les vecteurs propres sont classifiés selon les vecteurs d'onde $\vec{k}$. La symétrie de translation, qui est à l'origine de cette classification, n'apporte rien dans le cas de la diffusion incohérente en dehors de la réduction des types d'atomes symétriquement équivalents. Les vecteurs $\vec{k}$ définissent les phases relatives des mouvements des atomes. Etant donné, qu'il n'y a pas d'interférence entre les ondelettes provenant de différents atomes dans le cas de la diffusion incohérente, celle-ci n'est pas sensible à ces relations de phase. Par conséquent, et contrairement à la diffusion cohérente, la diffusion incohérente n'est pas soumise à des conditions de sélection du moment cristallin.

L'expression pour la section efficace différentielle partielle incohérente devient encore plus simple dans le cas d'un cristal de Bravais, c'est-à-dire avec un seul atome par maille.

$$
\left(\frac{d^{2} \sigma}{d \Omega d E_{f}}\right)_{\mathrm{inc}}^{1-\mathrm{ph}}=\frac{k_{f}}{k_{i}} Q^{2} \frac{N}{8 \pi} \frac{\sigma_{\mathrm{inc}}}{m} e^{-2 W_{d}(\vec{Q})} \frac{g(\omega)}{\omega}(1+n(\omega))
$$

ce que nous pouvons aussi écrire comme

$$
\left(\frac{d^{2} \sigma}{d \Omega d E_{f}}\right)_{\mathrm{inc}}^{1-\mathrm{ph}( \pm)}=\frac{k_{f}}{k_{i}} \frac{Q^{2}}{3} \sum_{\kappa} \frac{\sigma_{\mathrm{inc}}(\kappa)}{8 \pi m_{\kappa}} \sum_{j \in\left\{j_{\kappa}\right\}} e^{-2 W_{j}(\vec{Q})} \frac{F_{j}(\omega)}{\omega}\left[\frac{1}{2}\left(\cosh \left(\frac{\hbar \omega}{2 k_{\mathrm{B}} T}\right) \pm 1\right)\right] .
$$


avec

$$
\begin{aligned}
g(\omega) & =\frac{1}{3 N} \sum_{j} \sum_{i}\left|\vec{e}_{i}(j)\right|^{2} \delta\left(\omega-\omega_{i}\right) \\
& =\frac{1}{3 N} \sum_{i} \delta\left(\omega-\omega_{i}\right) \sum_{j}\left|\vec{e}_{i}(j)\right|^{2} \\
& =\frac{1}{3 N} \sum_{i} \delta\left(\omega-\omega_{i}\right)
\end{aligned}
$$

la densité d'états vibrationelle. Contrairement à l'expression (10.72) pour la diffusion cohérente à un phonon, l'expression (10.131) est proportionnelle au nombre de diffuseurs $N$. L'origine de cette dépendance explicite réside dans notre convention pour la normalisation de la densité d'états. En combinant (10.131) avec (10.132) nous nous apercevons immédiatement que la diffusion est proportionnelle au nombre de modes, qui lui est proportionnel au nombre d'atomes du système.

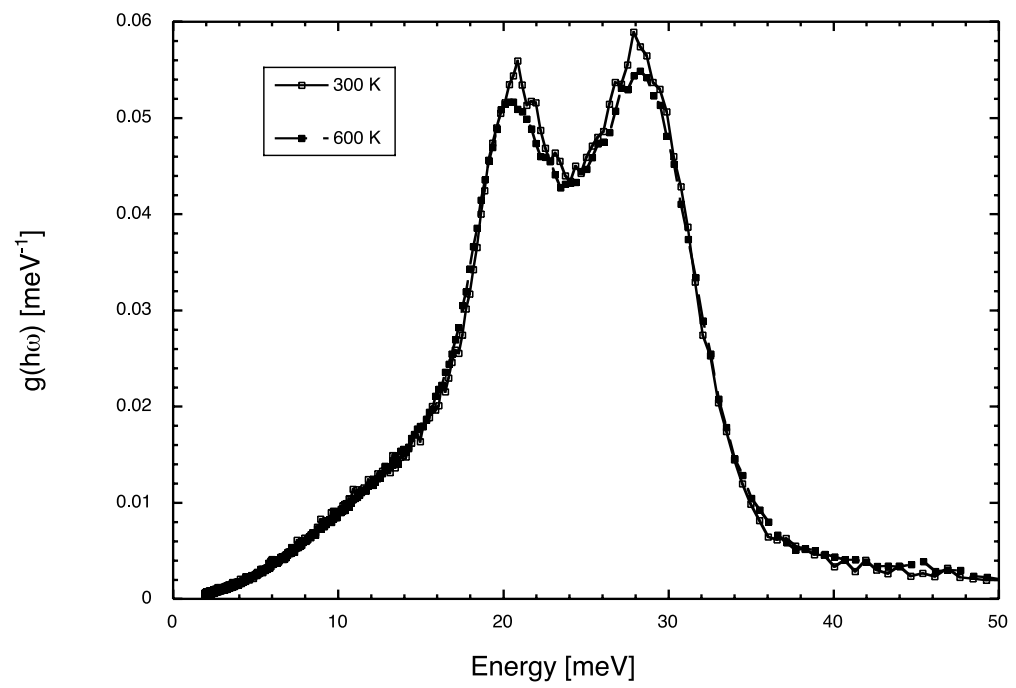

Figure 42. Densité d'états du vanadium à 300 et à $600 \mathrm{~K}$ obtenue par diffusion incohérente à un phonon sur l'instrument IN6 (se référer à la figure 45) avec une longueur d'onde incidente de $\lambda=4.16 \AA$ A . Les multiphonons ont été corrigés avec le processus décrit dans la section 11.5 (voir la figure 48). Pour un matériau très harmonique comme est le vanadium à ces températures, la densité d'états est plus ou moins indépendante de $T$.

\subsubsection{Rapport d'intensité entre diffusion élastique et inélastique}

Bien que les expressions ci-dessus donnent l'intensité de la diffusion en valeur absolue, il est intéressant de comparer la diffusion inélastique au signal élastique pour se faire une idée plus concrète de son importance. Nous allons effectuer cette comparaison pour un système simple à un seul composant qui est en plus un pur diffuseur incohérent, dans un réseau de symétrie cubique tel que le vanadium. La raison étant que pour un tel système, les résultats obtenus dans la précédente section et en particulier l'expression (10.131) s'appliquent rigoureusement. Dans ce cas, la diffusion élastique est selon (10.87) donnée par

$$
\left(\frac{d \sigma}{d \omega}\right)_{\mathrm{inc}}^{\mathrm{el}}=N \sigma_{i} e^{-2 W(Q)}
$$


Si on est intéressé uniquement par les plus basses fréquences alors on peut exploiter le fait que le spectre dans cette région est composé de modes acoustiques, c'est-à-dire de modes, qui présentent la dispersion

$$
\omega=\vec{k} \cdot c_{j, \hat{k}}
$$

où $c_{j, \hat{k}}$ représente la vitesse du son suivant la direction $\hat{k}$ pour la branche acoustique $j$. La densité d'états est alors donnée par

$$
g(\omega)=\left(\frac{V}{N}\right)\left(\frac{2 \pi^{2}}{c^{3}}\right) \omega^{2}=\frac{3 \omega^{2}}{\omega_{D}^{3}},
$$

où $c$ représente la vitesse du son moyennée sur les directions et sur les branches. $\omega_{D}$ tel que défini à partir de (10.135) est la fréquence de Debye du matériau. $V$ est le volume occupé par les $N$ atomes.

Pour appliquer l'équation (10.131) nous devons prendre en compte le facteur d'occupation. Pour des énergies d'excitation

$$
\hbar \omega \ll k_{\mathrm{B}} T: \quad n(\omega) \rightarrow \hbar \omega / k_{\mathrm{B}} T .
$$

Nous obtenons donc pour la section efficace inélastique en gain d'énergie

$$
\begin{aligned}
\left(\frac{d^{2} \sigma}{d E_{f} d \Omega}\right)_{\mathrm{inc}} & =\frac{3 N \sigma_{\mathrm{i}}}{8 \pi} \frac{k_{f}}{k_{i}} \frac{\hbar^{2} Q^{2}}{2 m} e^{-2 W(\vec{Q})}\left(\frac{k_{\mathrm{B}} T}{\left(\hbar \omega_{D}\right)^{3}}\right) \\
& =\frac{k_{f}}{k_{i}} 3 N \frac{\sigma_{\mathrm{i}}}{8 \pi} e^{-2 W(\vec{Q})}\left(\frac{E_{r}(Q) \cdot\left(k_{\mathrm{B}} T\right)}{\left(\hbar \omega_{D}\right)^{3}}\right),
\end{aligned}
$$

avec l'énergie de recul de l'atome définie comme dans la section 8.10 Nous allons rencontrer $E_{r}(Q)$ encore plusieurs fois lors de la discussion des multiphonons. Nous constatons que cette intensité est proportionnelle à la température et ne dépend plus de $\omega$. C'est un constat qui vaut pour tout système tridimensionnel, c'est-à-dire pour tout système dont la densité d'état est proportionnelle à $\omega^{2}$. Nous voyons en particulier que le rétrécissement du nombre d'états pour $\omega \rightarrow 0$ compense la divergence du facteur $n(\omega) / \omega .^{77}$

Ceci donne le rapport entre les signaux élastiques et inélastiques

$$
\left(\frac{d \sigma}{d \Omega}\right)_{\mathrm{inc}}^{\mathrm{el}} \int_{0}^{\hbar \omega_{l}}\left(\frac{d^{2} \sigma}{d(\hbar \omega) d \Omega}\right)_{\mathrm{inc}}^{\mathrm{el}} d \hbar \omega \approx \frac{4 \pi}{3}\left(\frac{\left(\hbar \omega_{D}\right)^{3}}{E_{r}(Q)\left(\hbar \omega_{l}\right)\left(k_{\mathrm{B}} T\right)}\right)
$$

où on a employé $k_{i} \approx k_{f}$ pour les faibles transferts d'énergie. Le rapport est donc défini par l'énergie caractéristique $\hbar \omega_{D}$ du spectre de vibration, l'énergie thermique $k_{\mathrm{B}} T$ et l'intervalle d'intégration $\hbar \omega_{l}$. Ces valeurs sont toutes dans la gamme entre zéro et $100 \mathrm{meV}$. Les énergies de Debye typiques se situent autour de $40 \mathrm{meV}$, alors que $k_{\mathrm{B}} T$ à température ambiante est environ de $30 \mathrm{meV}$. En prenant $Q=2 \AA^{-1}$ et une masse atomique de 50 uma, on obtient pour l'énergie de recul $\hbar^{2} Q^{2} / 2 m$ environ $0.3 \mathrm{meV}$. Cette estimation simple donne des intensités inélastiques (intégrées dans une gamme de 0 à $10 \mathrm{meV}$ ) 100 à 1000 fois plus petites que celles qu'on recueille dans le canal élastique. Ceci explique pourquoi il est si important de maintenir le bruit de fond à un faible niveau lors des expériences inélastiques. Notez aussi que le facteur de Debye-Waller, qui affecte de manière égale les intensités élastiques et inélastiques n'entre pas en ligne de compte dans le calcul de ce rapport.

\subsubsection{Densité d'états généralisée}

Les systèmes qui intéressent le scientifique sont en général complexes et ne satisfont donc pas à l'expression (10.131). C'est dommage dans la mesure où cette expression permettrait une interprétation simple des résultats en accédant directement à une quantité physique de grand intérêt, comme peut

${ }^{77} n(\omega) \rightarrow\left(\hbar \omega /\left(k_{\mathrm{B}} T\right)\right)^{-1}$ pour $\omega \rightarrow 0-\epsilon($ voir figure 37$)$. 
l'être la densité d'états. D'un point de vue purement formel nous pouvons sans problème obtenir une expression similaire à (10.131) pour un cristal à plusieurs atomes dans la maille

$$
\left(\frac{d^{2} \sigma}{d \Omega d E_{f}}\right)_{\mathrm{inc}}^{1-\mathrm{ph}(+)}=\frac{k_{f}}{k_{i}} Q^{2} \frac{N}{8 \pi} e^{-2 W(\vec{Q})} \frac{G(\omega)}{\omega}(1+n(\omega))
$$

en introduisant la densité d'états généralisée

$$
G(\omega)=\sum_{d=1}^{r} \frac{\sigma_{\mathrm{inc}}(d)}{m_{d}} F_{d}(\omega)
$$

et sous la condition que les facteurs

$$
e^{-2 W_{d}(\vec{Q})} \approx e^{-2 W(\vec{Q})}
$$

sont plus ou moins indépendants de $d$. Dans la densité d'états généralisée $G(\omega)$, les densités d'états partielles $F_{d}(\omega)$, correspondant aux différents atomes dans la maille primitive, sont pondérées avec ce que nous appelons leurs pouvoirs de diffusion

$$
\frac{\sigma_{\mathrm{inc}}(d)}{m_{d}}
$$

Il n'est guère surprenant qu'à masse égale, les atomes qui possèdent les longueurs de diffusion les plus importantes soient aussi les plus visibles dans la section efficace différentielle partielle.

Nous avons en principe la possibilité d'isoler les différentes contributions de la densité d'états généralisée, pourvu qu'il existe un moyen de modifier le contraste. La situation est analogue à l'isolement des fonctions de corrélation partielles (se référer à la section 8.4), dont les densités d'états partielles sont la manifestation directe. Modifier le contraste est possible si un élément chimique possède des isotopes avec des longueurs de diffusion très distinctes. C'est notamment le cas pour l'hydrogène et le deutérium. Mais alors, dans ce cas précis -qui est souvent exploité dans la recherche en matière molle et en biologie- les masses des isotopes sont très différentes. Cette différence influe beaucoup sur la fréquence des modes dont les vecteurs propres contiennent principalement des composantes de l'hydrogène ou du deutérium. L'exemple le plus parlant est l'eau. Le spectre des librations s'amortit beaucoup quand on passe de l'eau légère $\mathrm{H}_{2} \mathrm{O}$ à l'eau lourde $\mathrm{D}_{2} \mathrm{O}^{78}$.

Si on peut séparer les densités d'états partielles alors il est possible de remonter à la densité d'états vibrationelle $g(\omega)$. C'est la quantité qui intéresse avant tout le scientifique parce qu'elle rentre dans le calculs des fonctions thermodynamiques (voir chapitre I). La séparation des densités d'états partielles est malheureusement quelque chose d'exceptionnel. En général on devra se contenter de la densité d'états généralisée.

A la lumière des approximations que nous étions obligés d'introduire pour parvenir à l'expression (10.138), l'affirmation que la diffusion incohérente des neutrons donne un accès direct à $g(\omega)$ est à prononcer avec précaution. Il est certainement vrai que, même dans le cas d'un système polyatomique, il est toujours possible d'écrire la fonction de diffusion sous la forme

$$
S(\vec{Q}, \omega)=A(\vec{Q}, \omega) G_{\mathrm{eff}}(\omega),
$$

\footnotetext{
78 Il ne faut pas oublier que, en dehors des masses, les caractéristiques de diffusion de l'hydrogène et du deutérium sont très différentes (voir la section 5.8). Le deutérium est un diffuseur cohérent avec une section efficace incohérente non-négligable.
} 
avec en analogie avec l'expression (10.131)

$$
\left.A(\vec{Q}, \omega)=\frac{\hbar}{4 \pi} \frac{\left(\sigma_{\mathrm{inc}}(j) e^{-2 W_{j}(Q)}\right)_{\mathrm{av}}}{2 M_{\mathrm{av}}} \frac{Q^{2}}{\omega}[n(\omega)+1)\right]
$$

et

$$
M_{\mathrm{av}}=\frac{1}{N} \sum_{j} m_{j} \quad \text { et } \quad\left(\bar{b}^{2} e^{-2 W_{j}(Q)}\right)_{\mathrm{av}}=\frac{1}{N} \sum_{j} \sigma_{\text {inc }}(j) \exp \left(-2 W_{j}(Q)\right) \text {. }
$$

La densité d'états effective $G_{\text {eff }}(\omega)$ est bien définie du point de vue mathématique. Malheureusement elle peut être assez différente de la fonction d'états vibrationnelle $g(\omega)$, d'une façon encore pire que la densité d'états généralisée $G(\omega)$. Dans les cas où le but de l'expérience est de vérifier un modèle dynamique, la façon correcte de procéder est de calculer la densité d'états généralisée $G(\omega)$ ou encore mieux la fonction de diffusion $S(\vec{Q}, \omega)$, à partir de la théorie.

Sans modèle théorique on doit être conscient des erreurs faites en identifiant aveuglément la densité d'état effective $G_{\text {eff }}(\omega)$ avec la densité d'états vibrationnelle $g(\omega)$. Nous considérons cette source d'erreur suffisamment importante pour l'illustrer avec un exemple simple mais parlant : celui de la glace. La glace étant un cristal moléculaire typique, son spectre présente plusieurs bandes d'excitations (voir figure 43). Aux basses fréquences $(\hbar \omega<40 \mathrm{meV})$ on trouve les vibrations impliquant une translation du centre de gravité des molécules. Négligeons pour le moment le fait que les phases de la glace sont caractérisées par plus d'une molécule par maille primitive. Si on suppose un découplage complet entre les modes de translation et les modes de libration, alors le système peut être considéré comme atomique (l'atome étant la molécule $\mathrm{H}_{2} \mathrm{O}$ ) pour les basses fréquences, et le poids de chacun des modes translationnels dans la section efficace (10.129) est déterminé par $2 \sigma_{\text {inc }}(\mathrm{H}) / m\left(\mathrm{H}_{2} \mathrm{O}\right)$, tout en se rappelant que les vecteurs propres $e_{i}$ sont normalisés à l'unité et que la diffusion est dominée par la section efficace incohérente de l'hydrogène. La bande suivante d'excitations contient les librations. Comme les translations, les librations sont des modes externes c'est-à-dire qu'elles n'impliquent pas de distorsion des molécules $\mathrm{H}_{2} \mathrm{O}$. Pour des librations idéales, le centre de gravité est au repos (voir le découplage mentionné ci-dessus). Comme le centre de gravité est proche de l'atome d'oxygène, c'est une bonne approximation de considérer que les modes normaux n'ont des composantes non nulles que pour les atomes d'hydrogène. Le poids de chaque mode librationnel dans la section efficace est de ce fait déterminé par $\sigma_{\text {inc }}(\mathrm{H}) / m(\mathrm{H})$. Ceci signifie que, bien que les deux bandes contiennent le même nombre de modes, l'intensité de la bande librationnelle vue dans une expérience de diffusion sera multipliée par un facteur proche de 9 par rapport à une bande translationnelle hypothétique qui couvrirait le même domaine de fréquence. Si on analyse simplement la densité d'état généralisée comme étant la vraie densité d'états, cela peut introduire d'énormes erreurs dans l'interprétation. Des calculs plus élaborés, impliquant des simulations de dynamique moléculaire, confirment ces erreurs. [44].

\subsection{Approximation incohérente}

Comme nous l'avons déjà evoqué à plusieurs reprises, la densité d'états d'un système est d'une grande importance pour les propriétés physiques. Elle est en principe accessible par l'intermédiaire de la diffusion incohérente. Hélas, on est souvent confronté à des diffuseurs cohérents. Si on possède un monocristal on peut toujours mesurer toutes les branches de dispersion et en déduire la densité d'états. Dans la réalité cette approche est rarement utilisée du fait que, dès qu'il s'agit d'un cristal assez complexe, c'est-à-dire avec plusieurs atomes dans la maille primitive, le nombre de branches devient trop important. Pour déterminer la densité d'état d'un matériau, l'expérience doit capter l'ensemble des modes. Etant donné que la diffusion cohérente à un phonon est hautement sélective ceci n'est possible que si la mesure permet de couvrir -au minimum- la partie asymétrique de la zone de Brillouin. Ce 


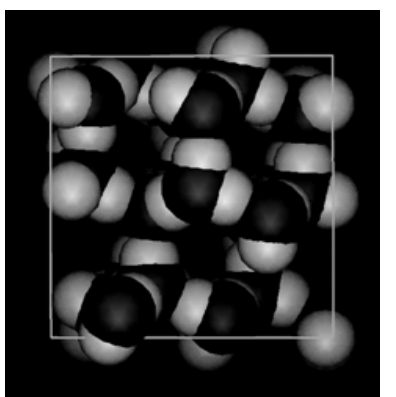

glace hexagonale

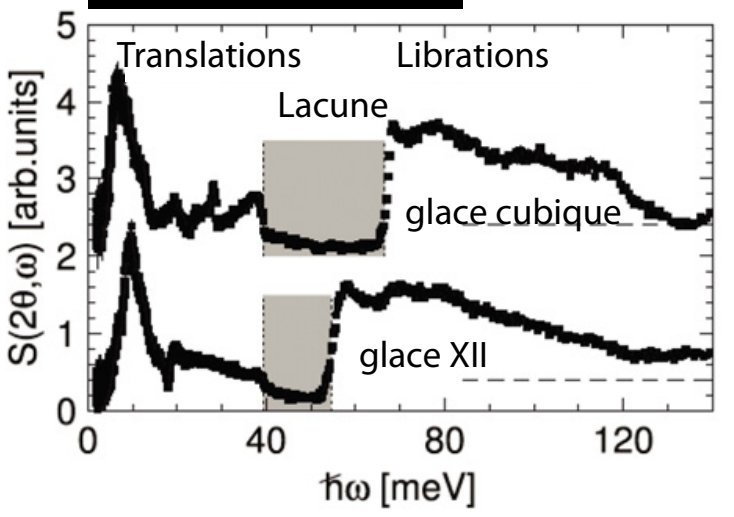

Densité d'états effective

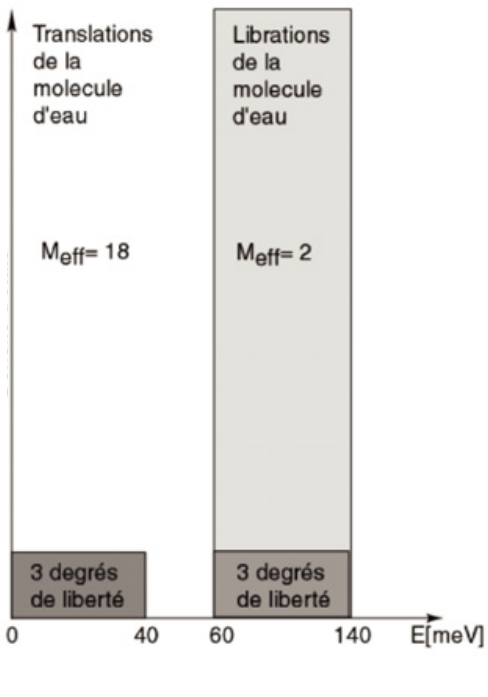

Figure 43. Intensité inélastique mesurée pour deux phases de la glace $\mathrm{H}_{2} \mathrm{O}$ sur l'instrument TOSCA à ISIS [45]. Dans la glace, les modes de translation des molécules d'eau sont bien séparés des librations. Les atomes en mouvement pour les modes de libration, se limitent aux deux atomes d'hydrogène. L'inverse de la masse effective, qui entre en jeu pour le calcul de l'intensité, est alors neuf fois plus élevé que celui des translations. Ne pas prendre en compte cette différence et déterminer la densité d'états vibrationelle à partir de l'expression (10.142) amènerait à une surestimation de la contribution des librations, dans le pire des cas d'un facteur neuf.

balayage peut s'avérer toujours insuffisant à cause de la projection des vecteurs propres sur $\vec{Q}$. Cette projection est par exemple, nulle si nous avons affaire à des modes purement transverses et si notre expérience ne couvre que la première zone de Brillouin. Même si le signal contient des contributions de tous les modes il n'est pas garanti que nous soyons en mesure de déterminer la densité d'état. Il faut toujours connaitre le poids exact de chaque contribution qui dépendra toujours de la projection des vecteurs propres sur $\vec{Q}$.

Etant donnée qu'on a besoin d'une moyenne sur tous les modes il semble logique de se tourner vers des mesures sur poudre. De telles mesures ont été discutées très tôt (voir par exemple [46]). Pour cerner un peu mieux le problème et en particulier pour identifier les principales sources d'erreurs nous proposons d'étudier un peu plus dans le détail l'influence d'une poudre sur la section efficace inélastique. Si nous nous limitons à des systèmes monoatomiques cubiques nous obtenons à partir de (10.72) pour la section efficace différentielle partielle cohérente

$$
\begin{aligned}
\left(\frac{d \sigma^{2}}{d(\hbar \omega) d \Omega}\right)_{\mathrm{coh}}^{1-\mathrm{ph}}= & \frac{k_{f}}{k_{i}} \frac{\sigma_{\mathrm{coh}}}{4 \pi} \frac{(2 \pi)^{3}}{V_{\text {maille }}} \sum_{\vec{G}} \sum_{i, \vec{k}} e^{-2 W(Q)} \frac{\left|\vec{Q} \cdot \vec{e}_{i}(\vec{k})\right|^{2}}{2 m \omega_{i}(\vec{k})} \\
& \times\left(1+n\left(\omega_{i}(\vec{k})\right)\right) \delta(\vec{k}-(\vec{Q}+\vec{G})) \delta\left(\omega-\omega_{i}(\vec{k})\right),
\end{aligned}
$$


où nous ne montrons que la partie liée à l'émission d'un phonon. La partie pour l'absorption pourra être déduite en parfaite analogie.

Pour effectuer des moyennes, il est en général préférable de transformer la somme sur $\vec{k}$ en intégrale $^{79}$

$$
\begin{aligned}
\left(\frac{d \sigma^{2}}{d(\hbar \omega) d \Omega}\right)_{\mathrm{coh}}^{1-\mathrm{ph}}= & N \frac{k_{f}}{k_{i}} \frac{\sigma_{\mathrm{coh}}}{8 \pi m} \sum_{i=1}^{3} \int_{\mathrm{BZ}} d^{3} k \frac{\left.\delta\left(\omega-\omega_{i}(\vec{k})\right)\right)}{\omega_{i}(\vec{k})}\left(1+n\left(\omega_{i}(\vec{k})\right)\right) \\
& \times \sum_{\vec{G}} e^{-2 W(Q)}\left|\vec{Q} \cdot \vec{e}_{i}(\vec{k})\right|^{2} \delta(\vec{k}-(\vec{Q}+\vec{G})) .
\end{aligned}
$$

Pour un transfert d'énergie donné, le vecteur $\vec{Q}$ est défini dans le référentiel du spectromètre par $\vec{k}_{i}$ et $\vec{k}_{f}$. Les vecteurs $\vec{k}$ ainsi que les vecteurs propres $\vec{e}_{i}(\vec{k})$ des phonons sont définis dans le référentiel de l'échantillon. Dans une poudre, ce référentiel change d'un grain à l'autre. Du point de vue de l'échantillon la direction de $\vec{Q}$ est donc arbitraire. Formellement le vecteur $\vec{Q}$ peut se trouver n'importe où sur une sphère de rayon $Q$. Pour décrire la diffusion correctement nous devons moyenner la section efficace sur toutes les directions de $\vec{Q}$. Nous définissons

$$
\left(\frac{d \sigma^{2}}{d(\hbar \omega) d \Omega}\right)_{\mathrm{coh}-|\mathrm{Q}|}^{1-\mathrm{ph}}=\frac{1}{4 \pi Q^{2}} \int_{0}^{\pi} \sin \theta_{Q} d \theta_{Q} \int_{0}^{2 \pi} d \Phi_{Q}\left(\frac{d \sigma^{2}}{d(\hbar \omega) d \Omega}\right)_{\mathrm{coh}}^{1-\mathrm{ph}}
$$

Dans cette expression les coordonnées sphériques se réfèrent, comme indiqué explicitement, à $\vec{Q}$ et non à $\vec{k}_{f}$. Effectuant cette moyenne pour l'expression (10.147) nous obtenons

$$
\begin{aligned}
\left(\frac{d \sigma^{2}}{d(\hbar \omega) d \Omega}\right)_{\mathrm{coh}-|\mathrm{Q}|}^{1-\mathrm{ph}}= & N \frac{k_{f}}{k_{i}} \frac{\sigma_{\mathrm{coh}}}{8 \pi m} \frac{1}{4 \pi Q^{2}} \sum_{i=1}^{3} \int_{\mathrm{BZ}} d^{3} k \frac{\left.\delta\left(\omega-\omega_{i}(\vec{k})\right)\right)}{\omega_{i}(\vec{k})}\left(1+n\left(\omega_{i}(\vec{k})\right)\right) \\
& \left.\times \sum_{\vec{G}} e^{-2 W(Q)}\left|\vec{e}_{i}(\vec{k}) \cdot(\vec{k}-\vec{G})\right|^{2} \delta(|\vec{k}-\vec{G}|-Q)\right) \\
= & N \frac{k_{f}}{k_{i}} \frac{\sigma_{\mathrm{coh}}}{8 \pi m} \frac{1}{4 \pi} \sum_{i=1}^{3} \int_{\mathrm{BZ}} d^{3} k \frac{\left.\delta\left(\omega-\omega_{i}(\vec{k})\right)\right)}{\omega_{i}(\vec{k})}\left(1+n\left(\omega_{i}(\vec{k})\right)\right) \\
& \times \sum_{\vec{G}} e^{-2 W(Q)\left|\vec{e}_{i}(\vec{k}) \cdot \hat{k}_{\vec{G}}\right|^{2} \delta\left(\left|\vec{k}_{\vec{G}}\right|-Q\right)}
\end{aligned}
$$

avec $\vec{k}_{\vec{G}}=\vec{k}-\vec{G}$ pour simplifier la notation. Par rapport à (10.147), les fonctions de Dirac des vecteurs $\vec{Q}$ deviennent des fonctions de Dirac de la norme $Q$.

${ }^{79}$ Nous utilisons le remplacement

$$
\sum_{\vec{k}} \ldots \rightarrow \frac{N}{V_{\mathrm{Bz}}} \int d^{3} k \ldots=N \frac{V_{\text {maille }}}{(2 \pi)^{3}} \int d^{3} k \ldots
$$


Si la diffusion était incohérente nous aurions obtenu en partant de (10.115)

$$
\begin{aligned}
\left(\frac{d \sigma^{2}}{d(\hbar \omega) d \Omega}\right)_{\mathrm{inc}-|\mathrm{Q}|}^{1-\mathrm{ph}}= & N \frac{k_{f}}{k_{i}} \frac{\sigma_{\text {inc }}}{8 \pi m} \frac{V_{\text {maille }}}{(2 \pi)^{3}} e^{-2 W(Q)} \\
& \times \sum_{i=1}^{3} \int_{\mathrm{BZ}} d^{3} k \frac{\left.\delta\left(\omega-\omega_{i}(\vec{k})\right)\right)}{\omega_{i}(\vec{k})}\left(1+n\left(\omega_{i}(\vec{k})\right)\right)\left\{\left|\vec{e}_{i}(\vec{k}) \cdot \vec{Q}\right|^{2}\right\}_{\mathrm{av}} \\
= & N \frac{k_{f}}{k_{i}} \frac{\sigma_{\mathrm{inc}}}{8 \pi m} \frac{1}{4 \pi} e^{-2 W(Q)} \frac{g(\omega)}{\omega}(1+n(\omega)) .
\end{aligned}
$$

C'est le résultat que nous connaissons. ${ }^{80} \mathrm{La}$ différence entre (10.149) et (10.150) réside en principe uniquement dans la sélection des modes qui contribuent à l'intégrale. La diffusion incohérente est extensive. Pour un composé à plusieurs atomes, le poids associé à un type d'atome est géré par sa longueur de diffusion incohérente. Tous les modes sont alors présents dans la section efficace. La diffusion cohérente est sélective. Même dans une poudre, un mode ne contribue à la section efficace que s'il existe un vecteur $\vec{G}$ du réseau réciproque tel que $|\vec{k}-\vec{G}|=\vec{Q}$. Si $Q$ est petit, le nombre de $\vec{G}$ qui remplissent cette condition sera très limité (se référer à la figure 44). Dans le cas extrême, tous les $\vec{Q}$ feront partie de la première zone de Brillouin et les vecteurs d'onde des modes seront soumis à la condition $|\vec{k}|=Q$. Il est alors évident que la plupart des modes nous échapperont. Le nombre de vecteurs réciproques $\vec{G}$ qui rentreront dans la section efficace augmentera considérablement avec $Q$. Malheureusement un $Q$ trop important n'est pas souhaitable pour d'autres raisons. Premièrement la résolution en énergie se dégrade, et deuxièmement parce que la contribution des multiphonons (voir section 11.4) devient de plus en plus forte.

La solution consiste à utiliser des valeurs de $Q$ raisonnablement grandes et d'assurer en parallèle le meilleur échantillonnage possible des modes en permettant une variation de $Q$. Dans la pratique, cette variation peut être produite en utilisant un instrument à multidétecteur. Pour un transfert d'énergie $\hbar \omega$ donné $Q$ variera en passant d'un détecteur à l'autre. L'expérience couvrira ainsi tout le volume contenu entre les sphères de rayon $Q_{\min }(\hbar \omega)$ et $Q_{\max }(\hbar \omega)$. Notons $p(\hbar \omega, Q)$ la probabilité ${ }^{81}$ que par diffusion, le changement d'impulsion du neutron possède la norme $Q$. La section efficace cohérente intégrée sur $V$ peut alors s'écrire comme

$$
\begin{aligned}
\left(\frac{d \sigma^{2}}{d(\hbar \omega)}\right)_{\mathrm{coh}-\mathrm{int}}^{1-\mathrm{ph}} & =\int_{V}\left(\frac{d \sigma^{2}}{d(\hbar \omega) d \Omega}\right)_{\mathrm{coh}}^{1-\mathrm{ph}} p_{\hbar \omega}(Q) d^{3} Q \\
& =4 \pi \int_{Q_{\min }}^{Q_{\max }}\left(\frac{d \sigma^{2}}{d(\hbar \omega) d \Omega}\right)_{\mathrm{coh}-|\mathrm{Q}|}^{1-\mathrm{ph}} Q^{2} p_{\hbar \omega}(Q) d Q \\
& =N \frac{k_{f}}{k_{i}} \frac{\sigma_{\mathrm{coh}}}{8 \pi m} \sum_{i=1}^{3} \int_{\mathrm{BZ}} d^{3} k \frac{\left.\delta\left(\omega-\omega_{i}(\vec{k})\right)\right)}{\omega_{i}(\vec{k})}\left(1+n\left(\omega_{i}(\vec{k})\right)\right)
\end{aligned}
$$

${ }^{80}$ Il vaut

$$
\sum_{i=1}^{3} \int_{\mathrm{BZ}} d^{3} k \ldots=3 V_{\mathrm{Bz}} \int g(\omega) \ldots d \omega .
$$

${ }^{81}$ Cette probabilité dépendra des détails de l'expérience. Nous en donnerons l'expression pour le cas d'un spectromètre à multidétecteur en géométrie de temps de vol directe. 


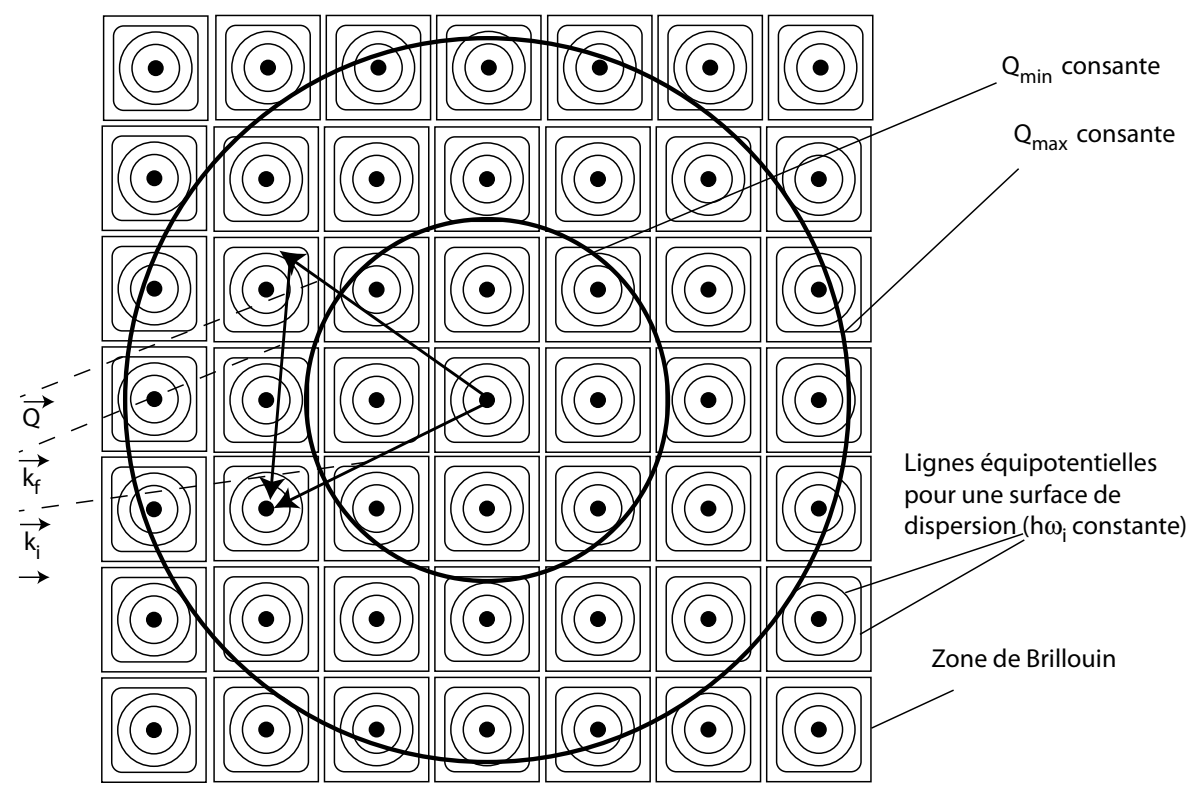

Figure 44. Coupe de l'espace réciproque pour un réseau cubique. Les courbes de dispersion des phonons sont indiquées de manière purement schématique par des lignes équipotentielles. Si on travaille avec un seul détecteur alors $Q$ est fixé par le transfert d'énergie $\hbar \omega$. Dans un cristal, on mesure un seul point $(\vec{Q}, \hbar \omega)$. Dans une poudre $\hat{Q}$ balaye toutes les directions dans le référentiel de l'échantillon. On moyenne la mesure sur une sphère de rayon $Q$. Si le rayon de cette sphère est choisi petit alors on ne sélectionnera qu'une partie des modes. Avec des instruments à multidétecteur (se référer à la figure 45) on couvrira un domaine étendu qui est contenu entre les sphères de rayons $Q_{\max }$ et $Q_{\min }$. Dans l'exemple montré ici, ce domaine correspond à une vingtaine de zones de Brillouin dans le plans et une centaine de zones dans le volume.

$$
\begin{aligned}
& \left.\times \int_{Q_{\min }}^{Q_{\max }} \sum_{\vec{G}} e^{-2 W(Q)}\left|\vec{e}_{i}(\vec{k}) \cdot \hat{k}_{\vec{G}}\right|^{2} \delta\left(\left|\vec{k}_{\vec{G}}\right|-Q\right)\right) Q^{2} p_{\hbar \omega}(Q) d Q \\
& =N \frac{k_{f}}{k_{i}} \frac{\sigma_{\mathrm{coh}}}{8 \pi m} \sum_{i=1}^{3} \int_{\mathrm{BZ}} d^{3} k \frac{\left.\delta\left(\omega-\omega_{i}(\vec{k})\right)\right)}{\omega_{i}(\vec{k})}\left(1+n\left(\omega_{i}(\vec{k})\right)\right) \\
& \times \sum_{(\vec{k}-\vec{G}) \in V} e^{-2 W(|\vec{k}-\vec{G}|)}\left|\vec{e}_{i}(\vec{k}) \cdot \hat{k}_{\vec{G}}\right|^{2}|\vec{k}-\vec{G}|^{2} p_{\hbar \omega}(|\vec{k}-\vec{G}|) .
\end{aligned}
$$

Pour un mode $(i, \vec{k})$ donné, l'intégration des termes qui dépendent de $\vec{Q}$ est donc contenu dans la fonction

$$
h_{i}(\vec{k})=\sum_{(\vec{k}-\vec{G}) \in V} e^{-2 W(|\vec{k}-\vec{G}|)}\left|\vec{e}_{i}(\vec{k}) \cdot \hat{k}_{\vec{G}}\right|^{2}|\vec{k}-\vec{G}|^{2} p_{\hbar \omega}(|\vec{k}-\vec{G}|) .
$$

Pour évaluer cette expression, nous négligeons à partir de maintenant la dépendance en $Q$ du facteur de Debye-Waller. Pour des volumes $V$ suffisamment grands, nous pouvons imaginer que les termes apparaissant dans $h_{i}(\vec{k})$ peuvent être remplacés par leurs moyennes

$$
h_{i}(\vec{k})=e^{-2 W(Q)} n \overline{\left|\vec{e}_{i}(\vec{k}) \cdot \hat{k}_{\vec{G}}\right|^{2}|\vec{k}-\vec{G}|^{2} p_{\hbar \omega}(|\vec{k}-\vec{G}|),}
$$


avec $n$ le nombre de termes, qui est identique à la fréquence d'apparition de la zone de Brillouin dans le volume $\mathrm{V}$

$$
n=\frac{V}{V_{\mathrm{Bz}}}=V \frac{V_{\mathrm{maille}}}{(2 \pi)^{3}}
$$

Dans cette moyenne, $\left|\vec{e}_{i}(\vec{k}) \cdot \hat{k}_{\vec{G}}\right|^{2}$ ne dépend que des directions et $|\vec{k}-\vec{G}|^{2} p_{\hbar \omega}(|\vec{k}-\vec{G}|)$ que de la norme. On peut donc supposer que les deux parties sont décorrélées

$$
\overline{\left|\vec{e}_{i}(\vec{k}) \cdot \hat{k}_{\vec{G}}\right|^{2}|\vec{k}-\vec{G}|^{2} p_{\hbar \omega}(|\vec{k}-\vec{G}|)}=\overline{\left|\vec{e}_{i}(\vec{k}) \cdot \hat{k}_{\vec{G}}\right|^{2}} \times \overline{|\vec{k}-\vec{G}|^{2} p_{\hbar \omega}(|\vec{k}-\vec{G}|)} .
$$

Pour un système monatomique cubique

$$
\overline{\left|\vec{e}_{i}(\vec{k}) \cdot \hat{k}_{\vec{G}}\right|^{2}}=\frac{1}{3}
$$

En parallèle

$$
\overline{|\vec{k}-\vec{G}|^{2} p_{\hbar \omega}(|\vec{k}-\vec{G}|)}=\frac{1}{V} \int_{Q_{\min }(\hbar \omega)}^{Q_{\max }(\hbar \omega)} p_{\hbar \omega}(Q) Q^{2} d Q .
$$

Ceci nous amène à

$$
h_{i}(\vec{k})=e^{-2 W(Q)} \frac{V_{\text {maille }}}{(2 \pi)^{3}} \frac{1}{3} \int_{Q_{\min }(\hbar \omega)}^{Q_{\max }(\hbar \omega)} p_{\hbar \omega}(Q) Q^{2} d Q,
$$

et finalement à

$$
\begin{aligned}
\left(\frac{d \sigma^{2}}{d(\hbar \omega)}\right)_{\mathrm{coh}-\mathrm{int}}^{1-\mathrm{ph}}= & N \frac{k_{f}}{k_{i}} \frac{\sigma_{\mathrm{coh}}}{8 \pi m} e^{-2 W(Q)} \frac{1}{3 V_{\mathrm{Bz}}} \sum_{i=1}^{3} \int_{\mathrm{BZ}} d^{3} k \frac{\left.\delta\left(\omega-\omega_{i}(\vec{k})\right)\right)}{\omega_{i}(\vec{k})}\left(1+n\left(\omega_{i}(\vec{k})\right)\right) \\
& \times \int_{Q_{\min }(\hbar \omega)}^{Q_{\max }(\hbar \omega)} p_{\hbar \omega}(Q) Q^{2} d Q \\
= & N \frac{k_{f}}{k_{i}} \frac{\sigma_{\mathrm{coh}}}{8 \pi m} e^{-2 W(Q)} \frac{g(\omega)}{\omega}(1+n(\omega)) \int_{Q_{\min }(\hbar \omega)}^{Q_{\max }(\hbar \omega)} p_{\hbar \omega}(Q) Q^{2} d Q
\end{aligned}
$$

Ce résultat est identique à l'expression que nous aurions obtenue en intégrant (10.150) pour la diffusion incohérente. ${ }^{82}$ Nous pouvons donc énoncer l'approximation incohérente comme [47]

$$
\left(\frac{d \sigma^{2}}{d(\hbar \omega)}\right)_{\mathrm{coh}-\mathrm{int}}^{1-\mathrm{ph}} \approx\left(\frac{d \sigma^{2}}{d(\hbar \omega)}\right)_{\mathrm{inc}-\mathrm{int}}^{1-\mathrm{ph}} .
$$

On pourra, dans cette approximation, employer le formalisme élaboré pour la diffusion incohérente pour déterminer la densité d'états d'un diffuseur cohérent, pourvu que la région échantillonnée en $\vec{Q}$ soit suffisamment large. Le terme de droite se rapporte à un échantillon incohérent hypothétique. On peut tester la validité de l'approximation incohérente en comparant les valeurs de $g(\omega)$ obtenues pour différentes régions d'échantillonnage dans l'espace des $\vec{Q}$. Une autre possibilité est de faire l'estimation des erreurs induites par l'approximation incohérente en employant des simulations sur ordinateur. On obtient déjà de bons résultats avec des modèles dynamiques d'un faible degré de sophistication. En général, tant l'expérience [48] que la simulation [49] montrent que les erreurs excèdent rarement 20\%, même dans les cas les plus défavorables.

\footnotetext{
82 Pour la diffusion incohérente, la moyenne sur $Q$ est triviale si nous négligeons, comme nous l'avons fait ici, la dépendance en $Q$ du facteur de Debye-Waller. Dans un calcul plus réaliste, cette dépendance est à prendre en compte (voir section 11.5.3).
} 


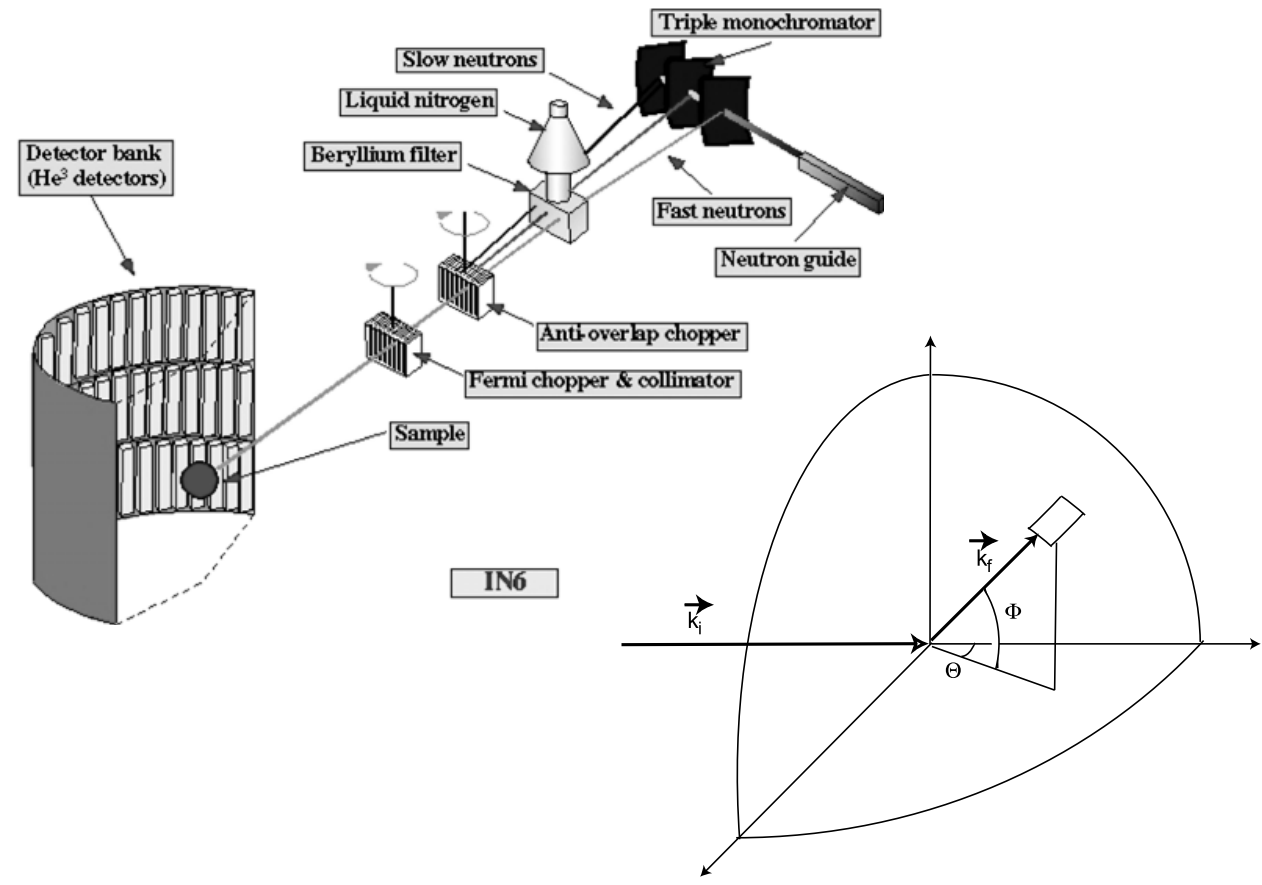

Figure 45. L'instrument IN6 à l'ILL est un spectromètre à temps de vol à géométrie directe. Le faisceau incident est monochromatique et pulsé, c'est-à-dire que $k_{i}$ est constant. $k_{f}$ est déterminé à partir du temps de vol, qui est le temps que met le neutron pour aller de l'échantillon aux détecteurs. Il est équipé de 334 détecteurs disposés en trois rangées. Les boîtes de détecteurs sont montées de telle manière à ce qu'elles maintiennent sur les trois rangées les mêmes angles par rapport au plan de diffusion. L'ouverture angulaire verticale des détecteurs $\Delta \Phi$ est determinée par leur hauteur. Des spectromètres de ce type permettent de balayer, pour un transfert d'énergie donné, un domaine de l'espace réciproque relativement large comme indiqué dans la figure 44.

Pour un instrument de type temps-de-vol à géometrie directe (voir [4], figure 45 ainsi que le chapitre de J. Ollivier et J.-M. Zanotti) avec énergie incidente fixe et multidétecteur ${ }^{83}$ couvrant un domaine angulaire compris entre les angles de diffusion $\theta_{\min }$ et $\theta_{\max }$, la section efficace intégrée possède la forme $^{84}$

$$
\left(\frac{d \sigma^{2}}{d(\hbar \omega)}\right)_{\theta_{\min }}^{\theta_{\max }}=\Delta \Phi \int_{\theta_{\min }}^{\theta_{\max }} \sin \theta\left(\frac{d \sigma^{2}}{d(\hbar \omega) d \Omega}\right) d \theta=\Delta \Phi \int_{\theta_{\min }}^{\theta_{\max }} \frac{Q}{k_{i} k_{f}}\left(\frac{d \sigma^{2}}{d(\hbar \omega) d \Omega}\right) d Q
$$

${ }^{83}$ Nous présumons que les données sont intégrées selon l'angle $\Phi$ le long des cônes de Debye-Scherrer, c'est-à-dire le long de la trajectoire $|Q|=$ constant. Si la partie des cônes couverte varie avec $\theta$ ceci doit être pris en compte lors de la normalisation des données.

${ }^{84}$ Avec $\vec{Q}=\vec{k}_{i}-\vec{k}_{f}$ et $\theta$ l'angle de diffusion on obtient

$$
Q=\sqrt{k_{i}^{2}+f_{f}^{2}+2 k_{i} k_{f} \cos \theta},
$$

et donc

$$
\frac{d Q}{d \theta}=\frac{1}{2 Q} 2 k_{i} k_{f} \sin \theta
$$

Ainsi

$$
Q d Q=k_{i} k_{f} \sin \theta d \theta .
$$


L'approximation incohérente se traduit dans ce cas en

$$
\int_{\theta_{\min }}^{\theta_{\max }}\left(\frac{\mathrm{d}^{2} \sigma}{\mathrm{d}(\hbar \omega) \mathrm{d} \Omega}\right)_{\text {coh }} \sin \theta \mathrm{d} \theta \approx \int_{\theta_{\min }}^{\theta_{\max }}\left(\frac{\mathrm{d}^{2} \sigma}{\mathrm{d}(\hbar \omega) \mathrm{d} \Omega}\right)_{\mathrm{inc}} \sin \theta \mathrm{d} \theta
$$

ou de manière équivalente

$$
\int_{Q_{\min }}^{Q_{\max }}\left(\frac{\mathrm{d}^{2} \sigma}{\mathrm{d}(\hbar \omega) \mathrm{d} \Omega}\right)_{\mathrm{coh}} Q \mathrm{~d} Q \approx \int_{Q_{\min }}^{Q_{\max }}\left(\frac{\mathrm{d}^{2} \sigma}{\mathrm{d}(\hbar \omega) \mathrm{d} \Omega}\right)_{\mathrm{inc}} Q \mathrm{~d} Q .
$$

Comparant (10.165) avec (10.152) nous identifions

$$
p_{\hbar \omega}(Q)=\Delta \Phi \frac{Q}{k_{i} k_{f}}
$$

Ceci nous permet de calculer

$$
\int_{Q_{\min }(\hbar \omega)}^{Q_{\max }(\hbar \omega)} p_{\hbar \omega}(Q) Q^{2} d Q=\frac{\Delta \Phi}{k_{i} k_{f}} \int_{Q_{\min }}^{Q_{\max }} Q^{3} d Q=\frac{\Delta \Phi}{4 k_{i} k_{f}}\left(Q_{\max }^{4}-Q_{\min }^{4}\right) .
$$

Ce qui donne avec l'aide de (10.160) la relation entre la section efficace intégrée et la densité d'états

$$
\left(\frac{d \sigma^{2}}{d(\hbar \omega)}\right)_{\mathrm{h}_{\min }}^{\mathrm{h}_{\max }}=\frac{N \Delta \Phi}{k_{i}^{2}} \frac{\sigma_{\mathrm{coh}}}{32 \pi m} e^{-2 W(Q)}\left(Q_{\max }^{4}-Q_{\min }^{4}\right) \frac{g(\omega)}{\omega}(1+n(\omega))
$$

\section{LA DIFFUSION INÉLASTIQUE AU-DELÀ DES EXPRESSIONS À UN PHONON}

Dans les expressions obtenues pour la fonction de diffusion, il n'apparait que des processus à un seul phonon : soit son absorption, soit son émission. Formellement c'est la conséquence du fait que nous n'ayons retenu dans le développement de

$$
\exp \left(\aleph_{j^{\prime}, j}(\vec{Q}, t)\right)=\exp \left(\left\langle\left(\vec{Q} \cdot \vec{u}_{j^{\prime}}\right)\left(\vec{Q} \cdot \vec{u}_{j}(t)\right)\right\rangle\right)
$$

que le terme linéaire en

$$
\aleph_{j^{\prime}, j}(\vec{Q}, t)=\left\langle\left(\vec{Q} \cdot \vec{u}_{j^{\prime}}\right)\left(\vec{Q} \cdot \vec{u}_{j}(t)\right)\right\rangle
$$

Nous avions motivé cette décision avec l'argument que les déplacements des ions étaient petits dans le régime de l'approximation harmonique. Pour être précis, nous aurions néanmoins dû rajouter que les déplacements devaient également être petits à l'échelle de l'inverse de la norme du vecteur $\vec{Q}$.

Les expressions obtenues seront donc valables pourvu que (i) nous nous trouvons à basse température et loin des points d'instabilité dans le diagramme de phase et que (ii) le transfert d'impulsion $|\vec{Q}|$ n'est pas trop important. Quand la dernière condition n'est pas respectée, nous devons prendre en compte les termes d'ordre supérieur ou -mieux encore- calculer les fonctions de diffusion partielles différentielles sans avoir recours à un développement. Nous insistons sur le fait que cela est nécessaire de façon indépendante de la validité de l'approximation harmonique. Les contributions multiphonons existent aussi bien pour les systèmes harmoniques que pour des systèmes anharmoniques. 


\subsection{Expression intégrale pour la fonction de diffusion}

Pour juger de la complexité d'un calcul intégral sans développement, nous rappelons que dans l'approximation harmonique

$$
S_{\kappa, \kappa^{\prime}}(\vec{Q}, \omega)=\frac{1}{2 \pi N \hbar} \sum_{j \in\left\{j_{\kappa}\right\}, j^{\prime} \in\left\{j_{k^{\prime}}\right\}} \int_{-\infty}^{\infty} d t e^{-i \omega t} e^{-i \vec{Q} \cdot\left(\vec{R}_{j^{\prime}}^{0}-\vec{R}_{j}^{0}\right)} e^{-W_{j^{\prime}}(\vec{Q})} e^{-W_{j}(\vec{Q})} e^{\left\langle\left(\vec{Q} \cdot \vec{u}_{j^{\prime}}\right)\left(\vec{Q} \cdot \vec{u}_{j}(t)\right)\right\rangle,}
$$

avec

$$
2 W_{j}(\vec{Q})=\left\langle\left(\vec{Q} \cdot \vec{u}_{j}\right)^{2}\right\rangle=\sum_{\alpha=x, y, z} \sum_{j=1}^{3 N} \frac{\left|Q_{\alpha} \cdot e_{i}(\alpha, j)\right|^{2} \hbar}{2 m_{j} \omega_{i}} \operatorname{coth}\left(\frac{1}{2} \frac{\hbar \omega}{k_{\mathrm{B}} T}\right),
$$

et

$$
\begin{aligned}
\aleph_{j^{\prime}, j}(\vec{Q}, t)= & \left\langle\left(\vec{Q} \cdot \vec{u}_{j^{\prime}}\right)\left(\vec{Q} \cdot \vec{u}_{j}(t)\right)\right\rangle \\
= & \frac{\hbar}{2} \sum_{\alpha, \beta} \frac{Q_{\alpha} Q_{\beta}}{\sqrt{m_{j} m_{j^{\prime}}}} \sum_{i, i^{\prime}} \sqrt{\frac{1}{\omega_{i} \omega_{i^{\prime}}} \times \delta_{i, i^{\prime}}} \\
& \times\left\{\left[e_{i}\left(\alpha, j^{\prime}\right) e_{i^{\prime}}^{*}(\beta, j)\left\langle\mathbf{a}_{i} \mathbf{a}_{i^{\prime}}^{+}\right) \exp \left(i \omega_{i^{\prime}} t\right)\right]+\left[e_{i}^{*}\left(\alpha, j^{\prime}\right) e_{i^{\prime}}(\beta, j)\left\langle\mathbf{a}_{i}^{+} \mathbf{a}_{i^{\prime}}\right\rangle \exp \left(-i \omega_{i^{\prime}} t\right)\right]\right\} \\
= & \frac{\hbar}{2} \sum_{i}\left\{\left[\frac{A_{i}\left(\vec{Q} ; j^{\prime}, j\right)}{\omega_{i}}\left(1+n\left(\omega_{i}\right)\right) \exp \left(i \omega_{i} t\right)\right]+\left[\frac{A_{i}\left(\vec{Q} ; j, j^{\prime}\right)}{\omega_{i}} n\left(\omega_{i}\right) \exp \left(-i \omega_{i} t\right)\right]\right\},
\end{aligned}
$$

ainsi que

$$
A_{i}\left(\vec{Q} ; j^{\prime}, j\right)=\frac{\left(\vec{Q} \cdot \vec{e}_{i}\left(j^{\prime}\right)\right) \cdot\left(\vec{Q} \cdot \vec{e}_{i}(j)\right)^{*}}{\sqrt{m_{j^{\prime}} m_{j}}}
$$

Il est donc possible de calculer numériquement $S_{\kappa, \kappa^{\prime}}(\vec{Q}, \omega)$ pourvu que nous connaissions la dynamique du réseau, c'est-à-dire pourvu que nous sachions déterminer les fréquences et les vecteurs propres de tous les modes harmoniques, par exemple à partir d'un calcul ab initio ou d'une simulation de dynamique moléculaire (voir chapitre I et le cours de M. Johnson et al.).

\subsection{L'oscillateur harmonique isotrope}

Comme souvent dans la physique de l'état solide une solution analytique de la fonction de diffusion peut être obtenue pour un simple oscillateur harmonique isotrope. Nous allons calculer cette fonction à partir de l'expression (11.2). Pour un seul oscillateur de fréquence $\omega_{0}$, qui de plus est isotrope, nous obtenons pour la fonction de Debye-Waller

$$
2 W(\vec{Q})=\left\langle(\vec{Q} \cdot \vec{u})^{2}\right\rangle=\frac{Q^{2} \hbar}{2 m \omega_{0}} \operatorname{coth}\left(\frac{1}{2} \frac{\hbar \omega_{0}}{k_{\mathrm{B}} T}\right)
$$


et pour la fonction de corrélation

$$
\begin{aligned}
\aleph(\vec{Q}, t) & =\langle(\vec{Q} \cdot \vec{u})(\vec{Q} \cdot \vec{u}(t))\rangle \\
& =\frac{Q^{2} \hbar}{2 m \omega_{0}}\left\{\left[\left(1+n\left(\omega_{0}\right)\right) \exp \left(i \omega_{0} t\right)\right]+\left[n\left(\omega_{0}\right) \exp \left(-i \omega_{0} t\right)\right]\right\} \\
& =\frac{Q^{2} \hbar}{2 m \omega_{0}} \frac{\cosh \left(\omega_{0}\left(i t+\frac{\hbar}{2 k_{\mathrm{B}} T}\right)\right)}{\sinh \left(\frac{\hbar \omega_{0}}{k_{\mathrm{B}} T}\right)} .
\end{aligned}
$$

L'échelle d'énergie et de température est fixée par $\hbar \omega_{0}$. L'amplitude de la fonction de Debye-Waller ainsi que de la fonction de corrélation est déterminée par $Q^{2} \hbar / 2 m \omega_{0}$. Pour un oscillateur harmonique à une dimension, les fluctuations de l'impulsion dans le niveau fondamental se calculent comme

$$
\Delta p=\sqrt{\left\langle n\left|\mathbf{p}^{2}\right| n\right\rangle}=\sqrt{\frac{1}{2} \hbar m \omega_{0}} .
$$

Ainsi,

$$
\frac{Q^{2} \hbar}{2 m \omega_{0}}=\frac{1}{4} \frac{(Q \hbar)^{2}}{(1 / 2) \hbar m \omega_{0}}=\left(\frac{1}{2} \frac{Q \hbar}{\Delta p}\right)^{2} .
$$

L'amplitude est donc régie par le rapport entre (i) le transfert d'impulsion du neutron et (ii) la fluctuation de l'impulsion de l'oscillateur harmonique dans son état fondamental. Cette expression peut aussi être écrite en terme de rapport d'énergie

$$
\begin{gathered}
\frac{Q^{2} \hbar}{2 m \omega_{0}}=\frac{\frac{\hbar^{2} Q^{2}}{2 m}}{\hbar \omega_{0}}=\frac{E_{r}}{\hbar \omega} . \\
E_{r}(Q)=\frac{\hbar^{2} Q^{2}}{2 m}
\end{gathered}
$$

est l'énergie de recul de la particule de masse $m$.

Le rapport $E_{r}(Q) / \hbar \omega_{0}$ peut être consideré comme le paramètre principal, qui, avec la température, gère la réponse dynamique de l'oscillateur harmonique.

Si nous posons

$$
\begin{aligned}
y & =\frac{Q^{2} \hbar}{2 m \omega_{0}} \frac{1}{\sinh \left(\frac{\hbar \omega_{0}}{k_{\mathrm{B}} T}\right)} \\
z(t) & =\omega_{0}\left(i t+\frac{\hbar}{2 k_{\mathrm{B}} T}\right)
\end{aligned}
$$

alors

$$
\langle(\vec{Q} \cdot \vec{u})(\vec{Q} \cdot \vec{u}(t))\rangle=y \cosh (z(t))
$$

et donc

$$
\begin{aligned}
S(\vec{Q}, \omega) & =\frac{1}{2 \pi \hbar} e^{-2 W(\vec{Q})} \int_{-\infty}^{\infty} d t e^{\langle(\vec{Q} \cdot \vec{u})(\vec{Q} \cdot \vec{u}(t))\rangle} \\
& =\frac{1}{2 \pi \hbar} \exp (-2 W(\vec{Q})) \int_{-\infty}^{\infty} d t \exp (y \cosh (z(t)))
\end{aligned}
$$


A ce point, nous appliquons l'identité

$$
\exp (y \cosh (z(t)))=\sum_{n=-\infty}^{n=\infty} \exp (n z(t)) I_{n}(y)
$$

avec $I_{n}=I_{-n}$ les fonctions de Bessel de première espèce ${ }^{85}$ (voir figure 46). Avec l'aide de cette identité on effectue l'intégration dans l'expression (11.15) et nous obtenons

$$
\begin{aligned}
\int_{-\infty}^{\infty} d t \exp (y \cosh (z(t))) & =\sum_{n=-\infty}^{n=\infty} I_{n}(y) \int_{-\infty}^{\infty} d t \exp (n z(t)) \\
& =\sum_{n=-\infty}^{n=\infty} I_{n}(y) \exp \left(\frac{\hbar \omega_{0}}{2 k_{\mathrm{B}} T}\right) \int_{-\infty}^{\infty} d t \exp \left(i n \omega_{0} t\right) \\
& =\sum_{n=-\infty}^{n=\infty} I_{n}(y) \exp \left(\frac{\hbar \omega}{2 k_{\mathrm{B}} T}\right)(2 \pi \hbar) \delta\left(\hbar \omega-n \hbar \omega_{0}\right) .
\end{aligned}
$$

Ceci nous amène à la fonction de diffusion

$$
S(\vec{Q}, \omega)=\exp (-2 W(\vec{Q})) \cdot \exp \left(\frac{\hbar \omega}{2 k_{\mathrm{B}} T}\right) \sum_{n=-\infty}^{n=\infty} I_{n}(y) \delta\left(\hbar \omega-n \hbar \omega_{0}\right) .
$$

Le neutron peut donc gagner ou perdre un nombre entier d'unités d'énergie $\hbar \omega_{0}$. Si nous ne retenons que le terme avec $n=0$, nous obtenons la diffusion élastique de l'oscillateur isotrope. Les termes $n= \pm 1$ correspondent à l'augmentation ou à la diminution d'un seul niveau d'excitation. Les autres termes impliquent des processus de diffusion qui correspondent à des passages de plusieurs niveaux. Il est très important de souligner qu'il ne s'agit pas d'une diffusion multiple dans le sens de la section 9.4. La création ou l'annihilation de plusieurs phonons fait partie d'un seul processus de diffusion. Elle ne dépend de ce fait, ni de la taille de l'échantillon, ni du libre parcours moyen des neutrons. Elle n'est pas non plus liée à des phénomènes d'anharmonicité. Avec des petites modifications (voir [15]), le résultat obtenu pour l'oscillateur isotrope s'applique directement à un système de plusieurs oscillateurs d'Einstein, donc d'oscillateurs qui ne sont pas couplés entre eux (voir chapitre I).

Etant donné que (voir figure 46)

$$
I_{n}(y) \rightarrow \frac{1}{n !}\left(\frac{y}{2}\right)^{n} \quad \text { pour } \quad y \rightarrow 0 \quad \text { et } \quad n>0,
$$

les premiers termes dans (11.20) dominent pour des petites valeurs de $Q$. Le développement de la fonction de diffusion en terme de processus peut donc être recalculé en conséquence.

85 Nous avions déjà rencontré les fonctions de Bessel sphériques dans la section 4.2. Les fonctions de Bessel sont des solutions de l'équation différentielle

$$
x^{2} \frac{d^{2} y}{d x^{2}}+x \frac{d y}{d x}+\left(x^{2}-n^{2}\right) y=0 .
$$

Les fonctions de Bessel de première espèce sont des solutions definies à $x=0$. Elles se calculent comme

$$
I_{n}(x)=\left(\frac{x}{2}\right)^{n} \sum_{p=0}^{\infty} \frac{(-1)^{p}}{2^{2 p} p !(n+p) !} x^{2 p} .
$$

Les représentations graphiques des fonctions de Bessel ressemblent à celles des fonctions sinus ou cosinus, mais s'aplatissent comme s'il s'agissait de fonctions sinus ou cosinus divisées par un terme de la forme $\sqrt{x}$. 


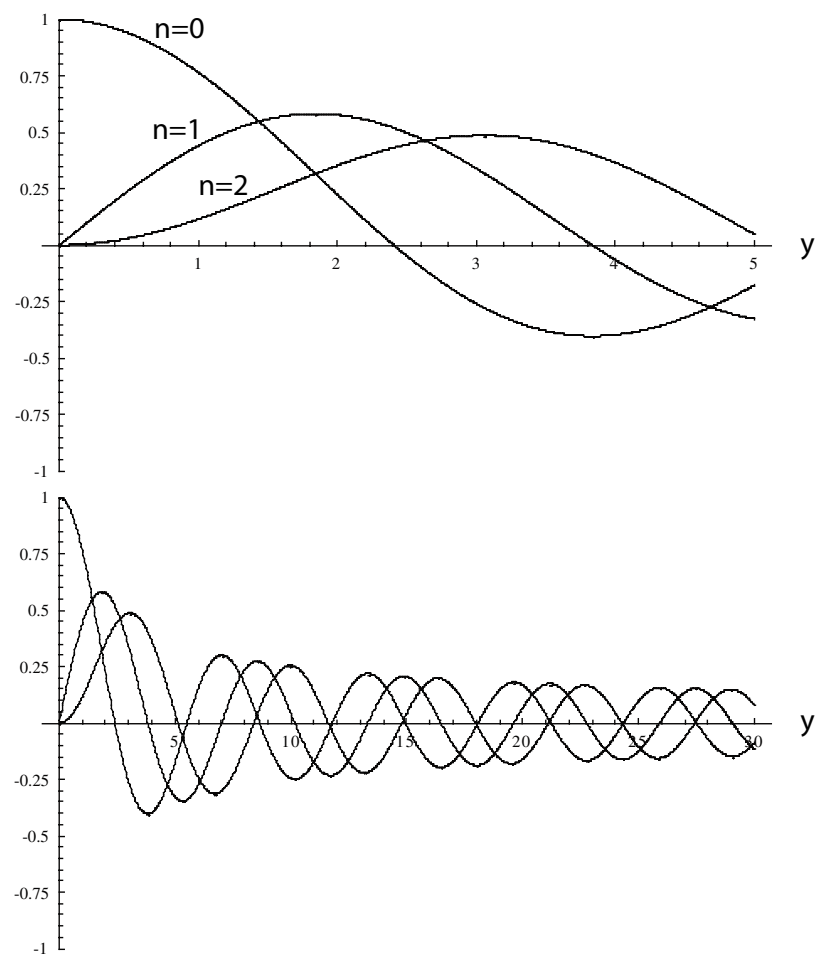

Figure 46. Fonctions de Bessel $I_{n}(y)$ de première espèce pour $n=0,1,2$.

\subsection{Approximation à temps courts ou approximation d'impulsion}

A l'autre extremité du spectre, c'est-à-dire pour $Q \rightarrow \infty$ et donc pour $y \rightarrow \infty$, les $I_{n}(y)$ deviennent tous comparables (voir figure 46). La somme dans l'expression (11.20) ne pourra donc plus être tronquée. Pour identifier les mécanismes qui opèrent lors de la diffusion à grands $Q$ nous devons choisir une autre approche, que nous allons brièvement rapporter ici.

Les ondes diffusées interfèrent et leurs phases respectives dépendront des positions relatives des atomes, qui sont une fonction du temps. Le front d'onde incident prendra un temps typique $t_{\text {sonde }}$ pour passer d'un atome à l'autre. Par exemple, des neutrons thermiques $\left(v \approx 2 \cdot 10^{3} \mathrm{~m} / \mathrm{s}\right)$ parcourront des distances inter-atomiques (quelques $\AA$ ) en quelques dixièmes de picosecondes. Le temps de parcours inter-atomique $t_{\text {sonde }}$ est ainsi très proche des temps caractéristiques $t_{\mathrm{ph}}$ des phonons de fréquences de quelques THz. En d'autres mots, le temps de parcours inter-atomique des neutrons correspond bien à la période des oscillations des atomes. C'est une autre manière d'expliquer pourquoi l'observation des phonons est assez aisée pour les neutrons. Si l'énergie des neutrons incident augmente, $t_{\text {sonde }}$ devient de plus en plus court. Pour observer des cycles complet d'oscillation il faudra une très bonne définition de la fréquence de l'onde incidente ainsi que de l'onde diffusée. L'observation des effets d'interférence requiert donc une résolution en énergie de plus en plus élevée. ${ }^{86}$ Dans la réalité, les

\footnotetext{
${ }^{86}$ L'argument de la résolution est important ici. Pour des rayons-X, le temps de trajet inter-atomique est beaucoup plus court $\left(t_{\mathrm{sonde}} \approx / c=0.3 \cdot 10^{-18} \mathrm{~s}\right)$. On pourra donc présumer (et on trouve cet argument dans la littérature précédant les sources de lumière de troisième génération [36]) que la diffusion des rayons-X ne peut donner accès qu'aux corrélations instantanées $(G(\vec{r}, t=0))$. Ce n'est évidemment plus vrai avec l'avènement des spectromètres aux rayons-X à très haute résolution. L'onde incidente est tellement bien définie dans son énergie $\left(E_{i} / \Delta E \approx 10^{7}\right)$, que les interférences restent observables même si les déphasages sont minimes à l'échelle de $t_{\text {sonde }}$.
} 
contraintes instrumentales font que c'est malheureusement le contraire qui se passe. La résolution absolue diminue avec l'énergie des neutrons.

Le fait que les termes à plusieurs excitations deviennent tous importants pour $Q \rightarrow \infty$ nous indique que la réponse spectrale va se déplacer vers des énergies élevées. Les grands transferts de moment $Q$ ainsi que d'énergie $\hbar \omega$ nécessiteront l'emploi de neutrons énergétiques. Pour une résolution de quelques pour-cents nous pouvons supposer que les interférences qui seront observables proviendront de $G(\vec{r}, t)$ pour des temps $t$ assez courts. Nous allons donc développer la fonction

$$
\langle(\vec{Q} \cdot \vec{u})(\vec{Q} \cdot \vec{u}(t))\rangle-\left\langle(\vec{Q} \cdot \vec{u})^{2}\right\rangle=\frac{Q^{2} \hbar}{2 m \omega_{0}}\left\{\frac{\cosh \left(\omega_{0}\left(i t+\frac{\hbar}{2 k_{\mathrm{B}} T}\right)\right.}{\sinh \left(\frac{\hbar \omega_{0}}{k_{\mathrm{B}} T}\right)}-\operatorname{coth}\left(\frac{1}{2} \frac{\hbar \omega}{k_{\mathrm{B}} T}\right)\right\}
$$

dans le temps. Si nous nous limitons à $G(\vec{r}, t=0)$, nous travaillons dans l'approximation statique qui donne une réponse élastique. Cette réponse est inexistante dans le cas d'un oscillateur harmonique.

En ne retenant les termes que jusqu'à l'ordre deux, nous incluons à la fois la partie réelle et la partie imaginaire de la fonction (11.22)

$$
\left\langle(\vec{Q} \cdot \vec{u})(\vec{Q} \cdot \vec{u}(t))-(\vec{Q} \cdot \vec{u})^{2}\right\rangle=\frac{Q^{2} \hbar}{2 m}\left[i t-\frac{t^{2} \omega_{0}}{2} \operatorname{coth}\left(\frac{1}{2} \frac{\hbar \omega_{0}}{k_{\mathrm{B}} T}\right)\right]
$$

Il s'agit d'une fonction gaussienne déplacée le long de l'axe imaginaire dans le plan complexe. Le calcul de la fonction de corrélation (11.15) implique la transformée de Fourier de (11.23) Avec

$$
\int_{-\infty}^{\infty} d t \exp \left(-a t^{2}+b t\right)=\sqrt{\frac{\pi}{a}} \exp \left(b^{2} / 4 a\right)
$$

la fonction de diffusion devient

$$
S(\vec{Q}, \omega)=\frac{1}{\sqrt{2 \pi \Delta^{2}}} \exp \left(-\frac{\left(\hbar \omega-E_{r}\right)^{2}}{2 \Delta^{2}}\right) .
$$

C'est une gaussienne centrée sur l'énergie de recul

$$
E_{r}=\frac{\hbar^{2} Q^{2}}{2 m}
$$

avec une largeur proportionnelle à la fonction de Debye-Waller

$$
\begin{aligned}
\Delta^{2} & =\frac{Q^{2} \hbar^{2}}{2 m}\left(\hbar \omega_{0}\right) \operatorname{coth} \frac{\hbar \omega_{0}}{2 k_{\mathrm{B}} T} \\
& =E_{r}\left(\hbar \omega_{0}\right) \operatorname{coth} \frac{\hbar \omega_{0}}{2 k_{\mathrm{B}} T} \\
& =2\left(\hbar \omega_{0}\right)^{2} W(\vec{Q}) .
\end{aligned}
$$

Avant d'interpréter le résultat (11.23) nous le déduirons de manière alternative, c'est-à-dire sans passer par l'expression analytique (11.22). Pour des temps très courts, le changement de position d'une particule est donné par sa vitesse $\vec{v}_{j}$ instantanée. Nous traduisons ceci dans le language de la mécanique quantique en écrivant l'opérateur de Heisenberg pour la position comme

$$
\overrightarrow{\mathbf{R}}_{j}(t)=\overrightarrow{\mathbf{R}}_{j}(t=0)+\frac{\mathbf{P}_{\mathbf{j}}(t)}{m_{j}} t
$$


Cette approximation est appelée l'approximation d'impulsion. Pour calculer la fonction de diffusion (10.2) nous devons évaluer la fonction de corrélation

$$
\left\langle\exp \left(-i \vec{Q} \cdot \overrightarrow{\mathbf{R}}_{j^{\prime}}(t=0)\right) \cdot \exp \left(i \vec{Q} \cdot \overrightarrow{\mathbf{R}}_{j}(t)\right)\right\rangle=\left\langle\exp \left(-i \vec{Q} \cdot \overrightarrow{\mathbf{R}}_{j^{\prime}}\right) \cdot \exp \left(i \vec{Q} \cdot \overrightarrow{\mathbf{R}}_{j}+i \vec{Q} \cdot \frac{\mathbf{P}_{j}(t)}{m_{j}} t\right)\right\rangle
$$

où le caractère d'opérateur est indiqué en caractère gras. Sachant que

$$
\left[\mathbf{R}_{\alpha, j}, \mathbf{P}_{\beta, j^{\prime}}\right]=i \hbar \delta_{\alpha, \beta} \delta_{j, j^{\prime}}
$$

nous pouvons évoquer l'identité (10.6) pour évaluer cette expression.

$$
\begin{aligned}
& \left\langle\exp \left(-i \vec{Q} \cdot \overrightarrow{\mathbf{R}}_{j^{\prime}}\right) \cdot \exp \left(i \vec{Q} \cdot \overrightarrow{\mathbf{R}}_{j}+i \vec{Q} \cdot \frac{\mathbf{P}_{j}(t)}{m_{j}} t\right)\right\rangle \\
& \quad=\left\langle\exp \left(-i \vec{Q} \cdot \overrightarrow{\mathbf{R}}_{j^{\prime}}+i \vec{Q} \cdot \overrightarrow{\mathbf{R}}_{j}+i \vec{Q} \cdot \frac{\mathbf{P}_{j}(t)}{m_{j}} t+\frac{1}{2}\left[\vec{Q} \cdot \overrightarrow{\mathbf{R}}_{j^{\prime}},\left(\vec{Q} \cdot \overrightarrow{\mathbf{R}}_{j}+\vec{Q} \cdot \frac{\mathbf{P}_{j}(t)}{m_{j}} t\right)\right]\right)\right\rangle \\
& \quad=\exp \left(i \frac{\hbar Q^{2} t}{2 m_{j}} \delta_{j, j^{\prime}}\right)\left\langle\exp \left(i \vec{Q} \cdot\left(\overrightarrow{\mathbf{R}}_{j}-\overrightarrow{\mathbf{R}}_{j^{\prime}}\right)+i \vec{Q} \cdot \frac{\mathbf{P}_{j}(t)}{m_{j}} t\right)\right\rangle
\end{aligned}
$$

à ce point, nous ne retenons que les termes $j=j^{\prime}$, c'est-à-dire que nous considérons la réponse complètement incohérente. En d'autres termes, nous présumons que la résolution de notre expérience est insuffisante pour observer des effets d'interférences à hautes fréquences émanant de la diffusion d'atomes distincts. La fonction de diffusion prend la forme

$$
S(\vec{q}, \omega)=\frac{1}{2 \pi \hbar N} \sum_{j} \int_{-\infty}^{\infty} d t \exp \left(i t\left(-\omega+\frac{\hbar Q^{2}}{2 m_{j}}\right)\right)\left\langle\exp \left(i \vec{Q} \cdot \frac{\mathbf{P}_{j}(t)}{m_{j}} t\right)\right\rangle
$$

Pour des atomes confinés dans des puits de potentiel harmoniques, la distribution des impulsions est une gaussienne

$$
\left\langle\exp \left(i \vec{Q} \cdot \frac{\mathbf{P}_{j}(t)}{m_{j}} t\right)\right\rangle=\exp \left(-t^{2} \frac{\left\langle\left(\vec{Q} \cdot \overrightarrow{\mathbf{P}}_{j}\right)^{2}\right\rangle}{2 m_{j}}\right)
$$

Cette expression peut être verifiée à l'aide de l'identité de Bloch (10.12), qui est applicable parce que $\overrightarrow{\mathbf{P}}(\alpha, j)$, comme $\overrightarrow{\mathbf{u}}(\alpha, j)$, s'expriment sous la forme d'une combinaison linéaire des opérateurs de création et d'annihilation. Nous retrouvons donc le résultat antérieur (11.25) avec la largeur cette fois exprimée comme

$$
\Delta^{2}=\frac{1}{m_{i}}\left\langle\left(\vec{Q} \cdot \overrightarrow{\mathbf{P}}_{j}\right)^{2}\right\rangle
$$

La largeur est en conséquence reliée à la distribution de la vitesse (ou de l'impulsion) des particules. Il n'est pas trop difficile de démontrer, en utilisant l'algèbre des opérateurs de création et d'annihilation, que (11.34) correspond à (11.27).

C'est un résultat que nous pouvons généraliser. Si pour des temps courts nous décrivons les états des particules comme des états libres avec une impulsion bien définie $\vec{P}_{j}$, alors

$$
\overrightarrow{\mathbf{P}}_{j}\left|\vec{P}_{j}\right\rangle=\vec{P}_{j}\left|\vec{P}_{j}\right\rangle
$$


et donc

$$
\left\langle\exp \left(i \vec{Q} \cdot \frac{\mathbf{P}_{j}(t)}{m_{j}} t\right)\right\rangle=\sum_{\vec{P}_{j}} n\left(\vec{P}_{j}\right) \exp \left(i \vec{Q} \cdot \frac{P_{j}}{m_{j}} t\right)
$$

avec $n\left(\vec{P}_{j}\right)$ la probabilité de trouver l'ion $j$ dans l'état $\mid \vec{P}_{j}>$. La fonction de diffusion correspondante (11.33) se calcul alors comme

$$
S(\vec{Q}, \omega)=\sum_{j} \sum_{\vec{P}_{j}} n\left(\vec{P}_{j}\right) \delta\left(\hbar \omega-E_{r}(Q)-\frac{\hbar}{m_{j}}\left(\vec{Q} \cdot \vec{P}_{j}\right)\right) .
$$

L'expérience de diffusion sonde donc, à des très grandes énergies, la distribution de l'impulsion des particules. Dans la figure 47 nous montrons $S(Q, \omega)$ pour une poudre de deutérium solide. Comme nous l'avions déjà mentionné, le deutérium est un solide quantique. Sa diffusion est, contrairement à l'hydrogène, principalement cohérente. Le caractère quantique de ce solide implique des fluctuations de position importante en dépit de la basse température. Le déplacement carré moyen $\overline{u^{2}}$ est de l'ordre de $0.25 \AA^{2}$ avec la fonction de Debye-Waller $W(Q)=\frac{1}{6} \overline{u^{2}}$. Pour des $\vec{Q}$ plus grands que quelques $\AA^{-1}$ il faudra donc s'attendre à des contributions multiphonons importantes. C'est confirmé par l'expérience. Quand $Q$ augmente, la réponse se déplace vers des énergies qui dépassent largement le spectre des phonons et elle acquiert un caractère de plus en plus incohérent. Le rapport entre l'énergie de recul et les énergies des phonons (voir (8.89) se rapproche de l'unité à partir de $Q \approx 4 \AA^{-1}$. Si nous restons avec des diffuseurs cohérents, un rapport plus favorable ne peut être obtenu que pour l'hélium. Néanmoins, même pour des valeurs de $\vec{Q}$ proche de $8 \AA^{-1}$ on est toujours loin du régime pour lequel l'approximation d'impulsion donne un très bon résultat. En particulier, la réponse ne ressemble pas encore à une gaussienne. Dans ce contexte, il est intéressant de regarder les approximations plus dans le détail (voir [50]). Des expériences pertinentes liées à cette approximation, dont le but est de mesurer la distribution des impulsions, sont menées avec des neutrons de très grandes énergies incidentes à la source à spallation ISIS en Angleterre.

\subsection{Multiphonons dans un monocristal}

Dès que les oscillateurs sont couplés et que les vecteurs propres cessent d'être triviaux, les expressions analytiques obtenues pour l'oscillateur simple ne seront plus applicables. Pour de très grands $Q$, les atomes se comporteront comme des particules individuelles essuyant des impacts. Nous pouvons donc espérer que l'approximation de la fonction de diffusion par la fonction de recul (11.24) continuera à donner des résultats satisfaisants, pourvu que nous effectuons une moyenne correcte sur les différents type d'atomes.

Pour des $Q$ intermédiaires nous n'avons guère d'autre choix que de baser le traitement sur l'expansion de la fonction de corrélation

$$
\exp \left(\aleph_{j^{\prime}, j}(\vec{Q}, t)\right)=\exp \left(\left\langle\left(\vec{Q} \cdot \vec{u}_{j^{\prime}}\right)\left(\vec{Q} \cdot \vec{u}_{j}(t)\right)\right\rangle\right)
$$

en incluant des termes supérieurs à un phonon. Les termes d'ordre deux auront la forme

$$
\aleph_{j^{\prime}, j}(\vec{Q}, t)^{2}=\left\langle\left(\vec{Q} \cdot \vec{u}_{j^{\prime}}\right)\left(\vec{Q} \cdot \vec{u}_{j}(t)\right)\right\rangle\left\langle\left(\vec{Q} \cdot \vec{u}_{j^{\prime}}\right)\left(\vec{Q} \cdot \vec{u}_{j}(t)\right)\right\rangle .
$$

En exprimant les opérateurs de position sous la forme d'opérateurs de création et d'annihilation (10.67) et en effectuant la transformée de Fourier (10.70) nous pouvons démontrer que la fonction de diffusion pour un cristal harmonique comprendra des termes non-nuls pourvu que les conditions

$$
\hbar \omega=\frac{\hbar^{2}}{2 m}\left(k_{i}^{2}-k_{f}^{2}\right)=\hbar\left( \pm \omega_{i}\left(\vec{k}_{1}\right) \pm \omega_{i^{\prime}}\left(\vec{k}_{2}\right)\right)
$$




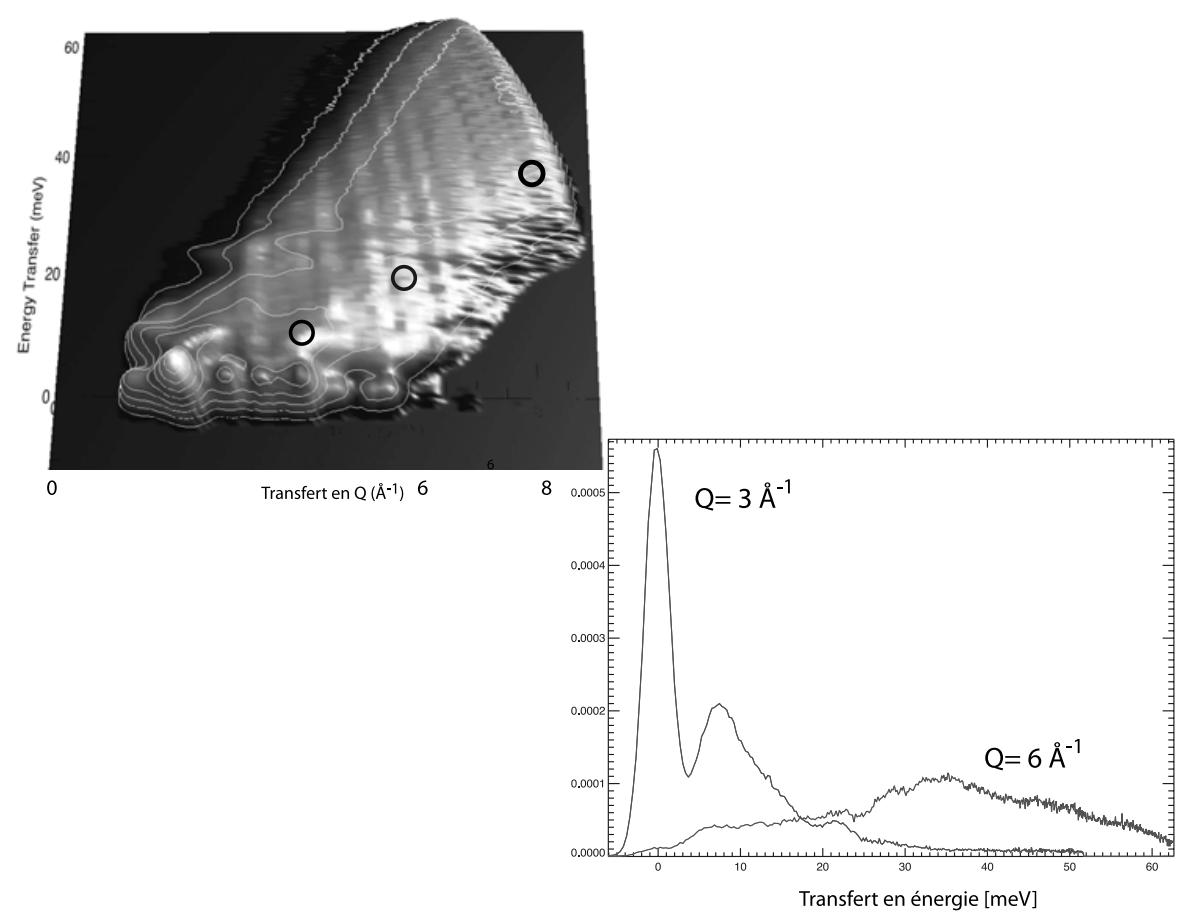

Figure 47. Illustration du passage de la diffusion à un phonon vers la diffusion de recul. Nous utilisons l'exemple du solide quantique $D_{2}$. Pour les détails expérimentaux voir [16]. La région en $Q$ et $\omega$ accessible à l'expérience montrée dans la figure en haut est beaucoup plus large que celle que nous avions présentée dans la figure 15. C'est l'avantage d'utiliser une longueur d'onde beaucoup plus courte $\left(1.1 \AA^{-1}\right.$ au lieu de $\left.2.2 \AA^{-1}\right)$. L'agrandissement du champ de vision est couplé à une dégradation de la résolution. Les phonons dans le deutérium solide possèdent des fréquences inférieures de $10 \mathrm{meV}$. Le signal observé à plus haute fréquence doit donc être attribué (i) aux transitions entre les niveaux de rotation de la molécule $\mathrm{D}_{2}$ ainsi qu'à (ii) la diffusion à plusieurs phonons. La diffusion à plusieurs phonons (diffusion multiphonons) augmente avec le transfert d'impulsion $Q$. Pour des valeurs de $Q$ très grandes, l'intensité inélastique devrait -selon 11.24- être centrée sur l'énergie de recul, qui est indiquée par les cercles noirs. Du fait de la contribution des transitions rotationnelles, cette parabole constitue plutôt une limite inférieure à la diffusion observée. Dans la figure à droite, nous montrons des coupes de $S(q, \omega)$ pour $Q=3$ et $Q=6 \AA^{-1}$. Nous observons que la diffusion cohérente se déplace de la région de la densité d'états $(\hbar \omega<10$ $\mathrm{meV}$ ) vers des énergies plus élevées et perd progressivement sa structuration, c'est-à-dire qu'elle ressemble de plus en plus à de la diffusion incohérente. L'intensité élastique est complètement supprimée vers des grands $Q$. La largeur à $Q=6 \AA^{-1}$ est compatible avec (11.27) si nous utilisons la fonction de Debye-Waller déterminée expérimentalement $\left(W(Q) \approx \frac{1}{6} \cdot 0.25 Q^{2}[16]\right)$ et un $\hbar \omega$ moyen entre 5 et $10 \mathrm{meV}$. Néanmoins la courbe à $6 \AA^{-1}$ ne ressemble pas encore trop à une gaussienne. Elle possède en particulier des structures beaucoup plus fines que la largeur de la fonction de recul.

$$
\vec{Q}=\vec{k}_{i}-\vec{k}_{f}=\vec{G} \pm \vec{k}_{1} \pm \vec{k}_{2}
$$

soient remplies. La diffusion implique donc soit (i) la création de deux phonons soit (ii) la création d'un phonon combinée avec l'annihilation d'un phonon soit (iii) l'annihilation de deux phonons. Pour chaque processus, l'énergie doit être conservée. En plus le moment cristallin doit être conservé à un vecteur $\hbar \vec{G}$ du réseau réciproque près. Contrairement à la diffusion à un phonon (voir (10.78) et (10.79)) la diffusion à plusieurs phonons n'est plus limitée à des valeurs de $\hbar \omega_{i}(\vec{k})$ discrets. Pour un angle de diffusion et pour un changement d'énergie donnés nous allons en principe toujours trouver des combinaisons $\left(\omega_{i}\left(\vec{k}_{1}\right), \omega_{i^{\prime}}\left(\vec{k}_{2}\right)\right)$ qui satisfont aux conditions (11.39) et (11.40). Le nombre de ces combinaisons 
déterminera en grande partie l'intensité de la diffusion à deux phonons pour un point $(\vec{Q}, \omega)$ donné. La continuité de la réponse à deux phonons complique considérablement son interprétation. La diffusion à multiples phonons n'a de ce fait guère de valeur pour l'expérimentateur. Souvent elle ne constitue qu'un bruit de fond indésirable, qui est sous-jacent aux pics à un phonon. Ce bruit, qui de plus dépend de la température, devra être correctement soustrait si nous voulons interpréter l'intensité des contributions à un phonon de façon quantitative. Cela ne pose pas beaucoup de problème si la résolution du spectromètre est bonne et si les pics à un phonon sont bien discernables.

Obtenir les expressions (11.40) pour la conservation du moment cristallin à partir des opérateurs $\vec{u}_{j}(t)$ devient très fastidieux, quand le nombre des facteurs $\aleph(Q, t)$ augmente. Comme nous l'avons déjà indiqué dans la section 10.5, ce calcul explicite n'est pas non plus nécessaire. La conservation de l'impulsion est une conséquence de la periodicité de l'Hamiltonien. C'est un résultat fondamental de la mécanique quantique qui stipule que toute opération de symétrie $S$ du système est couplée à un opérateur de mécanique quantique $T_{S}$ qui commute avec l'Hamiltonien (se référer au chapitre I). Pour une particule libre, un déplacement $\vec{r} \rightarrow \vec{r}+\vec{r}_{0}$ selon n'importe quelle direction, laisse l'Hamiltonien invariant. L'action de translation change la phase de la fonction d'onde de $\vec{k} \cdot \vec{r}_{0}$. Elle est donc décrite par l'opérateur

$$
T_{\vec{r}_{0}}=\exp \left(\frac{i}{\hbar} \vec{P} \cdot \vec{r}_{0}\right) .
$$

$T_{\vec{r}_{0}}$ commute évidemment avec $H=p^{2} / 2 m$. Il constitue alors une constante du mouvement. En d'autres termes, si la particule se trouve dans un état propre de $T_{\vec{r}_{0}}$ elle restera dans cet état pour toujours. Dans le cas de plusieurs particules n'interagissant qu'avec elles-mêmes, la translation du système dans son ensemble laissera l'Hamiltonien toujours invariant. Cette translation est décrite par

$$
T_{\vec{r}_{0}}=\exp \left(\frac{i}{\hbar} \sum_{i} \vec{P}_{i} \cdot \vec{r}_{0}\right) .
$$

Le fait que $\left[T_{\vec{r}_{0}}, H\right]=0$ entraîne la conservation de l'impulsion totale. Elle persiste naturellement aussi dans un cristal. Elle n'est néanmoins pas très utile dans le contexte de la diffusion. Ce qui nous intéresse ce sont les conséquences des translations par un vecteur $\vec{R}_{0}$ du réseau. Contrairement à une translation quelconque du système, ces translations particulières sont équivalentes à une permutation des variables. Elles établissent ainsi un lien à la fois entre les déplacements

$$
\vec{u}(\vec{R}) \rightarrow \vec{u}\left(\vec{R}-\vec{R}_{0}\right)
$$

et les impulsions

$$
\vec{P}(\vec{R}) \rightarrow \vec{P}\left(\vec{R}-\vec{R}_{0}\right)
$$

des particules. Une telle relation est absente pour une translation quelconque. Ces relations sont valables pour tout $\vec{R}$ désignant la position d'équilibre d'un atome. Pour exploiter cette symétrie supplémentaire, il nous faudra identifier l'opérateur quantique qui y est associé. C'est un opérateur unitaire

$$
T\left(\vec{R}_{0}\right)=\exp \left(i \mathbf{K}\left(\vec{R}_{0}\right)\right)
$$

qui doit assurer que

$$
\begin{aligned}
& \exp \left(i \mathbf{K}\left(\vec{R}_{0}\right)\right) \vec{u}(\vec{R}) \exp \left(-i \mathbf{K}\left(\vec{R}_{0}\right)\right)=\vec{u}\left(\vec{R}-\vec{R}_{0}\right) \\
& \exp \left(i \mathbf{K}\left(\vec{R}_{0}\right)\right) \vec{P}(\vec{R}) \exp \left(-i \mathbf{K}\left(\vec{R}_{0}\right)\right)=\vec{P}\left(\vec{R}-\vec{R}_{0}\right)
\end{aligned}
$$


L'opérateur $T\left(\vec{R}_{0}\right)$ rempli cette tâche si nous choisissons

$$
\mathbf{K}\left(\vec{R}_{0}\right)\left|\left\{n_{i}(\vec{k})\right\}\right\rangle=\sum_{\vec{k}} \sum_{i} n_{i}(\vec{k})\left(\vec{k} \cdot \vec{R}_{0}\right)\left|\left\{n_{i}(\vec{k})\right\}\right\rangle .
$$

Le lecteur soucieux d'en connaître la preuve est prié de se référer à [51]. Le fait que nous ayons formulé $\mathbf{K}\left(\vec{R}_{0}\right)$ dans la base des phonons n'implique pas que le cristal doit être harmonique. $\mathbf{K}\left(\vec{R}_{0}\right)$ est un opérateur qui extrait d'un état quantique du système représenté dans la base des phonons la somme des projections des vecteurs d'onde $\vec{k}$ sur $\vec{R}_{0}$. Il est appellé opérateur de moment cristallin.

Les translations $T\left(\vec{R}_{0}\right)$ commutent toutes entre elles. Si le système se trouve à un moment donné dans un état propre de l'Hamiltonien harmonique, alors cette état sera un état propre de toutes les translations $T\left(\vec{R}_{0}\right)$ avec les valeurs propres

$$
\exp \left(\sum_{\vec{k}} \sum_{i} n_{i}(\vec{k})\left(\vec{k} \cdot \vec{R}_{0}\right)\right)
$$

Du fait que les $T\left(\vec{R}_{0}\right)$ commutent avec l'Hamiltonien implique que le système devra y rester pour toujours. Si l'occupation des phonons change $n_{i}(\vec{k}) \rightarrow n_{i}^{\prime}(\vec{k})$, par exemple à cause des interactions anharmoniques, alors

$$
\exp \left(\sum_{\vec{k}} \sum_{i} n_{i}(\vec{k})\left(\vec{k} \cdot \vec{R}_{0}\right)\right)=\exp \left(\sum_{\vec{k}} \sum_{i} n_{i}^{\prime}(\vec{k})\left(\vec{k} \cdot \vec{R}_{0}\right)\right) \quad \forall \quad \vec{R}_{0}
$$

En d'autres termes

$$
\exp \left(\left[\sum_{\vec{k}} \sum_{i} n_{i}(\vec{k}) \vec{k}-n_{i}^{\prime}(\vec{k}) \vec{k}\right] \cdot \vec{R}_{0}\right)=1 \quad \forall \quad \vec{R}_{0}
$$

C'est l'expression qui définit les vecteurs du réseau réciproque (se référer au chapitre I). On en tire

$$
\left[\sum_{\vec{k}} \sum_{i} n_{i}(\vec{k}) \vec{k}-n_{i}^{\prime}(\vec{k}) \vec{k}\right]=\vec{G}
$$

Le moment cristallin est donc conservé à un vecteur du réseau réciproque près. C'est la propriété que nous avions déjà exploitée en définissant les processus anharmoniques dans le chapitre I.

Nous devons maintenant rajouter le neutron ou n'importe quelle autre particule qui n'interagit qu'avec les ions (par exemple un électron de Bloch). La présence du neutron ne perturbe pas la symétrie de permutation du réseau. Déplacé de $\vec{R}_{0}$, le neutron trouvera exactement les mêmes interactions qu'avant mais avec d'autres ions. L'opérateur de translation du neutron est donné par (11.41)

$$
T_{\vec{R}_{0}}^{\mathrm{n}}=\exp \left(\frac{i}{\hbar} \vec{P} \cdot \vec{R}_{0}\right) .
$$

La symétrie du réseau implique que le produit de $T_{\vec{R}_{0}}^{\mathrm{n}}$ et $T_{\vec{R}_{0}}^{\text {ions }}$ commute avec l'Hamiltonien. Si au départ le cristal se trouve dans un état propre du système harmonique et le neutron dans un état $\vec{P}=\hbar \vec{k}_{i}$, l'ensemble est un état propre de

$$
T_{\vec{R}_{0}}^{\mathrm{n}} T_{\vec{R}_{0}}^{\text {ions }} \quad \forall \quad \vec{R}_{0}
$$


avec comme valeurs propres

$$
\exp \left(\left[\vec{k}_{i}+\sum_{\vec{k}} \sum_{i} n_{i}(\vec{k}) \vec{k}\right] \cdot \vec{R}_{0}\right)
$$

à cause des interactions entre le neutron et les ions ainsi que des forces anharmoniques, ni l'état du neutron ni l'état du cristal ne sont stationnaires. Tous les deux vont évoluer dans le temps. En employant les mêmes arguments, qui nous ont amenés à (11.48), nous déduisons qu'entre les nombres quantiques de l'état initial et final il existe la relation

$$
\left[\sum_{\vec{k}} \sum_{i} n_{i}(\vec{k}) \vec{k}-n_{i}^{\prime}(\vec{k}) \vec{k}\right]+\vec{G}=\left(\vec{k}_{f}-\vec{k}_{i}\right) .
$$

Le changement d'impulsion du neutron est donc compensé par un changement du moment cristallin à un vecteur du réseau réciproque près. C'est un résultat d'une grande généralité. Il vaut pour des cristaux anharmoniques aussi bien que pour des cristaux harmoniques et inclut n'importe quel nombre de phonons échangés.

\subsection{Calcul itératif pour des systèmes monoatomiques incohérents}

La situation change si on a affaire à une mesure effectuée sur poudre. Dans ce cas la réponse à un phonon est déjà continue et ressemble, dans l'approximation incohérente, à la densité d'états. Elle donc a priori indiscernable de la réponse multiphonons. Sans modèle ou calcul de la dynamique du réseau, une correction de la contribution multiphonons est difficle. Une telle correction est néanmoins essentielle si nous voulons extraire des quantités physiques de la mesure, comme par exemple la densité d'états elle-même. Une procédure de correction itérative ne peut être développée que pour un système monoatomique incohérent. ${ }^{87}$ Cette condition peut apparaître extrêmement restrictive. Dans la réalité, on se rend néanmoins compte que la méthode donne des résultats assez convenables mêmes pour des systèmes complexes.

Pour un système monoatomique incohérent, la fonction de corrélation qui détermine la fonction de diffusion (11.2) a la forme

$$
\begin{aligned}
\aleph_{j, j}(\vec{Q}, t) & =\left\langle\left(\vec{Q} \cdot \vec{u}_{j}\right)\left(\vec{Q} \cdot \vec{u}_{j}(t)\right)\right\rangle \\
& =\frac{\hbar}{2} \frac{Q^{2}}{3 N} \frac{1}{m} \sum_{i}\left\{\left[\frac{1}{\omega_{i}}\left(1+n\left(\omega_{i}\right)\right) \exp \left(i \omega_{i} t\right)\right]+\left[\frac{1}{\omega_{i}} n\left(\omega_{i}\right) \exp \left(-i \omega_{i} t\right)\right]\right\},
\end{aligned}
$$

où nous avons utilisé les expressions $(10.111)$ et $(10.119)^{88}$. La dernière n'est strictement applicable que dans le cas d'un système cubique ou isotrope. L'expression (11.51) peut être formulée à l'aide de la densité d'états

$$
G(\omega)=\frac{1}{3 N} \sum_{i=1}^{3 N} \delta\left(\omega-\omega_{i}\right)
$$

\footnotetext{
87 Elle a été introduite assez tôt par A. Sjölander [52].

${ }^{88}$ Le facteur $(3 N)^{-1}$ provient du fait que les vecteurs propres $e_{i}(\alpha, j)$ ont $3 \mathrm{~N}$ composantes sur lesquelles l'amplitude de vibration est distribuée.
} 
On obtient

$$
\begin{aligned}
& \left\langle\left(\vec{Q} \cdot \vec{u}_{j}\right)\left(\vec{Q} \cdot \vec{u}_{j}(t)\right)\right\rangle \\
& \quad=\frac{\hbar}{2} \frac{Q^{2}}{m}\left\{\int_{0}^{\infty} d \omega \frac{g(\omega)}{\omega}[(1+n(\omega)) \exp (i \omega t)]+\int_{-\infty}^{0} d \omega \frac{g(\omega)}{-\omega}[n(-\omega) \exp (i \omega t)]\right\} .
\end{aligned}
$$

En exploitant l'identité

$$
1+n(\omega)=1+\frac{1}{\exp \left(\frac{\hbar \omega}{k_{\mathrm{b}} T}\right)-1}=\frac{\exp \left(\frac{\hbar \omega}{k_{\mathrm{b}} T}\right)}{\exp \left(\frac{\hbar \omega}{k_{\mathrm{b}} T}\right)-1}=\frac{1}{1-\exp \left(-\frac{\hbar \omega}{k_{\mathrm{b}} T}\right)}=-n(-\omega)
$$

nous aboutissons à l'expression relativement simple

$$
\aleph_{j, j}(\vec{Q}, t)=\left\langle\left(\vec{Q} \cdot \vec{u}_{j}\right)\left(\vec{Q} \cdot \vec{u}_{j}(t)\right)\right\rangle=\frac{\hbar Q^{2}}{2 m} \int_{-\infty}^{\infty} d \omega \frac{g(\omega)}{\omega}(n(\omega)+1) \exp (i \omega t) .
$$

Cette expression peut être formulée alternativement ${ }^{89}$ comme

$$
\aleph_{j, j}(\vec{Q}, t)=\frac{\hbar Q^{2}}{2 m} \int_{0}^{\infty} d \omega \frac{g(\omega)}{\omega}\left[i \sin (\omega t)+\cos (\omega t) \operatorname{coth}\left(\frac{\hbar \omega}{2 k_{\mathrm{b}} T}\right)\right] .
$$

En définissant

$$
f(t)=\frac{2 m}{\hbar Q^{2}} \aleph_{j, j}(\vec{Q}, t)=\int_{-\infty}^{\infty} d \omega \frac{g(\omega)}{\omega}(n(\omega)+1) \exp (i \omega t)
$$

la fonction de diffusion (voir (8.7) et (11.2)) s'écrit comme

$$
\begin{aligned}
& S(\vec{Q}, \omega) \\
& =\frac{\sigma_{\text {inc }}}{4 \pi} \frac{N}{2 \pi \hbar} \int_{-\infty}^{\infty} d t e^{-i \omega t} e^{-2 W(\vec{Q})} e^{\left\langle\left(\vec{Q} \cdot \vec{u}_{j}\right)\left(\vec{Q} \cdot \vec{u}_{j}(t)\right)\right\rangle} \\
& =\frac{\sigma_{\text {inc }}}{4 \pi} \frac{N}{2 \pi \hbar} \int_{-\infty}^{\infty} d t e^{-i \omega t} \exp (-2 W(\vec{Q})) \cdot \exp \left(\frac{\hbar Q^{2}}{2 m} f(t)\right) \\
& =\frac{\sigma_{\text {inc }}}{4 \pi} \frac{N}{2 \pi \hbar} \exp \left(-\frac{\hbar Q^{2}}{2 m} f(0)\right) \sum_{p=0}^{\infty} \frac{1}{p !} \int_{-\infty}^{\infty} d t e^{-i \omega t}\left(\frac{\hbar Q^{2}}{2 m} f(t)\right)^{p}
\end{aligned}
$$

89

$$
\begin{aligned}
(1 & +n(\omega)) \exp (i \omega t)+n(\omega) \exp (-i \omega t) \\
& =(1+n(\omega))(\cos (\omega t)+i \sin (\omega t))+n(\omega)(\cos (-\omega t)+i \sin (-\omega t)) \\
& =(1+n(\omega))(\cos (\omega t)+i \sin (\omega t))+n(\omega)(\cos (\omega t)-i \sin (\omega t)) \\
& =(1+2 n(\omega)) \cos (\omega t)+i \sin (\omega t) \\
& =\operatorname{coth}\left(\frac{\hbar \omega}{2 k_{\mathrm{b}} T}\right) \cos (\omega t)+i \sin (\omega t)
\end{aligned}
$$


où, dans la dernière ligne, nous avons exploité le fait que ${ }^{90}$ (voir (10.23))

$$
2 W(\vec{Q})=\frac{\hbar Q^{2}}{2 m} \int_{0}^{\infty} d \omega \frac{g(\omega)}{\omega}(2 n(\omega)+1)=\frac{\hbar Q^{2}}{2 m} f(0)=\aleph_{j, j}(\vec{Q}, t=0) .
$$

Nous en déduisons la fonction de diffusion intermédiaire

$$
I(\vec{Q}, t)=\frac{\sigma_{\text {inc }}}{4 \pi} N \exp \left(-\frac{\hbar Q^{2}}{2 m} f(0)\right) \sum_{p=0}^{\infty} \frac{1}{p !}\left(\frac{\hbar Q^{2}}{2 m} f(t)\right)^{p} .
$$

Si on ne retient que le premier terme de la somme, ce qui équivaut à $I(\vec{Q}, t=0)$, on obtient la diffusion élastique (à comparer avec (10.87))

$$
S_{(p=0)}(\vec{Q}, \omega)=N \frac{\sigma}{4 \pi} \exp (-2 W(\vec{Q})) \delta(\hbar \omega)
$$

Le terme suivant donne la réponse à un phonon (à comparer avec (10.131))

$$
\begin{aligned}
& S_{(p=1)}(\vec{Q}, \omega) \\
& \quad=\frac{\sigma_{\text {inc }}}{4 \pi} \frac{N}{2 \pi \hbar} \exp (-2 W(\vec{Q})) \int_{-\infty}^{\infty} d t e^{-i \omega t}\left(\frac{\hbar Q^{2}}{2 m} f(t)\right) \\
& \quad=\frac{\sigma_{\text {inc }}}{4 \pi} \frac{N}{2 \pi \hbar} \exp (-2 W(\vec{Q})) \int_{-\infty}^{\infty} d t e^{-i \omega t}\left(\frac{\hbar Q^{2}}{2 m} \int_{-\infty}^{\infty} d \omega^{\prime} \frac{g\left(\omega^{\prime}\right)}{\omega^{\prime}}\left(n\left(\omega^{\prime}\right)+1\right) \exp \left(i \omega^{\prime} t\right)\right) \\
& =\frac{\sigma_{\text {inc }}}{4 \pi} N \exp (-2 W(\vec{Q})) \frac{Q^{2}}{2 m} \int_{-\infty}^{\infty} d \omega^{\prime} \frac{g\left(\omega^{\prime}\right)}{\omega^{\prime}}\left(n\left(\omega^{\prime}\right)+1\right) \delta\left(\omega-\omega^{\prime}\right) \\
& =\frac{N}{8 \pi} Q^{2} \frac{\sigma_{\text {inc }}}{m} \exp (-2 W(\vec{Q})) \frac{g(\omega)}{\omega}(n(\omega)+1)
\end{aligned}
$$

Pour calculer les contributions à plusieurs phonons nous exploitons le fait que l'expression

$$
\int_{-\infty}^{\infty} d t e^{-i \omega t} f(t)^{p}=\int_{-\infty}^{\infty} d t e^{-i \omega t} f(t) \cdot f(t)^{p-1}
$$

est la transformée de Fourier d'un produit de fonction. Cette transformée de Fourier est donc la convolution des transformées de Fouriers individuelles. Avec

$$
\begin{aligned}
T_{0}(\omega) & =\frac{1}{2 \pi \hbar} \int_{-\infty}^{\infty} d t e^{-i \omega t}=\frac{1}{\hbar} \delta(\omega)=\delta(\hbar \omega), \\
T_{1}(\omega) & =\frac{1}{2 \pi \hbar} \int_{-\infty}^{\infty} d t e^{-i \omega t} f(t)=\frac{g(\omega)}{\hbar \omega}(n(\omega)+1) \\
& =\frac{g(\omega)}{2 \hbar \omega}\left(\operatorname{coth}\left(\frac{\hbar \omega}{2 k_{b} T}\right)+1\right),
\end{aligned}
$$

90 Attention, souvent la densité d'états n'est pas définie en $\omega$ mais en énergie et normalisée telle que

$$
\int_{0}^{\infty} g(\hbar \omega) d(\hbar \omega)=1
$$

Dans ce cas les expressions obtenues sont à corriger. Pour la fonction de Debye-Waller nous obtenons par exemple

$$
2 W(\vec{Q})=\frac{\hbar^{2} Q^{2}}{2 m} \int_{0}^{\infty} d(\hbar \omega) \frac{g(\hbar \omega)}{\hbar \omega}(2 n(\omega)+1)=\frac{\hbar^{2} Q^{2}}{2 m} \int_{0}^{\infty} d(\hbar \omega) \frac{g(\hbar \omega)}{\hbar \omega} \operatorname{coth}\left(\frac{\hbar \omega}{2 k_{\mathrm{B}} T}\right) .
$$


les termes supérieurs s'obtiennent de manière itératives comme

$$
\begin{aligned}
T_{p}(\omega) & =\int_{-\infty}^{\infty} d t e^{-i \omega t} f(t)^{p} \\
& =\int_{-\infty}^{\infty} T_{1}\left(\omega-\omega^{\prime}\right) T_{p-1}\left(\omega^{\prime}\right) d \omega^{\prime} .
\end{aligned}
$$

C'est un résultat très important. Les contributions à plusieurs phonons s'obtiennent par la convolution itérative de la densité d'états (correctement ponderée par le facteur d'occupation des modes) avec ellemême. C'est une simplification considérable par rapport à l'expression (11.58). Nous obtenons alors pour la fonction de diffusion

$$
S(\vec{Q}, \omega)=\frac{\sigma_{\mathrm{inc}}}{4 \pi} N \exp (-2 W(Q)) \sum_{p=0}^{\infty} \frac{1}{p !}\left(\frac{\hbar Q^{2}}{2 m}\right)^{p} T_{p}(\omega) .
$$

Par la suite, il est possible de calculer la totalité de la diffusion inélastique jusqu'aux ordres de phonons les plus élevés, une fois que la densité d'états $g(\omega)$ est connue. Dans la pratique, et sans connaissance préalable, $g(\omega)$ est déterminée de manière itérative. On calcule d'abord $g(\omega)$ à partir des données comme s'il n'existait pas de contributions d'ordres supérieurs en utilisant (11.69). On porte alors la première estimation de $g(\omega)$ dans les expressions (11.61) et (11.67) afin de déterminer, avec l'aide des expressions (11.68) et (11.69), la valeur attendue pour la section efficace double différentielle en allant jusqu'à un nombre maximum de phonons $p_{\max }$, qui est librement choisi. L'écart entre cette section efficace calculée et la section efficace actuellement mesurée est utilisé pour affiner l'estimation de $g(\omega)$. Cette procédure est se répète jusqu'à l'obtention de la convergence amenant à $g(\omega)$ d'une façon auto-cohérente. ${ }^{91}$

\subsubsection{Termes à p phonons symétrisés}

Les fonctions $T_{n}(\omega)$ ne sont pas symétriques par rapport à $\omega$. Cela ne pose à priori aucun problème. On préfère néanmoins souvent travailler avec une forme symétrisée quand on est confronté à des calculs numériques.[52,53]. En définissant

$$
T_{p}^{\mathrm{sym}}(\omega)=e^{-\frac{\hbar \omega}{2 k_{\mathrm{B}} T}} T_{p}(\omega)
$$

nous vérifions que

$$
\begin{aligned}
\int_{-\infty}^{\infty} T_{1}^{\mathrm{sym}}\left(\omega-\omega^{\prime}\right) T_{p-1}^{\mathrm{sym}}\left(\omega^{\prime}\right) d \omega^{\prime} & =\int_{-\infty}^{\infty} e^{-\frac{\hbar\left(\omega-\omega^{\prime}\right)}{2 k_{\mathrm{B}} T}} T_{1}\left(\omega-\omega^{\prime}\right) e^{-\frac{\hbar \omega^{\prime}}{2 k_{\mathrm{B}} T}} T_{p-1}\left(\omega^{\prime}\right) d \omega^{\prime} \\
& =e^{-\frac{\hbar \omega}{2 k_{\mathrm{B}} T}} \int_{-\infty}^{\infty} T_{1}\left(\omega-\omega^{\prime}\right) T_{p-1}\left(\omega^{\prime}\right) d \omega^{\prime} \\
& =e^{-\frac{\hbar \omega}{2 k_{\mathrm{B}} T}} T_{p}(\omega) \\
& =T_{p}^{\mathrm{sym}}(\omega)
\end{aligned}
$$

Les fonctions $T_{p}^{\mathrm{sym}}(\omega)$ peuvent donc être determinées comme les $T_{p}(\omega)$ de manière itérative à partir de

$$
T_{1}^{\mathrm{sym}}(\omega)=\frac{g(\omega)}{2 \hbar \omega \sinh \left(\frac{\hbar \omega}{2 k_{\mathrm{B}} T}\right)} .
$$

$T_{1}^{\text {sym }}(\omega)$ est bien symétrique par rapport à $\omega$, comme le sont toutes les autres $T_{p}^{\text {sym }}(\omega)$ qui sont obtenues à partir de $T_{1}^{\mathrm{sym}}(\omega)$ par convolutions successives.

91 Cette procédure est par exemple implémentée dans le programme MUPHOCOR de W. Reichardt [53]. 
En remplaçant les fonctions $T_{p}^{\mathrm{sym}}(\omega)$ dans (11.69), la section efficace différentielle partielle s'écrit comme

$$
\left(\frac{d^{2} \sigma_{\mathrm{inc}}}{d(\hbar \omega) d \Omega}\right)_{\mathrm{inc}}=\frac{k_{f}}{k_{i}} S(\vec{Q}, \omega)=\frac{N \sigma_{\mathrm{inc}}}{4 \pi} \frac{k_{f}}{k_{i}} e^{\frac{\hbar \omega}{2 k_{\mathrm{B}} T}} e^{-2 W(Q)} \sum_{p=1}^{\infty}\left(\frac{\hbar Q^{2}}{2 m}\right)^{p} \frac{T_{p}^{\mathrm{sym}}(\omega)}{p !}
$$

\subsubsection{Termes à p phonons normalisés}

Les expressions (11.69) et (11.73) décrivent toutes les deux la fonction de diffusion comme une suite de termes à $p$ phonons. Elles ont en commun que les fonctions $T_{p}(\omega)$ et $\tilde{T}_{p}(\omega)$ employées à cet effet, et qui peuvent être considerées comme des pseudo-densités d'états à $p$ phonons, ne sont pas normalisées. De ce fait il n'est pas évident d'identifier la grandeur physique qui pilote l'importance de chaque terme et ainsi la convergence de la suite. Nous pouvons remédier à ceci en normalisant les pseudo-densités d'états $T_{p}(\omega)$. A partir de l'expression (11.65) nous obtenons

$$
\int_{-\infty}^{\infty} d \omega T_{p}(\omega)=\int_{-\infty}^{\infty} d \omega \int_{-\infty}^{\infty} d t e^{-i \omega t} f(t)^{p}=\int_{-\infty}^{\infty} d t f(t)^{p} \delta(t)=f(0)^{p}
$$

Avec l'identité (11.61), ces normes sont directement liées aux fonctions de Debye-Waller

$$
f(0)^{p}=\left(2 W(Q) \frac{2 m}{\hbar Q^{2}}\right)^{p} .
$$

En définissant

$$
\tilde{T}_{p}(\omega)=\frac{T_{p}(\omega)}{f(0)^{p}}=T_{p}(\omega)\left(\frac{\hbar Q^{2}}{2 m} \frac{1}{2 W(Q)}\right)^{p}
$$

nous obtenons des fonctions normalisées

$$
\int_{-\infty}^{\infty} d \omega \tilde{T}_{p}(\omega)=1
$$

En remplaçant (11.76) dans (11.69), la fonction de diffusion s'exprime comme

$$
\begin{aligned}
S(\vec{Q}, \omega) & =\frac{\sigma_{\text {inc }}}{4 \pi} N \exp (-2 W(Q)) \sum_{p=0}^{\infty} \frac{1}{p !}\left(\frac{\hbar Q^{2}}{2 m} f(0)\right)^{p} \tilde{T}_{p}(\omega) \\
& =\frac{\sigma_{\text {inc }}}{4 \pi} N \exp (-2 W(Q)) \sum_{p=0}^{\infty} \frac{(2 W(Q))^{p}}{p !} \tilde{T}_{p}(\omega) .
\end{aligned}
$$

La suite convergera donc rapidement si la fonction de Debye-Waller donne pour le $Q$ en question une petite valeur. C'est le cas si le déplacement carré moyen $\overline{u^{2}}$ des atomes est petit. Au fur et à mesure que $Q$ ou $\overline{u^{2}}$ augmente, les termes à plusieurs phonons deviennent importants. C'est la situation que nous avions déjà rencontrée lors de la discussion de l'oscillateur harmonique isotrope. La fonction de Debye-Waller est donc un très bon indicateur pour le nombre de termes à prendre en compte lors de l'analyse des données.

\subsubsection{Intégration des termes à p phonons en angle et en $Q$}

Les expressions obtenues ci-dessus sont valables pour une valeur particulière de $Q$. Si l'on veut appliquer l'approximation incohérente ou si l'on doit sommer sur différents bancs de détecteurs pour une meilleure statistique, alors on souhaitera déterminer $g(\omega)$ à partir de données intégrées en angle de diffusion ou en $Q$. L'intégration de choix dans le contexte de la détermination des densités d'états est celle que nous avions présentée dans le contexte de l'approximation incohérente (voir l'équation 
(10.166)). Cette intégration appliquée à l'expression (11.73) mène, en utilisant (10.164), à

$$
\begin{aligned}
\left(\frac{d \sigma}{d(\hbar \omega)}\right)_{\mathrm{int}} & =\Delta \Phi \int_{\theta_{\min }}^{\theta_{\max }}\left(\frac{d^{2} \sigma_{\mathrm{inc}}}{d(\hbar \omega) d \Omega}\right)_{\mathrm{inc}} \sin \theta d \theta \\
& =\Delta \Phi \int_{Q_{\min }(\omega)}^{Q_{\max }(\omega)}\left(\frac{d^{2} \sigma_{\mathrm{inc}}}{d(\hbar \omega) d \Omega}\right)_{\mathrm{inc}} \frac{1}{k_{i} k_{f}} Q d Q \\
& =\Delta \Phi \frac{N \sigma_{\text {inc }}}{4 \pi} \frac{1}{k_{i}^{2}} e^{\frac{\hbar \omega}{2 k_{\mathrm{B}} T}} \sum_{p=1}^{\infty}\left(\frac{\hbar}{2 m}\right)^{p} \frac{T_{p}^{\mathrm{sym}}(\omega)}{p !} \int_{Q_{\min }(\omega)}^{Q_{\max }(\omega)} Q^{2 p} e^{-\gamma Q^{2}} Q d Q \\
& =\Delta \Phi \frac{N \sigma_{\mathrm{inc}}}{4 \pi} \frac{1}{k_{i}^{2}} e^{\frac{\hbar \omega}{2 k_{\mathrm{B}} T}} \sum_{p=1}^{\infty}\left(\frac{\hbar}{2 m}\right)^{p} \frac{T_{p}^{\mathrm{sym}}(\omega)}{p !} \frac{1}{2 \gamma^{p+1}} \int_{Q_{\min }(\omega)}^{Q_{\max }(\omega)}\left(\gamma Q^{2}\right)^{p} e^{-\gamma Q^{2}} d\left(\gamma Q^{2}\right)
\end{aligned}
$$

avec

$$
\gamma Q^{2}=2 W(Q)
$$

$Q_{\min }$ et $Q_{\max }$ dépendent naturellement de la fréquence $\omega$. Cette expression est rigoureusement exacte pour un diffuseur incohérent dans un réseau de Bravais cubique monoatomique.

Les intégrales de type

$$
I_{p}(y)=\int_{0}^{y} x^{p} e^{-x} d x
$$

qui apparaissent dans cette expression, peuvent être calculées de manière itératives

$$
I_{p}(y)=\int_{0}^{y} x^{p} e^{-x} d x=-y^{p} e^{-y}-p \int_{0}^{y} x^{p-1} e^{-x}=-y^{p} e^{-y}-p I_{p-1}(y) .
$$

Cette formule peut être facilement obtenue en employant l'intégration par parties. ${ }^{92}$ Les premiers termes se calculent comme

$$
\begin{aligned}
& I_{0}(y)=-e^{-y} \\
& I_{1}(y)=-(y+1) e^{-y} \\
& I_{2}(y)=-\left(y^{2}+2 y+2\right) e^{-y} \\
& I_{3}(y)=-\left(y^{3}+3 y^{2}+6 y+6\right) e^{-y} .
\end{aligned}
$$

${ }^{92}$ L'intégration par parties est basée sur l'identité

$$
\int_{a}^{b} u^{\prime}(x) v(x) d x=[u(x) v(x)]_{a}^{b}-\int_{a}^{b} u(x) v^{\prime}(x) d x .
$$

En posant

$$
\begin{aligned}
& u(x)=-e^{-x}, \\
& v(x)=x^{p}
\end{aligned}
$$

on obtient

$$
\int_{a}^{b} x^{p} e^{-x} d x=-\left[x^{p} e^{-x}\right]_{a}^{b}-n \int_{a}^{b} x^{p-1} e^{-x}
$$

et donc

$$
I_{p}(y)=\int_{0}^{y} x^{p} e^{-x} d x=-y^{p} e^{-y}-p \int_{0}^{y} x^{p-1} e^{-x}=-y^{p} e^{-y}-p I_{p-1}(y)
$$


Ainsi on aboutit au résultat final

$$
\left(\frac{d \sigma}{d(\hbar \omega)}\right)_{\mathrm{int}}=\Delta \Phi \frac{N \sigma_{\mathrm{inc}}}{8 \pi} \frac{1}{k_{i}^{2}} e^{\frac{\hbar \omega}{2 k_{\mathrm{B}} T}} \sum_{p=1}^{\infty}\left(\frac{\hbar}{2 m}\right)^{p} \frac{1}{\gamma^{p+1}}\left(I_{p}\left(\gamma Q_{\max }^{2}\right)-I_{p}\left(\gamma Q_{\min }^{2}\right)\right) \frac{T_{p}^{\mathrm{sym}}(\omega)}{p !}
$$

Si les déplacements carrés moyens sont petits à l'échelle de $Q_{\max }^{-2}$ on ne retiendra que le terme à un phonon

$$
\begin{aligned}
\left(\frac{d \sigma}{d(\hbar \omega)}\right)_{\mathrm{int}}^{p=1}= & \Delta \Phi \frac{N \sigma_{\mathrm{inc}}}{8 \pi} \frac{1}{k_{i}^{2}} e^{-\frac{\hbar \omega}{2 k_{\mathrm{B}} T}}\left(\frac{\hbar}{2 m}\right) \frac{1}{\gamma^{2}}\left(I_{1}\left(\gamma Q_{\max }^{2}\right)-I_{1}\left(\gamma Q_{\mathrm{min}}^{2}\right)\right) T_{1}^{\mathrm{sym}}(\omega) \\
= & \Delta \Phi \frac{N \sigma_{\mathrm{inc}}}{8 \pi} \frac{1}{k_{i}^{2}} e^{-\frac{\hbar \omega}{2 k_{\mathrm{B}} T}}\left(\frac{\hbar}{2 m}\right) \frac{1}{\gamma^{2}} \\
& \times\left(-\left(1+\gamma Q_{\max }^{2}\right) e^{-\gamma Q_{\max }^{2}}+\left(1+\gamma Q_{\min }^{2}\right) e^{-\gamma Q_{\min }^{2}}\right) T_{1}^{\mathrm{sym}}(\omega) .
\end{aligned}
$$

En plus nous pouvons dans ce cas développer le facteur

$$
I_{1}\left(\gamma Q^{2}\right)=-\left(1+\gamma Q^{2}\right) e^{-\gamma Q^{2}} \approx-\left(1+\gamma Q^{2}\right)\left(1-\gamma Q^{2}\right)=-1+\frac{1}{2} \gamma^{2} Q^{4}
$$

Ainsi

$$
\begin{aligned}
\left(\frac{d \sigma}{d(\hbar \omega)}\right)_{\mathrm{int}}^{p=1} & =\Delta \Phi \frac{N \sigma_{\mathrm{inc}}}{16 \pi} \frac{1}{k_{i}^{2}} e^{\frac{\hbar \omega}{2 k_{\mathrm{B}} T}}\left(\frac{\hbar}{2 m}\right)\left(Q_{\max }^{4}(\omega)-Q_{\min }^{4}(\omega)\right) T_{1}^{\mathrm{sym}}(\omega) \\
& =\Delta \Phi \frac{N \sigma_{\text {inc }}}{16 \pi} \frac{1}{k_{i}^{2}} e^{\frac{\hbar \omega}{2 k_{\mathrm{B}} T}}\left(\frac{\hbar}{2 m}\right)\left(Q_{\max }^{4}(\omega)-Q_{\text {min }}^{4}(\omega)\right) \frac{g(\omega)}{2 \hbar \omega \sinh \left(\frac{\hbar \omega}{2 k_{\mathrm{B}} T}\right)} \\
& =\Delta \Phi \frac{N \sigma_{\mathrm{inc}}}{32 \pi m} \frac{1}{k_{i}^{2}}(n(\omega)+1) \frac{g(\omega)}{\omega}\left(Q_{\text {max }}^{4}(\omega)-Q_{\text {min }}^{4}(\omega)\right) .
\end{aligned}
$$

Cette expression établit une relation simple entre les données expérimentales intégrées sur $Q d Q$ et la densité d'états $g(\omega)$. Elle est identique à (10.170) mis à part le facteur de Debye-Waller. Elle permet d'estimer la densité d'états initiale à partir de laquelle débutera le processus d'itération.

L'expression (11.95) est aussi intéressante d'un autre point de vue. Pour l'obtenir, nous avons pris en compte correctement la dépendance en $Q$ du facteur de Debye-Waller. De plus, le facteur de DebyeWaller a été retenu au même ordre de $\gamma$ que les multiphonons. Le résultat de cette procédure est que le facteur de Debye-Waller disparaît complètement de la section efficace à un phonon. Nous en tirons la conclusion suivante: Si l'on n'est pas en mesure de corriger les multiphonons il vaut mieux ne pas non plus corriger le facteur de Debye-Waller. Les deux corrections ont des effets inverses sur l'obtention de la densité d'états. Elle se compensent partiellement.

Nous voudrions renforcer ce point en sollicitant encore une fois la règle de somme $f$. Avec (voir (11.64))

$$
S_{(p=1)}(\vec{Q}, \omega) \frac{N}{8 \pi} Q^{2} \frac{\sigma_{\text {inc }}}{m} \exp (-2 W(\vec{Q})) \frac{g(\omega)}{\omega}(n(\omega)+1)
$$




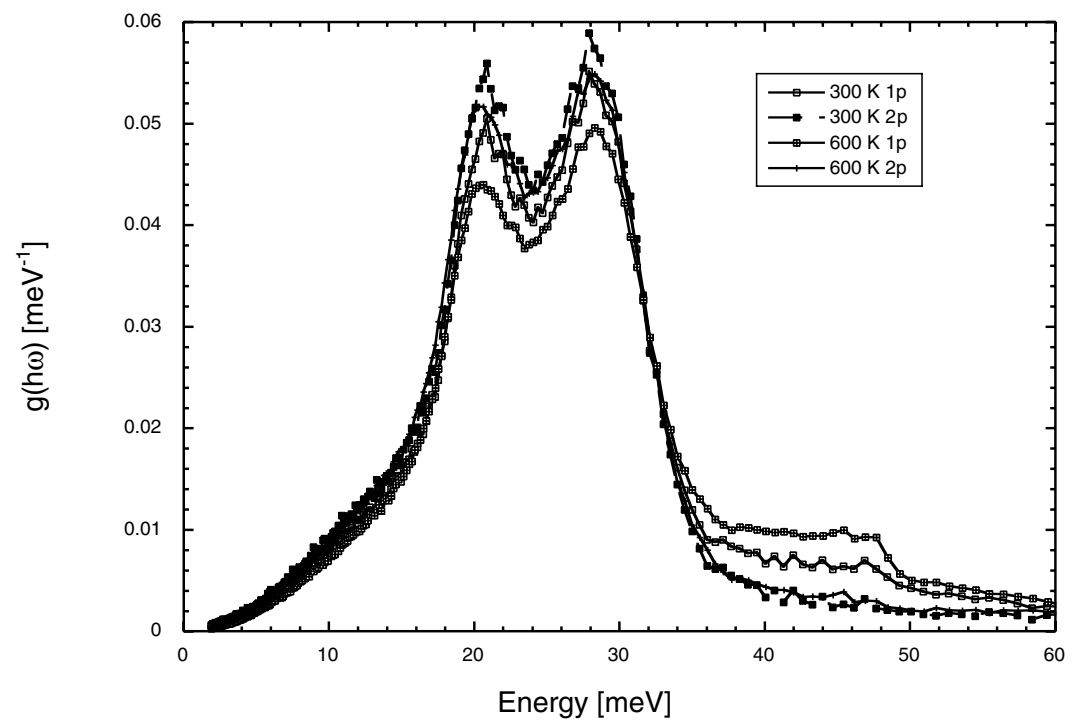

Figure 48. Densité d'états du vanadium à 300 et à $600 \mathrm{~K}$ obtenue de la diffusion incohérente à un phonon mesurée sur l'instrument IN6. Les courbes corrigées pour les multiphonons d'ordre deux sont comparées aux courbes qu'on obtient sans correction. La différence peut être attribuée à la contribution des processus à deux phonons. Cette contribution augmente avec la température. Si on ne la prenait pas en compte, on déduirait à tort qu'il existe une bande de fréquence entre 35 et $50 \mathrm{meV}$, qui -de plus- serait fortement anharmonique.

nous obtenons pour le moment d'ordre un

$$
\begin{aligned}
\int_{\infty}^{\infty} d(\hbar \omega)(\hbar \omega) S_{(p=1)}(\vec{Q}, \omega) & =\frac{N}{8 \pi} \hbar^{2} Q^{2} \frac{\sigma_{\text {inc }}}{m} \exp (-2 W(\vec{Q})) \int_{0}^{\infty} d \omega g(\omega) \\
& =N \overline{b^{2}} \frac{\hbar^{2} Q^{2}}{2 m} \exp (-2 W(\vec{Q})) \\
& =N \bar{b}^{2} E_{r}(\vec{Q}) \exp (-2 W(\vec{Q}))
\end{aligned}
$$

La diminution de ce moment par rapport au moment total selon (8.167) est due au facteur de DebyeWaller. Le résultat est correct si nous le complétons avec la contribution des multiphonons. Si nous décidions d'ignorer les multiphonons, alors nous ferions mieux aussi d'ignorer le facteur de DebyeWaller. Ainsi le premier moment sera reproduit correctement.

\subsection{Susceptibilité et anharmonicité}

Dans la section 8.11 nous avons appris (8.144) que la fonction de diffusion de n'importe quel système peut s'ecrire sous la forme

$$
S(\vec{Q}, \omega)=\frac{1}{\pi}(1+n(\omega)) \chi_{\vec{Q}}{ }^{\prime \prime}[\omega]
$$

avec $\chi_{\vec{Q}}^{\prime \prime}[\omega]$ la susceptibilité généralisée. Nous voudrions maintenant appliquer cette relation aux vibrations et aux phonons. Si nous prenons comme point de départ la fonction de diffusion 
cohérente (10.57)

$$
\begin{aligned}
S_{\mathrm{coh}}^{1-\mathrm{ph}}(\vec{Q}, \omega) & =\frac{1}{2} \sum_{i} \frac{\left|F_{i}(\vec{Q})\right|^{2}}{\omega_{i}}\left\{\left[\left(1+n\left(\omega_{i}\right)\right) \delta\left(\omega-\omega_{i}\right)\right]+\left[n\left(\omega_{i}\right) \delta\left(\omega+\omega_{i}\right)\right]\right\} \\
& =\frac{1}{2}(1+n(\omega)) \sum_{i} \frac{\left|F_{i}(\vec{Q})\right|^{2}}{\omega_{i}}\left\{\left[\delta\left(\omega-\omega_{i}\right)\right]+\left[\delta\left(\omega+\omega_{i}\right)\right]\right\}
\end{aligned}
$$

nous obtenons pour la susceptibilité

$$
\chi_{\vec{Q}}^{\prime \prime}[\omega]=\frac{\pi}{2} \sum_{i} \frac{\left|F_{i}(\vec{Q})\right|^{2}}{\omega_{i}}\left\{\delta\left(\omega-\omega_{i}\right)+\delta\left(\omega+\omega_{i}\right)\right\} .
$$

Le facteur de forme des vibrations est donné par (10.58)

$$
\left|F_{i}(\vec{Q})\right|^{2}=\left|\sum_{\kappa} \frac{\overline{b_{\kappa}}}{\sqrt{m_{\kappa}}} \sum_{j \in\left\{j_{\kappa}\right\}} e^{-i \vec{Q} \cdot \vec{R}_{j}^{0}} e^{-W_{j}(\vec{Q})}\left(\vec{Q} \cdot \vec{e}_{i}(j)\right)\right|^{2} .
$$

Il ne dépend de la température que par l'intermédiaire du facteur de Debye-Waller. Nous devons préciser que la susceptibilité ainsi définie se réfère aux fluctuations de la densité de la longueur de diffusion et non pas à la densité atomique tout court.

L'expression (11.98) est tout à fait analogue à celle que nous avons discutée dans le chapitre I pour un simple oscillateur harmonique de fréquence $\omega_{0}$. Pour un tel oscillateur, la susceptibilité liée aux fluctuations de la position $x$ se calcule comme

$$
\chi_{\hat{x}+\hat{x}}^{\prime \prime}[\omega]=\frac{\pi}{2 m \omega_{0}}\left[\left(\delta\left(\omega-\omega_{0}\right)-\delta\left(\omega+\omega_{0}\right)\right] .\right.
$$

Les expressions s'étendent facilement à la diffusion incohérente. Avec la fonction de diffusion incohérente à un phonon (10.129)

$$
S(\vec{Q}, \omega)_{\mathrm{inc}}^{1-\mathrm{ph}(+)}=Q^{2} \frac{1}{8 \pi} \sum_{\kappa} N_{\kappa} \frac{\sigma_{\mathrm{inc}}(\kappa)}{m_{\kappa}} e^{-2 W_{\kappa}(\vec{Q})} F_{\kappa}(\omega) \frac{(1+n(\omega))}{\omega}
$$

nous obtenons une relation entre la susceptibilité et les densités d'états partielles

$$
\chi_{\vec{Q}}^{\prime \prime}[\omega]=\frac{1}{8} Q^{2} \sum_{\kappa} N_{\kappa} \sigma_{\mathrm{inc}}(\kappa) \frac{e^{-2 W_{\kappa}(\vec{Q})}}{\omega m_{\kappa}} F_{\kappa}(\omega) .
$$

Cette expression ne dépend de la température que par l'intermédiaire des facteurs de Debye-Waller. Comme nous avons argumenté dans la section précédente, il vaut mieux négliger cette dépendance, si on n'inclut pas les multiphonons. Donc, pour un système harmonique, la susceptibilité

$$
\chi_{\vec{Q}}^{\prime \prime}[\omega]=\pi \frac{1}{1+n(\omega)} S_{\mathrm{inc}}(\vec{Q}, \omega)
$$

obtenue de la fonction de diffusion mesurée devrait être indépendante de la température pour un système harmonique. C'est un test facile à mettre en œuvre, qui évite toute détermination explicite de la densité d'états. Nous donnons un exemple dans la figure 49.

Le constat qu'un système n'est pas harmonique est souvent insuffisant pour déceler l'origine de l'anharmonicité. Si nous possédons un monocristal, qui permet l'étude détaillée des courbes de dispersions, nous pouvons aller plus loin dans l'interprétation. Comme nous avons expliqué dans le chapitre I, les processus anharmoniques ont principalement deux conséquences observables. Premièrement, les pics des phonons, pour un $\vec{k}$ et une branche $i$ donnés, acquièrent une largeur. Deuxièmement, leurs fréquences centrales changent avec la température. La susceptibilité d'un mode 


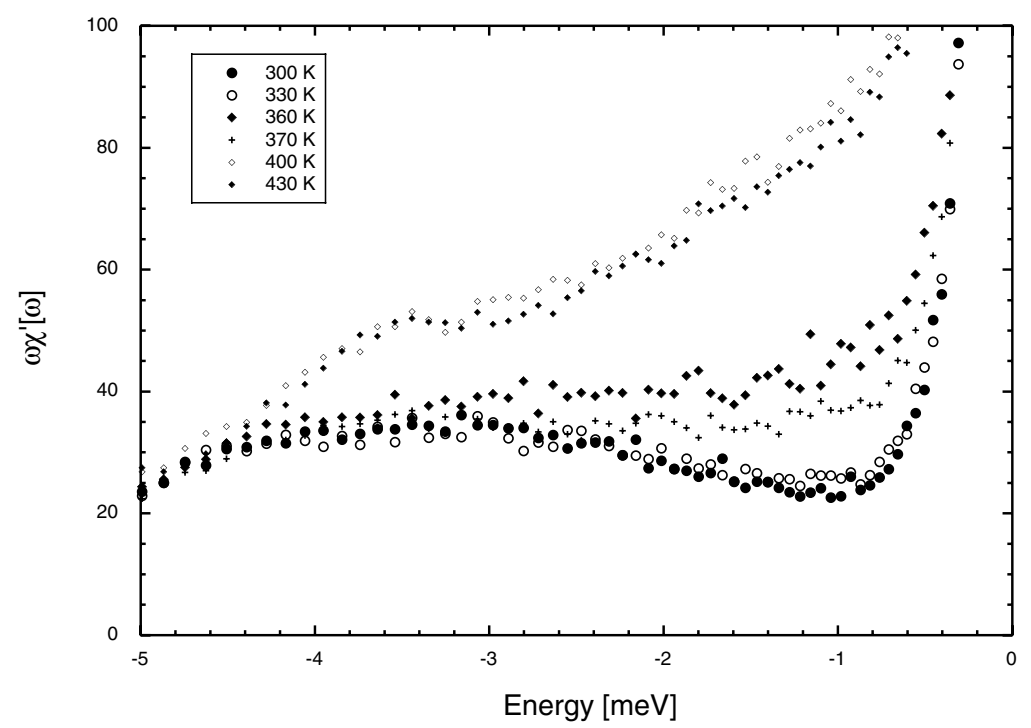

Figure 49. Susceptibilité (en unités arbitraires) multipliée par l'énergie pour le polymère $\mathrm{RbC}_{60}$ en fonction de la température en chauffant de 300 à $430 \mathrm{~K}$. La mesure a été effectuée sur l'instrument IN6 à l'ILL. Pour les détails se référer à [48]. Pour les basses températures, la susceptibilité est ne varie pas d'une température à l'autre. Au moment de la dépolymérisation autour de $350 \mathrm{~K}$, le système cesse d'être harmonique et la susceptibilité évolue. L'excès d'intensité à basse énergie peut être expliqué par la diffusion rotationelle des monomères.

amorti est donnée dans sa forme la plus générale par l'expression [54]

$$
\chi_{(i, \vec{k})}^{\prime \prime}(\omega)=A(i, \vec{k}) \frac{4 \omega_{0}(i, \vec{k}) \Gamma(i, \vec{k} ; \omega)}{\left[\omega^{2}-\omega_{0}(i, \vec{k})^{2}-2 \omega_{0}(i, \vec{k}) \Delta(i, \vec{k} ; \omega)\right]^{2}+4 \omega(i, \vec{k})^{2} \Gamma(i, \vec{k} ; \omega)^{2}}
$$

La dépendance en fréquence a priori inconnue de $\omega_{0}(j, \vec{k})$ et de $\Delta(j, \vec{k})$ rend cette expression peu pratique pour l'interprétation des données. L'approximation la plus simple, qui préserve les propriétés analytiques de la fonction de diffusion comme la parité est donnée par [55]

$$
\begin{aligned}
\Delta(i, \vec{k} ; \omega) & =\Delta(i, \vec{k}), \\
\Gamma(i, \vec{k} ; \omega) & =\frac{\omega}{\omega_{0}(j, \vec{k})} \Gamma(i, \vec{k}) .
\end{aligned}
$$

Ce choix nous amène à la susceptibilité pour un oscillateur amorti

$$
\chi_{(i, \vec{k})}^{\prime \prime}(\omega)=A(i, \vec{k}) \frac{4 \omega \Gamma(i, \vec{k})}{\left(\omega^{2}-\Omega(i, \vec{k})^{2}\right)^{2}+4 \omega^{2} \Gamma(i, \vec{k})^{2}} .
$$

où nous avons introduit la fréquence renormalisée $\Omega(i, \vec{k})$.

En introduisant les fréquences

$$
\omega_{\text {lor }}(i, \vec{k})^{2}=\Omega(i, \vec{k})^{2}-\Gamma(i, \vec{k})^{2}
$$


la susceptibilité de l'oscillateur amorti peut être écrite sous la forme alternative [56]

$$
\chi_{(i, \vec{k})}^{\prime \prime}(\omega)=\frac{A(i, \vec{k})}{\omega_{\operatorname{lor}}(i, \vec{k})}\left[\frac{\Gamma(i, \vec{k})}{\left.\left(\omega-\omega_{\text {lor }}(i, \vec{k})\right)^{2}+\Gamma(i, \vec{k})^{2}\right)}-\frac{\Gamma(i, \vec{k})}{\left.\left(\omega+\omega_{\operatorname{lor}}(i, \vec{k})\right)^{2}+\Gamma(i, \vec{k})^{2}\right)}\right],
$$

qui correspond pour $\Gamma(j, \vec{k}) \ll \omega_{\text {lor }}(j, \vec{k})$ à la somme de deux fonctions lorentziennes du côté gain et perte du spectre.

Cette forme est intéressante parce qu' elle nous ramène dans le cas de $\Gamma(i, \vec{k}) \rightarrow 0$ à l'expression (11.102) pour l'oscillateur harmonique, parce que

$$
\lim _{\epsilon \rightarrow 0} \frac{1}{\pi} \frac{\epsilon}{x^{2}+\epsilon^{2}}=\delta(x)
$$

Le facteur $A(i, \vec{k})$ peut donc être déterminé en substituant l'expression (11.103) dans la limite $\Gamma \rightarrow 0$ à (11.100) dans l'expression (11.99). On obtient alors

$$
A(j, \vec{k})=\left|F_{i}(\vec{Q} \mid \vec{k})\right|^{2}
$$

Le facteur de forme $\left|F_{i}(\vec{Q} \mid \vec{k})\right|^{2}$ est, pour les phonons, défini en (10.73). Nous obtenons pour la fonction de diffusion d'un ensemble d'oscillateurs amortis

$$
\begin{aligned}
S_{\mathrm{coh}}^{1-\mathrm{ph}}(\vec{Q}, \omega)= & \frac{1}{\pi} \frac{(2 \pi)^{3}}{2 V_{\text {maille }}} \sum_{\vec{G}} \sum_{i, \vec{k}} \frac{\left|F_{i}(\vec{Q} \mid \vec{k})\right|^{2}}{\omega_{\text {lor }}(i, \vec{k})}(1+n(\omega)) \\
& \times\left[\delta(\vec{k}-(\vec{Q}+\vec{G})) \frac{\Gamma(i, \vec{k})}{\left.\left(\omega-\omega_{\text {lor }}(i, \vec{k})\right)^{2}+\Gamma(i, \vec{k})^{2}\right)}\right. \\
& \left.-\delta(\vec{k}+(\vec{Q}+\vec{G})) \frac{\Gamma(i, \vec{k})}{\left.\left(\omega+\omega_{\text {lor }}(i, \vec{k})\right)^{2}+\Gamma(i, \vec{k})^{2}\right)}\right] .
\end{aligned}
$$

On doit être prudent quand on interprète les paramètres obtenus lors de l'ajustement des expressions (11.106) ou (11.110) aux données expérimentales. Pour des amortissements faibles, la différence entre $\Omega$ et $\omega_{\text {lor }}$ est petite. Quand $\Gamma$ augmente, la fréquence $\Omega$ est le paramètre physique pertinent. Dans le cas d'un mode mou (voir chapitre de Roland Currat) c'est ce paramètre qui s'extrapole correctement vers zéro à la température de transition [55].

\subsection{Résumé partiel}

Nous allons de nouveau succinctement résumer les principaux résultats obtenus dans les deux dernières sections.

Nous sommes partis des fonctions de diffusion partielles définies comme (10.2)

$$
S_{\kappa, \kappa^{\prime}}(\vec{Q}, \omega)=\frac{1}{2 \pi \hbar} \sum_{j \in\left\{j_{k}\right\}, j^{\prime} \in\left\{j_{\kappa^{\prime}}\right\}} \int_{-\infty}^{\infty} d t\left\langle e^{-i \vec{Q} \cdot \vec{R}_{j^{\prime}}(t=0)} e^{i \vec{Q} \cdot \vec{R}_{j}(t)}\right\rangle e^{-i \omega t}
$$

Dans un solide relaxé, le mouvement des ions est décrit par les déplacements des ions autour de positions d'équilibre

$$
\vec{u}_{j}(t)=\vec{R}_{j}(t)-\vec{R}_{j}^{0}, j=1, \ldots N
$$




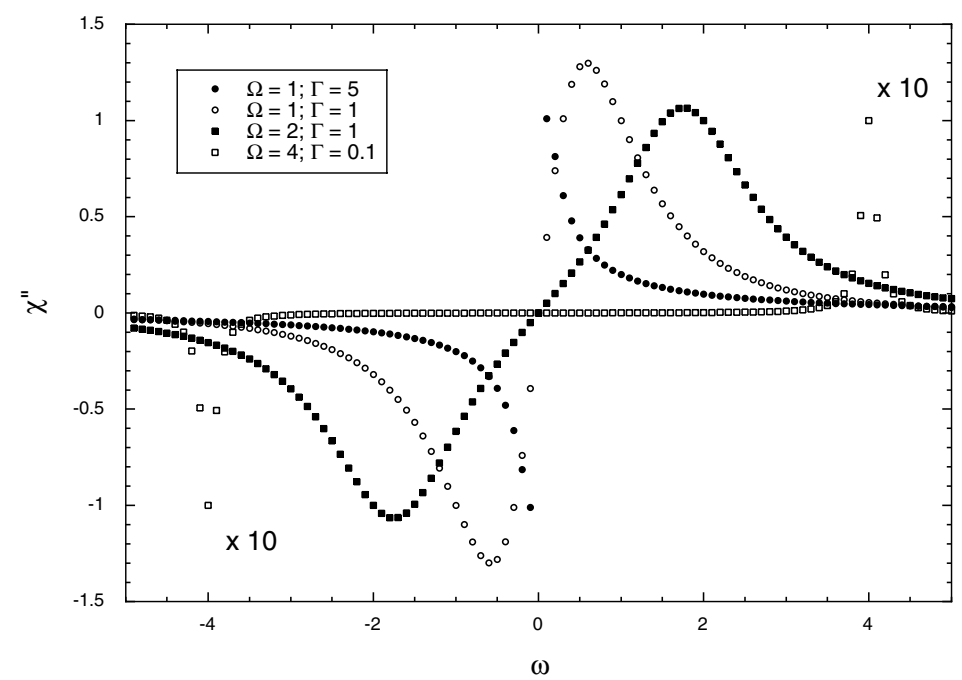

Figure 50. Susceptibilité pour l'oscillateur amorti selon (11.106). Pour $\Gamma \gg \Omega$ l'oscillateur est suramorti. Pour $\Gamma \ll \Omega$ on obtient deux pics de forme lorenztienne.

La fonction de corrélation, qui figure dans l'expression pour les fonctions de diffusion partielles, devient une fonction de corrélation des déplacements (10.5)

$$
\left\langle e^{-i \vec{Q} \cdot\left(\vec{R}_{j^{\prime}}^{0}+\vec{u}_{j^{\prime}}(t=0)\right)} e^{i \vec{Q} \cdot\left(\vec{R}_{j}^{0}+\vec{u}_{j}(t)\right)}\right\rangle=e^{-i \vec{Q} \cdot\left(\vec{R}_{j^{\prime}}^{0}-\vec{R}_{j}^{0}\right)}\left\langle e^{-i \vec{Q} \cdot \vec{u}_{j^{\prime}}(t=0)} e^{i \vec{Q} \cdot \vec{u}_{j}(t)}\right\rangle .
$$

Les propriétés algébriques des opérateurs de déplacement $\overrightarrow{\mathbf{u}}_{j}(t)$ nous permettent de transformer cette fonction dans l'approximation harmonique en (10.22)

$$
\left\langle e^{-i \vec{Q} \cdot \vec{u}_{j^{\prime}}} e^{i \vec{Q} \cdot \vec{u}_{j}(t)}\right\rangle=e^{-W_{j^{\prime}}(\vec{Q})} e^{-W_{j}(\vec{Q})} e^{\left\langle\left(\vec{Q} \cdot \vec{u}_{j^{\prime}}\right)\left(\vec{Q} \cdot \vec{u}_{j}(t)\right)\right\rangle},
$$

avec $W_{j}(\vec{Q})$ les facteurs de Debye-Waller.

Sous cette forme la moyenne statistique \langle\rangle s'est déplacée dans l'exposant. Par conséquent, cette expression se prête à un développement en série de la fonction de corrélation bi-linéaire en fonction de $\overrightarrow{\mathbf{u}}_{j}(t)$

$$
\aleph_{j^{\prime}, j}(\vec{Q}, t)=\left\langle\left(\vec{Q} \cdot \vec{u}_{j^{\prime}}\right)\left(\vec{Q} \cdot \vec{u}_{j}(t)\right)\right\rangle .
$$

Le premier terme dans ce développement décrit la diffusion élastique. Pour un cristal, la section cohérente élastique s'obtient comme (10.35)

$$
\left(\frac{d^{2} \sigma}{d \Omega d E_{f}}\right)_{\text {coh }}^{\mathrm{el}}=N_{\text {maille }} \frac{(2 \pi)^{3}}{V_{\text {maille }}}|F(\vec{Q})|^{2} \sum_{\vec{G}} \delta(\vec{Q}-\vec{G}) \delta(\hbar \omega)
$$

avec le facteur de forme (nucléaire) de la maille

$$
F(\vec{Q})=\sum_{d} \bar{b}_{d} \exp \left(-W_{d}(\vec{Q})\right) \exp (i \vec{Q} \cdot \vec{d})
$$

Le prochain terme dans le développement rend compte des contributions à une vibration (10.57)

$$
S_{\mathrm{coh}}^{1-\mathrm{ph}}(\vec{Q}, \omega)=\frac{1}{2} \sum_{i} \frac{\left|F_{i}(\vec{Q})\right|^{2}}{\omega_{i}}\left\{\left[\left(1+n\left(\omega_{i}\right)\right) \delta\left(\omega-\omega_{i}\right)\right]+\left[n\left(\omega_{i}\right) \delta\left(\omega+\omega_{i}\right)\right]\right\}
$$


avec

$$
\left|F_{i}(\vec{Q})\right|^{2}=\left|\sum_{\kappa} \frac{\bar{b}_{\kappa}}{\sqrt{m_{\kappa}}} \sum_{j \in\left\{j_{\kappa}\right\}} e^{-i \vec{Q} \cdot \vec{R}_{j}^{0}} e^{-W_{j}(\vec{Q})}\left(\vec{Q} \cdot \vec{e}_{i}(j)\right)\right|^{2}
$$

La fonction $F_{i}(\vec{Q})$ est le facteur de forme de la vibration $i$. Il détermine l'intensité avec laquelle le mode sera observé pour un transfert de $\vec{Q}$ donné. Pour un cristal, la symétrie de translation rajoute la conservation du moment cristallin (10.72)

$$
\begin{aligned}
S_{\text {coh }}^{1-\mathrm{ph}}(\vec{Q}, \omega)= & \frac{(2 \pi)^{3}}{2 V_{\text {maille }}} \sum_{\vec{G}} \sum_{i, \vec{k}} \frac{\left|F_{i}(\vec{Q} \mid \vec{k})\right|^{2}}{\omega_{i}(\vec{k})} \\
& \times\left[\left(1+n\left(\omega_{i}(\vec{k})\right)\right) \delta(\vec{k}-(\vec{Q}+\vec{G})) \delta\left(\omega-\omega_{i}(\vec{k})\right)\right. \\
& \left.+n\left(\omega_{i}(\vec{k})\right) \delta(\vec{k}+(\vec{Q}+\vec{G})) \delta\left(\omega+\omega_{i}(\vec{k})\right)\right]
\end{aligned}
$$

avec le facteur de forme du phonon

$$
\left|F_{i}(\vec{Q} \mid \vec{k})\right|^{2}=\left|\sum_{d=1}^{r} \frac{\overline{b_{d}}}{\sqrt{m_{d}}} e^{-W_{d}(\vec{Q})}\left(\vec{Q} \cdot \vec{e}_{i}(d \mid \vec{k})\right) e^{-i \vec{Q} \cdot \vec{d}}\right|
$$

La forme discrète de la réponse cohérente à un phonon permet la détermination des courbes de dispersion.

La diffusion incohérente pour un $\vec{Q}$ donné est par contre continue en fonction de $\omega$ même pour un cristal. Pour un système isotrope elle peut s'exprimer en fonction des densités d'états partielles (10.121)

$$
\left(\frac{d^{2} \sigma}{d \Omega d E_{f}}\right)_{\text {inc }}^{1-\mathrm{ph}(+)}=\frac{k_{f}}{k_{i}} \frac{Q^{2}}{3} \sum_{\kappa} \frac{\sigma_{\mathrm{inc}}(\kappa)}{8 \pi m_{\kappa}} \sum_{j \in\left\{j_{\kappa}\right\}} e^{-2 W_{j}(\vec{Q})} \frac{F_{j}(\omega)}{\omega}(1+n(\omega)), \quad \omega>0 .
$$

Pour un cristal (10.130)

$$
\left(\frac{d^{2} \sigma}{d \Omega d E_{f}}\right)_{\text {inc }}^{1-\mathrm{ph}}=\frac{k_{f}}{k_{i}} Q^{2} \frac{N}{8 \pi r} \sum_{d} \frac{\sigma_{\mathrm{inc}}(d)}{m_{d}} e^{-2 W_{k}(\vec{Q})} F_{d}(\omega) \frac{(1+n(\omega))}{\omega},
$$

Pour un système mono-atomique cette expression se simplifie. La diffusion incohérente à un phonon est directement determinée par la densité d'états vibrationelle $g(\omega)(10.131)$

$$
\left(\frac{d^{2} \sigma}{d \Omega d E_{f}}\right)_{\mathrm{inc}}^{1-\mathrm{ph}}=\frac{k_{f}}{k_{i}} Q^{2} \frac{N}{8 \pi} \frac{\sigma_{\mathrm{inc}}}{m} e^{-2 W_{d}(\vec{Q})} \frac{g(\omega)}{\omega}(1+n(\omega)) .
$$

La généralisation de cette formule à des systèmes polyatomiques est courante mais truffée de pièges. C'est particulièrement vrai si elle est, sous l'égide de l'approximation incohérente, appliquée à des diffuseurs cohérents. La prudence est de mise.

La réponse à un phonon est une bonne approximation de la diffusion inélastique pourvu que le déplacement des ions est petit à l'échelle de $Q^{-1}$. Au-delà de ce seuil, les contributions multiphonons deviennent importantes. Pour des systèmes incohérents mono-atomiques elles peuvent se calculer à partir de la densité d'états $g(\omega)$ en utilisant (11.69)

$$
S(\vec{Q}, \omega)=\frac{\sigma_{\text {inc }}}{4 \pi} N \exp (-2 W(Q)) \sum_{p=0}^{\infty} \frac{1}{p !}\left(\frac{\hbar Q^{2}}{2 m}\right)^{p} T_{p}(\omega)
$$


avec les termes à zéro et à un phonon

$$
\begin{aligned}
T_{0}(\omega) & =\frac{1}{2 \pi \hbar} \int_{-\infty}^{\infty} d t e^{-i \omega t}=\frac{1}{\hbar} \delta(\omega)=\delta(\hbar \omega) \\
T_{1}(\omega) & =\frac{1}{2 \pi \hbar} \int_{-\infty}^{\infty} d t e^{-i \omega t} f(t)=\frac{g(\omega)}{\hbar \omega}(n(\omega)+1) \\
& =\frac{g(\omega)}{2 \hbar \omega}\left(\operatorname{coth}\left(\frac{\hbar \omega}{2 k_{b} T}\right)+1\right)
\end{aligned}
$$

Cette prescription est souvent étendue aux systèmes polyatomiques et même cohérents. La prudence est de nouveau de mise.

Si le transfert en $Q$ est très élevé, les atomes réagissent aux collisions avec les neutrons en reculant. On est dans le régime de l'approximation d'impulsion (11.25)

$$
S(\vec{Q}, \omega)=\frac{1}{\sqrt{2 \pi \Delta^{2}}} \exp \left(-\frac{\left(\hbar \omega-E_{r}\right)^{2}}{2 \Delta^{2}}\right) .
$$

La réponse dynamique se résume à une gaussienne centrée sur l'énergie de recul

$$
E_{r}=\frac{\hbar^{2} Q^{2}}{2 m}
$$

avec une largeur proportionnelle à la fonction de Debye-Waller

$$
\Delta^{2}=2\left(\hbar \omega_{0}\right)^{2} W(\vec{Q})
$$

Il faut bien distinguer les effets multiphonons des effets anharmoniques. Les multiphonons sont présents dès que les déplacemens deviennent importants même si le potentiel est très harmonique. L'anharmonicité du potentiel influe sur tous les aspects de la réponse inélastique. Elle déplace notamment les fréquences des pics à un phonon et leur procure une largeur intrinsèque. Ces deux aspects sont captés par l'oscillateur anharmonique dont la susceptiblitité est donnée par (11.106)

$$
\chi_{(i, \vec{k})}^{\prime \prime}(\omega)=A(i, \vec{k}) \frac{4 \omega \Gamma(i, \vec{k})}{\left(\omega^{2}-\Omega(i, \vec{k})^{2}\right)^{2}+4 \omega^{2} \Gamma(i, \vec{k})^{2}} .
$$

\section{Remerciements}

Je voudrais remercier Stéphane Rols, Françoise Leclercq-Hugeux, Andrea Orecchini, Jérôme Combet, Mohamed Zbiri et Alexander Schober pour leur lecture critique de ce chapitre ainsi que Laurence Tellier qui a pris soin des corrections de français et de l'orthographe. Ce chapitre est dédié à Erwin Brunner, mon ancien professeur de mathématique.

\section{Références}

[1] J. Chadwick, Nature 129, 312 (1932).

[2] J. Chadwick, Proc. Roy. Soc. A 136, 692 (1932).

[3] Neutron Data Booklet, A-J. Dianoux and G. Lander (Eds.), Institut Laue-Langevin, Grenoble, France (2002).

[4] H. Schober, Neutron instrumentation in Neutron Applications in Earth, Energy and Environmental Sciences, pp. 37-104, L. Liang, R. Rinaldi and H. Schober (Eds.) Springer, New York (2008).

[5] P. Ageron, Nuclear Instr. and Meth. A 284, 197 (1989).

[6] A. Messiah, Méchanique Quantique, Dunod, Paris (1959).

[7] E. Balcar and S.W Lovesey, J. Phys. C: Solid State Phys. 194605 (1986). 
[8] O. Hittmair, Lehrbuch der Quantentheorie, Thiemig Verlag, München (1972).

[9] V.F. Sears, Neutron Optics, Oxford University Press, New York (1989).

[10] H. Rauch and S. Werner, Neutron Interferometry, Oxford University Press, Oxford (2000).

[11] S.K. Sinha, E.B. Sirota, S. Garoff, and H.B. Stanley, Phys. Rev. B 38, 2297 (1988).

[12] J. Daillant and A. Gibaud X ray and neutron reflectivity: Principles and Applications, (Springer Verlag, Berlin 1999).

[13] C. Cohen-Tannoudji , B. Diu, F. Laloë, Mécanique quantique - Tome 1 et Tome 2, Hermann (2007).

[14] Michel Le Bellac, Physique Quantique, deuxim̀e édition, EDP Science, 2007.

[15] S.W. Lovesey, Theory of Neutron Scattering from Condensed Matter, Vol. 1: Nuclear Scattering, Vol. 2: Polarization Effects and Magnetic Scattering, Oxford University Press, Oxford (1984).

[16] A. Frei, E. Gutsmiedl, C. Morkel, A.R. Müller, S. Paul, M. Urban, H. Schober, S. Rols, T. Unruh, and M. Hölzel, Phys. Rev. B 80, 064301 (2009).

[17] J.P. Pérez, Optique, Dunod, Paris (2004).

[18] L. Van Hove, Phys. Rev. 95, 249 (1954).

[19] J.H. Ferzinger and A. Leonard, Phys. Rev. 128, 2188 (1962).

[20] A. Rahman, K.S. Singwi, and A. Sjölander, Phys. Rev. 126, 986 (1962).

[21] P. Schofield, Phys. Rev. Lett 4, 239 (1960).

[22] P.A. Egelstaff, Adv. Phys. 11, 203 (1962).

[23] S.A. Egorov and J.L. Skinner, Chem. Phys. Lett. 293, 469 (1998).

[24] J. Jensen and A.R. Mackintosh, Rare Earth Magnetism: Structures and Excitations, Clarendon Press, Oxford (1991).

[25] A.C. Barnes, H.E. Fischer, and P.S. Salmon, J. Phys. IV France 111, 59, (2003).

[26] P.G. de Gennes, Physica 25, 825 (1959).

[27] H.R. Glyde, Excitations in Liquid and Solid Helium, Clarendon Press, Oxford (1994).

[28] G. Placzek, Phys. Rev. 86, 377 (1952).

[29] K. Protassov, Analyse statistique des données expérimentales, Collection Grenoble Science, Grenoble (2002).

[30] E. Farhi, V. Hugouvieux, M.R. Johnson, and W. Kob, J. Comp. Physics, 228, 5251 (2008).

[31] C.G. Shull, Phys. Rev. Lett. 21, 1585 (1968).

[32] V.F. Sears, Adv. Phys. 24, 1 (1975).

[33] B. Dorner, Journal of Neutron Research 13, 267 (2005).

[34] B. Dorner, Acta Cryst. A 28, 319 (1972).

[35] J. Felber, R. Gähler, R. Golub, and K. Prechtl, Physica B 252, 34 (1998).

[36] G.L. Squires, Introduction to the Theory of Thermal Neutron Scattering, Cambridge University Press, Cambridge (1978).

[37] P. Debye, Interferenz von Röntgenstrahlen und Wärmebewegung (in German), Ann. d. Phys. 348, 49 (1913).

[38] I. Waller, Zur Frage der Einwirkung der Wärmebewegung auf die Interferenz von Röntgenstrahlen (in German), Z. Physik A, Hadrons and Nuclei 17, 398 (1923).

[39] J.M. Carpenter and C.A. Pelizzari, Phys. Rev B 12, 2391 (1975).

[40] J.M. Carpenter and C.A. Pelizzari, Phys. Rev B 12, 2397 (1975).

[41] Götz Eckold, Symmetry Aspects of Excitations, Phonons in the International Tables of Crystallography, Vol. D, chapter 2.1 .

[42] J.M. Perez-Mato, M. Aroyo, J. Hlinka, M. Quilichini, and R Currat, Phys. Rev. Lett. 81, 2462 (1998).

[43] D. Strauch and B. Dorner, J. Phys. C: Solid State Phys. 19, 2853 (1986).

[44] J. Dawidowski, F. J. Bermejo, and J. R. Granada, Phys. Rev. B, 58, 706-715 (1998).

[45] MM. Koza, H. Schober, S.F. Parker, and J. Peters, Phys. Rev.B 77, 104306 (2008). 
[46] M.M. Bredov, B.A. Kotov, N.M. Okuneva, V.S. Oskotskii, and A.L. Shak-Budagov, Sov. Phys. Solid State 9, 214 (1967).

[47] V.S. Oskotskii, Sov. Phys. Solid State 9, 420 (1967).

[48] H. Schober, A. Tölle, B. Renker, R. Heid, and F. Gompf, Phys. Rev. B 56, 5937-5950 (1997).

[49] S.N. Taraskin and S. R. Elliott, Phys. Rev. B 55, 117-123 (1997).

[50] J.M.F. Gunn and M. Warner, Z. Physik B, Condensed Matter 56, 13 (1984).

[51] N.W. Ashcroft and N.D. Mermin, Physique des Solides, EDP Science (2002).

[52] A. Sjölander, Arkiv för Fysik 14, 315 (1958).

[53] W. Reichardt, Bericht des Forschungszentrums Karlsruhe (1984).

[54] H.R. Glyde and E.C. Svensson, in: Methods of Experimental Physics, Vol. 23, Part B, eds. D.L Price and K. Sköld, Academic Press, New York, (1987).

[55] B. Fåk and B. Dorner, Physica B 234-236, 1107 (1997).

[56] E.F. Talbot, H.R. Glyde, W.G. Stirling, and E.C. Svensson, Phys. Rev B 38, 11229 (1988). 